\title{
A Model Based Approach to System of Systems Risk Management
}

\author{
by
}

Andrew M.K. Kinder

\author{
A Doctoral Thesis \\ Submitted as partial fulfilment of the requirements \\ for the award of \\ Ph.D. of Loughborough University
}

$5^{\text {th }}$ November 2017

(C) by Andrew M. K. Kinder 2017 


\section{Abstract}

The failure of many System of Systems (SoS) enterprises can be attributed to the inappropriate application of traditional Systems Engineering (SE) processes within the SoS domain, because of the mistaken belief that a SoS can be regarded as a single large, or complex, system. SoS Engineering (SOSE) is a sub-discipline of SE; Risk Management and Modelling and Simulation (M\&S) are key areas within SoSE, both of which also lie within the traditional SE domain. Risk Management of SOS requires a different approach to that currently taken for individual systems; if risk is managed for each component system then it cannot be assumed that the aggregated affect will be to mitigate risk at the SoS level.

A literature review was undertaken examining three themes: (1) SoS Engineering (SoSE), (2) M\&S and (3) Risk.

Theme 1 of the literature provided insight into the activities comprising SoSE and its difference from traditional SE with risk management identified as a key activity.

The second theme discussed the application of M\&S to SoS, providing an output, which supported the identification of appropriate techniques and concluding that, the inherent complexity of a SoS required the use of M\&S in order to support SoSE activities.

Current risk management approaches were reviewed in theme 3 as well as the management of SoS risk. Although some specific examples of the management of SoS risk were found, no mature, general approach was identified, indicating a gap in current knowledge. However, it was noted most of these examples were underpinned by M\&S approaches.

It was therefore concluded a general approach SoS risk management utilising M\&S methods would be of benefit.

In order to fill the gap identified in current knowledge, this research proposed a new model based approach to Risk Management where risk identification was supported by a framework, which combined SoS system of interest dimensions with holistic risk types, where the resulting risks and contributing factors are captured in a causal network.

Analysis of the causal network using a model technique selection tool, developed as part of this research, allowed the causal network to be simplified through the replacement of groups of elements within the network by appropriate supporting models.

The Bayesian Belief Network (BBN) was identified as a suitable method to represent SoS risk. Supporting models run in Monte Carlo Simulations allowed data to be generated from which 
A Model Based Approach to System of Systems Risk Management

the risk BBNs could learn, thereby providing a more quantitative approach to SoS risk management. A method was developed which provided context to the BBN risk output through comparison with worst and best-case risk probabilities.

The model based approach to Risk Management was applied to two very different case studies: Close Air Support mission planning and the Wheat Supply Chain, UK National Food Security risks, demonstrating its effectiveness and adaptability.

The research established that the SoS Sol is essential for effective SoS risk identification and analysis of risk transfer, effective SoS modelling requires a range of techniques where suitability is determined by the problem context, the responsibility for SoS Risk Management is related to the overall SoS classification and the model based approach to SoS risk management was effective for both application case studies. 


\section{Acknowledgements}

Firstly I would like to thank my academic supervisors Mike Henshaw and Carys Siemieniuch who, from when I first tentatively enquired if they thought I was up to the challenge of a $\mathrm{PhD}$, have given nothing but encouragement and insightful advice for the duration of this six year journey. I must also include Stewart Robinson who kept me on track at our yearly reviews, providing advice and encouragement throughout.

Being part of the Engineering System of Systems group has been a privilege, seeing each of my peers achieve their PhDs has been a great motivator and all have provided support at various points in my research.

I undertook this research whilst also juggling a day job and thank the companies that supported and funded me throughout; ATEQ Consulting Ltd, JCSys Ltd and Lockheed Martin UK.

A lot has happened in these six years, my father Michael was desperate to see me graduate but was also battling serious illness and sadly passed away before I had managed to complete this thesis but he always told me he knew I would finish, so I am happy to prove him right. Also, never to see me complete were Oney, who always had faith in me, and my stepfather Chris, a lifelong academic who was still publishing in his eighties, which I found inspirational. Throughout these difficult times my mother Charlotte always asked how my studies were going and was sure, even when I wasn't, that I would complete them, so it was a pleasure to tell her that I had finally finished!

Combining the day job with research cut into many weekends and evenings for which, last but certainly not least, I wish to thank my amazing wife Fiona and twins Angus and Aimee who put up with this but always supported me; I promise to attend to that list of jobs now! 
A Model Based Approach to System of Systems Risk Management

\section{Key Words}

Risk Management, System of Systems, Model, Simulation, Modelling and Simulation, Systems Engineering, System of Systems Engineering, Bayesian 


\section{Table of Contents}

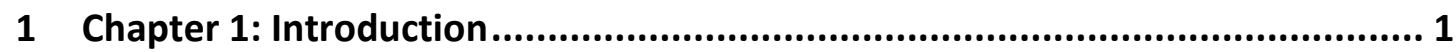

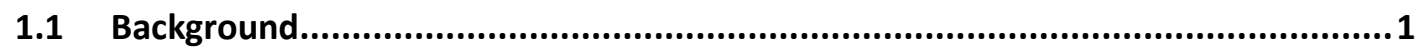

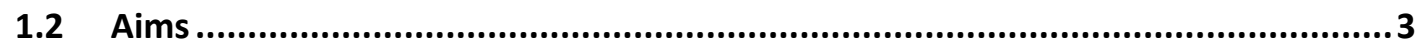

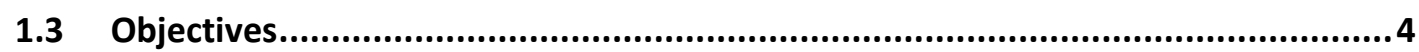

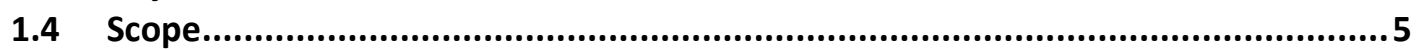

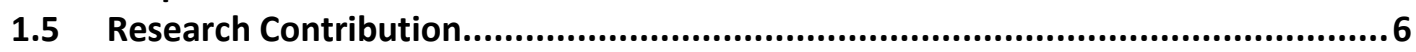

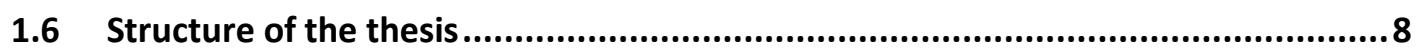

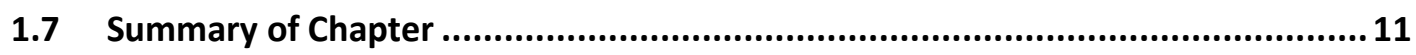

2 Chapter 2: Detailed Description of problem area ...................................... 12

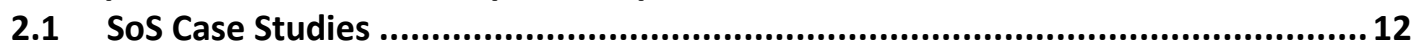

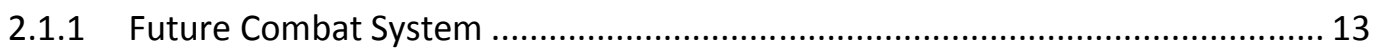

2.1.2 The National Programme for IT (NPfIT) in the NHS ...................................... 15

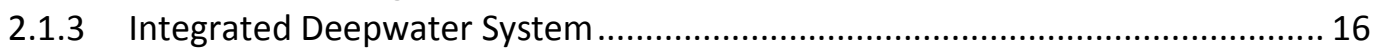

2.1.4 National Offender Management System (C-NOMIS) .................................... 17

2.1.5 Close Air Support: fatal friendly fire airstrike, Afghanistan 2014 ...................... 18

2.1.6 Hurricane Katrina Disaster Relief.............................................................. 19

2.1.7 Arizona and Southern California Power System .......................................... 21

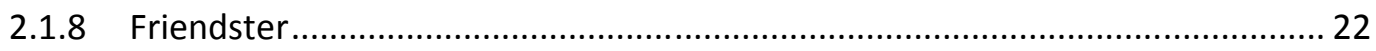

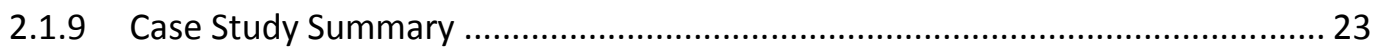

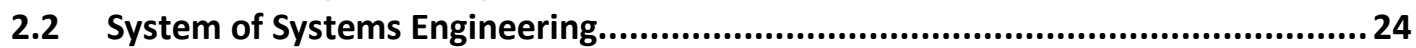

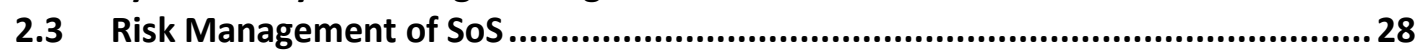

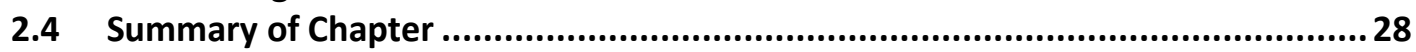

3 Chapter 3: Literature review................................................................. 30

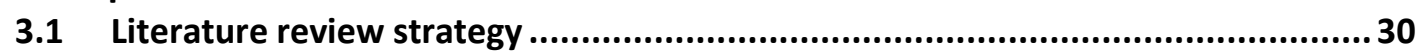

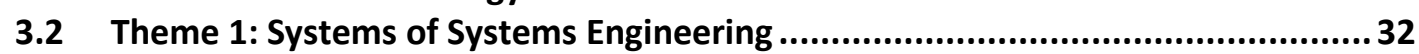

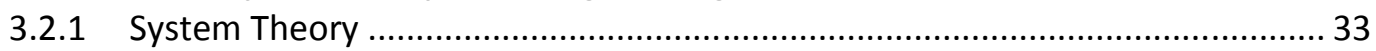

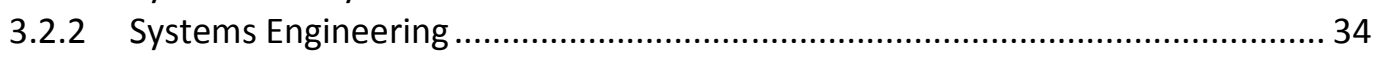

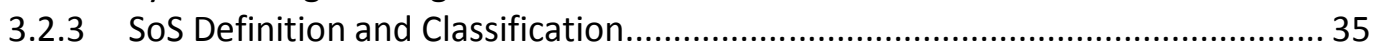

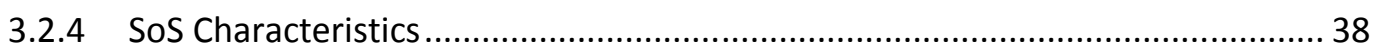

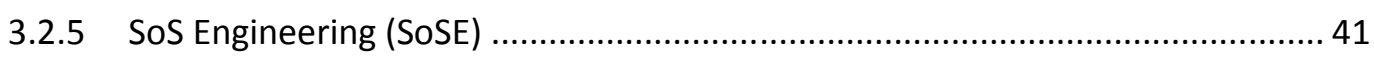

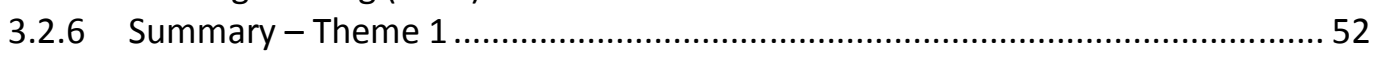

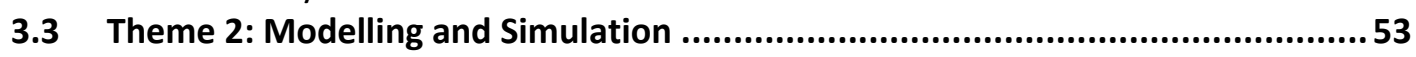

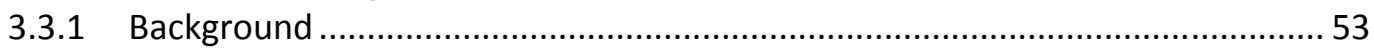

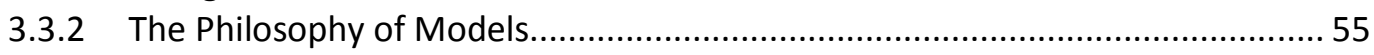

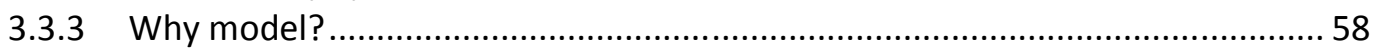

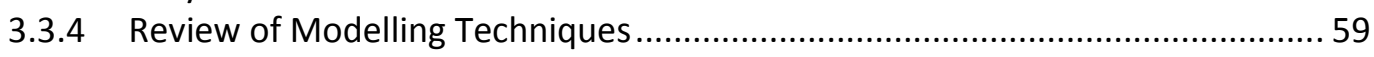

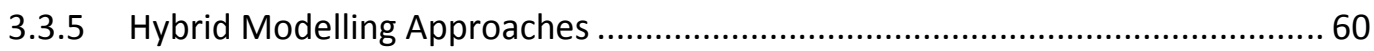

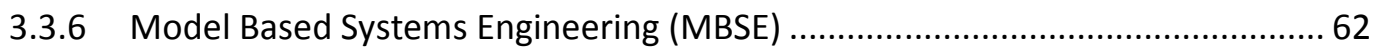

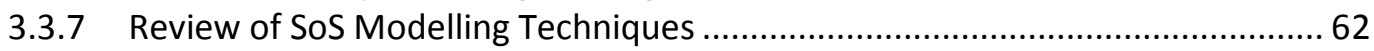

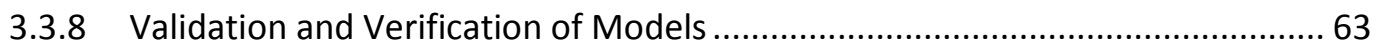

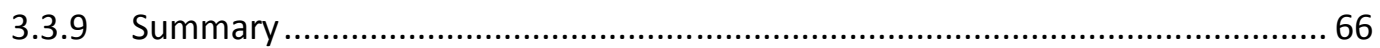

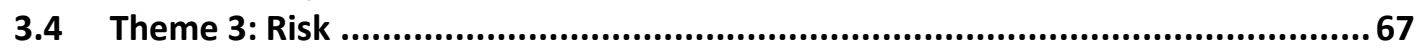

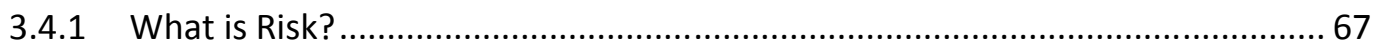

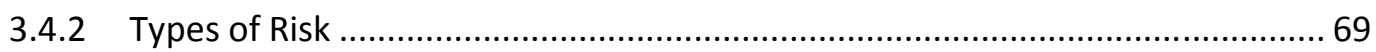

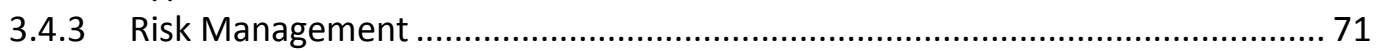

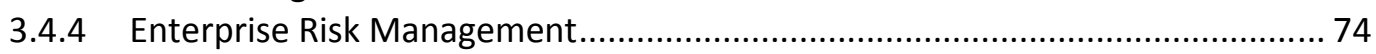




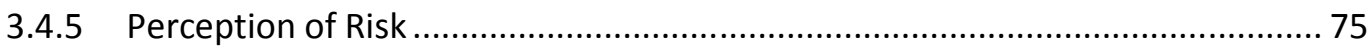

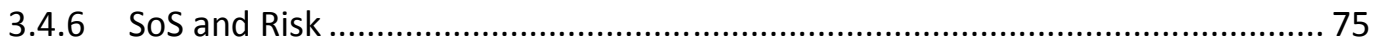

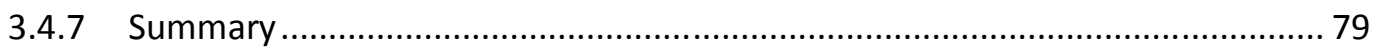

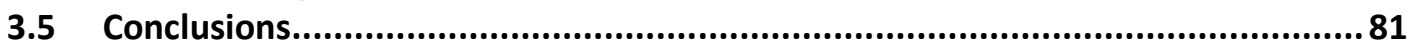

4 Chapter 4: Approaches to the research.................................................. 82

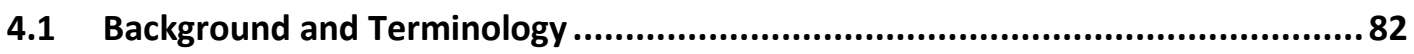

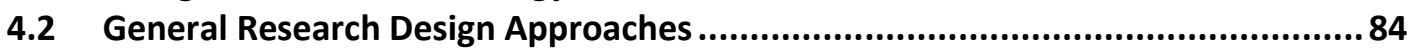

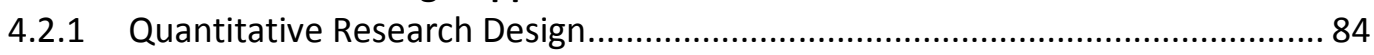

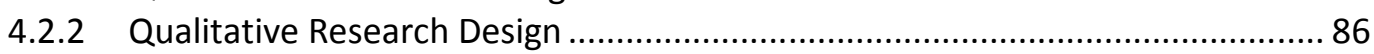

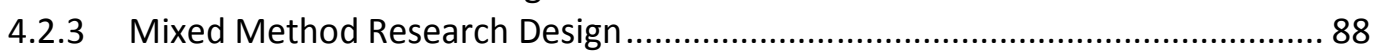

4.3 Taking a Systems Engineering Approach to Research.....................................89

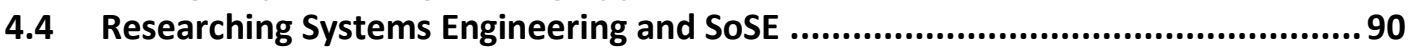

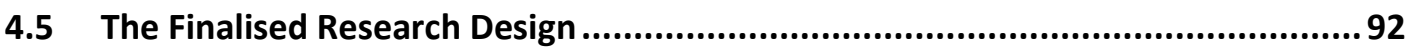

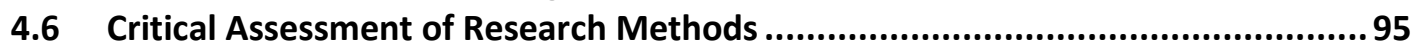

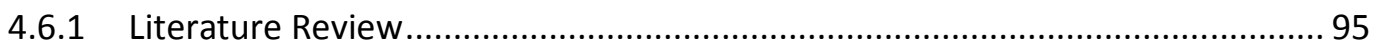

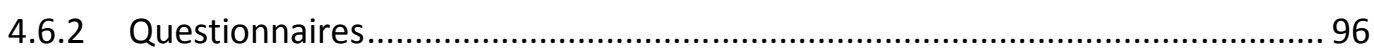

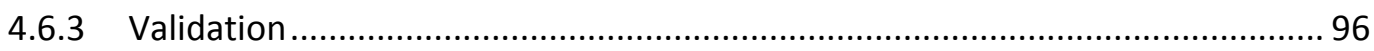

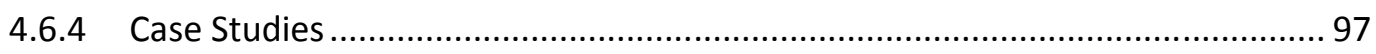

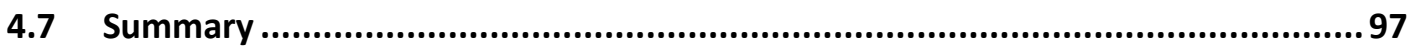

5 Chapter 5: Probabilistic Modelling............................................................. 99

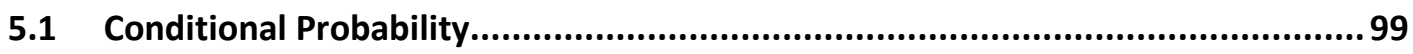

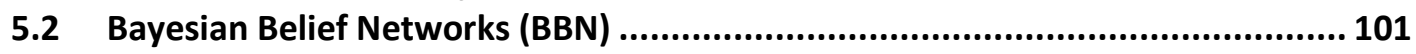

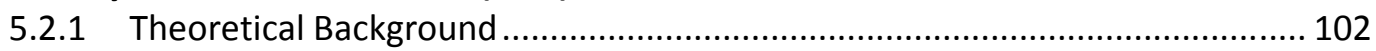

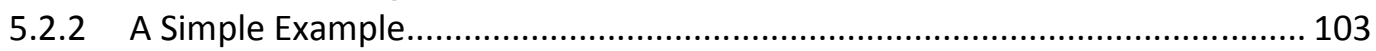

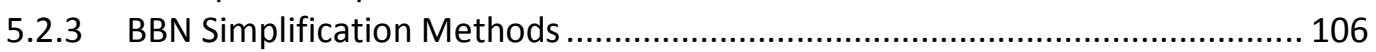

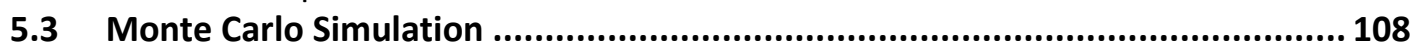

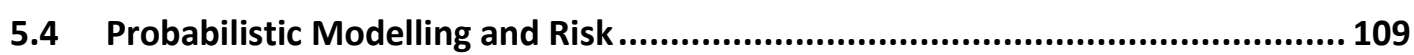

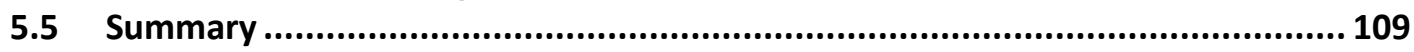

6 Chapter 6: A Method for the Selection of Appropriate SoS M\&S Techniques.110

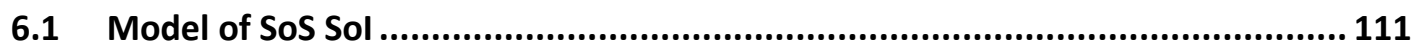

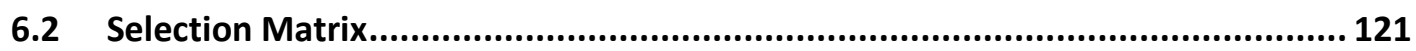

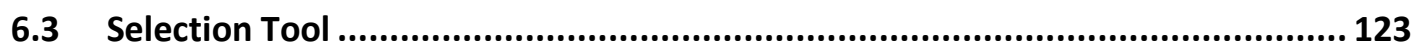

6.4 Future Development of the Spreadsheet based tool..................................... 127

6.5 Summary ..................................................................................................... 127

7 Chapter 7: SoS Risk Management ..........................................................129

7.1 Where does Risk Management fit in SoSE? ................................................. 130

7.2 Who performs SoS Risk Management? ............................................................. 131

7.3 Proposed SoS Risk Management Process ...................................................... 133

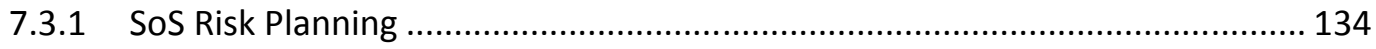

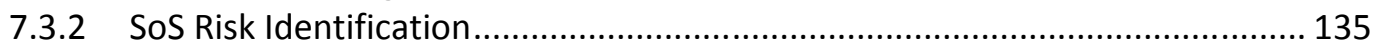

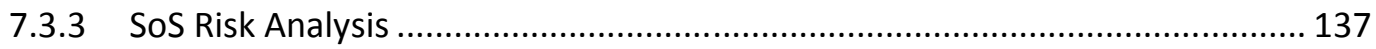

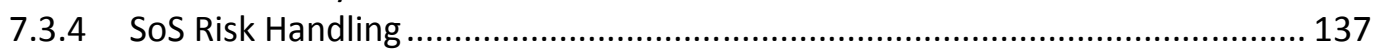

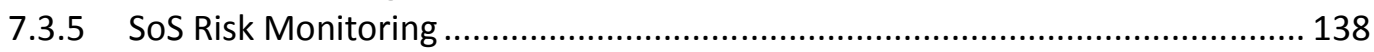

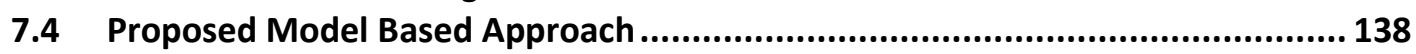

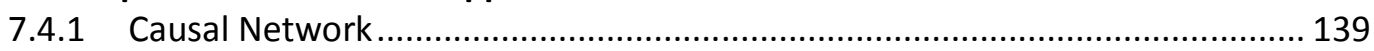

7.4.2 Central Bayesian Risk Model........................................................................ 140

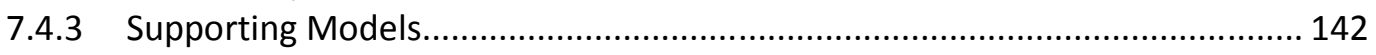

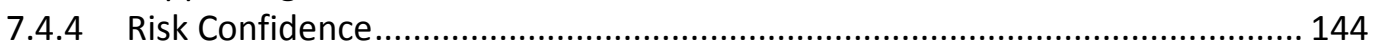

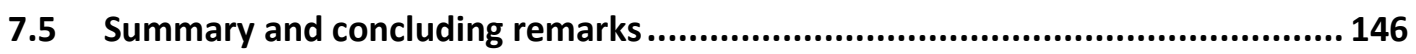


8 Chapter 8: Application 1: Close Air Support Mission Planning Risk Management 151

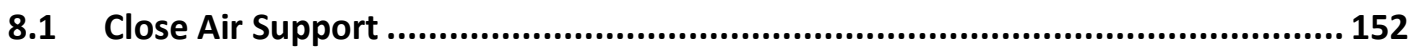

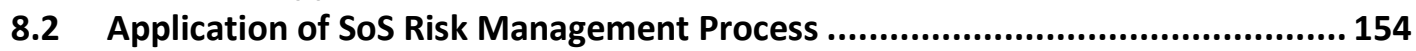

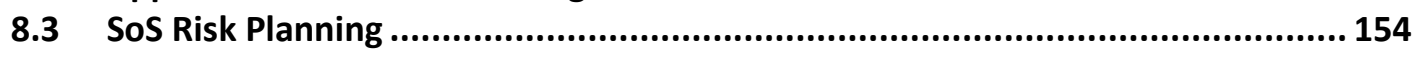

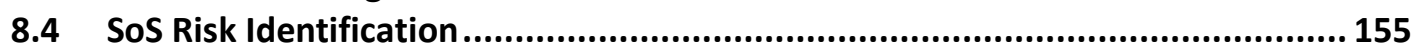

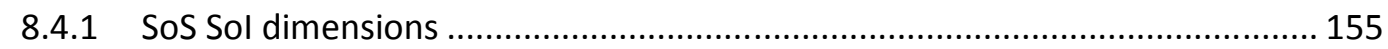

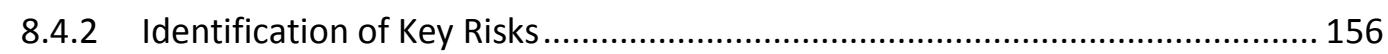

8.4.3 Identification of contributing factors....................................................... 159

8.4.4 Definition of causal network..................................................................... 161

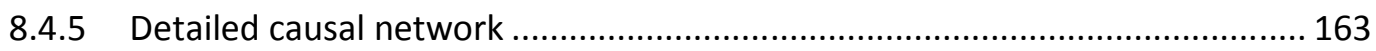

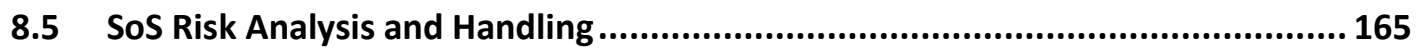

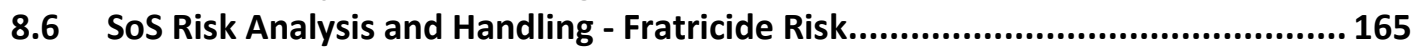

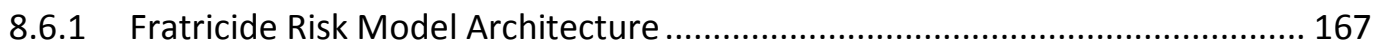

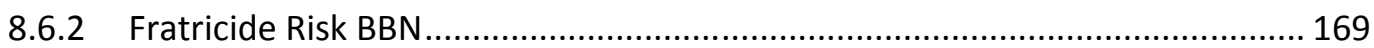

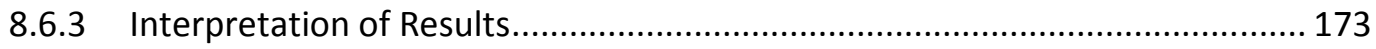

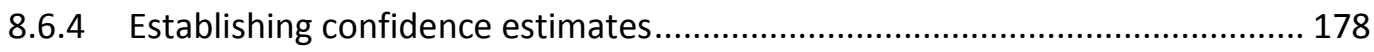

8.7 Analysis and Handling - Target Destruction Risk .......................................... 181

8.8 Analysis and Handling - Collateral Damage Risk ......................................... 182

8.9 Analysis and Handling - Additional Target Opportunity ................................. 182

8.10 Analysis and Handling - Loss of Aircraft Risk..............................................183

8.11 SoS Risk Analysis and Handling - Summary .............................................. 183

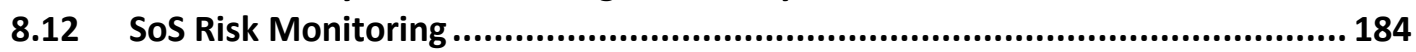

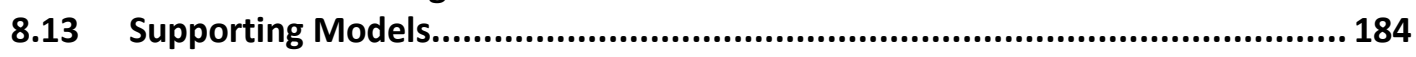

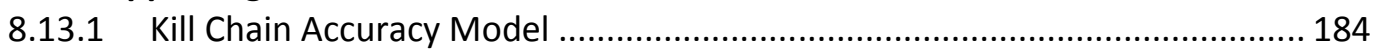

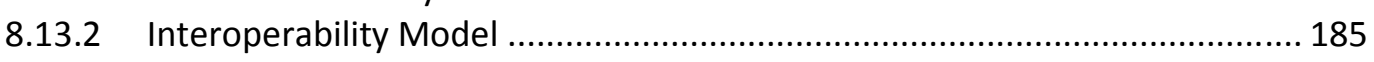

8.13.3 Blue Force Proximity and Civilian Proximity Model....................................... 186

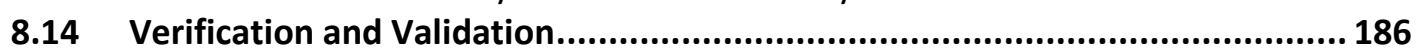

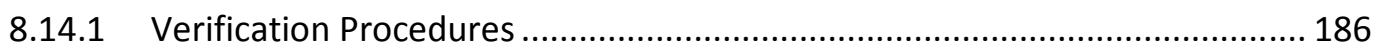

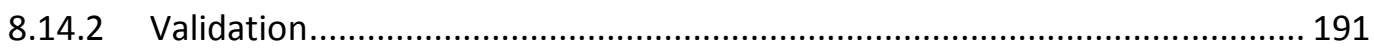

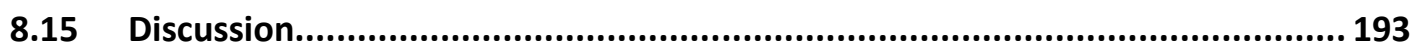

9 Chapter 9: Application 2: UK National Food Security - Wheat Supply Chain ..195

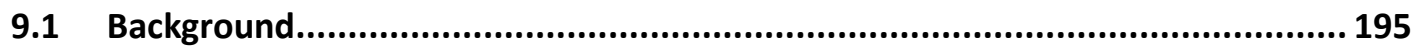

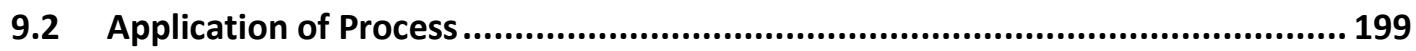

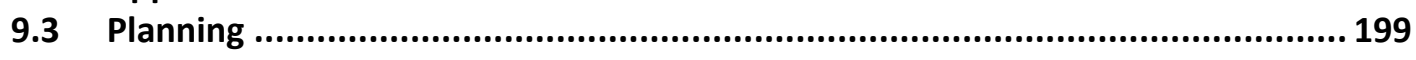

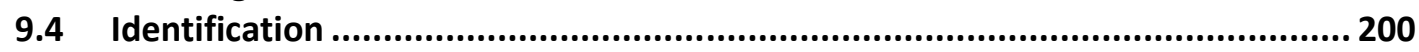

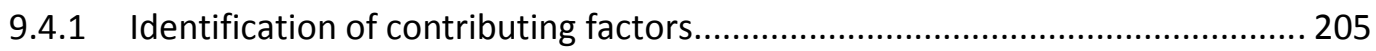

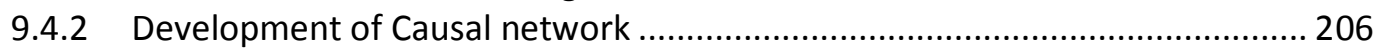

9.5 Analysis and Handling - Food (Wheat Based) Supply Shortage .......................... 208

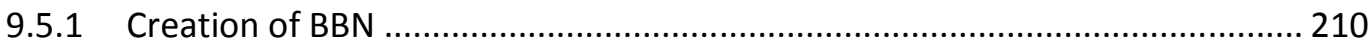

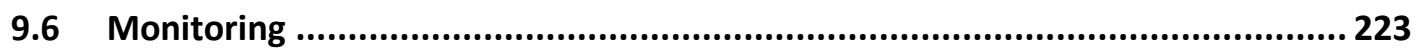

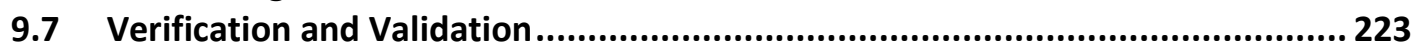

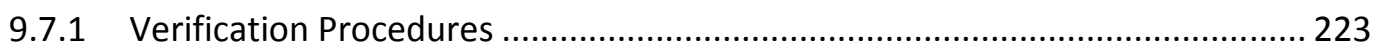

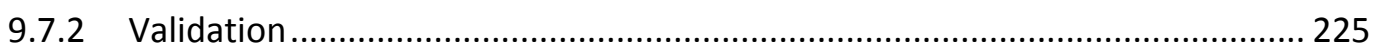

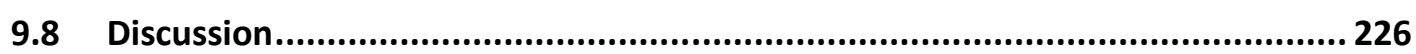

10 Chapter 10: Analysis of SoS Risk Management Approach ..........................227

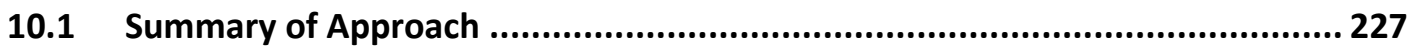

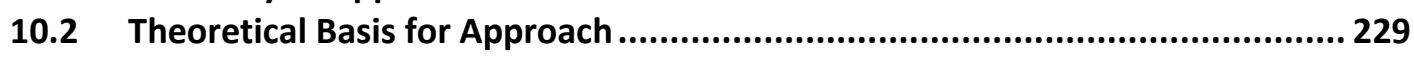

10.3 Critical Assessment of Risk Management Approach .................................. 231

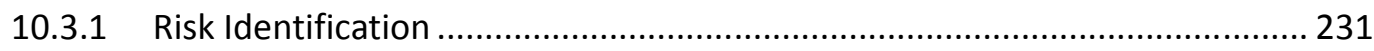


A Model Based Approach to System of Systems Risk Management

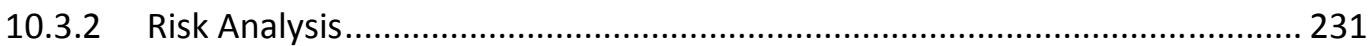

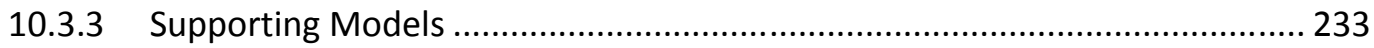

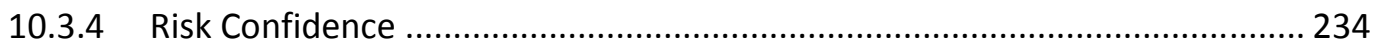

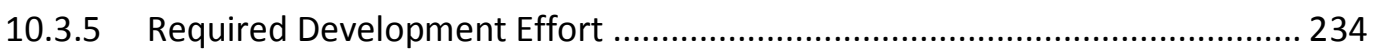

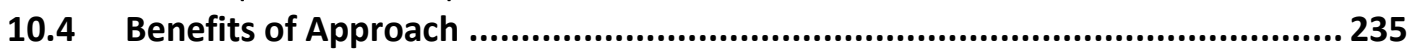

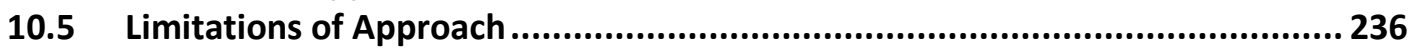

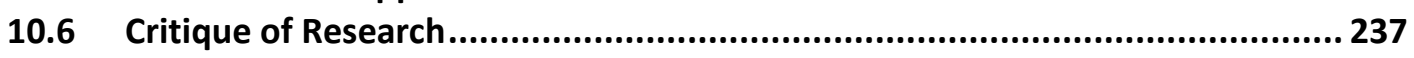

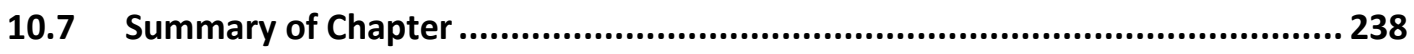

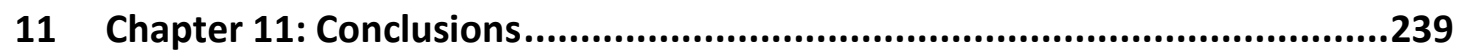

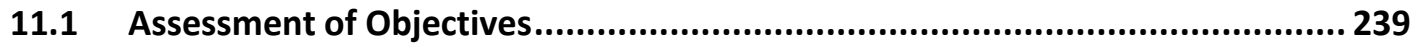

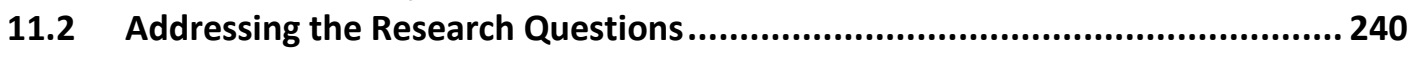

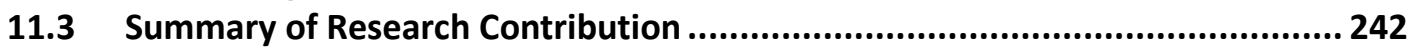

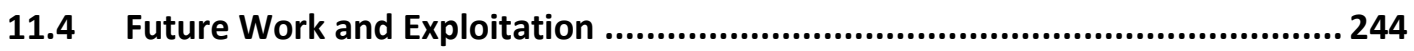

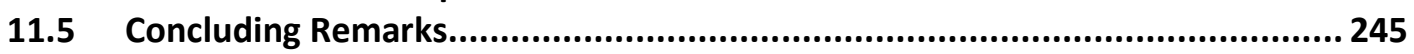

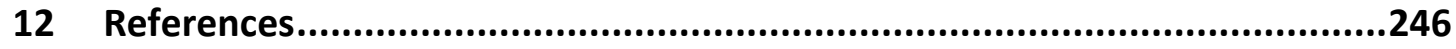

\section{Appendices}

Appendix A: SoS Sol Questionnaires

Appendix B: CAS Model Selection Matrix Tool Output

Appendix C: Application Case Study 1 Bayesian Belief Network Probability Tables

Appendix D: CAS Hypothetical Scenario Description

Appendix E: Further CAS Risk Analysis and Handling

Appendix F: Supporting Models

\section{Annexes}

Annex A: Paper 1 - System of Systems: "Defining the System of Interest"

Annex B: Paper 2 - System of Systems Modelling and Simulation - an Outlook and Open Issues

Annex C: Paper 3 - A Model Based Approach to System of Systems Risk Management

Annex D: System of Interest for System of Systems Dimensions: Workshop Report 


\section{Table of Tables}

Table 1 - Research Question to Objective Mapping............................................................... 5

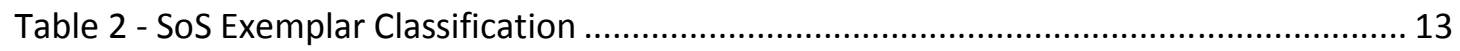

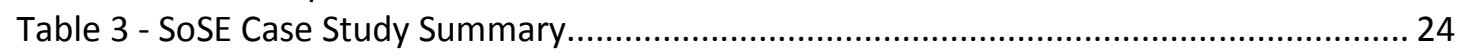

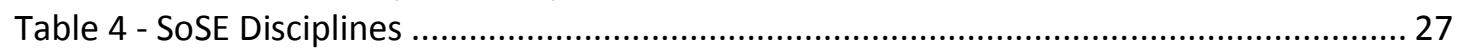

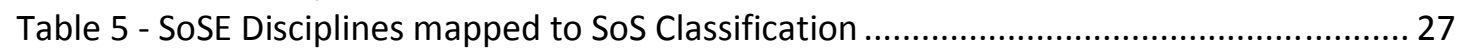

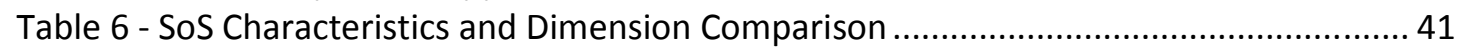

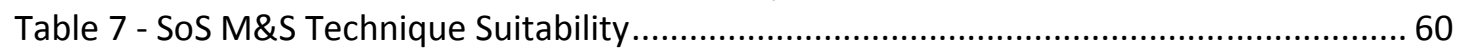

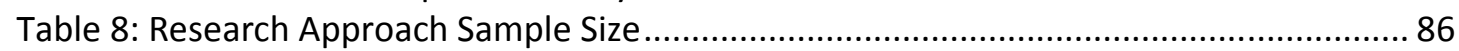

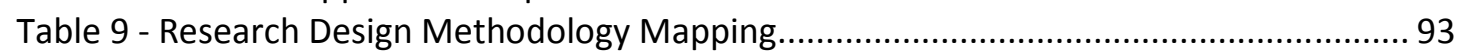

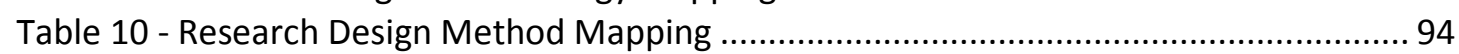

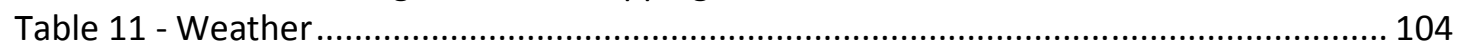

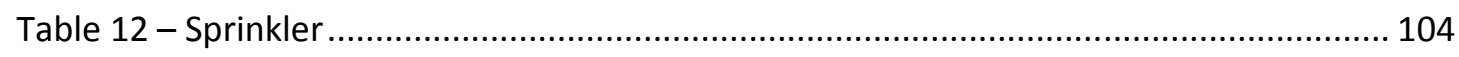

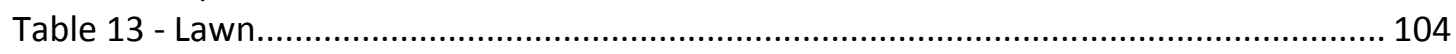

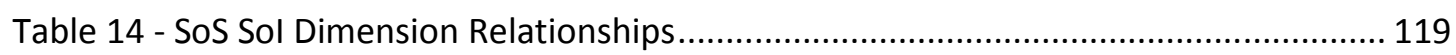

Table 15 - Case Study Applicability to SoS Sol Dimensions (Kinder et al., 2012) ................... 120

Table 16 - General M\&S attributes (Kinder et al., 2014)................................................... 123

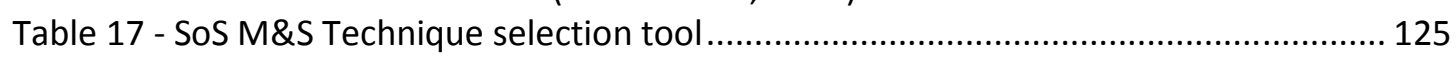

Table 18 - M\&S Method Stochastic and Uncertainty characteristics (Kinder et al., 2014)... 126

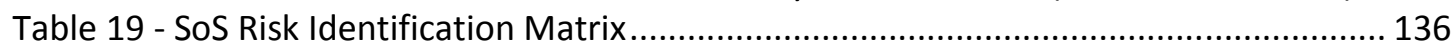

Table 20 - System and SoS Risk Management Comparison ............................................. 149

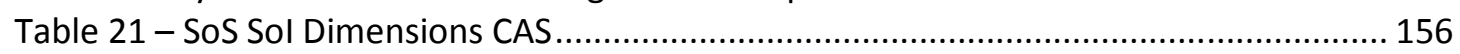

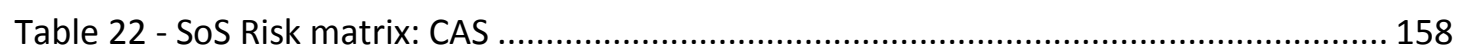

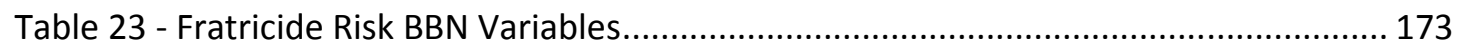

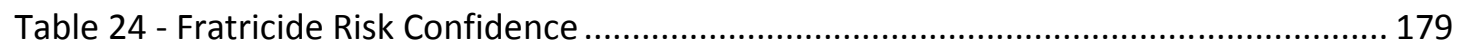

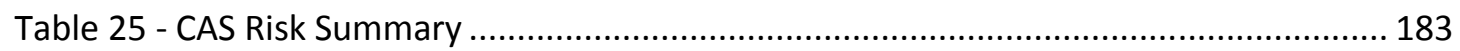

Table 26 - Verification Procedure - Fratricide Risk Identification....................................... 187

Table 27 - Verification Procedure - Fratricide Causal Network .......................................... 187

Table 28 - Verification Procedure - Fratricide BBN............................................................. 188

Table 29 - Verification Procedure - Supporting Models: Kill Chain Accuracy....................... 189

Table 30 - Verification Procedure - Supporting Models: Interoperability ............................ 189

Table 31 - Verification Procedure - Supporting Models: Blue Force and Civilian Proximity 190

Table 32 - Verification Procedure - Fratricide Risk Confidence BBN .................................. 191

Table 33 - Food Supply Chain Risk Summary (Diabat et al., 2012)...................................... 199

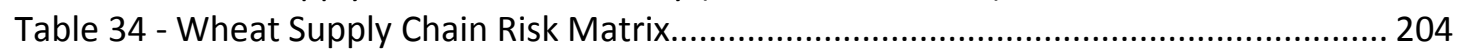

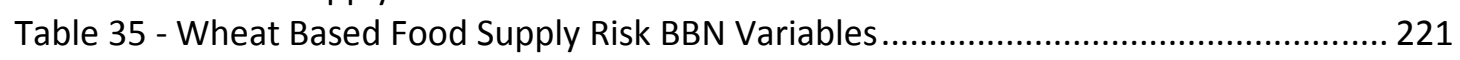

Table 36 - Verification Procedure - Food Shortage Risk Identification.................................... 223

Table 37 - Verification Procedure -Food Supply Risk Causal Network ................................. 224

Table 38 - Verification Procedure - Food Shortage BBN................................................... 224 


\section{Table of Figures}

Figure 1 - Combining Research Contributions to Address the Research Question .................. 7

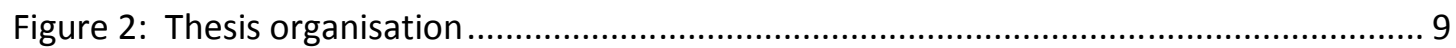

Figure 3 - Mapping Research Activities to Research Structure ....................................... 10

Figure 4 - Three-stage literature review process (Levy \& Ellis, 2006) ..................................... 30

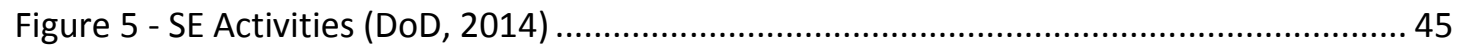

Figure 6 - SoSE mapping (US Department of Defense, 2008b) ........................................ 45

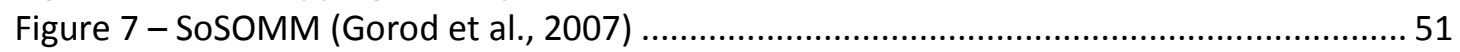

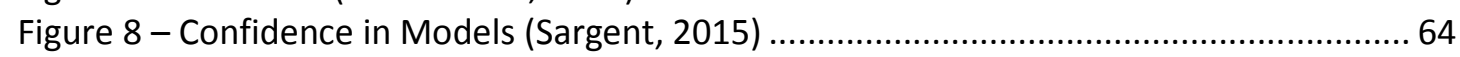

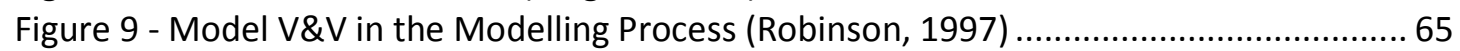

Figure 10 - DoD Risk Management Process (DASDSE, 2015) ............................................. 73

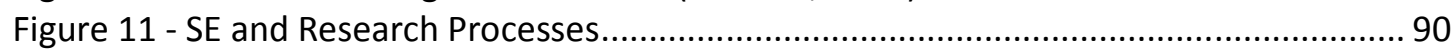

Figure 12 - Approach for the Development of the Rational Inductive Methodology (Sousa-

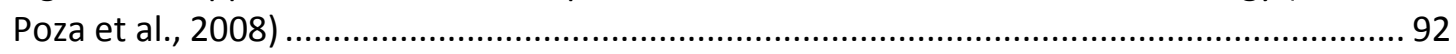

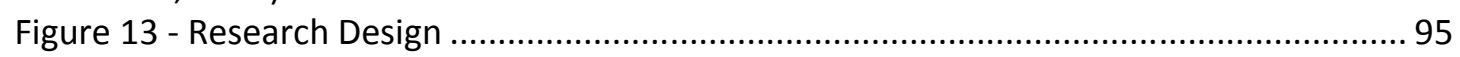

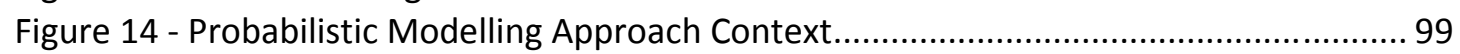

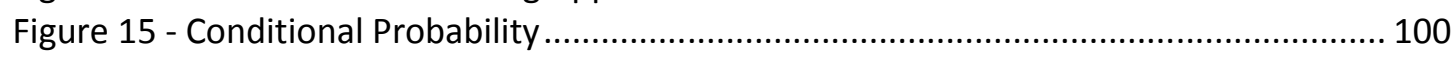

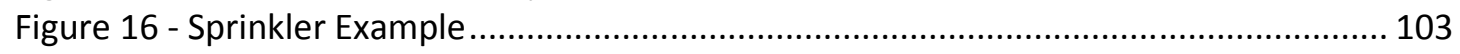

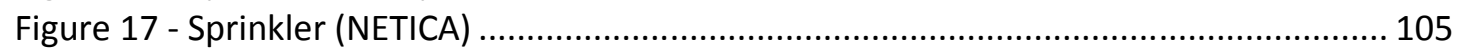

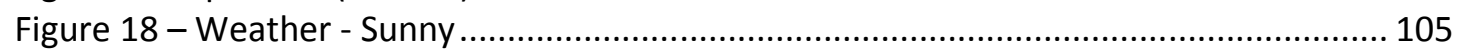

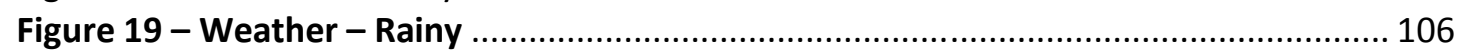

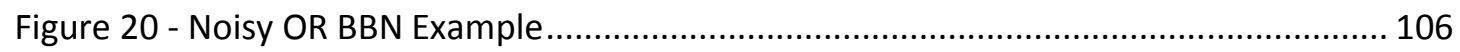

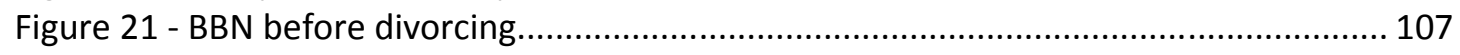

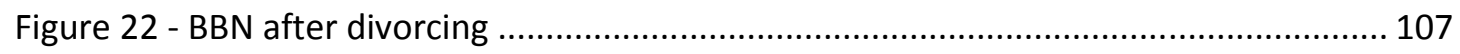

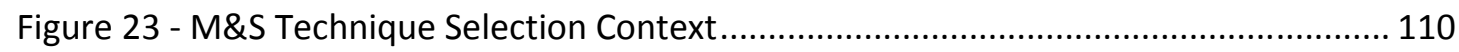

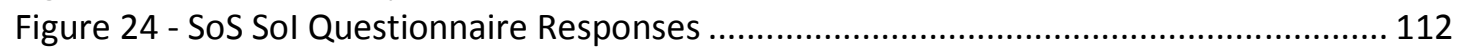

Figure 25 - SoS Sol Questionnaire Response Variance...................................................... 112

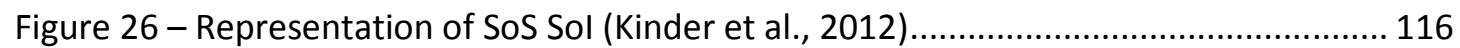

Figure 27 - SoS Risk Management Context..................................................................... 129

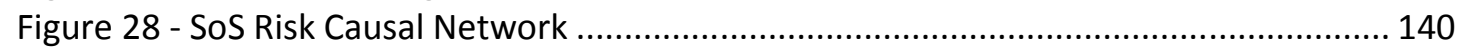

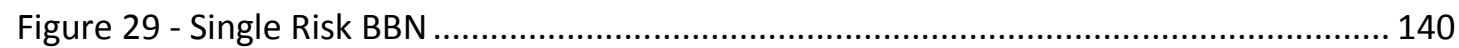

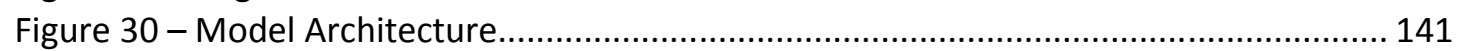

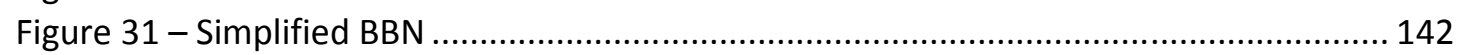

Figure 32 - Supporting Model - Formatted Output ......................................................... 143

Figure 33 - Supporting Model - Unformatted Output ......................................................... 144

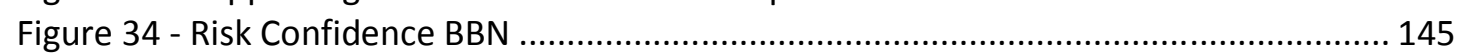

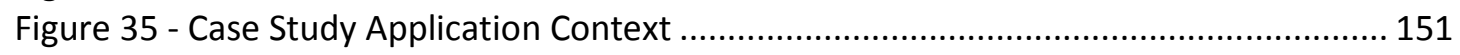

Figure 36 - Typical Close Air Support Scenario ............................................................. 153

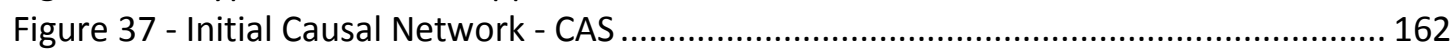

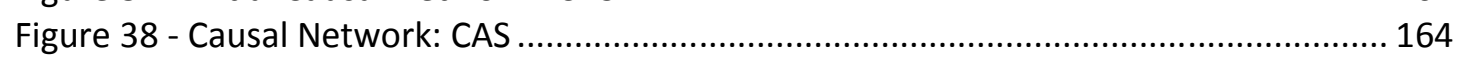

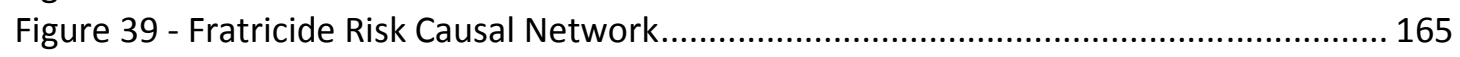

Figure 40 - Fratricide Risk - Model Architecture …............................................................. 168

Figure 41 - Fratricide Risk - Simplified Causal Network.................................................... 169

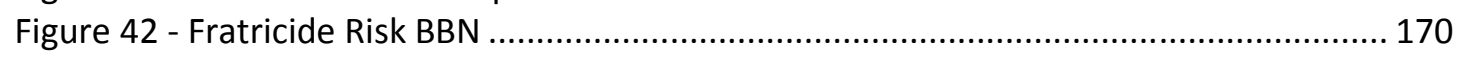

Figure 43 - Fratricide Risk BBN - Post Learning and Evidence Entry ................................ 174

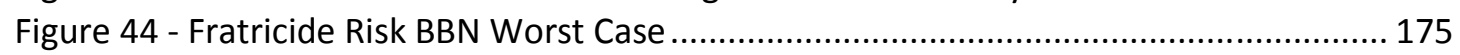

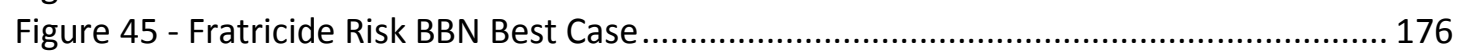

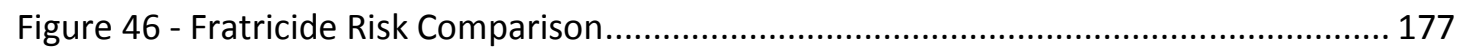

Figure 47 - Fratricide BBN with High Accuracy Kill Chain ................................................... 178 


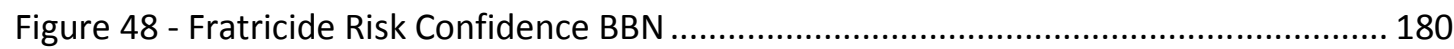

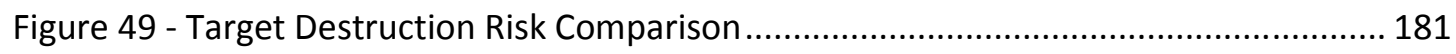

Figure 50 - Target Destruction BBN with High Accuracy Kill Chain ................................... 181

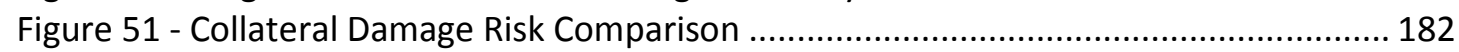

Figure 52 - Additional Target Opportunity Comparison................................................. 182

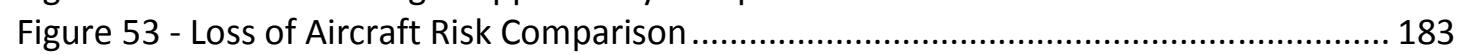

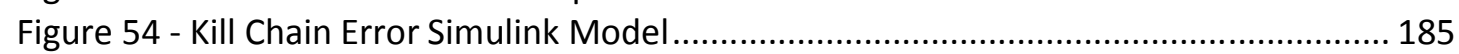

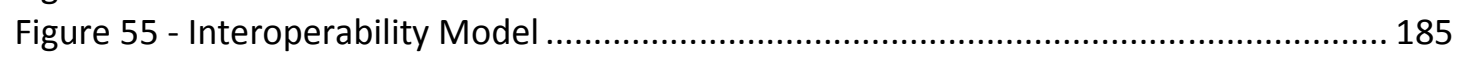

Figure 56 - Blue Force and Civilian Proximity Model..................................................... 186

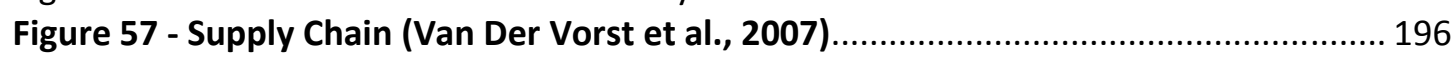

Figure 58 - Global Food System of Systems (Hipel et al., 2010) ........................................... 196

Figure 59 - Food (Wheat Based) Supply Chain - Initial Causal Network................................ 206

Figure 60 - Wheat Supply Chain Causal Network............................................................... 207

Figure 61 - Food (Wheat Based) Shortage Causal Network ............................................... 208

Figure 62 - Food (Wheat Based) Shortage - Model Architecture .......................................... 209

Figure 63 - Food (Wheat Based) Shortage - Simplified Causal Network ................................ 210

Figure 64 - Yield Model Technique Selection ........................................................................ 211

Figure 65 - Food (Wheat Based) Shortage Risk BBN Post Evidence Entry............................ 212

Figure 66 - Food (Wheat Based) Shortage Risk BBN - Worst Case ....................................... 213

Figure 67 - Food (Wheat Based) Shortage Risk BBN - Best Case ........................................ 214

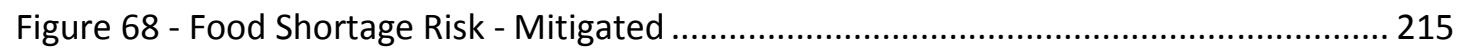

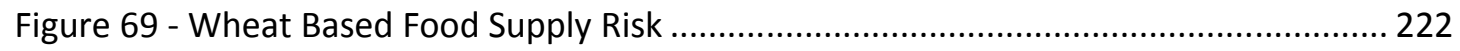

Figure 70 - Wheat Based Food Supply Risk with Infrastructure Mitigation ........................... 222

Figure 71 - Model Based SoS Risk Management Summary .................................................. 227 


\section{Glossary}

\begin{tabular}{|c|c|}
\hline Term & Definition \\
\hline ABM & Agent Based Model \\
\hline ABMS & Agent Based Modelling and Simulation \\
\hline ASTOR & Airborne Stand-off Radar \\
\hline ATO & Air Tasking Order \\
\hline BBN & Bayesian Belief Network \\
\hline C-NOMIS & National Offender Management System \\
\hline CAS & Close Air Support \\
\hline CML & COMPASS Modelling Language \\
\hline COMPASS & Comprehensive Modelling and Analysis for Advanced Systems of Systems \\
\hline coso & Committee of Sponsoring Organisations \\
\hline CREST & Centre for Renewable Energy Systems Technology \\
\hline CVaR & Conditional Value-at-Risk \\
\hline DANSE & Designing for adaptability and evolution in system of systems engineering \\
\hline DASDSE & Deputy Assistant Secretary of Defense Systems Engineering \\
\hline DEFRA & Department for Environment Food and Rural Affairs \\
\hline DES & Discrete Event Simulation \\
\hline DEVS & Discrete Event System Specification \\
\hline DoD & Department of Defence \\
\hline DODAF & DoD Architectural Framework \\
\hline ERM & Enterprise Risk Management \\
\hline ESoS & Engineering System of Systems \\
\hline FAC & Forward Air Controller \\
\hline FCS & Future Combat System \\
\hline FERC & Federal Energy Regulatory Commission \\
\hline GCSL & Goal Contracts Specification Language \\
\hline GP & General Practitioner \\
\hline $\mathrm{HQ}$ & Headquarters \\
\hline IDS & Integrated Deepwater System \\
\hline IEC & International Electrotechnical Commission \\
\hline IEEE & Institute of Electrical and Electronics Engineers \\
\hline
\end{tabular}


A Model Based Approach to System of Systems Risk Management

\begin{tabular}{|c|c|}
\hline INCOSE & International Council on Systems Engineering \\
\hline 10 & Interoperability \\
\hline IP & Internet Protocol \\
\hline iSMART & Interoperable Systems Management and Requirement Transformation \\
\hline ISO & International Organization for Standardization \\
\hline ISR & Intelligence, Surveillance and Reconnaissance \\
\hline IT & Information Technology \\
\hline JFAC & Joint Force Air Component \\
\hline JTAC & Joint Terminal Attack Controller \\
\hline JTRS & Joint Tactical Radio System \\
\hline LSI & Lead Systems Integrator \\
\hline M\&S & Modelling and Simulation \\
\hline MBSE & Model Based Systems Engineering \\
\hline MCDA & Multi-Criteria Decision Aid \\
\hline MDM & Multiple Domain Matrix \\
\hline MoD & Ministry of Defence \\
\hline MODAF & MoD Architectural Framework \\
\hline NATO & North Atlantic Treaty Organization \\
\hline NERC & North American Electric Reliability Corporation \\
\hline NHS & National Health Service \\
\hline NPfIT & National Programme for IT \\
\hline SA & Situational Awareness \\
\hline SAR & Search and Rescue \\
\hline SD & System Dynamics \\
\hline SE & Systems Engineering \\
\hline SEBoK & SE Body of Knowledge \\
\hline SIGINT & Signal Intelligence \\
\hline SME & Subject Matter Expert \\
\hline Sol & System of Interest \\
\hline SoS & System of Systems \\
\hline SoSCIE & SoS Engineering Collaborators Info Exchange \\
\hline SOSE & SoS Engineering \\
\hline SoSOMM & SoS Characteristics Operational Management Matrix \\
\hline SysML & Systems Modelling Language \\
\hline
\end{tabular}


A Model Based Approach to System of Systems Risk Management

TACC Tactical Air Control Centre

TDL Tactical Data Link

UAV Unmanned Air Vehicle

UK United Kingdom

US United States

V\&V Verification and Validation

VMF Variable Message Format

VSM Viable System Model 


\section{Chapter 1: Introduction}

This research has delivered a new model based risk management approach aimed specifically at System of Systems (SoS), where a gap in current knowledge has been identified; incorrect application of risk management has contributed to several high profile Sos failures.

This chapter provides the research motivation behind this $\mathrm{PhD}$ thesis and a brief description of the problem under consideration: this is followed by an outline of the aims and objectives for the research, planned research outputs, an overview of the research methodology used, the thesis and a summary of the research contribution made by this work carried out and reported on in this thesis.

\subsection{Background}

The Future Combat Systems (FCS) Program, launched in 2003, was the largest acquisition program in the US Army's history with estimated costs of $\$ 200$ (US) billion. On $23^{\text {rd }}$ June 2009 the US Undersecretary of Defence for Acquisition, Ashton Carter, cancelled the FCS programme, resulting in an estimated loss in the order of $\$ 1$ Billion (Axelband et al., 2012).

The Integrated Deepwater System (IDS) was a \$27 billion US Coastguard program that had the aim of combining several acquisition programs, to replace aging assets into a single, integrated program. In December 2011, Rear Admiral Jake Korn, assistant commandant for acquisition and chief acquisition officer published an article with the headline, "Deepwater R.I.P." (Korn, 2011) stating Deepwater was failing to support the modernisation of the US Coastguard capability and that no further work would be initiated under the contract.

The National Programme for IT (NPfIT), launched in 2002, was a top down approach to the introduction of a nation wide, integrated electronic patient care system. It was intended to connect 30,000 General Practitioners to 300 hospitals providing secure access to patient data by health professionals. However, in 2009 the UK Department of Health announced "that it was changing its approach to a more locally-led system allowing NHS organisations to introduce smaller, more manageable change in line with their local business requirements and capacity. The Department no longer intends to replace systems wholesale, and will instead in some instances build on trusts' existing systems" (National Audit Office, 2011). By 2012 all major contractors had either quit or been removed from the program and had incurred major financial losses. 
On June 92014 in Afghanistan, a B-1B Lancer bomber dropped its ordnance on five U.S. soldiers, including members of an elite Special Forces team; sadly all lost their lives. A U.S. military investigation found the incident was the result of poor communication, inadequate planning and "several other mistakes" (Lamothe, 2014).

Initiated in 2004 the C-NOMIS program was intended to provide a single capability to support offender management across both the Prison and Probation services through a single database. By 2007 the program was two years behind schedule and lifetime projected costs had risen from $f 234$ million to $f 690$ million (National Audit Office, 2009). In 2008 the program was restructured and was divided into five separate projects.

All the above are examples of high profile and high cost (both human and economic) System of Systems (SoS) failure, and hence either a failure of SoS Engineering (SoSE), or a failure to apply SoSE when appropriate. It is now widely accepted that aspects of the single System and the SoS are fundamentally different, requiring differing engineering approaches (Jamshidi, 2008), (Dahmann \& Baldwin, 2008). Definitions and more detailed discussion differentiating SoS and SOSE from traditional single system perspectives are provided in section 3.2 .

Jamshidi (2008) refers to SoSE as an "extension" of Systems Engineering; this infers that it is a sub-discipline rather than a discipline. The failure of many SoS enterprises can be attributed to the inappropriate application of traditional SE processes within the SoS domain because of the mistaken belief that a SoS can be regarded as a single large, or complex, system. Within this sub-discipline Modelling and Simulation (M\&S) and Risk Management are key areas, both of which also lie within the traditional Systems Engineering (SE) domain. This thesis is predicated on the premise that through modelling it is possible to predict SoS emergent behaviour; the greatest risk associated with a SoS is that this emergent behaviour will impact on the ability of the SoS to fulfil its purpose, with possible detrimental effects, although conversely, emergent behaviour may have a beneficial effect.

As discussed, a common theme throughout SoS failure is the lack of application of SoS Engineering (SOSE) approaches, particularly the failure to identify and manage SoS wide risks. Indeed, regarding the NPfIT and NOMIS examples, no reference to "System of Systems" at all is made in the reports produced by the National Audit Office (National Audit Office, 2011, 2012). 
A Model Based Approach to System of Systems Risk Management

Risk management typically focuses on a single system. For SoS, risk management must cross component system boundaries and take a holistic approach covering the entire SoS System of Interest (Creel \& Ellison, 2008).

This highlights two key problems:

1. SoSE, including SoS Risk Management, as an emerging discipline is immature and not clearly defined.

2. Traditional approaches to Systems Engineering and, in particular, Risk Management are still applied in the SoS domain, when these are not always adequate.

SoS Risk Management is a component of SOSE and could be applied to numerous aspects including SoS enterprise, physical SoS, development and acquisition. This research will focus upon the management of operational risk within a physical SoS, as this is a relatively unexplored area and the implicit requirement for rapid generation of risk assessments and management would inevitably require the use of an element of modelling and simulation. However, the results of this research could equally be applied to other areas, including acquisition.

\subsection{Aims}

The aim of this research was to establish the factors that differentiate Risk Management of SoS from "traditional" single systems and to develop a generic model based approach that would address these differences.

Hence, this research seeks to answer the following question:

How can risk be managed using a model based approach for a physical System of Systems in an operational environment?

This question raises the following lower level questions, which this research will seek to address:

1) How is the management of SoS risk different from managing risk at the single system level?

2) How is a model based approach beneficial?

3) How can a model based approach be developed and how can it quantify risk?

4) What are the types of risk associated with SoS?

5) How does the application of risk management relate to the SoS 'lifecycle'? 


\section{6) How can the impact of risk transfer be assessed within a SoS?}

Risks tend to be identified subjectively: using a formal model based approach the intent is to provide a more objective and realistic risk assessment. Risk mitigation approaches may be applied to a SoS model, enabling their effectiveness to be assessed. In the SoS domain, risks will not always be intuitive and, therefore, not identified. For an operational SoS, risk may arise rapidly, requiring responsive identification, assessment and mitigation: a tool providing this capability, based on the identified modelling techniques, will potentially be of great benefit.

\subsection{Objectives}

The fulfilment of the stated research aims will be accomplished through the completion of a set of objectives identified below. Table 1 provides a mapping between the research questions and the associated objectives.

\section{Objective 1: Review of the applicability of a wide range of modelling techniques to SoS}

This research is proposing a model-based approach to SoS Risk Management. To accomplish this a broad understanding of current modelling techniques is required and their applicability to modelling SoS: contributes to research sub questions 2 and 3.

\section{Objective 2: Review current risk management approaches}

A sound understanding of current system level Risk Management approaches is required before establishing a SoS approach: contributes to research sub questions 1 and 4 .

\section{Objective 3: Identify differences between system and SoS risk management}

With an understanding of system level Risk Management established, consideration will be given to how this differs from SoS Risk Management. This is accomplished through literature review and the use of case studies. Contributes to research sub questions 1, 4 and 5.

Objective 4: Identify an initial SoS application to explore whether a risk management model based approach would provide benefit

A practical application for the use of SoS Risk Management is identified: contributes to research sub questions 2 and 3.

Objective 5: Apply the SoS Risk Management process to the initial SoS application, developing models using appropriate identified modelling techniques. 
Having identified an application, appropriate modelling techniques are selected and the models implemented, verified and validated: contributes to research sub questions 2, 3 and 6.

Objective 6: Identify a second application (case study) in order to determine the adaptability of using the overall approach in a different domain

With an initial version of the approach developed a second case study is selected with the intention of adapting and developing models for this domain. The intention of this objective is to determine how adaptable the approach is, i.e. is each instance completely bespoke or are there common elements: contributes to research sub questions 2,3 and 6 .

\section{Objective 7: Use model to assess transfer of risk within a SoS}

The models underlying the approach as indicated under objectives 5 and 6 will be used to experiment with transferring risks between nodes in the model as a method to assess the impact of transfer of risk within a SoS: contributes to research sub question 6.

Objective 8: Define a SoS risk management methodology in which the approach can be encapsulated

The results of the previous objectives are analysed in order to determine if a general SoS risk management methodology can be derived, covering the Risk management process and the potential development of a support tool: contributes to research sub question 1.

The mapping of objectives to research questions is summarised in Table 1.

\begin{tabular}{|c|l|l|}
\hline \multicolumn{2}{|l|}{ Research Question } & Objective \\
\hline 1 & $\begin{array}{l}\text { How is this management of SoS risk different from managing risk at } \\
\text { the system level? }\end{array}$ & $2,3,7,8$ \\
\hline 2 & How is a model-based approach beneficial? & $1,4,5,6$ \\
\hline 3 & $\begin{array}{l}\text { How can a model-based approach be developed and how can it } \\
\text { quantify risk? }\end{array}$ & $1,4,5,6$ \\
\hline 4 & What are the types of risk associated with SoS? & 2,3 \\
\hline 5 & How do the types of risk relate to the SoS 'lifecycle'? & 3 \\
\hline 6 & How can impact of risk transfer be assessed within a SoS? & $5,6,7$ \\
\hline
\end{tabular}

Table 1 - Research Question to Objective Mapping

\subsection{Scope}

This research discusses SoS risk management throughout the lifecycle, although it should be noted that the proposed approach is focussed on the provision of support to development 
and planning activities: the development of a real time risk analysis and decision support capability is beyond the scope of this study.

The Close Air Support risk analysis, undertaken for case study 1 , utilised a set of models produced using heterogeneous applications; the resulting data was integrated through a manual process rather than automatically. Integration of all models into a single application would require a software development project in its own right and was not considered necessary in order to demonstrate the concept. In accordance with this general philosophy, the fidelity of each model was deemed to be sufficient to demonstrate the risk-modelling concept in order to minimise complexity and thereby reducing development effort to realistic levels. For the second case study, UK Food Security Risk Management, supporting models were identified but not developed because the intent was to determine the extensibility and adaptability of the proposed risk management approach beyond its application to a physical, operational SoS.

\subsection{Research Contribution}

This research has resulted in the following key contributions to knowledge:

- SoS, System of Interest (Sol) model

- SoS Risk Modelling Approach enabling the combination of outputs from heterogeneous models to provide an estimate of risk.

- SoS Risk Management Process

The SoS System of Interest Model underpins SoS Risk Modelling Approach technique selection and the SoS Risk Identification activities. It is described in section 6.1 and in an associated conference paper (Kinder et al,, 2012) which is attached in Annex A.

The proposed SoS Risk modelling approach uses a Monte Carlo simulation to run SoS heterogeneous models, the types of which are selected using the method described in chapter 6 . This generates probability distributions, which are then used to update a central Bayesian Belief Network, capturing the identified risks. At the 2014 Electronic, Electrical and Systems Engineering faculty PhD conference, positive feedback was received from members of the Centre for Renewable Energy Systems Technology (CREST), who also use BBN modelling, agreeing this can be considered as a unique approach in this context.

The management of risk in SoS is currently an immature discipline, requiring a holistic approach. However, current practice tends to assume that if all system level risk is managed 
then SoS risk will implicitly be managed, but this does not account for emergent behaviour at the SoS level. The research contributed to the understanding of SoS risk, through the development of a generic Risk Management process, described in chapter 7. This was achieved through the application of two case studies; development of a Close Air Support risk management tool (chapter 8) and risk analysis of UK Food Security from a SoS perspective (chapter 9).

A summary of the proposed risk management process, including the modelling approach, was presented at the $10^{\text {th }}$ annual SoSE conference in 2015 and published in an associated conference paper (Kinder et al., 2015) which is attached in Annex C.

The process was also presented, by invitation, to the INCOSE SoS Working Group and the Systems of Systems Engineering Collaborators Info Exchange (SOSCIE), which were well attended (the INCOSE webinar had over 50 attendees whilst the SOSCIE webinar had over 80) and received positive feedback (e.g.; "Good presentation and interesting application of a BN", “Worthwhile presentation. TKU”, "Interesting presentation. Thank you).

Figure 1 describes the relationship between the research components within the context of the proposed model based approach. This figure will be repeated and annotated where necessary throughout the thesis within chapters related to specific components, providing further context for the reader.

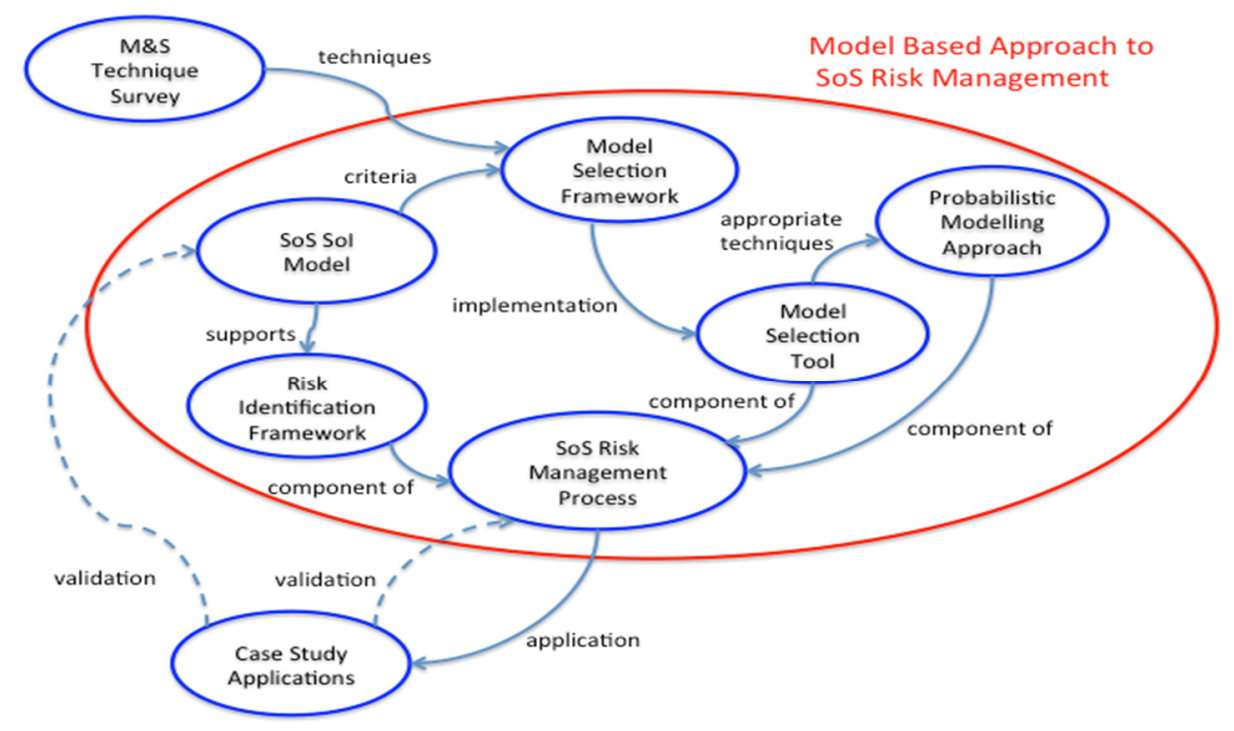

Figure 1 - Combining Research Contributions to Address the Research Question 
A Model Based Approach to System of Systems Risk Management

\subsection{Structure of the thesis}

Each chapter begins with an overview with its contents and concludes with a concise summary.

Chapter 1 introduces the thesis and provides necessary background information.

Chapter $\mathbf{2}$ describes the current State of the Art regarding Risk Management in the context of SoS, importance of this research, gaps, specific problems the research will alleviate and the research questions.

The literature review results, covering the three themes of, SoSE, Modelling and Simulation, and Risk Management, are documented in Chapter 3.

Chapter 4 describes the research methodological approach, methods used, the creation of SoS risk management method and process, illustrative case studies and the design approach. Chapter 5 provides theoretical background to the probabilistic modelling approaches, which underpin the model based SoS risk management approach.

Chapter 6 describes the development of a SoS System of Interest model, and provides a framework for a SoS modelling technique process.

The design approach, design and development of the SoS risk management method and process, are described in Chapter 7, with its application for Close Air Support and the UK Food Security prototype models documented in chapters 8 and 9 respectively.

Chapter 10 contains an overall analysis of the model-based approach, analysis of its effectiveness and adaptability, with any potential refinements or extensions identified as well as outlining the theoretical basis for the approach to SoS Risk and the combinations of models.

The research conclusions are documented in Chapter 11, analysing the achievement against the objectives, describing the contribution to knowledge and discussing potential for future exploitation and research.

The thesis structure is visualised below in Figure 2 (the lines are coloured for clarity only, the colour infers no other meaning). 


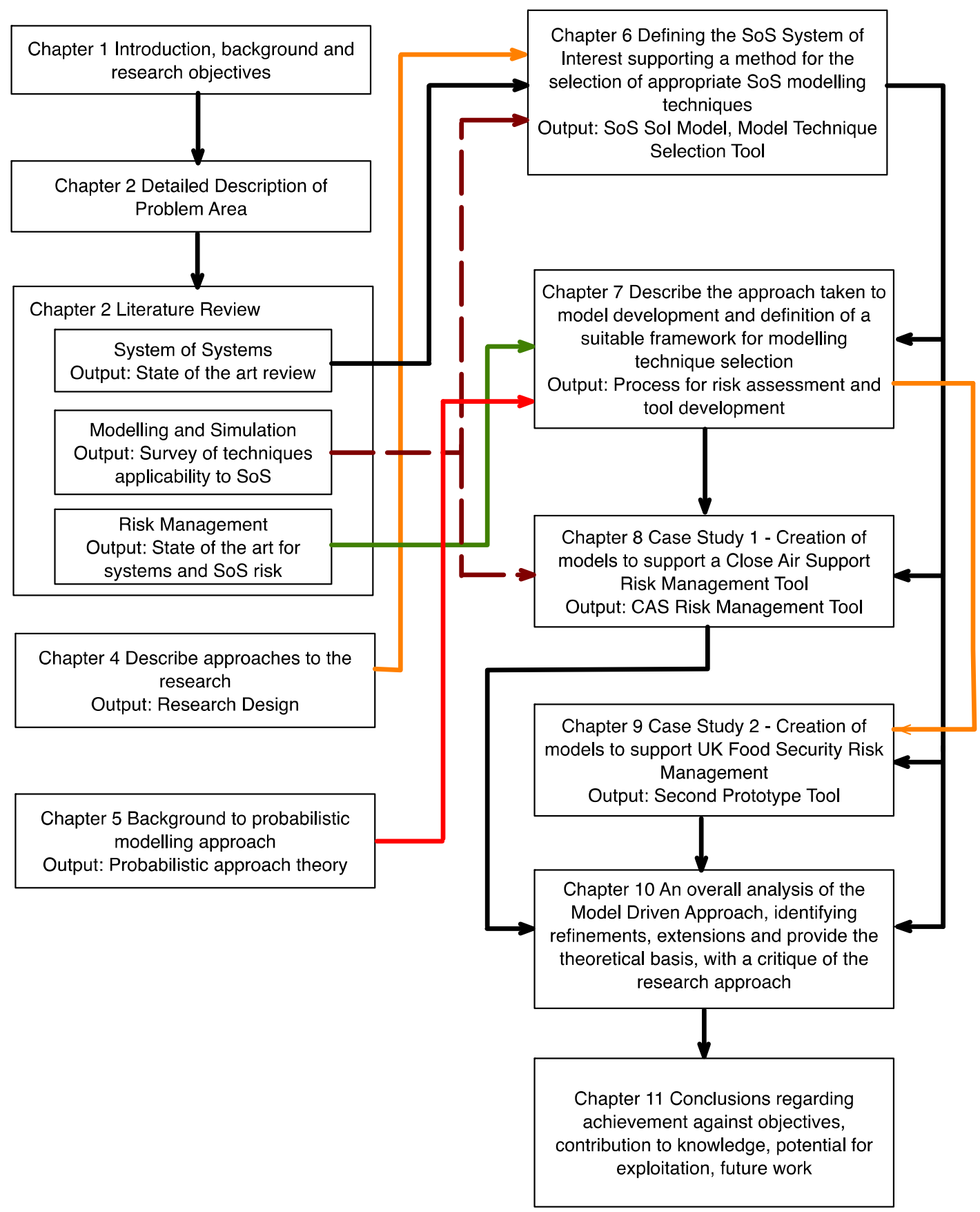

Figure 2: Thesis organisation

Figure 3 summarises the research design, indicating the relationships between research objectives, research outputs and the thesis structure, a detailed description of the research methodology is provided in Chapter 4. 


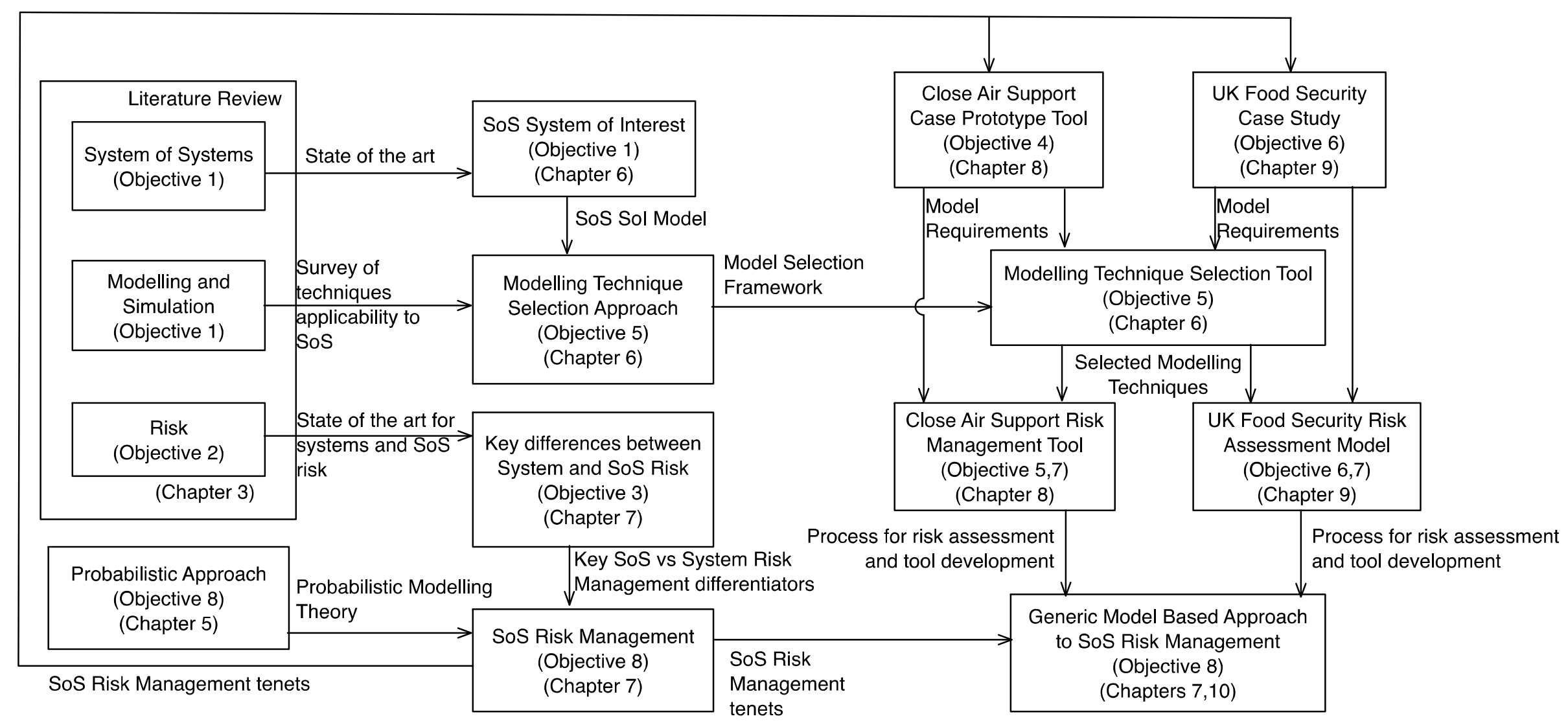

Figure 3 - Mapping Research Activities to Research Structure 
A Model Based Approach to System of Systems Risk Management

\subsection{Summary of Chapter}

The introductory chapter has provided an overview of the research background, identified the research aims and objectives, described the thesis structure and outlined the contribution to knowledge of the research. 


\section{Chapter 2: Detailed Description of problem area}

This chapter describes the current situation, regarding SoS, SoSE, modelling and simulation and risk management of systems and SoS. The potential impact of this research, gaps in current knowledge, specific problems the research will alleviate, and how research outcomes will address the gaps are also discussed.

\subsection{SoS Case Studies}

In order to demonstrate that this research does address a genuine problem the following examples are discussed, which highlight issues in the application, or indeed the lack of application, of SoSE.

- The Future Combat Systems Program (Axelband et al., 2012)

- The National Programme for IT (NPfIT) in the NHS (National Audit Office, 2011)

- Integrated Deepwater System Program (Government Accounting Office, 2011)

- National Offender Management System (C-NOMIS) (National Audit Office, 2009)

- Close Air Support, fatal friendly fire airstrike, Afghanistan 2014 (Lamothe, 2014)

- Hurricane Katrina Disaster Relief (Comfort, 2007)

- Arizona and Southern California Power Grid (FERC \& NERC, 2014)

- Friendster; one of the first and largest Online Social Networks (Garcia et al., 2013)

The exemplars listed above are chosen to ensure coverage of all types of SoS, it is anticipated that different aspects of SOSE may not be distributed evenly across all types; therefore this approach is intended to ensure that all these aspects are identified. The most widely accepted SoS classification (Dahmann \& Baldwin, 2008) identifies the following types (this is discussed further in section 3.2.3):

- Directed. Directed SoS are those in which the integrated system-of-systems is built and managed to fulfil specific purposes. It is centrally managed during long-term operation to continue to fulfil those purposes as well as any new ones the system owners might wish to address. The component systems maintain an ability to operate independently, but their normal operational mode is subordinated to the central managed purpose.

- Acknowledged. Acknowledged SoS have recognized objectives, a designated manager, and resources for the SoS; however, the constituent systems retain their independent ownership, objectives, funding, and development and sustainment 
approaches. Changes in the systems are based on collaboration between the SoS and the system.

- Collaborative. In collaborative SoS the component systems interact more or less voluntarily to fulfil agreed upon central purposes. The Internet is a collaborative system. The Internet Engineering Task Force works out standards but has no power to enforce them. The central players collectively decide how to provide or deny service, thereby providing some means of enforcing and maintaining standards.

- Virtual. Virtual SoS lack a central management authority and a centrally agreed upon purpose for the system-of-systems. Large-scale behaviour emerges-and may be desirable-but this type of SoS must rely upon relatively invisible mechanisms to maintain it.

Table 2 below identifies the classification of each exemplar, however it should be noted that a SoS may evolve, and therefore change type, or may simultaneously exhibit the behaviour of more than one type.

\begin{tabular}{|l|l|}
\hline SoS Case Study & SoS Classification \\
\hline The Future Combat Systems Program & $\begin{array}{l}\text { Directed; this was a centrally controlled } \\
\text { procurement programme }\end{array}$ \\
\hline $\begin{array}{l}\text { The National Programme for IT (NPfIT) in } \\
\text { the NHS }\end{array}$ & $\begin{array}{l}\text { Originally Directed but later moved towards } \\
\text { Acknowledged }\end{array}$ \\
\hline Integrated Deepwater System Program & $\begin{array}{l}\text { Directed, this was a centrally controlled } \\
\text { procurement programme }\end{array}$ \\
\hline $\begin{array}{l}\text { National Offender Management System } \\
\text { (C-NOMIS) }\end{array}$ & $\begin{array}{l}\text { Originally Directed but later moved towards } \\
\text { Acknowledged }\end{array}$ \\
\hline $\begin{array}{l}\text { Close Air Support, fatal friendly fire } \\
\text { airstrike, Afghanistan 2014 }\end{array}$ & $\begin{array}{l}\text { Acknowledged with elements Directed which } \\
\text { were under common command }\end{array}$ \\
\hline Hurricane Katrina Disaster Relief & $\begin{array}{l}\text { Collaborative initially but moving towards } \\
\text { Acknowledged as command and control was } \\
\text { established. However, within individual } \\
\text { agencies Acknowledged throughout relief and } \\
\text { recovery. }\end{array}$ \\
\hline $\begin{array}{l}\text { Arizona and Southern California Power } \\
\text { Grid }\end{array}$ & $\begin{array}{l}\text { Acknowledged within elements Directed } \\
\text { within individual regions }\end{array}$ \\
\hline Friendster & $\begin{array}{l}\text { Acknowledged with users forming Virtual } \\
\text { elements of the SoS }\end{array}$ \\
\hline
\end{tabular}

Table 2 - SoS Exemplar Classification

\subsubsection{Future Combat System}

The Future Combat Systems (FCS) Program, launched in 2003, was the largest acquisition program in the US Army's history with estimated costs of $\$ 200$ (US) billion. 
A Model Based Approach to System of Systems Risk Management

The program was cancelled in 2009, due to increasing costs, poor requirement definition, immature technology and unpredicted risks resulting in unacceptable development progress.

A key element of the project failure was the application of a System Engineering approach rather than SOSE which was more appropriate, as identified in a subsequent comprehensive project review (Axelband et al., 2012) stating:

"requirements at the system level rather than the system-of-systems level dominated the Operational Requirements Document, focusing design efforts at the system level and constraining critical trade space at the SoS level" and

"a Family of Systems Operational Requirements Document structure was used in lieu of SoS allocations."

The requirements were derived from war games (Army After Next and Objective Force) undertaken by the US Army based on fictional future scenarios, which were effectively predictions based on past events. This resulted in the requirement for lightweight vehicles that could be deployed rapidly in large numbers, as it was anticipated that most conflicts would involve combat between states.

The relatively low vehicle weight that was required put excessive constraints on the SoS component system design, eventually compromising the stated aim of the deployment of large numbers. However, with the conflicts in Iraq and Afghanistan, it became clear that the original concept was fundamentally inappropriate in modern conflicts, with smaller numbers of highly armoured vehicles required.

Another major contributor to the failure of FCS was the choice of communications medium. A key tenet of the FCS was that it would be highly networked providing high levels of situational awareness, thereby providing tactical advantage. However, this was based on immature technology, Joint Tactical Radio System (JTRS), and should have been identified as a SoS level risk. As development progressed the FCS design became dependent upon JTRS, which ultimately failed to reach the necessary maturity.

RAND (Axelband et al., 2012) make reference throughout the review to the lack of a mature SoS Risk Management approach, e.g.

"Traditional risk management has focused on a single system, and unfortunately there are no existing best practices for risk management tailored for the greater complexities of SoS acquisition." 
A Model Based Approach to System of Systems Risk Management

Disciplines required to enable effective SOSE in this case include:

- Requirements Management

- Project Management

- Risk Management

- Modelling and Simulation

\subsubsection{The National Programme for IT (NPfIT) in the NHS}

The NPfIT, launched in 2002, was a top down approach to the introduction of a nationwide, integrated electronic patient care system. It was intended to connect 30,000 GPs to 300 hospitals providing secure access to patient data by health professionals. The heart of the programme was the NHS Care Records Service (i.e. "the Spine"), making relevant parts of a patient's clinical record available NHS wide. Other component systems are now in use but operating independently.

However, in 2009 the Department of Health announced "that it was changing its approach to a more locally-led system allowing NHS organisations to introduce smaller, more manageable change in line with their local business requirements and capacity. The Department no longer intends to replace systems wholesale, and will instead in some instances build on trusts' existing systems." (National Audit Office, 2011).

By 2012 all major contractors had either quit or been removed from the program and had incurred major financial losses. It is widely conceded that the weight of program risk pushed onto the suppliers was excessive:

The architect of those deals, former NHS IT director-general Richard Granger, famously said he would "hold suppliers' feet to the fire until the smell of burning flesh is overpowering" (Glick, 2012).

Reasons for the project failure include, poor contract management, poor requirements elicitation and centralisation creating a single point of failure. Whilst requirements elicitation is generally a Systems Engineering centric activity an element is also required at the SoSE level, in cases where a capability is provided by the SoS emergent behaviour and not by an individual component system. It appears NPfIT failed at both levels. The failure of contract management in this case does suggest that it may be an essential element of SoSE and that a more "engineering" based approach may be suitable.

Disciplines required to enable effective SoSE in this case include: 
- Requirements Management

- Project Management

- Risk Management

- Contract Management

- Network Architecture

\subsubsection{Integrated Deepwater System}

The Integrated Deepwater System (IDS) was a US Coastguard program that intended to combine several acquisition programs, to replace aging assets, into a single, integrated SoS program.

The program was initiated in the late 1990's when the Coastguard determined that many of their deepwater ${ }^{1}$ assets would reach their end of life within several years of each other.

A private sector Lead Systems Integrator (LSI) was appointed to execute the SoS acquisition approach, responsible for designing, building and integrating the component systems.

By 2007 many of the component systems were running behind schedule and over budget, at which point the Coastguard abandoned the SoS acquisition approach and reverted to a traditional set of individual system acquisitions.

A report before the House of Representatives (Skinner, 2007) summarised the issues:

"We previously identified several common themes and risks in our audits of assets and information technology systems being acquired under the Deepwater contract. These include the dominant influence of expediency, unfavorable (sic) contract terms and conditions, poorly defined performance requirements, and inadequate management and technical oversight. These deficiencies contributed to schedule delays, cost increases, and asset designs that did not meet minimum Deepwater performance requirements."

In addition to this, there was also a lack of domain knowledge within the LSI, as raised by Richard L. Skinner, Inspector General, U.S. Department Of Homeland Security:

"According to the Coast Guard, its acquisition workforce did not have the requisite training, experience, and certification to manage an acquisition the size, scope, and complexity of the Deepwater Program".

An initial assessment of the evidence reviewed suggests that the LSI focused on the System Engineering of the individual component systems rather than concentrating efforts at the

\footnotetext{
${ }^{1}$ Deepwater is considered to be waters more than 50 miles from shore.
} 
A Model Based Approach to System of Systems Risk Management

SoS level. Combined with lack of appropriate domain knowledge, even with the benefit of hindsight, it seems inevitable that this project was destined to fail.

This is another example of a project that failed to distinguish the essential differences between Systems and SoS engineering.

Disciplines required to enable effective SOSE in this case include:

- Requirements Management

- Project Management

- Risk Management

- Modelling and Simulation

\subsubsection{National Offender Management System (C-NOMIS)}

Initiated in 2004 the C-NOMIS program was intended to provide a single capability to support offender management across both the Prison and Probation services through a single database.

By 2007 the program was two years behind schedule and lifetime projected costs had risen from $£ 234$ million to $£ 690$ million (National Audit Office, 2009).

In 2008 the program was restructured and was divided into five separate projects (National Audit Office, 2009):

- Replacement of several prison systems with the C-NOMIS application.

- Creation of a national probation case management system.

- Introduction of a read-only data share facility between the prison and probation services.

- The creation of a single offender risk assessment system.

- Replacement of the current prison Inmate Information System.

The public accounts committee identified several reasons for project failure (Public Accounts Committee, 2009).

- Lack of clear senior management and Ministerial ownership and leadership.

- There was little communication with stakeholders on project progress. With project plans failing to schedule engagement activities, many stakeholders were unsure how best to communicate with the project.

- Lack of skills and proven approach to project management and risk management. 
- Despite recognition that the project was high risk and had a challenging delivery schedule, there was no contingency within the budget, suggesting either a desire to keep costs down to achieve the go-ahead or a high degree of optimism.

- Lack of understanding of and contact with the supply industry at senior levels of the organisation.

- Lack of effective project team integration between clients, the supplier team and the supply chain.

Reasons for failure are similar to those encountered on the NPfIT project (2.1.2) and appear to be typical of a failed large scale IT project. Again, contract management is shown to be a problematic area.

Disciplines required to enable effective SoSE in this case include:

- Requirements Management

- Project Management

- Risk Management

- Contract Management

- Network Architecture

\subsubsection{Close Air Support: fatal friendly fire airstrike, Afghanistan 2014}

On June 92014 in Afghanistan, a B-1B Lancer bomber dropped its ordnance on five U.S. soldiers, including members of an elite Special Forces team; sadly all lost their lives. A U.S. military investigation found the incident was the result of poor communication, inadequate planning and several other mistakes (Lamothe, 2014).

The investigating officer stated; "The key members executing the close air support mission collectively failed to effectively execute the fundamentals, which resulted in poor situational awareness and improper target identification," and "While this complex combat situation presented a challenging set of circumstances, had the team understood their system's capabilities, executed standard tactics, techniques and procedures and communicated effectively, this tragic incident was avoidable."

The mission's purpose was to disrupt the Taliban thereby improving security in the Gaza Valley and Zabul's Arghandab district. However, the U.S. forces came under fire from insurgents whilst awaiting helicopter evacuation, at this point close air support was requested to neutralise the threat. The position of the troops under fire was not communicated effectively between the ground commander, joint terminal attack controller 
(JTAC) and the B-1B aircrew. Infrared markers were used to indicate the position of the troops requesting support, however it was not realised by any of these participants that the B-1B targeting system did not have the capability to detect these markers. The final source of confusion was the assumption that visible muzzle flashes indicated the insurgent position, when in fact it was the troops returning fire, which appeared to be corroborated by no detection of infrared markers.

The investigation revealed that, because of the stress and high tempo of the situation, decisions were not questioned at the time, the fatal error only became apparent following verbal radio communications immediately following the weapon strike.

Whilst the investigation apportioned blame through the command chain and stated that procedures were not adequately followed, this tragic situation can also be viewed as failure of a SoS, in this case due to degraded communications between component systems, poor situational awareness throughout the SoS and incorrect identification of a component systems capability.

Disciplines required to enable effective SoSE in this case include:

- Logistics Management

- Risk Management

- Network Architecture

- Command and Control

- Modelling and Simulation

\subsubsection{Hurricane Katrina Disaster Relief}

On August $23^{\text {rd }} 2005$ Doppler radar systems identified a tropical low-pressure system in the Caribbean, on the $24^{\text {th }}$ it was upgraded to a tropical storm, making landfall in south Florida on the $25^{\text {th }}$ the storm was now defined as a category 1 hurricane. Crossing the Gulf of Mexico and reaching Louisiana and Mississippi on the $27^{\text {th }}$ the hurricanes classification was raised to a category 3 (Comfort, 2007). The National Weather Service provided regular updates, which were provided to authorities from the local to federal level. However, the potential risk was not appreciated or acted upon at the time and co-ordination between agencies was limited. In the aftermath, different groups, including the coastguard, local emergency organisations and civilians, carried out rescue and clearance operations. However, these were largely uncoordinated because no overall authority was in control and none such had been planned in the event of a large-scale disaster. These disparate groups 
were also hampered by the lack of a common operating picture, resulting in a limited situational awareness (Townsend, 2006).

Many local state and local agencies capabilities were degraded, or even eliminated, as a result of the hurricane and the subsequent floods; for example numerous fire departments suffered "total destruction" of their facilities whilst others lost vehicles and stations. Additionally, the Orleans Parish Emergency Operations Centre was lost due to flooding, with the mayor of New Orleans being force to operate out of the upper floors of a hotel but without any communication capability for 48 hours. Indeed the entire communications network was disabled; flooding blocked access to the control centres, the " 911 " emergency system was inoperable and the backbone of the agencies radio system (the State of Louisiana's $800 \mathrm{MHz}$ radio system) ceased functioning. The Louisiana State Senator Robert Barham, chairman of the State Senate's homeland security committee, described the resulting situation; "People could not communicate. It got to the point that people were literally writing messages on paper, putting them in bottles and dropping them from helicopters to other people on the ground."

At this point a command structure could not be established which would have enabled the dissemination of information and co-ordination of the emergency agencies. Despite the lack of control and situational awareness a large-scale search and rescue (SAR) operation was undertaken by the Coast Guard, Urban Search and Rescue Task Forces, the Department of Defence and other Federal agencies. However, no plan was in place for the co-ordination of urban rescue (i.e. rescue from collapsed buildings) and traditional SAR, which included all other aspects. The lack of plan, along with the communications issues, caused co-ordination problems requiring agencies to determine their own priorities and work independently, i.e. conflicted Command and Control (Alberts, 2003).

Military and National Guard personnel were drafted in to assist with the SAR and relief operations, however the communications issues were exacerbated because the emergency responders, National Guard and active military used different equipment reducing interoperability. Initially military personnel were operating under separate command structures, which were organised geographically.

In order to rectify the communication issues the Federal Emergency Management Agency, the National Interagency Fire Centre and the Department of Defence began to provide communications assets including radios, satellite systems and command and control 
facilities. As communications were restored a more integrated command and control approach was established, providing co-ordinated Command and Control.

After a week the response had transitioned to a collaborative approach with an overall authority established providing full command and control of all remaining SAR and recovery activities.

Disciplines required to enable effective SoSE in this case include:

- Logistics Management

- Risk Management

- Network Architecture

- Command and Control

- Modelling and Simulation

\subsubsection{Arizona and Southern California Power System}

On the $8^{\text {th }}$ September 2011 an 11-minute disturbance in the Pacific Southwest power grid triggered a series of cascading failures throughout Arizona, Southern California, San Diego and Mexico, resulting in 2.7 million customers being without power for up to 12 hours. The power outage coincided with the start of rush hour, disrupting flights, transport, schools, businesses $^{2}$ as well as water treatment facilities resulting in coastal contamination. To further compound the problems it was also a hot day with many buildings reliant on air conditioning systems causing a peak in demand in the San Diego area.

The outage began with a single 500kilovolt transmission line failure, however the power grid was designed to withstand this, and had done when the same problem had previously occurred. The line in question is a major transmission corridor, which transports power from generators on Arizona to San Diego. When this line tripped power was redistributed through lower power lines, which were already running at high demand due to the hot weather, although the San Diego and Mexico areas were running at lower than peak generation levels. The redistribution resulted in large voltage deviations and equipment overloads to the north, triggering a ripple effect as the overloading of lines and transformers spread as sub-systems initiated automatic load shedding measures. At this point a "separation scheme" deployed which disconnected a nuclear power station from the grid, resulting in a total blackout of San Diego. This event took 11 minutes to unfold during which the reliability co-ordinators (i.e. the authority) issued no directives and local operators only took limited

\footnotetext{
${ }^{2}$ Sometimes referred to as "discovered" systems (Dahmann \& Henshaw, 2016)
} 
mitigating actions, the inability to stem this initial series of failures resulted in the further spread of outages and subsequent blackout. Time to restore power to customers took between 6 to 12 hours.

Two key areas were identified which prevented early mitigation of the failures (FERC \& NERC, 2014); operations planning and real-time situational awareness. If these functions had been adequately supported then it should have been possible to return the system to a secure state within 30 minutes.

With regards to planning, it was found that not enough consideration was given to the status of transmission lines, load and generation forecasting, the effect of component system contingencies on other systems within the network, as well as the reliability of individual component systems and the impact on the network. The earlier prediction of the impact on component systems would have enabled contingency measures to be instigated thereby mitigating the overloads and preventing the cascading failures.

The lack of situational awareness was mainly attributed to deficiencies in the real-time monitoring tools, based on models, which did not accurately reflect component systems and their functions. Recommendations made to improve the situation included greater visibility of models, through data sharing, between component system owners, ensuring the tools provide constant monitoring of systems and improvement of communications throughout all systems comprising the power grid.

Disciplines required to enable effective SoSE in this case include:

- Risk Management

- Network Architecture

- Modelling and Simulation

- Network Theory

\subsubsection{Friendster}

Friendster was one of the first social networking websites, having around 117 million users in 2002. However, it subsequently suffered an exodus of users to other sites, such as Facebook, and shut down in 2011, although it has since been re-established, but as an online gaming site rather than social networking.

One potential factor in the demise of Friendster was its change in role; it was initially set up as a dating site and subsequently adapted to become a more general social networking site. 
Although, at its peak, there were a large number of users, most were not connected to many other members, which could be related to its original use.

Through an examination of remaining residual data, Garcia, Mavrodiev and Schweitzer (2013) have determined that "the initial root of users of Friendster was much more tightly connected among themselves than towards other nodes, creating a denser subcommunity of old users".

This resulted in "weakly connected chains of friends that quickly disintegrated" according to Garcia $^{3}$.

In 2009, Friendster introduced changes in its user interface, which coincided with technical problems and the rise of popularity of Facebook. This exacerbated the decrease of active users in the community, ending in its closure in 2011.

Garcia, Mavrodiev and Schweitzer (2013) suggest that at the heart of successful social networking site are, so-called, K-cores. These are a subset of highly active users with many connections having "resilience and social influence". A site with a "critical mass" of K-cores will survive design changes and technical issues (up to certain level) as these key users have a lot invested in the site through the building up of personal networks, it is therefore in their own interest to remain a part of it.

In the case of Friendster, there were not sufficient K-cores to carry it through the difficulties encountered, as users with just a few connections could easily re-establish them through a competitor, most likely Facebook.

It is clear that Network Theory is the key related discipline in this instance and should be an essential underpinning element to SoSE. The findings of Garcia et al may provide a method to determine SoS resilience.

Disciplines required to enable effective SoSE in this case include:

- Network Theory

\subsubsection{Case Study Summary}

The case studies described above have identified the following set of disciplines, which were required to create, manage and control SoS, i.e. SoSE:

- Requirements Management (Rqmt Mgmt)

- Project Management (Proj Mgmt)

\footnotetext{
${ }^{3}$ http://www.wired.com/wiredenterprise/2013/02/friendster-autopsy/
} 
- $\quad$ Risk Management (Risk Mgmt)

- Contract Management (Cntrt Mgmt)

- Network Architecture (Net Arch)

- Logistics Management (Log Mgmt)

- Command and Control (C2)

- Network Theory (Net Thry)

- Modelling and Simulation (M\&S)

Table 3 summarises these results, cross-referencing the SoS exemplars with particular disciplines and, of particular significance to this research, highlighting the necessity of Risk Management as an element of SoSE which is indicated by its relevance to seven out of the eight case exemplars. It should be noted that adequate situational awareness is necessary for successful SoSE, indeed its lack was specifically identified as a contribution to SoS failure in some cases above. However, it is not a discrete discipline but rather an attribute, which cuts across all disciplines, hence it is not explicitly listed as a SoSE discipline.

\begin{tabular}{|l|c|l|l|l|l|l|l|l|l|}
\hline System & $\begin{array}{l}\text { Rqmt } \\
\text { Mgmt }\end{array}$ & $\begin{array}{l}\text { Proj } \\
\text { Mgmt }\end{array}$ & $\begin{array}{l}\text { Risk } \\
\text { Mgmt }\end{array}$ & $\begin{array}{l}\text { Cntrt } \\
\text { Mgmt }\end{array}$ & $\begin{array}{l}\text { Net } \\
\text { Arch }\end{array}$ & $\begin{array}{l}\text { Log } \\
\text { Mgmt }\end{array}$ & M\&S & $\begin{array}{l}\text { Net } \\
\text { Thry }\end{array}$ & C2 \\
\hline FCS & $\boldsymbol{V}$ & $\boldsymbol{V}$ & $\boldsymbol{V}$ & & & & $\boldsymbol{V}$ & & \\
\hline NPfIT & $\boldsymbol{V}$ & $\boldsymbol{V}$ & $\boldsymbol{V}$ & & & & $\boldsymbol{V}$ & & \\
\hline IDS & $\boldsymbol{V}$ & $\boldsymbol{V}$ & $\boldsymbol{V}$ & $\boldsymbol{V}$ & $\boldsymbol{V}$ & & & & \\
\hline C-NOMIS & $\boldsymbol{V}$ & & $\boldsymbol{V}$ & & $\boldsymbol{V}$ & $\boldsymbol{V}$ & $\boldsymbol{V}$ & & $\boldsymbol{V}$ \\
\hline Close Air Support & & $\boldsymbol{V}$ & $\boldsymbol{V}$ & & $\boldsymbol{V}$ & $\boldsymbol{V}$ & $\boldsymbol{V}$ & & $\boldsymbol{V}$ \\
\hline $\begin{array}{l}\text { Hurricane Katrina } \\
\text { Disaster Relief }\end{array}$ & $\boldsymbol{V}$ & $\boldsymbol{V}$ & & & & & & & \\
\hline Power System & & & $\boldsymbol{V}$ & & $\boldsymbol{V}$ & & $\boldsymbol{V}$ & $\mathbf{V}$ & \\
\hline Friendster & & & & & & & & $\boldsymbol{V}$ & \\
\hline
\end{tabular}

Table 3 - SoSE Case Study Summary

\subsection{System of Systems Engineering}

Systems Engineering (SE) is a relatively new discipline, in comparison to more longstanding engineering disciplines. For example, the Institute of Civil Engineers was founded in 1818 whereas the International Council on Systems Engineering (INCOSE) was founded in 1990. In contrast to this the Systems Engineering Guide for Systems of Systems (US Department of Defense, 2008b) was first published in 2008. 
With SoSE an immature sub-discipline, the engineering of a SoS has often been approached through the application of SE techniques where a SoS is regarded as a large-scale system with component systems viewed as sub-systems. Whilst this approach may be appropriate for certain aspects, such as interoperability of more clearly defined SoS, there are other aspects that are distinct and need to be treated accordingly.

In an effort to determine appropriate SoSE approaches the INCOSE SoS Working Group has identified the following issues, or 'pain points' (Dahmann, 2014):

- SoS Authorities: problems with overlapping authorities between the component systems

- Leadership: identification of SoS leadership is problematic due to the issue of overlapping authorities

- Constituent Systems' Perspectives: in-service and legacy component systems purpose may not align fully with that required for the SoS

- Capabilities and Requirement: SoS comprise component systems with their own requirements which may not fully align with the objectives of the SoS

- Autonomy, Interdependencies and Emergence: component systems are independent and may change independently of the SoS

- Testing, Validation, and Learning: Typically component systems are tested independently rather than within end to end SoS testing

These pain points illustrate that a holistic approach is required for SoSE, it cannot be assumed that if each component system is controlled and managed the aggregate affect will be that the SoS will fulfil it's intended purpose.

The case studies summarised in section 2.1.9 highlight specific disciplines which should be applied holistically at the SoS level and which require different approaches to those currently taken at the component system level.

Whilst SoSs that have well defined component systems, i.e. directed, may be suited to a more traditional SE approach, other classifications are less so. For example, in fulfilling a search and rescue mission several component systems will be required to act together. Some of these may have been designed to be interoperable, such as the coast guard command centre and a maritime rescue helicopter, however civilian vessels may also be involved with differing communication capabilities, in this case a traditional SE development cycle is clearly not applicable as the SoS is more of an ad-hoc arrangement, e.g. 
collaborative/acknowledged as in this case part of the SoS is formed rapidly in order to fulfil the specific purpose.

Table 4 lists the disciplines identified from the case studies summarised in section 2.1.9 that are required to enable effective SoSE with their relationship to the SoS pain points identified. Using this relationship, the description of the SoS pain points and comparison with traditional SE approaches the SoS perspective for each discipline is derived, providing a high level description of the required approach; hence Table 4 summarises the distinctions between SE and SoSE.

\begin{tabular}{|c|c|c|}
\hline SoSE Disciplines & Related SoS Pain Point & SoS perspective \\
\hline $\begin{array}{l}\text { Requirements } \\
\text { Management }\end{array}$ & $\begin{array}{l}\text { Capabilities and } \\
\text { Requirement } \\
\text { SoS Authorities } \\
\text { Leadership }\end{array}$ & $\begin{array}{l}\text { Equates to the SoS purpose, } \\
\text { requirements are traditionally levied at } \\
\text { the component system level however } \\
\text { the appropriate authority needs to be } \\
\text { established. }\end{array}$ \\
\hline $\begin{array}{l}\text { Project } \\
\text { Management }\end{array}$ & $\begin{array}{l}\text { Leadership } \\
\text { Constituent Systems' } \\
\text { Perspectives } \\
\text { Autonomy, } \\
\text { Interdependencies and } \\
\text { Emergence }\end{array}$ & $\begin{array}{l}\text { Management of heterogeneous } \\
\text { component systems lifecycle and } \\
\text { maturity is required. }\end{array}$ \\
\hline $\begin{array}{l}\text { Risk } \\
\text { Management }\end{array}$ & $\begin{array}{l}\text { SoS Authorities } \\
\text { Leadership } \\
\text { Constituent Systems' } \\
\text { Perspectives } \\
\text { Autonomy, } \\
\text { Interdependencies and } \\
\text { Emergence }\end{array}$ & $\begin{array}{l}\text { Holistic risk management approach is } \\
\text { required; management of risk at the } \\
\text { component system level will not } \\
\text { mitigate risk at the SoS level. }\end{array}$ \\
\hline $\begin{array}{l}\text { Contract } \\
\text { Management }\end{array}$ & $\begin{array}{l}\text { Capabilities and } \\
\text { Requirement } \\
\text { SoS Authorities }\end{array}$ & $\begin{array}{l}\text { Manage asynchronous contracts across } \\
\text { multiple component systems to ensure } \\
\text { delivery of SoS capability }\end{array}$ \\
\hline $\begin{array}{l}\text { Network } \\
\text { Architecture }\end{array}$ & $\begin{array}{l}\text { Autonomy, } \\
\text { Interdependencies and } \\
\text { Emergence }\end{array}$ & $\begin{array}{l}\text { Interactions between component } \\
\text { systems need definition although they } \\
\text { may be created on an ad-hoc basis and } \\
\text { change as the SoS evolves. }\end{array}$ \\
\hline $\begin{array}{l}\text { Logistics } \\
\text { Management }\end{array}$ & $\begin{array}{l}\text { Capabilities and } \\
\text { Requirement } \\
\text { SoS Authorities } \\
\text { Autonomy, } \\
\text { Interdependencies and } \\
\text { Emergence }\end{array}$ & $\begin{array}{l}\text { The management of the availability of } \\
\text { component systems is required, } \\
\text { particularly in operational situations. }\end{array}$ \\
\hline
\end{tabular}




\begin{tabular}{|l|l|l|}
\hline $\begin{array}{l}\text { Modelling and } \\
\text { Simulation }\end{array}$ & $\begin{array}{l}\text { Capabilities and } \\
\text { Requirement } \\
\text { Testing, Validation, } \\
\text { and Learning }\end{array}$ & $\begin{array}{l}\text { Due to the inherent complexity } \\
\text { associated with a SoS, M\&S will provide } \\
\text { understanding and decision support } \\
\text { capability, heterogeneous models will } \\
\text { be required to model all aspects. }\end{array}$ \\
\hline $\begin{array}{l}\text { Command and } \\
\text { Control }\end{array}$ & $\begin{array}{l}\text { SoS Authorities } \\
\text { Leadership }\end{array}$ & $\begin{array}{l}\text { Authority needs to be established at the } \\
\text { SoS level. }\end{array}$ \\
\hline Network Theory & $\begin{array}{l}\text { Autonomy, } \\
\text { Interdependencies and } \\
\text { Emergence }\end{array}$ & $\begin{array}{l}\text { Provides an understanding of the } \\
\text { interactions within the SoS }\end{array}$ \\
\hline
\end{tabular}

Table 4 - SoSE Disciplines

In order to draw some more general conclusions from the case study analysis above, Table 5 maps SoS classification (Dahmann \& Baldwin, 2008) to SoSE discipline. It can be seen that only Network Theory is identified as applicable to Virtual SoS, however this can be explained by the lack of defined control and purpose which prevents the application of more traditional disciplines. Risk Management, Project Management, Requirements Management, Network Architecture and Modelling and Simulation are applicable across Directed, Collaborative and Acknowledged types although the approach taken will differ within these types, for example a Directed SoS may be at an acquisition stage in the lifecycle but an Acknowledged SoS may be operational. It is also noted that the disciplines required for engineering a Acknowledged SoS are a combination of those necessary for Directed and Collaborative types; an explanation may be that SoS do not always remain in a single state (or type) tending to move from Directed to Acknowledged or to Acknowledged Collaborative. Hence, in summary, Table 5 provides a guide to the appropriate application of specific SoSE disciplines: of particular relevance to this thesis is the conclusion that Risk Management is not applicable to virtual SoS, and which is discussed further in chapter 7.

\begin{tabular}{|l|c|c|c|c|c|l|l|l|l|}
\hline SoS Classification & $\begin{array}{l}\text { Rqmt } \\
\text { Mgmt }\end{array}$ & $\begin{array}{l}\text { Proj } \\
\text { Mgmt }\end{array}$ & $\begin{array}{l}\text { Risk } \\
\text { Mgmt }\end{array}$ & $\begin{array}{l}\text { Cntrt } \\
\text { Mgmt }\end{array}$ & $\begin{array}{l}\text { Net } \\
\text { Arch }\end{array}$ & $\begin{array}{l}\text { Log } \\
\text { Mgmt }\end{array}$ & M\&S & $\begin{array}{l}\text { Net } \\
\text { Thry }\end{array}$ & C2 \\
\hline Directed & $\boldsymbol{V}$ & $\boldsymbol{V}$ & $\boldsymbol{V}$ & $\boldsymbol{V}$ & $\boldsymbol{V}$ & $\boldsymbol{V}$ & $\boldsymbol{V}$ & & $\boldsymbol{V}$ \\
\hline Acknowledged & $\boldsymbol{V}$ & $\boldsymbol{V}$ & $\boldsymbol{V}$ & $\boldsymbol{V}$ & $\boldsymbol{V}$ & $\boldsymbol{V}$ & $\boldsymbol{V}$ & & $\boldsymbol{V}$ \\
\hline Collaborative & $\boldsymbol{V}$ & $\boldsymbol{V}$ & $\boldsymbol{V}$ & & $\boldsymbol{V}$ & $\boldsymbol{V}$ & $\boldsymbol{V}$ & & $\boldsymbol{V}$ \\
\hline Virtual & & & & & & & & $\boldsymbol{V}$ & \\
\hline
\end{tabular}

Table 5 - SoSE Disciplines mapped to SoS Classification 


\subsection{Risk Management of SoS}

This section provides a brief overview of the issues surrounding the approach required for SoS Risk Management in contrast to the traditional system approach. A more detailed discussion is provided in section 3.4 and chapter 7.

The case studies discussed above (section 2.1) illustrate that Risk Management is a key element of SOSE. The DoD SOSE guide (US Department of Defense, 2008b) succinctly describes the subtle distinction between system and SoS risk management;

"Risk management for a SoS begins with the identification of SoS objectives and the identification of the risks that threaten the achievement of those objectives. While it is true that minor individual program risks could be major risks to the SoS, it is also true that significant system risks may have little or no impact on the SoS functionality. Furthermore there may be risk to a set of SoS objectives which are not risks to the constituent systems (e.g., unwanted emergent behaviour, infrastructure, integration risks, cost risk)."

A common perception is that if all SoS component system risks are managed, then any SoS risks will be managed without further intervention. The quote above demonstrates that SoS risk may be decoupled from individual system risk and should be managed separately.

When engineering 'traditional' systems, the likelihood of risk is typically determined through qualitative approaches resulting in the allocation of subjective (as opposed to objective) probability (Garvey, 2004). Pinto et al (2012) argue that, when engineering these traditional systems, the tools and methodologies are available to address defined problems and, as the system boundaries are fixed and expected behavior is known, scoping these problems and the associated risks, is relatively straightforward. However, the SoS boundary is not necessarily static, the component systems may not all be identified and behavior is emergent: therefore new tools and methodologies are required.

In addition, Conrow (2005) identifies the following SoS Risk Management issues, which are specific to this domain; multiple stakeholders and risk management processes, long life cycles, disparate technical risk assessment, integration risks, functional performance and interface complexity.

\subsection{Summary of Chapter}

This chapter discussed several exemplars covering all types of SoS; directed, collaborative, acknowledged and virtual (Dahmann \& Baldwin, 2008). For each exemplar specific disciplines are identified (in Table 4) which are required to enable effective SOSE in each 
A Model Based Approach to System of Systems Risk Management

case. These results are generalised in Table 5, which maps SoSE discipline to SoS type. Risk Management is identified as a key SoSE discipline being applicable to directed, collaborative and acknowledged types. However, as discussed above in section 2.3, Risk Management of SoS requires a different approach to that currently taken for individual systems and it cannot be assumed that if risk is managed for each component system then the aggregated effect will be to mitigate risk at the SoS level. 


\section{Chapter 3: Literature review}

This chapter describes the strategy and approach of the state of the art literature review, identifies and justifies the key themes, analyses the results of the review and concludes with a summary of the results.

\subsection{Literature review strategy}

A literature review can be described as "the use of ideas in the literature to justify the particular approach to the topic, the selection of methods, and demonstration that this research contributes something new" (Hart, 1998).

The state-of-the-art literature review approach was based on the three-stage review process as described by Levy \& Ellis (2006) in Figure 4 below.

\section{Processing}

\begin{tabular}{|c|c|c|}
\hline 1. Input & $\begin{array}{l}\text { 1. Know the literature } \\
\text { 2. Comprehend the literature } \\
\text { 3. Apply the literature }\end{array}$ & 3. Output \\
\hline & $\begin{array}{l}\text { 4. Analyse the literature } \\
\text { 5. Synthesise the literature } \\
\text { 6. Evaluate the literature }\end{array}$ & $\begin{array}{l}\text { - What is, and what is } \\
\text { not, helpful } \\
\text { - Gaps }\end{array}$ \\
\hline
\end{tabular}

Figure 4 - Three-stage literature review process (Levy \& Ellis, 2006)

The literature review initially utilised a combination of key word and recommended author searches providing familiarisation with the problem domain. However, as the researchers understanding increased and consequently the research question was refined, reducing the overall scope, specific themes were identified which allowed more targeted keyword searching. The initial results of the literature also identified key authors and journals to be searched providing greater coverage of the available knowledge base.

Following the initial literature search, backward and forward searches (Levy \& Ellis, 2006; Webster \& Watson, 2002) were employed to expand the review coverage and probe more deeply into the subjects under investigation. These techniques searched using author and reference attributes. The backwards search approach was employed to investigate the originating sources (e.g. journal articles) of concepts and theories, while the forward search was used to extend the knowledge by reviewing follow-up studies and developments related to the concepts and theories under investigation. 
The literature review was divided into the following three themes:

1. General definitions and differences between System and SOSE

2. Modelling and Simulation and its applicability to SoS

3. Risk Management and its application in the SoS domain

These themes were selected to ensure all aspects of the research questions were surveyed, as described in section 1.2. Each theme required a more general review with a relatively broad scope in order to provide context for the subsequent more specific review; e.g. a general review of different Modelling and Simulation techniques was undertaken and then each technique was reviewed in the context of SoS applicability.

For the first theme, some background publications regarding SE were surveyed but were limited to key sources, e.g. INCOSE, in order to enable the SOSE perspective to be compared. Numerous publications were available regarding SoS and SoSE, the review was initially undertaken using refined keyword combinations, developed from the initial search, as well as author, conference and publication attributes. Forward and backward searching techniques were also utilised to ensure greater survey coverage. This theme seeks to establish an understanding of the current state of SoSE, including its differentiation from traditional SE, how the SoS lifecycle differs from that of a single system, the characterisation and definition of SoS and identify the necessary components of SoSE. The output of this theme affords justification for the necessity of Risk Management as a specific SoSE activity as well as providing supporting information used in the development of the SoS System of Interest model, as described in section 6.1, with the associated conference paper (Kinder et al., 2012) attached in Annex A.

The initial survey for the Modelling and Simulation theme revealed a vast amount of publications, in order to bound the review a set of most widely used M\&S techniques was identified and only key publications were analysed in order to provide basic background understanding of this area. The number of publications relating to SoS M\&S was far lower with an uneven spread between techniques; e.g. Discrete Event Specification (DEVS) has been promoted as a general approach to SoS M\&S and hence numerous publications are available whereas the availability of publications relating Petri Nets and SoS applications is very limited. In order to provide context for the review a case study was used (Data Looping in Tactical Data Link architectures) which provided a framework of heterogeneous techniques to be compared from a SoS perspective, the results of this review were published 
in the International Journal of SoSE (Kinder et al., 2014), the paper is attached in Annex C. This theme is essential to support the "model-based" aspect of this research, directly fulfilling research objective 1 (Review of a wide range of modelling techniques applicability to SoS) and providing input to objectives 5 (Identify appropriate modelling techniques and implement and test tool), 6 (Identify a case study and determine the adaptability of the tool) and 7 (Use model to assess transfer of risk within a SoS).

The third theme also required a background knowledge review, in this case related to Risk and Risk Management, and again limiting the review to key publications. The number of publications found which related to the management of risk specifically within SoS was very low, as a result the search was widened to include SoS case study examples and in particular lessons learnt reports, these in turn were searched for reference to risk and risk management. The results of the theme 3 review support research objectives 2 (Review current risk management approaches), 3 (Identify differences between system and SoS risk management), 7 (Use model to assess transfer of risk within a SoS) and 8 (Define a SoS risk management methodology).

The literature sources included the following:

- $\quad$ IEEE IEXPLORE

- INCOSE

- Science Direct

- Elseiver

- Google/Google Scholar

- Scopus

- Loughborough University library

\subsection{Theme 1: Systems of Systems Engineering}

The first literature theme reviews the current state of SoSE, surveying SoS definitions, SoS characteristics, SoSE approaches and lifecycle models. Key elements of SoSE are identified as well as gaps in existing knowledge. 
A brief review of system theory and systems engineering are also provided which are required to support subsequent arguments and discussions.

\subsubsection{System Theory}

Karl Ludwig Von Bertalanffy (1937) first proposed the "system" concept in 1937, defining a system as a "complex of interacting elements". This concept spawned a proliferation of system approaches and disciplines, including Systems Engineering. It should be noted that, this section is included in order to provide basic coverage of foundational knowledge, which contributes to the subsequent sections, hence the depth of inquiry is necessarily limited.

Beyond the basic system concept, Bertalanffy proposed General Systems Theory (Von Bertalanffy, 1968) which espoused the following system laws and which provide the basis for systems engineering and thinking methodologies:

- Holism: the whole system is primary and the components are secondary, the whole is more than the sum of its parts.

- Open/Closed Systems: open systems exchange matter, information and energy with their environment, whereas closed systems are unchanged by their environments.

- Boundaries: defines the limit of a system. Closed system boundaries are static, open systems are dynamic.

- Inputs/Outputs: input of information and output back into the environment by open systems; this is related to "boundaries".

- Feedback: outputs are fed back into the system as it transforms and adapts to the environment.

- Single/Multiple Outcomes: relates to the system goal.

- Equifinality: the relationship between the initial conditions and final outcome.

- Entropy: the measure of disorder.

- Hierarchies: the relationship of the system to its component parts.

- Interrelated Components: by definition, systems are comprised of connected elements.

- Dynamic Equilibrium: system equilibrium is attained if all component parts are in a state of equilibrium.

- Internal Elaboration: closed systems move towards entropy and disorganisation whereas open systems move towards diversity and increased complexity. 
Adams (2011) assessed system theory in the context of SoS and identified additional relevant principles, including:

- Emergence: the whole is more than the sum of its parts; constitutive characteristics are not explainable from the characteristics of the individual parts.

- Satisficing: rather than optimising an option chosen that is "good enough".

- Requisite variety: is calculated by the number of system states raised to the power of system elements and is directly related to complexity. The controller of a system must exhibit at least the same level of variety as the system under control (Ashby, 1961).

- Requisite parsimony: human beings can only deal simultaneously with between five and nine observations at one time (Miller, 1956).

\subsubsection{Systems Engineering}

This section is intended to provide a basic set of state of the art definitions regarding systems and SE upon which subsequent sections and chapters may refer to. It is not intended to provide a detailed literature review of the domain.

ISO/IEC 15288, Systems and software engineering - System life cycle processes, defines a system as "combination of interacting elements organized to achieve one or more stated purposes" where a system element is defined as a "member of a set of elements that constitute a system" (British Standards, 2011).

INCOSE provides similar system definition; "an integrated set of elements, or assemblies that accomplish a defined objective" (INCOSE, 2015).

These definitions build upon Bertalanffy's definition (a complex of interacting elements) through the addition of an association to a system purpose or objective.

Systems Engineering (SE) is the "interdisciplinary approach governing the total technical and managerial effort required to transform a set of stakeholder needs, expectations, and constraints into a solution and to support that solution throughout its life" (British Standards, 2011).

In order to undertake SE the System of Interest (Sol) must be established, INCOSE defines the Sol for a system as; "The system whose life cycle is under consideration", whilst ISO/IEC 15288 (British Standards, 2011) takes a hierarchical view; "the top system in the system 
A Model Based Approach to System of Systems Risk Management

structure is called a system-of-interest and consists of lower level systems. Except the lowest level is identified as being made up of system elements."

\subsubsection{SoS Definition and Classification}

Ackoff made one of the first references to "Systems of Systems", in 1971, proposing the grouping of disparate system concepts into single systems, which could be applied in the management of organisations (Ackoff, 1971). Even earlier, in 1964, Berry used the term "Systems within Systems" (Berry, 1964) when examining the evolution of cities through urban models. Clearly a distinction between single systems and SoS has been identified for some time; indeed SoSs been have developed for many years, for example the British railway network, which can be classified as a SoS, was developed in the mid-nineteenth century (Kemp et al., 2013). Another example is the Joint Tactical Information Distribution System (JTIDS) (NATO, 2008), which began development in the late 1960's and enabled the exchange of situational awareness information between platforms. This was a key development in the evolution of the SoS, it allowed greater interaction between component systems, resulting in closer coupling even within SoS created on a more ad-hoc basis. When JTIDS became operational in the 1980's it provided a step change in tactical awareness however there were many interoperability problems, which reduced the potential effectiveness of this new capability. This resulted in the creation of iSMART process, which provided a SoSE approach to solving interoperability issues (Lockheed Martin, 2016).

However, this was a very specific solution tailored to the particular problem space, it was not until Maier's seminal publication; Architecting principles for Systems of Systems (Maier, 1998), that a more general differentiation between systems and SoSs was articulated, providing a sound basis for SoSE research. Maier argued that the two key properties, which differentiate the SoS from a system, are managerial and operational independence of the component systems. This countered the generally accepted view at the time that SoSs are geographically distributed but managed and developed conventionally (Eisner et al., 1991; Shenhar, 1995). Operational independence requires component systems to have the ability to operate independently. Managerial independence requires component system to be acquired, operated and integrated independently. Maier also observed that SoSs exhibit different characteristics, proposing the following categorisation based on managerial control:

- Directed: Directed systems-of-systems are those in which the integrated system-ofsystems is built and managed to fulfill specific purposes. It is centrally managed 
A Model Based Approach to System of Systems Risk Management

during long-term operation to continue to fulfill those purposes, and any new ones the system owners may wish to address. The component systems maintain an ability to operate independently, but their normal operational mode is subordinated to the central managed purpose.

- Collaborative: Collaborative systems-of-systems are distinct from directed systems in that the central management organization does not have coercive power to run the system. The component systems must, more or less, voluntarily collaborate to fulfill the agreed upon central purposes.

- Virtual: Virtual systems-of-systems lack both a central management authority and centrally agreed upon purposes. Large-scale behavior emerges, and may be desirable, but the supersystem must rely upon relatively invisible mechanisms to maintain it.

Dahmann et al (Dahmann \& Baldwin, 2008) argued that a further SoS type exists which is similar to a Directed SoS in that they have a common managerial authority and purpose but are in fact a mix of new and legacy systems which can also operate independently. This additional type is classified as an "Acknowledged" SoS and is defined below:

- Acknowledged. Acknowledged SoS have recognized objectives, a designated manager, and resources for the SoS; however, the constituent systems retain their independent ownership, objectives, funding, and development and sustainment approaches. Changes in the systems are based on collaboration between the SoS and the system.

Maier (Maier, 1998) states that a Virtual SoS may be formed deliberately or accidently; as a result Kemp et al (Kemp et al., 2013) have proposed a fifth classification, "Accidental", applicable to emergent SoS, which are entirely unplanned:

- Accidental. Accidental SoS are completely unplanned and unpredicted but becomes a reality

It is noted that an Accidental SoS is very similar to a Virtual SoS and indeed the distinction between them may not be of value. The definition of an Accidental SoS could be regarded as retrospective; once an Accidental SoS has been identified then, at that point, it also fits the definition of a Virtual SoS. A potential further refinement could explicitly classify deliberate and accidental Virtual SoS. 
However, the ability to identify a system as a SoS and then to classify it as a specific type enables more appropriate methods of development, control and management to be applied, or at least provides an awareness that traditional approaches may not be suitable. However, this is less applicable to the Virtual SoS and, by definition, not applicable to the Discovered SoS. The misclassification of a SoS or monolithic system can have major impacts (Maier, 1998); for example if a system is declared a monolithic SoS rather than a monolithic system the resulting architecture will incorporate unnecessary redundancy as every component system will be required to operate independently hence the overall system cost and complexity will also rise. If the converse is true and the system is incorrectly classified, as a monolithic system, then the system architecture will consist of tightly coupled component systems, which may not be appropriate if an adaptable, evolutionary system is required.

Incorrect classification of a SoS (e.g. Directed rather than Acknowledged) may result in owners, developers or operators over or underestimating the level of control they process, leading to the adoption of inappropriate mechanisms for ensuring collaboration between component systems (Maier, 1998).

Since Maier's SoS definition numerous alternatives have been proposed, with many specific to particular domains. Through a literature review Jamshidi (2008) identified six different definitions alone and proposed the following alternative:

"SoSs are large-scale integrated systems which are heterogeneous and independently operable on their own, but are networked together for a common goal" (Jamshidi, 2008).

The DoS Defense Acquisition Guidebook (DoD, 2014) provides the following definition:

"A SoS is described as a set or arrangement of systems that results when independent and useful systems are integrated into a larger system that delivers unique capabilities."

The ISO System and Software engineering standard (British Standards, 2011) closely follows Maier's original definition, describing a SoS as:

"..an SOI whose elements are themselves systems. A SoS brings together a set of systems for a task that none of the systems can accomplish on its own". It is also states "SoS are characterized by the managerial and operational independence of the constituent systems".

The INCOSE SE Guide cites the two characteristics identified by Maier but removes the stipulation that both must be exhibited to enable a classification as a SoS, thereby deviating from the definition provided in ISO 15288: 
A Model Based Approach to System of Systems Risk Management

"..an SOI whose elements are managerially and/or operationally independent systems" (INCOSE, 2015).

There are now numerous examples of SoS managed by a single organisation and there are also constituent systems that, although they can theoretically be operated independently, provide limited independent functionality (Brook, 2016). Brook argues that Maier's original definition is now overly prescriptive and proposes the following less restrictive definition:

"A SoS is a system which results from the coupling of a number of constituent systems at some point in their life cycles" (Brook, 2016).

This definition neatly encompasses the evolutionary nature of the SoS, which avoids the current confusion regarding the relevance of managerial and operational independence, and is the preferred definition within this thesis.

Despite the number of SoS definitions available, it is often asked if a SoS consists of component systems, which can be regarded as sub-systems, then can a SoS be treated as a large system?

Brook provides a compelling response through the application of systemic and systematic viewpoints (Brook, 2016). The Systemic view considers the whole system behaviour and its interaction with the environment whilst the systematic view is concerned with the composition of the system and the interactions with its component parts. Brook argues that SoS and individual systems are the same when viewed systemically because the internal structure is hidden and only the externally viewed behaviour is observed, although it is noted that emergence and the potential unpredictability of SoS is not considered. When viewed systematically "the differences between systems and SoS are major and deepseated", in this case Brook argues that SoSE is focused upon the building of complex systems through the coupling of individual systems. Brooks' SoS definition, as stated above, combines both systemic and systematic aspects and is therefore more generic than most alternatives, but also well founded.

\subsubsection{SoS Characteristics}

As illustrated in the discussion above, the search for a succinct, all encompassing definition of a SoS is somewhat elusive, with many attempting to encapsulate Maier's properties. Boardman and Sauser (Boardman \& Sauser, 2006) took a different approach, proposing the differentiation of SoS vice system through the following set of distinguishing characteristics 
providing a more sophisticated approach to classification thereby reducing the risk of misclassification; Autonomy, Belonging, Connectivity, Diversity and Emergence.

Autonomy aligns somewhat with Maier's key SoS property, operational independence. From the system perspective a constituent that is deemed to serve the system, and is designed for a specific purpose, is regarded as a system part, hence the system itself is autonomous rather than the constituent parts. However, from the SoS perspective the component systems act autonomously whilst contributing to the SoS purpose.

Belonging is related to the collaboration between system components. A system part inherently belongs to the parent system whereas component systems comprising a SoS develop belonging through an acceptance of the benefit of participation and contribution to the SoS purpose.

Connectivity between components is essential to the function of a system or SoS. Within a system, parts are tightly coupled with interactions encapsulated and explicitly specified. The connectivity between component systems is provided on a dynamic basis with connections made on a real-time basis in order to support the SoS purpose as required.

Diversity allows a SoS to adapt to external conditions or a change in its purpose, in accordance with Ashby's Law of Requisite Variety (Ashby, 1961) (section 3.2.1), which states that "for a system to maintain stability in an operating environment it must possess at least the same number of degrees of freedom or dimensionality of that environment". The diversity is provided by the autonomy, belonging and dynamic connectivity of the component systems. In contrast, a system comprises a static set of parts where connectivity is fixed.

Emergence is deliberately designed into a system; the resulting behaviour is intended to fulfil the systems purpose with testing used to eliminate unwanted behaviour. Within a SoS emergence arises through the characteristics autonomy, belonging, connectivity and diversity. Boardman and Sauser suggest that a SoS should support emergence but eliminate undesirable behaviour.

Of the five characteristics, autonomy and connectivity provide a more intuitive distinction and are therefore more likely to be correctly determined. Belonging and diversity are somewhat esoteric and may be difficult to assess when considering actual systems or SoS. Emergence can be observed but again, it may be difficult to differentiate between behaviour arising from a tightly coupled system and that from a SoS, the suggested ability for a SoS to eliminate unwanted behaviour may not be easily predicted. Hence, although these proposed 
A Model Based Approach to System of Systems Risk Management

characteristics provide richer descriptions and differentiation of system and SoS, in practice their application does not appear to be straightforward.

Through a review of current knowledge Keating et al (Keating \& Katina, 2011) identified the following characteristics:

- Managerial Independence (Maier, 1998).

- Operational Independence (Maier, 1998).

- Evolutionary development: "changes have to be made to the SoS as more knowledge is acquired and circumstances shift" (Keating \& Katina, 2011). This is in contrast to component systems where development is described as "front loaded".

- Emergent behaviour: develops over time through the interaction of component systems (Keating, 2009)

- Geographical distribution (Eisner et al., 1991); it is noted that this is frequently attributed to Maier (Maier, 1998), however Maier states that component systems are commonly but not always geographically distributed, hence this is not necessarily a defining characteristic.

- Interoperability: "the ability of two or more systems or components to exchange information and to use the information that has been exchanged" (IEEE, 1989). It is emphasised that a SoS functions through the interaction of its component systems.

- Complementarity (Keating \& Katina, 2011): "provides multiple perspectives of any given system", it is stated that this should result in the most appropriate solution adopted and that component systems will compliment one another within the SoS.

- Holism (Kovacic et al., 2008): a philosophical view, which states the SoS must be regarded as a whole and that reductionism is not appropriate to the understanding of SoS behaviour.

Kinder et al (Kinder et al., 2012) ${ }^{4}$ take an alternative approach, rather than attempting to define SoS characteristics, a SoS System of Interest model is developed identifying nine "dimensions" which enable a SoS to be described and classified. The Sol model has been developed as a key element of the model based approach to SoS Risk Management; the dimensions are listed below but are described in greater detail in section 6.1:

- Component Systems (including specific and general system types),

- Classification

\footnotetext{
${ }^{4}$ Paper is attached in Annex A
} 
A Model Based Approach to System of Systems Risk Management

- Interactions

- Nature of Relationships

- Lifecycle

- Variability

- Functions

- Systems Owners and Operations

- Concept of Operation / Use / Employment

Table 6 provides a direct comparison between the SoS characteristics and dimensions described above.

\begin{tabular}{|l|l|l|}
\hline $\begin{array}{l}\text { Sol Dimensions (Kinder et al., } \\
\text { 2012) }\end{array}$ & $\begin{array}{l}\text { Characteristics } \\
\text { (Boardman \& Sauser, } \\
\text { 2006) }\end{array}$ & $\begin{array}{l}\text { Keating (Charles B Keating \& } \\
\text { Katina, 2011) }\end{array}$ \\
\hline $\begin{array}{l}\text { Component Systems (including } \\
\text { specific and general system } \\
\text { types), }\end{array}$ & Autonomy & $\begin{array}{l}\text { Managerial Independence } \\
\text { Operational Independence } \\
\text { Geographical distribution }\end{array}$ \\
\hline Classification & Belonging & Holism \\
\hline Interactions & Connectivity & Interoperability \\
\hline Nature of Relationships & & Evolutionary development \\
\hline Lifecycle & Diversity & Emergent Behaviour \\
\hline Variability & Emergence & \\
\hline Functions & & Complementarity \\
\hline $\begin{array}{l}\text { Systems Owners } \\
\text { Operations }\end{array}$ & & \\
\hline $\begin{array}{l}\text { Concept of Operation / Use / } \\
\text { Employment }\end{array}$ & & \\
\hline
\end{tabular}

Table 6 - SoS Characteristics and Dimension Comparison

\subsubsection{SoS Engineering (SOSE)}

As stated previously, in section 1.1, SoSE is an immature, emerging sub-discipline of SE. All aspects of SoSE are still the subjects of debate; indeed even a globally accepted definition of SoSE has yet to be agreed.

Although not intended to provide a comprehensive approach to SoSE, Maier (1998) suggested the following heuristics relating to the design of SoS architecture: 
- Stable Intermediate Forms: system designers need to consider intermediate states of the SoS as it evolves and their stability.

- Policy Triage: determine points at which leverage over system evolution is possible, e.g. communication standards.

- Leverage at the Interfaces: the primary point at which the designer has control over the system.

- Ensuring Cooperation: consider why component systems need to collaborate and ensure design provides support.

These heuristics are valid if a SoS is "designed", i.e. they appear more applicable to a directed SoS whereby control over the architecting and design phases is possible. However, when considering a collaborative or virtual SoS, this is not necessarily feasible as the SoS may be formed on an ad-hoc basis in response to an emerging, potentially urgent, need. Hence, although these heuristics provided early guidance on factors that should be considered when undertaking SoSE, they cannot be universally applied to all potential cases and cannot be considered comprehensive.

The development of SOSE is still immature with no single coherent approach currently agreed across all domains. Indeed, Keating et al (Keating \& Katina, 2011) argue that the SoSE field is diverging between hard and soft system approaches stating that; there is a lack of an accepted definition of SOSE, the relationship and differentiation of SOSE to SE is tenuous and there is an overemphasis on information technology (interoperability) and lack of emphasis on human, social, organisational, political, policy dimensions.

The lack of accepted SoSE definitions is a valid concern, with few available. The DoD definition below appears more relevant to directed and acknowledged SoS, implying the use of traditional SE approaches thereby limiting the necessary scope of SoSE. Whereas the second definition is more generic hence the scope of SoSE is not limited in this case.

The process of planning, analyzing, organizing, and integrating the capabilities of a mix of existing and new systems into a system-of-systems capability that is greater than the sum of the capabilities of the constituent parts (DoD, 2014).

The evolving, designed combination of systems to form a system of systems in networks that are safe, secure, efficient and able to respond to changing requirements and operational situations (Barot et al., 2013).

The key to defining SoSE is describing the meaning of "engineering" however, the dichotomy is that the definition of discrete activities may result in an overly prescriptive definition, 
A Model Based Approach to System of Systems Risk Management

applicable only within a specific domain whereas a generic definition will provide a definition of "engineering". Another problem with attempting to create a specific (tight) definition is that the SoSE approach will differ for each SoS classification, which without an excessively cumbersome definition will not provide this distinction. For these reasons it is suggested that a "tight" definition for SoSE is not appropriate, as a truly universal definition will never be achieved and that a "loose", generic definition is the more suitable approach with the detail concerning specific activities covered in appropriate standards and guides.

DoD SE Guide for SoS

The DoD SE Guide for SoS (US Department of Defense, 2008b) was first published in 2008 and provided the first comprehensive set of SoSE guidelines, describing how each aspect of SE should be applied from a SoS perspective. However, the guide relates to the defence domain and is focussed upon acknowledged SoS, although it is stated that future updates will include directed SoS but these have not, as yet, been forthcoming. The guide identifies the following seven core elements of SoSE, with the first three (highlighted) deemed critical to SOSE:

- Translating capability objectives - translating capability into technical requirements and identifying new needs as the SoS evolves.

- Understanding systems and relationships - understanding component system support to SoS objectives, interactions and asynchronous development paths.

- Monitoring and assessing changes - understanding how changes in component systems affect the SoS.

- Assessing performance to capability objectives - determine metrics and methods for monitoring SoS performance and feedback to component systems to enable system level changes.

- Developing and evolving a SoS architecture - determine the relationships, functions and dependencies of component systems.

- Addressing requirements and solution options - determine user needs and identify solution options, liaise at the system level to ensure systems can support these.

- Orchestrating upgrades to SoS - orchestrate component systems to ensure support to the SoS is maintained.

Each core element is mapped to one or many of the sixteen SE technical and technical management processes as defined in the SE chapter of the DoD Acquisition Guide (DoD, 
A Model Based Approach to System of Systems Risk Management

2014). The sixteen processes are shown in Figure 5 and the mapping to the SOSE core elements in Figure 6.

Of particular relevance to this research is the mapping of Risk Management, which is indicated, as applicable to all seven SoSE elements, and therefore can be considered a key discipline within SOSE.

As stated previously these SoSE guidelines are intended for development of an Acknowledged SoS, which, by definition, will have a designated manager, hence an element of control over all component systems is possible. However, when considering collaborative and virtual SoS no centralised control is available, in these cases the proposed DoD SoSE, which is closely associated with the traditional SE approach, becomes less appropriate. In these cases, where a SoS is created on a more ad-hoc basis it is not possible to influence individual component system design and the interfaces between them at the time of SoS creation. The "engineering" of the collaborative SoS is more related to the selection of appropriate component systems, which will provide the highest likelihood of achieving the SoS purpose or mission. However, the classification of a SoS is not always a single discrete state, as stated previously frequently sub-groups of component systems within a SoS may be classified differently to the overall classification. For example, this may occur when a group of component systems are assembled on an ad-hoc basis but interact through an established communications medium such as tactical data links or the internet protocol (IP). 
Technical Processes

Requirements Development takes all inputs from relevant stakeholders and translates the inputs into technical requirements.

Logical Analysis is the process of obtaining sets of logical solutions to improve understanding of the defined requirements and the relationships among the requirements (e.g.r functional, behavioral, temporal).

Design Solution translates the outputs of the Requirements Development and Logical Analysis processes into alternative design solutions and selects a final design solution.

Implementation is the process that actually yields the lowest-level system elements in the system hierarchy. The system element is made, bought, or reused.

Integration is the process of incorporating the lower-level system elements into a higher-level system element in the physical architecture.

Verification confirms that the system element meets the design-to or build-to specifications. It answers the question "Did you build it right?".

Validation answers the question of "Did you build the right thing".

Transition is the process applied to move ... the end-item system to the user.

Technical Management Processes

Decision Analysis provides the basis for evaluating and selecting alternatives when decisions need to be made.

Technical planning ensures that the systems engineering processes are applied properly throughout a system's life cycle.

Technical Assessment measures technical progress and the effectiveness of plans and requirements.

Requirements Management provides traceability back to user-defined capabilities.

Risk Management ... helps ensure program cost, schedule, and performance objectives are achieved at every stage in the life cycle and to communicate to all stakeholders the process for uncovering, determining the scope of, and managing program uncertainties.

Configuration Management is the application of sound business practices to establish and maintain consistency of a product's attributes with its requirements and product configuration information.

Data Management addresses the handling of information necessary for or associated with product development and sustainment.

Interface Management ensures interface definition and compliance among the elements that compose the system, as well as with other systems with which the system or system elements must interoperate.

Figure 5 - SE Activities (DoD, 2014)

\begin{tabular}{|c|c|c|c|c|c|c|c|c|c|c|c|c|c|c|c|c|}
\hline & \multicolumn{16}{|c|}{ Technical Management Processes } \\
\hline & 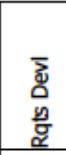 & 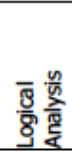 & 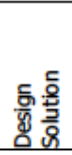 & 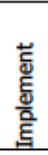 & 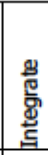 & $\frac{\grave{c}}{\grave{N}}$ & 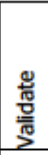 & 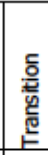 & 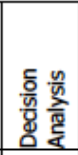 & 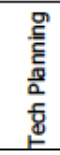 & 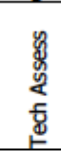 & 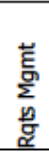 & $\begin{array}{l}\vec{E} \\
\frac{\sigma}{2} \\
\frac{.}{\underline{j}} \\
\end{array}$ & 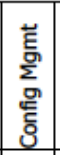 & 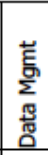 & 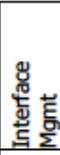 \\
\hline $\begin{array}{l}\text { Translating Capability } \\
\text { Objectives }\end{array}$ & $x$ & & & & & & & & & & & $x$ & $\mathrm{x}$ & $x$ & $x$ & \\
\hline $\begin{array}{l}\text { Understanding Systems \& } \\
\text { Relationships }\end{array}$ & & $x$ & & & & & & & & & & & $\mathrm{x}$ & $x$ & $x$ & $x$ \\
\hline $\begin{array}{l}\text { Assessing Performance to } \\
\text { Capability Objectives }\end{array}$ & & & & & & & $x$ & & $x$ & & $\mathrm{x}$ & & $\mathrm{x}$ & & $x$ & \\
\hline $\begin{array}{l}\text { Developing and Evolving an } \\
\text { SoS Architecture }\end{array}$ & $x$ & $\mathrm{x}$ & $\mathrm{x}$ & & & & & & $\mathrm{x}$ & $\mathrm{x}$ & & $\mathrm{x}$ & $\mathrm{x}$ & $x$ & $\mathrm{x}$ & $\mathrm{x}$ \\
\hline $\begin{array}{l}\text { Monitoring and Assessing } \\
\text { Changes }\end{array}$ & & & & & & & & & $x$ & & & & $\mathrm{x}$ & $\mathrm{x}$ & $x$ & $\mathrm{x}$ \\
\hline $\begin{array}{l}\text { Addressing Requirements and } \\
\text { Solution Options }\end{array}$ & $x$ & & $\mathrm{x}$ & & & & & & $x$ & $\mathrm{x}$ & & $\mathrm{x}$ & $\mathrm{x}$ & $x$ & $x$ & $x$ \\
\hline Orchestrating Upgrades & & & & $\mathrm{x}$ & $\mathrm{x}$ & $\mathrm{x}$ & $x$ & $x$ & $x$ & & $\mathrm{x}$ & $x$ & $\mathrm{x}$ & & $x$ & $\mathrm{x}$ \\
\hline
\end{tabular}

Figure 6 - SoSE mapping (US Department of Defense, 2008b) 
ISO/IEC/IEEE 15288:2015 Annex G - Application of system life cycle processes to a system of systems

The Systems and software engineering standard, ISO 15288 (British Standards, 2011) now includes an annex providing an informative overview of SOSE. The SoS classifications as defined in the DOD SOSE guide are re-iterated along with a brief description of emergence and the potential effects. The remainder of the annex discusses the relationship of SoS to the following processes:

- Agreement processes

- Organisational project-enabling processes

- Technical Management processes

- Technical processes

Agreement processes are described as the "modes of developmental and operational control among the organizations responsible for the SoS", it is also clarified that complete control of component systems is only possible for the directed SoS and that for acknowledged and collaborative SoS reciprocal co-operation is required and this becomes more informal for virtual SoS.

Organisational project-enabling and technical management processes are described as "planning, analyzing, organizing, and integrating the capabilities of a mix of existing and new systems into a SoS capability". It is also emphasised that the component systems development lifecycles are not necessarily synchronised and this is an issue that needs to be considered at the SoS level. Risk management is also identified as an activity that should be undertaken at the system and SoS level.

Technical processes include traditional SE activities, requirements analysis, architecture definition, design, implementation, verification, validation, operations, maintenance and disposal but applied at the SoS level.

15288:2015 Annex G currently provides only high-level descriptions of the SoS processes, which are generally replications of those performed at the system level but with general caveats stating that these also need to be carried out at the SoS level.

\section{DANSE Methodology}

The DANSE (Designing for adaptability and evolution in system of systems engineering) project "created and evaluated methodology and tools for SoS evolution and adaptation" (DANSE Consortium, 2015). 
A key aspect of DANSE is the development of a SoS modelling and simulation approach, which is discussed under theme 2 of this literature review. However, DANSE also proposes a SoSE lifecycle methodology, providing a more innovative approach than merely retargeting traditional SE activities at the SoS level, which is "driven by the realization that a SoS is constantly changing". An iterative, evolutionary approach is proposed consisting of whereby the lifecycle comprises three stages; SoS Initiation Phase, SoS Creation Phase and SoS Operations Phase.

During the Initiation phase the SoS comes into existence through the connection of a group of component systems enabling the sharing of information and resources. These interactions result in emergent behaviour at the SoS level. It is stated that the SoS "manager" will attempt to gain control over the SoS at this point either through bottom up consensus, or top down authority and will begin to identify goals, contracts and needs. However, with the exception of virtual SoS, it is noted a need will tend to arise requiring a "manager" to select a set of component systems to form an initial SoS rather than the SoS forming first which the DANSE description does not appear to consider, although it is accepted that an initial formation of the SoS will always occur.

The Creation Phase is described as optional and is required when the initial SoS emergent behaviour is severely deficient, requiring the SoS to be "re-envisioned and developed in a top-down approach". In this phase models of alternate architectures are produced in order to determine a solution, which provides the required emergent behaviour. The component systems are also modelled in order to establish any necessary changes to support the desired emergent behaviour, however there is an assumption that influence over these system configurations or design will be possible which is not necessarily the case.

In the Operational Phase the SoS operates providing the desired emergent behaviour, however the "manager" will monitor for additional potential needs and influence the component systems in order that the emergent behaviour is suitably modified. Again, this assumes influence over the development of the component systems is possible.

In addition to three lifecycle phases, the following six engineering activities are described:

- Model SoS behaviour

- Operate the SoS

- Define potential needs

- Analyse possible architecture changes

- Influence and Implement Changes 
A Model Based Approach to System of Systems Risk Management

- Constituent Systems engineering

Modelling SoS behaviour utilises the DoD Architectural Framework (DoDAF) to model the SoS architecture with SysML used to model component (constituent) systems. DoDAF and SysML are discussed within the paper (Kinder et al., 2014) attached in Annex C.

Operation of the SoS is described as running in parallel with modelling, potentially allowing prediction of emergent behaviour thereby providing to support in managing the SoS.

The definition of potential needs requires either SoS or component system capabilities to be updated in order to fulfil needs that arise.

Additional needs may impact the SoS architecture hence an activity is described which requires the modelling of architecture changes enabling evaluation of potential options.

It is acknowledged within the DANSE methodology that the SoS manager is unlikely to have the authority to force component systems owners to perform modifications, however it is assumed that it will be possible to influence these owners, with modelling artefacts providing support for this. As stated previously the "manager" may not even have any influence but may be restricted to the selection of component systems rather than modification.

Finally, the engineering of changes to the component systems themselves is required. These are not specifically a SoSE activity but are necessary where changes to the component systems are required in order to support a SoS emergent need and are embedded within the DANSE lifecycle.

\section{COMPASS}

COMPASS (COMPASS Consortium, 2014a) is a, now complete, European Union project, which is intended to provide "Comprehensive Modelling and Analysis for Advanced Systems of Systems". The modelling aspect of the COMPASS project is discussed under theme 2 of the literature review in section 3.3.7, however a SOSE approach is also proposed comprising an Ontology, Architectural Framework and Process Library.

The Ontology "defines the concepts relevant to the approach, along with the relationships between these concepts" and covers the following areas:

- SoS Requirements

- Process and Competency 
- Architectures and Architectural Frameworks

- SoS Integration

- Traceability

- Refinement

The COMPASS Architectural Framework comprises Viewpoints, which allows creation of views, in combination with ontology elements, providing visualisation of the SoS architecture. The Process Library comprises processes, which address particular aspects of SoSE.

The COMPASS approach to SoSE considers the traditional SE lifecycle (Concept Development, Development, Production, Utilization, Support and Retirement (INCOSE, 2015)) and suggests that SoSE is only applicable at the concept development stage and for integration within the development stage. Specifically, operational requirement identification and potential architecture definition are undertaken at the concept development stage, and test scenario development, constrained by individual component system design decisions as part of the integration activity, underpinned by a Model Based Systems Engineering (MBSE) approach (COMPASS Consortium, 2014b). This is in contrast to the DANSE approach, which does not attempt to consider SoSE activities in the context of the traditional SE lifecycle but rather from a purely SoS viewpoint through the proposed iterative SoS lifecycle.

COMPASS also provides a provides a maturity, lifecycle definition that is applicable to SoS and component systems, as follows:

- New: requires initial development, no current capabilities.

- Middle-Life: enhance a current SoS or develop a new SoS utilising current capabilities.

- Aging: existing SoS requiring major restructuring and change to component systems. These classifications are also applied at the component systems level, providing a greater understanding of the extent of collaboration and flexibility between the systems comprising the SoS. A key risk to SoS development is the effect of asynchronous development of the component system on the overall SoS capabilities; distinguishing between the different maturity states enables this impact to be modelled and hence, assessed. 
A method of classifying component systems is also provided, enabling the degree of openness towards integration into the SoS to be modelled, thereby enabling the general level of SoS agility and adaptability to be assessed. The classifications are as follows:

- Closed: the component system is integrated into the SoS with no changes to the component system itself.

- Legacy: systems that have been in existence for a "long" period and may comprise out-dated technology; these are treated as closed systems.

- Extensible: system allows dynamic installation of components, e.g. plug-ins.

- Open: changes may be made to the component system.

- Forbidden: system provides interfaces that cannot be used.

\section{$\underline{\text { SoS Characteristics Operational Management Matrix (SoSOMM) }}$}

The SoSOMM approach (Gorod et al., 2007) is based upon the ISO Network Management Model (International Organization for Standardization, 1989), which comprises the following five areas defining terminology, structure and activities required for the management of netcentric systems:

- Fault Management: detection and correction of abnormal operation.

- Configuration Management: identifies, controls, provides data to/from open systems enabling operation and termination of interconnection services.

- Accounting Management: establishes charges and costs of use.

- Performance Management: enables effectiveness of services to be evaluated.

- Security Management: supports the application of security policies.

Whilst these areas, referred to as FCAPS, are intended for the management of IT systems, there are clear similarities to the management of a SoS; both being distributed systems. The SoSOMM approach maps FCAPS to be following areas, which are generalised for SoS applicability:

- Risk Management: monitor, identify, assess, analyse and mitigate SoS risk.

- Configuration Management: carry out command and control, structural and software management.

- Resource Management: provide usage information of Systems' resources within the SoS. 
- Performance Management: monitor and measure performance of individual systems for overall SoS performance to be maintained at the appropriate level.

- Policy Management: provide SoS access to authorised processes and protect SoS from illegal access.

Having established the five areas applicable to SoS, the management matrix is then constructed using the Boardman-Sauser SoS characteristics (Boardman \& Sauser, 2006) (as discussed in section 3.2.4), as illustrated in Figure 7 (Gorod et al., 2007). However, whilst this appears a novel approach it does not appear to have been developed further since publication in 2007.

\begin{tabular}{|c|c|c|c|c|c|}
\hline $5^{5^{N}}$ & $\begin{array}{c}\text { Risk } \\
\text { Management }\end{array}$ & $\begin{array}{l}\text { Configuration } \\
\text { Management }\end{array}$ & $\begin{array}{l}\text { Performance } \\
\text { Management }\end{array}$ & $\begin{array}{c}\text { Policy } \\
\text { Management }\end{array}$ & $\begin{array}{c}\text { Business } \\
\text { Management }\end{array}$ \\
\hline Autonomy & $\begin{array}{l}\text { Preserves } \\
\text { autonomous } \\
\text { capabilities }\end{array}$ & $\begin{array}{c}\text { Governs autonomous } \\
\text { behavior }\end{array}$ & $\begin{array}{l}\text { "Satisficing" } \\
\text { constituent system } \\
\text { performance in order } \\
\text { to meet SoS goals }\end{array}$ & $\begin{array}{l}\text { Ensures security of } \\
\text { constituent systems }\end{array}$ & $\begin{array}{l}\text { Provides information } \\
\text { on autonomous } \\
\text { resources }\end{array}$ \\
\hline Belonging & $\begin{array}{l}\text { Provides } \\
\text { environmental } \\
\text { perspective }\end{array}$ & $\begin{array}{c}\text { Unifies } \\
\text { configuration of } \\
\text { constituent systems }\end{array}$ & $\begin{array}{c}\text { Allows cost/benefit } \\
\text { analysis }\end{array}$ & $\begin{array}{l}\text { Restricts/allows } \\
\text { constituent systems } \\
\text { access to the SoS }\end{array}$ & $\begin{array}{l}\text { Provides information } \\
\text { on constituent } \\
\text { system functions }\end{array}$ \\
\hline Connectivity & $\begin{array}{l}\text { Maintains acceptable } \\
\text { integration }\end{array}$ & $\begin{array}{c}\text { Establishes and } \\
\text { maintains consistency } \\
\text { in integration }\end{array}$ & $\begin{array}{l}\text { "Satisficing" to } \\
\text { maximum } \\
\text { capability }\end{array}$ & $\begin{array}{l}\text { Protects against } \\
\text { unauthorized } \\
\text { integration }\end{array}$ & $\begin{array}{l}\text { Tracks connected } \\
\text { resources }\end{array}$ \\
\hline Diversity & $\begin{array}{c}\text { Evaluates } \\
\text { diverse activities }\end{array}$ & $\begin{array}{l}\text { Governs diverse } \\
\text { behavior }\end{array}$ & $\begin{array}{c}\text { "Satisficing" } \\
\text { diverse activities }\end{array}$ & $\begin{array}{l}\text { Restricts/allows } \\
\text { diverse behavior }\end{array}$ & $\begin{array}{l}\text { Provides information } \\
\text { on inventory of } \\
\text { diverse resources }\end{array}$ \\
\hline Emergence & $\begin{array}{l}\text { Verifies and validates } \\
\text { achievement of new } \\
\text { behaviors }\end{array}$ & $\begin{array}{l}\text { Shapes and bounds } \\
\text { emergent behavior }\end{array}$ & $\begin{array}{l}\text { Permits "Satisficing" } \\
\text { path of emergence }\end{array}$ & $\begin{array}{c}\text { Influences emergent } \\
\text { behavior }\end{array}$ & $\begin{array}{l}\text { Tracks emergent } \\
\text { capabilities }\end{array}$ \\
\hline
\end{tabular}

Figure 7 - SoSOMM (Gorod et al., 2007)

Whilst Keating et al (Keating \& Katina, 2011) argue that SoSE cannot be progressed without establishing a sound theoretical foundation Dahman and Roedler (2016) take a more pragmatic approach stating that as "current standards and other authoritative references provide a strong foundation for the SOSE fundamentals" a move towards SoSE 
standardisation is now possible. The argument for the development of a sound theoretical basis for SoSE is persuasive, however the engineering of real world SoS has been on going for many years hence SOSE approaches have been developed, admittedly in a somewhat uncoordinated manner, out of necessity. As a theoretical approach emerges, it is anticipated that current approaches will be re-evaluated against and, through an iterative process, be refined until the practical approaches eventually align with the theoretical underpinning. At this point, it is emphasised that this research has been undertaken using a sound methodological approach which is described in detail in chapter 4.

\subsubsection{Summary-Theme 1}

This section provides background information regarding system theory and engineering. References to systems theory are made both in the support of SE and SOSE, for example "holism" is particularly applicable to SoSE risk management, which requires a holistic approach.

Many definitions of a SoS exist and have evolved as understanding of the domain has progressed, the preferred definition for this research, which was recently proposed, is:

"A SoS is a system which results from the coupling of a number of constituent systems at some point in their life cycles" (Brook, 2016).

There does not appear to be a generally agreed definition for SoSE, with many being domain specific, however the T-Area SoS has proposed the following, which can be applied generically and is therefore appropriate for this research:

The evolving, designed combination of systems to form a system of systems in networks that are safe, secure, efficient and able to respond to changing requirements and operational situations (Barot et al., 2013).

The SoS classifications; directed, acknowledged, collaborative and virtual (Dahmann \& Baldwin, 2008) are now well established and are referenced throughout this research, although a SoS classification may evolve and parts of a SoS may simultaneously have different classifications.

Several SoSE methodologies are reviewed, Risk Management is identified as a key element of SoSE however, although it is agreed a holistic approach is required, a detailed approach is not provided in any of the surveyed literature, indicating that this is a gap in current knowledge. 
It is also noted that within the period of this research, definitions and approaches have evolved, for example the preferred SoS definition was proposed in 2016, which emphasises the current immaturity of SoSE.

The output of the theme 1 literature review demonstrates the necessity for the development of a SoS Risk Management approach and identifies activities and lifecycle phases where it should be applied.

\subsection{Theme 2: Modelling and Simulation}

Theme 2 of the literature review discusses the general nature of M\&S and seeks to establish a set of definitions, to which later sections refer back, as well as providing a deeper understanding of the philosophy behind the need for models and their use. The bulk of this part of the literature review consists of a survey, examining a wide range of M\&S techniques and their applicability to the SoS domain, the results of it were published in the International Journal of System of Systems (Kinder et al., 2014), and is included as Annex C to this thesis. The section also reviews hybrid modelling, Model Based Systems Engineering (MBSE), SoS specific modelling techniques and M\&S Verification and Validation (V\&V), concluding with a general summary.

\subsubsection{Background}

The are numerous definitions for a model; for example " $A$ model is a physical, mathematical or otherwise logical representation of a system, entity, phenomenon or process" (US Department of Defense, 2008a), “An approximation, representation, or idealization of selected aspects of the structure, behavior, operation, or other characteristics of a real-world process, concept, or system" (IEEE, 1989) and "a representation of one or more concepts that may be realized in the physical world" (Friedenthal et al., 2009). However, all definitions can essentially be distilled down to:

"A model is a representation of a system or process."

The term "simulation" is directly related to "model"; although somewhat inconsistently defined throughout literature, a common theme running through the many definitions is the generation of dynamic behaviour over time, e.g. "A method for implementing a model over time" (US Department of Defense, 2008a), "A model that behaves or operates like a given system when provided a set of controlled inputs" (IEEE, 1989), and "the implementation of a model (or models) in a specific environment that allows the model's execution (or use) over time" (INCOSE, 2015). 
A Model Based Approach to System of Systems Risk Management

Although the terms "model" and "simulation" are frequently used interchangeably, indeed the term "simulation model" is sometimes used (Carson, 2005), within this thesis where reference is made to "dynamic model" this can be regarded as equivalent to "simulation", i.e. the model executes over time. It should also be noted that some modelling techniques only provide meaningful results when executed; for example the Agent Based Modelling and Simulation (ABMS) approach requires the definition of a set of autonomous agents, however it is only when the model is executed that the agents interact and emergent behaviour is observable.

Different models (or simulations) and modelling techniques may be classified through the following key characteristics (Fritzson, 2010; Kinder et al., 2014) and is "useful for selecting the right type of model for the intended purpose and scope" (SEBoK, 2017):

- Continuous: "A mathematical or computational model whose output variables change in a continuous manner" (US Department of Defense, 2008a)

- Discrete: a model "has entities only possessing one of many values within a finite range" (Sulistio et al., 2004)

- Deterministic: "no random events occurring, so repeating the same simulation will always return the same simulation results" (Sulistio et al., 2004)

- Stochastic (Probabilistic): "random events occurring, so repeating the same simulation often returns different simulation results" (Sulistio et al., 2004)

- Static: "model of a system in which there is no change" (US Department of Defense, 2008a)

- Dynamic: "model of a system characterized by continuous change, activity or progress" (US Department of Defense, 2008a), "time as part of the simulation" (Sulistio et al., 2004)

Kinder et al (2014) (attached as Annex C) provide another perspective through the definition of a set of group classifications ${ }^{5}$, which are listed below and include reference to specific modelling techniques:

\section{- Graph-based models to create structure:}

- Enterprise Architecture Framework

- Modelling Languages

○ Petri Nets

\footnotetext{
${ }^{5}$ These are inspired by consideration of SoS, whereas the preceding classifications are more general.
} 
- Inference testing for consistency, patterns, and completeness of models above:

- Artificial Neural Networks

- Network Models (Graph Theory)

- Probabilistic simulation based on Directed Acyclic Graphs:

- Bayesian Belief Networks

- Markov Models

- Decision Trees

\section{- System simulation:}

- Discrete Event Simulation

- Discrete Event System Specification (DEVS)

- Agent-Based Modelling and Simulation

○ System dynamics

- Surrogate Models

- Game Theory

\subsubsection{The Philosophy of Models}

All effective models are required to fulfil a specific purpose. Considering scientific models, their purpose is to deductively or inductively support or disprove a theory. Hesse (1963) suggested that in fact, without models, theories cannot fulfil all functions associated with them and cannot be genuinely predictive, hence theory and model are inextricably bound; the model is the theory.

Hesse also identified some fundamental aspects of models, through discussion of positive, neutral, negative analogies. Using billiard balls as the basis for a model of the dynamical theory of gases, properties that are specific to the billiard balls but have no relevance to the model, such as colour, are described as negative analogies. Properties that are specific to the model and are directly analogous to the system under consideration are positive analogies; an example in this case is the billiard ball properties of impact and motion. Properties of the model that are not determined as positive or negative are deemed neutral analogies and are considered to be those characteristics that may result in new discovery. Whilst this discussion is more related to scientific theories it is also applicable to more general modelling; indeed having identified negative analogies within a model it may be possible to remove some capability thereby reducing complexity without diminishing the models effectiveness. The concept of neutral analogies is also applicable in the systems modelling domain, in that these analogies may reveal unexpected emergent behaviour; for 
A Model Based Approach to System of Systems Risk Management

example fluid dynamics modelling has been applied to traffic flow analysis, the understanding of the similarities between these domains, i.e. the analysis of the neutral analogies, has resulted in new theories related to traffic management (Bretti et al., 2007).

Analogical reasoning will generally support an inductive argument whereby if the premises are true then it is unlikely the conclusion is false (Bartha, 2016). When considering system models, it is likely that the majority will also support inductive argument, as the models will be abstractions of the system under consideration where the abstractions are themselves determined through analogical reasoning. Indeed, systems models may also contain stochastic elements; therefore likelihood is associated with the model output, which supports an inductive, rather than deductive, argument.

Hesse (1963) also makes the distinction between formal and material analogies; a formal analogy occurs when there is a direct correspondence between interpretations of the same formal theory. A material analogy describes "pre-theoretic analogies between observables" such as pitch (property of sound) and light (property of light), where similarities are observed before derivation of a theory, and is necessary when a theory is required to have:

1. Strong falsifiability or predictive power (i.e. identifies relations between new observation predicates)

2. Justification in terms of choice criteria for selecting theories, which depend on models in (1).

Therefore, from the discussion above it can be concluded that a model is essential where a theory is based upon a material analogy; the model is necessary in order to provide the necessary "falsifiability or predictive power".

Three kinds of theories (and hence models) are identified:

1. Formal theories (mathematical models) which are weakly predictive and describe a set of observations but does not predict unobserved behaviour or correlations between them, an example of this is a mathematical model, that describes a hypothesis designed to fit a set of experimental data.

2. Conceptual models which are strongly predictive but not justified by further choice criteria. A conceptual model suggests interactions and correlations between observations, which are the result of neutral analogies, however the model is "imaginary" as the predictions are not based upon current theory. 
3. Material analogue-models which are strongly predictive and justified by further choice criteria, namely similarity (horizontally) and causality (vertically), as illustrated in the example below:

$\begin{array}{lll}\text { Sound waves } & - & \text { Light waves } \\ \text { Loudness } & - & \text { Intensity } \\ \text { Pitch } & - & \text { Colour }\end{array}$

Although the discussion above relates to scientific modelling, parallels can be drawn with system modelling. For example a static model representing a SoS using MODAF viewpoints (MOD, 2012) could be described as a "formal model" in that it is not predictive and describes currently held knowledge (or data set).

An agent-based model could be considered as a conceptual model, in the sense of the description above; it predicts emergent behaviour but the causal interactions between the agents are not necessarily understood. This is in contrast with a Discrete Event Simulation; in which all interactions between elements within the model have to be understood in order for the model to be implemented and could therefore be likened to a material analogue model. These viewpoints provide an alternative approach to model classification, through the consideration of potential predictability and the level of understanding of causality.

Box (Box, 1954) discussed the use of scientific models from a different perspective, arguing that "parsimony" is a critical attribute. A model will be comprised of a set of parameters, where the number of these is small the model is said to be parsimonious. This is desirable because unnecessary complexity can obscure the "truth", greater precision generally results and "indiscriminate elaboration" can become never ending. Box argued that parsimonious models are necessary approximations and could therefore be considered "wrong", hence his widely quoted statement "All models are wrong but some are useful". Sterman (Sterman, 2002) extends this stating, "All decisions are based on models, and all models are wrong", and in the context of mental models, is arguing that human perception is based upon mental models which are simplifications, abstractions and therefore incomplete and incorrect. In order to become less reliant upon mental models, more sophisticated externally constructed models are necessary providing a common reference, rather than a collection, of perhaps contradictory perceptions, thereby providing a general justification for the use of models. 
A Model Based Approach to System of Systems Risk Management

\subsubsection{Why model?}

As discussed above the primary perceived use of models is to predict behaviour, however there are many other potential uses as identified by Epstein (2008):

\section{Explain (very distinct from predict)}

2. Guide data collection

3. Illuminate core dynamics

4. Suggest dynamical analogies

5. Discover new questions

6. Promote a scientific habit of mind

7. Bound (bracket) outcomes to plausible ranges

8. Illuminate core uncertainties.

9. Offer crisis options in near-real time

10. Demonstrate tradeoffs / suggest efficiencies

11. Challenge the robustness of prevailing theory through perturbations

12. Expose prevailing wisdom as incompatible with available data

13. Train practitioners

14. Discipline the policy dialogue

15. Educate the general public

16. Reveal the apparently simple (complex) to be complex (simple)

Although Epstein discusses the use of models from a social science perspective the uses he identifies above are equally applicable in the engineering, and hence SoSE, domain. One of his key points is the differentiation of explanation from prediction; from a SoS perspective a model may provide understanding of the interactions between component systems rather than predict a timeline of events, which could be dependent upon availability of these systems and other factors which may be unknown. "Explanation" can also be viewed as "characterising an existing system" (INCOSE, 2015) whereby a potentially poorly documented system is modelled, providing a clear baseline understanding thereby enabling modifications to be undertaken more effectively. Models are also effective throughout all phases of the system development lifecycle including requirements, architecture and design definition as well as verification, validation and operational support (INCOSE, 2015).

Another essential use of models, particularly within engineering, is to provide a medium of communication between all stakeholders associated with a project. For example, a highly 
A Model Based Approach to System of Systems Risk Management

technical or mathematical concept may require discussion however the stakeholders will likely include non-technical end users, hence a model providing a visual element provides common understanding at all levels and thereby facilitates meaningful dialogue.

Models also provide technical and management metrics, within the SE (and SOSE) these may provide the following support (SEBoK, 2017):

- Assess progress;

- Estimate effort and cost;

- Assess technical quality and risk;

- Assess model quality.

\subsubsection{Review of Modelling Techniques}

A substantial part of the literature review for this theme involved the survey of a range of modelling techniques in the context of their applicability to SoS modelling. The result of this review was published in the International Journal of SOSE (Kinder et al., 2014) and is included in its entirety within Annex B of this thesis.

A key output from this survey was a matrix which identified specific M\&S techniques and identified their suitability (low or high) in relation to a set of SoS System of Interest "dimensions" as proposed by Kinder et al (2012); this forms the basis of a technique selection approach and is elaborated further in chapter 6 .

Included, for reference, is the summary table and explanatory text from this paper:

Table 7 summarises the suitability of the assessed M\&S techniques to more general SoS modelling, considering all SoS classifications and all phases of the lifecycle. This demonstrates that some bottom up techniques, such as DEVS, Petri Nets and ABMS, whilst being highly applicable to the case study, which was concerned with the operation (or execution) phase, are less appropriate throughout the lifecycle. These techniques are also less suited to SoS with higher variability. This is because individual component systems are modelled; as a result if these systems change in the SoS lifetime then the model becomes redundant, exhibiting low resilience to variability. However, top down techniques, such as System Dynamics are generally used to model more strategic dimensions rather than individual component systems and hence are more resilient to variability. 
A Model Based Approach to System of Systems Risk Management

\begin{tabular}{|c|c|c|c|c|c|c|c|c|c|}
\hline & $\begin{array}{l}\text { Compon- } \\
\text { ent } \\
\text { Systems }\end{array}$ & $\begin{array}{l}\text { Inter- } \\
\text { actions }\end{array}$ & Lifecycle & $\begin{array}{l}\text { Variab- } \\
\text { ility }\end{array}$ & $\begin{array}{l}\text { Classifi } \\
\text {-cation }\end{array}$ & $\begin{array}{l}\text { Func- } \\
\text { tions }\end{array}$ & $\begin{array}{l}\text { System } \\
\text { Owners \& } \\
\text { Operationss }\end{array}$ & $\begin{array}{l}\text { Concept } \\
\text { of Oper- } \\
\text { ation }\end{array}$ & $\begin{array}{l}\text { Relation- } \\
\text { ship }\end{array}$ \\
\hline $\begin{array}{l}\text { Discrete Event } \\
\text { Simulation }\end{array}$ & HIGH & HIGH & LOW & LOW & LOW & HIGH & LOW & LOW & HIGH \\
\hline Petri Nets & $\mathrm{HIGH}$ & $\mathrm{HIGH}$ & LOW & LOW & LOW & LOW & LOW & LOW & $\mathrm{HIGH}$ \\
\hline Agent Based Model & HIGH & $\mathrm{HIGH}$ & LOW & LOW & LOW & $\mathrm{HIGH}$ & LOW & LOW & LOW \\
\hline System Dynamics & LOW & LOW & $\mathrm{HIGH}$ & $\mathrm{HIGH}$ & $\mathrm{HIGH}$ & LOW & $\mathrm{HIGH}$ & $\mathrm{HIGH}$ & LOW \\
\hline Surrogate Models & $\mathrm{HIGH}$ & LOW & $\mathrm{HIGH}$ & LOW & LOW & LOW & LOW & LOW & LOW \\
\hline $\begin{array}{l}\text { Artifical Neural } \\
\text { Network }\end{array}$ & LOW & LOW & $\mathrm{HIGH}$ & HIGH & HIGH & $\mathrm{HIGH}$ & LOW & LOW & LOW \\
\hline $\begin{array}{l}\text { Bayesian Belief } \\
\text { Network }\end{array}$ & LOW & LOW & HIGH & $\mathrm{HIGH}$ & HIGH & LOW & HIGH & $\mathrm{HIGH}$ & LOW \\
\hline Markov Models & LOW & LOW & $\mathrm{HIGH}$ & $\mathrm{HIGH}$ & $\mathrm{HIGH}$ & LOW & $\mathrm{HIGH}$ & $\mathrm{HIGH}$ & LOW \\
\hline Game Theory & LOW & LOW & $\mathrm{HIGH}$ & $\mathrm{HIGH}$ & $\mathrm{HIGH}$ & LOW & HIGH & $\mathrm{HIGH}$ & LOW \\
\hline Decision Trees & LOW & LOW & $\mathrm{HIGH}$ & $\mathrm{HIGH}$ & $\mathrm{HIGH}$ & LOW & LOW & LOW & LOW \\
\hline Network Models & LOW & HIGH & LOW & LOW & $\mathrm{HIGH}$ & LOW & LOW & LOW & $\mathrm{HIGH}$ \\
\hline $\begin{array}{l}\text { Enterprise } \\
\text { Architecture } \\
\text { Framework }\end{array}$ & HIGH & HIGH & HIGH & LOW & HIGH & LOW & HIGH & HIGH & HIGH \\
\hline $\begin{array}{l}\text { Modelling } \\
\text { Languages }\end{array}$ & HIGH & HIGH & LOW & LOW & LOW & HIGH & HIGH & HIGH & HIGH \\
\hline
\end{tabular}

Table 7 - SoS M\&S Technique Suitability

It should be noted that this M\&S review did not take a traditional approach, instead a framework enabling the comparison of techniques and their applicability to SoS was developed. Hence, the framework itself, as well as the survey results, is considered to be an additional research output.

\subsubsection{Hybrid Modelling Approaches}

Hybrid modelling involves the combination of a least two modelling techniques or methodologies within a single model. The appropriate M\&S technique, or combination thereof, is driven by the context and the problem space (Kinder et al., 2014), however even though a wide range of potential techniques are available, most hybrid approaches only combine two or three different techniques as illustrated in the following examples.

Teose et al (2007) propose embedding System Dynamics (SD) within Agent Based Models (ABM) to model Complex Adaptive Systems, the rationale for this approach being; "heterogeneity allows for the simulation of novel, complex intervention strategies at the 
level of agents that might otherwise be difficult or impossible to express succinctly in system dynamics terminology".

Ross et al (2014) define three SoS views, Social, Physical and Social-Physical. For the social view "the modeller is interested in individual attributes and because the interactivities at a given time within the social level are unknown" $A B M$ is the suggested approach. The physical view concerns "engineered systems with known interactions", for which Discrete Event Simulation (DES) is appropriate, and for the Social-Physical "known system-level interactions between the physical and social constituents" are modelled which is suitable for SD modelling. The combination of these views provides a holistic SoS modelling approach.

DES and SD have also been integrated within a hybrid business simulation approach (Rabelo et al., 2005) intended to support policymaking and control. It is recognised that business enterprise modelling requires support for both long-term strategic decision-making and short-term detailed analysis. The proposed approach utilises SD for the strategic modelling, e.g. corporate growth, and DES for short-term analysis, e.g. plant operations.

Lynch et al (2014) describe a "multi-paradigm modelling framework" which defines three levels of granularity, macro, meso, and micro. These levels relate to the knowledge of the problem domain and what assumptions are made, the framework uses these levels to indicate the whether SD, DES or ABM is to be used for specific parts of the model.

A different approach is taken by Macharis (2000) who proposes a framework that "blends" SD modelling with Multi-Criteria Decision Aid (MCDA). MCDA enables complex decision rules to be implemented, which are dependent on specific variables; Macharis suggests that these rules are embedded within the SD models, thereby enabling development of dynamic multicriteria analysis models.

Whilst the approaches described above enable hybrid modelling approaches through the combination of a limited number of techniques Kinder et al (2014) propose a modelling approach, initially intended to support SoS Risk Management, which enables a wide range of heterogeneous techniques to be combined into a single model through integration into a central Bayesian Belief Network; this approach is described fully in section 7.4. This technique moves away from the "one size fits all" approach often suggested and allows appropriate techniques to be utilised for the specific context and problem space, which is necessary to fully support SoS modelling. 


\subsubsection{Model Based Systems Engineering (MBSE)}

Although traditional SE approaches will generally make use of models throughout the lifecycle to some extent, MBSE "is the formalized application of modelling to support system requirements, design, analysis, verification and validation activities beginning in the conceptual design phase and continuing throughout development and later life cycle phases" (INCOSE, 2007). MBSE ensures that project models are coordinated between lifecycle phases and disciplines, promoting the use of models rather than traditional documentation where appropriate, for example requirements definition. SysML (Object Management Group, 2016a) is the modelling language, which is promoted for use within the process, with MoDAF or DoDAF used to provide a SoS modelling capability (Lochow, 2016). MBSE could be applied to support the engineering of directed SoS however, without a dynamic capability the prediction of emergent behaviour is limited. MoDAF and DoDAF are widely used throughout the defence industry to support SoSE activities, but again the lack of dynamism is restrictive. As MBSE does not provide a hybrid modelling approach or a mechanism to select and implement heterogeneous models it's application to SoSE must be considered limited.

\subsubsection{Review of SoS Modelling Techniques}

As discussed above (section 3.3.6), MBSE does not provide a capability to fully support all SoSE activities; the COMPASS and DANSE methodologies were developed to overcome this shortcoming and are discussed below.

The COMPASS project (COMPASS Consortium, 2014a) goal was to provide an approach to SoS modelling, which could be utilised throughout a SoS, overcoming the inherent managerial and operational dependence. As part of this approach the COMPASS Modelling Language (CML) was developed, specifically for use in the context of SoS, enabling representation of both constituent systems and the architecture composing the SoS. Although CML is based upon several current languages, Vienna Development Method, Communication Sequential Processes and Circus (a concurrent language for refinementbased reasoning for modern systems) it is an entirely new language. Whilst a language entirely focussed upon SoS modelling does provide obvious advantages, the fact that it is new, and hence unknown, may limit its uptake with practitioners tending to rely on more familiar, proven approaches. Although CML enables very detailed models of SoS to be created, the amount of information required in order to provide this detail implies that $\mathrm{CML}$ 
will tend to be restricted to modelling directed and acknowledged SoS where there are less associated unknowns.

The DANSE methodology (DANSE Consortium, 2015) takes a different approach to COMPASS, generally utilising available modelling techniques and tools, providing extensions and plug-ins where necessary, although a goal contracts specification language (GCSL) is developed. The Unified Profile for DoDAF and MoDAF (Object Management Group, 2016b) is used at the architectural level with SysML modelling the constituent systems. Joint modelling allows different models and simulations to be combined, allowing the incorporation of dynamic models (e.g. Modelica, Simulink) into the overall model. GCSL enables automatic statistical checking of the joint model. Overall DANSE provides a comprehensive SoS modelling capability although it is explicitly stated that the methodology is only applicable to Directed, Acknowledged, and Collaborative SoS types, which is necessary because a SoS "controller" is required to co-ordinate the DANSE process. However, as is the case with COMPASS, extensive knowledge of the component system interactions is required to enable the detailed modelling required.

\subsubsection{Validation and Verification of Models}

General definitions of Validation and Verification are first provided in order to avoid any misunderstanding, as these terms are not always used consistently.

Validation "ensures a system is able to accomplish its intended use, goals and objectives (i.e., meet stakeholder requirements) in the intended operational environment. The right system is built" (British Standards, 2011).

Verification is "a set of activities that compares a system or system element against the required characteristics. This includes, but is not limited to, specified requirements, design description and the system itself. The system is built right" (British Standards, 2011).

As discussed in section 3.3.2, it is widely accepted that "all models are wrong but some are useful" (Box, 1954). This appears to result in a dichotomy, how can an entity be validated and verified to determine "correctness" when it is intrinsically wrong? However, "wrong" could be interpreted as "inaccurate" which then infers a model will possess a level of accuracy, in which case the aim of $V \& V$ is to ensure a model is sufficiently accurate, or provides sufficient fidelity and resolution, in relation to its purpose (Robinson, 1997).

The understanding and definition of a models' purpose is critical in determining the level of accuracy required and hence this will bound the necessary $V \& V$ activities. As shown below in 
Figure 8 (Sargent, 2015) the value of a model is related to the confidence a user has in it, which is provided through $V \& V$, however there is a trade-off between the level of confidence and associated cost, which should be considered. Indeed, the outcome of model V\&V should not result in a model deemed as absolutely incorrect or correct, but rather as a degree of credibility (e.g. on a 0 to 100 scale) (Balci, 1995).

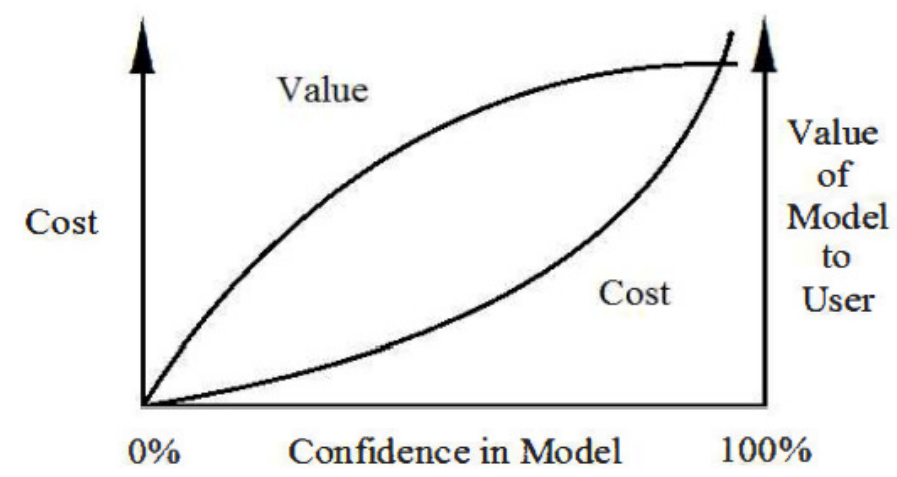

Figure 8 - Confidence in Models (Sargent, 2015)

The approach generally taken when undertaking model $V \& V$ is through the formulation of a simulation study, "which starts by developing an understanding of the real world and the problem to be tackled. Following this a model is developed, first as a conceptual model and then as a computer model" (Robinson, 1997). Figure 9 (Robinson, 1997) illustrates model $V \& V$ activities and their relationship to the modelling process, noting that the following differing types of validation are undertaken:

- Conceptual Model Validation: determine the scope and level of detail that supports the model purpose and ensure assumptions are correct.

- Data Validation: data required for model implementation, validation and experimentation are sufficiently accurate.

- White-box Validation: a "micro" check, ensuring the constituent parts of the computer model represent the real world with sufficient accuracy, in relation to the purpose.

- Black-box Validation: a "macro" check ensuring the model as a whole represents the real world with sufficient accuracy, in relation to the purpose. 


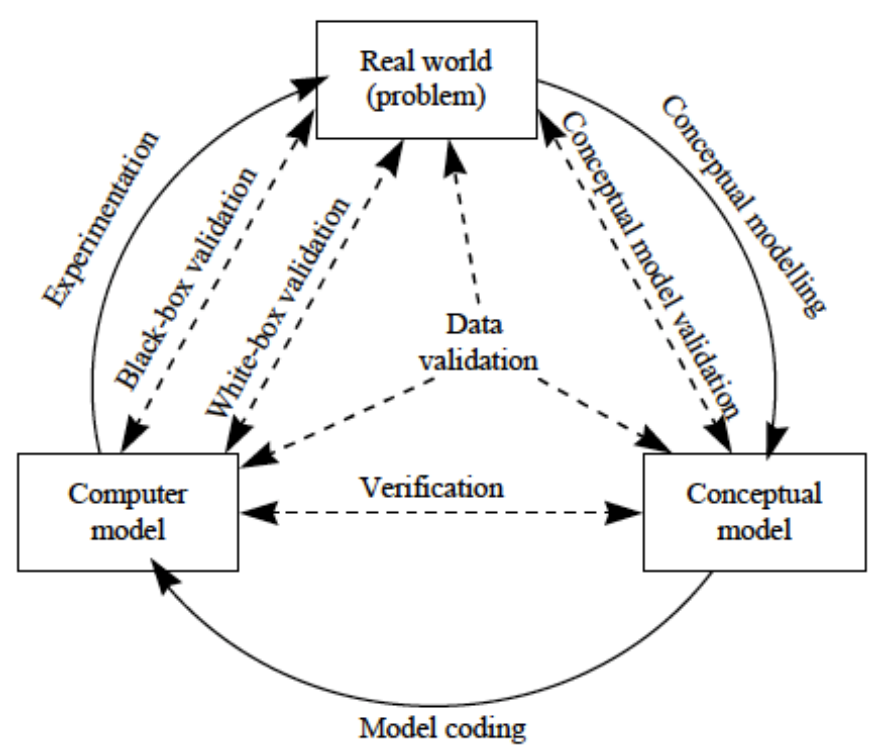

Figure 9 - Model V\&V in the Modelling Process (Robinson, 1997)

Although the activities described above are intended to enable model $V \& V$, there are several difficulties that require consideration. One approach to $V \& V$ is the comparison of a model with the real world system it represents, however it is often the case that the actual system does not yet exist (Robinson, 1997). A further complication is the differing perceptions of reality held by different individuals, and which may be dependent upon their role or personal bias; hence a model may be valid to one individual and not another (Law, 2009; Robinson, 1997). Where a real system or data is available it cannot always be assumed it is valid, for example a small sample size may reduce its legitimacy (Law, 2009; Robinson, 1997), it must also be borne in mind that model prediction based on historical data does not necessarily guarantee accuracy, and may in fact be misleading (Taleb, 2007).

Validation of the conceptual model involves determination that the underlying assumptions and theories are correct and that the structure, causal relationships and representation of the problem entity is reasonable (Sargent, 2015). Mathematical and statistical analysis, e.g. fitting distributions to data, may be used to validate the theories and assumptions whereas determination of a reasonable representation will generally be through expert analysis (Sargent, 2015).

Model verification will generally require a static or dynamic testing approach (Sargent, 2015); static testing involves walk through checking of implementation code whereas dynamic testing involves executing the model with a range of input values and confirming the output is as expected, or at least appears reasonable. The key objective of model verification is the confirmation that the implemented model is "true" to the conceptual 
model (Robinson, 1997). White box testing is subtly different in that it confirms the model is "true to the real world" (Robinson, 1997).

Black box testing considers the overall model behaviour and will employ a dynamic testing approach whereby, with specific input conditions, the model output is compared with real system outputs, or if this is not available, with either expected output as determined from expert matter input or other models (Robinson, 1997).

These methods of model V\&V illustrate that an accepted approach is the use of expert opinion to assess model accuracy and hence value, throughout all stages of model development. This raises an important point; although models will provide quantitative output it should be understood that the underlying implementation is likely to be underpinned by a subjective element, i.e. subject matter expert opinion.

\subsubsection{Summary}

Section 3.3 established a set of basic definitions for a model, which is a representation of a system or process and a simulation is a model, which executes over time.

The following set of model characteristics were identified and are used within the SoS M\&S technique selection framework as described in the journal paper (Kinder et al., 2014) attached in Annex B:

- Continuous

- Discrete

- Deterministic

- Stochastic (Probabilistic)

- Static

- Dynamic

The philosophy of modelling was discussed, providing a theoretical foundation to the M\&S aspects of this research, in particular positive, neutral, negative analogies (Hesse, 1963) are used to classify characteristics of models that are relevant to the theory (or question) a model supports. The following types of model are identified: Formal theories (mathematical models), Conceptual models and Material analogue-models which are classified in accordance with their predictive capabilities and their ability to be derived from a similar model (or theory). 
Many uses of modelling are identified however, key are prediction and explanation, which are both required in support of Risk Management.

A survey of M\&S technique applicability to SoS was undertaken, the results of which were published in the International Journal of SoSE (Kinder et al., 2014) and is attached in Annex $A$, and which also proposed a M\&S method selection framework.

Modelling SoS requires the use of appropriate M\&S techniques, which are suitable for the problem space under investigation. Hybrid modelling uses a combination of techniques, hence a survey was undertaken to ascertain the suitability of current approaches to SoS. It was found that all approaches are constrained to a small (up to three) set of M\&S techniques rather than allowing the selection of any appropriate technique, which justified the development of the SoS Risk Management M\&S process as described in this research (section 7.4).

Finally, model Validation and Verification was discussed which is an essential element of the model driven approach proposed in this thesis, a key point was that subject matter expert assessment is deemed a valid approach to model V\&V and which is the approach generally taken for models implemented for this research project.

\subsection{Theme 3: Risk}

Theme 3 examines the basic definition of risk and considers its relationship to uncertainty. Traditional risk management is surveyed in order to provide an understanding of current processes, enabling comparison with specific SoS risk management approaches.

\subsubsection{What is Risk?}

The ISO Guide relating to risk management vocabulary (International Organization for Standardization, 2009b) defines risk simply as the,

"effect of uncertainty on objectives".

Where "effect" is defined as,

"An effect is a deviation from the expected - positive and/or negative"

And "uncertainty" is defined as,

"Uncertainty is the state, even partial, of deficiency of information related to, understanding or knowledge of an event, its consequence, or likelihood".

This implies risk is uncertainty, although an explanatory note does state, 
"Risk is often expressed in terms of a combination of the consequences of an event (including changes in circumstances) and the associated likelihood of occurrence".

Whilst this definition may capture current practice, whereby risks are often identified on a subjective basis often without quantification, it also highlights a common pitfall, the confusion of risk and uncertainty.

A key point that this definition does raise is that risk encompasses both detrimental and beneficial effects. However, risk management frequently only focuses on negative effects (Hillson, 2003), although this research will consider both positive and negative effects, i.e. threats and opportunities.

In common with most definitions of risk, the Risk Management Guide for DoD Acquisition definition does directly link risk to probability (DASDSE, 2015), although noting that it only focuses on negative risk:

"Risks are future events or conditions that may have a negative effect on achieving program objectives for cost, schedule, and performance. Risks are defined by (1) the probability (greater than 0 , less than 1) of an undesired event or condition and (2) the consequences, impact, or severity of the undesired event, were it to occur."

An important aspect described in the definition above is that risk is associated with probability, thereby distinguishing risk from uncertainty.

Combining the definitions above the following general definition of risk is proposed:

"Risks are future events or conditions that may have a positive or negative effect on achieving objectives. Risks are defined by likelihood of an event or condition and the consequences, impact, or severity of the event, were it to occur."

To illustrate the difference between uncertainly and risk, consider tossing a coin (assumed to be unbiased). The probability of heads is 0.5 , as is the probability of tails. In this case all outcomes are known (heads, tails), as are the associated probabilities, this is therefore classified as risk.

In contrast, for the financial markets, all outcomes cannot be identified and therefore all probabilities cannot be determined, this is classified as uncertainty.

However, the distinction is frequently not as clear cut: for example, the weather forecast will attempt to identify outcomes and assigned probabilities, such as a $70 \%$ likelihood of rain tomorrow. The percentage in this case is typically derived from the product of the confidence that precipitation will occur somewhere within the forecast area and the 
A Model Based Approach to System of Systems Risk Management

percentage of the area the will receive measurable precipitation, for example "the forecaster is only $50 \%$ sure that precipitation will occur, and expects that, if it does occur, it will produce measurable rain over about $80 \%$ of the area, the chance of rain is $40 \% "$ (National Weather Service, 2017). Hence, the overall probability in this instance is a best estimate $^{6}$ based upon an inherent confidence; therefore there is an element of uncertainty associated with the probability.

This is an important point - it is exceptionally unlikely that absolute probabilities will be known, or derivable, indeed Taleb (2007) states, "..."computable" risks are largely absent from real life. They are laboratory contraptions!"

If risk models are to provide quantified risk, then that quantification must be honest, as indicated by an associated confidence. If a risk model is perceived as honest then decision makers will trust and utilise the model's output.

\subsubsection{Types of Risk}

Risk tends to be categorized into logical groupings allowing risk analysis to be undertaken within the appropriate context. For example, the ISO/IEC Risk Management standard (ISO/IEC, 2006) identifies the following types of risk:

- Technical

- Legal

- Organizational

- Safety

- Economic

- Engineering

- Cost

- Schedule

Ganguly et al (2010) describe a risk assessment framework for analyzing risks associated with a SE Process which identifies the following types of risk based on a literature survey and SME interview.

- Economic/Financial

- Time/Schedule Risks

\footnotetext{
6 "When we issue a forecast we usually give our best estimate of what will happen, but often we know more about the confidence or uncertainty surrounding that forecast." (Met Office, http://research.metoffice.gov.uk/research/nwp/ensemble/uncertainty.html)
} 
- Organizational Risks

- Technology \& Design Risks

- Socio-political \& Legal Risks

- Quality Risks

These are broadly similar to those listed the ISO/IEC standard (ISO/IEC, 2006), although it is noted 'Safety' is not explicitly differentiated and is not discussed as part of the framework, however it must be considered a key area and is a specialized element of risk management. Hopkin (2002) takes a more holistic view of risk and suggests the following broader categories:

- Hazard Risks (e.g. Threat and perils)

- Control Risks (e.g. Doubt about mission achievement, project management control)

- Opportunity Risks (Opportunity to enhance or inhibit mission achievement)

The categories proposed by Hopkin are essentially a way of classifying specific risks types, as identified above, providing a useful perspective above the individual component system viewpoint and therefore appears suitable at the SoS level.

In addition to risk categories, sources of risk need to be considered, for example the DoD SoSE guide (US Department of Defense, 2008b) identifies following potential sources of risk from a SoS perspective;

- SoS scalability

- Quality of service

- Technology maturity

- Coordination of SoS risk management activities across the individual systems

- Ability of constituent systems to provide needed SoS functions on time

However, the SoSE Guide is very acquisition focused hence further work is required to identify a wider range of SoS risk types, particularly relating to operational SoS requiring dynamic risk management. An approach that is explored in this thesis is to use the SoS System of Interest model (Kinder et al., 2012) ${ }^{7}$, to identify risk types through the consideration of each dimension and is described further in section 6.1, i.e.

- Component Systems

- Classification

\footnotetext{
${ }^{7}$ This paper is included in Annex A
} 
- Interactions

- Nature of Relationships

- Lifecycle

- Variability

- Functions

- System Owners and Operations

- Concept of Operation/Use/Employment

\subsubsection{Risk Management}

The INCOSE SE Guide (INCOSE, 2015) describes Risk Management as:

"a disciplined approach to dealing with the uncertainty that is present throughout the entire system lifecycle."

The Risk, Issue, and Opportunity Management Guide for Defense Acquisition Programs (DASDSE, 2015) defines the following key activities;

- Risk Planning: What is the program's risk management process?

- Risk Identification: What can go wrong?

- Risk Analysis: What are the likelihood and consequence of the risk?

- Risk Handling: Should the risk be accepted, avoided, transferred, or mitigated?

- Risk Monitoring: How has the risk changed?

Risk planning is the development and documentation of the Risk Management process. The process is typically documented in a formal risk management plan, which defines a risk register providing a common medium for all stakeholders to record risk. The program manager may establish a Risk Management Board, which comprises members from different stakeholder groups.

Risk identification is undertaken by all stakeholders and considers risks in accordance with the risk categories defined in the risk management plan, e.g. schedule, cost or performance. The central risk manager will assess each identified risk and update the risk register accordingly. Risks may be identified informally through individual subjective assessment or through more formal approaches such as brainstorming, expert interview, Delphi method, technical review, failure mode and effects analysis and fault tree analysis. It is noted that ISO/IEC 31010 (International Organization for Standardization, 2009a) identifies and assesses numerous techniques applicable to risk management activities. 
Risk analysis provides an estimate of the likelihood and consequence of a risk and will typically consist of a combination of qualitative and quantitative approaches. The consequence of a risk is measured in the context of its category, for example a financial risk will be quantified in either additional costs or potential savings. However, the INCOSE SE guide states; "Risk generally needs to be analysed subjectively because adequate statistical data are rarely available" (INCOSE, 2015) identifying interviews and models as suitable approaches. Probabilistic models (discussed in chapter 5) such as Monte Carlo simulations and Bayesian Belief Networks may be used (DASDSE, 2015; International Organization for Standardization, 2009a) to reflect uncertainty in potential outcomes. The output of the analysis will be documented in the risk register, however all stakeholders, enabling comparison of risks regardless of the source, should use a common framework.

Risk Handling requires the acceptance, avoidance, transfer, or mitigation of risk. Acceptance allows a risk to be realised although it will still be necessary to monitor it and the effect of the consequences. Avoidance requires the project to take an alternative path whereby the associated costs do not exceed that of accepting the risk. Transfer may result in a different program assuming ownership of a risk, or from customer to contractor or between subsystems. Risk mitigation will reduce the likelihood or the impact of consequence through approaches such as early prototyping, incremental development, demonstrations, test and review.

Risk Monitoring will typically require the central program authority and associated contractors meeting regularly and assessing all risks currently defined in the project risk register and updating as required.

These activities are performed on a continuous basis, Figure 10 (DASDSE, 2015) is included from the guide visualising this process. 


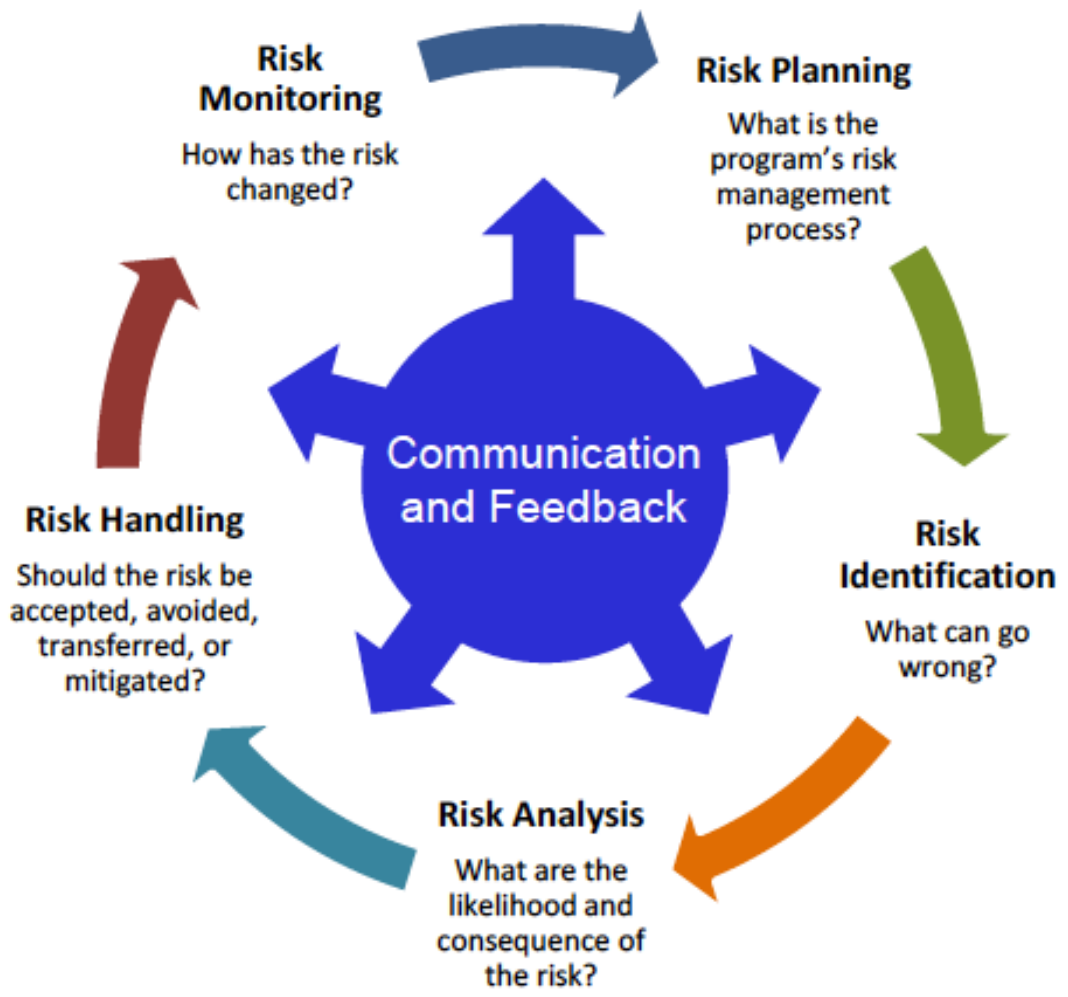

Figure 10 - DoD Risk Management Process (DASDSE, 2015)

The Risk management ISO 31000 standard (International Organization for Standardization, 2009c) outlines the following activities in the Risk Management process and align closely with the process described above:

- Communication and Consultation

- Establish the context

- Risk identification

- Risk analysis

- Risk evaluation

- Risk treatment

- Monitoring and Review

This short analysis highlights two key points.

1. Risk Management is an iterative process, with risk monitoring a continuous activity.

2. Risk identification and assessment are part of the Risk Management process. Note, this is not always consistent; Risk Management and Risk Assessment are on occasion defined as separate processes. For the purposes of this research Risk management shall include assessment. 


\section{A Model Based Approach to System of Systems Risk Management}

The Risk Management process can be generically applied, however the implementation of each activity differs dramatically between disciplines, e.g. engineering, finance, insurance, or indeed within disciplines themselves. This research is primarily concerned with the Risk analysis and evaluation stages, where both qualitative and quantitative approaches are taken.

When developing systems, risks are often analysed and assessed through subjective scoring techniques, rather than rigorous and scientific techniques, which as (Hubbard, 2009) comments, are still not widespread. This results in the use of subjective (as opposed to objective) probabilities when engineering systems (Garvey, 2004). However, the requirement for more rigorous techniques is dependent upon the complexity of the system and risk context under consideration.

Statistical modelling approaches typically used for the quantification of risk include Monte Carlo simulation, Bayesian Belief Networks, Markov analysis and Decision trees (International Organization for Standardization, 2009a).

\subsubsection{Enterprise Risk Management}

Enterprise Risk Management (ERM) is defined as:

"The culture, capabilities, and practices, integrated with strategy-setting and its execution, that organizations rely on to manage risk in creating, preserving, and realizing value" (COSO, 2016).

Where ERM differs from traditional Risk Management is its focus on culture, which aims to ensure that people within an enterprise respond appropriately to risk and understand risk in the context of overall objectives and strategy.

The holistic view of risk, coupled with its attention to culture, identifies ERM as applicable to the SoS domain, particularly where the human element requires consideration.

A further distinguishing factor of ERM is the relationship of risk to value, which determines an organisation's appetite for risk, hence "managing risk within risk appetite enhances an organization's ability to create, preserve, and realize value" (COSO, 2016).

However, ERM does assume central managerial control through an independent board of directors, hence risk strategy is determined and flowed "top down" within an enterprise. 


\subsubsection{Perception of Risk}

Risk is frequently determined as a subjective estimate of likelihood utilising experience of an individual or team, which is subject to intuitive biases and emotion (Kinder et al., 2015). The "affect heuristic", relates the assessment of risk to the perceived "goodness" or "badness" of an activity (Slovic \& Peters, 2006), whereby if an activity is regarded negatively, then the benefits are perceived to be low and the risk high; conversely an activity regarded positively will elicit a perception of high benefit and low risk. The resulting implication is that there is often an emotional aspect to risk assessment.

The "conspiracy of optimism" (Flyvbjerg et al., 2009) is another situation where the perception of risk is influenced by emotion or subconscious peer pressure, and where the likelihood or impact of risk is underestimated due to financial, managerial or political pressures through bias in favour of the desired outcome.

Prospect Theory (Kahneman, \& Tversky, 2016) illustrates a further perceptive bypass whereby the rationality of decision making is influenced by the presentation of the possible choices and their relationship to risk.

These potential emotional biases provide a strong justification for the use of modelling to support risk management, allowing risk to be assessed from a more objective perspective. Indeed Haimes (2012) states "in the context of risk management, no effective risk management policy options can be developed; nor can the associated tradeoffs among all critical costs, benefits, and risks be evaluated; and neither can the impacts of current decisions on future options be assessed, without having constructed a model, or a set of interdependent models, that represent the essence of the system".

\subsubsection{SoS and Risk}

Most literature and guidance regarding Risk Management is focussed upon individual systems (Conrow, 2005), however when managing SoS risk a holistic view of the interactions between risk components and consequences is required (Gandhi et al., 2012a).

Although the DoD SoSE guide (US Department of Defense, 2008b) is acquisition focussed, it does succinctly describe the subtle distinction between system and SoS risk management;

"Risk management for a SoS begins with the identification of SoS objectives and the identification of the risks that threaten the achievement of those objectives. While it is true that minor individual program risks could be major risks to the SoS, it is also true that significant system risks may have little or no impact on the SoS functionality. Furthermore 
A Model Based Approach to System of Systems Risk Management

there may be risk to a set of SoS objectives which are not risks to the constituent systems (e.g., unwanted emergent behaviour, infrastructure, integration risks, cost risk)."

A common perception is that if all SoS component system risks are managed, then any SoS risks will be managed without further intervention. The quote above demonstrates that SoS risk may be decoupled from individual system risk and should be managed separately.

The DoD SoSE guide (US Department of Defense, 2008b) states SoS Risks are managed by the "SOSE SE team" and an integrated Risk Management board, implying managerial control and hence limiting the applicability of the guidance to directed and, to some extent, acknowledged SoS, although this is appropriate as most DoD programmes conform with these classifications. However, of relevance to all types of SoS is the guide's assertion that SoS Risk Management is dependent on the determination of SoS objectives; SoS risks are those which may prohibit the fulfilment of those objectives.

Conrow (Conrow, 2005) identified the following seven issues associated with the management of risk within SoS, he also provided suggested mitigation:

1. Multiple stakeholders: may have heterogeneous behaviour and objectives, a central RM process will help alleviate these issues.

2. Multiple RM processes: incompatibilities could exist between processes, e.g. risk levels may not align, perception and tolerance of risk may differ. A proposed solution is the definition of a "Rosetta Stone" allowing comparison of the different outputs.

3. Long life cycles: these can extend from years to decades, component systems may have differing levels of maturity, hence managing risk will need to simultaneously consider risk at different points in the individual development lifecycles requiring the process to evolve in parallel with the SoS.

4. Common technical risk classes: e.g. design, functional performance, integration, resource availability, support, and technology. Technical risks are frequently managed separately, however through grouping of the technical classes, risk planning, identification, analysis and handling may be more co-ordinated.

5. Integration risk: this is exacerbated due to the inherent complexity but may be alleviated by consideration of integration issues throughout the lifecycle.

6. Functional performance risk: requires demonstration that functional performance requirements have been met, however use of $M \& S$ and demonstrations can be used throughout development and integration. 
7. Interface complexity: it is suggested that the probability of risk arising is directly related to the complexity, which is inherently high. Mapping the interface risks to technical risk classes may assist with their evaluation.

Although these issues are valid, they appear more applicable to directed SoS and could equally be considered relevant to complex systems. This approach is rooted in traditional RM thinking, however Gandhi et al (2012a) suggest a more innovative approach through a framework utilising SoS characteristics (Boardman \& Sauser, 2006), as discussed in section 3.2.4, which considers systemic risk and, in the context of SoS, "is a risk that could be greater than the sum of its individual constituent risks". A number of constituent risks are identified: "Schedule Risk, Technical Risks, Financial Risks, Vendor Risk, Culture Risk, Reputation Risk, Intellectual Property Risk, Flexibility Risk, Compliance Risk, and Quality Risk". The systemic risk approach is described in context of the following SoS characteristics (Gandhi et al., 2012a):

- Autonomy: attempt to understand the effect of all known risks affecting the autonomy of all systems within a SoS

- Belonging: attempt to understand the holistic effect of all the known risks on all the systems within the SoS

- Connectivity: look at a network and attempt to consider the effects of all known risks on the network of systems as part of a SoS

- Diversity: the diversity associated with the risks is taken into consideration and the varying effects they have on the different constituents of the SoS are taken into consideration

- Emergence: consider multiple risks and the dynamics between the various systems in the SoS

However, this approach does not appear to have been developed further, but it does suggest there is merit in viewing SoS risk through a more holistic framework. The quantity of research undertaken which relates to SoS risk is limited but examples of note include, an approach for SoS RM of extreme events (Bristow et al., 2012) which utilises modelling to "help to foster pluralistic understanding of a system with multiple models". Both cascading and collective failures are considered and risk is treated as dynamic whereby risk "depends not only on changing surroundings, but also on changing perceptions of multiple participants 
based on how they set their systems' boundaries". A qualitative model is produced initially, capturing the different Risk Management outcomes effect on severity and probability of consequences from the participants' perspective. The most likely outcomes in the context of the associated risk problem are then identified using a Graph Model for Conflict Resolution (GMCR) approach which draws upon Game Theory but "incorporates some plausible restrictions on knowledge and rationality, making it appropriate for advising individuals in a multi- decision-maker context" (Kilgour \& Hipel, 2005).

Game Theory is also used, in conjunction with the Viable System Model (VSM) approach, to manage risk related to SoS cyber security (Spyridopoulos et al., 2014). VSM “models the organisational structure of viable and autonomous systems. The model initially divides the enterprise in three fundamental parts (Operations, Management and Environment), which are connected to each other in order to maintain the viability of the whole system." Each system component is assessed through the effect of interactions being disrupted, with Agent Based Modelling used to enable this.

A SoS approach is taken to the analysis of risk associated with safety critical plants (Zio \& Ferrario, 2013) which utilises Muir Web and Monte Carlo simulation. Muir Web "is a system analysis technique to model a complex system and the relationships among its elements" and enables the representation of dependencies between component systems or system elements. Monte Carlo simulation is used to determine the probability that a safety critical plant (e.g. a nuclear plant) enters an unsafe state, taking into account internal safety systems and external infrastructure.

Risk management in supply chains is another area where it is acknowledged that systemic risks occur hence a SoS approach is appropriate (Ghadge et al., 2013), whereby interrelations between component systems are represented within a multiple domain matrix (MDM) with ABM used "to validate the assumptions drawn from the structure of the supply chain network as well as to comprehend the system's emergent behaviour under uncertainty".

In the context of SoS safety a risk modelling approach is proposed which utilises fault tree models based upon information derived from associated MODAF architectural models (Aitken et al., 2011) providing a capability to determine the probability of specified events occurring.

A more general, and somewhat esoteric, method involves the use of a Conditional Value-atRisk (CVaR) approach to SoS RM (Shah et al., 2015). CVaR is a "portfolio management 
A Model Based Approach to System of Systems Risk Management

approach developed in financial engineering" which "limits worst cases losses in performance of cost" and is intended to support the selection of component systems which provide optimum SoS capability whilst minimising the risk of cascading failure. However, this technique does require the quantification of component system performance and an overall SoS performance index, which may be problematic and difficult to interpret, for example if SoS performance is predicted to be $70 \%$ vice $60 \%$ what does this actually mean? A SoS engineer would generally require a model to provide an understanding of the specific problem space and not just an abstracted output value, although an aggregated measure may be of value when used in conjunction with other approaches.

\subsubsection{Summary}

In common with the previous literature review themes, theme 3 first established fundamental definitions related to risk management.

The following definition of risk was derived from several sources and encompasses the key aspects of positive and negative effects and the linkage of an event to likelihood:

Risks are future events or conditions that may have a positive or negative effect on achieving objectives. Risks are defined by likelihood of an event or condition and the consequences, impact, or severity of the event, were it to occur.

This definition, through the association of risk with likelihood, differentiates uncertainty where the likelihood of an event is unknown.

Types of risk were discussed, for example the ISO/IEC Risk Management standard (ISO/IEC, 2006) identifies the following types of risk:

- Technical

- Legal

- Organizational

- Safety

- Economic

- Engineering

- Cost

- Schedule

The following broader categories (Hopkin, 2002) were also identified which allows risk to be classified from a holistic viewpoint which is appropriate when considering SoS risk:

- Hazard Risks 
A Model Based Approach to System of Systems Risk Management

- Control Risks

- Opportunity Risks

Current Risk Management approaches were reviewed with the conclusion that it is an iterative process; with risk monitoring a continuous activity and that risk identification and assessment are part of the Risk Management process. However, Risk is frequently determined through subjective techniques, which are influenced by human bias and perception, it was therefore argued that this provides a strong justification for the use of modelling to support risk management, allowing risk to be assessed from a more objective perspective.

When considering SoS risk a holistic approach is desirable (Gandhi et al., 2012b); risk cannot just be considered at the component system level with the assumption that if risk at this level is mitigated then SoS risk will also (US Department of Defense, 2008b). It is noted that, although ERM is not explicitly applied within the SoS domain it does advocate a holistic approach, hence there is potentially some synergy between ERM and SoS Risk Management approaches.

Few Risk Management approaches were discovered which were specifically intended for SoS use, although the DoD SoSE SE Guide (US Department of Defense, 2008b) does provide some basic guidance relevant to directed SoS, however most of those found were limited to narrow problem spaces. Gandhi et al (2012a) proposed an interesting approach which considered risk in the context of SoS characteristics (Boardman \& Sauser, 2006), however this does not appear to have been developed further, although the use of a framework is comparable to the approach proposed by this research, as described in section 7.3. Other approaches utilised modelling techniques including game theory (Spyridopoulos et al., 2014), agent based modelling (Ghadge et al., 2013), MODAF architectural models (Aitken et al., 2011) and a Conditional Value-at-Risk (CVaR) approach (Shah et al., 2015). It is of note that these approaches all utilised modelling to some extent, which is driven by the inherent complexity associated with SoS.

This review has revealed a gap in current knowledge regarding the management of SoS risk and thereby a lack of appropriate methods, resulting in the use of traditional approaches which do not provide the necessary holistic perspective. 
A Model Based Approach to System of Systems Risk Management

\subsection{Conclusions}

Theme 1 of the literature provided insight into the activities comprising SoSE and its difference from traditional SE with risk management identified as a key activity.

The second theme discussed the application of M\&S to SoS, providing an output, which supported the identification of appropriate techniques and concluding that, the inherent complexity of a SoS required the use of M\&S in order to support SoSE activities.

Current risk management approaches were reviewed in theme 3 as well as the management of SoS risk. Although some specific examples of the management of SoS risk were found, no mature general approach was identified, indicating a gap in current knowledge. However, it was noted most of these examples were underpinned by M\&S approaches.

It was therefore concluded a general approach SoS risk management utilising M\&S methods would be of benefit.

The selection of appropriate models to represent the essence of the system's multiple perspectives determines the effectiveness of the entire risk assessment, management, and ultimately communication process. (Haimes, 2015) 


\section{Chapter 4: Approaches to the research}

This chapter provides general background information and terminology definitions regarding the research philosophy and approaches adopted in this thesis, examines approaches that are suitable in the Systems and SoS Engineering domain before describing and justifying the specific research design constructed to enable the research questions, defined in section 1.2 , to be answered.

\subsection{Background and Terminology}

Academic research in the System Engineering and SoSE domains has extended beyond the realms of physical science into human and social science as well as business schools (Brown, 2009). Brown (2009) argues that research in these areas is not always methodologically sound and that one reason for this is the lack of understanding, and inconsistent use, of terminology regarding research methodology. Therefore, in order to establish a consistent and unambiguous understanding of key terminology within this thesis, definitions and descriptions are provided below.

Ontology: the study of the nature of reality, which can be divided into objective and subjective perspectives.

Epistemology: the study of knowledge, or how knowledge is obtained from the perspective of a particular ontology.

Worldview: combine both philosophical perspectives, ontology and epistemology, and are described as "a basic set of beliefs that guide action" (Guba, 1990). The research herein refers to worldviews, as opposed to paradigms (Lincoln et al., 2011; Mertens, 2010); epistemologies and ontologies (Crotty, 2012), or broadly conceived research methodologies (Neuman, 2003). The worldview is the fundamental viewpoint from which the research approach is determined.

Postpositivist Worldview: most commonly associated with the 'traditional' empirical scientific approach whereby a quantitative approach is used to observe and measure objective reality. This is a deterministic philosophy where causes are identified which influence outcomes, usually measured through appropriate experimentation. This approach is also reductionist; a research question may be broken down into a set of discrete variables, which may then be quantitatively tested. This approach is essentially positivist where independence of the worldview is assumed; postpositivism acknowledges that theories and 
experiments are not independent of the researchers worldview where a key assumption is that a hypothesis cannot be proved, only falsified, which is an empirical falsification approach (Popper, 2002). It should be noted that the Postpositivist Worldview, as proposed by Creswell (2014), also encompasses the positivist view.

Constructivist Worldview: a subjective perspective and is more typically associated with social science research. Individuals subject views are collected, the context and environment, which influence an individual's subjectivity, is considered and understanding of the data is developed through induction (Crotty, 2012). A basic differentiation between Postpositivism and Constructivism is that the former is generally rooted in mathematics and numbers, while the latter is expressed linguistically.

Transformative Worldview: a subjective, politically orientated perspective. The starting point for transformative research is usually a key current political issue and requires a collaborative approach with a group of individuals whom are affected by the issue under consideration. The research considers inequalities within these groups and resulting asymmetric power relationships, political action is then linked to the inequalities (Mertens, 2010).

Pragmatist Worldview: not limited to a specific ontology, where both quantitative and qualitative assumptions may be made. Cresswell (2014) states that this ontological position (or worldview) "arises out of actions, situations, and consequences rather than antecedent conditions" and is concerned with applications and solutions to problems (Patton, 1990). This allows research investigators to utilise both qualitative and quantitative data as appropriate in order to understand the research problem, and hence allows the most suitable research techniques and procedures to be selected.

Research Methodology: Having considered the research from an epistemological viewpoint, and an appropriate worldview determined, a suitable research methodology is required. Research methodology is the process of research; it not only defines the type of research design but also includes the approach to collecting and analysing data (DeForge, 2010). It is important to distinguish between 'Methodology' and 'Methods'; there is a tendency in Systems Engineering research to directly describe methods and techniques when discussing research methodology, rather than through the appropriate worldview which considers fundamental philosophical viewpoint of the research, thereby providing the necessary methodological rigour (Brown, 2009). 
Research Design: Having considered the research approach from epistemological and ontological viewpoints, i.e. through the appropriate worldview, a suitable Research Design is constructed which will take a Qualitative, Quantitative or Mixed Method approach, in line with the applicable methodology. The Research Design can be described as the general plan for answering the research question (Saunders et al., 2009), identifying the required activities, suitable methods, the order of activities and the dependencies between them, it provides a logical structure enabling the research questions to be answered (DeForge, 2010). The Research Design approach is effectively an instantiation of the chosen Research Methodology, which determines the appropriate Research Methods, dependent on its qualitative, quantitative or mixed nature.

\subsection{General Research Design Approaches}

The three alternative research design approaches; quantitative, qualitative and mixed method are described below.

\subsubsection{Quantitative Research Design}

The postpositivist worldview necessitates a quantitative research approach, whereby relationships are investigated between a discrete set of variables through empirical measurement and observation to test a proposed theory. Typically, quantitative data will be obtained either through experimentation or survey methods.

Cresswell (2014) describes a survey design as "a quantitative or numeric description of trends, attitudes, or opinions of a population by studying a sample of that population". The purpose of a survey design is to generalize characteristics, attitude or behaviour (Creswell, 2014) from a population sample and infer these across the general population. The survey will either be a cross section or longitudinal, i.e. data collected at one point in time or over a period of time respectively. The design will also determine the medium over which data is collected, for example, mail, telephone, the Internet, personal interviews, or group administration (Fowler, 2009).

The sampling design may be single or multistage (clustering). Where individuals or elements can be identified within a population the single-stage sampling approach is appropriate. The multistage approach is typically used where individuals cannot be directly identified within a population, in this case organisations or groups are first identified, individuals are then identified from within these. Individuals may either be selected randomly or on a nonprobabilistic basis (convenience sample), which is determined by their convenience and 
availability (Creswell, 2014). Although the random selection is generally preferred in social science research, the selection of specific individuals selected by their expertise within a particular discipline may be appropriate when eliciting responses within a specialist field, such as Systems Engineering.

The questionnaire should elicit values for variables, which relate directly to research questions and which in turn map to elements in the questionnaire. This mapping should be documented, providing full traceability from the research question and survey output, thereby ensuring full coverage.

Analysis of the returned questionnaires requires the compilation of basic statistics such as the number of returns, the ample size, mean, standard deviations and range of responses. Response bias requires consideration of the effect of non-responses on the outcome; one approach is direct contact with non-respondents to determine if their answers would have differed substantially from those received to the extent that they would be statistically significant.

Experimentation generates numerical data through rigorous data collection, the classical design is the pre-post test control group (DeForge, 2010); individuals are randomly assigned to control or experimental groups and compared to an outcome variable, this is also referred to as a "true experiment" (Creswell, 2014). The key feature of this approach is the random assignment of individuals to the experimental and control groups, which reduces the likelihood of bias and threat to the research validity. However, like survey design, participants may also be chosen on a non-random basis, where participants of a pre-existing group may determine the sample; this is referred to as a quasi-experiment. The sample size of groups is determined by a power analysis (Lipsey, 1990), which takes into account the level of statistical significance of the experiment, the amount of power for the statistical test of the null hypothesis and the effect size (the difference in means of the control and test groups). The variables to be measured consist of independent variables, which influence the outcome, dependent variables and intervening variables, which mediate the effect of the independent variable upon the dependent variable. Moderating variables affect the strength of the relationship between dependent and independent variables and control variables

Other types of experimental designs, beyond the classic pre-post test control group, include the following: post-test only control group, alternate treatments, multiple treatments and controls, Solomon four group, Latin square, crossover, factorial, block, and repeated measures (longitudinal, nested (hierarchical), mixed) (DeForge, 2010). 
Data is collected in accordance with a pre-defined procedure and appropriate statistical analysis performed. The final stage of the experiment is the interpretation of the data in the context of the research question and hypothesis to determine if they are supported or refuted.

\subsubsection{Qualitative Research Design}

Qualitative research utilises textual and image data rather numerical data. The following are key characteristics of this type of research (Creswell, 2014):

- Natural Setting: less laboratory based, information collected from participants in the field

- Researcher as key instrument: researcher tends to be the data collection instrument

- Multiple sources of data: several techniques are often use, such as interview, questionnaire, observation or document

- Inductive and deductive data analysis: themes are built bottom up, from the data. When themes are established they are examined deductively to determine if further evidence is required to support them

- Participants' meanings: focus on the meaning of the problem from the participants' perspective rather than the researchers' or current literature.

- Emergent design: the initial plan is likely to change, as the field is explored and data is collected the plan evolves accordingly

- Reflexivity: the research reflects on their own experiences and how these influence the direction of the research

- Holistic account: a complex picture of the problem is developed requiring multiple viewpoints

Another key difference from quantitative research is that participants or artefacts are purposefully selected, as opposed to the random selection of large sample groups. The sample size is related to the chosen research approach, demonstrated in Table 8 below:

\begin{tabular}{|l|l|}
\hline Qualitative Research Approach & Typical Sample Size \\
\hline Phenomenology & $3-10$ individuals \\
\hline Grounded theory & $20-30$ individuals \\
\hline Ethnography & 1 single culture-sharing group \\
\hline Case study & $4-5$ case studies \\
\hline Narrative & $1-2$ individuals \\
\hline
\end{tabular}

Table 8: Research Approach Sample Size 
Data is collected either through observation of behaviour, whereby notes are recorded in an unstructured or semi-structured way, or through interviews involving unstructured or openended questions. Data may also include qualitative documents (e.g. newspaper articles, private journals, etc.) and audio or visual material (e.g. photographs, film, audio recordings, artwork, etc.). The data recording procedures require development of observational or interview protocols, which describe the structure of the data to be recorded, pragmatic details to be recorded such as time and location information and outline questions. However, this is less prescriptive than the quantitative approach.

Data analysis will typically be undertaken in parallel with subsequent data collection and will be used to inform it, for example it may result in a change in the interview questions in order to explore an issue exposed by the analysis. Depending on the complexity of the data recorded, it may be analysed either manually or using a software application, such as a spreadsheet or data analysis program. It is of note that, at this point, the qualitative data is being analysed from a quantitative perspective. It is anticipated that several themes and patterns will emerge from the data as a result of the analysis; again this is a key distinction from quantitative research design as in this case a bottom up approach is taken whereby theories are developed from the data as opposed to data corroborating a theory. Essential to the provision of credible output from qualitative research is validation of the findings, typically through application of a validity strategy; key strategies are identified below:

- Triangulation using data from different sources or perspectives which converge to provide justification for emerging themes

- Member checking by returning to participants with research findings for validation

- Rich, thick descriptions providing detailed descriptions of context or themes can provide further validity of the findings

- Bias of the researcher should be clearly and honestly provided

- Negative or discrepant information which provides contradictory evidence should be discussed, providing realism to the outcome

- Time in the field provides a deeper understanding, through prolonged close association of the study domain.

- Peer debriefing through the review of study providing an additional viewpoint of an individual familiar with the project

- External auditor to review the entire project but does not have immediate knowledge of it; the aim is to provide an objective view. 


\subsubsection{Mixed Method Research Design}

As the name suggests mixed method research design combines both quantitative and qualitative approaches, the argument being that this results in a stronger understanding of the problem and a minimising of the weaknesses of the individual approaches (Pinto, 2010).

Cresswell (2014) identifies the following basic mixed method designs:

- Convergent Parallel Mixed Methods Design

- Explanatory Sequential Mixed Methods Design

- Exploratory Sequential Mixed Methods Design

The convergent parallel mixed methods approach performs quantitative and qualitative data collection in parallel, the results of which are compared to determine if they support or conflict with each other. The key element of this approach is that the same variables or constructs are used to collect both types of data; however combining the quantitative and qualitative data is perhaps the most challenging aspect. Data combination generally takes a "side by side" approach whereby one set of data is analysed and the other is then compared as each finding is discussed, another approach is transformation, where the qualitative data is first converted to a quantitative format, or vice-versa, and direct combination is then possible.

The explanatory sequential mixed methods approach begins with a quantitative study, the results of which are analysed in order to inform the design of the following qualitative stage. For example, the second stage may comprise interviews to discuss the results of the initial quantitative study. The intent of this approach is to use the qualitative results to explain and expand upon the qualitative results rather than to combine them.

The exploratory sequential mixed methods approach is the reverse of the explanatory sequential mixed methods, whereby an initial qualitative study is built upon by a successive quantitative study. For example, a qualitative grounded theory approach will generate a theory that may then be tested quantitatively.

However, research often requires more flexibility of the design allowing further complex combinations quantitative and qualitative phases, Cresswell (2014) identifies the following more advanced mixed method designs:

- Embedded mixed methods: qualitative data is embedded within a larger quantitative study, or vice versa 
- Transformative mixed methods: a social justice theory (e.g. feminism) as a framework for the study

- Multiphase mixed methods: several mixed method, qualitative or quantitative studies carried out within a larger research project over a sustained period

\subsection{Taking a Systems Engineering Approach to Research}

$\mathrm{SE}$ is an iterative process used to develop a system, which provides a solution to address an identified problem; i.e. "an iterative process of top-down synthesis, development, and operation of real-world system that satisfies, in a near optimal manner, the full range of requirements for the system" (Eisner, 2008).

The SE approach is "an interdisciplinary approach and means to enable the realisation of successful systems. It focuses on defining customer needs and required functionality early in the development cycle, documenting requirements, and then proceeding with design synthesis and system validation while considering the complete problem: operations, cost and schedule, performance, training and support, test, manufacturing, and disposal." (INCOSE, 2015).

Although the SE process is not a research methodology, elements of the process align with typical research activities, for example determining the research questions is similar to requirements elicitation. The SE lifecycle was defined in ISO/IEC 15288 (British Standards, 2011) as: Concept stage - Development stage - Production stage - Utilization stage - Support stage - Retirement stage.

However, it is the concept and development stages which are relevant to the research process, later stages are perhaps more relevant to any future exploitation of the research output.

The concept stage defines the problem space, characterizes the solution space, explores ideas, refines stakeholder needs and explores concepts (INCOSE, 2015). This broadly equates to the definition of the research question, the literature review and the refinement of the original research question.

The development stage defines the system requirements, creates a solution description, implements the system and then verifies and validates it (INCOSE, 2015). This stage equates to the definition of the research objectives and then the definition of the tool ${ }^{8}$ (or tools) to collect and analyse data, which in the case of this research is both questionnaires and

\footnotetext{
${ }^{8}$ Or more generally referred to as the "instrument".
} 
models. The research conclusion validates and verifies the research output against the research questions and objectives.

The SE concept and development stages are visualised alongside the research process in Figure 11 below.

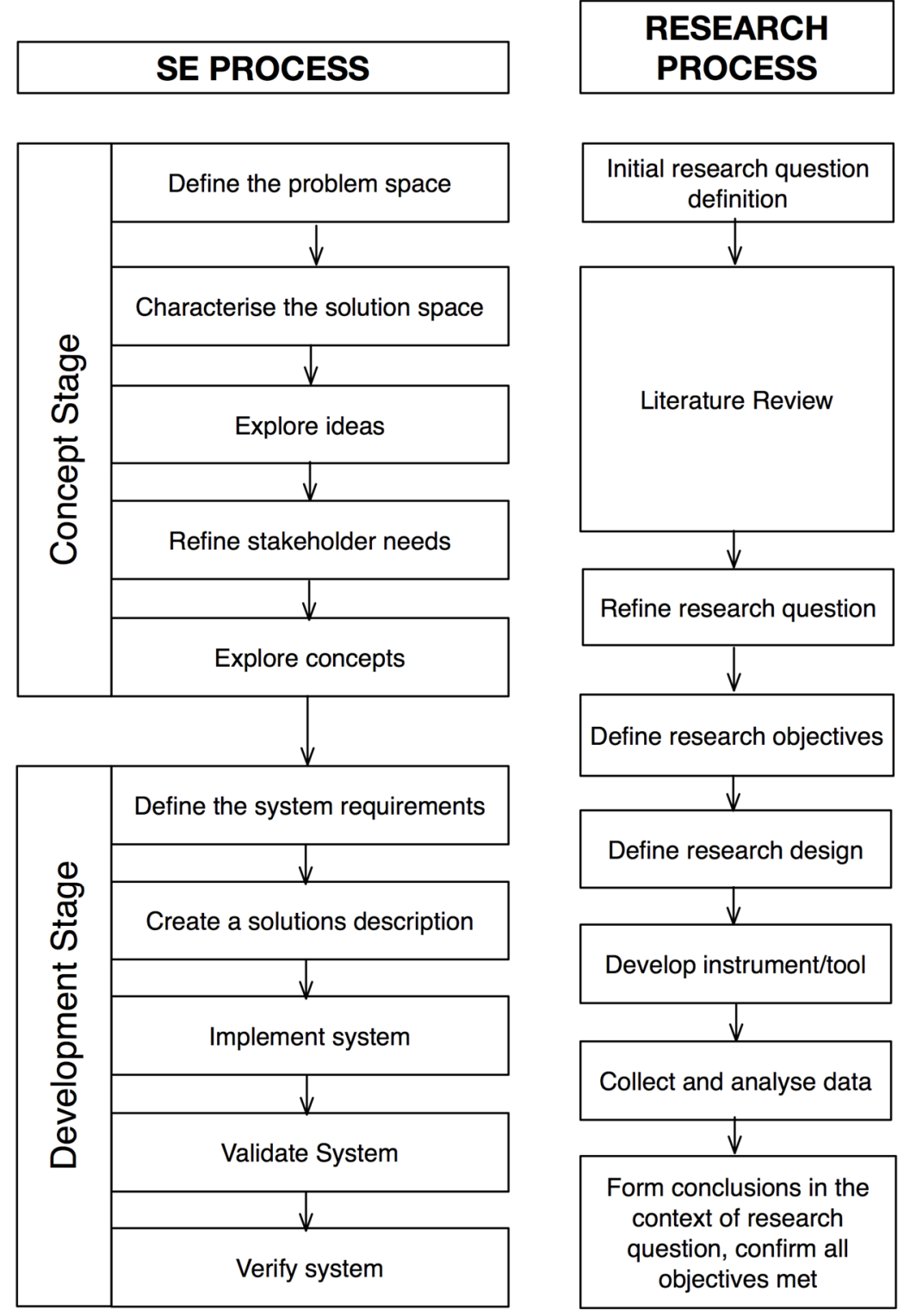

Figure 11 - SE and Research Processes

\subsection{Researching Systems Engineering and SoSE}

Much of the background material described above concerning research design originates in the social science domain, particularly qualitative and mixed method research, with quantitative designs typically used in more "traditional" scientific research. However Systems Engineering is a discipline that requires both subjective and objective viewpoints; 
for example a project may be planned and monitored using quantitative key performance indicators but it will also need to consider organisations and the individuals that comprise them, hence research in this domain will similarly need to encompass both views. The inherent complexity of a SoS also impacts the research approach; SoS behaviour needs to be considered holistically although causal factors may be at a low level within individual component systems, hence the suitability of a reductionist approach is reduced (Henshaw et al., 2013).

SE and SOSE are relatively immature research areas, with few specific methodologies currently identified; this is evidenced by the low number of published papers which discus this topic (Brown, 2009; Ferris, 2009). SoSE methods are generally derived through ontological studies with little attention being paid to epistemological and methodological approaches (Sousa-Poza et al., 2008). With a lack of defined methodology there is a tendency for SE and SOSE research to confuse the identification of methods with the foundational methodology; this results in research designs merely describing a set of methods without reference to a worldview. However, if the appropriate methodology is identified at the outset then the basic research approach can be justified, producing more credible output through the demonstration of an understanding of research philosophy, whereby the methodology properly aligns with the research questions; the methods used will then be driven by the chosen methodology.

SE research that has identified a methodology has taken a qualitative case study approach where the researcher has no control over events and the research questions are of the form "How?" and "Why?" (Martin \& Davidz, 2007). Other SE research has utilised a grounded theory approach, which collects data in a systematic manner and then derives theory from it (Valerdi \& Davidz, 2008). Sousa-Poza et al (2008) argue that for SoSE research the empirical deductive approach is not appropriate due to the SoS inherent complexity, instead a Rationalist Inductive approach is suggested. Figure 12 (Sousa-Poza et al., 2008) visualises the mapping between the proposed methodology and the research method. 


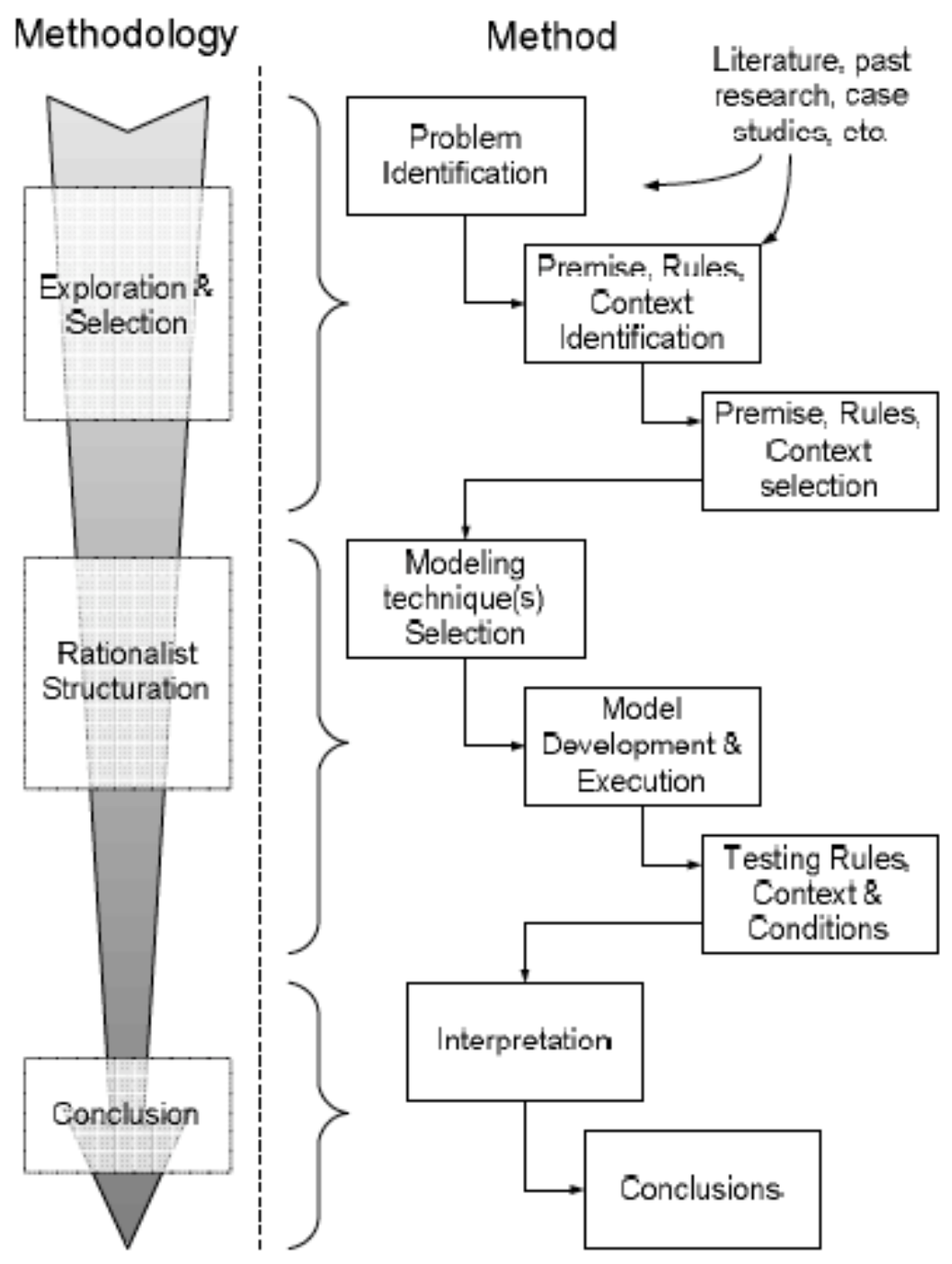

Figure 12 - Approach for the Development of the Rational Inductive Methodology (Sousa-Poza et al., 2008)

\subsection{The Finalised Research Design}

Having established appropriate research design terminology and potential approaches, the first step in determining a research design tailored to this study is to consider the research questions, as defined in section 1.2. These questions either involve an assessment of risk management approaches, for which the Constructivist Worldview is appropriate, or the development of a modelling approach for which Pragmatist Worldview is suitable; Table 9 provides a mapping of research question to worldview and hence methodology, a mapping to research objective is also provided. 


\begin{tabular}{|l|l|l|l|l|}
\hline \multicolumn{2}{|l|}{ Research Questions } & Worldview & Methodology & $\begin{array}{l}\text { Objective } \\
\text { (QL = Qualitative QN } \\
\text { = Quantitative) }\end{array}$ \\
\hline 1 & $\begin{array}{l}\text { How is this management of } \\
\text { SoS risk different from } \\
\text { managing risk at the } \\
\text { system level? }\end{array}$ & Constructivist & Qualitative & 2(QL), 3(QL), 8(QL) \\
\hline 2 & $\begin{array}{l}\text { How is a model-based } \\
\text { approach beneficial? }\end{array}$ & Pragmatist & Mixed Method & $\begin{array}{l}\text { 1(QL), 4(QL), } \\
\text { 5(QL,QN), 6(QL,QN) }\end{array}$ \\
\hline 3 & $\begin{array}{l}\text { How can a model-based } \\
\text { approach be developed } \\
\text { and how can it quantify } \\
\text { risk? }\end{array}$ & $\begin{array}{l}\text { Pragmatist } \\
\text { associated with SoS? }\end{array}$ & Mixed Method & 1(QL), 4(QL), \\
5(QL,QN), 6(QL,QN)
\end{tabular}

Table 9 - Research Design Methodology Mapping

Each research objective can be associated to an activity for which an appropriate research method should be selected. Having identified an appropriate research methodology for each objective, a research method is selected which is consistent with the methodology, as provided in Table 10 below. It is noted that the Embedded Mixed Method approach was specifically selected as the development of the case study tools required qualitative input but was verified through quantitative test cases, however the validation required a more subjective assessment from purposefully selected subject matter experts. 
A Model Based Approach to System of Systems Risk Management

\begin{tabular}{|c|c|c|c|}
\hline \multicolumn{2}{|c|}{ Objective } & \multirow{2}{*}{$\begin{array}{l}\text { Methodology } \\
\text { Qualitative }\end{array}$} & \multirow{2}{*}{$\begin{array}{l}\text { Method } \\
\text { Literature Review, } \\
\text { Assess using case } \\
\text { study }\end{array}$} \\
\hline 1 & $\begin{array}{l}\text { Review of a wide range of modelling } \\
\text { techniques applicability to SoS }\end{array}$ & & \\
\hline 2 & $\begin{array}{l}\text { Review current risk management } \\
\text { approaches }\end{array}$ & Qualitative & Literature Review \\
\hline 3 & $\begin{array}{l}\text { Identify differences between system and } \\
\text { SoS risk management }\end{array}$ & Qualitative & $\begin{array}{l}\text { Literature Review, } \\
\text { Case Study, } \\
\text { Modelling }\end{array}$ \\
\hline 4 & $\begin{array}{l}\text { Identify an application for which a risk } \\
\text { management model based tool will } \\
\text { provide benefit }\end{array}$ & Qualitative & Pragmatic selection \\
\hline 5 & $\begin{array}{l}\text { Identify appropriate modelling } \\
\text { techniques and implement and test tool }\end{array}$ & $\begin{array}{l}\text { Embedded Mixed } \\
\text { Method }\end{array}$ & $\begin{array}{l}\text { Modelling, SE } \\
\text { approach to tool } \\
\text { development, Case } \\
\text { Study }\end{array}$ \\
\hline 6 & $\begin{array}{l}\text { Identify a case study and determine the } \\
\text { adaptability of the tool }\end{array}$ & $\begin{array}{l}\text { Embedded Mixed } \\
\text { Method }\end{array}$ & $\begin{array}{l}\text { Pragmatic selection, } \\
\text { SE approach to tool } \\
\text { development, Case } \\
\text { Study }\end{array}$ \\
\hline 7 & $\begin{array}{l}\text { Define a SoS risk management } \\
\text { methodology }\end{array}$ & Qualitative & $\begin{array}{l}\text { Triangulation from } \\
\text { case study results }\end{array}$ \\
\hline
\end{tabular}

Table 10 - Research Design Method Mapping

The complete research design is described in Figure 13, showing how the key studies in the research map to objectives, methods and methodologies. The Rationalist Inductive approach (Figure 12) was also shown above as it is does align with the overall approach taken in this research.

As described in section 4.3, a Systems Engineering approach was taken throughout this research, both at a high level when performing the research activities and also at a low level when developing the prototype tools for each case study. 


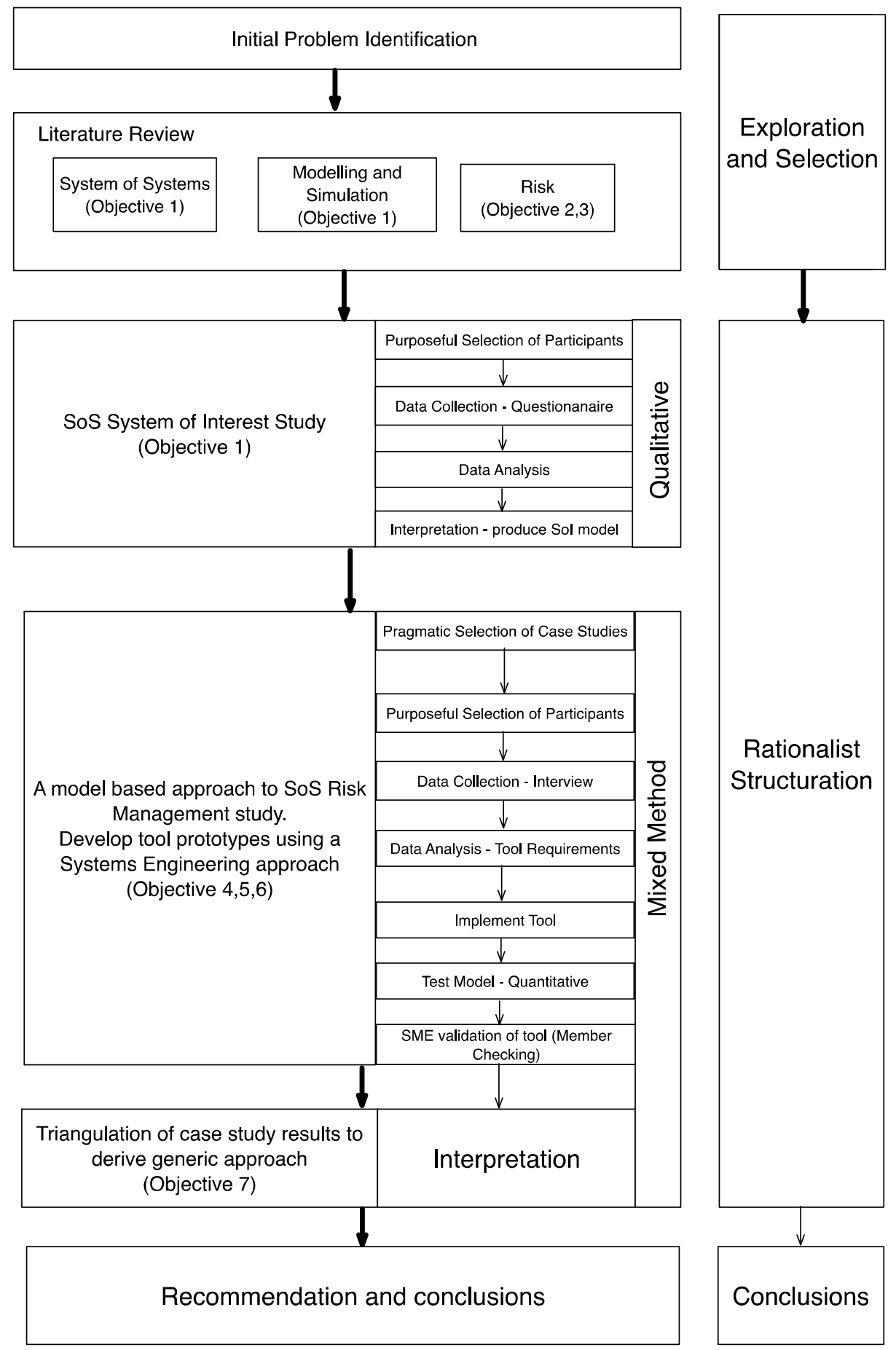

Figure 13 - Research Design

\subsection{Critical Assessment of Research Methods}

\subsubsection{Literature Review}

The state-of-the-art literature review approach was based on the three-stage review process (Levy \& Ellis, 2006) and is described in section 3.1, it was divided into the following three themes; (1) General definitions and differences between System and SOSE, (2) Modelling 
and (3) Simulation and its applicability to SoS and Risk Management and its application in the SoS domain. The results of the review provided a sound basis for the development of the model based risk management process enabling identification of knowledge gaps and areas of alignment with current approaches.

\subsubsection{Questionnaires}

A questionnaire was used to identify the SoS Sol dimensions, which is described in section 6.1, with the results attached in Appendix A and Annex D. For each proposed dimension the following question was asked; "This dimension is relevant to the Sol of a SoS?" Participants were required to respond in accordance with the following scale, 1=Strongly Disagree, 2=Disagree, $3=$ Neutral, $4=$ Agree, $5=$ Strongly Agree. Following analysis of the results a workshop was held with a sub-group of participants, which further refined the proposed dimensions.

Questionnaires were also used in conjunction with semi-structured interviews in order to support the validation of case study outputs by SMEs, as described in chapters 8 and 9 . This approach ensured that key points required for adequate validation were covered and consistent between case studies.

\subsubsection{Validation}

Validation of the case study outputs was required in order to demonstrate the efficacy of the proposed risk management process. Creswell (2014) identifies the following terms relating to the validity of qualitative research; trustworthiness, authenticity, and credibility which may be provided through the strategies described in section 4.2.2. These strategies are listed below but in this instance a description of the action taken to fulfil them is provided.

- Triangulation - data was used from different case studies, which in turn were validated by different SMEs. The case studies were chosen to ensure a wide difference in application.

- Member checking - this was in part also provided by SME validation and also by peer review and citation of published research output, in particular the SoS Sol model.

- Rich, thick descriptions - detailed descriptions of the case study and associated outputs are provided in chapters 8 and 9 .

- Bias of the researcher is addressed through the selection of the second case study. Whilst the researcher was very familiar with the problem space addressed by the 
close air support case study (chapter 8), the second case study was completely unfamiliar (chapter 9). This ensured that, with respect to domain familiarity, the risk management process was applied without bias, demonstrating that risks were identified and mitigated as a result of the process rather than through knowledge of the domain.

- Negative or discrepant information - chapter 10 provides a critical analysis of the proposed risk management approach, identifying areas which require further development.

- Time in the field - the researcher leveraged substantial experience of SoSE, specifically relating to interoperability issues in the military domain, which also informed the first case study (chapter 8).

- Peer debriefing, External auditor - it is suggested that this was provided by the research supervisors, examiners, SMEs and publication reviewers.

\subsubsection{Case Studies}

Section 2.1 outlines several case studies, providing examples of all SoS type within the context of the research question.

A further two heterogeneous case studies; Close Air Support and UK National Food Security - Wheat Supply Chain were selected for application of the risk management process and are described in chapters 8 and 9 respectively.

\subsection{Summary}

A SoS is inherently complex, therefore a reductionist research approach, whereby a problem is distilled down to a set of discrete variables which can then be quantitatively measured, is not appropriate. Typically, SoS research will require a combination of both quantitative and qualitative elements, utilising a mixed method approach (Creswell, 2014).

The research approach to the development of a model-based approach to SoS Risk Management, utilises a mixed method approach, specifically an exploratory sequential mixed method approach (Creswell, 2014).

Research was conducted through the analysis of appropriate case studies, the building of models and SME interviews. The analysis of case studies and SME interview provides qualitative data, which enabled the definition of the Risk Management model requirements. The models provide a set of quantitative data, which is validated against the model requirements and further SME input. A SoS System of Interest model, as described in section 
A Model Based Approach to System of Systems Risk Management

6.1, was developed through the canvasing of subject matter experts using questionnaires and a follow up workshop, the report of which is included in Annex D. 


\section{Chapter 5: Probabilistic Modelling}

Although there are numerous probabilistic modelling techniques, such as Markov Chains and Decision Trees, the proposed model-based approach to SoS Risk Management specifically utilises Bayesian Belief Networks (BBNs) and Monte Carlo simulation as key elements within the model architecture, hence this chapter is intended to provide an understanding of both techniques, describing the theoretical underpinning, practical use and current use within traditional Risk Management processes.

Figure 14 illustrates how probabilistic modelling relates to other research output components providing context for the information provided in this chapter. It should be noted that this chapter does not define the probabilistic modelling approach (as described in section 7.4) per se, but does provide a basic theoretical background in support of it.

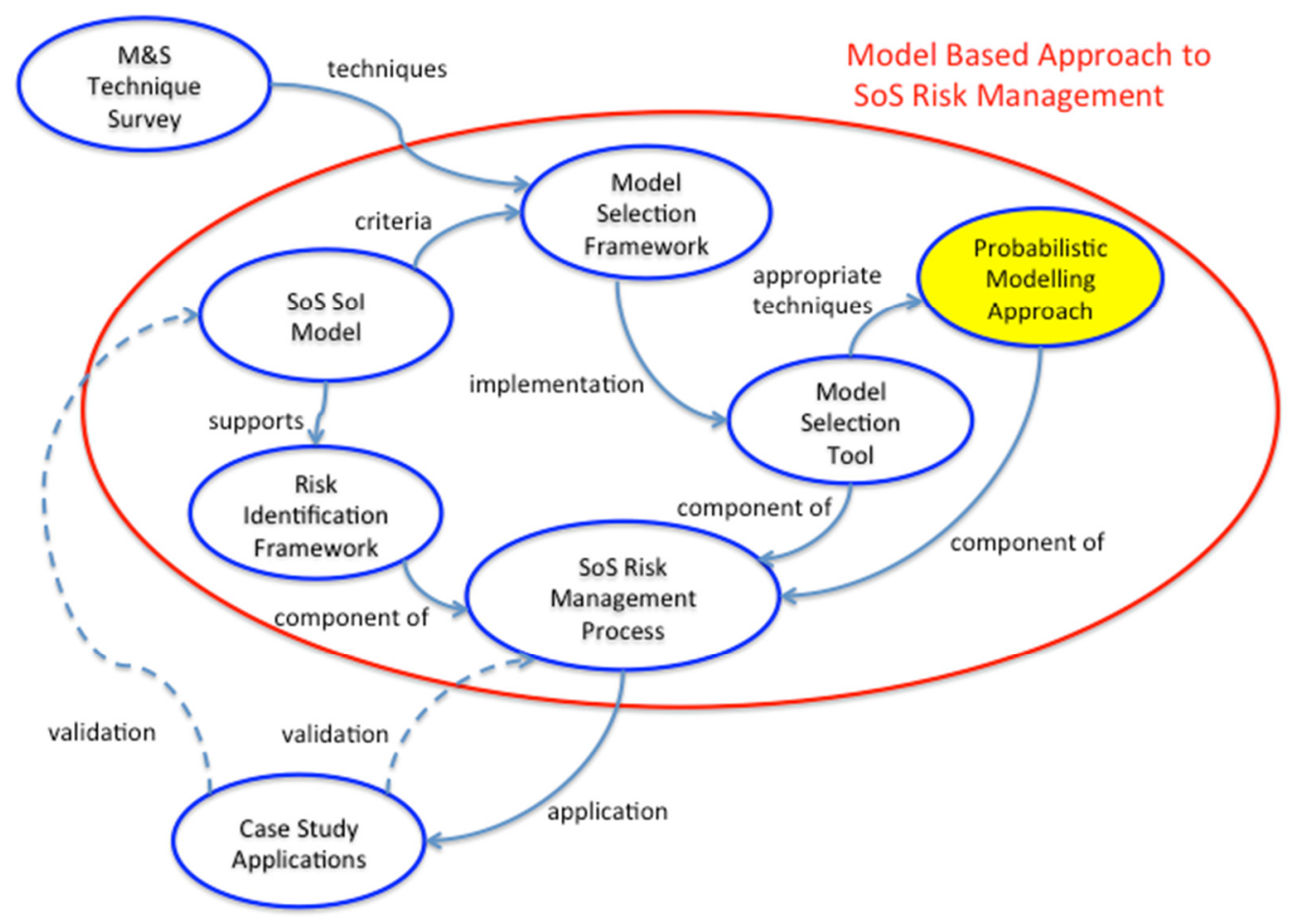

Figure 14 - Probabilistic Modelling Approach Context

\subsection{Conditional Probability}

Where the probability of event $B$ is dependent upon that of event $A$ occurring, the probabilities are said to be conditional. This is illustrated in Figure 15; inside the blue rectangle all worlds in which $A$ is true are represented, outside the blue rectangle all worlds in which $A$ is false are represented. The same logic applies for $B$, i.e. inside the red rectangle, 
A Model Based Approach to System of Systems Risk Management

all worlds in which $B$ is true are represented, outside the red rectangle all worlds in which $B$ is false are represented.

Hence

$$
\begin{aligned}
& 0<=P(A)<=1 \\
& P(\text { true })=1 \text { and } P(\text { false })=0
\end{aligned}
$$

The intersection of $A$ and $B, P(A \wedge B)$, represents the probability of both $A$ and $B$ being true, therefore the probability of $A$ or $B$ being true is:

$$
P(A \vee B)=P(A)+P(B)-P(A \wedge B)
$$

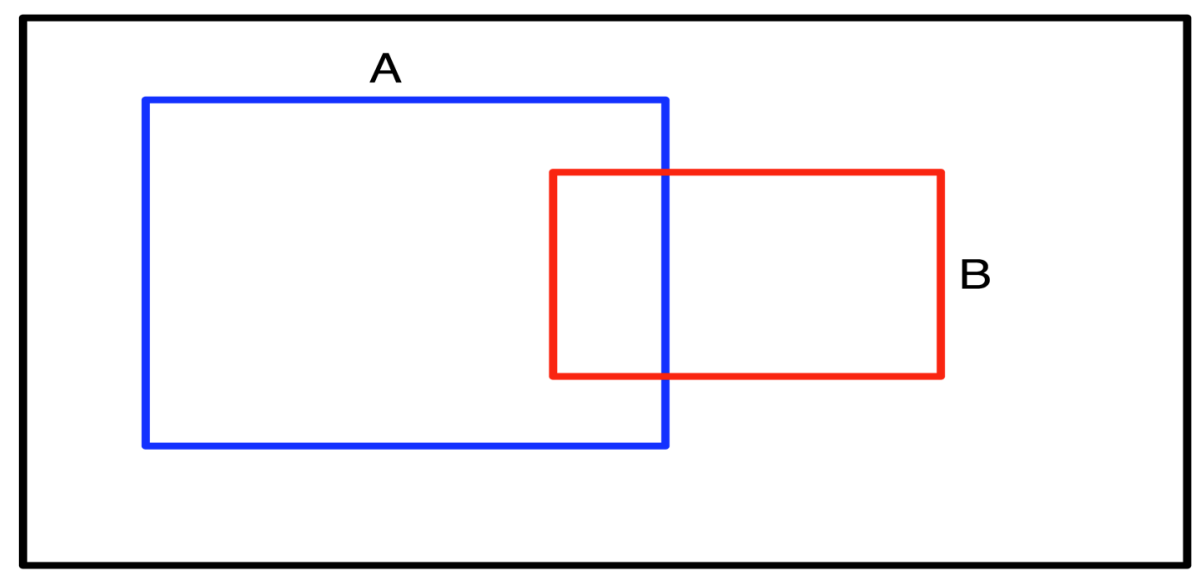

Figure 15 - Conditional Probability

The conditional probability is defined as:

$$
P(A \mid B)=\quad \text { Fraction of worlds in which } B \text { is true that also have } A \text { true }
$$

For example, if $P(A)$ represents the probability of having a stomach-ache and $P(B)$ represents the probability of having food poisoning then it follows that if one has food poisoning then the probability of having a stomach ache will be increased, hence:

$P(A \mid B) \quad=\quad$ worlds with food poisoning and stomach-ache
worlds with food poisoning
$=\quad \frac{\text { Area of } A \text { and } B \text { region }}{\text { Area of } B \text { region }}$
$=\frac{P(A \wedge B)}{P(B)}$

Therefore:

$$
P(A \wedge B) \quad=\quad P(A / B) P(B)
$$


It follows that:

$$
P(A \wedge B)=\quad=\quad P(B \mid A) P(A)
$$

Hence

$\begin{array}{lll}P(B \mid A) & = & \frac{P(A \wedge B)}{P(A)} \\ P(B \mid A) & = & \frac{P(A \mid B) P(B)}{P(A)}\end{array}$

Equation (1) is Bayes Theorem.

Bayes Theorem was initially derived by the Reverend Thomas Bayes and presented posthumously to the Royal Academy two years after his death, in 1763. Although simple, this theorem is extremely powerful, describing the relationship of conditional and prior probabilities of two events. This characteristic enables either prior estimates of probability, based on contextual knowledge, or actual evidence to be used, which provides predictive and diagnostic support for the determination of probability (Pearl, 1986). For example, the probability of the ground being wet will be very high if it is known that it has rained previously, however the theorem also allows for the weather forecast to be taken into account if rain has not yet fallen.

\subsection{Bayesian Belief Networks (BBN)}

A BBN is a graphical representation of the probabilistic relationships between a set of random variables using a Directed Acyclic Graph (DAG) and is underpinned by Bayes Theorem. A network consists of a set of interconnected variables with each interconnection representing the causal relationship between variables and with each variable having multiple states. The probabilities relate to the likelihood of a particular state occurring in relation to the states of the connected nodes. A particular advantage of representing conditional probabilities in a graphical format is that it may be intuitively interpreted and does not require a deep understanding of the underlying theory.

The network of variables can be considered a causal network; it is the representation of the causal links as conditional probabilities that differentiate the BBN from the basic causal network (Jensen \& Nielsen, 2007).

The BBN is therefore fundamentally different to the Markov Network which is an undirected graph whose links represent symmetrical probabilistic dependencies (Pearl, 2014), rather than causal links. The modelling of causal linkages is a key requirement of the model-based 
A Model Based Approach to System of Systems Risk Management

approach for SoS Risk Management (as described in section 7.4), hence BBNs are utilised in preference to Markov Networks, for this reason Markov Networks are not described further in this chapter.

\subsubsection{Theoretical Background}

Jensen and Nielsen (Finn V. Jensen; Thomas Dyhre Nielsen, 2007) identify the BBN components as follows:

- A set of variables and a set of directed edges between the variables

- Each variable has a finite set of mutually exclusive states

- The variables together with the directed edges form a Directed Acyclic Graph

- To each variable $A$ with parents $B_{1}, \ldots, B_{n}$ there is attached a conditional probability table $P\left(A \mid B_{1}, \ldots, B_{n}\right)$

A key strength of BBNs is propagation, whereby probability distributions are calculated and the network updated in response to entry of evidence, learning or adjustment of probability distribution within a single node. The updated probabilities are calculated through the application of the chain rule, which is derived below.

The formula for conditional probability (section 5.1) can be re-arranged to give the product rule:

$P(A, B) \quad=p(A / B) p(B)$

Expanding this for three variables gives:

$P(A, B, C) \quad=P(A \mid B, C) P(B, C)$

$$
=P(A \mid B, C) P(B / C) P(C)
$$

Generalizing for $\mathrm{n}$ variables gives the chain rule:

$P(A 1, A 2, \ldots, A n)=P(A 1 \mid A 2, \ldots, A n) P(A 2 \mid A 3, \ldots, A n) P(A n-1 \mid A n) P(A n) \ldots(2)$

Consider a BBN with a joint distribution $P\left(X_{1}, X_{2}, X_{3}, \ldots, X_{n}\right)$ over the associated set of variables $\left\{X_{1}, X_{2}, X_{3}, \ldots X_{n}\right\}$ which is defined as a probability table $P\left(X_{1} \wedge X_{2} \wedge X_{3}, \wedge \ldots \wedge, X_{n}\right)$ for all values that the set of variables can assume.

For simple case with two binary variables the joint distribution would be defined by the following probability tables; $P\left(X_{1} \wedge X_{2}\right), P\left(\bar{X}_{1} \wedge X_{2}\right), P\left(X_{1} \wedge \bar{X}_{2}\right)$ and $P\left(\bar{X}_{1} \wedge \bar{X}_{2}\right)$ (Lampis, 2010). Applying the chain rule (2) to the variables $\left\{X_{1}, X_{2}, X_{3}, \ldots, X_{n}\right\}$ gives the joint distribution: 
$P\left(X_{1}, \ldots, X_{n}\right)=\prod_{i} P\left(X_{i} \mid P a_{i}\right)$

where $P a_{i}$ are the parents of $X_{i}$ (Finn V. Jensen; Thomas Dyhre Nielsen, 2007)

As stated previously the property of propagation is extremely powerful, supporting experimentation within the network, however propagation in general has been proved to be a NP hard (non-deterministic polynomial time hard) problem (Cooper, 1990). This has necessitated the development of propagation algorithms (Lauritzen \& Spiegelhalter, 1988; Pearl, 1986) supporting efficient belief and evidence updates throughout a network. Several software applications are currently available which support the creation of BBNs and implement these algorithms allowing propagation within them. NETICA is one such tool and implements "message passing in a junction tree (or "join tree") of cliques", a full description of this algorithm can be found in Lauritzen \& Spiegelhalter (1988).

\subsubsection{A Simple Example}

In order to illustrate the definition and use of a BBN a simple, often cited, example is used; this involves a lawn with two states; wet or dry. The state of the lawn will obviously be dependant on the weather. However, the lawn has a sprinkler, which will also determine if the lawn is wet or dry. In addition, the state of the sprinkler will be dependant on the weather. From this scenario the variables and the causal relationships in the network can be derived, as illustrated below in Figure 16, where "Weather" is termed the parent of the child variables "Sprinkler" and "Lawn".

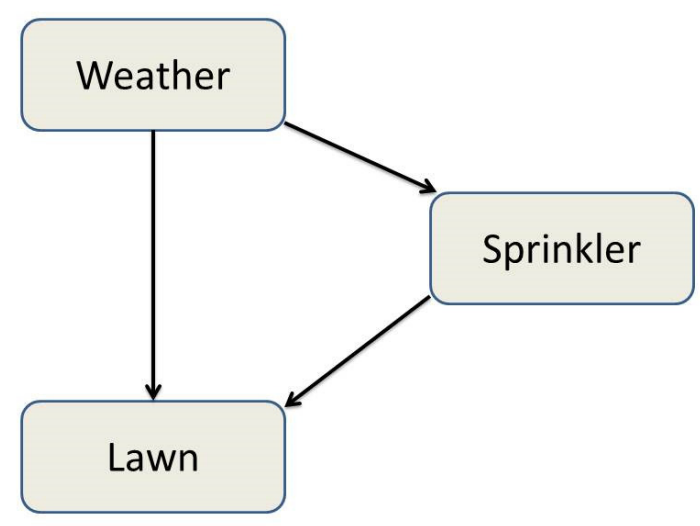

Figure 16 - Sprinkler Example

With the causal links established the next stage is to populate the probability tables associated with each node, either using probability values derived from evidence or, more subjectively, from expert opinion. Each state is assigned a probability, the summation of 
A Model Based Approach to System of Systems Risk Management

which must always be 1.0 since a variable must be in one of its possible states at any time and each state is mutually exclusive. An example probability table for Weather is defined in Table 11, for Sprinkler in Table 12 and for Lawn in Table 13.

\begin{tabular}{|l|l|}
\hline Weather \\
\hline Sunny & 0.3 \\
\hline Cloudy & 0.4 \\
\hline Rainy & 0.3 \\
\hline
\end{tabular}

Table 11 - Weather

\begin{tabular}{|l|l|l|}
\hline & \multicolumn{2}{|l|}{ Sprinkler } \\
\hline Weather & On & Off \\
\hline Sunny & 0.7 & 0.3 \\
\hline Cloudy & 0.5 & 0.5 \\
\hline Rainy & 0.05 & 0.95 \\
\hline
\end{tabular}

Table 12 - Sprinkler

\begin{tabular}{|l|l|l|l|}
\hline & & Lawn \\
\hline Weather & Sprinkler & Wet & Dry \\
\hline Sunny & On & 0.99 & 0.01 \\
\hline Sunny & Off & 0.1 & 0.9 \\
\hline Cloudy & On & 0.999 & 0.001 \\
\hline Cloudy & Off & 0.2 & 0.8 \\
\hline Rainy & On & 1.0 & 0.0 \\
\hline Rainy & Off & 0.99 & 0.01 \\
\hline
\end{tabular}

Table 13 - Lawn

The network is now populated and can be used to answer questions such as; "what is the probability of the grass being wet if the weather is sunny?"

With the nodes, arcs and probabilities entered, the software determines the conditional probabilities, as shown below in Figure 17. 


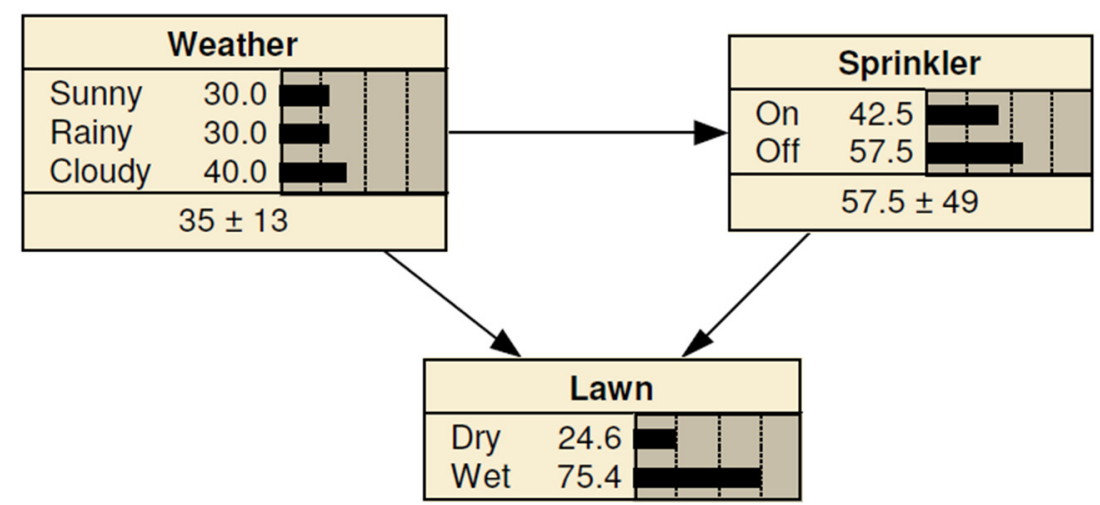

Figure 17 - Sprinkler (NETICA)

It is now possible to answer the question we posed above. We know that day must be sunny, so that 'evidence' is entered into the network. It can now be seen that the probability of the lawn being wet is now $72.3 \%$ and that the probability of the sprinkler being on has risen from $42.5 \%$ to $70.0 \%$. Intuitively this result appears correct, as on a sunny day the probability of the sprinkler being on should increase.

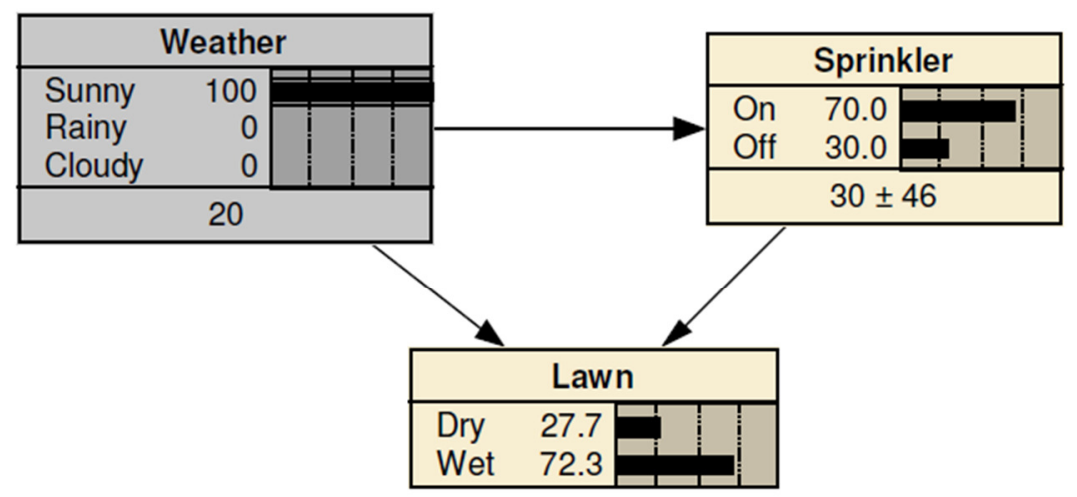

Figure 18 - Weather - Sunny

As a further test of the network we pose the question; what is the probability of the grass being wet when it is raining? The weather node evidence is changed to reflect this and the probability of the lawn being wet rises to $99 \%$, with the probability of the sprinkler being off rising to $95 \%$, as demonstrated below in Figure 19. Again, this result appears reasonable. It could be argued that the probability of the lawn being wet should be higher; this could either be rectified by altering the lawn probability table or by refining the network. For example, if the rain is very light and the temperature is high it may evaporate before reaching the ground; in this case the network could be refined through the addition of rain density and air temperature variables. 


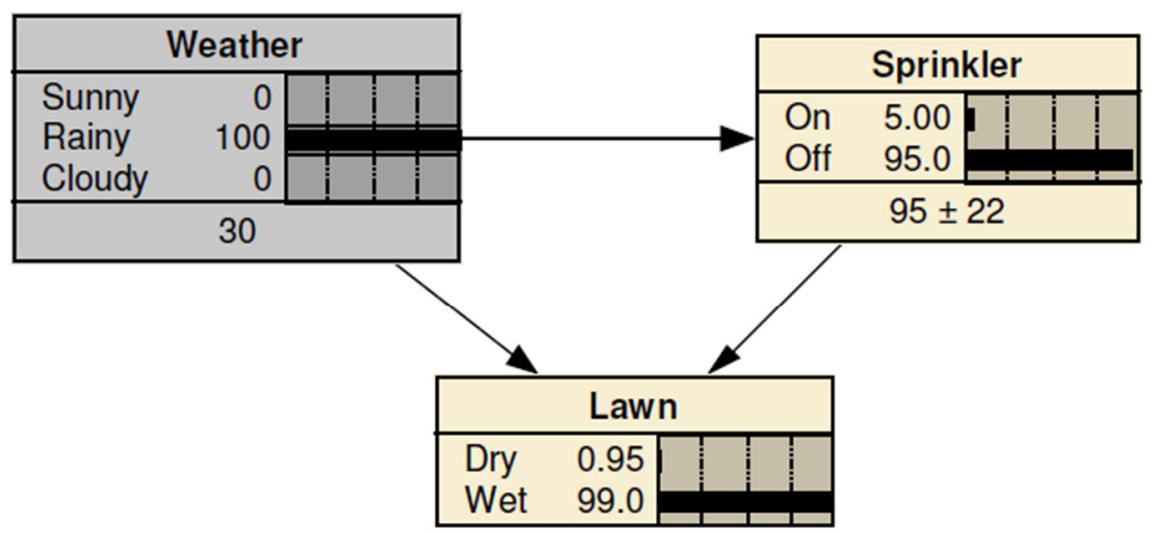

Figure 19 - Weather - Rainy

\subsubsection{BBN Simplification Methods}

The example described above in section 5.2 .2 is an extremely simple example; typically a BBN will contain far more variables, this leads to the number of entries contained in probability tables increasing with the number of parents, hence for a variable with $x$ states and $y$ parents the number of entries will be $x^{y}$.

However, there are a number of simplification approaches, which may be applied, thereby reducing the probability tables to a manageable size.

\section{$\underline{\text { Noisy-OR }}$}

The Noisy-OR technique limits the growth in the number of probabilities to a linear relationship with the number of parents.

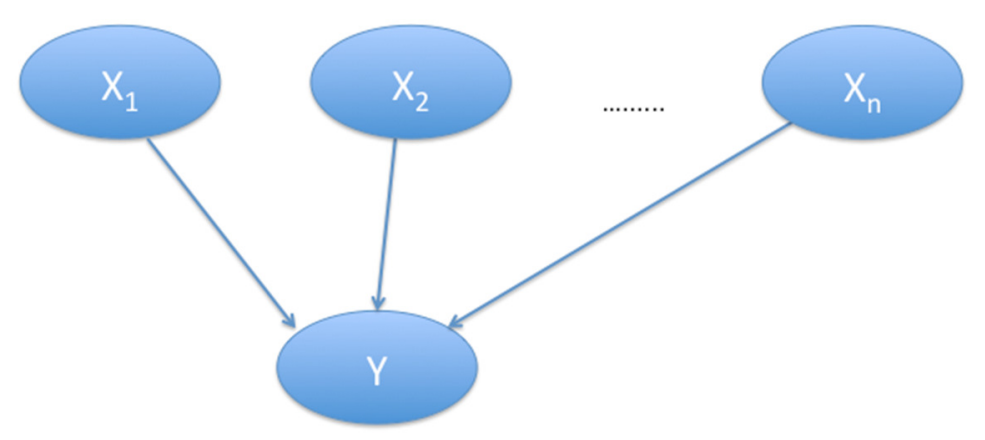

Figure 20 - Noisy OR BBN Example

If the BBN depicted in Figure 20 comprises variables $\{X 1, X 2, \ldots, X n\}$ which are all two-state, then a Noisy-OR model assumes that the parent causes of $Y$ are independent. In this case the probability that none of them caused $Y$ is the product of all probabilities that each did 
A Model Based Approach to System of Systems Risk Management

not cause $Y$, it then follows that the probability of any parents causing $Y$ is this value subtracted from 1. The probability table now contains $n$ entries as opposed to $x^{n}$, which would be the case without application of the Noisy-OR model.

\section{Divorcing}

Another simplification technique is "divorcing" which utilises the addition of mediating variables, for example variable $Y$, shown in Figure 21, has four parents, if each parent is a three state variable then the variable $Y$ probability table will contain $3^{4}$ entries. Figure 22 introduces the mediating three-state variable $Z$ where the associated probability table will contain $3^{2}$ entries; this results in the variable $Y$ probability table reduced to $3^{3}$ entries. Further simplification could be undertaken through the addition of an additional mediating variable between $\left\{X_{3}, X_{4}\right\}$ and $Y$, which would reduce the size of the probability table for $Y$ to $3^{2}$.

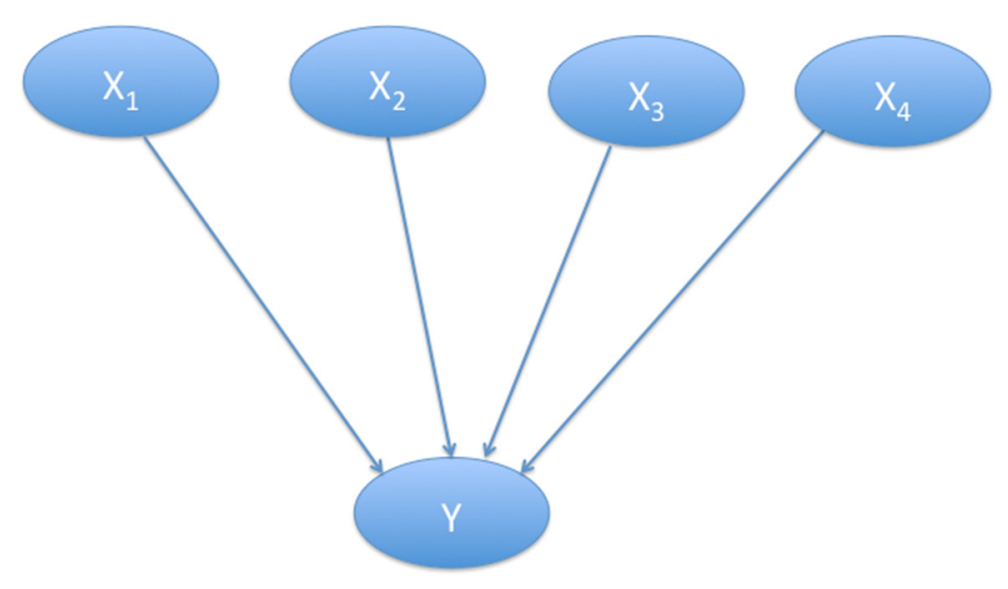

Figure 21 - BBN before divorcing

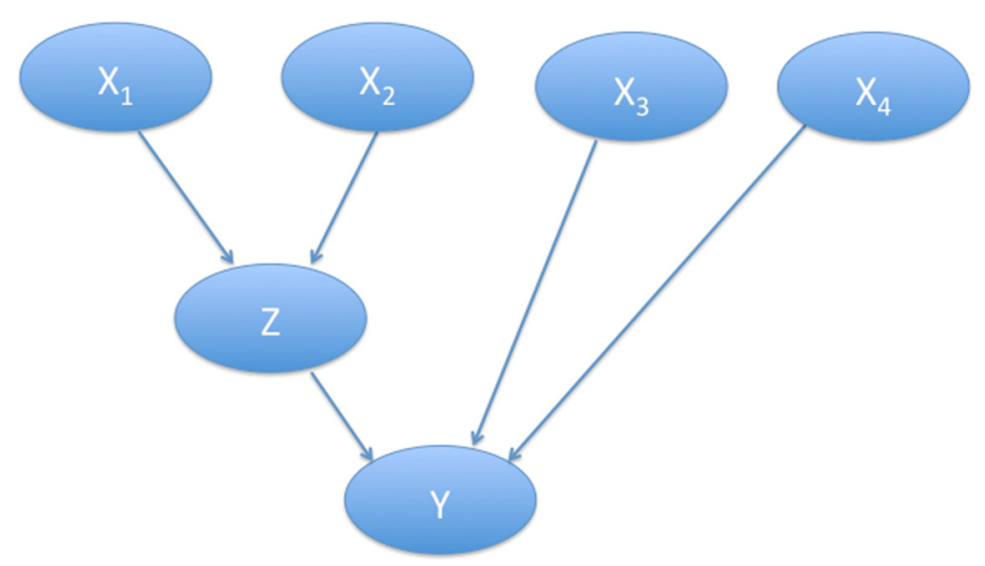

Figure 22 - BBN after divorcing 


\subsection{Monte Carlo Simulation}

Monte Carlo ${ }^{9}$ simulation requires a model to either accept a range or distribution of inputs, or internally represent distributions and when executed over multiple iterations the output is in the form of a distribution. Scientists at the nuclear research laboratory in Los Alamos, New Mexico first used this approach in the 1940's (Metropolis \& Ulam, 1949), and it is now an established statistical modelling technique being used in financial planning, business, reliability engineering, and safety engineering for quantitative risk analysis and control (Taylor \& Ranganathan, 2013).

Typically a model that is run within a Monte Carlo simulation will have the same structure as a single point, deterministic model, it is the replacement of certain single value variables with distributions or random numbers that is the key differentiator.

The strong law of large numbers states that the larger the sample size the closer the distribution is to the theoretical distribution (Day, 2008). This is manifested through the running of a model over multiple iterations, as the number of iterations increases then the randomness in the output reduces and the outliers become less relevant.

The central limit theory states that a mean set of variables drawn independently from an arbitrary distribution will have an approximately normal distribution.

Consider an example, whereby the rolling of two dice is modelled and the sum of the outcome is output. For a very low number of iterations (e.g. 50) the set of results will form a random distribution, however as the number of iterations is increased substantially (at least 5000) the output distribution resembles a normal bell curve (Day, 2008), in accordance with the central limit theory.

Day (2008) proposed the following stages for Monte Carlo simulation development in the context of developing a model of profit margins for rental properties:

1) Define an initial single point model.

2) Decide on appropriate probability distributions, their attributes and interrelationships, possibly through historical data and update the model if required.

3) Use random numbers to generate large numbers of potential scenarios, these may be inputs to the model or internalized variables within the model.

4) Plot the output data on a scatter chart.

\footnotetext{
${ }^{9}$ The name Monte Carlo is sometimes attributed to the famous casino, although some sources state that is taken from the codename of the classified project on which it was first used.
} 
5) Count results in ranges.

6) Calculate the descriptive statistics of the distribution.

7) Plot a histogram of the results.

Whilst the procedure outlined above was applied in a specific case it does demonstrate the key attributes of a Monte Carlo simulation, inherent randomness, multiple iterations and interpretation of the output distribution and outlines typical development steps, which may be generally applied.

\subsection{Probabilistic Modelling and Risk}

The international standard which identifies Risk assessment techniques (International Organization for Standardization, 2009a), lists Bayesian and Monte Carlo analysis as suitable statistical methods. It should be noted that Markov techniques are also identified but, as stated in section 5.2, BBNs are used in preference to Markov techniques for the application described within this thesis.

The use of BBNs to support the risk analysis of complex systems has increased substantially over the last twenty years with applications including military installations, fire protection systems, banking organisations, the nuclear power industry and human reliability (Weber et al., 2012). In these examples BBNs were used to represent human, technical and organizational barriers and quantify low probability but high impact risks, as well as enabling the input of both historical data and information elicited from subject matter experts.

Monte Carlo is perhaps the most widely used statistical modelling technique in support of quantitative risk analysis, being applied in numerous domains including the nuclear industry, the construction industry, public health, financial markets, insurance, meteorology and ecology.

\subsection{Summary}

This chapter has provided basic theoretical underpinning and a guide to the application of BBNs and Monte Carlo Simulations, both of which are identified as fundamental components of the model based approach to SoS Risk Management as proposed in this thesis. 


\section{Chapter 6: A Method for the Selection of Appropriate SoS M\&S Techniques}

The proposed model based approach to SoS Risk Management, by definition, requires the use of a range of modelling techniques. However, critical to the success of this approach is the selection of techniques appropriate for the problem space being explored. Whilst it is accepted that in some cases the appropriate technique may be an obvious fit, there will typically be circumstances where the practitioner may be only familiar with a limited range of modelling techniques and hence the selection will be constrained to this knowledge base. This chapter describes the development of a SoS System of Interest model (Kinder et al., 2012) which was utilised as a framework supporting a model technique selection matrix (Kinder et al., 2014). From the matrix, a tool was developed in Microsoft Excel, allowing the practitioner to define SoS modelling requirements and from which appropriate techniques are automatically identified.

Figure 23 provides context for the research output components described in this chapter, illustrating their relationship to each other and to components described outside this chapter.

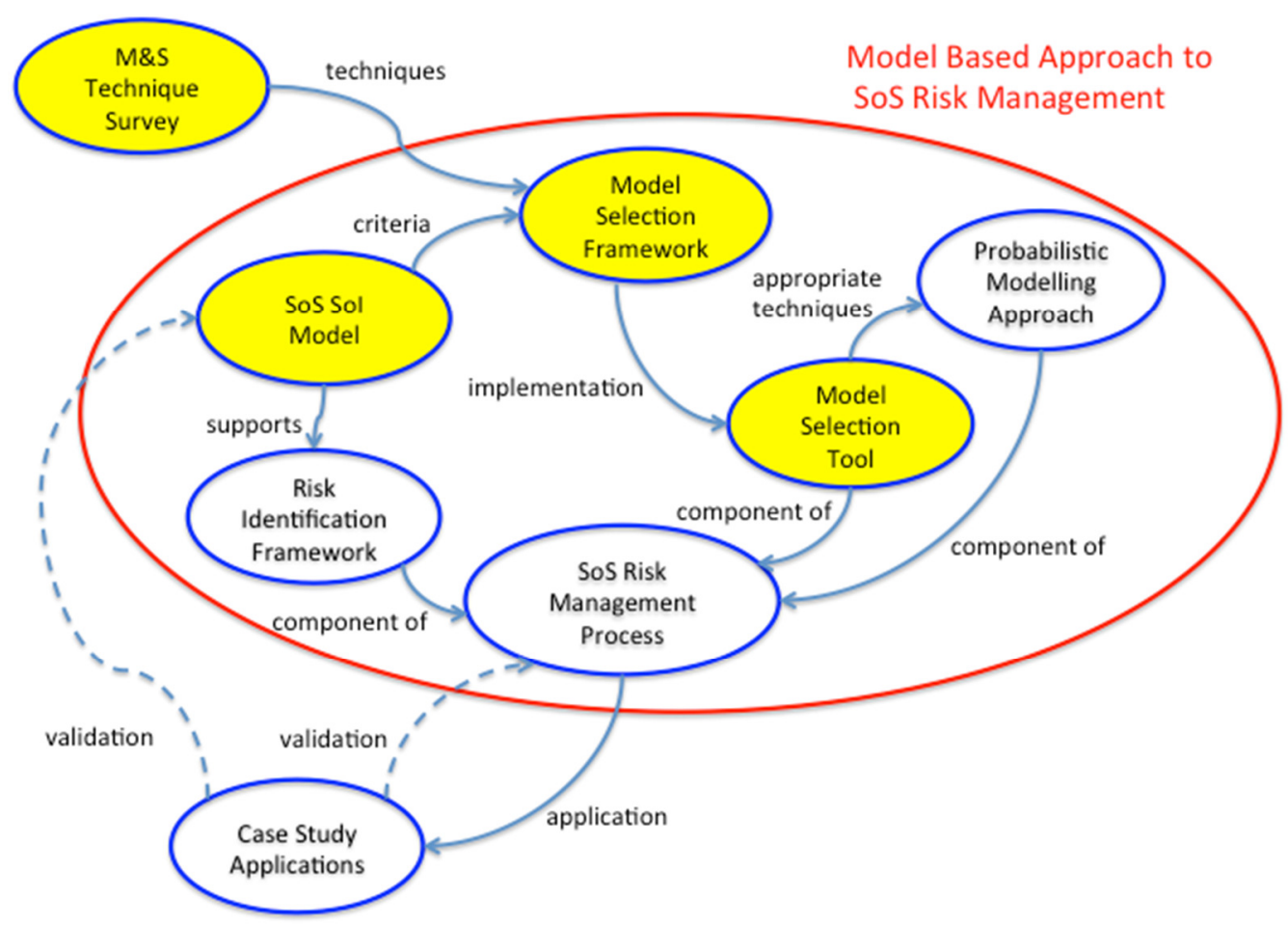

Figure 23 - M\&S Technique Selection Context 
A Model Based Approach to System of Systems Risk Management

\subsection{Model of SoS Sol}

"A distinguishing feature between Systems Engineering and System of Systems Engineering is the difficulty of defining the System of Interest (Sol). But failure to properly identify the Sol is one of the causes of unpredicted emergent behaviour, which usually arises from the combined actions, and interactions of the constituents of a SoS" (Kinder et al., 2012).

As stated in section 3.2.2, in order to undertake SE the Sol must be established, this is equally applicable to SOSE. However, the literature review undertaken for Theme 1 - SOSE did not reveal any approaches, which currently support the determination of a SoS Sol.

The definition of a set of "dimensions" which could be used to represent the SoS Sol was first suggested at the ESoS academic retreat held in 2011. Under this research, the concept was developed further using a qualitative research approach. A questionnaire designed to elicit an agreed set of dimensions was circulated to 11 purposefully selected participants with deep knowledge of the subject under consideration, the results of which are attached in Appendix A. The majority of participants were from an academic background (ranging from $\mathrm{PhD}$ candidate to professor), specialising in SE and SoSE, although one contributor was industry based being the chief scientist within a multinational defence company. Specifically the group consisted of one professor, three senior lecturers, one visiting fellow, an industrial chief scientist and five PhD researchers who as a whole had specific experience of M\&S, human behaviour, culture, enterprise architecture, interoperability and engineering approaches in the context of SoS. Selection of participants with both SE and SoS knowledge was required to minimise bias towards a traditional SE approach in the responses whilst also allowing suitable elements of SE to be identified. A workshop was subsequently held where a subgroup of 5 participants discussed the survey results and agreed a final set of dimensions; a report containing the results analysis and workshop discussion is attached in Annex $\mathrm{D}$ and summarised below. For each proposed dimension the following question was asked; "This dimension is relevant to the Sol of a SoS?" Participants were required to respond in accordance with the following scale; $1=$ Strongly Disagree, $2=$ Disagree, $3=$ Neutral, 4=Agree, 5=Strongly Agree.

Whilst it is acknowledged the sample size was relatively low, the results of the research were subsequently published in a peer reviewed conference paper (Kinder et al., 2012) as attached in Annex A, was re-iterated in a peer reviewed journal paper (Kinder et al., 2014), attached in Annex B, and also published in a second conference paper (Kinder et al., 2015) attached in Annex C. In addition the Sol SoS model was presented at INCOSE SoS Working 
Group and the SoSCIE, webinars which had over 50 and over 80 attendees respectively, who were highly knowledgeable of the SoS domain. Throughout these reviews and presentations the SoS Sol model was positively supported, providing further validation of the approach.

Figure 24 illustrates the questionnaire responses; the overall score was determined by simply adding all response values.

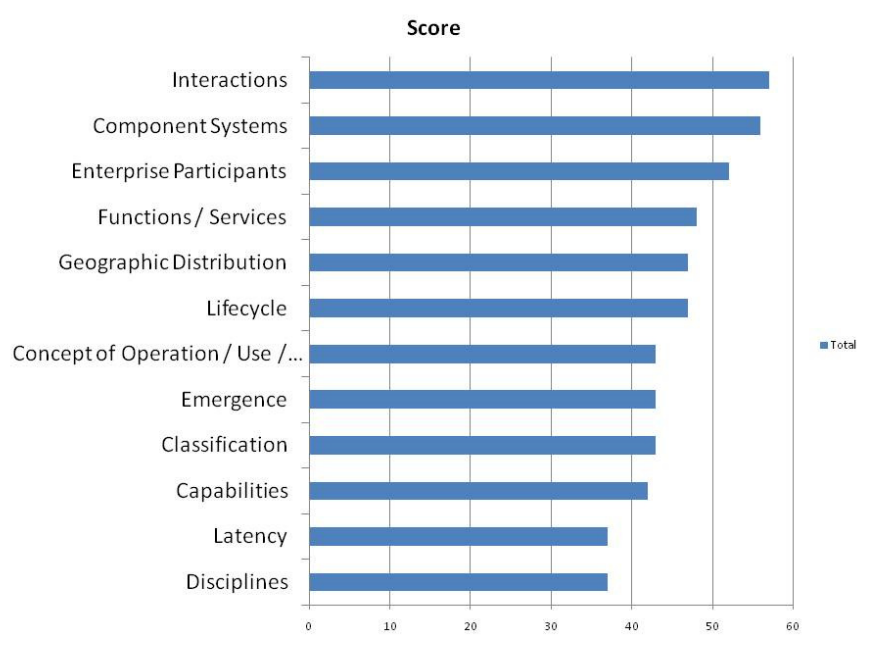

Figure 24 - SoS Sol Questionnaire Responses

In order to determine the level of consensus across the responses a graph of the variance of each set of responses was produced, as shown in Figure 25.

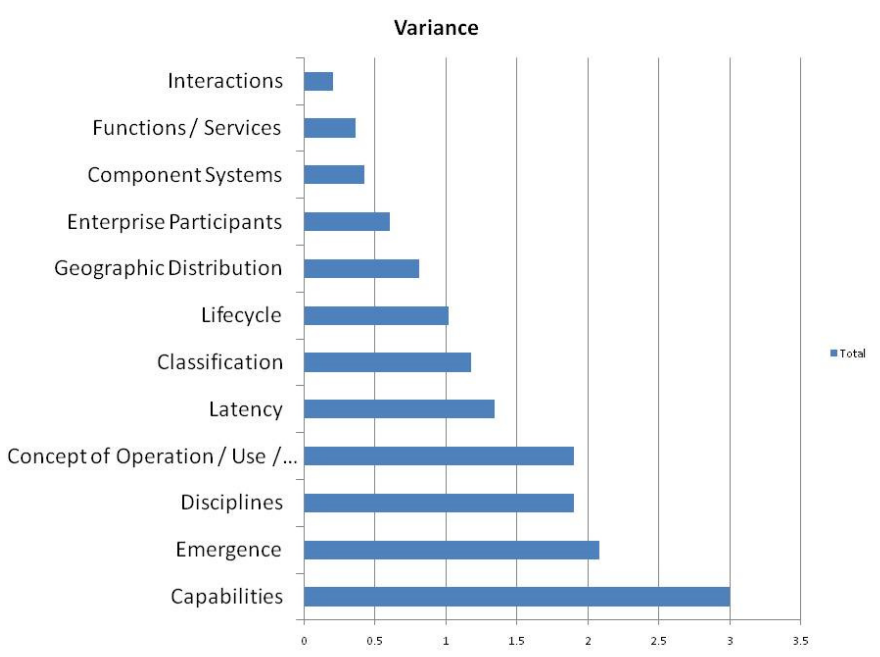

Figure 25 - SoS Sol Questionnaire Response Variance

A low variance indicates a high level of agreement between participants. This analysis provided the basis for discussion at the subsequent workshop, whereby dimensions with high scores and low variance were generally accepted, low scores with low variance were 
rejected and those with higher scores but higher variance were the targets of more discussion.

Although Geographic Distribution scored relatively highly, workshop discussions identified that, although relevant, this dimension is subsumed by the Interactions dimension rather than requiring a specific dimension. This aligns with Maier's classification of SoS (Maier, 1998), as discussed in section 3.2.3 which acknowledges geographic distribution as a typical, rather than a defining, characteristic.

Enterprise Participants was accepted as a valid dimension, however the workshop reached the conclusion that it was more related to organisations and management; hence it was renamed to Systems Owners and Operations.

Functions/Services was also accepted as a valid dimension, however the workshop agreed that the term "Services" should not be taken forward as services potentially imply Service Oriented Architecture, which was not the intention. It was also agreed that the Functions dimension adequately covered Capability.

Whilst it was agreed Emergence is a critical issue with regards to a SoS it was decided that is a characteristic rather than a separate Sol dimension and is a result of the relationships between the other dimensions.

Latency is directly related to the SoS interactions, therefore this dimension was deemed superfluous.

Each component system comprises a number of disciplines, hence it was agreed that the Disciplines dimension was unnecessary as the component systems dimension already encompassed the SoS attribute.

The agreed set of dimensions, as listed below, were presented at the 7th International Conference on System of Systems Engineering in 2012, the accompanying paper (Kinder et al., 2012) is attached in Annex A:

- Component Systems (including specific and general system types)

- Classification

- Interactions

- Nature of Relationships

- Lifecycle

- Variability

- Functions 
A Model Based Approach to System of Systems Risk Management

- Systems Owners and Operations

- Concept of Operation / Use / Employment

Hence the definition of a SoS Sol was proposed: The system of systems whose life cycle is under consideration described by all dimensions that contribute to the resultant emergent behaviour.

Descriptions of each dimension are provided in the Annex A paper (Kinder et al., 2012), elements of which are quoted from in the summary below.

\section{Component Systems (including specific and general system types)}

Component systems (also known as constituent systems) comprise the SoS and will generally be identified for the directed SoS and to some extent the acknowledged SoS, although early in the development lifecycle specific systems may not yet be identified. The same situation also arises for SoS classified as collaborative and virtual, however in this case, it is possible that generic system types may be identified before it is possible to instantiate them with specific systems.

\section{Classification}

The SoS classifications (Dahmann \& Baldwin, 2008); directed, acknowledged, collaborative and virtual, may populate this dimension, although any suitable alternative may be used. However, it is noted that a particular SoS may simultaneously exhibit the behaviours of more than one classification, depending on the make-up of component systems and the current point in the lifecycle.

$\underline{\text { Interactions (including types) }}$

A SoS exists only because of interactions between constituent systems. With no interaction the SoS merely becomes a set of independent systems exhibiting no overall emergent behaviour, so the interactions must provide some definition of the SoS Sol. The ability of the SoS to fulfil its purpose is dependent upon the effectiveness and availability of these interactions.

Interactions may be defined generically or specifically allowing SoS development to be supported from initial high-level information exchange requirements through to detailed interaction contents, protocol and media definitions.

The availability of interactions is important and related to performance and agility, i.e. a SoS may be formed or reconfigured relatively quickly if component systems share a common 
means to interact. For two component systems to interact there must be at least one homogeneous interaction medium, if there is none then an intermediate system must act as a "translator" or gateway. Interactions can also be constrained by geographical dispersion, therefore this should be considered.

\section{$\underline{\text { Nature of Relationships }}$}

This dimension is used to define the category of relationship between component systems, such as 'peer-to-peer', hierarchical control or distributed control.

\section{Lifecycle}

This dimension considers the lifecycle of the SoS as a whole as well as the respective timelines of the component systems, providing an insight into dynamism within the SOI. For some SoS, it may not be possible to define a cycle, as such, but rather an evolution in which the SoS passes through identifiable phases, as discussed in section 3.2.5, where it may be appropriate to use an alternative lifecycle model such as that proposed by the DANSE project (DANSE Consortium, 2015).

It was also suggested that there is a link between the point in the lifecycle and the ability to populate a dimension, for example, until the execution phase of a SoS the actual component systems may not be known but the generic types should be. It is argued that the lifecycle is an overarching dimension, affecting all other dimensions. As the SoS passes through the lifecycle phases the dimensions evolve both in relation to each other and to external influences.

\section{$\underline{\text { Variability }}$}

Variability is the frequency of change of a SoS and is related to time in the sense that it may reveal some sort of characteristic frequency representing change in the SoS. Stability is linked to the behaviours of the Sol such as "Stable over time", "Unstable, rapidly grows" and "Boundary changes". Factors such as an evolving purpose or a response to environmental factors will influence the variability.

\section{Functions}

A SoS performs a combination of functions in order to accomplish its purpose. Functions may be performed by individual component systems, by subsets of component systems or by the entire SoS. The Function dimension defines both specific functions and more generic function types. The aggregate effect of the functions gives the SoS emergent behaviour; it is 
this behaviour that should fulfil the SoS purpose. Analysis of these functions may enable unexpected behaviour to be anticipated.

\section{Systems Owners and Operations}

This dimension is closely related to organisations, management and enterprise (multiorganisational) relationships. It is dependent on the classification type, for example within a directed SoS the owners and operations will be well defined but for a more ad-hoc SoS this dimension will not be so well defined. However, the definition of System Owners and Operations includes generic types enabling the dimension to be defined to a certain extent for less rigidly defined systems.

\section{Concept of Operation / Use / Employment}

Given that the individual systems can perform operations independently the use/mission of the SoS is critical for defining the Sol for the SoS as opposed to the individual constituent systems. This dimension is potentially the starting point for populating the SOI dimensions.

Having established the SoS Sol dimensions, the relationships between them were defined, as illustrated in Figure 26 (Kinder et al., 2012) below.

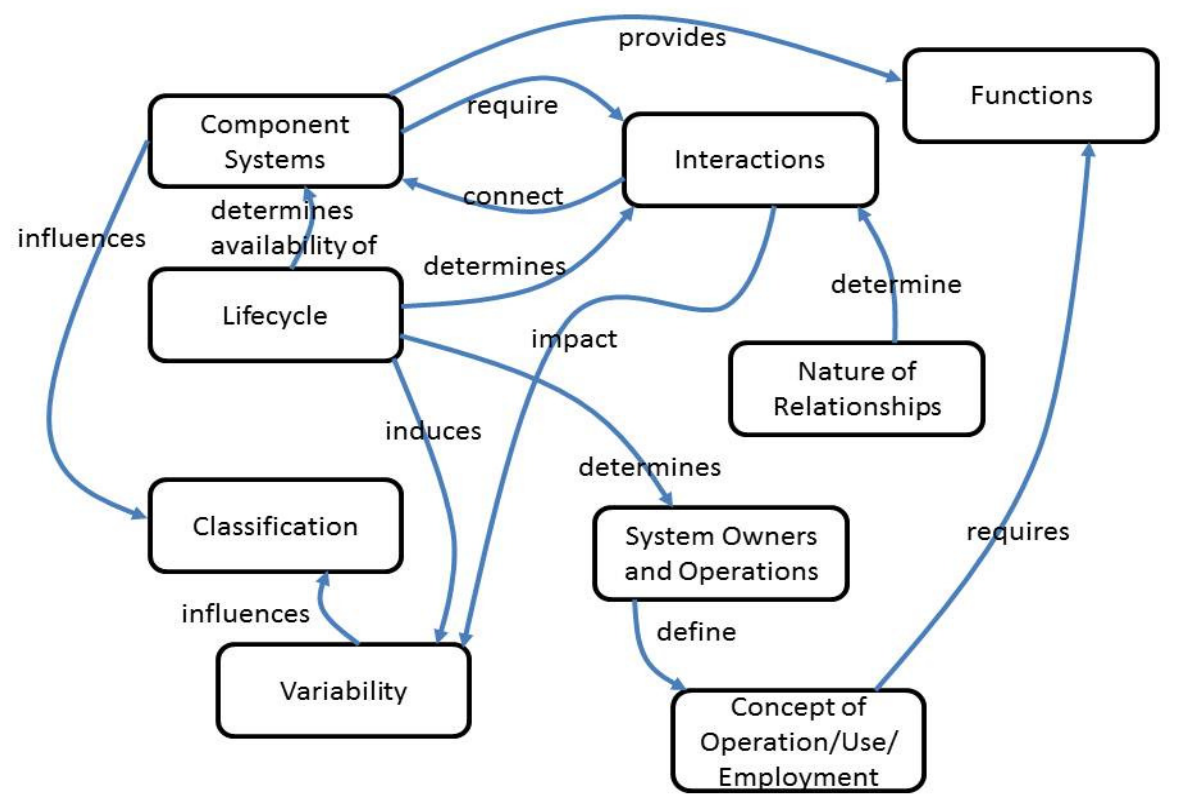

Figure 26 - Representation of SoS Sol (Kinder et al., 2012)

Descriptions of, and the rationale behind each relationship are provided in Table 14.

\begin{tabular}{|l|l|l|l|}
\hline $\begin{array}{l}\text { Source } \\
\text { Dimension }\end{array}$ & $\begin{array}{l}\text { Connecting } \\
\text { Dimension }\end{array}$ & Relationship & Description \\
\hline
\end{tabular}




\begin{tabular}{|c|c|c|c|}
\hline $\begin{array}{l}\text { Source } \\
\text { Dimension }\end{array}$ & $\begin{array}{l}\text { Connecting } \\
\text { Dimension }\end{array}$ & Relationship & Description \\
\hline $\begin{array}{l}\text { Component } \\
\text { Systems }\end{array}$ & Interactions & require & $\begin{array}{l}\text { A SoS exists only because of } \\
\text { interactions between } \\
\text { constituent systems (Kinder } \\
\text { et al., 2012). Hence } \\
\text { Component Systems require } \\
\text { interactions in order to form } \\
\text { a SoS. }\end{array}$ \\
\hline $\begin{array}{l}\text { Component } \\
\text { Systems }\end{array}$ & Functions & provides & $\begin{array}{l}\text { Component systems provide } \\
\text { both individual functions in } \\
\text { support of the SoS purpose } \\
\text { and functions, which arise } \\
\text { through interaction of the } \\
\text { component systems. }\end{array}$ \\
\hline $\begin{array}{l}\text { Component } \\
\text { Systems }\end{array}$ & Classification & influences & $\begin{array}{l}\text { The identification and } \\
\text { maturity of the component } \\
\text { systems influences the SoS } \\
\text { classification. Where all } \\
\text { component systems are } \\
\text { known and "built and } \\
\text { managed to fulfil specific } \\
\text { purposes" (Dahmann \& } \\
\text { Baldwin, 2008) }\end{array}$ \\
\hline Interactions & $\begin{array}{l}\text { Component } \\
\text { Systems }\end{array}$ & connect & $\begin{array}{l}\text { As stated above, connection } \\
\text { of component systems } \\
\text { provides SoS capability; } \\
\text { therefore interactions are } \\
\text { required to connect them. }\end{array}$ \\
\hline Interactions & Variability & impact & $\begin{array}{l}\text { The availability and stability } \\
\text { of the interactions between } \\
\text { the component systems } \\
\text { impacts the variability, i.e. if } \\
\text { the interactions are unstable } \\
\text { and subject to frequent } \\
\text { change then the variability } \\
\text { will increase. }\end{array}$ \\
\hline Lifecycle & $\begin{array}{l}\text { Component } \\
\text { Systems }\end{array}$ & $\begin{array}{l}\text { determines } \\
\text { availability of }\end{array}$ & $\begin{array}{l}\text { It is assumed that at points } \\
\text { later in the lifecycle, the } \\
\text { identification and availability } \\
\text { of component systems will } \\
\text { increase, as discussed in } \\
\text { section } 3.2 .5 \text {, hence the } \\
\text { point in the lifecycle } \\
\text { determines availability of } \\
\text { component systems. }\end{array}$ \\
\hline Lifecycle & Interactions & determines & $\begin{array}{l}\text { As above, it is assumed that } \\
\text { at points later in the }\end{array}$ \\
\hline
\end{tabular}




\begin{tabular}{|c|c|c|c|}
\hline $\begin{array}{l}\text { Source } \\
\text { Dimension }\end{array}$ & $\begin{array}{l}\text { Connecting } \\
\text { Dimension }\end{array}$ & Relationship & Description \\
\hline & & & $\begin{array}{l}\text { lifecycle, the identification } \\
\text { and availability of } \\
\text { component systems will } \\
\text { increase. }\end{array}$ \\
\hline Lifecycle & $\begin{array}{l}\text { System Owners } \\
\text { and Operations }\end{array}$ & determines & $\begin{array}{l}\text { Throughout the SoS lifecycle } \\
\text { the System Owners and } \\
\text { Operations may change, for } \\
\text { example for a directed SoS } \\
\text { the implementers may be } \\
\text { considered system owners, } \\
\text { when the SoS enters service } \\
\text { the end users will take } \\
\text { ownership. }\end{array}$ \\
\hline Lifecycle & Variability & induces & $\begin{array}{l}\text { This relationship assumes } \\
\text { that earlier in the lifecycle } \\
\text { the SoS will be more likely to } \\
\text { change, thereby increasing } \\
\text { variability, as the SoS } \\
\text { matures the rate of change } \\
\text { (i.e. variability) will decrease. }\end{array}$ \\
\hline Variability & Classification & influences & $\begin{array}{l}\text { Variability is an indication of } \\
\text { the rate of change within a } \\
\text { SoS, this relationship } \\
\text { suggests that SoS which are } \\
\text { more closely managed } \\
\text { (directed and acknowledged) } \\
\text { are more likely to exhibit a } \\
\text { low level of variability } \\
\text { whereas this is more likely to } \\
\text { increase for collaborative } \\
\text { and virtual SoS. }\end{array}$ \\
\hline $\begin{array}{l}\text { Nature of } \\
\text { Relationships }\end{array}$ & Interactions & determine & $\begin{array}{l}\text { The Nature of Relationships } \\
\text { may, at least to some extent, } \\
\text { determine the interactions } \\
\text { between the component } \\
\text { systems. }\end{array}$ \\
\hline $\begin{array}{l}\text { System Owners } \\
\text { and Operations }\end{array}$ & $\begin{array}{l}\text { Concept of } \\
\text { Operation/Use/ } \\
\text { Employment }\end{array}$ & define & $\begin{array}{l}\text { The system owners and } \\
\text { operations will directly } \\
\text { define the SoS Concept of } \\
\text { Operation/Use/Employment } \\
\text { although these may also } \\
\text { emerge and evolve through } \\
\text { the use of the SoS. }\end{array}$ \\
\hline $\begin{array}{l}\text { Concept of } \\
\text { Operation/Use/ } \\
\text { Employment }\end{array}$ & Functions & requires & $\begin{array}{l}\text { The SoS Concept of } \\
\text { Operation/ Use/Employment } \\
\text { requires a set of functions in }\end{array}$ \\
\hline
\end{tabular}




\begin{tabular}{|l|l|l|l|}
\hline $\begin{array}{l}\text { Source } \\
\text { Dimension }\end{array}$ & $\begin{array}{l}\text { Connecting } \\
\text { Dimension }\end{array}$ & Relationship & Description \\
\hline & & & order to support them. \\
\hline
\end{tabular}

Table 14 - SoS Sol Dimension Relationships

The SoS Sol representation was tested against two cases studies (as documented in the paper provided in Annex A):

- a typical example of a military Counter Air Mission

- National Programme for IT in the NHS (NPfIT), as discussed in section 2.1.2.

Table 15 (Kinder et al., 2012) illustrates how elements of the case studies were mapped to the dimensions, indicating that, at least in these cases, the model of SoS Sol does fulfil its intended purpose. Further discussion of the mapping activity is provided in the paper attached in Annex A.

\begin{tabular}{|c|c|c|}
\hline \multirow{2}{*}{ Dimensions } & \multicolumn{2}{|l|}{ Case Studies } \\
\hline & Counter Air Mission & $\begin{array}{l}\text { National Programme for IT in the } \\
\text { NHS (NPfIT) }\end{array}$ \\
\hline $\begin{array}{l}\text { Component } \\
\text { Systems }\end{array}$ & $\begin{array}{l}\text { Counter Air Aircraft (Typhoons) } \\
\text { Command and Control Unit (E3-D, } \\
\text { TACC) } \\
\text { JRE Gateway (KC-135) } \\
\text { TDL gateway (Type 45) } \\
\text { Air Defence Artillery (Type 45) } \\
\text { ISR (E3-D) } \\
\text { CVS Aircraft Carrier } \\
\text { Satellite (Skynet 5) }\end{array}$ & $\begin{array}{l}\text { Choose and Book } \\
\text { Electronic Prescription Service (EPS) } \\
\text { National Network for the NHS (N3) } \\
\text { NHS Mail } \\
\text { Picture Archiving and } \\
\text { Communication System (PACS) } \\
\text { The Spine }\end{array}$ \\
\hline Interactions & $\begin{array}{l}\text { Digital Communications (Link 11/16, } \\
\text { Satellite) } \\
\text { Voice Communications } \\
\text { Track Data } \\
\text { Command and Control } \\
\text { Imagery }\end{array}$ & $\begin{array}{l}\text { N3 Network } \\
\text { eMAIL } \\
\text { Appointment Data } \\
\text { Image Processing } \\
\text { Patient records } \\
\text { Prescriptions (electronic) }\end{array}$ \\
\hline Lifecycle & $\begin{array}{l}\text { Requirement, Planning, Assembly, } \\
\text { Execution } \\
\text { Dispersion }\end{array}$ & $\begin{array}{l}\text { Concept, Design and Development, } \\
\text { Operation, Reconfigure/Upgrade, } \\
\text { Disposal }\end{array}$ \\
\hline
\end{tabular}




\begin{tabular}{|c|c|c|}
\hline Variability & $\begin{array}{l}\text { Assets may change during mission. } \\
\text { The purpose may evolve as the } \\
\text { mission progresses. This SoS may } \\
\text { exhibit a high level of variability. }\end{array}$ & $\begin{array}{l}\text { Intuitively one assumes variability is } \\
\text { low because of the directed nature } \\
\text { of this SoS. However, due to the } \\
\text { poor definition of requirements } \\
\text { component systems were frequently } \\
\text { changed which resulted in a higher } \\
\text { level of variability than would } \\
\text { perhaps be expected. }\end{array}$ \\
\hline Classification & $\begin{array}{l}\text { Collaborative/Acknowledged. } \\
\text { Directed elements, e.g. Link 11/16 } \\
\text { networks }\end{array}$ & $\begin{array}{l}\text { Originally Directed but later moved } \\
\text { towards Acknowledged. }\end{array}$ \\
\hline Func & $\begin{array}{l}\text { Surveillance, } \\
\text { Targeting, Detect, Identify, Intercept, } \\
\text { Destroy, Aircraft Control, Battle } \\
\text { Management. }\end{array}$ & $\begin{array}{l}\text { Choose and Book appointment, } \\
\text { Change appointment, Produce } \\
\text { prescription, Picture Archiving }\end{array}$ \\
\hline $\begin{array}{l}\text { Systems } \\
\text { Owners and } \\
\text { Operations }\end{array}$ & $\begin{array}{l}\text { Nations (Governments), Services (Air } \\
\text { force, Navy, Army), Overarching } \\
\text { control, e.g. NATO. }\end{array}$ & $\begin{array}{l}\text { Project Team, NHS Connecting for } \\
\text { Health } \\
\text { Local Authority / Strategic Health } \\
\text { Authority } \\
\text { Project Board, Board of Directors / } \\
\text { PCT } \\
\text { Executive Group }\end{array}$ \\
\hline $\begin{array}{l}\text { Concept of } \\
\text { Operation }\end{array}$ & $\begin{array}{l}\text { The SoS provides protection from air } \\
\text { and missile threats. }\end{array}$ & $\begin{array}{l}\text { Provide a single, centrally mandated } \\
\text { electronic care record for patients } \\
\text { and to connect } 30,000 \text { GPs to } 300 \\
\text { hospitals. }\end{array}$ \\
\hline $\begin{array}{l}\text { Nature of } \\
\text { Relationships }\end{array}$ & Hierarchical (military C2) & $\begin{array}{l}\text { Plural system of procurement. } \\
\text { Client-Server. }\end{array}$ \\
\hline
\end{tabular}

Table 15 - Case Study Applicability to SoS Sol Dimensions (Kinder et al., 2012)

Regarding the Counter Air Mission case study, the proposed framework showed the relationship between component systems and interactions, emphasising the need for interoperability. Whilst this in itself can already be determined with current frameworks (e.g. MODAF (Ministry of Defence Architecture Framework)), the proposed framework allows further elaboration of each system, in accordance with the life cycle and the SoS purpose and shows the relationships to other aspects of the SoS (Kinder et al., 2012).

The model of SoS Sol may have enabled the identification of several issues that resulted in failure of the National IT Programme for the NHS. Those closest to the healthcare delivery were not able to provide adequate input into the SoS requirements definition, resulting in specific local requirements not being met. The SoS Sol representation links Lifecycle, System Owners and Operations, Concept of Operation/Use/Employment and Functions. This 
relationship illustrated that system owners indirectly influence the functions provided by the SoS. By definition all health trusts (System Owners) are part of the SOI and, therefore, in accordance with the proposed model, would be provided with input into the definition of Lifecycle, System Owners and Operations, Concept of Operation / Use / Employment (Kinder et al., 2012).

Incompatibility between systems was a major issue. The SoS Sol representation links Component Systems, Interactions and Lifecycle, allowing component system types and interaction types to be defined early in the lifecycle and, if correctly modelled, enabling any incompatibilities to be detected and prevented. The relationship of Lifecycle in this case was intended to enable component systems at different phases of development to form part of the Sol (Kinder et al., 2012).

Application of the model of SoS Sol for these case studies demonstrated the potential utility through the identification of issues and desired behaviour as discussed above. However, the SoS Sol identifies dimensions from a generic viewpoint, thereby enabling each to be instantiated appropriately in accordance with the maturity of the SoS under consideration, e.g. component systems may be identified at an abstract level or as specific systems. It should also be noted that use of all dimensions is not mandated when defining a particular Sol, only those applicable need be instantiated, which also supports the generic approach.

Of relevance to this research is the use of the SoS Sol model in support of key research outputs, specifically the SoS M\&S technique selection matrix and associated tool, described in sections 6.2 and 6.3 respectively as well as providing the basis for a SoS risk identification framework, described in section 7.3.2.

\subsection{Selection Matrix}

The survey of M\&S techniques and their applicability to the SoS domain, as discussed in section 3.3.4, is documented in the journal paper (Kinder et al., 2014) attached in Annex C. The output of the survey produced a matrix (reproduced in Table 7, section 3.3.4), which cross references M\&S technique to SoS Sol dimension, indicating the level of applicability.

The following general process for M\&S method selection was proposed (Kinder et al., 2014):

1) Determine the model requirements, through the identification of the basic functions.

2) Cross-reference the model functions to the Sol dimensions and determine the applicable dimensions. 
A Model Based Approach to System of Systems Risk Management

3) From the applicability matrix (Table 7, section 3.3.4), select M\&S techniques, which are applicable across dimensions.

4) From the model requirements determine if dynamic behaviour is required; re-assess the list of selected models using Table 16 information. 


\begin{tabular}{|c|c|c|c|}
\hline & Dynamic & $\begin{array}{l}\text { Ease of } \\
\text { creation }\end{array}$ & Verifiable \\
\hline DES/DEVS & $\mathrm{HIGH}$ & LOW & $\mathrm{HIGH}$ \\
\hline Petri Nets & HIGH & LOW & $\mathrm{HIGH}$ \\
\hline ABMS & $\mathrm{HIGH}$ & LOW & $\mathrm{HIGH}$ \\
\hline $\begin{array}{l}\text { System } \\
\text { Dynamics }\end{array}$ & HIGH & LOW & HIGH \\
\hline $\begin{array}{l}\text { Surrogate } \\
\text { Models }\end{array}$ & HIGH & LOW & HIGH \\
\hline ANN & HIGH & $\mathrm{HIGH}$ & $\mathrm{HIGH}$ \\
\hline BNN & LOW & HIGH & HIGH \\
\hline $\begin{array}{l}\text { Markov } \\
\text { Models }\end{array}$ & LOW & HIGH & HIGH \\
\hline $\begin{array}{l}\text { Game } \\
\text { Theory }\end{array}$ & LOW & LOW & LOW \\
\hline $\begin{array}{l}\text { Decision } \\
\text { Trees }\end{array}$ & LOW & HIGH & HIGH \\
\hline $\begin{array}{l}\text { Network } \\
\text { Models }\end{array}$ & LOW & HIGH & HIGH \\
\hline EAF & LOW & $\mathrm{HIGH}$ & LOW \\
\hline $\begin{array}{l}\text { Modelling } \\
\text { Languages }\end{array}$ & LOW & HIGH & LOW \\
\hline
\end{tabular}

Table 16 - General M\&S attributes (Kinder et al., 2014)

\subsection{Selection Tool}

Following the definition of the selection matrix and the associated process for M\&S method selection it became apparent that development of a spreadsheet based tool based on the framework would be straightforward, and which could offer a basic level of automation as well as enabling documentation of the process in a standard format.

The tool fulfilled the following requirements:

1) Allow the user to identify the model functions and manually identify which SoS Sol dimensions (section 6.1) and General M\&S attributes (Table 16) are applicable.

2) Automatically identify appropriate $M \& S$ techniques.

3) Automatically generate a ranking, indicating the suitability of the selected techniques.

An example of an instantiated spreadsheet is provided in Table 17 - SoS M\&S Technique selection tool 
The first column, titled 'Model', allows the user to define the model name, in this case "Kill Chain" (which is fully described in Chapter 8).

The second column entitled "Model Components" allows the main functions of the model to be entered; in this example seven are identified. This forms a matrix with the SoS Sol dimensions, which is manually populated by the user, thereby fulfilling the first requirement. During development of the CAS tool prototype models (described in chapter 8) additional characteristics of "stochastic" and "uncertainty" were added, which are shown in Table 17 - SoS M\&S Technique selection tool

The "stochastic" characteristic was necessary to support model functions requiring an element of randomness, for example a Gaussian distribution may represent the accuracy of a sensor. The "uncertainty" characteristic is used to indicate the model function requires probabilistic modelling, where the output of the model provides an indication of probability or likelihood, such as risk. 


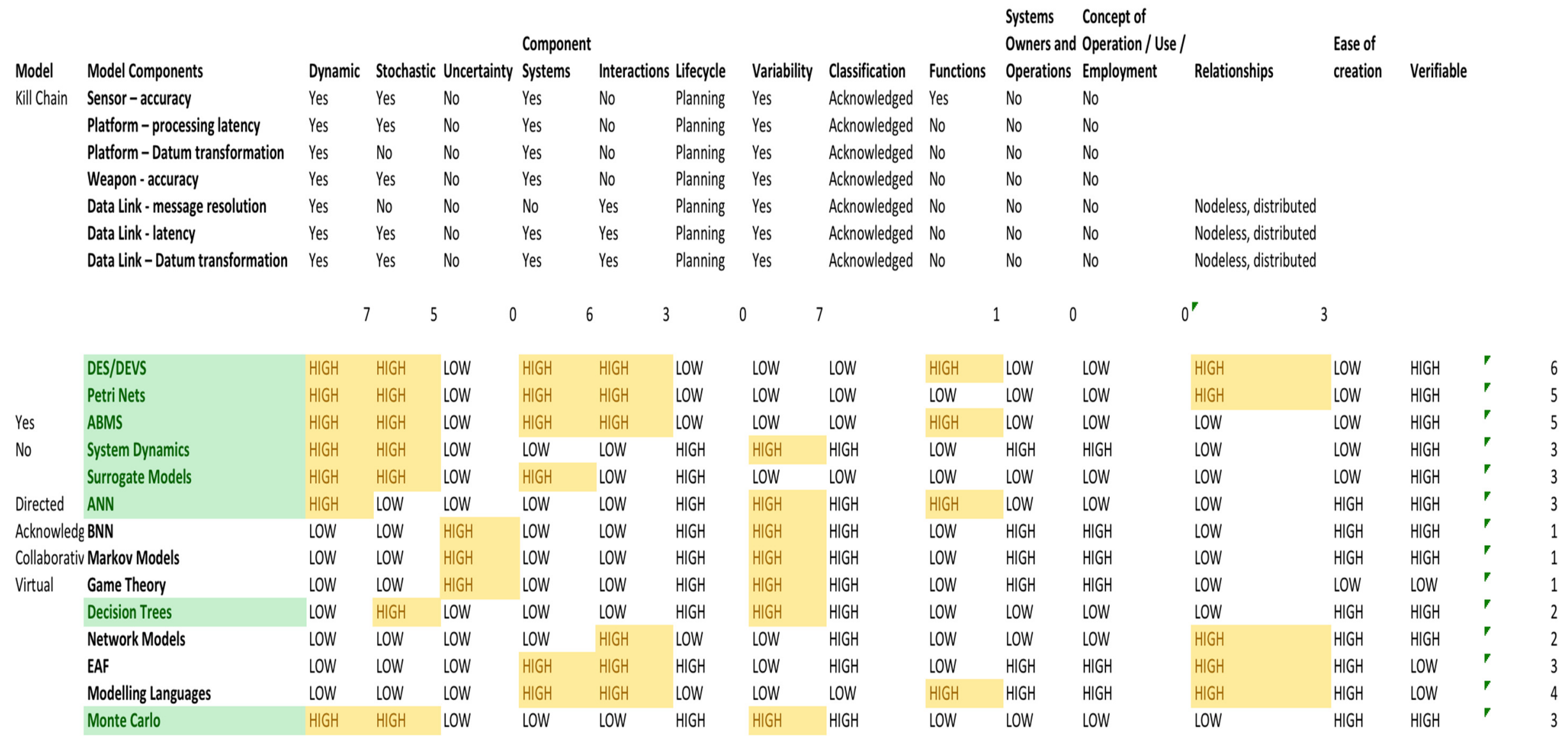

Table 17 - SoS M\&S Technique selection tool 
Below the list of model components, the list of potential M\&S methods is defined and a matrix cross referencing SoS Sol dimensions and M\&S characteristics inserted, as described in Table 7 and Table 16. This matrix also includes the "stochastic" and "uncertainty" characteristics, with the mapping to M\&S methods shown in Table 18.

\begin{tabular}{|l|c|c|}
\hline & Stochastic & Uncertainty \\
\hline DEVS & & \\
\hline Petri Nets & & \\
\hline ABMS & & \\
\hline $\begin{array}{l}\text { System } \\
\text { Dynamics }\end{array}$ & & \\
\hline $\begin{array}{l}\text { Surrogate } \\
\text { Models }\end{array}$ & & \\
\hline ANN & & \\
\hline BNN & & \\
\hline $\begin{array}{l}\text { Markov } \\
\text { Models }\end{array}$ & & \\
\hline $\begin{array}{l}\text { Game } \\
\text { Theory }\end{array}$ & & \\
\hline $\begin{array}{l}\text { Decision } \\
\text { Trees }\end{array}$ & & \\
\hline $\begin{array}{l}\text { Network } \\
\text { Models }\end{array}$ & & \\
\hline EAF & & \\
\hline $\begin{array}{l}\text { Modelling } \\
\text { Languages }\end{array}$ & & \\
\hline
\end{tabular}

Table 18 - M\&S Method Stochastic and Uncertainty characteristics (Kinder et al., 2014)

Conditional formatting is used to indicate which elements in the matrix are relevant, i.e. for a each column if at least one model component is applicable to a dimension or characteristic then all instances in the matrix and in the same column which are defined as ' $\mathrm{HIGH}$ ' are coloured amber, providing an overview of the model applicability to dimensions and characteristics.

Conditional formatting is also used to highlight appropriate M\&S methods green, fulfilling the second requirement. The rule for highlighting is as follows:

Highlight method cell green if:

- Any element in model technique row is amber and dynamic, stochastic or uncertainty capability is required

- Any SoS Sol dimension is amber, no dynamic or stochastic capability is required and method does not support dynamic or stochastic capability

The suitability ranking is calculated by summing the number of relevant $M \& S$ characteristics and SoS Sol dimension supported by the modelling technique, fulfilling the third 
A Model Based Approach to System of Systems Risk Management

requirement. If the highest ranked technique does not support all required attributes this indicates that a hybrid modelling approach is required (reference section 3.3.5) or alternatively the modelling requirements should be decomposed further.

The tool was used in the development of prototype models which are described in chapter 8.

\subsection{Future Development of the Spreadsheet based tool}

It is acknowledged that the application of just two case studies does not provide strong evidence of the generalizability of model of SoS Sol (Kinder et al., 2012), hence future development should consider the application of additional exemplars, however this has been undertaken to some extent by the development of prototype models, as described in chapter 8.

The model selection framework could be expanded through the addition of further M\&S techniques, beyond those listed in Table 7.

The tool is currently spreadsheet based, however providing it as a web service would be possible thereby providing a widely available SoSE support capability.

\subsection{Summary}

This chapter introduced a unique approach to the determination of the SoS Sol through definition of the following dimensions (Kinder et al., 2012) ${ }^{10}$ :

- Component Systems (including specific and general system types)

- Classification

- Interactions

- Nature of Relationships

- Lifecycle

- Variability

- Functions

- Systems Owners and Operations

- Concept of Operation / Use / Employment

\footnotetext{
${ }^{10}$ This paper is attached in Annex A.
} 
A Model Based Approach to System of Systems Risk Management

A model illustrating the relationships between the SoS Sol dimensions (Kinder et al., 2012) was developed, as shown in Figure 26, with descriptions of the relationships provided in Table 14.

The model of SoS Sol provides a basis for a SoS M\&S technique selection framework in combination with the survey described in section 3.3.4. The selection framework supports the selection of appropriate M\&S techniques appropriate for SoS within a particular context. This framework was further developed into a spreadsheet-based tool, which provides a basic level of automation and a common format for populating the framework. 
A Model Based Approach to System of Systems Risk Management

\section{Chapter 7: SoS Risk Management}

Whereas section 3.4.6 discussed current approaches to SoS Risk Management, finding very limited application ${ }^{11}$, this chapter describes a proposed model based approach to SoS Risk Management, discusses how it may be integrated into SoSE activities and who within the SoS has responsibility for this task. A published paper summarising the model based approach to SoS Risk Management (Kinder et al., 2015) is attached in Annex B.

Analysis of the case studies in section 2.1 and Theme 1 (section 3.2.5) of the literature review identified Risk Management as a key component of SoSE, being referred to in the DoD SE Guide for SoS (US Department of Defense, 2008b), the Systems and software engineering standard, ISO 15288 (British Standards, 2011) and the SoSOMM approach (Gorod et al., 2007).

Figure 27 provides context for the research output components described in this chapter, illustrating their relationship to each other and to components described outside this chapter.

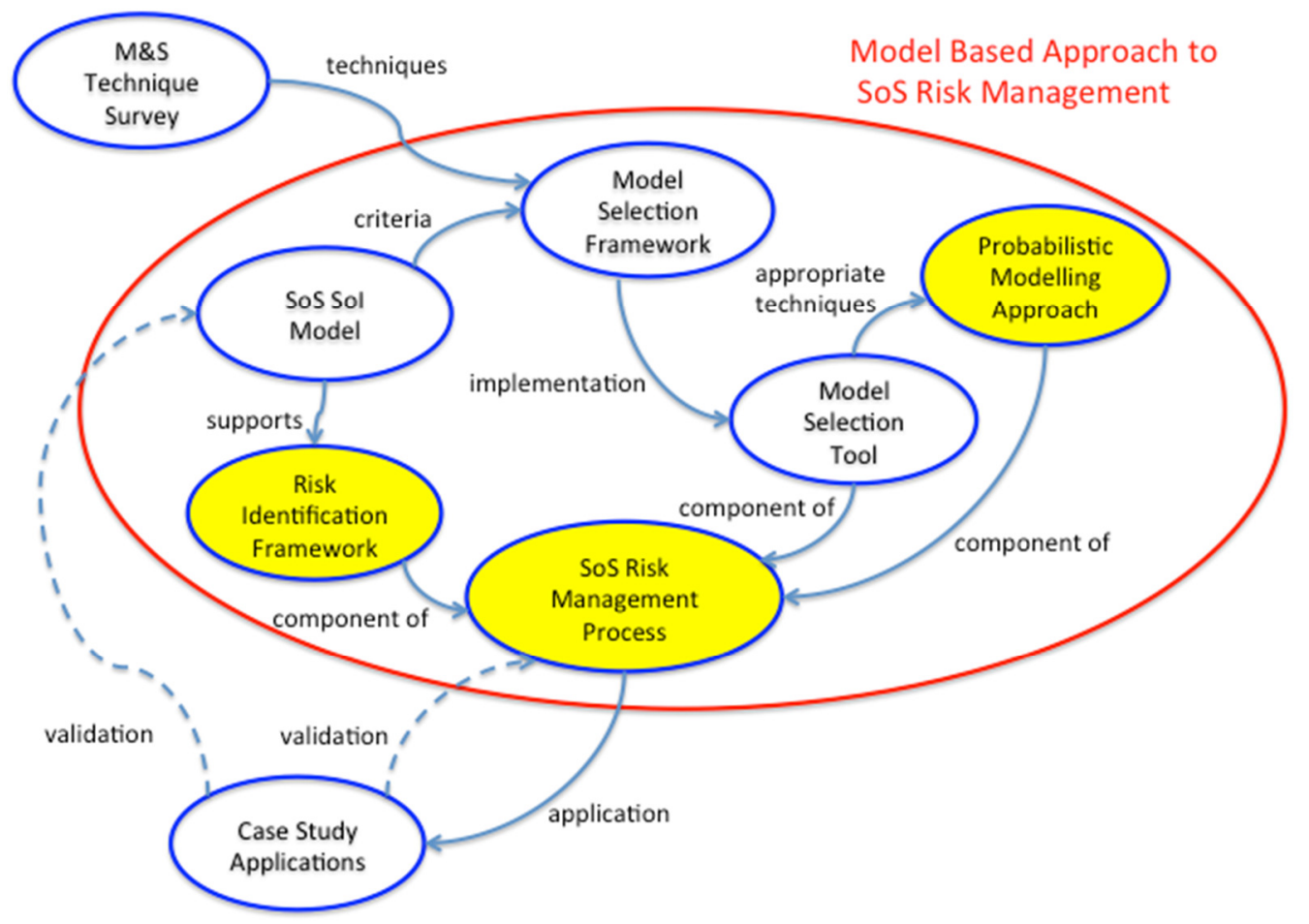

Figure 27 - SoS Risk Management Context

\footnotetext{
${ }^{11}$ Although the DoD SE Guide for SoS (US Department of Defense, 2008b) does provide some basic guidance applicable to acknowledged SoS
} 


\subsection{Where does Risk Management fit in SoSE?}

The literature review established that Risk Management, in the context of SE, is defined as: "a disciplined approach to dealing with the uncertainty that is present throughout the entire system lifecycle" (INCOSE, 2015).

This definition of Risk Management could be equally applied to SoS, in that risk throughout the entire SoS lifecycle must be considered, however a key difference between SE and SOSE is the lifecycle definition (section 3.2.5). Development of a single system will typically comprise a linear series of activities undertaken within specific lifecycle phases; concept, development, production, utilization, support, and retirement (British Standards, 2011) and where Risk Management is undertaken as a continuous activity within each phase.

A SoS can be defined as "A SoS is a system which results from the coupling of a number of constituent systems at some point in their life cycles" (Brook, 2016).

This definition succinctly illustrates that a SoS consists of a number of component systems, where each may be at different phases within its development lifecycle and where there may be limited influence over these systems at the SoS level; hence Risk Management of SoS does not directly align with the traditional SE lifecycle. A more appropriate lifecycle approach is the DANSE model, comprising the phases, initiation, creation and operations (section 3.2.5) and which accounts for asynchronous development of the component systems, although it does not encompass virtual SoS where there is no central managerial control.

How Risk Management integrates into the SoSE approach is largely driven by the SoS classification; directed, acknowledged, collaborative and virtual (Dahmann \& Baldwin, 2008) (section 3.2.3).

During its Initiation phase the SoS comes into existence through the connection of a group of component systems enabling the sharing of information and resources, resulting in emergent behaviour at the SoS level. Risk Management at this point will attempt to identify associated risks, which may result in undesired emergent behaviour, thereby preventing the SoS fulfilling its purpose. However, risk encompasses both detrimental and beneficial effects (section 3.4.1), therefore risks that result in unexpected, but beneficial, emergent behaviour need to be identified and potential exploitation of this behaviour considered.

The creation phase is optional, only being required when the initial SoS emergent behaviour is severely deficient, this may be a pre-emptive activity as a result of risks identified within 
the initiation phase, however the same risk management process will be undertaken as for the creation phase.

In the Operational Phase the SoS provides the desired emergent behaviour, with the "manager" monitoring for additional potential needs and influencing the component systems where modification emergent behaviour is required. Risk Management will be continuously undertaken in these phase to both assess risks which may impact the new SoS objectives and those which may impact current objectives, such as changes to component systems.

\subsection{Who performs SoS Risk Management?}

A directed SoS is centrally managed and an acknowledged SoS has a designated manager (Dahmann \& Baldwin, 2008), which enables a single body to perform Risk Management, the DoD SE Guide for SoS (US Department of Defense, 2008b) recommends the creation of an integrated Risk Management Board, encouraging participation of members from component systems. Having overall control of the SoS, the board will generally take a top down approach to Risk Management.

In a Collaborative SoS "component systems interact more or less voluntarily to fulfil agreed upon central purposes" (Dahmann \& Baldwin, 2008), hence no single controlling authority exists. In this case an advisory body may perform Risk Management but has no authority over the component systems, alternatively it may be performed using a bottom up approach through collaboration of the component systems.

For SoS that are rapidly created in response to an immediate need, risk management may be undertaken by those responsible for planning in the creation phase, an example of this is a Close Air Support mission, which is described in chapter 8.

Virtual SoS "lack a central management authority and a centrally agreed upon purpose for the system-of-systems" (Dahmann \& Baldwin, 2008), hence it is not possible to identify associated risks in the traditional sense. The SoS purpose emerges from the resulting behaviour, therefore risks cannot be anticipated although it could be argued that once a purpose is established the SoS may evolve to minimise risk, or alternatively if a risk materialises the SoS may evolve to fulfil a different purpose. It is therefore suggested that Risk Management cannot be undertaken for a virtual SoS, where the materialisation of both positive and negative risks will result in the evolution of the SoS and any risk mitigation will be undertaken by individual users or elements. 
However, it is noted that a SoS may simultaneously exhibit different classifications where component systems form sub-groups; for example a SoS supporting disaster relief (Hurricane Katrina, discussed in section 2.1.6) will typically consist of component systems under the control of a single agency which are classified as directed, working with similarly classified groups but as a whole the SoS may be working collaboratively until overall control of all agencies is established.

Considering the case studies discussed in section 2.1, within the FCS program risk was managed by a combination of individual integrated product teams, the Risk Working Group, and the Risk Review Board, which this resulted in a "single-system focus rather than SoS approach" (Axelband et al., 2012) as a result of identifying risks at the component system level rather than holistically at the SoS level.

The US Coastguard Deepwater program attempted to manage risk through a traditional approach, where the prime contractor assumed that if risks associated with component systems were managed then those at the SoS level would not arise, a misconception addressed by the DoD SE Guide for SoS (US Department of Defense, 2008b), as discussed in section 3.4.3.

Project managers were the focal point for Risk Management on the NPfIT (section 2.1.2) in the NHS, although it appears likelihood or probability were not determined for identified risks (Qinetiq, 2005) and it is assumed an overarching view of SoS risk was not considered, which could have been accomplished through the formation of an integrated Risk Management Board, as discussed above.

Poor Risk Management was identified as a major contributor to the failure of the C-NOMIS project (section 2.1.4), where identified risks were not managed and the governance structure did not encourage accurate reporting of issues (National Audit Office, 2009); again there appeared to be no specific SoS risk approach and the creation of an integrated Risk Management Board would have been appropriate.

The Close Air support (CAS) mission fratricide, as described in section 2.1.5, arose as a result of unidentified risks. In this case traditional project based risk management was not appropriate, instead the planning phase should have incorporated appropriate risk identification activities. The management of CAS risk, using the proposed SoS modelling approach, is described in chapter 8.

The disaster relief effort in response to Hurricane Katrina required agencies to co-ordinate collaboratively, however there were initially many obstacles to achieving this, particularly 
communication issues, with risks being mitigated as they materialised. Eventually a central command structure was established but there did not appear to be a coherent risk management strategy. However, in light of the lessons learnt the US Department of Homeland Security produced a set of fifteen National Planning Scenarios (Townsend, 2006), enabling risk management to be performed pre-emptively for future disaster relief.

The Arizona and Southern California Power Grid blackout (section 2.1.7) was the result of a cascading failure, risk management was undertaken by individual authorities which together formed an acknowledged SoS, however each area is intended to operate in accordance with the North American Electric Reliability Corporation's (NERC) mandatory Reliability Standards (FERC \& NERC, 2014).

The Friendster social networking website (section 2.1.8) provided the infrastructure, through enabling interactions, for users to form virtual SoS. The website itself could be regarded as an acknowledged SoS; hence a level of control existed. Although documentation is not available, it is assumed that management of the website included an element of Risk Management which identified functionality required to maintain its popularity, however eventually the change in users behaviour resulted in an unsustainable drop in use and the associated virtual SoS dissipated, hence the website was shut down. This illustrated that where elements of a SoS are classified as virtual, risk management can only be applied to those elements that form directed, acknowledged or collaborative SoS groups, where influence of the component systems is possible.

In summary, the answer to the question "who should perform SoS risk management?" is anyone with the authority and/or power to implement mitigation. This could be one role, or many roles that are distributed across the SoS, depending on the nature of the SoS concerned. So individual system owners may also have a, possibly, unrecognised responsibility to consider SoS risks as well as system-level risks.

\subsection{Proposed SoS Risk Management Process}

The SoS Risk Management process comprise the same basic activities as those required when engineering individual systems ${ }^{12}$, (section 3.4 .3 ) and is recapped below:

- Risk Planning

- Risk Identification

\footnotetext{
12 The DoD SoSE guide (US Department of Defense, 2008b) directly references the Risk, Issue, and Opportunity Management Guide for Defense Acquisition Programs (DASDSE, 2015)
} 
- Risk Analysis

- Risk Handling

- Risk Monitoring

It is the approach taken for each of these activities, the tempo of the process and the level of influence over component systems that differentiates SoS risk management from that required within traditional $\mathrm{SE}$.

The tempo of the Risk Management process is driven by the required timeliness. For example, a 'traditional' system will typically be developed over an extended period of time, typically months or years, a SoS may be formed rapidly in response to an urgent need such as disaster relief. In this case risk management may be required to become a near real-time, decision support activity (Kinder et al., 2015), integrated into the SoS lifecycle (Haimes, 2012).

The following sections consider each Risk Management activity and describe the approach required from a SoS perspective.

\subsubsection{SoS Risk Planning}

Directed and Acknowledged SoS both have a designated manager, enabling central control of SoS risk activities, including planning activities. This aligns with the traditional Risk Management approach, which allows roles to be identified, definition of process and procedures, allocation of resources and establishes traceability of risk to SoS objectives. A directed SoS will generally be developed over a relatively long period of time, following a similar lifecycle to traditional system development (section 3.2.5), however an acknowledged SoS may be formed in short order, for example a Close Air Support (CAS) mission as described in chapter 8 . In this case risk management should be incorporated into the planning phase and established in pre-defined procedures, for CAS missions they are defined in "Tactics, Techniques And Procedures For Close Air Support Operations" (NATO, 2005).

Another example of a pre-defined procedure is the UK Police Services, Gold, Silver, Bronze (GSB) command and control model (National Police Improvement Agency, 2009) which provides "a framework for delivering a strategic, tactical and operational response to an incident or operation", particularly where a multi-agency response is required. The Gold, Silver and Bronze hierarchy is related to role rather than rank and enables a command and control structure to be quickly established for complex situations requiring rapid response 
through its generic and adaptable approach. The publication of GSB guidance has provided awareness amongst potential partner agencies enabling them to work collaboratively with the Police Service in situations where specific pre-planning is precluded, thereby reducing risks associated with poorly defined lines of command, communications and interoperability.

Within a collaborative SoS, component systems work together on a voluntary basis in order to fulfil a central purpose (Dahmann \& Baldwin, 2008), hence the traditional risk planning approach is not necessarily applicable. In this situation a voluntary body may be established to undertake this task, and will have to achieve agreement before roles are assigned. Ideally a procedure is established which defines the SoS risk management process, however without any central control it will be the responsibility of component systems to follow the process and co-ordinate throughout it. For collaborative SoS formed rapidly in response to an unpredicted need, it is likely risks will be addressed as they arise until the SoS develops a more acknowledged structure. It is suggested that any lessons learnt are captured in order to either update any existing procedures or to create one if required, however this would require co-operation between the component systems and the identification or creation of a group or authority to hold the procedure.

Risk planning for virtual SoS is by definition not possible as there is no control and no agreed purpose.

\subsubsection{SoS Risk Identification}

Types of risk are discussed in section 3.4.2, where it was concluded that current risk categories are not necessarily always applicable in the SoS domain, although this is dependent upon SoS classification. For a directed SoS, risk types such as Technical, Programmatic, Schedule and Cost are likely to be applicable, however when considering an acknowledged SoS, which may be formed in short order to fulfill a specific aim, only the Technical and Schedule risk apply and these will have different interpretations to the usual project based approach. For example, in this context technical risks may be more related to interoperability and schedule related to time sensitive aspects of a mission.

Therefore an alternative approach is suggested whereby, rather than using the traditional SE risk types as a basis for the identification of risk, a framework is used which incorporates the SoS Sol dimensions (Kinder et al., 2012), described in section 6.1 and Annex A, and the holistic risk types; hazard, control and opportunity (Hopkin, 2002) which were identified as 
suitable for SoS application in theme 3 of the literature review (section 3.4.2). The framework is illustrated below in Table 19, which contains examples of potential risks.

\begin{tabular}{|c|c|c|c|}
\hline & $\begin{array}{l}\text { Hazard Risks (Threat } \\
\text { and perils) }\end{array}$ & $\begin{array}{l}\text { Control Risks (Doubt } \\
\text { about mission } \\
\text { achievement, project } \\
\text { management control) }\end{array}$ & $\begin{array}{l}\text { Opportunity Risks } \\
\text { (Opportunity to } \\
\text { enhance or inhibit } \\
\text { mission } \\
\text { achievement) }\end{array}$ \\
\hline $\begin{array}{l}\text { Component } \\
\text { Systems }\end{array}$ & $\begin{array}{l}\text { Emergent behaviour } \\
\text { inhibits purpose }\end{array}$ & $\begin{array}{l}\text { System immaturity } \\
\text { System unavailability }\end{array}$ & $\begin{array}{l}\text { Emergent behaviour } \\
\text { enhances purpose }\end{array}$ \\
\hline Classification & $\mathrm{N} / \mathrm{A}$ & Misclassification & $\mathrm{N} / \mathrm{A}$ \\
\hline Interactions & $\begin{array}{l}\text { Poor interoperability } \\
\text { Bandwidth insufficient }\end{array}$ & $\begin{array}{l}\text { Poor interoperability } \\
\text { interrupts command and } \\
\text { control }\end{array}$ & $\begin{array}{l}\text { Bandwidth can } \\
\text { support additional } \\
\text { interaction medium }\end{array}$ \\
\hline $\begin{array}{l}\text { Nature of } \\
\text { Relationships }\end{array}$ & $\begin{array}{l}\text { Failure dependent on } \\
\text { a single node }\end{array}$ & $\begin{array}{l}\text { Hierarchical command } \\
\text { structure inhibits agility }\end{array}$ & Agility increased \\
\hline Lifecycle & $\begin{array}{l}\text { Immaturity of } \\
\text { component systems }\end{array}$ & Lack of coordination & $\begin{array}{l}\text { Lifecycles of } \\
\text { component systems } \\
\text { align }\end{array}$ \\
\hline Variability & SoS instability & $\begin{array}{l}\text { Instability inhibits } \\
\text { control }\end{array}$ & High agility \\
\hline Functions & $\begin{array}{l}\text { Functions not } \\
\text { available }\end{array}$ & $\begin{array}{l}\text { Ownership of function } \\
\text { not defined }\end{array}$ & $\begin{array}{l}\text { Additional } \\
\text { functionality exists }\end{array}$ \\
\hline $\begin{array}{l}\text { Systems } \\
\text { Owners and } \\
\text { Operations }\end{array}$ & Lack of co-operation & $\begin{array}{l}\text { Lack of management } \\
\text { authority }\end{array}$ & $\begin{array}{l}\text { High level of co- } \\
\text { operation }\end{array}$ \\
\hline $\begin{array}{l}\text { Concept of } \\
\text { Operation / } \\
\text { Use / } \\
\text { Employment }\end{array}$ & $\begin{array}{l}\text { Concept of operation } \\
\text { not supported }\end{array}$ & $\begin{array}{l}\text { No clear concept of } \\
\text { operation }\end{array}$ & $\begin{array}{l}\text { Adaptable for } \\
\text { changing concept of } \\
\text { operation }\end{array}$ \\
\hline
\end{tabular}

Table 19 - SoS Risk Identification Matrix

It is emphasized that this is not intended to be prescriptive approach to SoS risk identification, the framework is intended to provide a starting point for the identification process and could utilize techniques, such as brainstorming or the Delphi method (section 3.4.3) as appropriate. It should be noted that, as well as identifying potential risks, the factors contributing to them would also be identified at this stage. However, the risk analysis modeling activities (section 7.3.3) may reveal additional risks and contributing factors which may than be analysed further.

Having identified risks in the context of the SoS dimensions, the ownership of each risk can now be considered. The key criterion for determining ownership is the ability of an entity to 
affect influence over the risk. This will be dependent upon the SoS classification, for example the identification of a risk owner within a directed SoS will be relatively straightforward as lines of responsibility should be well defined, whereas within a collaborative SoS a level of consensus may have to be reached in order to agree ownership.

\subsubsection{SoS Risk Analysis}

The key objectives of risk analysis are to estimate the likelihood that a risk will occur, estimate the consequences and determine the risk level (US Department of Defense, 2006) (section 3.4.3).

These objectives are equally relevant for both SoS and system risk analysis. In the engineering of systems risks are typically quantified through, subjective expert opinion and derived from a mental model. However, human processing of problems involving four variables "showed a significant decline in accuracy and speed of solution" from those involving three variables, whilst, problem solving involving five variables was at "chance level" (Halford et al., 2011). A SoS is inherently complex, hence when analysing SoS risk, it is assumed many variables will require consideration thereby exceeding the limits of human processing and breaking the law of requisite parsimony. Perception of risk is influenced by emotional response and bias, which also may impair the objective assessment of risk, as discussed in section 3.4.4 (Kinder et al., 2015).

It is therefore proposed that, in order to provide, rigorous and trustworthy quantified SoS risk assessment, a modelling approach must be taken, removing the reliance on subjective judgement and mental models of complex causal relationships between risk factors (Kinder et al., 2015).

The model based approach is elaborated further in section 7.4.

\subsubsection{SoS Risk Handling}

Following identification, analysis and quantification of a risk, it must be determined if the risk is to be accepted, avoided, transferred, or mitigated. This decision process is equally applicable to risk handling within single systems and SoS.

A risk will be accepted if the cost of mitigation outweighs the cost incurred if the risk materialises, in the context of SoS "cost" may be financial or temporal. As discussed, a directed SoS may be developed over a relatively long period, with many parallels to traditional SE, in this case the managerial structure will exhibit a level of financial control, hence cost may be assessed and decisions taken based on this. For SoS created rapidly, short 
term financial concerns are less relevant; it is the ability of the SoS to meet its objectives within the required timescales that are key. For example, a SoS undertaking a search and rescue mission will be extremely time sensitive, hence if the mitigation of a risk is excessively time consuming then the risk may be accepted in the interests of achieving the objective. However, the long term financial impact of short term risk mitigation should be considered where possible. It is proposed that the models created in support of the risk analysis activities may enable the impact of accepting risk to be more accurately quantified, particularly where temporal aspects require quantification.

Transfer of risk may occur within the SoS itself or risk may be transferred to an external entity. For example, when considering a disaster relief scenario, responsibility for a specific task may be re-allocated to a different agency (or component system) with greater capability in a specific area, such as thermal location of earthquake survivors. In this case, where the risk is transferred outside the Sol, then from the SoS perspective it is mitigated, in contrast to an internal transfer where the risk not mitigated. An advantage provided by a model based approach is the ability to compare potential risk transfer strategies, enabling the most suitable to be identified; this is explored further through the case studies in chapter 8 . As discussed previously, the SoS classification determines the options available for risk transfer, which is itself dependent upon the ability to influence component systems or the level of cooperation between them.

\subsubsection{SoS Risk Monitoring}

As with traditional risk management, identified risks require monitoring, involving reassessment of likelihood and severity. Where a high level of control is available over the component system development, monitoring will be undertaken in the same way as traditional risk monitoring (section 3.4.3), however where a SoS is formed rapidly in a collaborative environment this approach does not provide the necessary agility. In this case a potential solution would be the provision of a decision support capability providing automated monitoring of risks; the proposed modelling approach could potentially provide the basis for this. Again, this is explored further through the case study applications described in chapter 8.

\subsection{Proposed Model Based Approach}

Several modeling approaches to SoS Risk Management were discussed under theme 3 of the literature review, in section 3.4.6, however these were found to be tightly coupled to their associated problem space, without scope for wider generalisation within the SoS domain. 


\section{A Model Based Approach to System of Systems Risk Management}

Hence, the following model-based approach was developed, utilizing the model selection technique described in chapter 6 providing a generic approach which can be tailored for each problem space allowing the integration of appropriate models, rather than constraining the approach to a single or small set of techniques (a constraint encountered in hybrid modeling approaches, as discussed in section 3.3.5). However, BBNs and Monte Carlo Simulation were identified as an appropriate technique for modelling risk and therefore form a key element of the model-based approach.

The approach comprises the following steps and is described further in the following subsections:

- Create causal network incorporating all identified risks and the contributing factors

- Create a Bayesian Belief Network (BBN) for each risk, including all linked contributing factors

- Create supporting models of contributing factors where required

- Run supporting models in a Monte Carlo simulation, use model output to update BBN

- Create Risk confidence model

\subsubsection{Causal Network}

The risk identification activity (section 7.3.2) by definition will determine risks applicable to the SoS, however it will also identify factors that impact the likelihood of a risk materialising. Within the model based approach, the risks and their relationship to the contributing factors are captured in a causal network, which are one aspect of BBNs and are described in section 5.2. At this stage no probabilities are assigned, the model purely identifies causal effects, providing a qualitative perspective. A simplified generic example is provided in Figure 28 , it should be noted that contributing factors may be layered and one factor may influence one or many other factors or risks. However, the number of contributing factors and risks will generally be higher, as demonstrated in the case studies described in chapter 8 . 


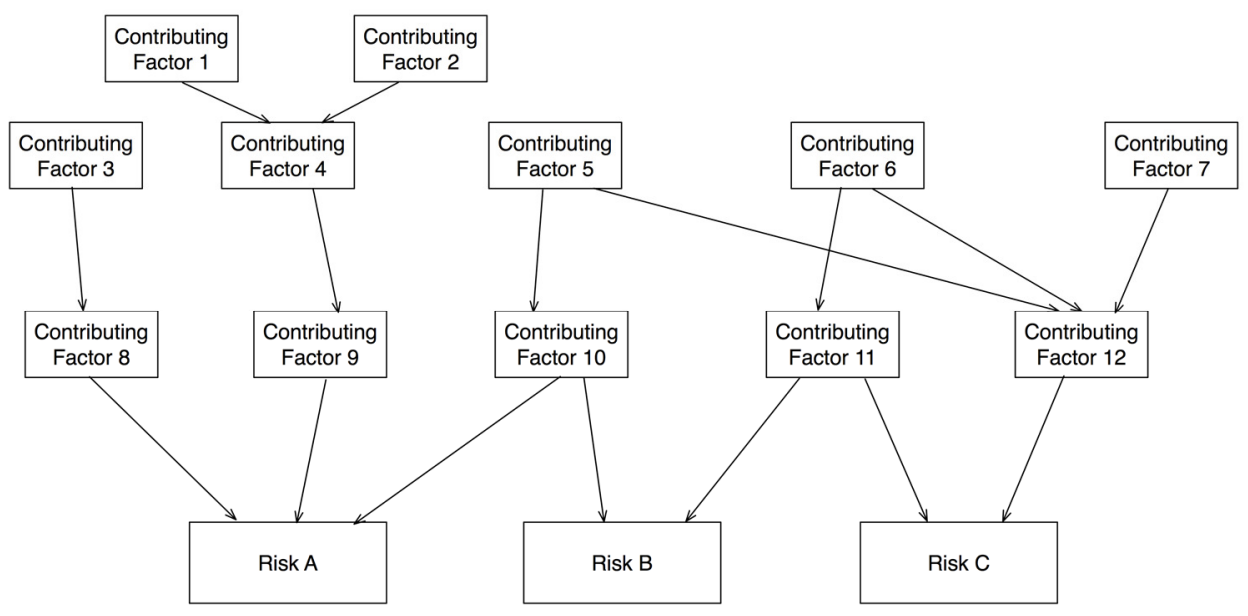

Figure 28 - SoS Risk Causal Network

\subsubsection{Central Bayesian Risk Model}

Having created the causal network capturing the complete risk picture, BBNs are constructed enabling risk to be quantified. However, creation of a single BBN derived directly from the causal network may be overly cumbersome, hence an alternative approach is the creation of a separate BBN for each individual risk. Focussing on single risks dramatically simplifies each BBN requiring only the relevant contributing factors to be included. Considering "Risk A" shown in Figure 28, the structure of the associated BBN now only includes connected contributing factors, as shown in Figure 29. It is emphasised that the effectiveness of the BBNs risk analysis capability is reliant on the identification of all relevant contributing factors, as described in section 7.3.2.

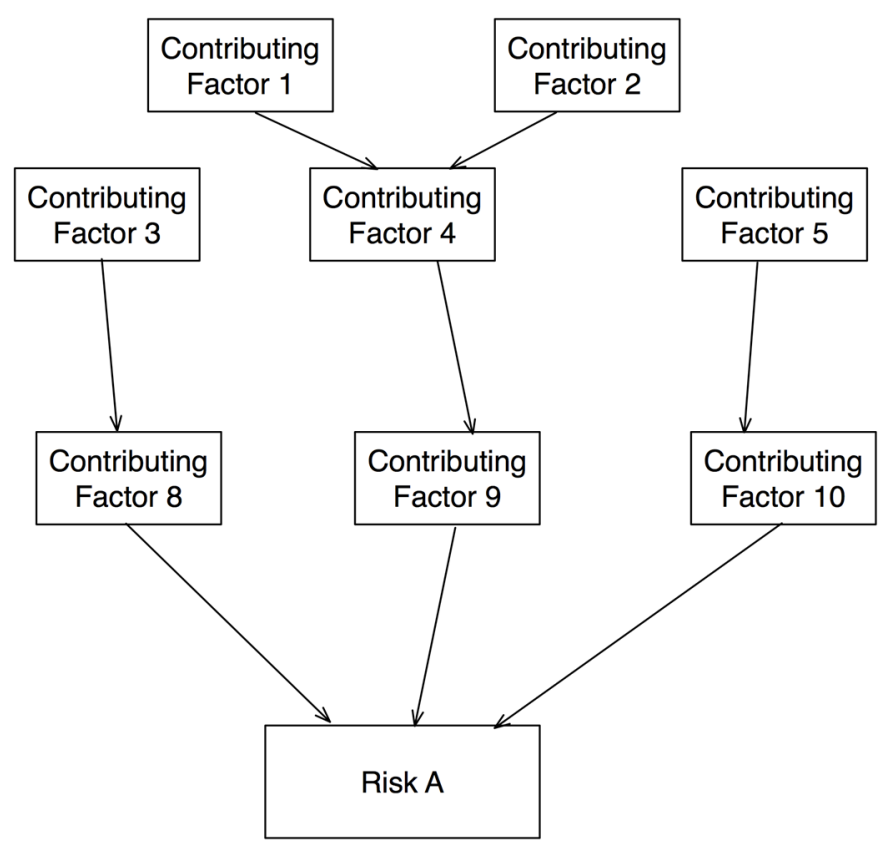

Figure 29 - Single Risk BBN 
However, before determining the final structure of the selected risk BBN each contributing factor, or groups of factors need to be assessed using the model technique selection criteria as described in section 6.2. This process may allow further simplification of the BBN whereby some contributing factors are combined into a single supporting model (described in section 7.4.3), reducing the number of nodes in the BBN. However, all contributing factors, which are directly connected to the risk node, must also be represented with the BBN by an equivalent node, otherwise no mechanism will exist to influence the risk probability. Figure 30 illustrates how contributing factors could be grouped and supporting models identified. In order to identify appropriate groupings the following procedure should be followed.

1. Identify top level modelling requirements for contributing factor directly connected to risk.

2. Insert model requirements into model selection matrix tool (section 6.3) and select relevant SoS Sol dimensions (section 6.1); the tool will identify appropriate modelling technique(s).

3. For each parent contributing factor, assess if the factor could be represented as a model variable, if this is not the case then perform step 2 .

4. If the same modelling technique is identified for both parent and child then group as a single supporting model.

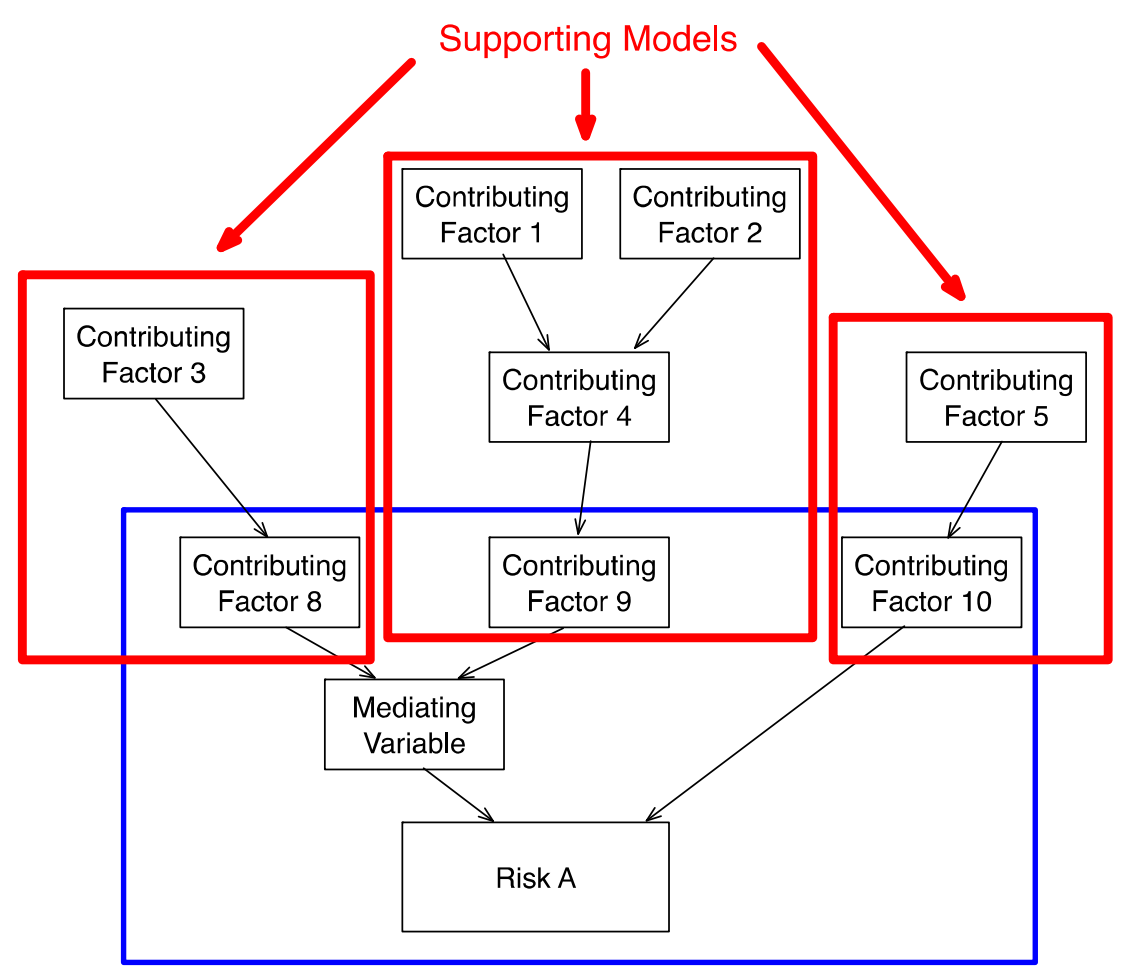

BBN

Figure 30 - Model Architecture 
Having determined the grouping of the contributing factors the model architecture can be defined, as shown in Figure 31.

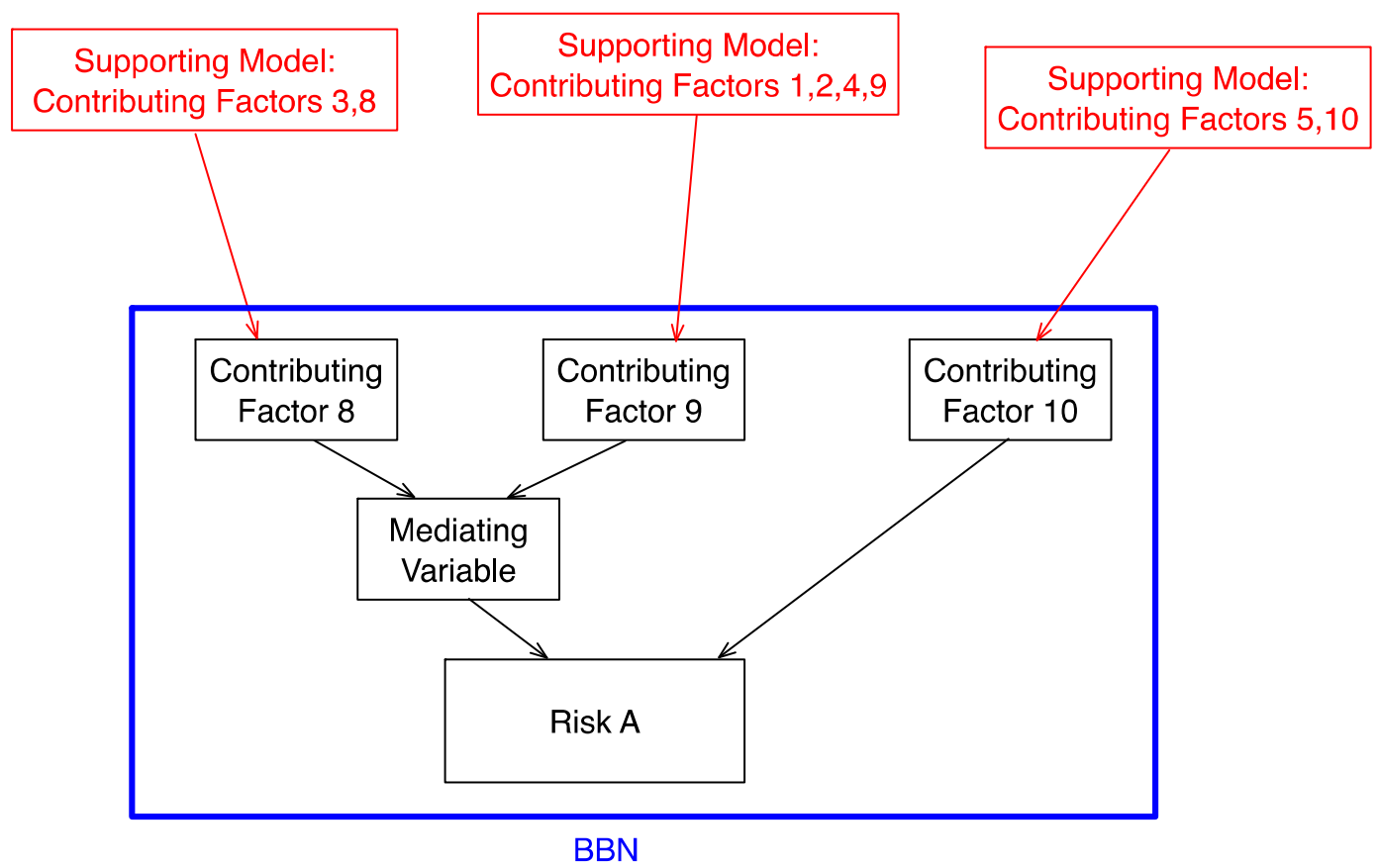

Figure 31 - Simplified BBN

As illustrated in Figure 31, the model architecture is now simplified to a five variable BBN and three supporting models. The mediating variable is introduced, through the divorcing method (section 5.2.3), in order to reduce the size of the Risk A probability table.

For each node in the BBN a set of appropriate values is determined. For the 'Risk A' node potential values could simply be Low, Medium and High. The values for the remaining nodes are dependent on the context, for example a binary true, false or a set of range values (actual examples are provided in the prototype models described in chapter 8 ). However, it is essential that clear definitions be provided for all state values otherwise the BBN cannot be meaningfully interpreted.

Having established the values for each node, probabilities are assigned for each value. The nodes linked to supporting models will be updated by 'learning' from the model outputs, as described in section 7.4.3. For the remaining nodes, the probability tables (section 5.2) are manually populated requiring domain subject matter expertise input.

\subsubsection{Supporting Models}

The risk model architecture identifies the central BBN and a set of supporting models. The advantage of this approach lies in the ability to support a truly heterogeneous modelling 
approach utilising combinations of techniques that are appropriate to specific "contributing factors". The key is the integration of these techniques with the central BBN, which is achieved through the alignment of the model output with the BBN variable values and the execution of the models within a Monte Carlo simulation.

The generic example shown in Figure 32 includes a BBN variable, which has three values: Low, Medium and High. However, in order to provide the required range of outputs, the supporting model must incorporate a stochastic element, for example this may be a Gaussian distribution reflecting the error bounds for a sensor. For each model an explicit definition of the variable and the associated variables should be provided, this ensures the risk model output is interpreted correctly and the supporting model output updates the BBN probabilities accurately. For example, the values may relate to a range where low is less then 10 , medium is between 10 and 20 and high is greater than 20 . In this case there are two possible approaches, the supporting model could output enumerate values of low, medium or high when executed or it could output a raw value. Where the tool ${ }^{13}$ used to generate the BBN supports both options, the input of the raw value is preferable, decoupling the model output from the BBN value definition to a greater extent. In this situation the BBN application will enable the input range for each value to be defined and when reading, or learning from, the supporting model output it will automatically determine which BBN value is applicable, as illustrated in Figure 33.

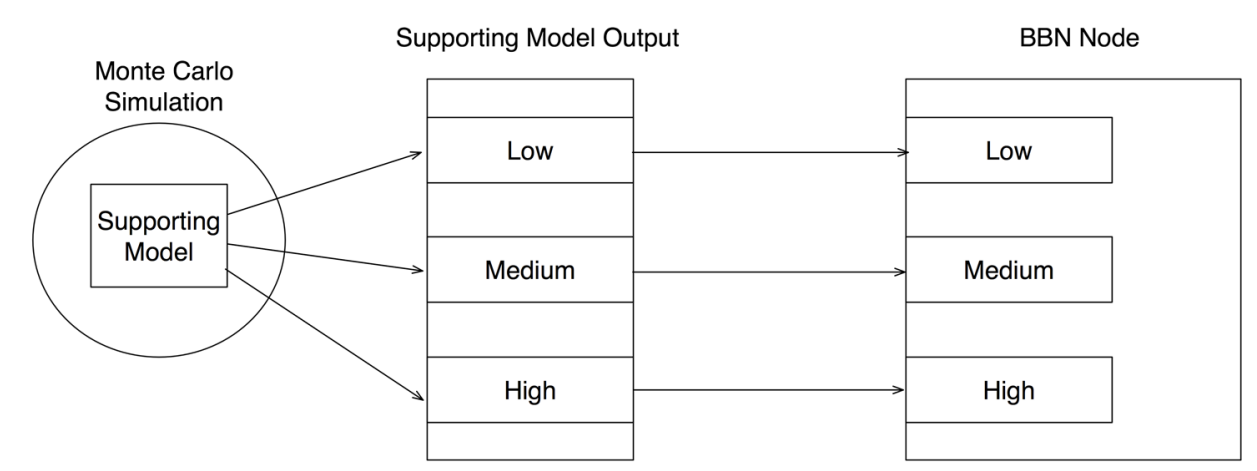

Figure 32 - Supporting Model - Formatted Output

\footnotetext{
${ }^{13}$ Netica ${ }^{\circledR}$ is the application used throughout this research project and supports both options.
} 


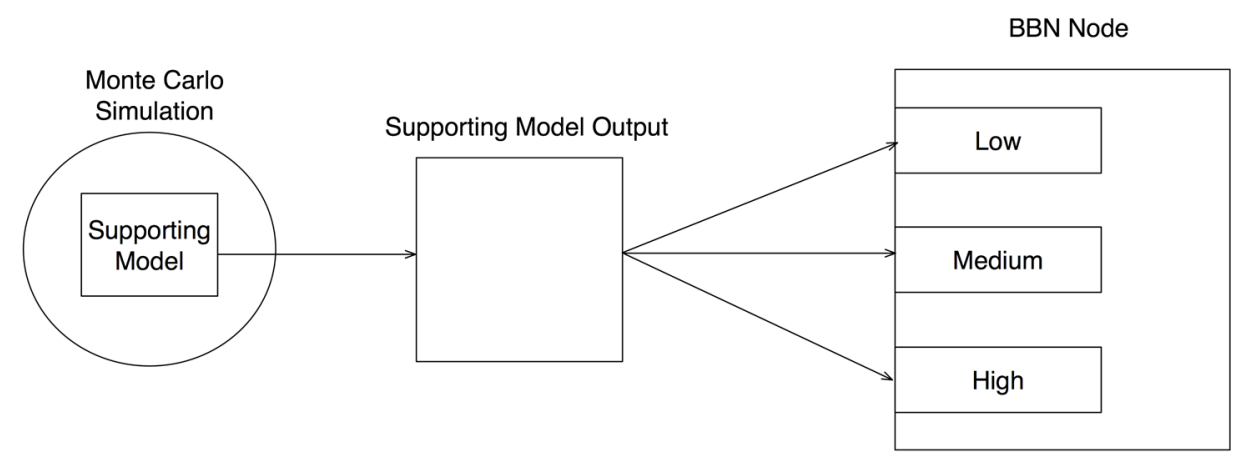

Figure 33 - Supporting Model - Unformatted Output

In order to learn from the supporting model output an adequately sized data sample is required. On each execution of the supporting model a single output value is generated, hence the model must be executed repeatedly which is achieved by running it as a Monte Carlo simulation (section 5.3). The Netica ${ }^{\circledR}$ application requires model output data to be stored in an external text file and enables learning for single or multiple BBN nodes.

It should also be noted that this approach also allows the central BBN to learn from historical data, although the data requires appropriate formatting.

\subsubsection{Risk Confidence}

Probabilities associated with risk are generally "best estimates", however stakeholders will not act upon these values unless there is an element of trust in them, which is best conveyed through an associated confidence value. For the proposed model based approach it is accuracy of the supporting models and the evidence, which determine the confidence of the risk estimates. However, as stated in the literature review, the outcome of model V\&V should not result in a model deemed as absolutely incorrect or correct, but rather as a degree of credibility (Balci, 1995). Although a 0 to 100 scale is suggested for the representation of model credibility (or confidence), this may present difficulties with consistent grading of different models. It is therefore proposed that a more pragmatic and simplistic approach is taken, whereby model confidence is graded as low, medium or high and is defined as follows:

- Low confidence: the model is based upon unsubstantiated or undocumented assumptions; evidence of validation and verification is insufficient, lack of subject matter expert matter input.

- Medium confidence: key assumptions are substantiated, although less critical assumptions are unsubstantiated but are documented; evidence of validation and verification is provided although coverage may not be complete, subject matter 
expert input evident but may not be comprehensive or level of expertise not authoritative.

- High confidence: all assumptions are substantiated; comprehensive evidence of validation and verification is provided with full coverage; authoritative subject matter expert input evident throughout model development.

As stated above the risk confidence is also dependent on the confidence of the evidence entered into the BBN, for which the following levels are proposed:

- Low confidence: the evidence is based upon unsubstantiated or undocumented assumptions and is purely subjective.

- Medium confidence: evidence is derived from subject matter expert input evident or historical data but may not be comprehensive or level of expertise not authoritative.

- High confidence: evidence is provided directly from current known information.

With a model and evidence confidence classifications defined, a method is required to combine the classifications of each supporting model such that a confidence of the overall risk assessment is provided; a BBN approach is proposed to enable this. Taking the original risk BBN, a "mirror" BBN can be created, where each variable will now represent confidence, i.e. low, medium and high and is illustrated in Figure 34 , which is derived from the risk BBN shown in Figure 31.

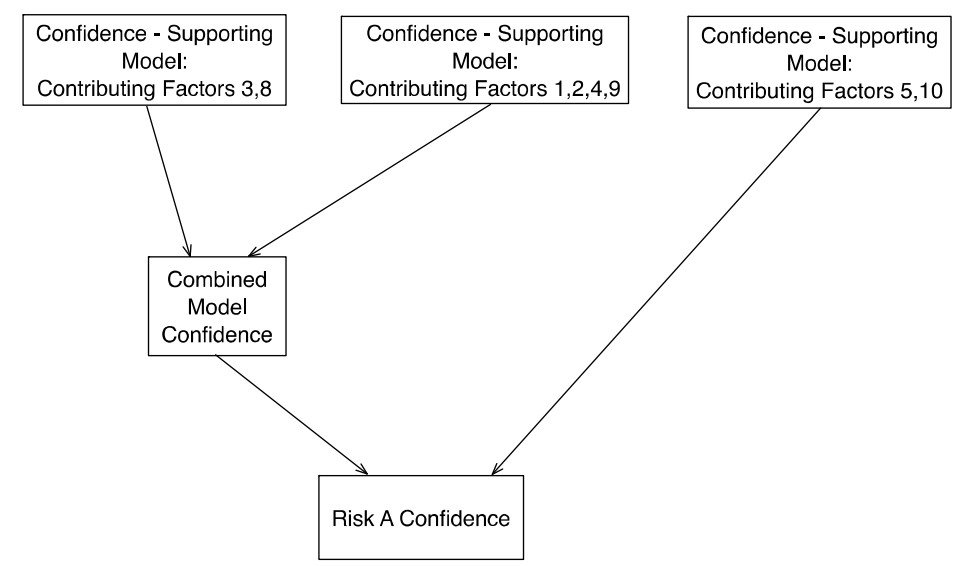

Figure 34 - Risk Confidence BBN

This approach should be undertaken for each risk BBN, thereby providing a confidence indicator for each assessed risk. 
A Model Based Approach to System of Systems Risk Management

\subsection{Summary and concluding remarks}

This chapter discussed the relationship of Risk Management to SoSE, concluding that it should be undertaken continuously within each lifecycle phase and that the DANSE lifecycle model (DANSE Consortium, 2015) provides the most suitable basis for this. The responsibility for SoS Risk Management was related to the overall SoS classification whereby directed and acknowledged SoS allow a single body to perform Risk Management but a collaborative SoS would require an advisory body or alternatively a bottom up cooperative approach. Of note, was the conclusion that in acknowledged and collaborative SoS Risk Management could be incorporated into planning activities, which is explored in the CAS case study described in chapter 8. It was also concluded that Risk Management could not be applied to virtual SoS due to the "lack a central management authority and a centrally agreed upon purpose for the system-of-systems" (Dahmann \& Baldwin, 2008). A series of case studies, described in section 2.1, were analysed in the context of SoS Risk Management which illustrated the necessity for a specific approach rather than applying the traditional SE approach.

The activities associated with Risk Management process were discussed, it was concluded that although the same process applied to SoSE Risk Management the approach to each activity differs from that traditionally undertaken, the key differences are summarised in Table 20, although it should be noted that Virtual SoS is not included as it was concluded that Risk Management is not applicable to this type of SoS. 


\begin{tabular}{|c|c|c|c|c|}
\hline Activity & System & Directed SoS & Acknowledged SoS & Collaborative SoS \\
\hline \multirow[t]{3}{*}{ Planning } & $\begin{array}{l}\text { Risk Management Plan } \\
\text { and Strategy }\end{array}$ & Risk Management Plan & $\begin{array}{l}\text { Risk Management Plan or } \\
\text { Operating Procedure }\end{array}$ & $\begin{array}{l}\text { Operating Procedure or ad-hoc } \\
\text { organisation }\end{array}$ \\
\hline & Program manager & Centrally Managed & Designated Manager & Collective agreement \\
\hline & Protracted timescales & Protracted timescales & $\begin{array}{l}\text { Timescales are variable; } \\
\text { protracted or short }\end{array}$ & $\begin{array}{l}\text { Timescales are variable; protracted } \\
\text { or short }\end{array}$ \\
\hline \multirow[t]{4}{*}{ Identification } & By all stakeholders & $\begin{array}{l}\text { SoS risk identified by Component } \\
\text { System stakeholders and board }\end{array}$ & $\begin{array}{l}\text { SoS risk identified by } \\
\text { Component System } \\
\text { stakeholders and board }\end{array}$ & $\begin{array}{l}\text { SoS risk identified by Component } \\
\text { System stakeholders }\end{array}$ \\
\hline & $\begin{array}{l}\text { Common framework } \\
\text { required }\end{array}$ & $\begin{array}{l}\text { Common framework required } \\
\text { SoS Sol framework proposed }\end{array}$ & $\begin{array}{l}\text { Common framework } \\
\text { required } \\
\text { SoS Sol framework proposed }\end{array}$ & $\begin{array}{l}\text { Common framework may not exist } \\
\text { SoS Sol framework proposed }\end{array}$ \\
\hline & Risk register & Risk register & $\begin{array}{l}\text { Risk register or mission } \\
\text { planning process }\end{array}$ & $\begin{array}{l}\text { Risks recorded by each component } \\
\text { system }\end{array}$ \\
\hline & Central co-ordination & Central co-ordination & $\begin{array}{l}\text { Central co-ordination but } \\
\text { risks at component system } \\
\text { level may take priority }\end{array}$ & $\begin{array}{l}\text { Co-ordination through collaboration, } \\
\text { not central }\end{array}$ \\
\hline \multirow[t]{2}{*}{ Analysis } & $\begin{array}{l}\text { Common framework } \\
\text { required }\end{array}$ & $\begin{array}{l}\text { Common framework required } \\
\text { SoS Sol framework proposed }\end{array}$ & $\begin{array}{l}\text { Common framework } \\
\text { required } \\
\text { SoS Sol framework proposed }\end{array}$ & $\begin{array}{l}\text { Common framework may not exist } \\
\text { SoS Sol framework proposed }\end{array}$ \\
\hline & $\begin{array}{l}\text { Output updates single } \\
\text { risk register }\end{array}$ & $\begin{array}{l}\text { Output updates single risk } \\
\text { register }\end{array}$ & $\begin{array}{l}\text { Output updates single risk } \\
\text { register or used to inform } \\
\text { mission planning }\end{array}$ & $\begin{array}{l}\text { Output held at Component System } \\
\text { level and shared }\end{array}$ \\
\hline
\end{tabular}




\begin{tabular}{|c|c|c|c|c|}
\hline \multirow[t]{2}{*}{ Activity } & System & Directed SoS & Acknowledged SoS & Collaborative SoS \\
\hline & $\begin{array}{l}\text { Probabilistic models may } \\
\text { be used }\end{array}$ & $\begin{array}{l}\text { Probabilistic and supporting } \\
\text { model approach proposed }\end{array}$ & $\begin{array}{l}\text { Probabilistic and supporting } \\
\text { model approach proposed; a } \\
\text { decision support capability } \\
\text { may be required }\end{array}$ & $\begin{array}{l}\text { Probabilistic and supporting model } \\
\text { approach proposed; a decision } \\
\text { support capability may be required }\end{array}$ \\
\hline $\begin{array}{l}\text { Handling } \\
\text { Acceptance }\end{array}$ & $\begin{array}{l}\text { Central authority to } \\
\text { accept risk }\end{array}$ & $\begin{array}{l}\text { Central authority to accept risk } \\
\text { with influence over component } \\
\text { systems }\end{array}$ & $\begin{array}{l}\text { Central authority to asses } \\
\text { risk but limited influence } \\
\text { over component systems }\end{array}$ & $\begin{array}{l}\text { No central authority, risk acceptance } \\
\text { though consensus }\end{array}$ \\
\hline \multirow[t]{2}{*}{$\begin{array}{l}\text { Handling } \\
\text { Avoidance }\end{array}$} & $\begin{array}{l}\text { Project takes alternative } \\
\text { path }\end{array}$ & $\begin{array}{l}\text { Project takes alternative path bit } \\
\text { requires coordination of } \\
\text { component systems }\end{array}$ & $\begin{array}{l}\text { Project takes alternative } \\
\text { path but requires } \\
\text { cooperation of component } \\
\text { systems but individual } \\
\text { objectives may override }\end{array}$ & $\begin{array}{l}\text { Component systems agree an } \\
\text { alternative approach but individual } \\
\text { objectives may override }\end{array}$ \\
\hline & $\begin{array}{l}\text { Cost of alternative path } \\
\text { assessed }\end{array}$ & Cost of alternative path assessed & $\begin{array}{l}\text { Cost of alternative path may } \\
\text { be difficult to assess. Cost } \\
\text { may be temporal rather than } \\
\text { financial. }\end{array}$ & $\begin{array}{l}\text { Cost of alternative approach may be } \\
\text { determined and agreed by } \\
\text { component. Cost may be temporal } \\
\text { rather than financial. }\end{array}$ \\
\hline \multirow[t]{2}{*}{$\begin{array}{l}\text { Handling } \\
\text { Transfer }\end{array}$} & Between programs & $\begin{array}{l}\text { Between SoS but influence to } \\
\text { effect this may be limited }\end{array}$ & $\begin{array}{l}\text { Between SoS but influence } \\
\text { to effect this may be limited }\end{array}$ & $\begin{array}{l}\text { Between SoS but influence to effect } \\
\text { this would be undertaken by an } \\
\text { external body }\end{array}$ \\
\hline & $\begin{array}{l}\text { Between customer and } \\
\text { contractor }\end{array}$ & $\begin{array}{l}\text { Between customer and prime } \\
\text { contractor }\end{array}$ & $\begin{array}{l}\text { Between customer and } \\
\text { prime contractor, although } \\
\text { this may be resisted due to } \\
\text { lack of influence over } \\
\text { component systems. } \\
\text { Between missions. }\end{array}$ & $\begin{array}{l}\text { Customer and contractor } \\
\text { relationship does not exist. }\end{array}$ \\
\hline
\end{tabular}




\begin{tabular}{|c|c|c|c|c|}
\hline Activity & System & Directed SoS & Acknowledged SoS & Collaborative SoS \\
\hline & Between subsystems & $\begin{array}{l}\text { Between component systems - } \\
\text { managerial influence }\end{array}$ & $\begin{array}{l}\text { Between component } \\
\text { systems - through } \\
\text { cooperation, SoS manager } \\
\text { has less influence }\end{array}$ & $\begin{array}{l}\text { Between component systems - } \\
\text { through collaboration }\end{array}$ \\
\hline \multirow[t]{2}{*}{$\begin{array}{l}\text { Handling } \\
\text { Mitigation }\end{array}$} & $\begin{array}{l}\text { Prototyping, modelling at } \\
\text { system level }\end{array}$ & $\begin{array}{l}\text { Prototyping, modelling at SoS } \\
\text { level. } \\
\text { Model based approach required }\end{array}$ & $\begin{array}{l}\text { Prototyping, modelling at } \\
\text { SoS level } \\
\text { Model based approach } \\
\text { required } \\
\text { Decision support tool may be } \\
\text { required }\end{array}$ & $\begin{array}{l}\text { Prototyping, modelling at SoS level, } \\
\text { would need to be undertaken } \\
\text { through collaboration } \\
\text { Model based approach required }\end{array}$ \\
\hline & Test and review & Test and review at SoS level & Test and review at SoS level & $\begin{array}{l}\text { Errors may emerge rather than be } \\
\text { detected through formal testing }\end{array}$ \\
\hline Monitoring & $\begin{array}{l}\text { Regular meeting } \\
\text { between program } \\
\text { authority and } \\
\text { contractors }\end{array}$ & $\begin{array}{l}\text { Regular meeting between SoS } \\
\text { authority and component system } \\
\text { contractors }\end{array}$ & $\begin{array}{l}\text { Regular meeting between } \\
\text { SoS authority and } \\
\text { component system } \\
\text { authorities }\end{array}$ & $\begin{array}{l}\text { Regular meeting between } \\
\text { component system stakeholders }\end{array}$ \\
\hline
\end{tabular}

Table 20 - System and SoS Risk Management Comparison 
Specific approaches to each SoS Risk Management activity were discussed and summarised as follows:

- Planning: directed SoS may adopt traditional approach, acknowledged SoS may incorporate this activity within mission planning and collaborative SoS may utilise pre-defined procedures.

- Identification: utilises a framework cross referencing SoS Sol dimension (Kinder et al., 2012) with hazard, control and opportunity risk categories (Hopkin, 2002). Both risks and contributing factors are identified and are captured in a causal network.

- Analysis: it was argued that, due to the inherent complexity of a SoS, the use of modelling and simulation is necessary rather than subject matter expert mental models which may be influenced by perception and bias. The model-based approach allows a level of quantification and reduces the subjectivity of the risk analysis.

- Handling: risks are accepted, avoided, transferred, or mitigated. Acceptance occurs if cost of mitigation outweighs the cost incurred if the risk materialises, the modelbased approach can be used to evaluate this impact and can also be used to determine the effect of risk transfer.

- Monitoring: the model-based approach could enable the implementation of a automated risk monitoring capability where rapid decision making is required, where a SoS is centrally controlled a more traditional approach may be taken.

A model-based approach was described which captures all risks and contributing factors within a causal network. BBNs are defined for each risk, derived from the initial causal network. Contributing factors are identified which can be modelled using appropriate techniques using the model selection matrix tool (section 6.3). The supporting models are run iteratively in Monte Carlo simulations generating output data from which the associated BBN may learn. An associated risk confidence model provides a confidence level for each estimated risk. 


\section{Chapter 8: Application 1: Close Air Support Mission Planning Risk Management}

Having described the proposed model based approach to SoS Risk Management in chapter 7, two very different case study applications were identified; Close Air Support and UK Food Security - Wheat Supply Chain, through which the process was exercised, thereby assessing its efficacy and adaptability. The Close Air Support application case study is described in this chapter and the UK Food Security - Wheat Supply Chain case study in chapter 9.

Close Air Support was selected as an appropriate example of a SoS requiring Risk Management to be undertaken within a short planning cycle where component systems could not be influenced, hence the traditional approaches were not suitable. It was also a subject area where the author has technical knowledge and access to operational subject matter expertise.

Figure 35 provides the context for these application case studies within this research, which in addition to exercising the proposed process, also provide further validation of the SoS Sol model (described in section 6.1) through its use in determining the Sol for further examples of SoS.

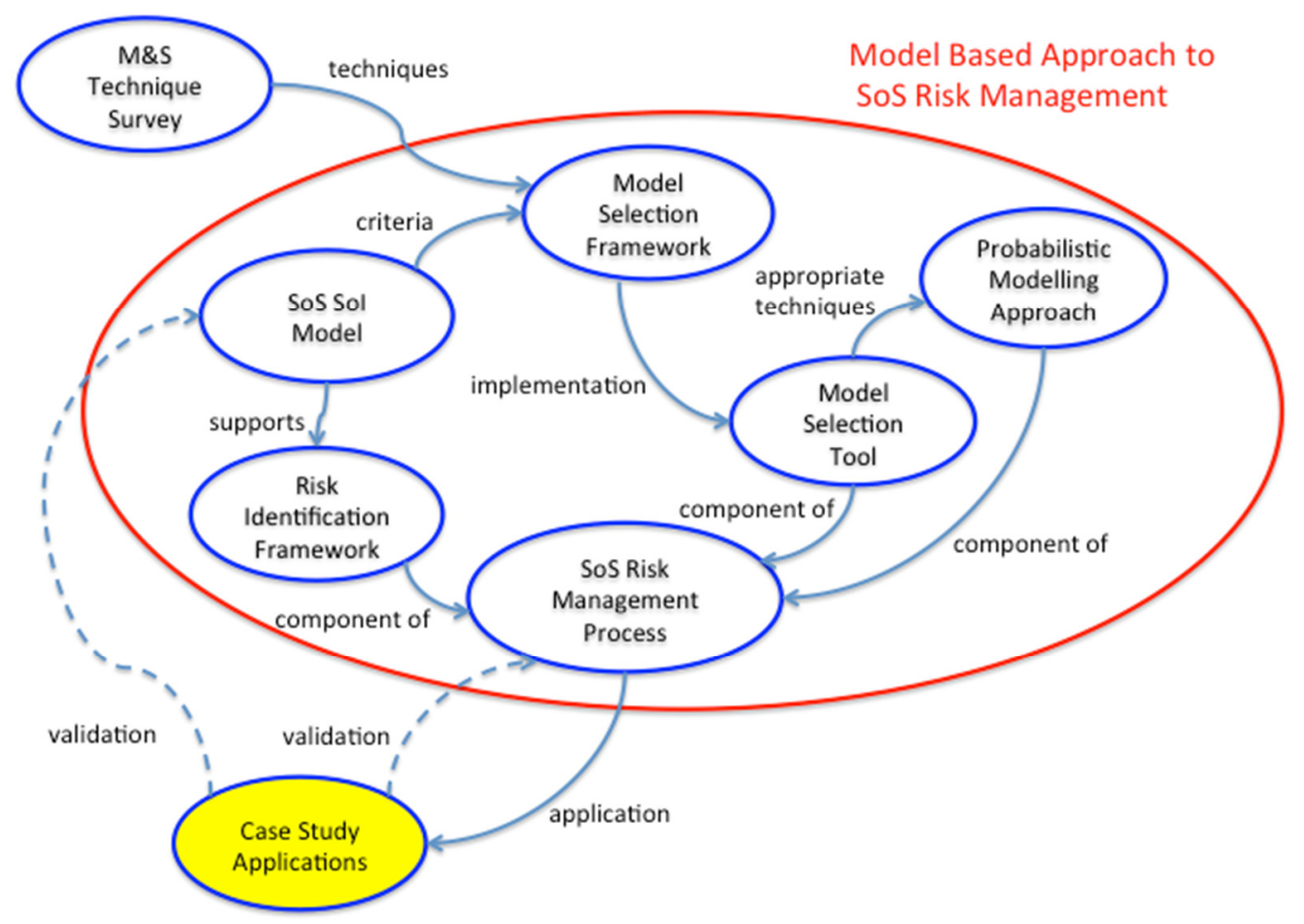

Figure 35 - Case Study Application Context 
A Model Based Approach to System of Systems Risk Management

\subsection{Close Air Support}

The NATO publication; Tactics, Techniques and Procedures for Close Air Support Operations (NATO, 2005) defines Close Air Support as;

“..air action against hostile targets which are in close proximity to friendly forces and requires detailed integration of each air mission with the fire and movement of those forces." CAS missions are differentiated between 'immediate' and 'pre-planned'. An immediate CAS mission will require an urgent response in a situation where it is probable troops are under fire. In this situation the planning phase will typically involve assigning the closest available assets with less constraints placed on their mission suitability, e.g. accuracy of munitions. With minimal planning time available, the mission risk increases. Pre-planned missions fit into the typical planning cycle, usually in the order of 24-72 hours, where the suitability of platforms and munitions may be tailored to match the mission requirements. However the planning time is still limited and it not always possible to reduce risk to optimal levels.

It was therefore proposed that application of the model based Risk Management process would provide a set of models which could provide a capability, potentially integrated into a single tool, that mission planners could utilise in order to undertake rapid risk assessment and analysis, indeed the following quote emphasises the need for rapid decision making in these circumstances;

"Four minutes can seem like an eternity to a soldier in the middle of a complex ambush. Aircraft arriving even a few seconds earlier can make the difference between life and death" (Schaefer III, 2012).

Several subject matter experts with current or previous CAS planning experience were informally interviewed at the case study initiation. Those with planning experience felt the proposed tool would be beneficial, stating that current planning is typically "based on judgement calls but has to account for many variables." In addition, it was also pointed out "personnel burn out when on station for long periods hence the ability to exercise clear judgement in high pressure situations can be compromised."

Figure 36 illustrates a typical CAS scenario, where blue (friendly) land forces require air support, provided by Tornado GR4 aircraft. The Forward Air Controller (FAC), also referred to as Joint Terminal Attack Controller (JTAC), guides the fighter into the target with coordinates provided from a sensor, such as a laser rangefinder. Planning and control of the mission is undertaken at the brigade HQ Tactical Air Control Centre (TACC), receiving higher level orders from the battalion $\mathrm{HQ}$. Intelligence, Surveillance and Intelligence (ISR) 
information is provided by assets such as the E3-D surveillance aircraft and unmanned air vehicles (UAV). Tactical information, which includes blue (friendly) force and target position is exchanged using digital communication systems, in this case Link 16, Variable Message Format (VMF) and Bowman, where data is exchanged in fixed format messages using radio frequency transmissions. Communication between HQs utilises Falcon, an IP based system providing secure voice, instant messaging and email capability. Additionally, traditional radio based voice communications are utilised throughout. It should also be noted that the UAV provides a "forwarding" capability, which allows data flow between heterogeneous networks through the translation of data formats.

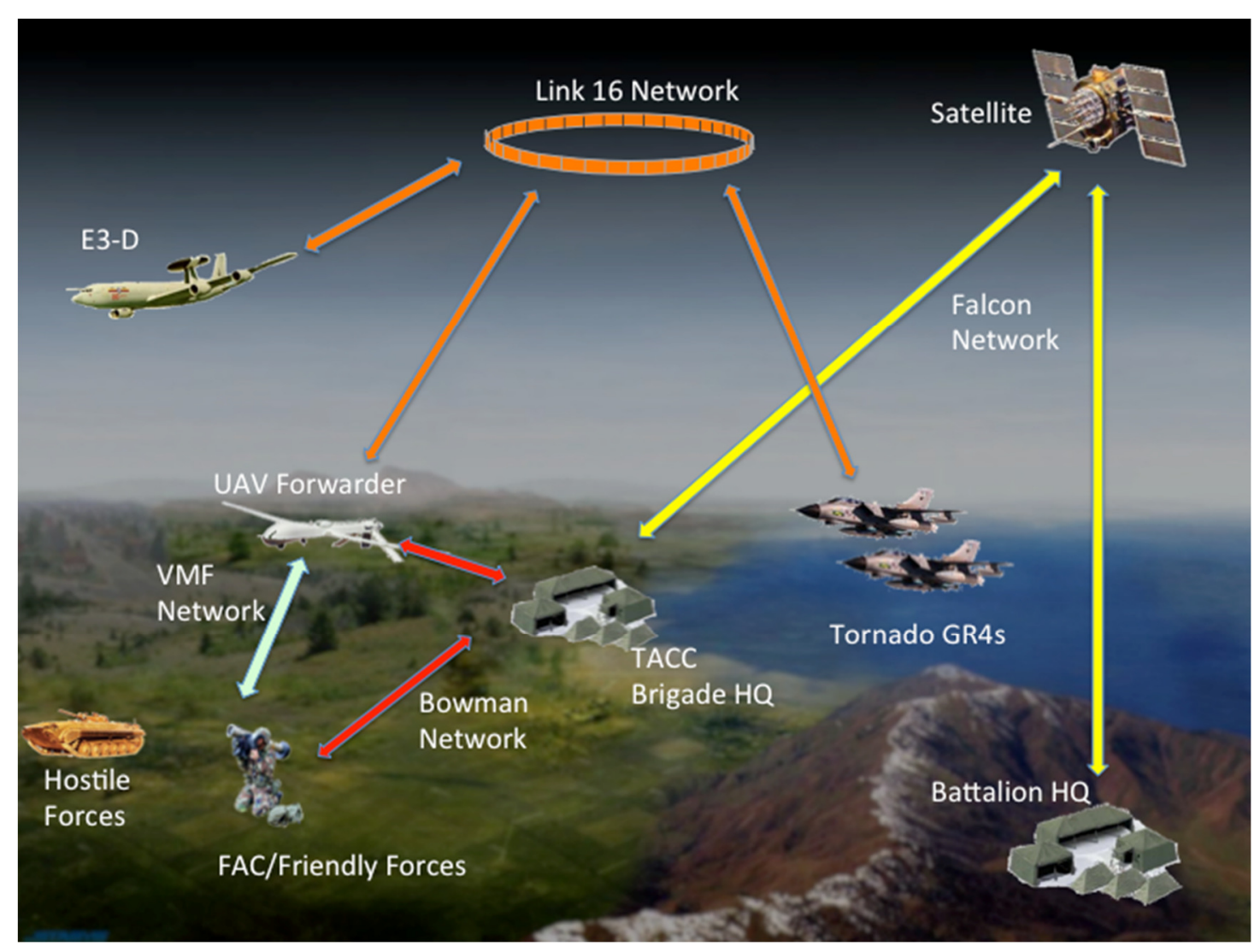

Figure 36 - Typical Close Air Support Scenario

Initial discussions regarding the CAS process were undertaken on an informal basis with a subject matter expert (SME) with current knowledge as well as experience of CAS planning.

The SME confirmed that CAS planning and the subsequent compilation of the Air Tasking Order (ATO) is a complex task, and which requires a support system, providing the following overview:

- Pre-planned CAS, ATO process is on a 24-72 hours cycle.

- Local commanders submit requests, e.g.; 
○ "Destroy bridge"

○ "Take snipers out"

- Requests are Joint Force Air Component (JFAC) approved

- High priority requests go into ATO

- For immediate or responsive CAS;

- Tasking is communicated by 9 line brief or radio

- Aircraft on holding patterns or high alert

- Video is used for visual confirmation of target

- Fratricide risk is reduced by forwarding of blue force information on Link 16

- Misses tend to be short or long, rather than to the side

- Pre-planned CAS uses Intelligence data.

A more specific hypothetical scenario was defined as a basis for the risk management activities, a description is provided in Appendix $D$.

\subsection{Application of SoS Risk Management Process}

The process described in section 7.3 was applied in the context of this case study. Although this research is principally focussed upon risk identification and analysis, all risk management activities were considered in order to determine applicability of the modelbased approach throughout.

\subsection{SoS Risk Planning}

A CAS mission is fulfilled by a SoS, which may be primarily classified as acknowledged (discussed in section 8.4). Centralised control of the SoS is provided by the CAS mission planning role, which utilises standard operating procedures, for example the NATO Tactics, Techniques and Procedures for Close Air Support Operations (NATO, 2005), although typically national procedures take precedence which identify risks and mitigation strategies. As stated previously, CAS planning is undertaken within relatively short timescales and, in some circumstances requires an almost immediate response, it is therefore unrealistic to expect a full model based risk analysis to be undertaken requiring the associated models to be specified, implemented and validated. Hence, in this case, the model-based approach should be used to undertake analysis in "slow time" outside the operational environment, thereby allowing models to be implemented within appropriate timescales and which may be subsequently integrated into a decision support tool, supporting rapid risk assessment. 


\subsection{SoS Risk Identification}

The risk identification matrix, as shown in Table 19, provides a framework for the identification activity enabling risk to be considered from a SoS perspective. However, as a precursor it was found that population of the Sol SoS dimension table provided valuable context, where the dimensions could be instantiated either generically or specifically.

\subsubsection{SoS Sol dimensions}

For the purposes of this case study the SoS Sol dimensions are populated in accordance with the scenario illustrated in Figure 36 and described in Appendix D, where generic assets and systems are identified with examples of specific instances.

\begin{tabular}{|c|c|}
\hline Dimensions & Instantiation \\
\hline $\begin{array}{l}\text { Component } \\
\text { Systems }\end{array}$ & $\begin{array}{l}\text { Fighter Aircraft (e.g. Tornado GR4) } \\
\text { Command and Control Unit (e.g. E3-D, TACC) } \\
\text { Tactical Data Link (TDL) gateway (e.g. UAV) } \\
\text { ISR (e.g. E3-D) } \\
\text { Forward Air Controller (FAC) using sensor to track target, e.g. Laser Range } \\
\text { Finder } \\
\text { Brigade HQ (TACC) } \\
\text { Battalion HQ } \\
\text { Satellite } \\
\text { Weapon (may be regarded as a component system if a "smart" missile as } \\
\text { opposed to a "dumb" ballistic bomb) }\end{array}$ \\
\hline Interactions & $\begin{array}{l}\text { TDLs (e.g. Link 16, VMF, Bowman) } \\
\text { Voice Communications } \\
\text { Track Data } \\
\text { Command and Control } \\
\text { Imagery }\end{array}$ \\
\hline Lifecycle & Initiation (planning), Creation, Operations (mission execution) \\
\hline Variability & $\begin{array}{l}\text { Assets may change during mission. } \\
\text { The purpose may evolve as the mission progresses. This SoS may exhibit a } \\
\text { high level of variability. }\end{array}$ \\
\hline Classification & $\begin{array}{l}\text { Collaborative/Acknowledged. } \\
\text { Directed elements, e.g. Link 11/16 networks }\end{array}$ \\
\hline Functions & $\begin{array}{l}\text { Surveillance, Reconnaissance, Targeting, Detect, Identify, Intercept, } \\
\text { Destroy, Aircraft Control, Battle Management. }\end{array}$ \\
\hline $\begin{array}{l}\text { Systems } \\
\text { Owners and }\end{array}$ & Nations (Governments), Services (Air force, Navy, Army), Overarching \\
\hline
\end{tabular}




\begin{tabular}{|l|l|}
\hline Dimensions & Instantiation \\
\hline Operations & control, e.g. NATO. \\
\hline $\begin{array}{l}\text { Concept of } \\
\text { Operation }\end{array}$ & $\begin{array}{l}\text { The SoS provides protection of blue forces through elimination of hostile } \\
\text { threat whilst avoiding collateral damage. }\end{array}$ \\
\hline $\begin{array}{l}\text { Nature of } \\
\text { Relationships }\end{array}$ & Hierarchical (military C2) \\
\hline
\end{tabular}

Table 21 - SoS Sol Dimensions CAS

\subsubsection{Identification of Key Risks}

Having established the SoS Sol, the SoS risk matrix was populated using SME knowledge, elicited through informal interview. The identified risks can be classified in two categories; key risks (coloured red) and contributing factors. A key risk is a potential outcome that directly affects the SoS purpose, either negatively or positively. A contributing factor is an event or condition that influences the likelihood of a key risk occurring. The outcome of the risk identification exercise is documented below in Table 22.

\begin{tabular}{|l|l|l|l|}
\hline & $\begin{array}{l}\text { Hazard Risks (Threat } \\
\text { and perils) }\end{array}$ & $\begin{array}{l}\text { Control Risks (Doubt } \\
\text { about mission } \\
\text { achievement, project } \\
\text { management control) }\end{array}$ & $\begin{array}{l}\text { Opportunity Risks } \\
\text { (Opportunity to } \\
\text { enhance or inhibit } \\
\text { mission } \\
\text { achievement) }\end{array}$ \\
\hline $\begin{array}{l}\text { Component } \\
\text { Systems }\end{array}$ & $\begin{array}{l}\text { Loss of CAS Fighter } \\
\text { Aircraft } \\
\text { Loss of FAC or Blue } \\
\text { Forces (Fratricide) } \\
\text { Blue forces in close } \\
\text { proximity to the } \\
\text { weapon blast } \\
\text { zone/target. } \\
\text { Fighter aircraft on low } \\
\text { altitude flight path. }\end{array}$ & $\begin{array}{l}\text { Required component } \\
\text { systems are not } \\
\text { available. }\end{array}$ & \\
\hline Classification & & $\begin{array}{l}\text { SoS incorrectly classified } \\
\text { resulting in } \\
\text { inappropriate control } \\
\text { approach. }\end{array}$ & \\
\hline
\end{tabular}




\begin{tabular}{|c|c|c|c|}
\hline & $\begin{array}{l}\text { Hazard Risks (Threat } \\
\text { and perils) }\end{array}$ & $\begin{array}{l}\text { Control Risks (Doubt } \\
\text { about mission } \\
\text { achievement, project } \\
\text { management control) }\end{array}$ & $\begin{array}{l}\text { Opportunity Risks } \\
\text { (Opportunity to } \\
\text { enhance or inhibit } \\
\text { mission } \\
\text { achievement) }\end{array}$ \\
\hline Interactions & $\begin{array}{l}\text { Poor interoperability } \\
\text { due to; lack or } \\
\text { incompatibility of } \\
\text { digital data links, } \\
\text { spoken language } \\
\text { barrier or degraded } \\
\text { voice } \\
\text { communications. } \\
\text { Poor visibility due to } \\
\text { weather and light } \\
\text { conditions. } \\
\text { Kill chain error } \\
\text { accumulation caused } \\
\text { by inherent system } \\
\text { inaccuracies and } \\
\text { latency. } \\
\text { Loss of } \\
\text { communications } \\
\text { through jamming. } \\
\text { Loss of GPS through } \\
\text { jamming. } \\
\text { Different geodetic co- } \\
\text { ordinate systems } \\
\text { resulting in positional } \\
\text { translation errors. }\end{array}$ & $\begin{array}{l}\text { Poor interoperability } \\
\text { interrupts command and } \\
\text { control. } \\
\text { Terrain has a } \\
\text { detrimental on } \\
\text { communications, where } \\
\text { line of sight required. } \\
\text { Coalitions of different } \\
\text { nations may result in } \\
\text { interoperability issues } \\
\text { due to system and } \\
\text { operating procedure } \\
\text { incompatibility. }\end{array}$ & $\begin{array}{l}\text { Bandwidth can } \\
\text { support additional } \\
\text { interaction medium. } \\
\text { Disruption of hostile } \\
\text { system } \\
\text { communications } \\
\text { providing tactical } \\
\text { advantage. } \\
\text { Common digital } \\
\text { communication } \\
\text { system enhance SA } \\
\text { picture. }\end{array}$ \\
\hline $\begin{array}{l}\text { Nature of } \\
\text { Relationships }\end{array}$ & $\begin{array}{l}\text { Breakdown of } \\
\text { command, possibly } \\
\text { through national or } \\
\text { political tension. }\end{array}$ & & \\
\hline Lifecycle & & $\begin{array}{l}\text { Component systems } \\
\text { unavailable when } \\
\text { required. }\end{array}$ & \\
\hline Variability & & $\begin{array}{l}\text { SoS cannot adapt to } \\
\text { respond to an } \\
\text { unexpected threat. }\end{array}$ & $\begin{array}{l}\text { Availability for quick } \\
\text { response to urgent } \\
\text { arising need. }\end{array}$ \\
\hline
\end{tabular}




\begin{tabular}{|c|c|c|c|}
\hline & $\begin{array}{l}\text { Hazard Risks (Threat } \\
\text { and perils) }\end{array}$ & $\begin{array}{l}\text { Control Risks (Doubt } \\
\text { about mission } \\
\text { achievement, project } \\
\text { management control) }\end{array}$ & $\begin{array}{l}\text { Opportunity Risks } \\
\text { (Opportunity to } \\
\text { enhance or inhibit } \\
\text { mission } \\
\text { achievement) }\end{array}$ \\
\hline Functions & $\begin{array}{l}\text { Inaccurate targeting } \\
\text { (Misses tend to be } \\
\text { short or long, rather } \\
\text { than to the side) } \\
\text { Incorrect target } \\
\text { identification, e.g. } \\
\text { identifying friendly } \\
\text { asset as hostile. } \\
\text { Target marking, } \\
\text { provided by smoke, } \\
\text { phosphorous or laser, } \\
\text { is ineffective due to } \\
\text { environmental } \\
\text { conditions, e.g. light } \\
\text { level, dust or } \\
\text { cloud/fog. } \\
\text { Hostile defensive } \\
\text { capability endangers } \\
\text { CAS aircraft }\end{array}$ & $\begin{array}{l}\text { Degraded surveillance } \\
\text { capability resulting in } \\
\text { poor Situational } \\
\text { Awareness } \\
\text { Inaccurate intelligence } \\
\text { regarding hostile force } \\
\text { capabilities. } \\
\text { Poor intelligence } \\
\text { providing incorrect or } \\
\text { incomplete information. }\end{array}$ & $\begin{array}{l}\text { Advanced sensor } \\
\text { capability enabling } \\
\text { attack in poor } \\
\text { visibility conditions. } \\
\text { Defensive capability } \\
\text { enabling attack } \\
\text { through hostile fire } \\
\text { Data fusion } \\
\text { capability provides } \\
\text { clear SA picture }\end{array}$ \\
\hline $\begin{array}{l}\text { Systems } \\
\text { Owners and } \\
\text { Operations }\end{array}$ & $\begin{array}{l}\text { Excessive operator } \\
\text { workload may occur in } \\
\text { very active } \\
\text { environments. }\end{array}$ & $\begin{array}{l}\text { Conflict between } \\
\text { national tactics and } \\
\text { procedures. } \\
\text { Where different nations } \\
\text { are involved the } \\
\text { language barrier may } \\
\text { cause communication } \\
\text { problems. }\end{array}$ & \\
\hline $\begin{array}{l}\text { Concept of } \\
\text { Operation / } \\
\text { Use / } \\
\text { Employment }\end{array}$ & $\begin{array}{l}\text { Collateral Damage } \\
\text { Target Destruction } \\
\text { Failure } \\
\text { Civilians in close } \\
\text { proximity to target. }\end{array}$ & & $\begin{array}{l}\text { Additional target } \\
\text { opportunity }\end{array}$ \\
\hline
\end{tabular}

Table 22 - SoS Risk matrix: CAS

In Table 22 the key (hazard and opportunity) risks were identified (highlighted in red), these are summarised below and the risk owner and criticality are also indicated:

- Loss of CAS Fighter Aircraft where the impact is catastrophic and the owner is the aircraft itself 
- Loss Blue Forces (Fratricide) from friendly fire, the impact is catastrophic with the blue land forces the risk owner

- Collateral Damage, i.e. civilian casualties, the impact is also catastrophic in this case. Although civilians are directly impacted by this risk they do not own it because they have no influence over it, the risk ownership may in fact move as the mission progresses, for example in the planning stage the mission planner will influence the risk but at the point of weapons release the aircraft pilot may abort the attack if civilians are sighted with the blast range. From a SoS perspective the risk ownership could be regarded as relating to the SoS lifecycle

- Target Destruction Failure; the primary target was not destroyed; the impact is dependent on the blue force situation therefore range from mission critical to catastrophic. Again, the ownership of this risk will be related to the point in the mission (or SoS lifecycle)

- Additional target opportunity; the CAS Fighter aircraft is able to respond rapidly to identification of a further target in close vicinity, to the primary target. The risk impact could be regarded as "beneficial". The mission commander, responsible for retargeting aircraft, would own the risk.

\subsubsection{Identification of contributing factors}

Each of these risks were analysed in the context of the contributing factors that directly influence them, which was the first step in defining the causal network (section 7.3.2) representing the relationships between risks and contributing factors and is described below.

The loss of the CAS aircraft is directly influenced by the following contributing factors:

- Situational Awareness (SA) Picture Quality: if SA is poor then location of hostile forces may be inaccurate or unknown thereby exposing the aircraft to unexpected attack.

- Enemy Capability: if the hostile weapon and defensive systems are highly capable this may increase the risk of loss of the CAS aircraft.

- Flight Path Altitude: the mission may require a low altitude flight path, potentially through mountainous terrain; this increases the risk of anti-aircraft weapon or ground impact.

- Defensive Capability: defensive aids, such as chaff and flare, and a jamming capability may reduce the risk of hostile detection and interception. 
A Model Based Approach to System of Systems Risk Management

The loss of FAC or Blue Forces (Fratricide) is directly influenced by the following contributing factors:

- Blue Force Proximity to blast zone: the distance of the blue forces from the target, and hence blast zone, directly relates to the risk of fratricide.

- Kill Chain Accuracy: target co-ordinates may be passed from the FAC, over TDLs, via forwarding systems to the CAS aircraft. Errors and latency (particularly if the target is moving) will introduce errors into the target position, potential increasing the risk of fratricide due to the weapon impact being closer to the blue force position than expected.

- SA Picture Quality: if SA is poor then location of blue and red forces may be inaccurate or unknown thereby exposing blue force to increased risk of friendly fire.

- Blue Force Visibility: visual observation of blue forces may be required, if this is impaired due to weather or environmental conditions, or lack of capability to indicate position then risk of fratricide will increase.

The risk of collateral damage is influenced by the following contributing factors:

- Kill Chain Accuracy: as stated above, target co-ordinates may be passed through a series of systems which each introduce errors, thereby increasing the risk of collateral damage due to the weapon impact being closer to civilian locations than expected.

- SA Picture Quality: if SA is poor then the location of civilians may be inaccurate or unknown thereby increasing the risk of friendly fire.

- Civilian Proximity to blast zone: the distance of civilians from the target, and hence blast zone, directly relates to the risk of collateral damage.

- Civilian Visibility: visual observation of blue forces may be required, if this is impaired due to weather or environmental conditions, or lack of capability to indicate position then risk of fratricide will increase.

The risk of failure to destroy the primary target is influenced by the following contributing factors:

- Kill Chain Accuracy: as stated above, if errors are introduced into the target coordinates used at the point of weapon launch, the likelihood of successful 
destruction of the risk will be diminished, the extent of which relates to the magnitude of these errors.

- SA Picture Quality: if SA is poor then the target location may be inaccurate thereby reducing the likelihood of destruction.

- Target Visibility: if visual identification of the target is required by the fighter aircraft to ensure accurate weapon aiming then the visibility of the target will influence the likelihood of a successful outcome.

The (opportunity) risk of successfully prosecuting an additional target is influenced by the following contributing factors:

- Kill Chain Accuracy: if the kill chain allows propagation of accurate and timely target information then the likelihood of rapidly prosecuting a secondary target will be enhanced.

- SA Picture Quality: with a high quality picture the position of secondary targets will be known throughout the SoS.

- Target Visibility: the likelihood of prosecuting a secondary target will be increased where it is highly visible.

\subsubsection{Definition of causal network}

The analysis above enabled an initial causal network to be defined, illustrating the relationships between the risks (highlighted in yellow) and the primary contributing factors (which are indicated by clear boxes), as shown in Figure 37.

Having established the initial causal relationships, analysis was undertaken to determine the relationships between the primary contributing factors and the remaining influencing factors, and is described below. This is further elaborated in the detailed causal network described in Section 8.4 .5 below. 


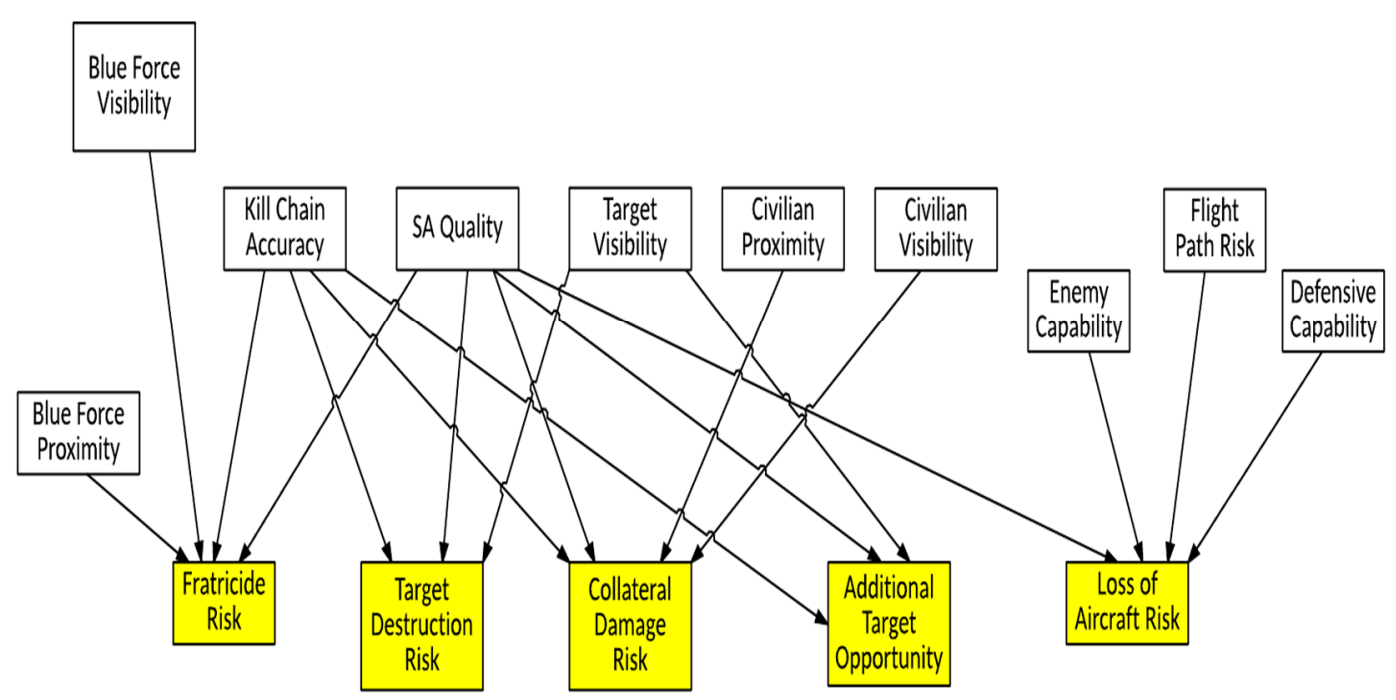

Figure 37 - Initial Causal Network - CAS

\subsubsection{SA quality}

SA picture quality is influenced by a variety of factors; interoperability is key in distributing the SA picture, enabling information to be exchanged between component systems. The Intelligence, Surveillance and Reconnaissance (ISR) capability is the source of the SA information, therefore to maintain a high quality picture, trusted and accurate information is required. The scenario density, i.e. the number of objects comprising the SA picture, may also impact the quality; where the density is high, component systems ability to fuse and filter large quantities of data will affect the clarity of the picture, this situation may also impose a high operator workload where manual maintenance of the picture is required, leading to potential errors in the identification and classification of information. Interoperability includes the dissemination of information through voice communications, over digital TDL communications systems and using IP based networks, in some cases line of sight may be required. The compatibility of different TDL systems throughout the SoS is required to ensure effective interoperability in addition to the data capacity of each TDL, which should support the scenario density. Voice communications not only require technical connectivity, e.g. transmission frequencies are agreed within the SoS, but also will be affected by any natural language barriers where coalition operations are undertaken. Compatibility of geodetic reference systems is also necessary, with appropriate translations performed, to ensure positional information can be exchanged between component systems. 
A Model Based Approach to System of Systems Risk Management

\subsubsection{Kill chain accuracy}

Where a ground target is moving, latency has a major impact upon kill chain accuracy, for example a delay of 5 seconds could result in an error of approximately 65 metres, potentially placing the blue force on the edge of the weapon blast radius. Kill chain accuracy is also affected by the granularity of the position information represented in the TDL fixed format messages and is dependent on the TDL type. The capability of the sensor used to detect the target is fundamental to kill chain accuracy, it is clear that errors introduced at the start of the chain will be propagated, and potentially exacerbated throughout it.

\subsubsection{Target visibility}

Where a target is not camouflaged, situated in open terrain with clear atmospheric conditions and of reasonable size it will be highly visible. However, this is not a typical situation, generally a target will require "marking" as an aid to the incoming CAS aircraft. Target marking may be achieved through a number a methods including; smoke, laser or phosphorous. Laser marking requires compatible technology on the CAS aircraft and/or the weapon; smoke and phosphorous provide an entirely visual guide. Environmental conditions such as cloud, dust and light level will constrain visual marking effectiveness. Civilian visibility and blue force visibility is also dependent upon these environmental factors.

\subsubsection{Enemy capability}

Enemy capability is provided by weapon sophistication, e.g. guided ground to air missile and the effectiveness of targeting and tracking capability which may range from basic visual targeting to complex sensor, e.g. Radar systems.

\subsubsection{Detailed causal network}

Having established the causal relationships between the risks and all contributing factors, a more detailed causal network was produced which reflected this analysis and is shown below in Figure 38. 


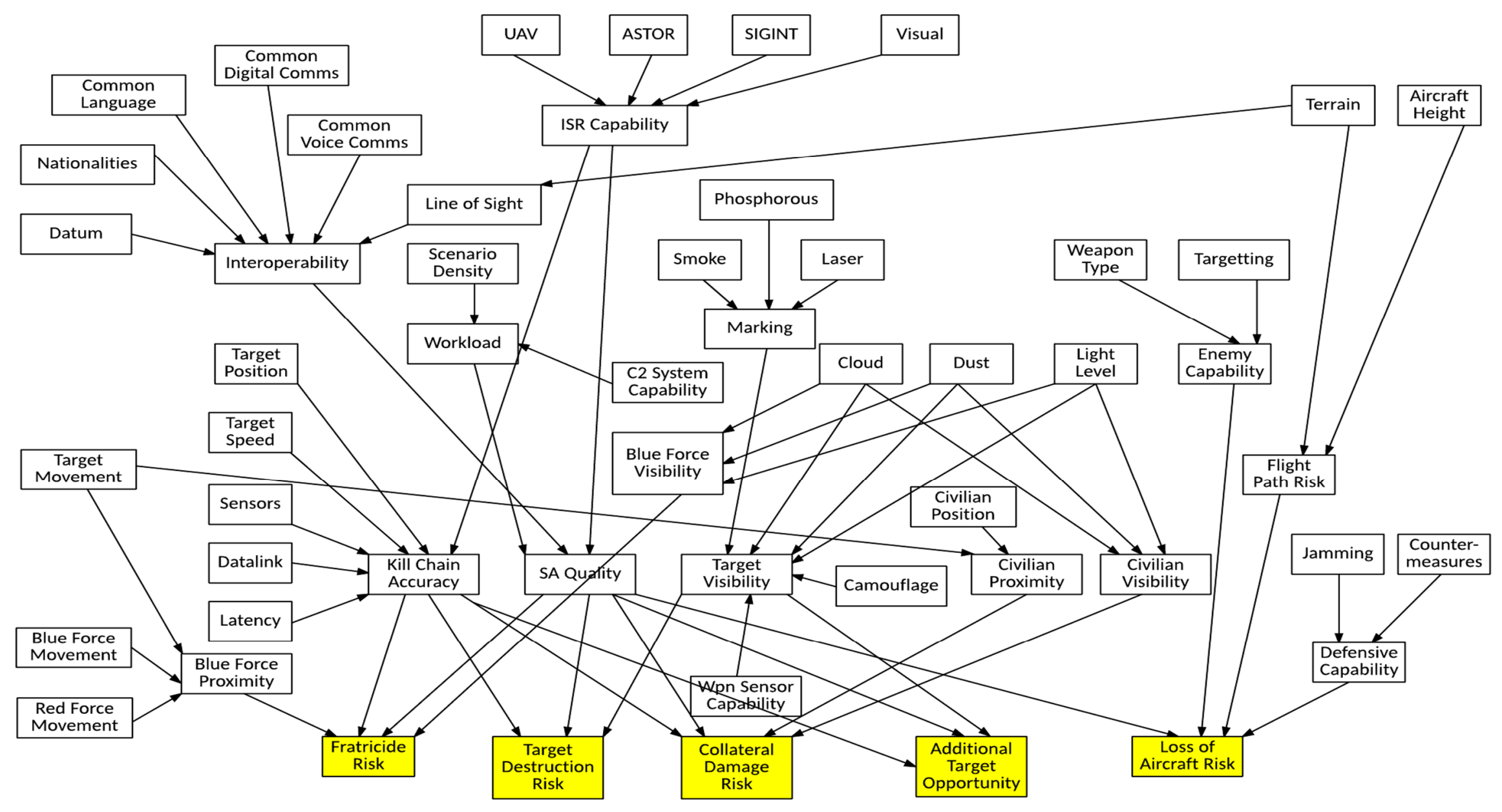

Figure 38 - Causal Network: CAS 


\subsection{SoS Risk Analysis and Handling}

Having established the causal risk network (Figure 38), each risk and the associated contributing factors were considered in accordance with the process defined in section 7.4. It should be noted that a comprehensive description of the Fratricide Risk analysis and handling is included within the main body of this document, however the remaining risk analysis is summarised with further detail appended within Appendix E. This approach was taken to avoid unnecessary repetition and duplication of information, which would otherwise arise though the application of the same process for each risk and does not provide further understanding of the process, although it would be of benefit in a future study requiring these research outputs as starting point.

\subsection{SoS Risk Analysis and Handling - Fratricide Risk}

The causal network relating to fratricide risk was extracted from the complete network (Figure 38) and is shown below in Figure 39, where Blue Force Proximity, Kill Chain Accuracy, SA Quality and Blue Force Visibility are the primary contributing factors.

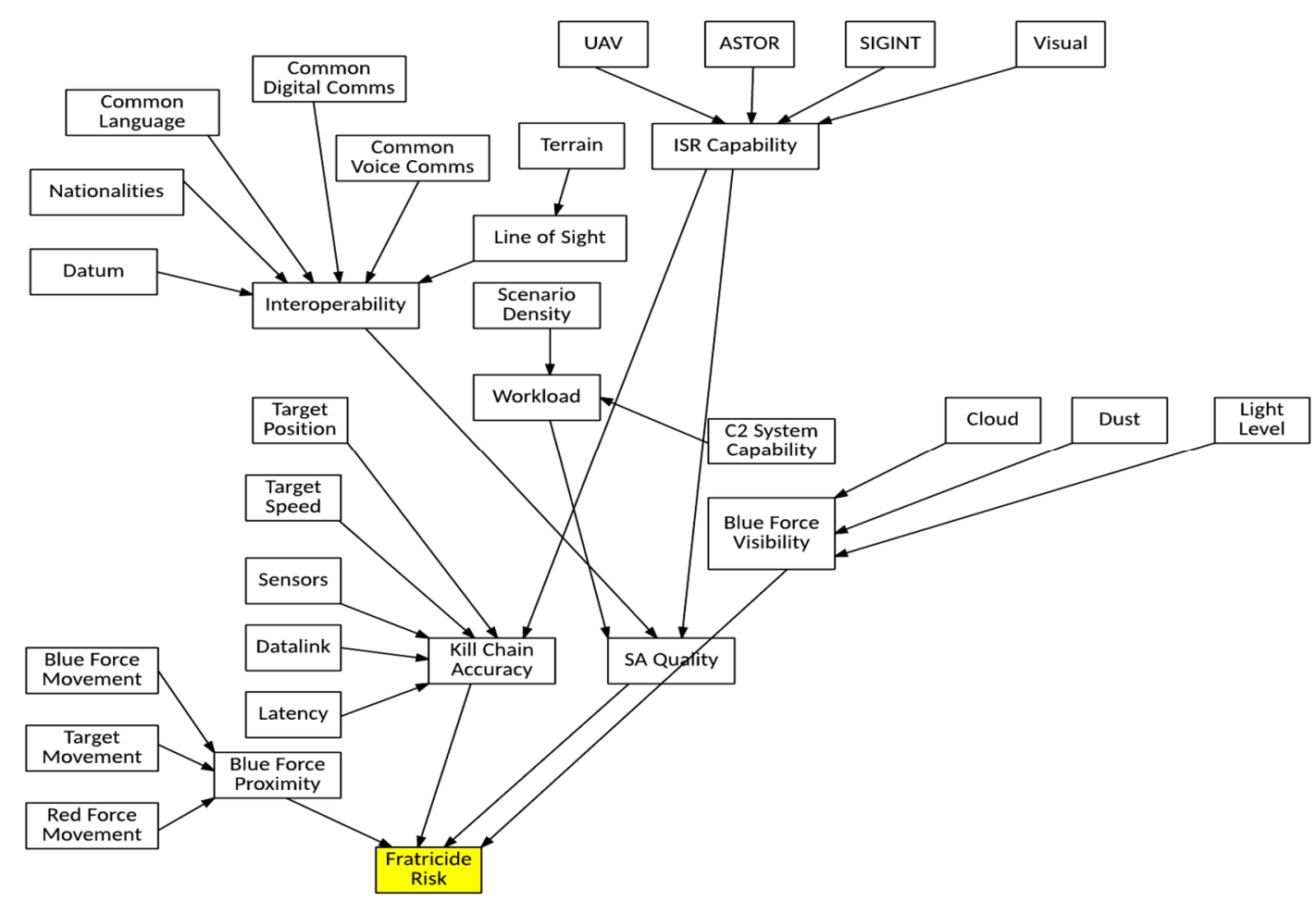

Figure 39 - Fratricide Risk Causal Network

\section{Blue Force Proximity}

Blue force proximity was influenced by three contributing factors; blue force movement, red force movement and target movement. The model selection matrix tool (section 6.3) was populated accordingly; with a screen shot provided in Appendix B. The tool indicated that, in 
this case, the most suitable M\&S technique would be DES/DEVS, ABMS or System Dynamics. ABMS allows individual agents to be encoded with a simple set of rules, which can relate to movement, therefore it was decided to utilise this technique in this instance as it appeared to enable relatively straightforward implementation of the desired functionality, with the additional benefit of providing visualisation of the agent movements. As all contributing factors could be represented using ABMS, it was possible to combine them into a single "Blue force Proximity" model (in accordance with the rules defined in section 7.4.2).

\section{Kill Chain Accuracy}

Kill chain accuracy was influenced by the following contributing factors; latency, TDL characteristics, sensors, target speed, target position and ISR capability. However, ISR capability was interpreted as sensor accuracy and was therefore combined with the "Sensor" contributing factor for the purposes of model selection matrix tool population; the output of which is provided in Appendix B, and which indicated DES/DEVS as the most appropriate technique. A kill chain is effectively a sequence of transmissions, transformations and translations hence it was appropriate to produce a single model incorporating all contributing factors, thereby modelling the entire chain.

\section{$\underline{\text { SA Quality }}$}

SA Quality was influenced by three factors, Interoperability, Workload and ISR Capability. Initial population of the M\&S technique selection tool produced an output, which identified an overly broad range of potential techniques to be of use and is included in Appendix B. Examining this output revealed that removing Interoperability, to be analysed separately, would narrow the number of suitable techniques, and was confirmed by application of the selection tool, the output of which is located in Appendix B, identifying BBN, Markov Chain or Game Theory as appropriate techniques. Hence, as a BBN was used to represent the central risk model it was decided to incorporate workload and ISR capability into this, eliminating the necessity for an additional model.

$\underline{\text { Interoperability }}$

Interoperability was influenced by the following contributing factors, Geodetic Datum, Nationalities, Common Language, Common Digital Communications and Common Voice Communications. The M\&S selection tool was populated and indicated that System Dynamics was a suitable approach for modelling Interoperability and all contributing factors; the tool output is included in Appendix B. In the context of this case study the Interoperability model was intended to represent overall capability and concept of 
operation, requiring the use of aggregated information rather than detailed representation of individual component systems, which resulted in the identification of System Dynamics rather than a technique such as DES or ABMS. Of note, was the selection of the 'Stochastic' requirement rather than 'Uncertainty' for the contributing factors, the rationale being that knowledge relating to these factors would be available but potentially with a margin of error, for example the digital communications capability within the SoS may have been known but the estimated data capacity would have an associated error.

Blue Force Visibility

The final contributing factor under consideration, relating to Fratricide, was Blue Force Visibility. In this case the contributing factors were environmental; although they could not be considered to be inside the Sol SoS boundary they were key factors determining the visibility. It was expected that cloud and dust predictions would be obtained from meteorological forecast information, with light levels directly determined from the expected mission time in conjunction with meteorological conditions. The populated selection tool spreadsheet, included in Appendix B, indicated that a BBN approach was suitable, hence it was decided to incorporate Blue Force Visibility and the associated contributing factors directly into the central BBN.

\subsubsection{Fratricide Risk Model Architecture}

Having established the appropriate modelling approach for each contributing factor, the overall fratricide risk model architecture was defined and is shown below in Figure 40. The red containers indicate the boundary of the supporting models with the blue container indicating the BBN boundary, although it should be noted that any factor directly connected to the BBN were also be replicated within the BBN itself, e.g. Interoperability. 


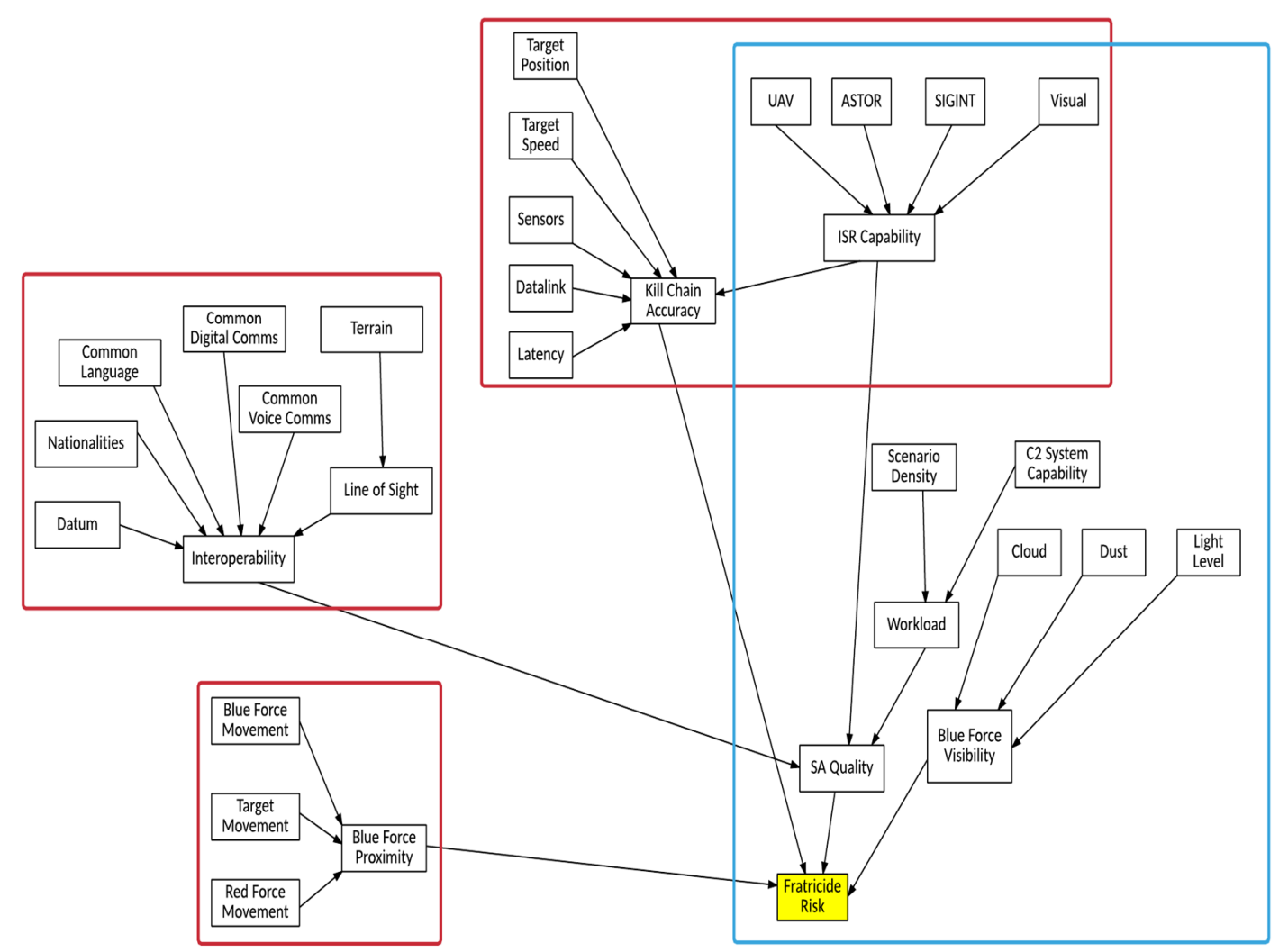

Figure 40 - Fratricide Risk - Model Architecture

Of note, is the inclusion of ISR capability in both the central BBN and the Kill Chain Accuracy supporting model. This demonstrates the ability of the proposed approach to consider contributing factors in different contexts; in this case ISR capability related to the sensor capability and also has a more general impact on SA quality.

The analysis above enabled the causal network to be simplified, as shown below in Figure 41, in accordance with the process defined in section 7.4.2, where a red box indicates a node is informed by the following associated supporting models:

- Blue Force Proximity-ABMS

- Kill Chain Accuracy - DES

- Interoperability - System Dynamics 


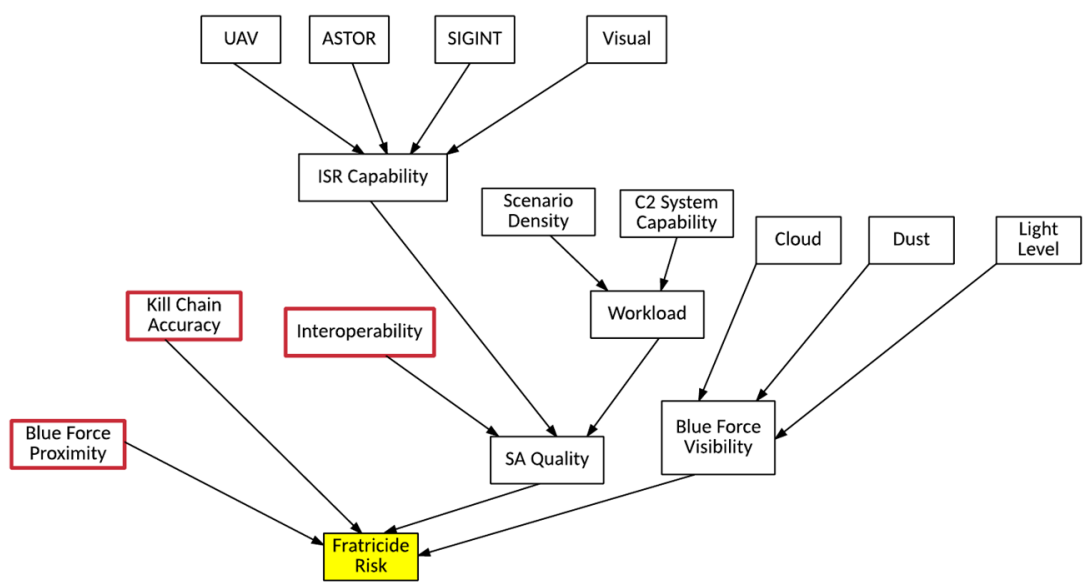

Figure 41 - Fratricide Risk - Simplified Causal Network

\subsubsection{Fratricide Risk BBN}

Having established the model architecture, the Fratricide Risk BBN was implemented in accordance with the Causal Network and is shown below in Figure 42. The BBN variables and their associated states are described in Table 23; definition of the nodes and their states is an essential part of the analysis process; without supporting descriptions the variables within the BBN may not be interpreted correctly, generic states, e.g. low, medium and high, are meaningless without specific definitions.

It should be noted that Table 23 describes the variables derived directly from the causal network shown in Figure 41, however additional mediating variables are also included, as discussed in section 5.2.3, reducing the size and complexity of the variable probability tables and are listed below:

- Surveillance Capability

- Intelligence Capability

- ISR Capability

- SoS Capability

- Information Quality

- Atmospheric Visibility

- Blue Force Risk

The probability tables associated with parent variables are contained in Appendix $C$ and were populated using SME input where it was found that the addition of the mediating variables, listed above, greatly reduced the complexity of estimating these values, thereby providing the necessary requisite parsimony (described in section 3.2.1). 


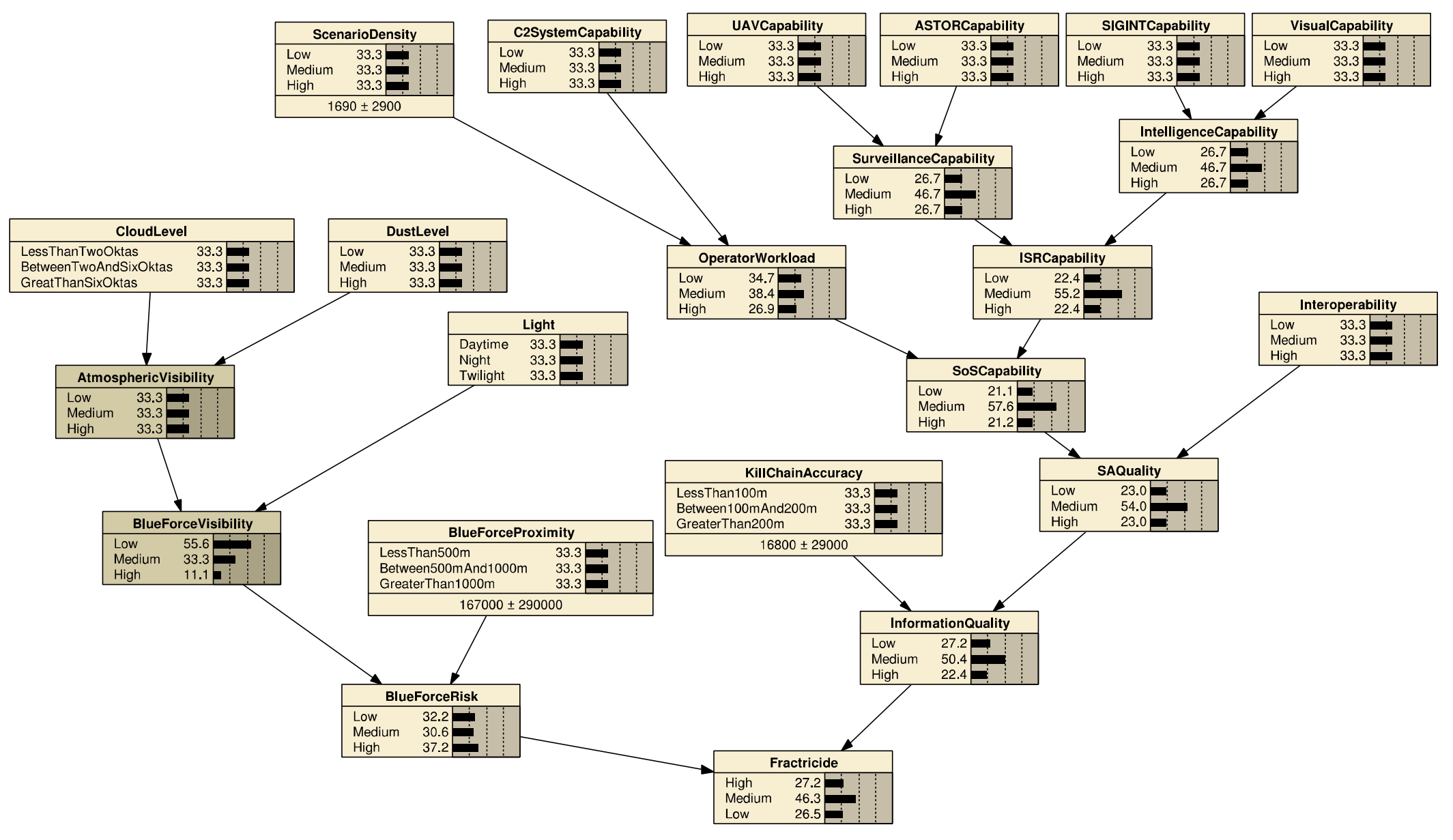

Figure 42 - Fratricide Risk BBN 


\begin{tabular}{|c|c|c|}
\hline Variable & Description & States \\
\hline ScenarioDensity & $\begin{array}{l}\text { An assessment of the } \\
\text { number of tracks likely to } \\
\text { be present within a } \\
\text { mission }\end{array}$ & $\begin{array}{l}\text { Low: Less than } 20 \text { tracks } \\
\text { Medium: Less than } 50 \text { tracks } \\
\text { High: } 50 \text { or more tracks }\end{array}$ \\
\hline C2SystemCapability & $\begin{array}{l}\text { An assessment of the } \\
\text { Command and Control } \\
\text { system capability, } \\
\text { specifically the level of } \\
\text { automation }\end{array}$ & $\begin{array}{l}\text { Low: manual track picture } \\
\text { management required } \\
\text { Medium: basic level of } \\
\text { automation and track filtering } \\
\text { High: high level of automation }\end{array}$ \\
\hline WorkLoad & $\begin{array}{l}\text { Overall workload derived } \\
\text { from scenario density and } \\
\text { C2 system capability }\end{array}$ & $\begin{array}{l}\text { Low: overall workload has no } \\
\text { detrimental impact upon } \\
\text { system performance } \\
\text { Medium: workload impacts } \\
\text { system performance to a } \\
\text { limited extent } \\
\text { High: workload has a } \\
\text { detrimental affect upon } \\
\text { system performance }\end{array}$ \\
\hline UAVCapability & $\begin{array}{l}\text { Unmanned Air Vehicle } \\
\text { capability }\end{array}$ & $\begin{array}{l}\text { Low: no UAV capability } \\
\text { Medium: basic video feed } \\
\text { from UAV } \\
\text { High: video and sensor } \\
\text { information sourced from UAV }\end{array}$ \\
\hline ASTORCapability & $\begin{array}{l}\text { Airborne Stand-off Radar } \\
\text { capability }\end{array}$ & $\begin{array}{l}\text { Low: no ASTOR capability } \\
\text { Medium: basic short range } \\
\text { Radar capability } \\
\text { High: medium/long range, } \\
\text { high definition Radar } \\
\text { capability }\end{array}$ \\
\hline SIGINTCapability & $\begin{array}{l}\text { Signal Intelligence; } \\
\text { intelligence gather } \\
\text { through the interception } \\
\text { of signals capability }\end{array}$ & $\begin{array}{l}\text { Low: no SIGINT capability } \\
\text { Medium: basic signal } \\
\text { interception } \\
\text { High: all signals monitored and } \\
\text { associated intelligence } \\
\text { gathered }\end{array}$ \\
\hline
\end{tabular}


A Model Based Approach to System of Systems Risk Management

\begin{tabular}{|c|c|c|}
\hline Variable & Description & States \\
\hline VisualCapability & $\begin{array}{l}\text { Intelligence provided from } \\
\text { direct sightings }\end{array}$ & $\begin{array}{l}\text { Low: no visual reports } \\
\text { Medium: Non-current visual } \\
\text { information } \\
\text { High: Real time visual } \\
\text { information }\end{array}$ \\
\hline ISRCapability & $\begin{array}{l}\text { Overall combined } \\
\text { Intelligence, Surveillance, } \\
\text { Reconnaissance capability }\end{array}$ & $\begin{array}{l}\text { Low: Minimal ISR information } \\
\text { provided } \\
\text { Medium: Basic ISR information } \\
\text { provided } \\
\text { High: Comprehensive ISR } \\
\text { information provided }\end{array}$ \\
\hline CloudLevel & Cloud cover & $\begin{array}{l}\text { Less than } 2 \text { Oktas } \\
\text { Between } 2 \text { and } 6 \text { Oktas } \\
\text { Greater than } 6 \text { Oktas }\end{array}$ \\
\hline DustLevel & Atmospheric dust level & $\begin{array}{l}\text { Low: No dust } \\
\text { Medium: Dust present with } \\
\text { impact on visibility } \\
\text { High: Dust present having a } \\
\text { major detrimental effect on } \\
\text { visibility }\end{array}$ \\
\hline Light & $\begin{array}{l}\text { Light level related to the } \\
\text { time of day }\end{array}$ & $\begin{array}{l}\text { Daytime } \\
\text { Night } \\
\text { Twilight }\end{array}$ \\
\hline BlueForceVisibility & $\begin{array}{l}\text { Overall blue (friendly) } \\
\text { force visibility }\end{array}$ & $\begin{array}{l}\text { Low: Blue Force heavily } \\
\text { visually obscured } \\
\text { Medium: Blue Force visible } \\
\text { with some obscuration } \\
\text { High: Clear view of Blue Forces }\end{array}$ \\
\hline BlueForceProximity & $\begin{array}{l}\text { Distance of Blue Force } \\
\text { from target }\end{array}$ & $\begin{array}{l}\text { Less than } 500 \mathrm{~m} \\
\text { Between } 500 \mathrm{~m} \text { and } 1 \mathrm{Km} \\
\text { Greater than } 1 \mathrm{Km}\end{array}$ \\
\hline KillChainAccuracy & $\begin{array}{l}\text { Total positional error } \\
\text { introduced by the kill } \\
\text { chain }\end{array}$ & $\begin{array}{l}\text { Less than } 100 \mathrm{~m} \\
\text { Between } 100 \text { and } 200 \mathrm{~m} \\
\text { Greater than } 200 \mathrm{~m}\end{array}$ \\
\hline
\end{tabular}


A Model Based Approach to System of Systems Risk Management

\begin{tabular}{|c|c|c|}
\hline Variable & Description & States \\
\hline Interoperability & $\begin{array}{l}\text { Overall level of } \\
\text { interoperability }\end{array}$ & $\begin{array}{l}\text { Low: poor interoperability } \\
\text { throughout SoS } \\
\text { Medium: limited } \\
\text { interoperability between SoS } \\
\text { components } \\
\text { High: unhindered exchange of } \\
\text { information possible } \\
\text { throughout SoS }\end{array}$ \\
\hline SAQuality & $\begin{array}{l}\text { Overall measure of the SA } \\
\text { quality, reflecting the } \\
\text { accuracy, completeness } \\
\text { and timeliness of the } \\
\text { tactical information }\end{array}$ & $\begin{array}{l}\text { Low: information not } \\
\text { complete, accurate or current } \\
\text { Medium: information mostly } \\
\text { complete, accuracy and } \\
\text { latency is reflected in "honest" } \\
\text { confidence indications } \\
\text { High: information is complete } \\
\text { with high associated } \\
\text { confidence }\end{array}$ \\
\hline Fratricide & $\begin{array}{l}\text { The risk of Fratricide as a } \\
\text { result of the CAS mission }\end{array}$ & $\begin{array}{l}\text { Low: Fratricide is extremely } \\
\text { unlikely to occur, no further } \\
\text { risk mitigation is required } \\
\text { Medium: There is a potential } \\
\text { for Fratricide, approaches for } \\
\text { risk mitigation should be sort } \\
\text { and applied if possible or risk } \\
\text { should be accepted prior to } \\
\text { mission execution } \\
\text { High: There is a significant } \\
\text { potential for Fratricide, risk } \\
\text { mitigation is necessary before } \\
\text { mission commencement }\end{array}$ \\
\hline
\end{tabular}

Table 23 - Fratricide Risk BBN Variables

\subsubsection{Interpretation of Results}

The BBN was next updated with evidence derived from a hypothetical scenario and the outputs from the supporting models, which are described in section 8.13. The hypothetical scenario description and assumptions are provided in Appendix D.

The updated BBN is shown below in Figure 43, indicating High Risk probability of $12.1 \%$, Medium Risk of $43.7 \%$ and Low Risk of $44.3 \%$. However, the question arose, "what does this mean?" 


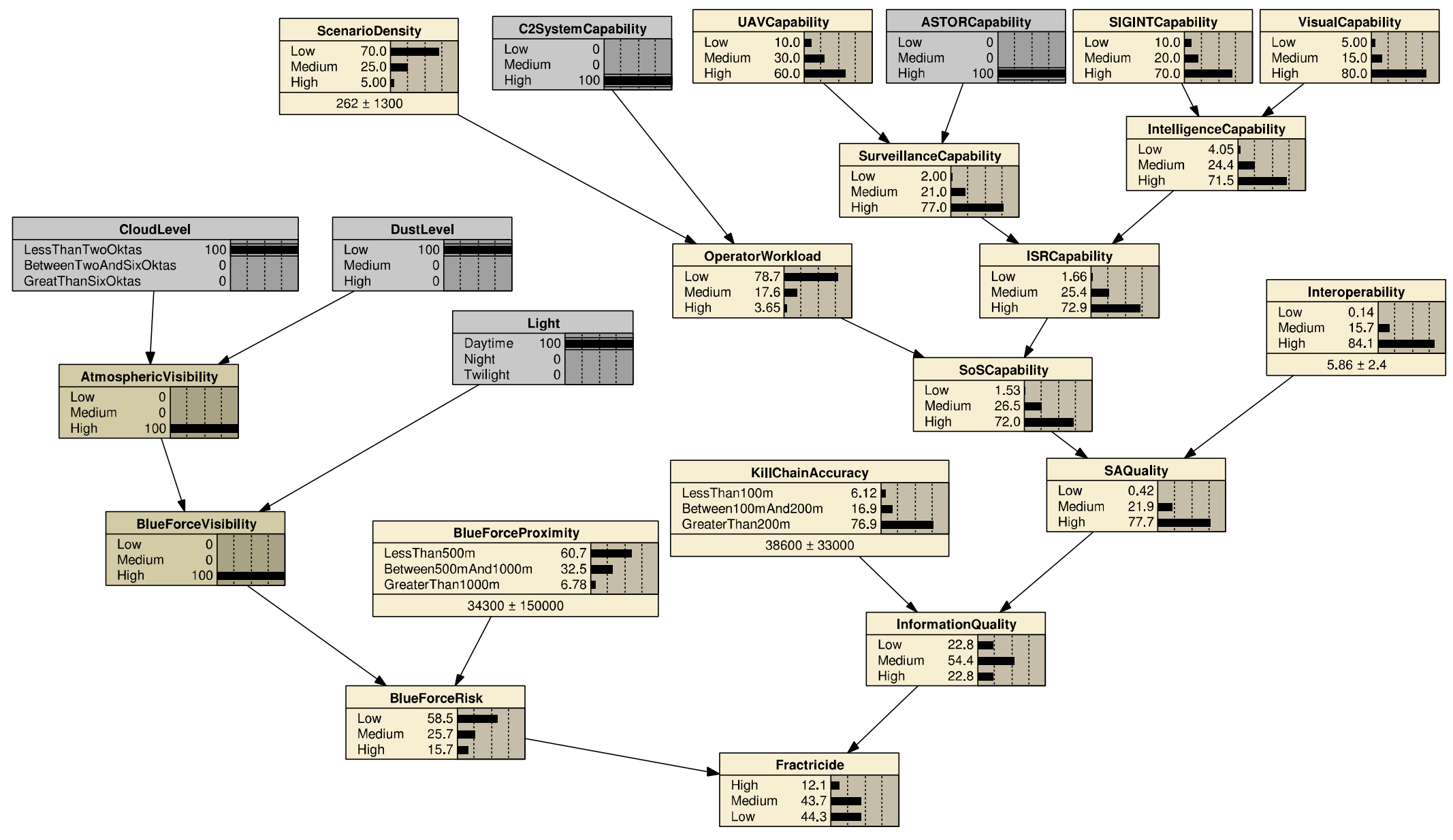

Figure 43 - Fratricide Risk BBN - Post Learning and Evidence Entry 


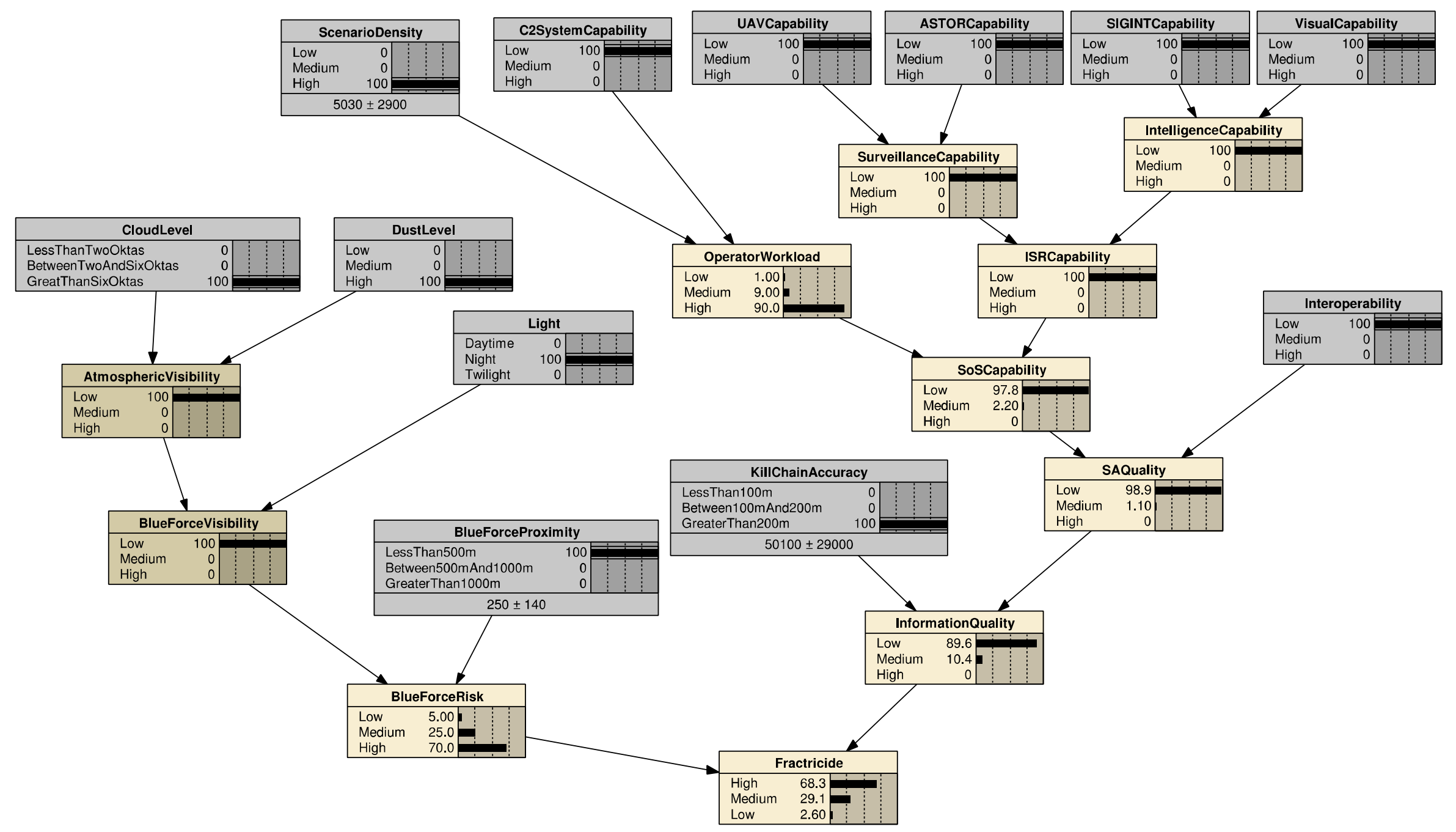

Figure 44 - Fratricide Risk BBN Worst Case 


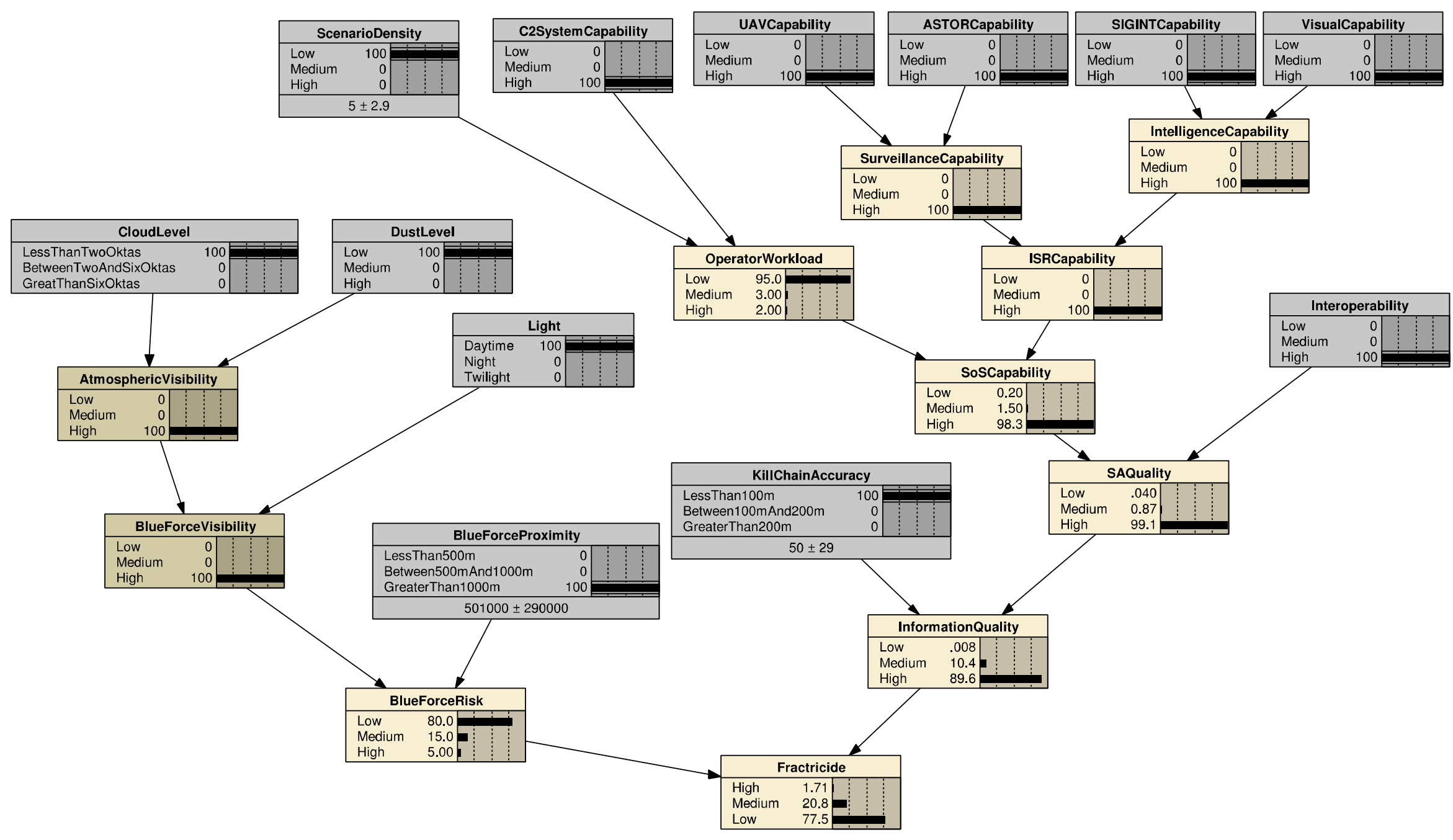

Figure 45 - Fratricide Risk BBN Best Case 
In order to meaningfully interpret this output an approach was taken which enabled a relative comparison of the results with best and worst-case scenarios, hence two further sets of evidence were entered as shown in Figure 44 and Figure 45.

The worst-case scenario evidence indicated low system capability, poor visibility or close vicinity to the target. Conversely, the best-case scenario evidence indicated high system capability, high visibility and large distance from the target.

The process of entering worst and best case evidence also proved to be valuable verification activity, with several errors in the probability tables discovered as a result and subsequently corrected, as well as providing scenarios that were easily validated by SMEs.

Figure 46 provides a side-by-side comparison of the results and enabling an assessment of the risk profile, used to describe the low, medium and high risk probability levels, where the question is posed, "is the profile closer to best or worst case?"

In this case it was observed that the risk profile was a closer match to the best-case profile, with the overall conclusion that the likelihood of high risk was small, however the increased potential of medium risk did suggest some further risk mitigation would be beneficial but should not prohibit mission execution. The interpretation of these results was judgement based, requiring further SME input and would be dependent on the risk appetite, which in a CAS mission, could be influenced by operational or political needs.

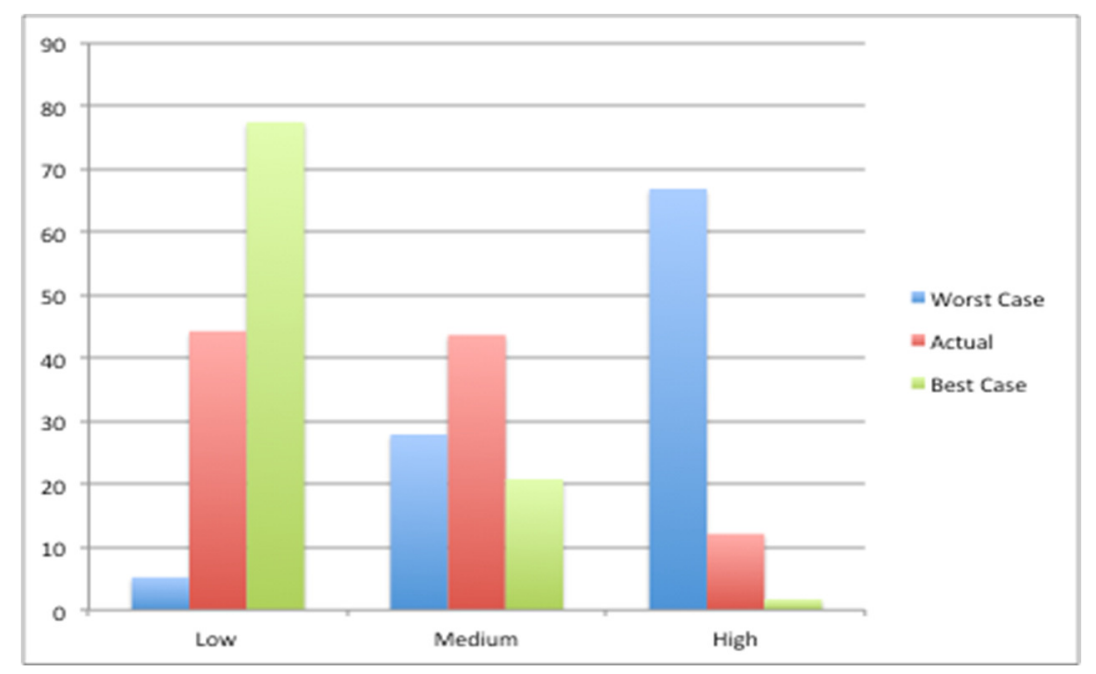

Figure 46 - Fratricide Risk Comparison 
Examining the updated BBN (Figure 43) it was observed that the likelihood of blue force proximity to the target being less than $500 \mathrm{~m}$ was relatively high whereas the kill chain accuracy was more likely to be poor ( $>200 \mathrm{~m})$. An advantage of a BBN representation is the ability to test "what if" scenarios, in this case the KillChainAccuracy variable state of "Less Than $100 \mathrm{~m}$ " was set to $100 \%$, indicating a low error level throughout the kill chain and resulted in the desired increase in the likelihood of low risk of fratricide, as shown below in Figure 47.

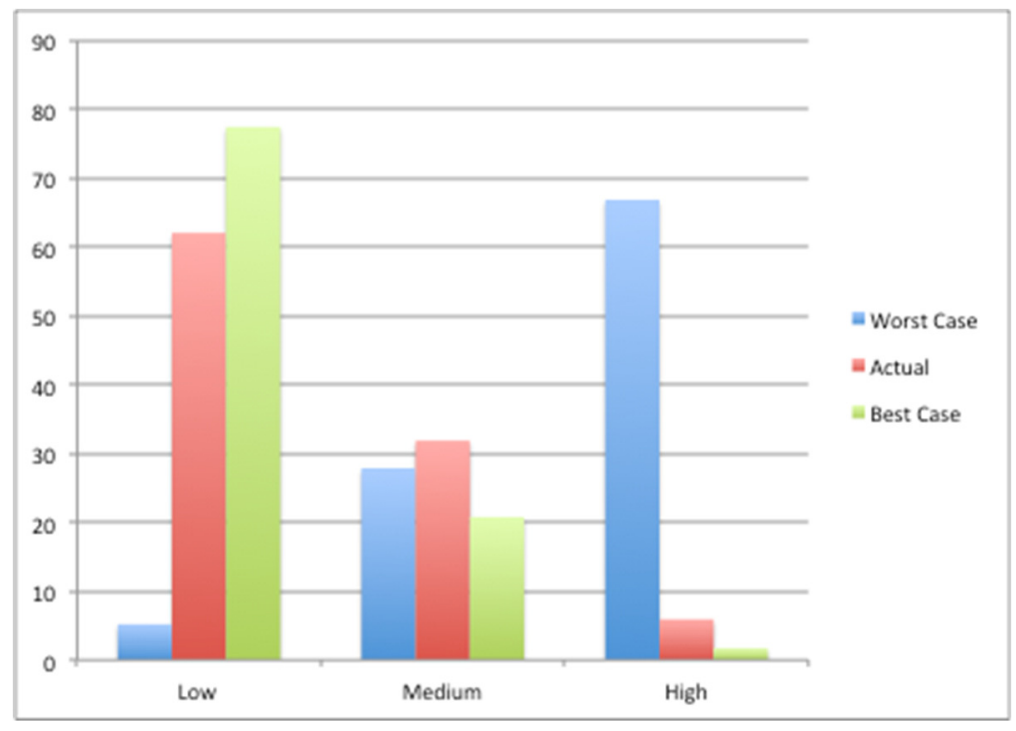

Figure 47 - Fratricide BBN with High Accuracy Kill Chain

Although this was a crude check, it did indicate that an increase in accuracy throughout the kill chain would reduce the risk of fratricide in this case ${ }^{14}$ but would require more detailed analysis within the kill chain model to identify specific elements that could be influenced.

\subsubsection{Establishing confidence estimates}

Having determined the Fratricide risk level, the final stage in the analysis process was the derivation of the associated confidence, requiring the construction of an equivalent confidence BBN as shown in Figure 48, with the rationale for the BBN variable confidence estimates provided in Table 24.

\footnotetext{
${ }^{14}$ This was validated by the SME who stated kill chain accuracy was deliberately reduced during a CAS exercise in order to reduce risk
} 


\begin{tabular}{|c|c|c|}
\hline Variable & Input Type & Confidence Estimate \\
\hline ScenarioDensity & Estimate & $\begin{array}{l}\text { Medium: input based on current scenario } \\
\text { density levels, with the assumption that this } \\
\text { will not significantly change }\end{array}$ \\
\hline C2SystemCapability & Evidence & High: Available C2 system capability is known \\
\hline UAVCapability & Evidence & High: Available UAV system capability is known \\
\hline ASTORCapability & Evidence & $\begin{array}{l}\text { High: Available ASTOR system capability is } \\
\text { known }\end{array}$ \\
\hline SIGINTCapability & Evidence & High: Current SIGINT capability is known \\
\hline VisualCapability & Estimate & $\begin{array}{l}\text { Low: estimate of visual report availability is } \\
\text { uncertain }\end{array}$ \\
\hline CloudLevel & $\begin{array}{l}\text { Meteorological } \\
\text { forecast }\end{array}$ & High: forecast has a high confidence level \\
\hline DustLevel & $\begin{array}{l}\text { Meteorological } \\
\text { forecast }\end{array}$ & $\begin{array}{l}\text { High: forecast, which directly impacts dust } \\
\text { levels, has a high confidence level }\end{array}$ \\
\hline Light & $\begin{array}{l}\text { Evidence (derived } \\
\text { from planned } \\
\text { mission time) }\end{array}$ & $\begin{array}{l}\text { High: light level is derived from the planned } \\
\text { mission execution time of day }\end{array}$ \\
\hline BlueForceProximity & Supporting Model & Low: model is simplistic \\
\hline KillChainAccuracy & Supporting Model & Low: model is simplistic \\
\hline Interoperability & Supporting Model & Low: model is simplistic \\
\hline
\end{tabular}

Table 24 - Fratricide Risk Confidence 


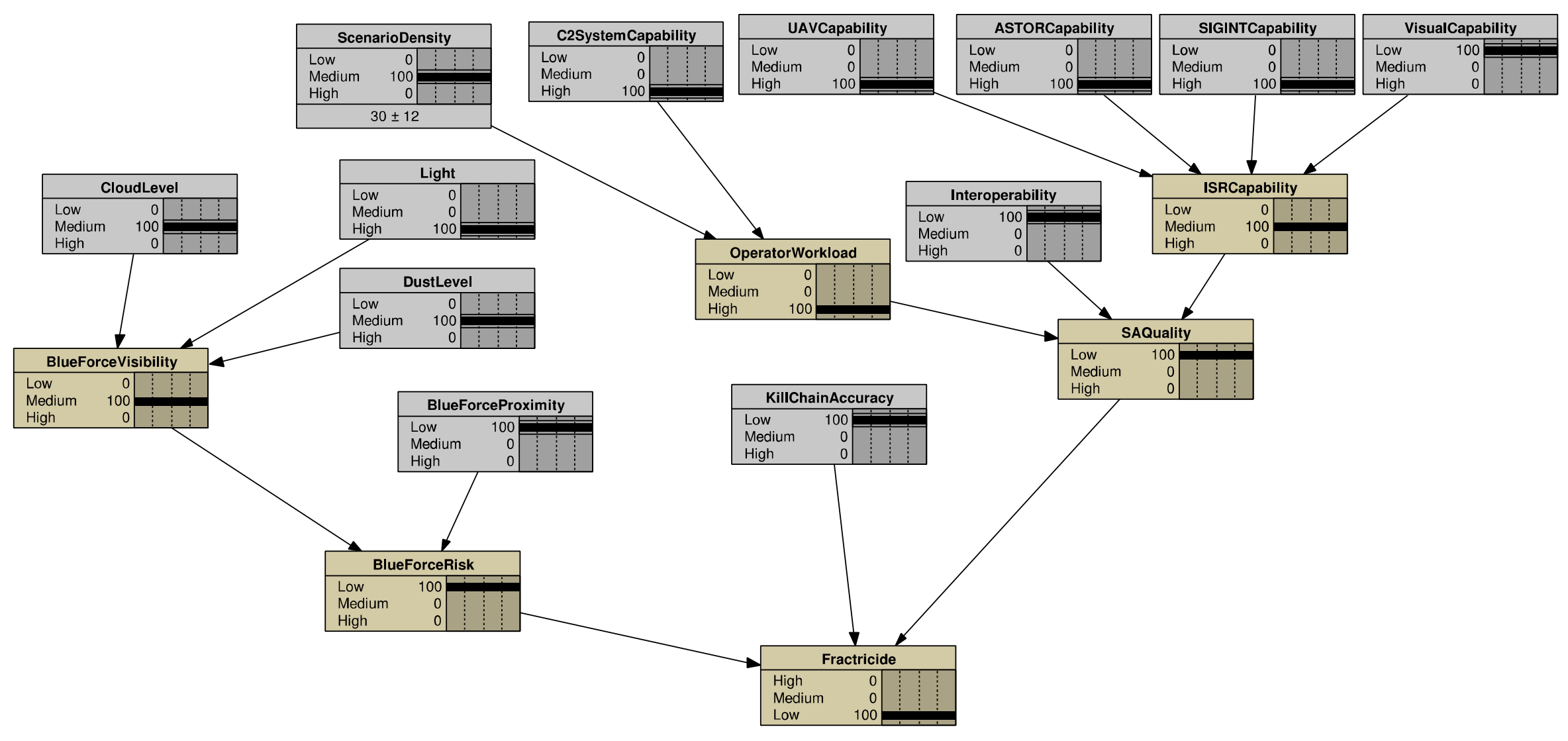

Figure 48 - Fratricide Risk Confidence BBN 


\subsection{Analysis and Handling - Target Destruction Risk}

Having determined the BBN and supporting models relating to Fratricide, target destruction risk was considered, the detailed analysis can be found in Appendix E. The results of the analysis are shown below in Figure 49, indicating that although the risk profile was more aligned to the best-case results the probability of a low chance of target destruction failure was $30 \%$ lower than the optimum value. It was therefore concluded that the level of risk was acceptable but any potential mitigation should be explored. The associated risk analysis confidence was determined as "medium".

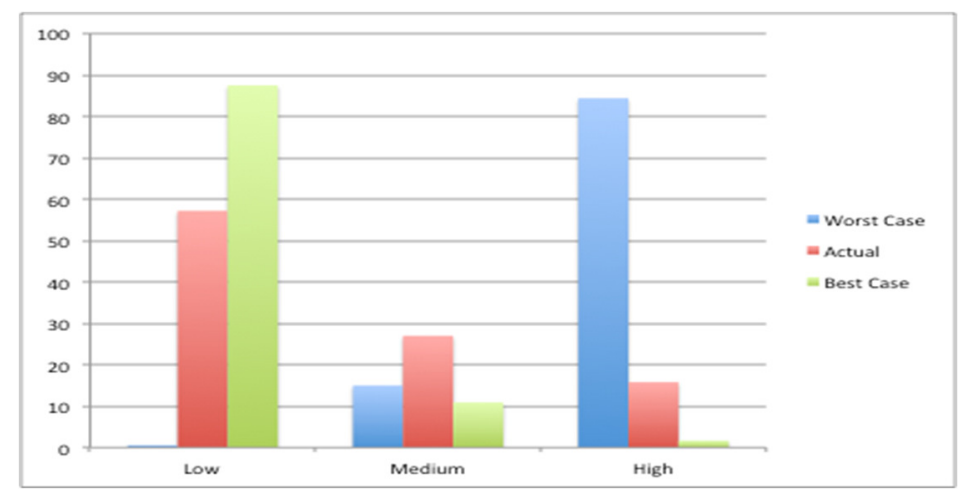

Figure 49 - Target Destruction Risk Comparison

Examining the Target Destruction Risk BBN (Appendix E) it was noted that the inaccuracy associated with the kill chain was contributing to the reduced probability of low risk of target destruction failure, as it also similarly had for fratricide risk. Using the same approach as taken previously, the BBN was updated by setting the probability for minimum kill chain error to $100 \%$, improving the overall risk level, which was now closely comparable with the best case values, as shown in Figure 50, implying that further analysis of the kill chain component systems with an aim to reducing error growth would be beneficial.

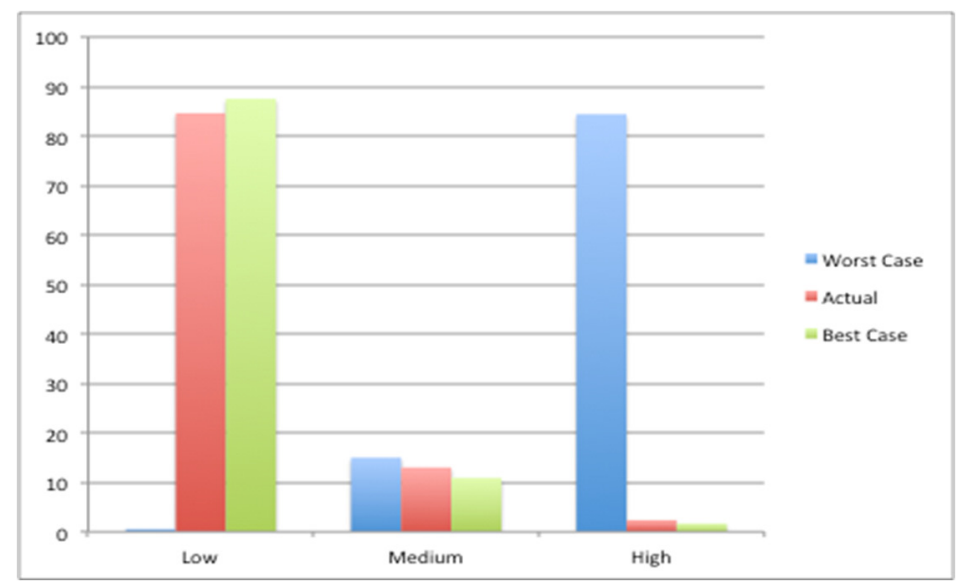

Figure 50 - Target Destruction BBN with High Accuracy Kill Chain 


\subsection{Analysis and Handling - Collateral Damage Risk}

Detailed Collateral Damage Risk analysis is provided in Appendix E.

The resultant side-by-side comparison of the actual, best-case and worst-case results are shown below in Figure 51, revealing that although the actual results were more closely aligned to the best-case values, the probability of low risk was approximately $20 \%$ under the optimum value, indicating risk mitigation would be beneficial but the risk level would not prohibit a mission execution attempt if no improvement was possible. However, the risk confidence was determined as "low" in this case.

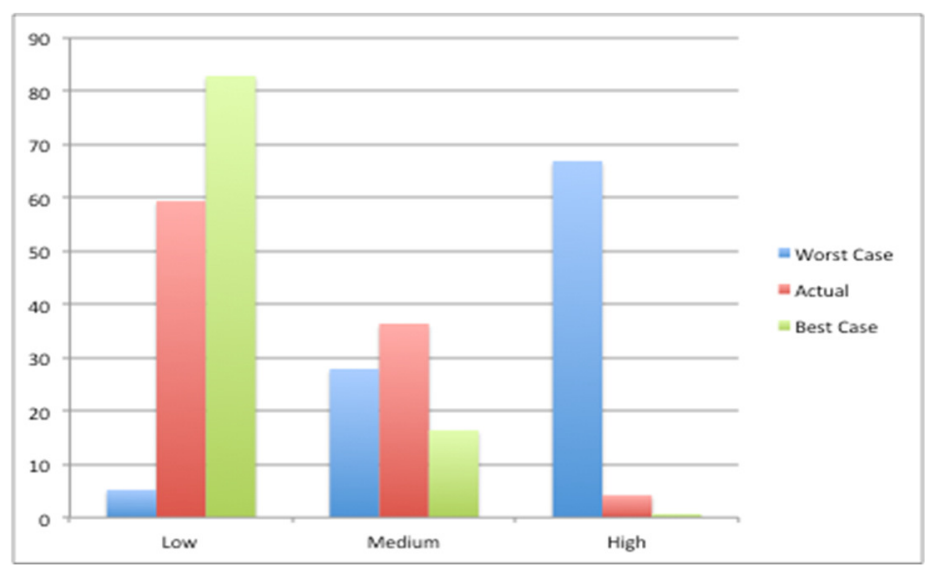

Figure 51 - Collateral Damage Risk Comparison

\subsection{Analysis and Handling - Additional Target Opportunity}

Detailed Additional Target Opportunity Risk analysis is provided in Appendix E.

The resultant side-by-side comparison is shown below in Figure 52, which revealed a relatively close correlation between the actual and best-case results, although further experimentation was undertaken in order to identify potential approaches to improvement in the actual risk profile. In this case the risk confidence was determined to be "medium".

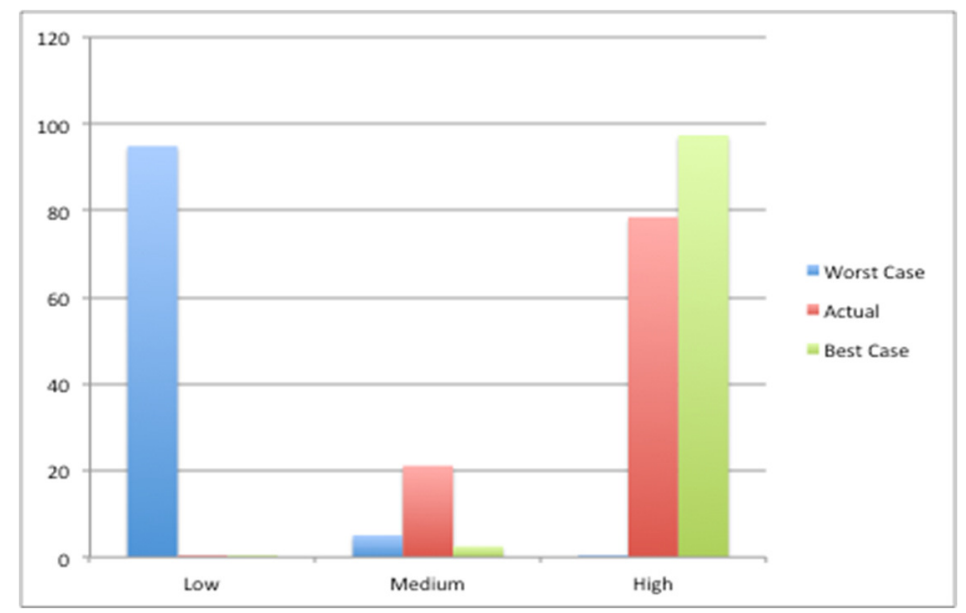

Figure 52 - Additional Target Opportunity Comparison 


\subsection{Analysis and Handling - Loss of Aircraft Risk}

Detailed Loss of Aircraft Risk analysis is provided in Appendix E.

The final side-by-side comparison is shown below in Figure 53 revealing that, although the analysis results aligned more closely to the best case risk profile, the probability of low risk was approximately $30 \%$ lower than the optimum level, indicating that further risk mitigation was desirable, although the risk confidence was determined to be "low".

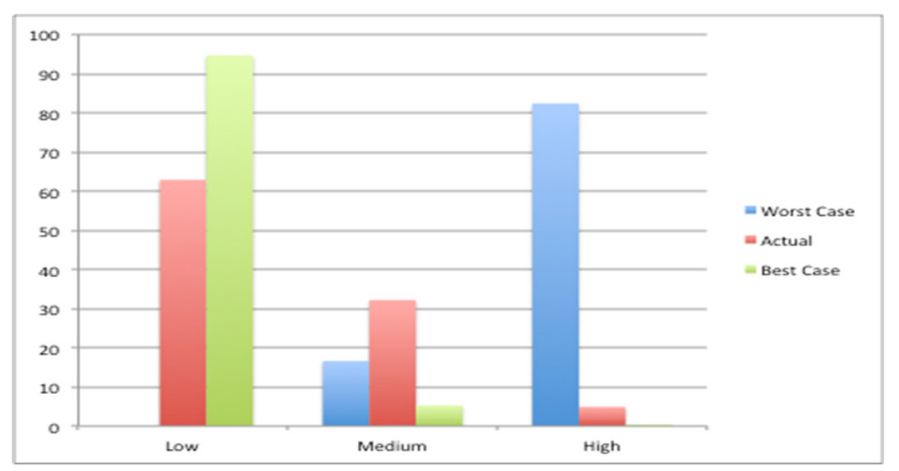

Figure 53 - Loss of Aircraft Risk Comparison

A potentially straightforward risk mitigation approach in this case was to raise the mission flight altitude of over 1000ft, resulting in an improved risk profile, reducing the gap between best-case and actual low risk level to $15 \%$.

\subsection{SoS Risk Analysis and Handling - Summary}

Table 25 below, summarises the risk analysis and handling outcome for each of the risks under consideration.

\begin{tabular}{|l|l|l|l|l|}
\hline Risk & Assessment & Impact & Confidence & Handling \\
\hline Fratricide & $\begin{array}{l}\text { Medium/Low } \\
\text { Risk }\end{array}$ & Catastrophic & Low & Mitigation Required \\
\hline $\begin{array}{l}\text { Failure to } \\
\text { Destroy Target }\end{array}$ & Low Risk & $\begin{array}{l}\text { Mission } \\
\text { Critical/ } \\
\text { Catastrophic }\end{array}$ & Medium & $\begin{array}{l}\text { Accept Risk, Mitigation } \\
\text { Beneficial but not } \\
\text { essential }\end{array}$ \\
\hline $\begin{array}{l}\text { Collateral } \\
\text { Damage }\end{array}$ & Cow Risk & $\begin{array}{l}\text { Accept Risk, Mitigation } \\
\text { Beneficial but not } \\
\text { essential }\end{array}$ \\
\hline $\begin{array}{l}\text { Loss of Aircraft } \\
\text { Target } \\
\text { Opportunity }\end{array}$ & $\begin{array}{l}\text { Medium/Low } \\
\text { Risk } \\
\text { target } \\
\text { opportunity }\end{array}$ & Catastrophic & Low & \begin{tabular}{l} 
Mitigation Required \\
High likelihood \\
\hline
\end{tabular}
\end{tabular}

Table 25 - CAS Risk Summary 


\subsection{SoS Risk Monitoring}

The monitoring of risk associated with CAS mission planning requires rapid analysis and assessment of risks, hence the implementation and testing of BBNs with associated supporting models from scratch is not a practical approach due to these time constraints. However, using the model-based approach as a basis for a decision support tool, which could be updated with appropriate information and parameters, is a feasible future exploitation of this research.

\subsection{Supporting Models}

The risk analysis activity identified a requirement for four supporting models; Kill Chain Accuracy, Interoperability, Blue Force Proximity and Civilian Proximity. However, during implementation Blue Force Proximity and Civilian Proximity were combined into a single model, which was found to be a more efficient approach.

A brief description of each model is provided below, with greater detail provided in Appendix F.

\subsubsection{Kill Chain Accuracy Model}

The Kill Chain Accuracy model was designed to simulate the accumulation of spatial and temporal error from "sensor" to "shooter". A target position may be determined from a land-based sensor, such a laser range finder, which is then transmitted through a series of systems via interconnecting data links to the weapon delivery aircraft and potentially to the weapon itself, depending on its capability. The model allowed a fixed position to be defined which was input into a sensor model module, introducing typical sensor errors. Each successive system was represented by a separate module, with each adding further errors either assumed or derived from available unclassified specifications. The model was implemented as a discrete event simulation using Simulink. The top-level view of the Simulink model is shown below in Figure 54. The model was executed over 5000 iterations (i.e. a Monte Carlo simulation), where the output consisting of $x, y$ co-ordinates and latency was written to the Simulink/MATLAB ${ }^{\circledR}$ workspace allowing it to be manually copied into an Excel spreadsheet, and where the distance of the position from target "truth" was calculated for each. Finally, the results were copied to a text file and formatted such that the Netica BBN application could "learn" from it. 


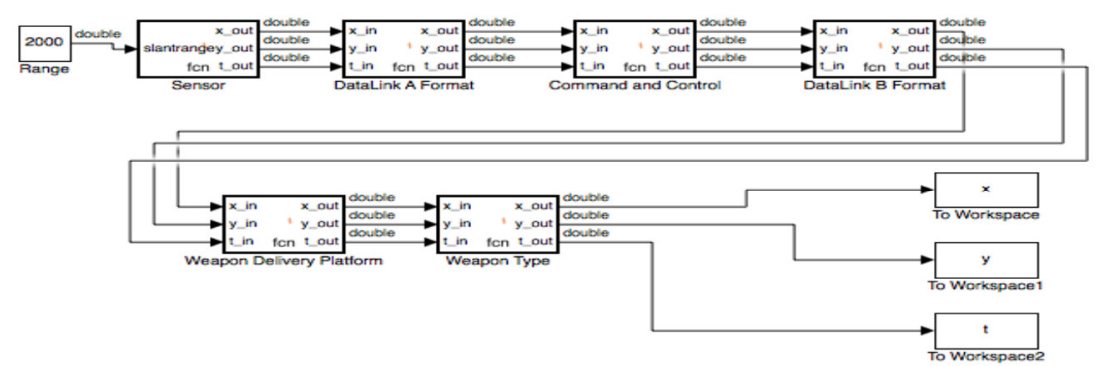

Figure 54 - Kill Chain Error Simulink Model

\subsubsection{Interoperability Model}

The Interoperability System Dynamics model represented overall levels of IO throughout the SoS from a generalised perspective, considering aggregated behaviour rather than modelling individual information exchanges, the model itself is shown below in Figure 55 and was implemented using the Vensim ${ }^{\circledR}$ application. System Dynamics normally models causal effect over a period of time, however in this case a more unconventional approach was taken, where each time step was used to generate a different 10 "level" as a result of the randomness defined for the model variables. The model was run over 5000 time steps, thereby generating a suitably sized result set which was reformatted into textual format and then input, i.e. learned, by the BBNs.

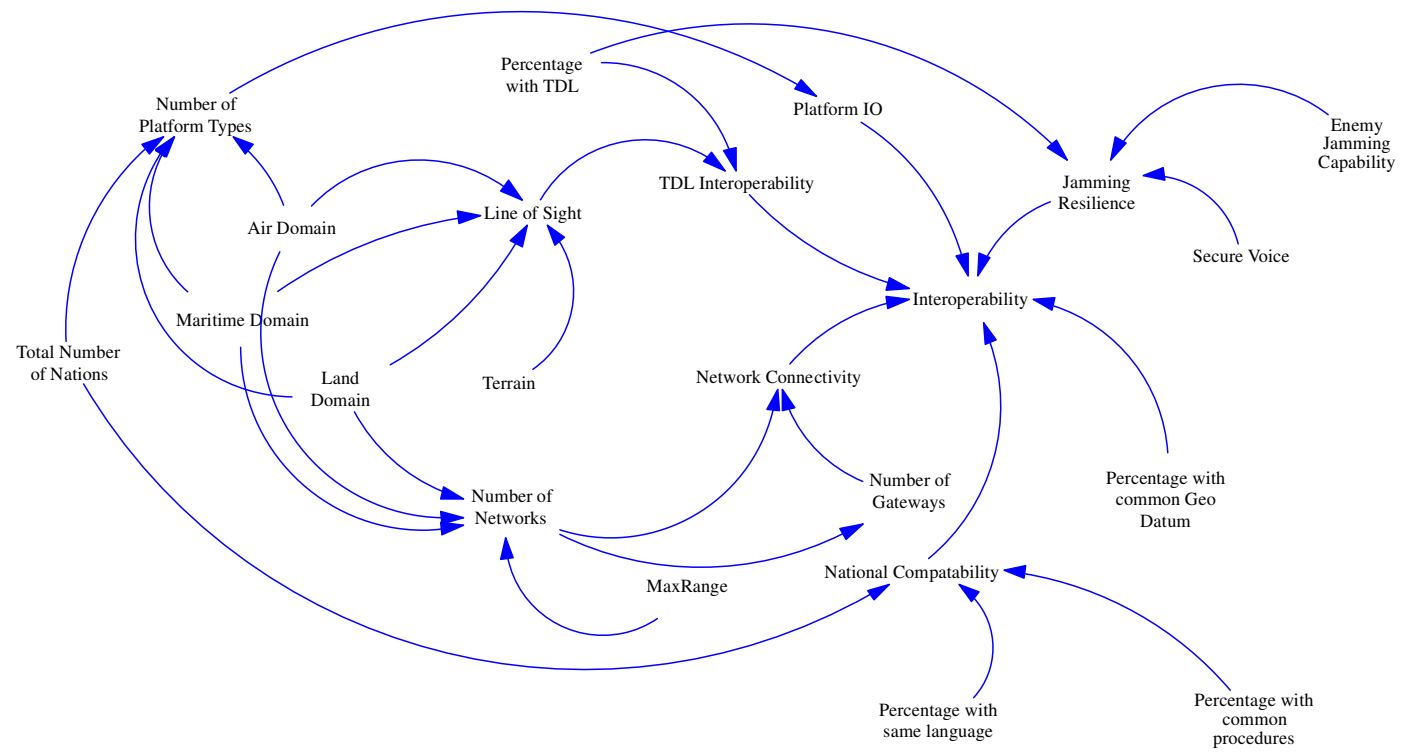

Figure 55 - Interoperability Model 


\subsubsection{Blue Force Proximity and Civilian Proximity Model}

The agent based proximity model enabled the movement of a target, blue forces and civilians to be simulated. Based on intelligence data, the model could be initialised with predicted positions within areas of uncertainty for forces and civilians, the motion of which could then be simulated if appropriate and with an element of randomness. The model was written in ReLogo, an ABMS language, using the Repast Simphony toolset; a screen shot of the initialised state is shown below in Figure 56. The model was run 1000 times with the output data exported into text files for BBN learning. It should be noted that the number of model iteration was limited to 1000 because higher numbers appeared to inhibit the creation of output data files.

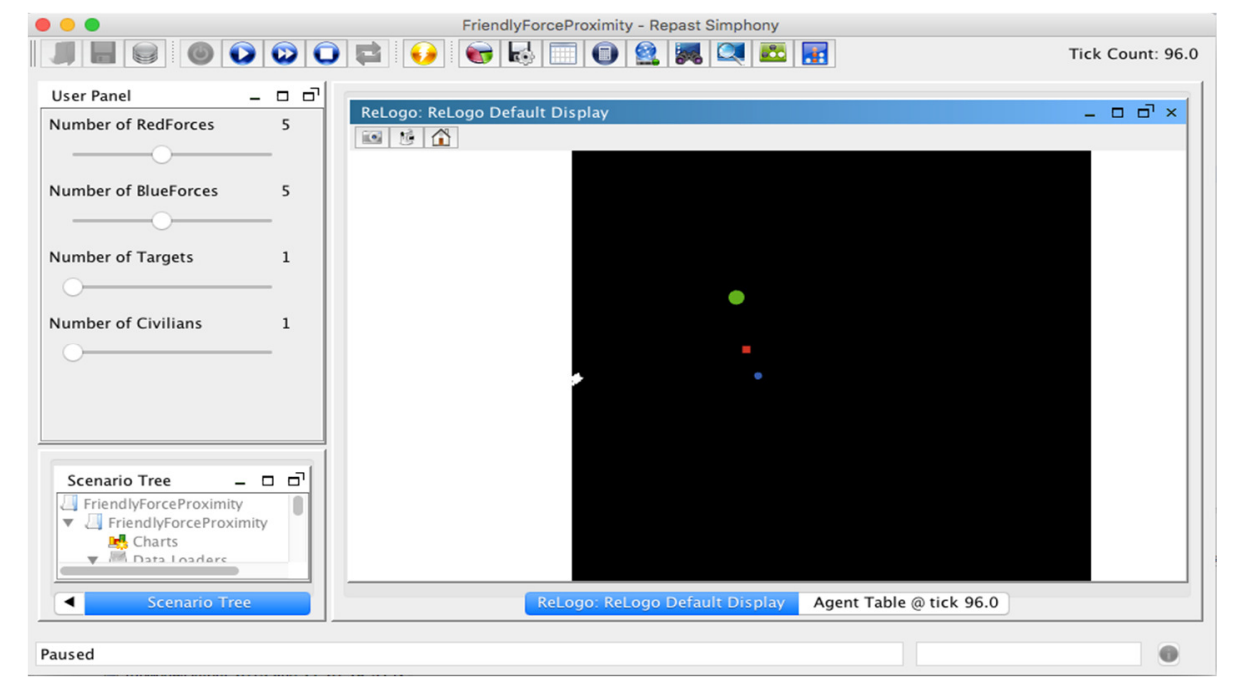

Figure 56 - Blue Force and Civilian Proximity Model

\subsection{Verification and Validation}

The application case study was broken down into the following components in order to logically structure Verification and Validation activities; Risk Identification, Causal Network, BBNs, Supporting Models, Confidence BBNs and Risk Assessment Output. The researcher undertook all verification activities, whilst validation was based upon elicitation of SME input.

\subsubsection{Verification Procedures}

The procedure for verification of each element of the application case study is provided below in tabular form. It should be noted that the level of verification was in accordance with the "demonstration of concept" approach, it is acknowledged that a fully developed solution would require substantially greater verification effort. 


\begin{tabular}{|c|c|c|}
\hline \multicolumn{3}{|c|}{ Verification Procedure - Risk Identification } \\
\hline Step & Action & Result \\
\hline 1 & $\begin{array}{l}\text { Manually check risks identified in Table } 22 \text { include those } \\
\text { discussed with SME. }\end{array}$ & Confirmed, all included \\
\hline 2 & $\begin{array}{l}\text { Manually check risks identified in Table } 22 \text { include those } \\
\text { derived from NATO Tactics, Techniques and Procedures } \\
\text { for Close Air Support Operations (NATO, 2005). }\end{array}$ & Confirmed, all included \\
\hline 3 & $\begin{array}{l}\text { Manually check Table } 22 \text { and confirm any risks not } \\
\text { identified in steps } 1 \text { and } 2 \text { are valid. }\end{array}$ & $\begin{array}{l}\text { Interoperability } \\
\text { risks/contributing factors } \\
\text { are additional and valid }\end{array}$ \\
\hline 4 & $\begin{array}{l}\text { Manually check Table } 22 \text { and confirm all SoS dimensions } \\
\text { (as defined in section 6.1) are included. }\end{array}$ & Confirmed, all included \\
\hline 5 & $\begin{array}{l}\text { Manually check Table } 22 \text { and confirm risks align } \\
\text { correctly with SoS Sol dimensions. }\end{array}$ & $\begin{array}{l}\text { Confirmed, aligns with } \\
\text { dimensions }\end{array}$ \\
\hline
\end{tabular}

Table 26 - Verification Procedure - Fratricide Risk Identification

\begin{tabular}{|c|c|c|}
\hline \multicolumn{3}{|c|}{ Verification Procedure - Causal Network } \\
\hline Step & Action & Result \\
\hline 1 & $\begin{array}{l}\text { Manually check all risks and contributing factors are } \\
\text { included in the causal network as defined in Table } 22 \text {. }\end{array}$ & $\begin{array}{l}\text { Factors relating to } \\
\text { Lifecycle, Classification } \\
\text { and Nature of } \\
\text { Relationships are not } \\
\text { included. Justified } \\
\text { because of their generic } \\
\text { nature. }\end{array}$ \\
\hline 2 & $\begin{array}{l}\text { Manually check the causal network as defined in Figure } \\
38 \text { to identify risks or contributing factors that are not } \\
\text { defined in Table } 22 \text {. }\end{array}$ & \begin{tabular}{|lrr}
$\begin{array}{l}\text { Confirmed } \\
\text { reflects }\end{array}$ risks & network \\
contributing & and \\
identified & factors
\end{tabular} \\
\hline 3 & $\begin{array}{l}\text { Check that there are no unconnected nodes in the } \\
\text { causal network, as shown in Figure } 38 .\end{array}$ & $\begin{array}{l}\text { Confirmed that all nodes } \\
\text { are connected }\end{array}$ \\
\hline 4 & $\begin{array}{l}\text { Confirm that connections within the causal network are } \\
\text { appropriate and logically correct. }\end{array}$ & $\begin{array}{l}\text { Confirmed all connections } \\
\text { correct }\end{array}$ \\
\hline 5 & $\begin{array}{l}\text { Manually check the Fratricide causal network, as } \\
\text { defined in Figure } 41 \text { and confirm it includes the } \\
\text { Fratricide Risk and all contributing factors connected to } \\
\text { it, as defined in Figure } 38 \text {. }\end{array}$ & $\begin{array}{l}\text { Confirmed network is } \\
\text { complete }\end{array}$ \\
\hline
\end{tabular}

Table 27 - Verification Procedure - Fratricide Causal Network 


\begin{tabular}{|c|c|c|}
\hline \multicolumn{3}{|c|}{ Verification Procedure - Fratricide BBN } \\
\hline Step & Action & Result \\
\hline 1 & $\begin{array}{l}\text { Using the Netica application, open the Fratricide BBN } \\
\text { (as shown in Figure } 38 \text { ). }\end{array}$ & Fratricide BBN displayed \\
\hline 2 & $\begin{array}{l}\text { Manually check the Fratricide BBN and confirm all } \\
\text { nodes defined in the causal network (Figure } 38 \text { ) are } \\
\text { included. }\end{array}$ & $\begin{array}{l}\text { Confirmed all nodes are } \\
\text { present }\end{array}$ \\
\hline 3 & $\begin{array}{l}\text { Manually check the Fratricide BBN and confirm that } \\
\text { additional nodes (not defined in Figure } 38 \text { ) are valid } \\
\text { divorcing nodes. }\end{array}$ & $\begin{array}{l}\text { Confirmed all additional } \\
\text { nodes are valid }\end{array}$ \\
\hline 4 & $\begin{array}{l}\text { Manually check each variable within the Fratricide } \\
\text { BBN and confirm that the states match those defined } \\
\text { in Table } 23 .\end{array}$ & $\begin{array}{l}\text { Kill chain did not match. } \\
\text { Table corrected. Confirmed } \\
\text { all correct }\end{array}$ \\
\hline 5 & $\begin{array}{l}\text { Check all parent nodes in the Fratricide BBN have } \\
\text { correctly defined conditional probability tables. }\end{array}$ & Confirmed tables correct \\
\hline 6 & $\begin{array}{l}\text { For all "edge" variables enter evidence, setting each } \\
\text { state in turn to } 100 \% \text { probability and confirming } \\
\text { probability changes are propagated throughout the } \\
\text { network as expected. }\end{array}$ & $\begin{array}{l}\text { Errors found } \text { were } \\
\text { corrected and step re-run, } \\
\text { confirming } \\
\text { propagation }\end{array}$ \\
\hline 7 & $\begin{array}{l}\text { Enter the "worst case" scenario evidence as shown in } \\
\text { Figure } 44 \text {, confirming probability changes are } \\
\text { propagated throughout the network as expected. }\end{array}$ & $\begin{array}{l}\text { Worst case BBN results as } \\
\text { expected }\end{array}$ \\
\hline 8 & $\begin{array}{l}\text { Enter the "best case" scenario evidence as shown in } \\
\text { Figure } 45 \text {, confirming probability changes are } \\
\text { propagated throughout the network as expected. }\end{array}$ & $\begin{array}{l}\text { Best case BBN results as } \\
\text { expected }\end{array}$ \\
\hline 9 & $\begin{array}{l}\text { Select the "Learn" option, then select the Kill Chain } \\
\text { Accuracy Model Output file; ensure the Kill Chain } \\
\text { Accuracy variable in the BBN is updated accordingly. }\end{array}$ & BBN updated as expected \\
\hline 10 & $\begin{array}{l}\text { Select the "Learn" option, then select the } \\
\text { Interoperability Model Output file; ensure the } \\
\text { Interoperability variable in the BBN is updated } \\
\text { accordingly. }\end{array}$ & BBN updated as expected \\
\hline 11 & $\begin{array}{l}\text { Select the "Learn" option, then select the Proximity } \\
\text { Model, Blue Force Proximity Output file; ensure the } \\
\text { Blue Force Proximity variable in the BBN is updated } \\
\text { accordingly. }\end{array}$ & BBN updated as expected \\
\hline
\end{tabular}

Table 28 - Verification Procedure - Fratricide BBN 


\begin{tabular}{|l|l|l|}
\hline \multicolumn{3}{|l|}{ Verification Procedure - Supporting Models: Kill Chain Accuracy } \\
\hline Step & Action & Result \\
\hline 1 & $\begin{array}{l}\text { Open the Kill Chain Accuracy model using the MATLAB } \\
\text { application. }\end{array}$ & Kill chain model opened \\
\hline 2 & $\begin{array}{l}\text { View the Simulink model and confirm all related } \\
\text { elements identified in the causal network (Figure 38) } \\
\text { are represented. }\end{array}$ & $\begin{array}{l}\text { Kill chain Simulink model } \\
\text { displayed }\end{array}$ \\
\hline 3 & $\begin{array}{l}\text { Perform a visual inspection of the code within each } \\
\text { Simulink element to confirm logical correctness. }\end{array}$ & $\begin{array}{l}\text { Errors rectified; final } \\
\text { version of code correct }\end{array}$ \\
\hline 4 & $\begin{array}{l}\text { Run the model with inputs between 0 and 100000 and } \\
\text { check output from each element is in accordance with } \\
\text { expected output. }\end{array}$ & Outputs as expected \\
\hline 5 & $\begin{array}{l}\text { Run the model, with input range set to 2000, over 5000 } \\
\text { iterations and confirm data is output into result matrix. }\end{array}$ & $\begin{array}{l}\text { Output dataset } \\
\text { successfully created }\end{array}$ \\
\hline 6 & Confirm result matrix contains 5000 entries. & 5000 entries confirmed \\
\hline 7 & $\begin{array}{l}\text { Export result matrix into Excel and confirm spread of } \\
\text { results around target position is as expected. }\end{array}$ & $\begin{array}{l}\text { Spread of results as } \\
\text { expected }\end{array}$ \\
\hline
\end{tabular}

Table 29 - Verification Procedure - Supporting Models: Kill Chain Accuracy

\begin{tabular}{|c|c|c|}
\hline \multicolumn{3}{|c|}{ Verification Procedure - Supporting Models: Interoperability } \\
\hline Step & Action & Result \\
\hline 1 & $\begin{array}{l}\text { Open the Interoperability model using the Vensim } \\
\text { application. }\end{array}$ & Model displayed \\
\hline 2 & $\begin{array}{l}\text { View the System Dynamics model and confirm all related } \\
\text { elements identified in the causal network (Figure 38) are } \\
\text { represented. }\end{array}$ & $\begin{array}{l}\text { Confirmed, all } \\
\text { represented }\end{array}$ \\
\hline 3 & $\begin{array}{l}\text { Perform a visual inspection of the code within each } \\
\text { variable to confirm logical correctness. }\end{array}$ & $\begin{array}{l}\text { Model refined, final } \\
\text { version confirmed to be } \\
\text { accepted }\end{array}$ \\
\hline 4 & $\begin{array}{l}\text { Perform a visual inspection of the causal links in the } \\
\text { model to confirm logical correctness. }\end{array}$ & $\begin{array}{l}\text { Model refined, final } \\
\text { version confirmed to be } \\
\text { accepted }\end{array}$ \\
\hline 5 & $\begin{array}{l}\text { Enter "worst case" values, which would result in poor } \\
\text { interoperability and confirm model output reflects this. }\end{array}$ & $\begin{array}{l}\text { Initial test resulted in } \\
\text { further refinement, } \\
\text { verification tests re-run, } \\
\text { final version confirmed } \\
\text { to be correct }\end{array}$ \\
\hline 6 & $\begin{array}{l}\text { Enter "best case" values, which would result in poor } \\
\text { interoperability and confirm model output reflects this. }\end{array}$ & Results as expected \\
\hline
\end{tabular}

Table 30 - Verification Procedure - Supporting Models: Interoperability 


\begin{tabular}{|c|c|c|}
\hline \multicolumn{3}{|c|}{ Verification Procedure - Supporting Models: Blue Force and Civilian Proximity } \\
\hline Step & Action & Result \\
\hline 1 & $\begin{array}{l}\text { Open the Proximity model using the Repast Symphony } \\
\text { application. }\end{array}$ & $\begin{array}{l}\text { Proximity mode } \\
\text { components displayed }\end{array}$ \\
\hline 2 & $\begin{array}{l}\text { Ensure agents are defined which represent blue forces, } \\
\text { civilians, target and weapon positions. }\end{array}$ & $\begin{array}{l}\text { Agent classes are } \\
\text { defined }\end{array}$ \\
\hline 3 & $\begin{array}{l}\text { Perform a visual inspection of the code within each agent } \\
\text { to confirm logical correctness. }\end{array}$ & Code appears correct \\
\hline 4 & $\begin{array}{l}\text { Perform a visual inspection of the UserObserver Class and } \\
\text { confirm all agents are correctly created and initialized. }\end{array}$ & Code appears correct \\
\hline 5 & $\begin{array}{l}\text { Initialise the model for a single run, ensure all agents are } \\
\text { displayed and can be distinguished from one another. }\end{array}$ & $\begin{array}{l}\text { All agents displayed, } \\
\text { differentiated by colour }\end{array}$ \\
\hline 6 & $\begin{array}{l}\text { Run the model and confirm that the agents movement is } \\
\text { as expected. }\end{array}$ & Movement as expected \\
\hline 7 & $\begin{array}{l}\text { Confirm that when the weapon "hits" the target the } \\
\text { agent movement is frozen. }\end{array}$ & $\begin{array}{l}\text { Model initially } \\
\text { corrected, and re- } \\
\text { verified as correct }\end{array}$ \\
\hline 8 & $\begin{array}{l}\text { Initialise and perform a single run of the model, confirm } \\
\text { the agent start positions and movement have changed } \\
\text { within the defined ranges. }\end{array}$ & $\begin{array}{l}\text { Start positions different } \\
\text { for each run }\end{array}$ \\
\hline 9 & $\begin{array}{l}\text { Initialise the model in batch mode and configure it for } \\
5000 \text { runs. }\end{array}$ & $\begin{array}{l}\text { Model set up as } \\
\text { required }\end{array}$ \\
\hline 10 & $\begin{array}{l}\text { Run the model and confirm } 5000 \text { output data sets are } \\
\text { created. }\end{array}$ & $\begin{array}{l}\text { Model completes run, } \\
\text { no data set created. } \\
\text { Reset to run } 1000 \text { times, } \\
\text { data set successfully } \\
\text { created. Accepted as } \\
\text { limitation of Repast. }\end{array}$ \\
\hline 11 & $\begin{array}{l}\text { Export the data sets into Excel and confirm that blue } \\
\text { force and civilian distances from the target are } \\
\text { distributed as expected. }\end{array}$ & Distribution as expected \\
\hline
\end{tabular}

Table 31 - Verification Procedure - Supporting Models: Blue Force and Civilian Proximity 


\begin{tabular}{|c|c|c|}
\hline \multicolumn{3}{|c|}{ Verification Procedure - Fratricide Risk Confidence BBN } \\
\hline Step & Action & Result \\
\hline 1 & $\begin{array}{l}\text { Using the Netica application, open the Fratricide Risk } \\
\text { Confidence BBN (as shown in Figure 48). }\end{array}$ & Fratricide BBN displayed \\
\hline 2 & $\begin{array}{l}\text { Manually check the Fratricide Confidence BBN and } \\
\text { confirm all nodes defined Fratricide BBN (Figure 38) are } \\
\text { included. }\end{array}$ & $\begin{array}{l}\text { Confirmed all nodes are } \\
\text { present }\end{array}$ \\
\hline 4 & $\begin{array}{l}\text { Manually check each variable within the Fratricide } \\
\text { Confidence BBN and confirm that the states for all are } \\
\text { Low, Medium and High }\end{array}$ & $\begin{array}{l}\text { Confirmed all states are } \\
\text { correct }\end{array}$ \\
\hline 5 & $\begin{array}{l}\text { Check all parent nodes in the Fratricide Confidence BBN } \\
\text { have correctly defined conditional probability tables. }\end{array}$ & Confirmed tables correct \\
\hline 6 & $\begin{array}{l}\text { For all "edge" variables enter evidence, setting each state } \\
\text { in turn to } 100 \% \text { probability and confirming probability } \\
\text { changes are propagated throughout the network as } \\
\text { expected. }\end{array}$ & $\begin{array}{l}\text { BBN updated as } \\
\text { expected }\end{array}$ \\
\hline 7 & $\begin{array}{l}\text { Enter the "worst case" evidence, where all edge variables } \\
\text { are set Low }=100 \% \text {, confirming probability changes are } \\
\text { propagated throughout the network as expected. }\end{array}$ & $\begin{array}{l}\text { Worst case results as } \\
\text { expected }\end{array}$ \\
\hline 8 & $\begin{array}{l}\text { Enter the "best case" scenario evidence, where all edge } \\
\text { variables are set High }=100 \% \text {, confirming probability } \\
\text { changes are propagated throughout the network as } \\
\text { expected. }\end{array}$ & $\begin{array}{l}\text { Best case BBN results as } \\
\text { expected }\end{array}$ \\
\hline
\end{tabular}

Table 32 - Verification Procedure - Fratricide Risk Confidence BBN

\subsubsection{Validation}

The approach taken to validate the application case study components was to elicit subject matter expertise through a semi-structured interview, which established the realism of the case study and the risks identified as well as the value of the proposed risk management approach within the SMEs domain.

The interview questions and responses were as follows:

1. Describe your experience and knowledge relating to Close Air Support mission planning and how this may be applicable to risk management.

Response: The SME stated that he is currently in a Navy reservist role seconded to the Joint Force Air Component, which is responsible for command and control of the planning of air missions including CAS. He has been involved with several live exercises involving a CAS element, one of which involved investigations into reducing kill chain length in order to increase accuracy and reduce latency. 
2. In the provided Close Air Support risk matrix, are all major risks and contributing factors identified? If not, which risks and factors are missing and is their consequence greater or less than those already identified?

Response: The SME agreed all identified were valid, although he stated that the risk of Aircraft Loss would exist at medium altitude if enemy air defences were highly capable. The researcher stated that the BBN does cater for this, but the risk identification should be aligned accordingly. Regarding the risk of inaccurate targeting the SME stated that the run-in direction has to be planned such that any friendlies are not likely to be down the bearing, thereby mitigating the risk. The SME noted the time to identify hostile elements and convey this information to the incoming aircraft would impact risk, although the researcher explained that the interoperability model was intended to reflect this, at least implicitly.

The "footprint" of the forces on the ground, both blue and red, was a factor influencing risk; the researcher agreed that future enhancements of the BBNs and proximity models should reflect this.

Night vision goggle illumination states were also identified as a possible future model enhancement, relating to target visibility.

The SME queried why target opportunity was identified as a key risk, interpreting "risk" as always having a detrimental impact. The researcher explained that risk could be interpreted as opportunity, which was accepted by the SME but did not find the concept intuitive.

3. In the provided causal network, are the links between contributing factors and risks reasonable and correct from your experience?

Response: The SME agreed that the causal network was reasonable and correct from his experience.

4. Do the risk matrix and causal network positively support the identification of risks within CAS planning? If not, why do they not support this task?

Response: The SME stated "yes" he regarded them as providing positive support.

5. Does the Fratricide BBN update as expected when evidence is entered?

Response: the researcher demonstrated propagation using the Fratricide BBN, the SME thought this was an effective way of modelling and demonstrating risk and that the results were appropriate and reasonable. 
6. Are the Kill Chain Accuracy, Interoperability and Proximity models sufficiently realistic, bearing mind they are intended to demonstrate a concept at this stage?

Response: the researcher described each supporting model; the SME agreed that these were appropriate and sufficiently realistic.

7. Is the Fratricide Risk analysis output provided in an understandable, meaningful and usable format?

Response: the SME required further explanation of the best and worst case output and was satisfied that the output made sense, however it was agreed this output would require presenting in a more intuitive format in any exploited solution, e.g. a "traffic light" visual display.

8. Do you think the SoS Risk Management process and its outputs could provide benefit to CAS mission planning activities? If so, how and where?

Response: The SME stated, "yes" provided the risk level and consequence were accurate. The researcher explained that the associated confidence level should support interpretation of the results, the SME agreed.

9. Bearing in mind that this approach is currently a concept, do you see any limitations of the approach?

Response: The SME stated that time to perform the analysis would be a limitation in the CAS context. The researcher agreed and stated that the outputs could be used as the basis for decision support tool enabling rapid risk assessment. The SME thought this would be of great benefit, particularly if integrated into a hand-held device enabling near real tine risk assessment in operational environments.

\subsection{Discussion}

The SoS Sol dimensions provided an effective framework, enabling the CAS mission to be viewed from a SoS perspective. Extending this framework into a risk identification matrix encouraged risks to be considered in the context of the SoS rather than at the individual system level. Although some risks could be identified without need for the SoS framework, it was still found to be of benefit in determining risk relationship to the SoS and distinguishing between SoS and systems risks.

The model technique selection tool, was key in identifying appropriate methods, however the population of the spreadsheet was not a purely mechanistic activity requiring a decision to determine general modelling approach required to support the risk analysis activity. Model choice was also driven by practitioner knowledge and potential re-use, which could reduce development effort. 
A Model Based Approach to System of Systems Risk Management

The causal network was the foundation for all subsequent analysis. Extracting a subset of the causal network for each risk was initially effective, simplifying the required modelling and thought processes. However, this approach ultimately resulted in duplication of effort when producing BBNs, where risks were linked to common elements within the causal network.

The descriptions of BBN variables were essential in eliciting SME input, providing context for the population of the conditional probability tables, evidence input and supporting model learning. The worst, best case risk comparison provided context for the analysis results, without which it was difficult to meaningfully interpret them. Hence the interpretation was based on comparison of the extreme values but still required subjective judgement.

Finally, the use of an equivalent BBN proved to be an effective method to determine risk confidence. Although the population of it was based on subjective input, it allowed elicitation of practitioner input and allowed both the evidence and supporting model output to be assessed. 


\section{Chapter 9: Application 2: UK National Food Security - Wheat Supply Chain}

This chapter applies the model-based SoS Risk Management process in the context of UK National food security and specifically the supply of wheat based food products, with the intention of demonstrating the adaptability of process within a domain, which is markedly different from the first application case study.

In contrast to the CAS case study, UK Food security provided a more enterprise SoS based example, allowing the flexibility of the approach to be tested, the UK Met Office and a supply chain specialist provided subject matter expertise.

\subsection{Background}

Food security exists "when all people, at all times, have physical and economic access to sufficient, safe and nutritious food to meet their dietary needs and food preferences for an active and healthy life" (FAO, 1996).

Although there are several levels of food security: household, regional, national and global (DEFRA, 2006), this case study focused on national food security and specifically the supply of wheat based foodstuffs; expert advice was provided by the UK Met Office. The concentration on wheat supply enabled the scope of this case study to be maintained at a manageable level whilst still demonstrating the adaptability of the model based approach.

The Department for Environment Food and Rural Affairs (DEFRA) identified several key themes, which influence food security (DEFRA, 2006):

- Availability

- Access

- Affordability

- Nutrition and quality

- Safety

- Resilience

- Confidence

Although the UK's food security level is generally high, global pressures on food production, such as population growth and climate change, are increasing, leading the Environment, Food and Rural Affairs Committee to state "Ensuring that all UK citizens have access to sufficient healthy and safe food at an affordable price is therefore a challenge which is likely 
to become more acute in the future unless action is taken" (Environment Food and Rural Affairs Committee, 2015). Hence, an alternative approach providing the ability to manage risk related to food security could potentially be of great benefit.

Food is delivered through a supply chain, which can be defined as "a sequence of (decisionmaking and execution) processes and (material, information and money) flows that aim to meet final customer requirements, that take place within and between different stages along a continuum, from production to final consumption" and is illustrated below in Figure $\mathbf{5 7}$ (Van Der Vorst et al., 2007).

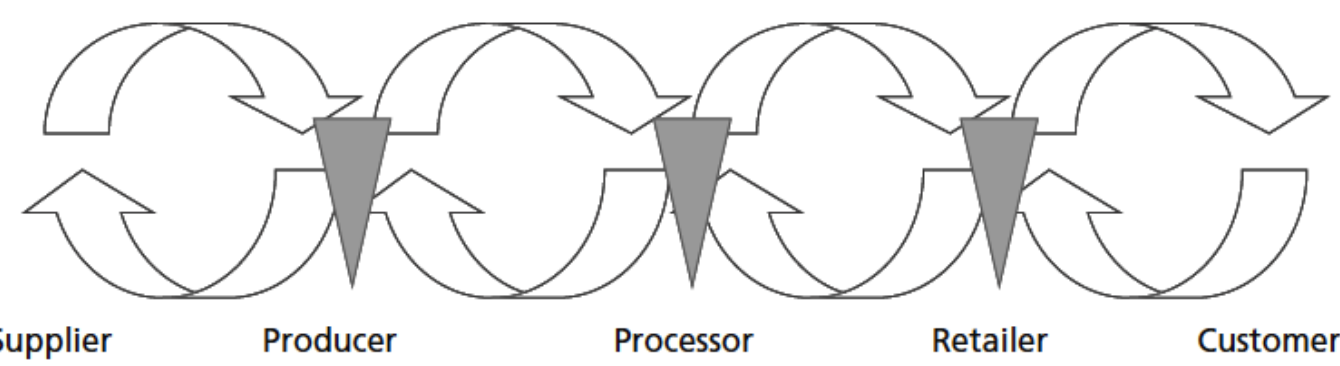

Figure 57 - Supply Chain (Van Der Vorst et al., 2007)

Hipel et al (2010) state that "agriculture is essentially the management of a secure food supply" and may be viewed as a SoS which "exploits environmental systems, but also utilizes large-scale industrial systems and international trade systems", they illustrate the relationship between these systems in Figure 58. The argument that a SoS perspective is appropriate is supported by both Maiers (1998) definition, identifying managerial and operational independence of the component systems and Brooks (2016) wider definition citing coupling of the component systems as some point in their lifecycle.

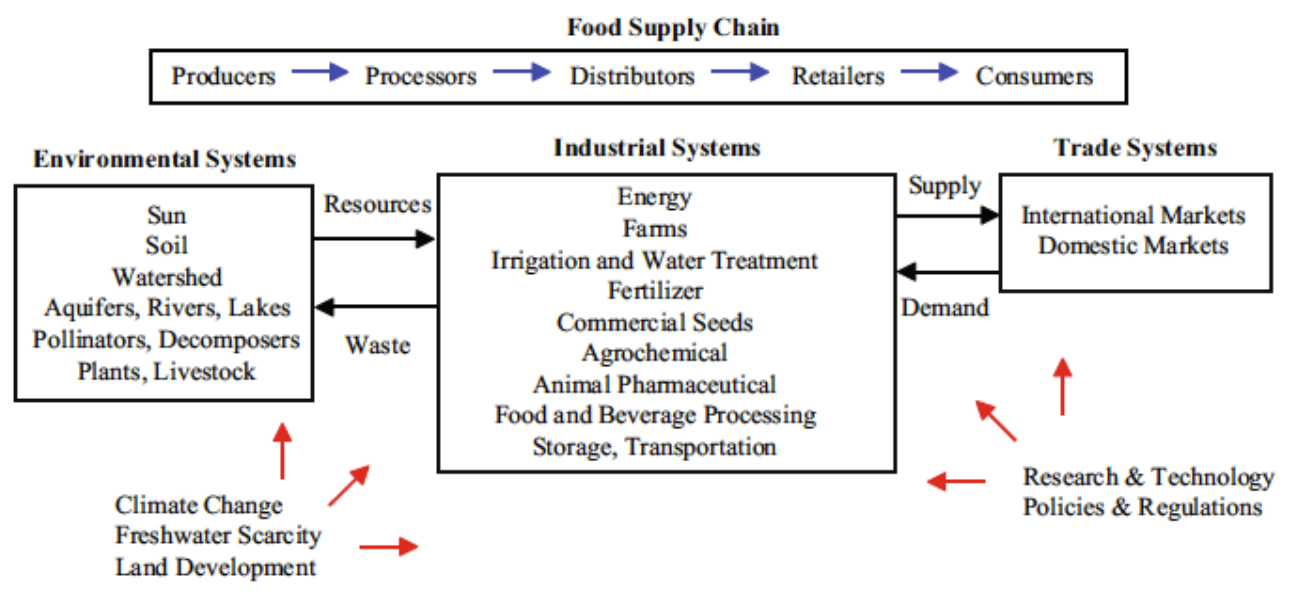

Figure 58 - Global Food System of Systems (Hipel et al., 2010) 
Initial discussions with the Met Office highlighted the following issues that were considered pertinent in the context of this case study:

- Storage of grain from previous years would impact the stock levels, not just current yearly yield.

- Disease is related to climate; hence weather patterns will affect this although not necessarily extreme weather. However extreme weather should be considered as opposed to unusual variations in the weather.

- Stock information is an issue; in the West the market drives this but figures are not exact, estimate $10-20 \%$ held as stock. In contrast China, for example, the government has control of stocks where up to $80 \%$ of the yield is stored to protect against economic fluctuations.

- Long term modelling considers mean production output; contributing factors include the adoption of enhancing productivity technology and population level.

- Rather than climate data, production data may be more pertinent, specifically consider the following:

- Yield is weather related.

- The Area harvested is related to previous prices, whereby farmers will sow less if less return is expected.

- Yield gaps will have a dramatic effect on production.

- The Met Office were specifically interested in determining key factors influencing wheat price, for example climate change and its actual impact as opposed to general perception. It was stated that determining orders of magnitude of factors would be of great benefit.

- It was stated that climate models currently exist and could potentially be referenced as supporting models. This highlighted a key point relating to the model based process, whereby re-use of available models should always be considered when identifying necessary supporting models. 
A Model Based Approach to System of Systems Risk Management

Specifically considering risk in agriculture, Musser and Patrick (2002) identified the following sources:

- Production risk: variations in crop yields due to weather, disease and pests.

Potential mitigation includes diversification of crop type, irrigation, pesticide, fertilizer, soil testing and crop insurance.

- Marketing risk: variations in commodity prices and demand which may be mitigated by marketing contracts (fixing price and quantity), direct sales or forwarding marketing techniques (e.g. futures, options).

- Financial risk: cash flow enabling bill payment, funds to cover day to day farming costs and bankruptcy, with mitigations including financial record keeping, cash flow planning, maintaining financial reserves and investments.

- Legal and environmental risk: lawsuits initiated by companies or individuals, contractual disputes, changes in government regulations, which may be mitigated by liability insurance, legal representation and machinery maintenance.

- Human Resources risk: the threat that owners, family or employees will not be available for labour and management activities which may be mitigated by business and succession planning, health and disability insurance and sound human resource management.

Considering risk more generically across entire food supply chain the following were identified below in Table 33 (Diabat et al., 2012). 


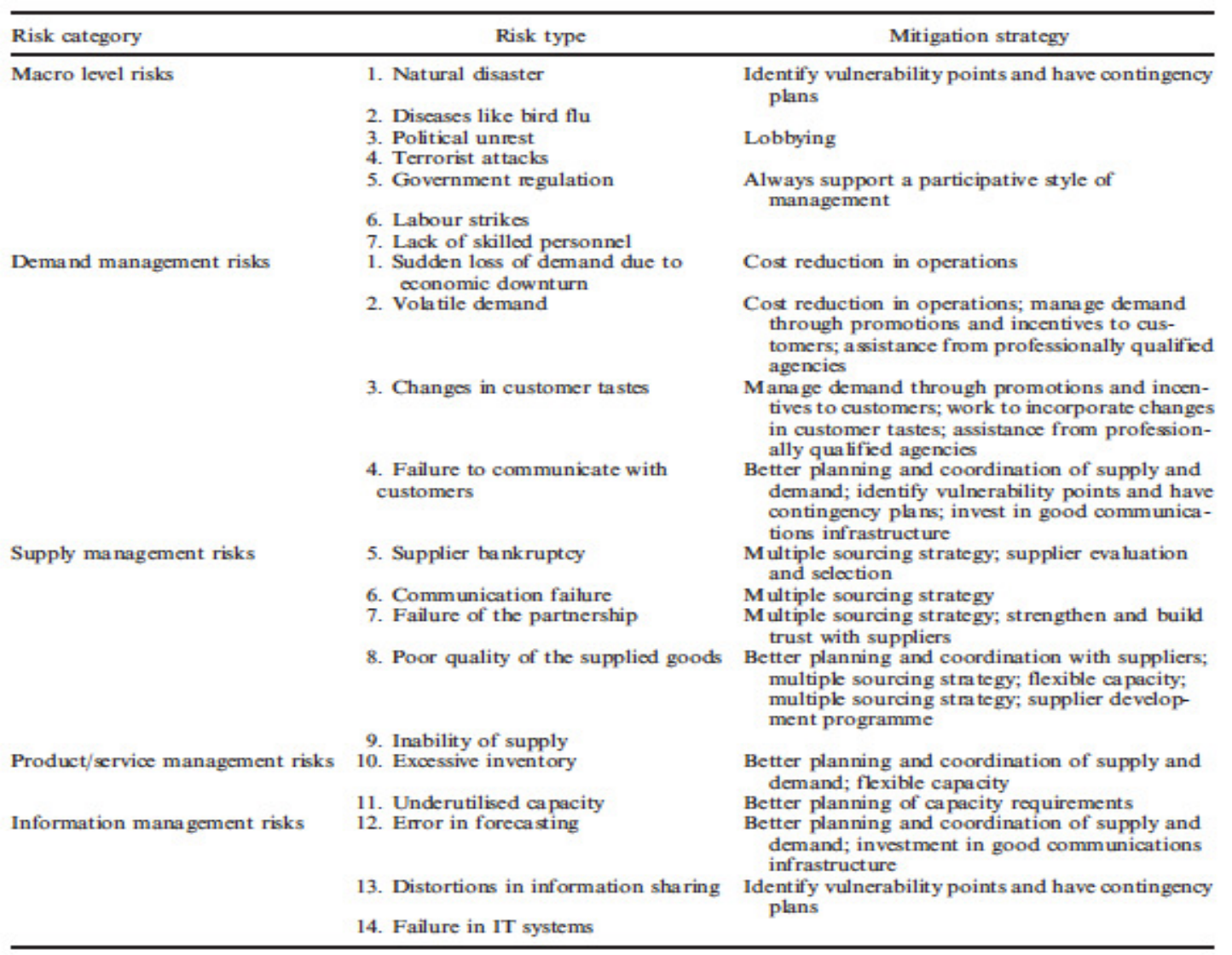

Table 33 - Food Supply Chain Risk Summary (Diabat et al., 2012)

\subsection{Application of Process}

Having established that the UK wheat supply chain could legitimately be viewed as a SoS, the process described in section 7.3 was applied in the context of this case study. The first case study was intended to demonstrate detailed application of the process with the second study showing the adaptability of the process. Hence, the scope of this second application was reduced accordingly, not including support model implementation and with only one risk fully analysed, although all were identified. In accordance with the reduction in scope the description of the analysis is also restricted to key points, whereas the first case study included sufficient detail to demonstrate the risk management process. It should also be noted that the risk confidence analysis was also omitted from this second application having been sufficiently demonstrated under the CAS case study.

\subsection{Planning}

In this case risk planning at the SoS level would typically be undertaken by government agencies, such as DEFRA, through policy and incentive initiatives and, to some extent, through contractual agreement between component systems within the supply chain. Planning would also need to consider both long and short-term risk management. 
A Model Based Approach to System of Systems Risk Management

\subsection{Identification}

The risk identification matrix, as shown below in Table 34, was populated through SME consultation and consultation of the material referenced above in section 9.

\begin{tabular}{|c|c|c|c|c|}
\hline SoS Dimension & Instantiation & Hazard & Control & Opportunity \\
\hline \multirow[t]{4}{*}{ Component Systems } & Supplier (seed, fertilizer) & $\begin{array}{l}\text { Business failure } \\
\text { High Cost } \\
\text { Low supply }\end{array}$ & $\begin{array}{l}\text { Planned supply does not } \\
\text { meet demand } \\
\text { Reliance on small number of } \\
\text { suppliers }\end{array}$ & $\begin{array}{l}\text { GM seed development, } \\
\text { increases yield } \\
\text { Agri-chemical advance } \\
\text { improves effectiveness }\end{array}$ \\
\hline & Farm & $\begin{array}{l}\text { Business failure, bankruptcy } \\
\text { impact if supply base not } \\
\text { broad }\end{array}$ & $\begin{array}{l}\text { Government regulation } \\
\text { increases costs } \\
\text { Labour availability related to } \\
\text { national workforce and } \\
\text { immigration level }\end{array}$ & $\begin{array}{l}\text { Increased demand } \\
\text { New technology increases } \\
\text { efficiency } \\
\text { Diversification } \\
\text { Government regulation } \\
\text { reduces costs }\end{array}$ \\
\hline & Distributor & $\begin{array}{l}\text { Fuel supply interruption } \\
\text { Industrial action }\end{array}$ & $\begin{array}{l}\text { Fuel costs increase (oil price } \\
\text { and tax) }\end{array}$ & $\begin{array}{l}\text { Fuel costs decrease (oil price } \\
\text { and tax) }\end{array}$ \\
\hline & Manufacturer (industrial bakery) & $\begin{array}{l}\text { Industrial action } \\
\text { Business failure }\end{array}$ & $\begin{array}{l}\text { Labour cost increase } \\
\text { Over production } \\
\text { Drop in demand } \\
\text { Raw materials cost increase }\end{array}$ & $\begin{array}{l}\text { Labour cost decrease, } \\
\text { possibly through technology } \\
\text { Demand increase }\end{array}$ \\
\hline
\end{tabular}


A Model Based Approach to System of Systems Risk Management

\begin{tabular}{|c|c|c|c|c|}
\hline SoS Dimension & Instantiation & Hazard & Control & Opportunity \\
\hline & Processor (milling) & Industrial action & $\begin{array}{l}\text { Labour cost increase } \\
\text { Over production } \\
\text { Drop in demand } \\
\text { Raw materials cost increase } \\
\text { Poor quality }\end{array}$ & $\begin{array}{l}\text { Labour cost decrease, } \\
\text { possibly through technology } \\
\text { Demand increase }\end{array}$ \\
\hline & Storage & Stock level exhausted & $\begin{array}{l}\text { Stock level does not } \\
\text { compensate for low yield }\end{array}$ & $\begin{array}{l}\text { Excess stock level supplies } \\
\text { additional market }\end{array}$ \\
\hline & $\begin{array}{l}\text { Retailer (supermarket chains which } \\
\text { are SoS and individual small scale } \\
\text { retailers) }\end{array}$ & Business failure & & $\begin{array}{l}\text { Competition reduces cost of } \\
\text { end product }\end{array}$ \\
\hline & $\begin{array}{l}\text { Water Supply (national which is a } \\
\text { SoS and local irrigation systems) }\end{array}$ & $\begin{array}{l}\text { Drought } \\
\text { Supply contamination } \\
\text { Flooding }\end{array}$ & Water Storage capacity & \\
\hline & $\begin{array}{l}\text { Energy Supply (national grid (SoS) } \\
\text { and local e.g. wind turbine)) }\end{array}$ & $\begin{array}{l}\text { Power grid failure } \\
\text { Cyber attack effecting power } \\
\text { grid } \\
\text { Terrorist attack }\end{array}$ & $\begin{array}{l}\text { High energy prices increases } \\
\text { costs } \\
\text { High oil price increases } \\
\text { energy costs }\end{array}$ & $\begin{array}{l}\text { Low price reduces costs } \\
\text { Local green power source } \\
\text { provides additional energy }\end{array}$ \\
\hline Interactions & $\begin{array}{l}\text { Currency (flow of payments } \\
\text { between food chain components) }\end{array}$ & $\begin{array}{l}\text { Business cash flow problem } \\
\text { Cyber attack }\end{array}$ & $\begin{array}{l}\text { Unfavourable exchange rate } \\
\text { increases costs } \\
\text { Government introduces or } \\
\text { increases tax/levy on } \\
\text { transactions } \\
\text { Economic downturn }\end{array}$ & Favourable exchange rate \\
\hline
\end{tabular}


A Model Based Approach to System of Systems Risk Management

\begin{tabular}{|c|c|c|c|c|}
\hline SoS Dimension & Instantiation & Hazard & Control & Opportunity \\
\hline & $\begin{array}{l}\text { Transport (Wheat, Feed) - although } \\
\text { this is a SoS itself }\end{array}$ & $\begin{array}{l}\text { Industrial action } \\
\text { Fuel shortage }\end{array}$ & High oil price increases costs & Low oil price reduces costs \\
\hline & $\begin{array}{l}\text { Order (purchase agreements or } \\
\text { contracts between food chain } \\
\text { components) }\end{array}$ & & $\begin{array}{l}\text { Administration error } \\
\text { Failure to communicate } \\
\text { between producers, suppliers } \\
\text { and retailers in terms of } \\
\text { planning }\end{array}$ & \\
\hline & $\begin{array}{ll}\text { Internet - } & \text { supports } \\
\text { communications } & \text { between } \\
\text { component systems } & \end{array}$ & Cyber attack & $\begin{array}{l}\text { Interoperability between } \\
\text { component systems poor }\end{array}$ & $\begin{array}{l}\text { Increased networking } \\
\text { improves efficiency }\end{array}$ \\
\hline & Raw materials - wheat, flour & Supply failure & Increased costs & Reduced costs \\
\hline & End Product e.g. bread & Supply failure & Increased costs & Reduced costs \\
\hline Variability & $\begin{array}{l}\text { Low (the supply chain is relatively } \\
\text { stable over time, not subject to } \\
\text { frequent rapid changes) }\end{array}$ & $\begin{array}{l}\text { Sudden change may disrupt } \\
\text { supply chain SoS }\end{array}$ & & $\begin{array}{l}\text { Stable SoS allows efficiencies } \\
\text { to be maximised, reducing } \\
\text { costs }\end{array}$ \\
\hline Classification & $\begin{array}{l}\text { Generally Collaborative, with } \\
\text { acknowledged and directed } \\
\text { elements. Some component } \\
\text { systems are SoS their own right }\end{array}$ & & $\begin{array}{l}\text { Incorrect classification of } \\
\text { entire SoS and/or subgroups } \\
\text { of component systems } \\
\text { leading to incorrect } \\
\text { management/engineering } \\
\text { approach }\end{array}$ & \\
\hline Functions & Seed production & Disease & Seed supply does not meet & Surplus seed supply reduces \\
\hline
\end{tabular}


A Model Based Approach to System of Systems Risk Management

\begin{tabular}{|c|c|c|c|c|}
\hline SoS Dimension & Instantiation & Hazard & Control & Opportunity \\
\hline & & & $\begin{array}{l}\text { demand } \\
\text { Seed cost increase }\end{array}$ & costs \\
\hline & Sow & $\begin{array}{l}\text { Extreme weather delays or } \\
\text { prevents sowing }\end{array}$ & $\begin{array}{l}\text { Co-ordinating of sowing does } \\
\text { not provide an even supply }\end{array}$ & \\
\hline & Harvest & $\begin{array}{l}\text { Extreme weather delays } \\
\text { harvest } \\
\text { Extreme weather results in } \\
\text { crop failure }\end{array}$ & $\begin{array}{l}\text { Weather allows a small } \\
\text { window for harvesting } \\
\text { resulting in lack of availability } \\
\text { of resources (e.g. mechanical } \\
\text { harvesters) }\end{array}$ & $\begin{array}{l}\text { Favourable weather during } \\
\text { harvesting maximises yield } \\
\text { Export if excess yield }\end{array}$ \\
\hline & Process (wheat) & Wheat supply failure & $\begin{array}{l}\text { Demand cannot be met } \\
\text { Overproduction }\end{array}$ & $\begin{array}{l}\text { Technical advance in process } \\
\text { improves efficiency }\end{array}$ \\
\hline & Produce (end product) & Supply failure & Change in consumer taste & $\begin{array}{l}\text { Technical advance in process } \\
\text { improves efficiency }\end{array}$ \\
\hline & Sell (end product) & Product supply failure & $\begin{array}{l}\text { Change in consumer taste } \\
\text { Price war reduces consumer } \\
\text { choice (through failure of } \\
\text { smaller retailers) }\end{array}$ & $\begin{array}{l}\text { Competition reduces price } \\
\text { Reduced costs allows lower } \\
\text { price }\end{array}$ \\
\hline
\end{tabular}


A Model Based Approach to System of Systems Risk Management

\begin{tabular}{|c|c|c|c|c|}
\hline SoS Dimension & Instantiation & Hazard & Control & Opportunity \\
\hline \multirow[t]{5}{*}{$\begin{array}{l}\text { Systems Owners and } \\
\text { Operations }\end{array}$} & Distributor & Business failure & & \\
\hline & Processor & Business failure & & \\
\hline & Retailer & Business failure & $\begin{array}{l}\text { Monopoly results in price } \\
\text { increases }\end{array}$ & $\begin{array}{l}\text { Influence public taste to } \\
\text { healthier diet }\end{array}$ \\
\hline & Consumer & $\begin{array}{l}\text { GM crops reduces demand } \\
\text { Affordability results in low } \\
\text { quality food purchases } \\
\text { affecting general health }\end{array}$ & Change in customer tastes & \\
\hline & Government & $\begin{array}{l}\text { Average wage drop makes } \\
\text { products unaffordable } \\
\text { Inflation increases prices }\end{array}$ & $\begin{array}{l}\text { Increases taxes/levies adding } \\
\text { to costs }\end{array}$ & $\begin{array}{l}\text { Introduces policy which } \\
\text { reduces costs } \\
\text { Introduces subsidies }\end{array}$ \\
\hline $\begin{array}{l}\text { Concept of Operation / } \\
\text { Use / Employment }\end{array}$ & $\begin{array}{l}\text { Ensure all people, at all times, have } \\
\text { physical and economic access to } \\
\text { sufficient, safe and nutritious wheat } \\
\text { based food to meet their dietary } \\
\text { needs and food preferences } \\
\text { (adapted from the FAO food } \\
\text { security definition (FAO, 1996)). }\end{array}$ & $\begin{array}{l}\text { Food (wheat based) shortage } \\
\text { Low economic access } \\
\text { Poor health }\end{array}$ & $\begin{array}{l}\text { Poor planning resulting in } \\
\text { uneven food supply } \\
\text { Change in customer tastes }\end{array}$ & High economic access \\
\hline Relationships & Distributed, market driven & & Monopoly, increases costs & Competition, reduces costs \\
\hline
\end{tabular}

Table 34 - Wheat Supply Chain Risk Matrix 
A Model Based Approach to System of Systems Risk Management

From Table 34 it can be seen that the key (hazard and opportunity) risks, highlighted in red, were identified as:

- Food (Wheat Based) Shortage: consumers have no or reduced access to wheat based food products.

- Low Economic Access: the price of food-based products reduces consumer access.

- High Economic Access: the price of food-based products enhances consumer access.

- Poor Health: the general quality of food-based products is reduced resulting in an adverse effect on general population health.

\subsubsection{Identification of contributing factors}

As undertaken for the previous case study, each risk was analysed in the context of the contributing factors that directly influence it, in order to begin definition of the causal network (described in section 7.3.2).

Food (Wheat Based) Shortage risk is influenced by the following contributing factors:

- Retail Supply: a reduction or break of foodstuff supply to retail outlets directly equates to food shortage.

- Power Grid Failure: power failures may result in short term food shortages due to disruption of the supply chain.

- Banking Systems Failure: any disruption of payment systems may cause short-term disruption of the supply chain. More systemic failure of the banking system will have a detrimental long-term impact on the supply chain.

- Transport Availability: disruption on transport systems will have a short term, detrimental impact upon the supplier chain.

Low and High ${ }^{15}$ economic access to wheat based food risks are influenced by the following contributing factors:

- Retail Supply: in this context the geographic availability of retail supplies is a factor, whereby consumer costs incurred in obtaining foodstuffs, e.g. travel costs will impact the overall affordability.

\footnotetext{
${ }^{15}$ Economic access is separated into two risks in order to distinguish between the hazard and opportunity risks
} 
- Retail Cost: the cost of products in stores is directly related to the economic access, i.e. affordability to the consumer. It should be noted that Retail Cost is the result of cumulative costs throughout the supply chain.

- State of Economy: where average wages are relatively high, a larger proportion of the population will have economic access to products.

The Poor Quality Diet risk is influenced by the following contributing factors:

- Retail Supply: in this context the type of foodstuff being produced will impact national health.

- State of Economy: a weak economy may reduce average consuming spending power, which in turn may result in the lowering of retail costs through the production of lower quality products.

\subsubsection{Development of Causal network}

The initial causal network was constructed based on the analysis above and is shown below in Figure 59.

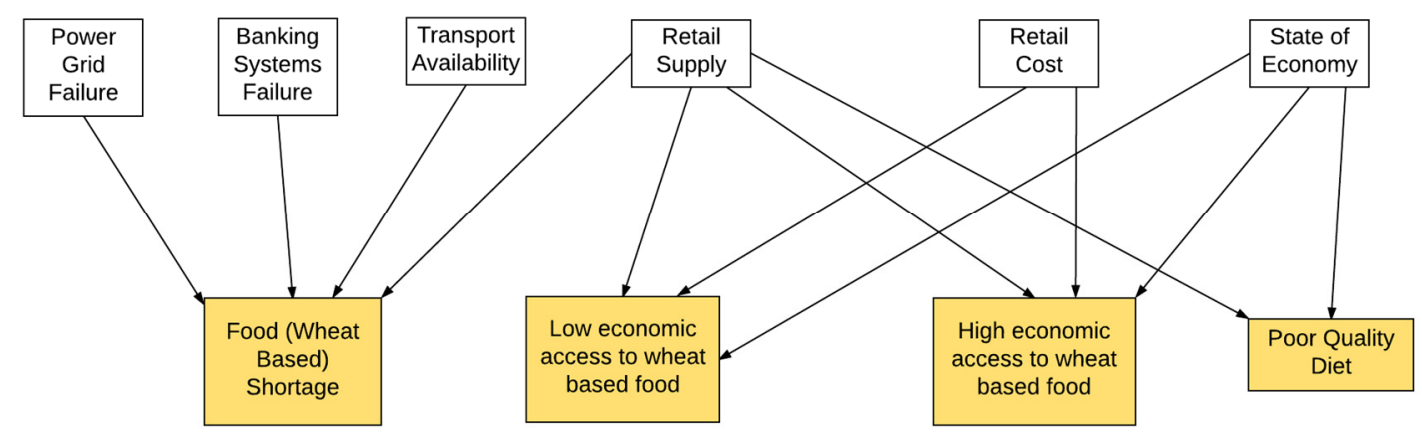

Figure 59 - Food (Wheat Based) Supply Chain - Initial Causal Network

With the initial causal relationships established, further analysis was undertaken to determine the relationships between the primary contributing factors and the remaining influencing factors, as described below.

Power Grid Failure was influenced by the supply level (i.e. demand is met) and disrupting factors such as industrial action or cyber or terrorist attack. Banking systems failure, from an infrastructure perspective, is most likely to be induced by a cyber attack. Transport availability may be affected by industrial action or fuel shortages. Retail Supply risk is more complex and, although directly affected by supply to retail outlets and the stability of 
business (particularly large supermarket chains), it is also affected by risks throughout the supply chain, similarly retail costs are accumulated through the entire supply chain. The state of the economy is potentially an extremely complex indicator, however in the context of this case study key factors were considered to be inflation and the average wage. The results of the complete analysis are shown in the causal network shown below in Figure 60 .

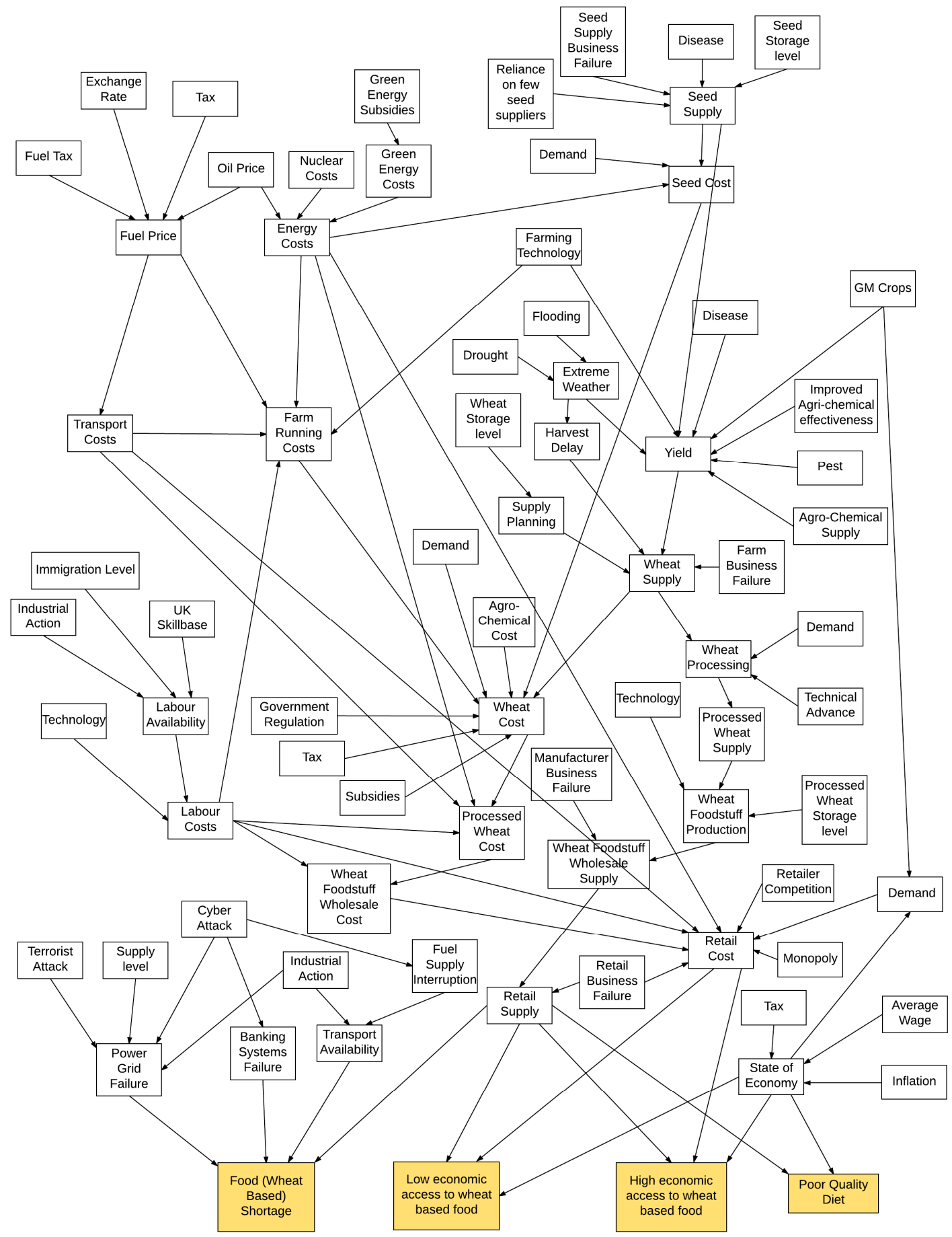

Figure 60 - Wheat Supply Chain Causal Network 
A Model Based Approach to System of Systems Risk Management

\subsection{Analysis and Handling - Food (Wheat Based) Supply Shortage}

The causal network relating to wheat based food shortage was extracted from the complete network (Figure 60) and is provided below in Figure 61; enabling all relevant contributing factors to be more easily identified.

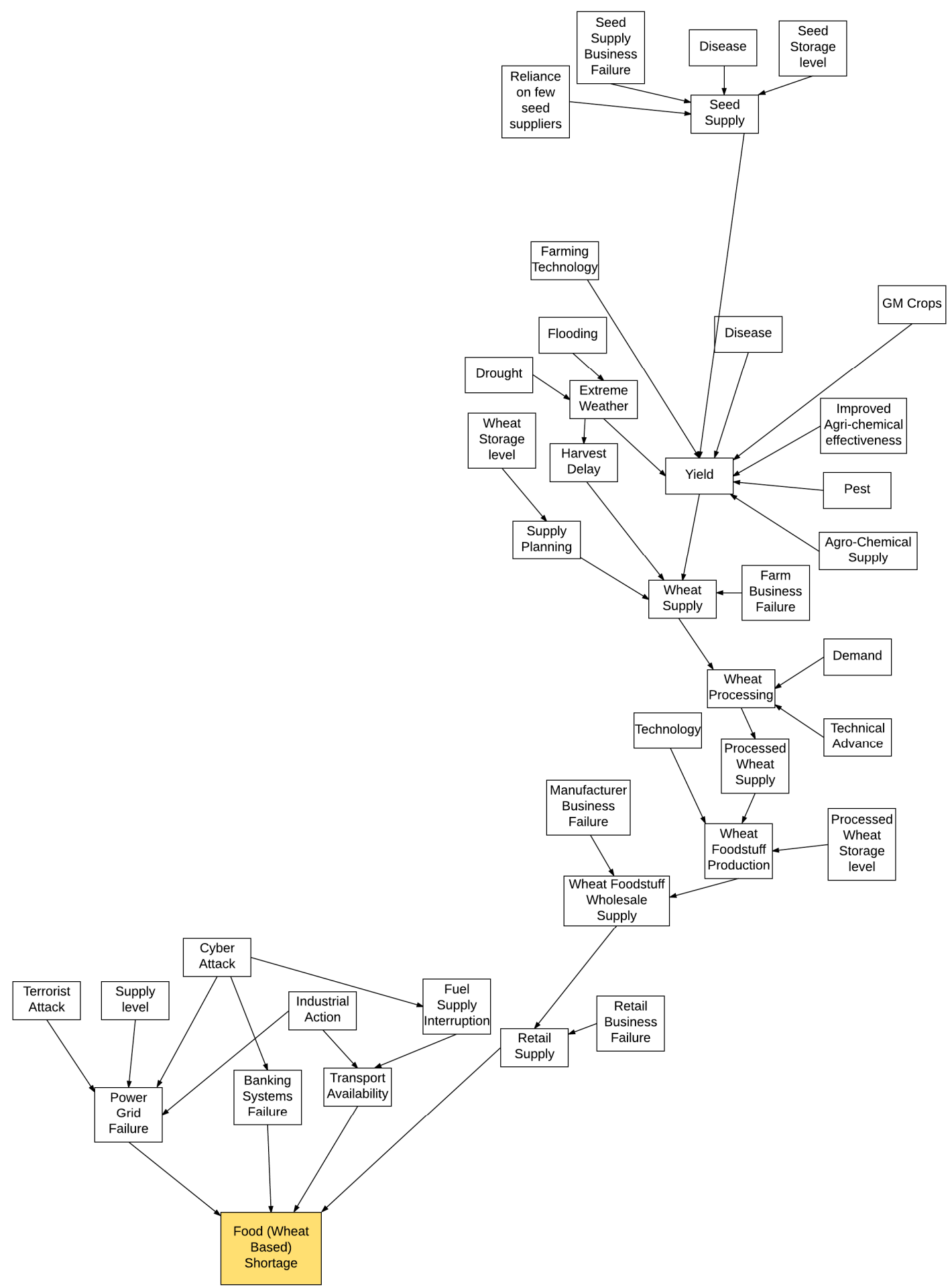

Figure 61 - Food (Wheat Based) Shortage Causal Network 
In accordance with the model based SoS Risk Management process the selection tool (section 6.3) was populated for each group of contributing factors, resulting in the model architecture shown below in Figure 62. The red boxes indicate single or groupings of factors and are annotated with the proposed modelling technique. For this case study a single example of the tool output is included, in Figure 64, showing the Yield factor analysis.

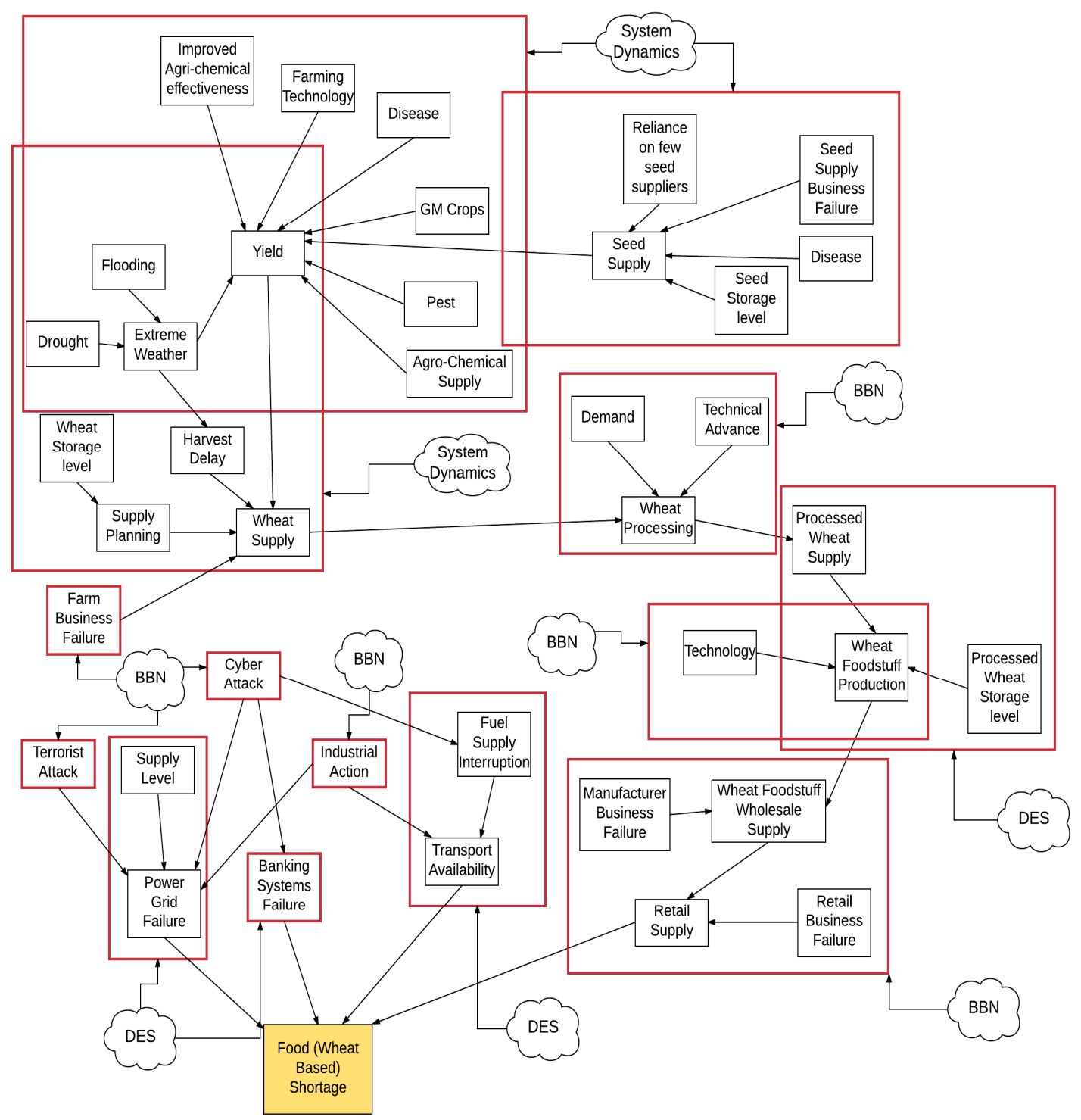

Figure 62 - Food (Wheat Based) Shortage - Model Architecture

Having determined the model architecture a simplified causal network could be derived, as shown below in Figure 63. It should be noted that the following additional nodes were introduced in order to allow integration of supporting models: Transport Risk, Wheat Foodstuff Production Risk, Wheat Processing Efficiency and Wheat Supply Risk. The nodes in the network outlined in red represent those linked to supporting models and are annotated to indicate the model technique required. 


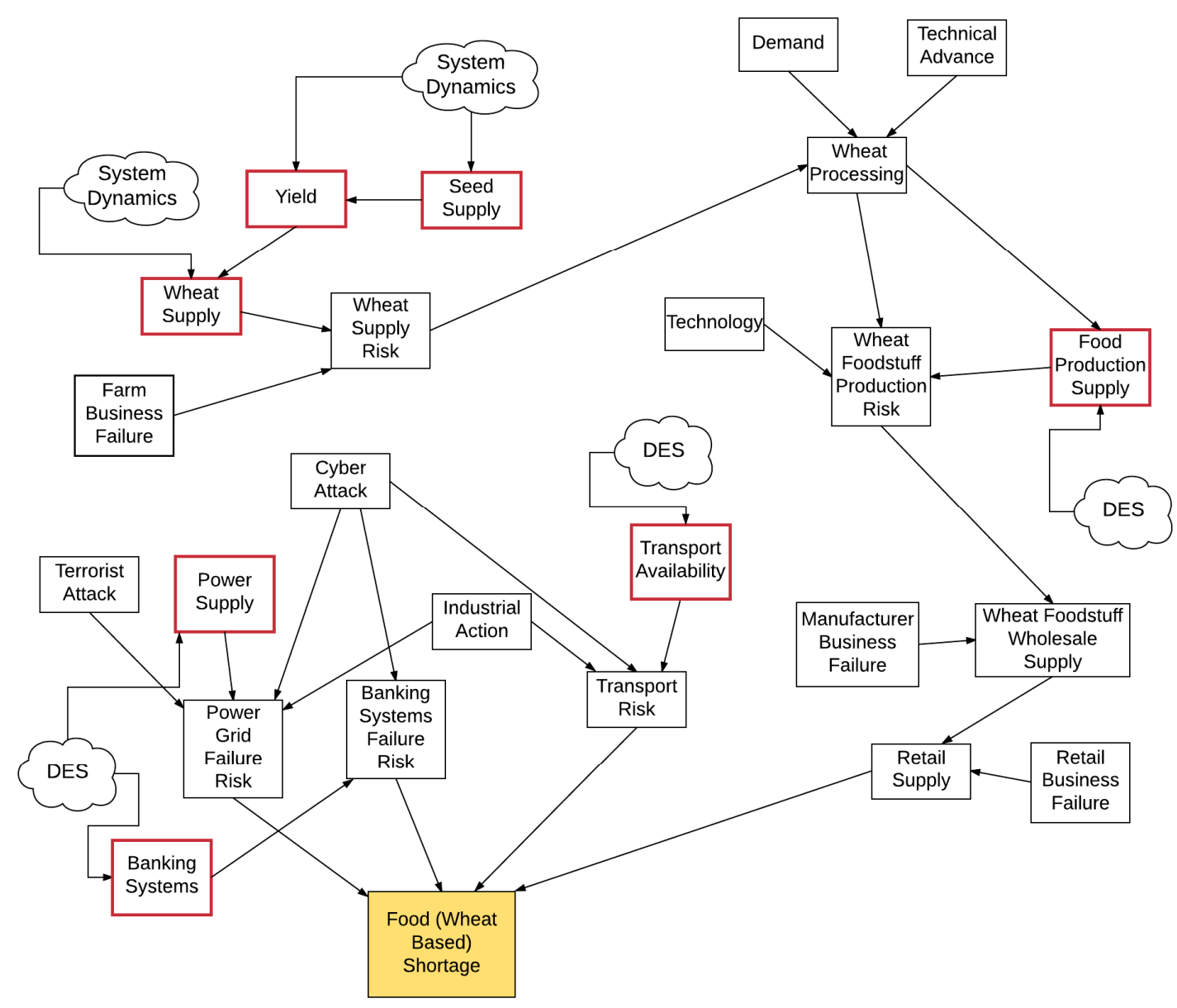

Figure 63 - Food (Wheat Based) Shortage - Simplified Causal Network

\subsubsection{Creation of BBN}

The next stage in the model based SoS Risk Management process required the creation of a BBN based upon the simplified causal network, the resultant BBN is shown in Figure 65 with descriptions of the variables provided in Table 35.

As stated previously, with the reduced scope of this study, supporting models were not implemented, however the BBN (Figure 65) was created with representative data. 


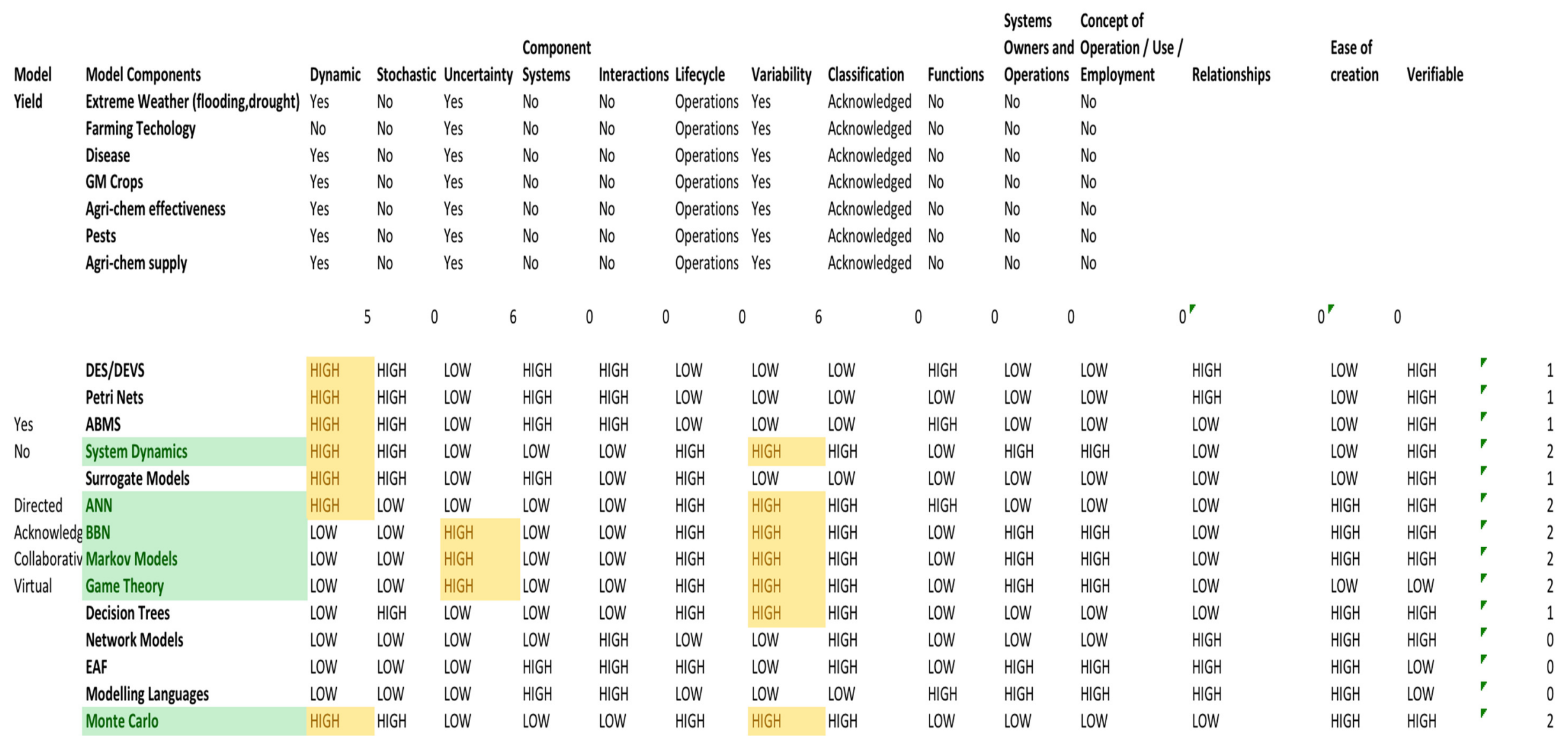




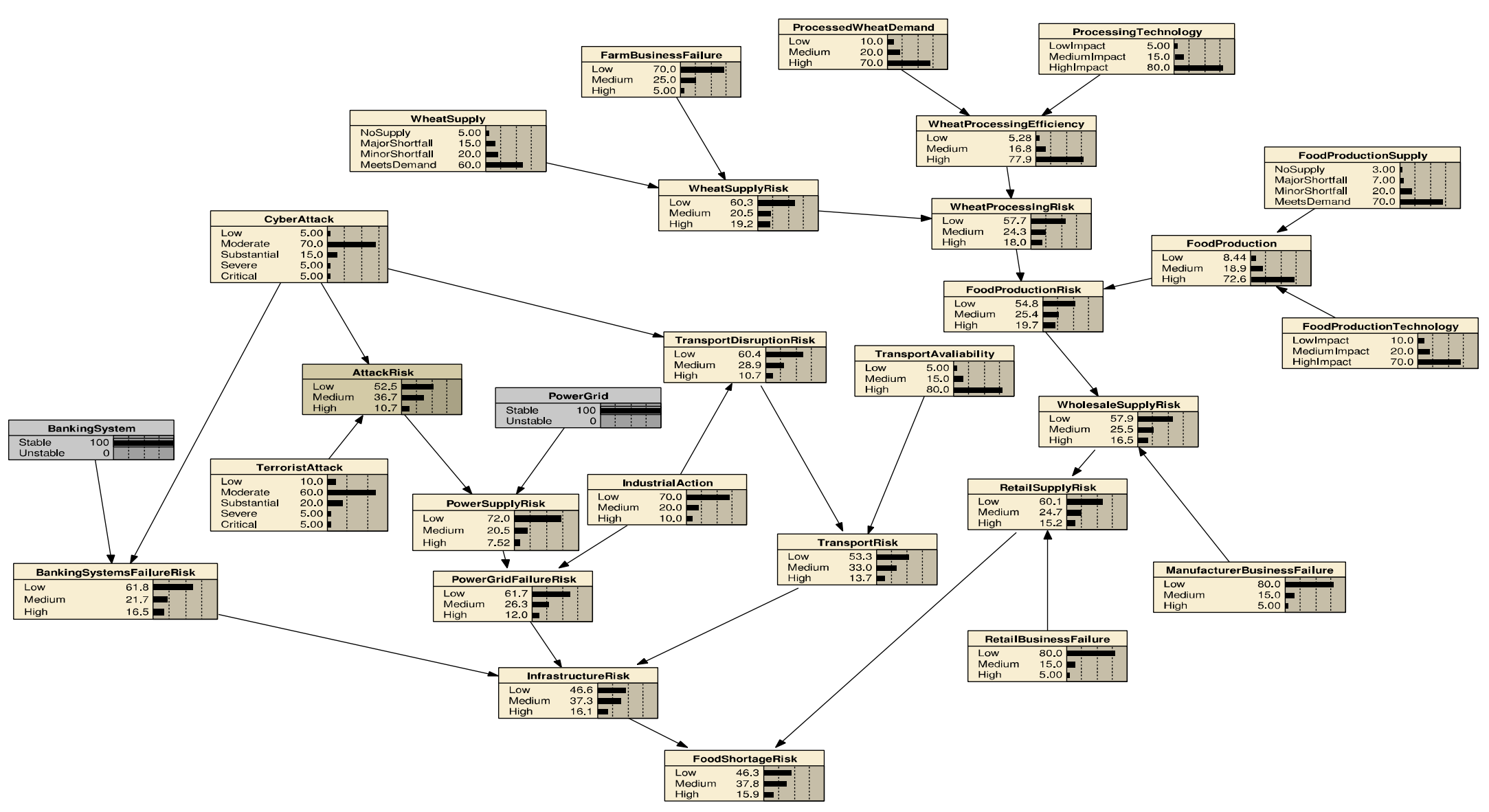

Figure 65 - Food (Wheat Based) Shortage Risk BBN Post Evidence Entry 


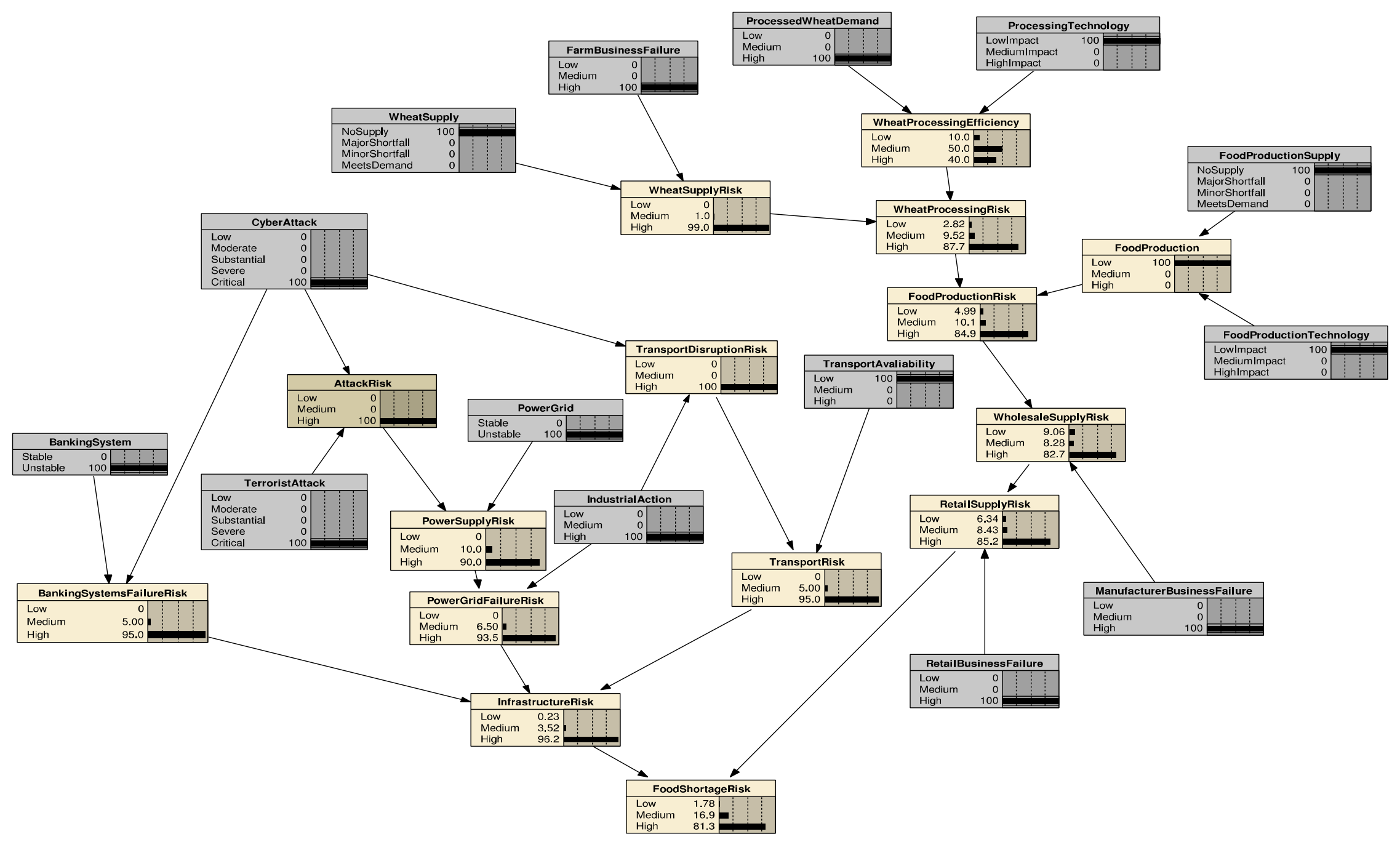

Figure 66 - Food (Wheat Based) Shortage Risk BBN - Worst Case 


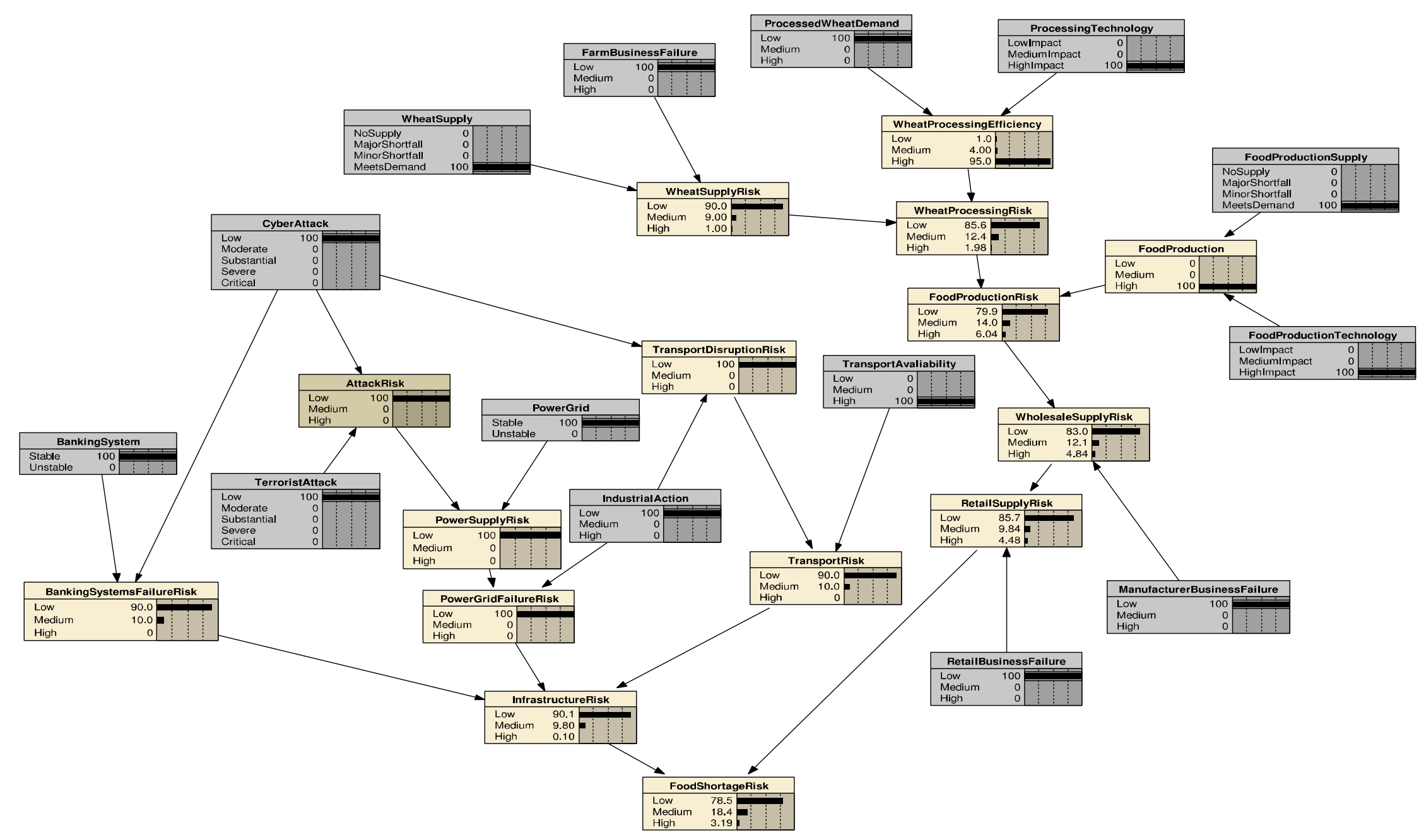

Figure 67 - Food (Wheat Based) Shortage Risk BBN - Best Case 


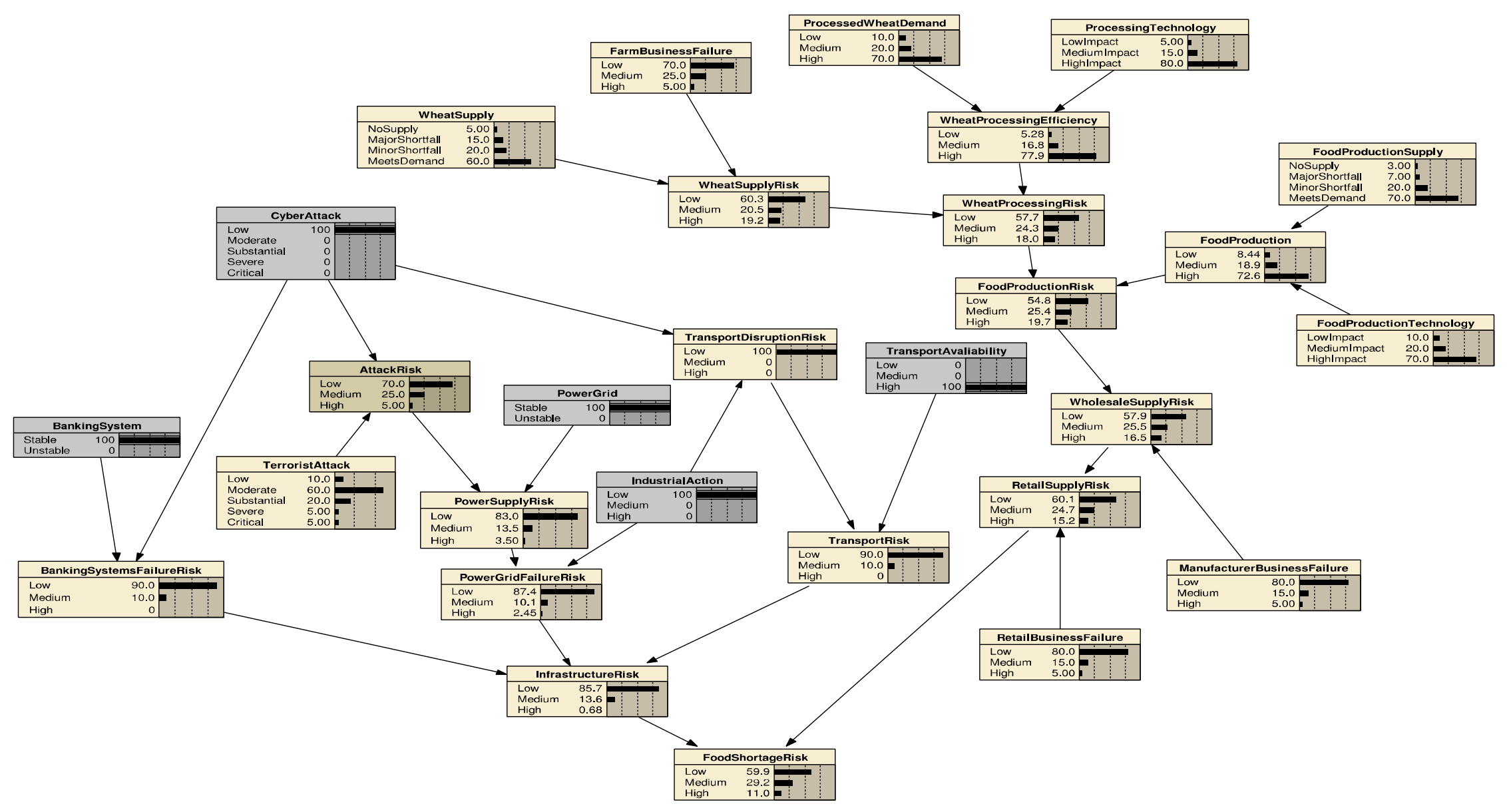

Figure 68 - Food Shortage Risk - Mitigated 
Table 35 below provides a description of each variable, and their associated states, comprising the Food Shortage Risk BBN.

\begin{tabular}{|c|c|c|}
\hline Variable & Description & States \\
\hline WheatSupply & $\begin{array}{l}\text { An assessment of the } \\
\text { wheat supply level } \\
\text { based on supporting } \\
\text { model output }\end{array}$ & $\begin{array}{l}\text { No Supply: Break in supply, less than } 10 \% \text { of demand } \\
\text { Major Shortfall: Substantial reduction in output, between } 70 \% \text { and } 90 \% \text { of demand } \\
\text { Minor Shortfall: Substantial reduction in output, between } 70 \% \text { and } 90 \% \text { of demand } \\
\text { Meets Demand: greater than } 90 \% \text { of demand }\end{array}$ \\
\hline FarmBusinessFailure & $\begin{array}{l}\text { An assessment of the } \\
\text { national rate of farm } \\
\text { business failure }\end{array}$ & $\begin{array}{l}\text { Low: Farming Industry stable, low rate of business failure offset by new business } \\
\text { Medium: Increased level of farm failure not fully offset by new business, localised impact } \\
\text { on food production } \\
\text { High: Severe level of farm business failure with national impact on food production }\end{array}$ \\
\hline WheatSupplyRisk & $\begin{array}{l}\text { The level of risk relating } \\
\text { to wheat supply. }\end{array}$ & $\begin{array}{l}\text { Low: Wheat supply is uninterrupted and at demanded levels } \\
\text { Medium: Wheat supply is restricted and is below demanded level } \\
\text { High: Wheat supply is severely restricted and is substantially below demanded level }\end{array}$ \\
\hline ProcessedWheatDemand & $\begin{array}{l}\text { An assessment of the } \\
\text { demand for process } \\
\text { wheat, e.g. flour }\end{array}$ & $\begin{array}{l}\text { Low: Demand is less lower than average } \\
\text { Medium: Demand equates to average levels } \\
\text { High: Demand is higher than average }\end{array}$ \\
\hline ProcessingTechnology & $\begin{array}{l}\text { An assessment of the } \\
\text { impact technology has } \\
\text { on wheat processing } \\
\text { efficiency }\end{array}$ & $\begin{array}{l}\text { Low Impact: No likely technological advances } \\
\text { Medium Impact: Technological advances are expected to provide limited efficiency } \\
\text { improvement } \\
\text { High Impact: A technological advance is expected to provide a major efficiency } \\
\text { improvement }\end{array}$ \\
\hline
\end{tabular}




\begin{tabular}{|l|l|l|}
\hline Variable & Description & States \\
\hline WheatProcessingEfficiency & Divorcing Variable & $\begin{array}{l}\text { Low: Wheat processing is inefficient } \\
\text { Medium: Wheat processing requires optimization } \\
\text { High: Wheat processing is close to optimum efficiency }\end{array}$ \\
\hline WheatProcessingRisk & $\begin{array}{l}\text { The risk associated with } \\
\text { the supply of processed } \\
\text { wheat (e.g. flour) }\end{array}$ & $\begin{array}{l}\text { Low: Processed wheat supply is uninterrupted and at demanded levels } \\
\text { Medium: Processed wheat supply is restricted and is below demanded level } \\
\text { High: Processed wheat supply is severely restricted and is substantially below demanded } \\
\text { level }\end{array}$ \\
\hline FoodProductionSupply & $\begin{array}{l}\text { The level of risk relating } \\
\text { to processed wheat } \\
\text { supply. }\end{array}$ & $\begin{array}{l}\text { No Supply: Break in supply, less than } 10 \% \text { of demand } \\
\text { Major Shortfall: Substantial reduction in output, between 70\% and } 90 \% \text { of demand } \\
\text { Minor Shortfall: Substantial reduction in output, between 70\% and 90\% of demand } \\
\text { Meets Demand: greater than 90\% of demand }\end{array}$ \\
\hline FoodProductionTechnology & $\begin{array}{l}\text { An assessment of the } \\
\text { impact technology has } \\
\text { on food production } \\
\text { efficiency }\end{array}$ & $\begin{array}{l}\text { Low Impact: No likely technological advances } \\
\text { Medium Impact: Technological advances are expected to provide limited efficiency } \\
\text { improvement } \\
\text { High Impact: A technological advance is expected to provide a major efficiency } \\
\text { improvement }\end{array}$ \\
\hline FoodProduction & $\begin{array}{l}\text { Divorcing Variable } \\
\text { Low: Food production is substantially below demand } \\
\text { Medium: Food production is close to demand } \\
\text { High: Food production is meeting or exceeding demand }\end{array}$ \\
\hline
\end{tabular}




\begin{tabular}{|c|c|c|}
\hline Variable & Description & States \\
\hline FoodProductionRisk & $\begin{array}{l}\text { The risk associated with } \\
\text { the supply of wheat } \\
\text { based foodstuff }\end{array}$ & $\begin{array}{l}\text { Low: Wheat based foodstuff supply is uninterrupted and at demanded levels } \\
\text { Medium: Wheat based foodstuff supply is restricted and is below demanded level } \\
\text { High: Wheat based foodstuff supply is severely restricted and is substantially below } \\
\text { demanded level }\end{array}$ \\
\hline ManufacturerBusinessFailure & $\begin{array}{l}\text { An assessment of the } \\
\text { national rate of wheat } \\
\text { based foodstuff } \\
\text { manufacturing business } \\
\text { failure }\end{array}$ & $\begin{array}{l}\text { Low: Foodstuff manufacturing Industry stable, low rate of business failure offset by new } \\
\text { business } \\
\text { Medium: Increased level of foodstuff manufacturing business failure not fully offset by } \\
\text { new business, localised impact on food production } \\
\text { High: Severe level of foodstuff manufacturing business failure with national impact on } \\
\text { food supply }\end{array}$ \\
\hline WholesaleSupplyRisk & $\begin{array}{l}\text { The risk associated with } \\
\text { the wholesale supply of } \\
\text { wheat based foodstuff }\end{array}$ & $\begin{array}{l}\text { Low: Wholesale foodstuff supply is uninterrupted and at demanded levels } \\
\text { Medium: Wholesale foodstuff supply is restricted and is below demanded level } \\
\text { High: Wholesale foodstuff supply is severely restricted and is substantially below } \\
\text { demanded level }\end{array}$ \\
\hline RetailBusinessFailure & $\begin{array}{l}\text { An assessment of the } \\
\text { national rate of } \\
\text { foodstuff retail business } \\
\text { failure }\end{array}$ & $\begin{array}{l}\text { Low: Foodstuff retail Industry stable, low rate of business failure offset by new business } \\
\text { Medium: Increased level of foodstuff retail business failure not fully offset by new } \\
\text { business, localised impact on food production } \\
\text { High: Severe level of foodstuff retail business failure with national impact on food supply }\end{array}$ \\
\hline RetailSupplyRisk & $\begin{array}{l}\text { The risk associated with } \\
\text { the retail supply of } \\
\text { wheat based foodstuff } \\
\text { (i.e. supply to } \\
\text { consumer) }\end{array}$ & $\begin{array}{l}\text { Low: Retail foodstuff supply is uninterrupted and at demanded levels } \\
\text { Medium: Retail foodstuff supply is restricted and is below demanded level } \\
\text { High: Retail foodstuff supply is severely restricted and is substantially below demanded } \\
\text { level }\end{array}$ \\
\hline
\end{tabular}




\begin{tabular}{|c|c|c|}
\hline Variable & Description & States \\
\hline CyberAttack & $\begin{array}{l}\text { Assessment of the } \\
\text { likelihood of a cyber } \\
\text { attack }\end{array}$ & $\begin{array}{l}\text { Low: Attack is unlikely } \\
\text { Moderate: Attack is possible but not likely } \\
\text { Substantial: Attack is a strong possibility } \\
\text { Severe: Attack is highly likely } \\
\text { Critical: Attack is expected imminently } \\
\text { (Security Service MI5, 2017) }\end{array}$ \\
\hline BankingSystem & $\begin{array}{l}\text { Basic assessment of the } \\
\text { national banking } \\
\text { system stability }\end{array}$ & $\begin{array}{l}\text { Stable: Banking system is operating normally } \\
\text { Unstable: Banking system is unstable, major national bank(s) are threatened with } \\
\text { collapse }\end{array}$ \\
\hline BankingSystemsFailureRisk & $\begin{array}{l}\text { Assessment of the } \\
\text { likelihood of banking } \\
\text { system failure } \\
\text { restricting payment } \\
\text { flow through supply } \\
\text { chain }\end{array}$ & $\begin{array}{l}\text { Low: Banking system fully supporting payment flow though supply chain } \\
\text { Medium: Infrastructure problems delaying or prohibiting payments within the supply } \\
\text { chain } \\
\text { High: National bank(s) likely to require government intervention to prevent collapse } \\
\text { and/or serious infrastructure problems prohibiting payments }\end{array}$ \\
\hline TerroristAttack & $\begin{array}{l}\text { Assessment of the } \\
\text { likelihood of a terrorist } \\
\text { attack }\end{array}$ & $\begin{array}{l}\text { Low: Attack is unlikely } \\
\text { Moderate: Attack is possible but not likely } \\
\text { Substantial: Attack is a strong possibility } \\
\text { Severe: Attack is highly likely } \\
\text { Critical: Attack is expected imminently } \\
\text { (Security Service MI5, 2017) }\end{array}$ \\
\hline
\end{tabular}


A Model Based Approach to System of Systems Risk Management

\begin{tabular}{|c|c|c|}
\hline Variable & Description & States \\
\hline AttackRisk & Divorcing variable & $\begin{array}{l}\text { Low: Low risk of a cyber or terrorist attack } \\
\text { Medium: Possibility of a cyber or terrorist attack } \\
\text { High: Cyber or terrorist attack is probable }\end{array}$ \\
\hline PowerGrid & $\begin{array}{l}\text { Basic assessment of the } \\
\text { power grid state, } \\
\text { supporting power } \\
\text { supply }\end{array}$ & $\begin{array}{l}\text { Stable: Power grid is supplying power in line with demand } \\
\text { Unstable: Power grid is not meeting demand through reduced output }\end{array}$ \\
\hline PowerSupplyRisk & Divorcing variable & $\begin{array}{l}\text { Low: Low risk of power supply disruption } \\
\text { Medium: Possibility of power supply disruption } \\
\text { High: Probability of power supply disruption }\end{array}$ \\
\hline IndustrialAction & $\begin{array}{l}\text { Likelihood of industrial } \\
\text { action }\end{array}$ & $\begin{array}{l}\text { Low: Industrial action unlikely } \\
\text { Medium: Possibility of industrial action } \\
\text { High: Industrial action is highly likely }\end{array}$ \\
\hline PowerGridFailureRisk & $\begin{array}{l}\text { Assessment of the risk } \\
\text { of reduced or } \\
\text { interrupted power } \\
\text { supply }\end{array}$ & $\begin{array}{l}\text { Low: Power supply is likely to be maintained, meeting demand } \\
\text { Medium: Possibility of brief power interruption } \\
\text { High: Extended power supply reduced output or break in supply is highly likely }\end{array}$ \\
\hline TransportDisruptionRisk & Divorcing variable & $\begin{array}{l}\text { Low: Low risk of transport disruption } \\
\text { Medium: Possibility of transport disruption } \\
\text { High: Probability of transport disruption }\end{array}$ \\
\hline
\end{tabular}




\begin{tabular}{|l|l|l|}
\hline Variable & Description & States \\
\hline TransportAvailability & $\begin{array}{l}\text { Assessment of the state } \\
\text { of the transport } \\
\text { network }\end{array}$ & $\begin{array}{l}\text { Low: Transport network is operating normally } \\
\text { Medium: Transport network is likely to interrupt the supply chain for short periods } \\
\text { High: Transport network is likely to experience major problems, severely disrupting the } \\
\text { supply chain }\end{array}$ \\
\hline TransportRisk & $\begin{array}{l}\text { The risk associated with } \\
\text { transport network } \\
\text { failure supporting the } \\
\text { supply chain }\end{array}$ & $\begin{array}{l}\text { Low: Transport network is fully supporting the supply chain } \\
\text { Medium: Transport network is likely to interrupt the supply chain } \\
\text { High: Transport network is likely to severely disrupt the supply chain }\end{array}$ \\
\hline InfrastructureRisk & $\begin{array}{l}\text { The risk associated with } \\
\text { the infrastructure } \\
\text { (banking, transport, IT) } \\
\text { supporting the supply } \\
\text { chain }\end{array}$ & $\begin{array}{l}\text { Low: The supply chain support infrastructure is fully operational } \\
\text { Medium: The supply chain support infrastructure is likely to interrupt the supply chain } \\
\text { High: The supply chain support infrastructure is likely to severely disrupt the supply chain }\end{array}$ \\
\hline FoodShortageRisk & $\begin{array}{l}\text { The risk of consumer } \\
\text { wheat based food } \\
\text { shortages }\end{array}$ & $\begin{array}{l}\text { Low: Wheat based food supply is available, meeting demand } \\
\text { Medium: Wheat based food is available but not fully meeting consumer demand } \\
\text { High: Wheat based food is unavailable or at available at extremely low levels }\end{array}$ \\
\hline
\end{tabular}

Table 35 - Wheat Based Food Supply Risk BBN Variables 
As undertaken for the previous application case study, in order to provide context for the interpretation of the risk analysis results, the Wheat Based Food Supply Risk BBN was updated with worst and best case inputs, as shown in Figure 66 and Figure 67 respectively.

The combination of the best and worst outputs with the results obtained from the original BBN (Figure 65) is presented below in Figure 69, indicating that although the actual results are more closely aligned to the best case profile the probability of "low" risk is substantially lower than the optimum, i.e. approximately $30 \%$. However, it is emphasised that the interpretation of these results is judgement based, utilising SME input.

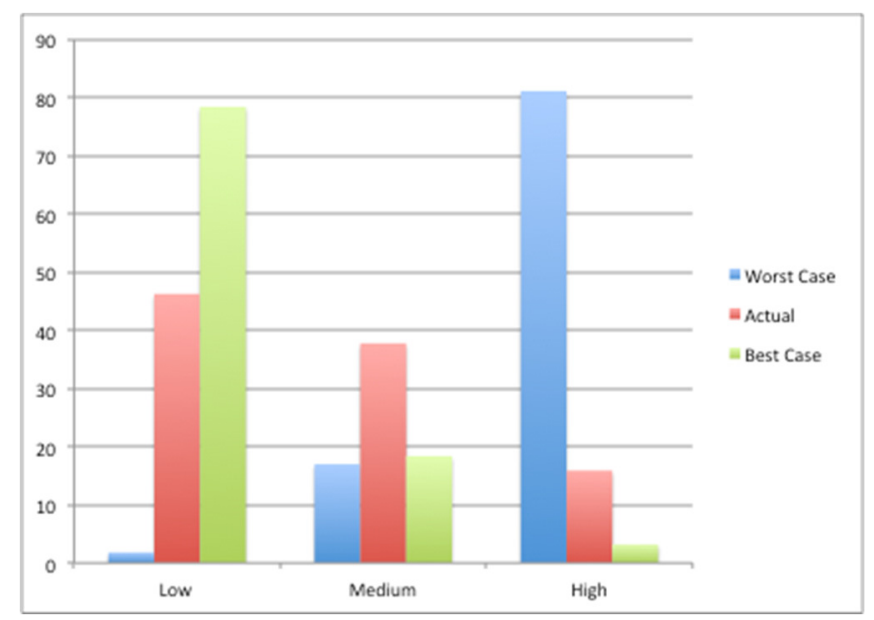

Figure 69 - Wheat Based Food Supply Risk

Using the BBN to investigate potential risk mitigation, one approach considered reducing risk relating to the infrastructure supporting the supply chain. The BBN was therefore updated, as shown in Figure 68 to reflect this through entering evidence indicating certainty of low cyber attack and transportation risk, resulting in a noticeable improvement in the risk profile, as shown below in Figure 70, thereby illustrating the model based approach capability allowing differing scenarios and mitigation approaches to be explored.

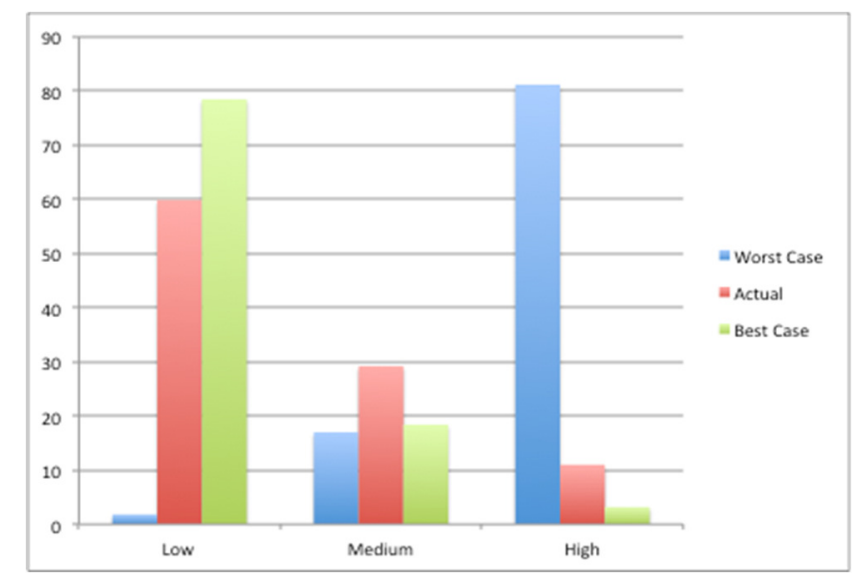

Figure 70 - Wheat Based Food Supply Risk with Infrastructure Mitigation 


\subsection{Monitoring}

On-going monitoring of wheat supply chain risk could be undertaken using the models created within the analysis activity, updated with current data as it became available. Additional supporting models could be defined and refinements of those already implemented would also be possible, enabled by the longer timescales associated with risk management in this context, which considers risks in the medium to long term.

\subsection{Verification and Validation}

The application case study was broken down into the following components in order to logically structure Verification and Validation activities; Risk Identification, Causal Network, BBNs, and Risk Assessment Output. The researcher undertook all verification activities, whilst validation was based upon elicitation of SME input.

\subsubsection{Verification Procedures}

The procedure for verifying each element of the application case study is described below in tabular form.

\begin{tabular}{|l|l|l|}
\hline \multicolumn{2}{|l|}{ Verification Procedure - Risk Identification } \\
\hline Step & Action & Result \\
\hline 1 & $\begin{array}{l}\text { Manually check risks identified in Table 34 include } \\
\text { those discussed with SME and Met Office. }\end{array}$ & Confirmed, all included \\
\hline 2 & $\begin{array}{l}\text { Manually check Table 34 and confirm any risks not } \\
\text { identified in step 1 are valid. }\end{array}$ & $\begin{array}{l}\text { Additional risks appear } \\
\text { valid; confirmed they } \\
\text { were derived from peer } \\
\text { reviewed sources }\end{array}$ \\
\hline 3 & $\begin{array}{l}\text { Manually check Table 34 and confirm all SoS } \\
\text { dimensions (as defined in section 6.1) are included. }\end{array}$ & Confirmed, all included \\
\hline 4 & $\begin{array}{l}\text { Manually check Table 34 and confirm risks align } \\
\text { correctly with SoS dimensions. }\end{array}$ & $\begin{array}{l}\text { Confirmed, all dimensions } \\
\text { included }\end{array}$ \\
\hline
\end{tabular}

Table 36 - Verification Procedure - Food Shortage Risk Identification

\begin{tabular}{|l|l|l|}
\hline \multicolumn{2}{|l|}{ Verification Procedure - Causal Network } \\
\hline Step & Action & Result \\
\hline 1 & $\begin{array}{l}\text { Manually check all risks and contributing factors are } \\
\text { included in the causal network as defined in Table 34. }\end{array}$ & $\begin{array}{l}\text { Factors relating to } \\
\text { Lifecycle and Classification } \\
\text { are not included. Justified } \\
\text { because of their generic } \\
\text { nature. }\end{array}$ \\
\hline 2 & $\begin{array}{l}\text { Manually check the causal network as defined in Figure } \\
60 \text { to identify risks or contributing factors that are not }\end{array}$ & $\begin{array}{l}\text { Confirmed that network } \\
\text { reflects risks and }\end{array}$ \\
\hline
\end{tabular}




\begin{tabular}{|l|l|l|}
\hline & $\begin{array}{l}\text { defined in Table 34. } \\
\text { contributing factors } \\
\text { identified }\end{array}$ \\
\hline 3 & $\begin{array}{l}\text { Check that there are no unconnected nodes in the } \\
\text { causal network, as shown in Figure 60. }\end{array}$ & $\begin{array}{l}\text { Confirmed that all nodes } \\
\text { are connected }\end{array}$ \\
\hline 5 & $\begin{array}{l}\text { Confirm that connections within the causal network are } \\
\text { appropriate and logically correct. }\end{array}$ & $\begin{array}{l}\text { Confirmed all connections } \\
\text { correct }\end{array}$ \\
\hline 5 & $\begin{array}{l}\text { Manually check the Food Shortage causal network, as } \\
\text { Fratricide Risk and all contributing factors connected to } \\
\text { it, as defined in Figure 60. }\end{array}$ & $\begin{array}{l}\text { Confirmed network is } \\
\text { complete }\end{array}$ \\
\hline
\end{tabular}

Table 37 - Verification Procedure -Food Supply Risk Causal Network

\begin{tabular}{|c|c|c|}
\hline \multicolumn{3}{|c|}{ Verification Procedure - Food Shortage BBN } \\
\hline Step & Action & Result \\
\hline 1 & $\begin{array}{l}\text { Using the Netica application, open the Food Shortage } \\
\text { BBN (as shown in Figure 65). }\end{array}$ & Food Shortage BBN display \\
\hline 2 & $\begin{array}{l}\text { Manually check the Food Shortage BBN and confirm } \\
\text { all nodes defined in the causal network (Figure 61) are } \\
\text { included. }\end{array}$ & $\begin{array}{l}\text { Confirmed all nodes are } \\
\text { present }\end{array}$ \\
\hline 3 & $\begin{array}{l}\text { Manually check the Food Shortage BBN and confirm } \\
\text { that additional nodes (not defined in Figure 61) are } \\
\text { valid divorcing nodes. }\end{array}$ & $\begin{array}{l}\text { Confirmed all additional } \\
\text { nodes are valid }\end{array}$ \\
\hline 4 & $\begin{array}{l}\text { Manually check each variable within the Food } \\
\text { Shortage BBN and confirm that the states match those } \\
\text { defined in Table } 35 \text { - Wheat Based Food Supply Risk } \\
\text { BBN Variables. }\end{array}$ & Confirmed that all match \\
\hline 5 & $\begin{array}{l}\text { Check all parent nodes in the Food Shortage BBN have } \\
\text { correctly defined conditional probability tables. }\end{array}$ & Confirmed tables correct \\
\hline 6 & $\begin{array}{l}\text { For all "edge" variables enter evidence, setting each } \\
\text { state in turn to } 100 \% \text { probability and confirming } \\
\text { probability changes are propagated throughout the } \\
\text { network as expected. }\end{array}$ & $\begin{array}{lr}\text { Errors found } & \text { were } \\
\text { corrected and step } & \text { re-run, } \\
\text { confirming } & \text { correct } \\
\text { propagation } & \end{array}$ \\
\hline 7 & $\begin{array}{l}\text { Enter the "worst case" scenario evidence as shown in } \\
\text { Figure } 66, \text { confirming probability changes are } \\
\text { propagated throughout the network as expected. }\end{array}$ & $\begin{array}{l}\text { Worst case BBN results as } \\
\text { expected }\end{array}$ \\
\hline 8 & $\begin{array}{l}\text { Enter the "best case" scenario evidence as shown in } \\
\text { Figure } 67, \text { confirming probability changes are } \\
\text { propagated throughout the network as expected. }\end{array}$ & $\begin{array}{l}\text { Best case BBN results as } \\
\text { expected }\end{array}$ \\
\hline
\end{tabular}

Table 38 - Verification Procedure - Food Shortage BBN 
A Model Based Approach to System of Systems Risk Management

\subsubsection{Validation}

The approach taken to validate the application of the model based approach in this case study components was to elicit subject matter expertise through semi-structured interviews, which established the realism of the case study and the risks identified as well as the value of the proposed risk management approach within the SMEs domain.

The interview questions were as follows:

1. Describe your experience and knowledge relating to supply chains in the agricultural domain and how this may be applicable to risk management.

Response: Supply chain and forecasting manager in a large UK agricultural supply company with experience of the food supply chain

2. In the provided Wheat Supply Chain Risk Matrix risk matrix, are all major risks and contributing factors identified? If not, which risks and factors are missing and is their consequence greater or less than those already identified?

Response: All major risks are captured. However, day to day issues with the wheat supply chain are congestion in stores as farmers who hold out for better prices for un-contracted wheat and imbalance in the type of wheat, i.e. a lot of UK wheat is exported for animal feed and bread/beer wheat is imported and most importantly the global price affected by global weather and futures trading.

3. In the provided causal network, are the links between contributing factors and risks are reasonable and correct from your experience?

Response: The causal network was regarded as accurate and a very useful artefact, providing understanding of the supply chain.

4. Do the risk matrix and causal network positively support the identification of risks within Wheat Supply Chain and Food Security planning? If not, why do they not support this task?

Response: Yes, they enabled all risks to be identified.

5. Does the Food (Wheat Based) Shortage Risk BBN update as expected when evidence is entered?

Response: Yes, the updated values appeared reasonable. 
6. Is the Food (Wheat Based) Shortage Risk analysis output provided in an understandable, meaningful and usable format?

Response: It was agreed the format was acceptable.

7. Do you think the SoS Risk Management process and its outputs could provide benefit to Wheat Supply Chain and Food Security or planning activities? If so, how and where?

Response: It could be valuable in testing "what if" scenarios and mitigation approaches. However, accurate prediction of future events would not be possible due to the complexity and inherent uncertainty of the many interrelating factors. "The complexity of forecasting worldwide short/med/long term weather as well as commodity prices which can also be political (China, India, Russia, North Africa \& Baltics) makes it difficult enough, but there is also the sentiment of commodity traders, merchants and farmers all second guessing the market."

8. Bearing in mind that this approach is currently a concept, do you see any limitations of the approach?

Response: As stated above, due to the complexity of the contributing factors it would not be possible to produce accurate predictive models.

\subsection{Discussion}

The risk identification matrix based upon the SoS Sol dimensions proved to be an effective approach to identifying food security and supply chain risk. However, it was necessary to take a relatively high level perspective in comparison to the CAS application case study and would require development of potentially highly complex supporting models.

The factors influencing wheat supply chain risk are highly complex, it would not therefore be practical or possible to implement accurate predictive models but the BBNs and supporting models would provide an effective tool to understanding risks and the causes which in turn would support the development of mitigation strategies.

Whereas the CAS analysis considered risk over a short period, i.e. mission length in the order of hours, in this case risk was applicable to the medium and long term, potentially over several years. To accommodate the view of risk development over time, the risk management approach would require extending to create a set of risk results rather than a single output; this could be achieved through the use of dynamic BBNs. 


\section{Chapter 10: Analysis of SoS Risk Management Approach}

This chapter provides an overall summary of the approach, having been refined as a result of both application case studies, followed by a description of the underlying theoretical basis. Although each application case study provided a critical assessment of the approach in specific contexts a more general assessment is provided in this chapter, followed by a critique of the research itself and discussion of its benefits and limitations.

\subsection{Summary of Approach}

Figure 71 below summarises the entire model based risk management process and illustrates the relationship to the traditional activities namely, planning, identification, analysis, handling and monitoring where each is distinguished by colour and linked to a box enclosing the related part of the process.

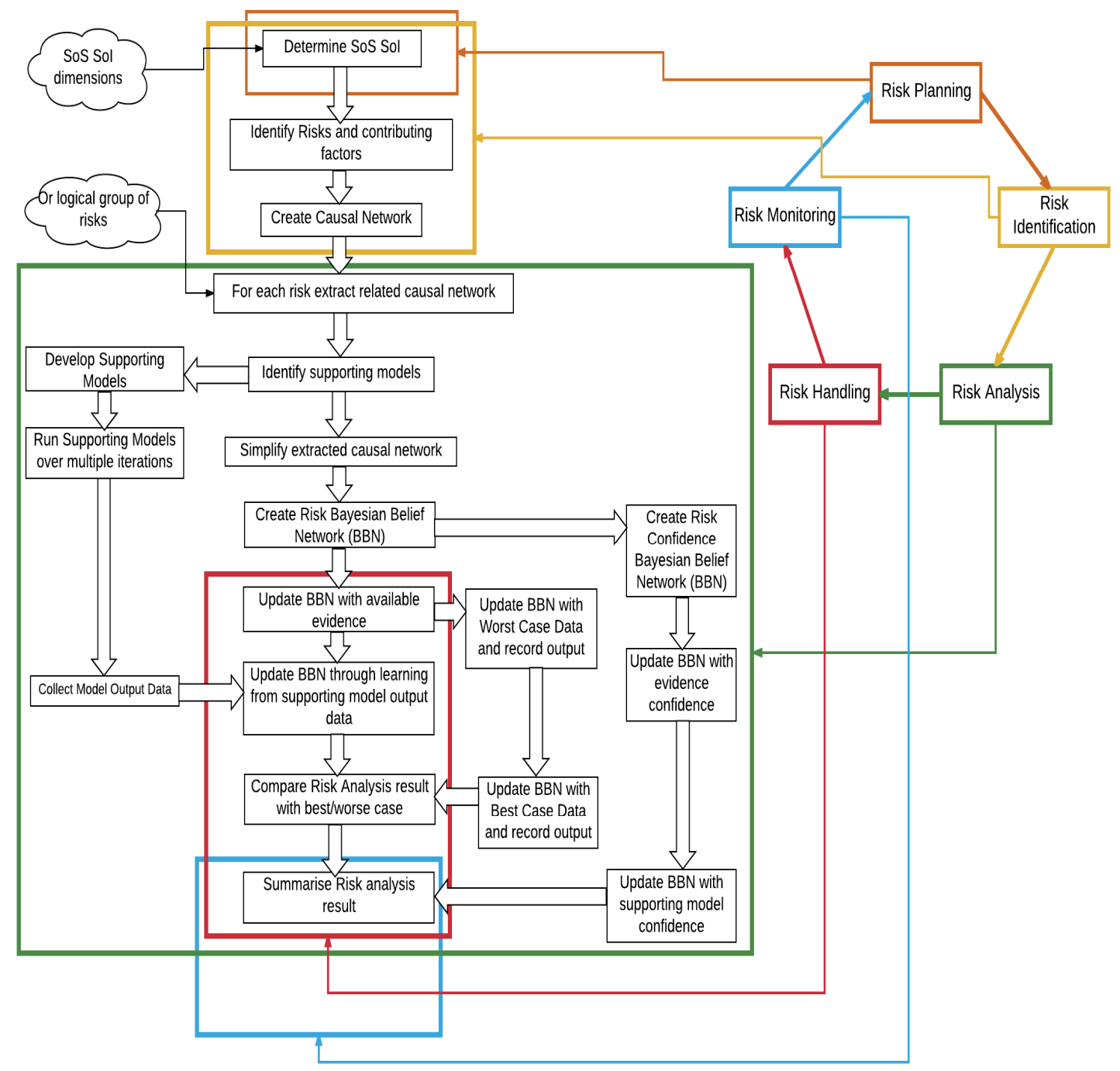

Figure 71 - Model Based SoS Risk Management Summary 
A Model Based Approach to System of Systems Risk Management

\section{Risk Planning}

Risk planning will primarily decide on the management approach and potentially identify the proposed model based approach, at this stage it will also need to establish the Sol.

\section{Risk Identification}

The initial part of the process is intended to support risk identification through the framework comprised of the SoS Sol dimensions (Kinder et al., 2015), as described in section 6.1, and the holistic risk categories, hazard, control and opportunity (Hopkin, 2002). The framework allows each Sol element to be assessed in the context of the holistic risk types, ensuring full analysis coverage and the SoS perspective is maintained. Having established all risks, and factors that influence them, a causal network is constructed, establishing the relationship between them.

\section{Risk Analysis}

The bulk of the process addresses risk analysis. Initially a "subset" of the overall causal network is extracted relating to the specific risk under analysis. However, where multiple risks relate to common sections of the causal network they may be analysed simultaneously in order to minimise duplication of effort. The risk specific causal network is then analysed to determine where nodes, or groups of nodes, may be represented in separate "supporting" models, which enables the causal network to be simplified through the reduction in the quantity of nodes. A BBN is then created representing the simplified causal network, ensuring each variable and state is clearly defined which is critical to providing unambiguous context when populating the BBN conditional probability tables.

\section{Supporting Models}

In parallel, the supporting models are developed and assessed according to the confidence in the assumptions, expert input and $V \& V$ effort. The models are executed over multiple iterations (in the order of 5000) producing a set of output data, which may be "learned" by the BBN.

\section{Interpretation of Analysis Results}

The BBN is also updated though the direct input of available evidence. In order to provide context for the interpretation of the BBN risk output, risk levels are established for worst and best case scenarios. The decision to mitigate risk based on the analysis results in comparison with the worst/case values is judgement based using expert input. 
A Model Based Approach to System of Systems Risk Management

\section{Risk Confidence}

Confidence in the risk analysis output is determined through the creation of a BBN that mirrors the risk BBN but each variable represents the confidence of the evidence or supporting model.

\section{Risk Handling}

Representing the risks and contributing factors in BBNs and supporting models allow alternative risk handling and mitigation approaches to be assessed in order to determine the most effective.

\section{Risk Monitoring}

Risk monitoring may be undertaken by continuous observation of the BBN output, providing the BBN is frequently updated with current information.

\subsection{Theoretical Basis for Approach}

This section brings together the theoretical basis for the proposed SoS RM approach, through the definition of a set of principles and the identification of the theory underpinning these.

The core principles of the SoS Risk Management process are as follows:

1) The process should establish the SoS Sol

2) It should view risk from a holistic perspective

3) It should support risk management for SoS types with a defined purpose

4) It should represent the relationship between risks and contributing factors

5) It should use Bayesian methods to assess the probability of risk

6) It should represent risks using suitable modelling techniques

7) It should support the integration of heterogeneous modelling techniques

Principle 1 is fundamentally underpinned by General Systems Theory, specifically Holism, Open/Closed Systems, Boundaries, Single/Multiple Outcomes, Entropy, Hierarchies and Interrelated Components (Von Bertalanffy, 1968). Holism is also directly related to principle 2.

The following SoS Sol dimensions (Kinder et al., 2012), are discussed in section 6.1 and support principles 1 and 2:

- Component Systems (including specific and general system types) 
A Model Based Approach to System of Systems Risk Management

- Classification

- Interactions

- Nature of Relationships

- Lifecycle

- Variability

- Functions

- Systems Owners and Operations

- Concept of Operation / Use / Employment

The following risk types (Hopkin, 2002) underpin principle 2, in combination with the SoS Sol dimensions listed above, enabling the definition of a SoS risk identification framework:

- Hazard Risks (e.g. Threat and perils)

- Control Risks (e.g. Doubt about mission achievement, project management control)

- Opportunity Risks (Opportunity to enhance or inhibit mission achievement)

Principle 3 specifically relates to the SoS types directed, acknowledged and collaborative (Dahmann \& Baldwin, 2008).

Principles 4 and 5 are supported by Bayesian Network theory (Pearl, 2014), which encompasses the definition of causal networks and the propagation of probabilities within a BBN. This also partially supports principle 7 through the theory of Bayesian learning.

Several theories support principle 6. The philosophy of models and analogies (Hesse, 1963) defines the fundamentals of modelling theory and is discussed in section 3.3.2. The justification for this principle is the law of requisite parsimony (Miller, 1956) whereby the use of models enables risks to be analysed through the consideration of suitable sized groups of variables or observations, and requisite variety which requires models to provide a level of variety of at least the same level as the system represented. The set of potential modelling techniques is described in the paper (Kinder et al., 2014) attached in Annex A which provides a summary of basic underlying principles.

Principle 7 utilises Monte Carlo simulation which is itself underpinned by the strong law of large numbers (Day, 2008). 


\subsection{Critical Assessment of Risk Management Approach}

\subsubsection{Risk Identification}

SoS Risk identification utilises a framework defined by SoS Sol dimensions (Kinder et al., 2012) cross referenced to risk types, hazard, control and opportunity (Hopkin, 2002). Whilst this was generally found to be a successful method for the identification of risks and their contributing factors, the dimensions classification and lifecycle did not map particularly well to risks, which could be regarded as SoS characteristics derived from the other dimensions.

The scope of the "Interactions" dimension was somewhat unclear; it could be interpreted as including the information exchanged by component systems or the connection itself. For the purposes of the case studies, both interpretations were used, although in some cases the connection could also be considered a component system in itself, e.g. the Internet which is in fact a SoS in its own right, leading to a further conclusion that the "component system" dimension should encompass both component systems and component SoS. A possible clarification of the "Interactions" dimension is by defining "what" is transferred, the direction and an associated quality parameter.

Whilst many of the risks and contributing factors were not dependent upon the framework for identification it did provide a structured method of documenting the risk identification output and ensured risks were considered from a SoS perspective.

As is the case with traditional risk management approaches, the identification of risks was dependent upon expert input elicited through techniques such as brainstorming, interview or the Delphi method.

Construction of the causal network also required expert knowledge to determine the relationship between the risks and contributing factors. Whilst this was found to improve understanding of the interdependencies contributing to risk, the complexity of the resultant network could potentially become unmanageable where a large number of risks and factors were identified, requiring careful management of the scope of the identification activity.

\subsubsection{Risk Analysis}

Risk analysis was performed for each risk separately requiring the elements of the causal network to be extracted relevant to the risk under consideration, enabling analysis to be performed on a smaller scale sub-network. Whilst this approach was successful in reducing complexity it did result in duplication of effort where risks were closely related, with large sections of the causal network having common elements. In these cases, the risk 
management process should be refined to permit analysis of multiple risks simultaneously where duplicated effort would otherwise occur.

Analysis of each individual risk causal network utilised the model selection tool (described in section 6.3) in order to determine where supporting models could be utilised and simplify the causal network. The approach was effective in identifying appropriate modelling techniques, although population of the tool was influenced to some extent by the perspective required by the practitioner. For example, Interoperability (section 8.13) was modelled from a top down perspective using System Dynamics but could have used a Discrete Event Simulation, bottom up approach instead. However, the Discrete Event technique would have resulted in a model of far greater complexity, requiring detailed representation of all component systems and interactions. In this case the risk analysis required a general, high-level assessment of interoperability making the System Dynamics the most suitable option. This illustrates that, although the tool provided benefit as a decision support tool, practitioner knowledge was required in order provide input and interpret the output. The selection tool was designed specifically to identify modelling techniques appropriate to SoS, however many factors influencing risk were environmental, e.g. meteorological or economic, and therefore not directly suitable for analysis by the tool. In these cases modelling approaches or currently available models required identification through expert knowledge.

Using BBNs to represent risks and contributing factors was found to be extremely effective, in particular the propagation of probabilities throughout the BBN allowed different mitigation strategies to be tested. Key in defining an effective BBN was the provision of clear and unambiguous definitions for each variable and the associated states. As well as enabling meaningful interpretation of the BBN it also provided context for the elicitation of SME information required for the BBN population, which included input evidence and definition of the conditional probability tables. The use of expert knowledge was a pragmatic approach to fully defining the BBN with each table requiring assessment in order to determine the most appropriate distribution. However, it did result in the quantitative representation of subjective information, which emphasised that although the approach does reduce subjectivity in the risk assessment it does not eliminate it. A common misconception in this situation is that quantitative representation is based upon quantitative data and hence high accuracy is assumed, however the introduction of the confidence assessment provided a means to prevent this incorrect interpretation, although the confidence assessment was itself based on subjective assessment. 
When the first application case study (chapter 8 ) analysis results were initially reported, it was found that interpretation of them was problematic with no context to determine if the output presented an acceptable level of risk. This lack of context was overcome by producing risk analysis results for worst and best case scenarios, which then enabled a relative comparison of the actual analysis output. This solution was successful in providing a more meaningful output. However; it did still require an expert based judgement to determine if mitigation was required.

A key difference between the application of the process in the two case studies was that the smaller scope of the CAS Sol enabled the derivation of predictive results whereas the complexity and uncertainty which was inherent in the food supply chain case study limited the predictive capability of the models.

\subsubsection{Supporting Models}

The method proposed to support the integration of supporting models with the central risk BBN was demonstrated successfully within application case study 1, providing a source of quantitative data thereby reducing subjectivity. The most flexible approach proved to be the creation of "continuous" variables with the BBN where the associated states defined bands of input values; the BBN could then learn from a set of raw values output from a supporting model and thereby decoupling the BBN and model.

However, producing a set of outputs over a distribution required the models to contain some stochastic variables and output the data in a specific format. Whilst this was achievable when producing bespoke models under case study 1 , in some cases it would be desirable to reuse pre-existing models, however a method would be required to ensure these could be adapted to produce the required output, or alternatively the existing output could be adapted. This is particularly relevant to case study 2 which required input from climate and economic models which are in existence and highly complex therefore developing these as part of the risk analysis activity would be unnecessary and impractical. Indeed, the complexity and inherent uncertainty of these supporting models was an area of concern raised by the SME validating the case study 2 .

Verification and validation ( $\mathrm{V} \& \mathrm{~V})$ of the models was undertaken in order to determine the associated confidence, and which relied on definitions provided in section 7.4.4. Unlike V\&V of an engineered system, whereby the performance and behaviour are specified and can therefore be tested, a predictive model by definition produces outputs that are not predefined and hence validation is more subjective, typically requiring expert input to 
confirm that model behaviour appears reasonable, according to their knowledge and experience. This method is an accepted approach (as discussed in section 3.3.8) and proved to be effective and pragmatic when applied in case study 1.

\subsubsection{Risk Confidence}

An indication of confidence in the risk assessment result is essential if stakeholders are to have trust in it; therefore it was necessary to provide an overall confidence value and a method to derive it. Hence, the method described in section 7.4.4 was proposed which required the creation of a $B B N$, which mirrored the risk $B B N$ under consideration, enabling risk confidence estimates for evidence and supporting models to be combined into a single overall risk confidence level. Although this method supported the requirement to derive a risk confidence indication there was a level of subjectivity associated with assigning the supporting model and evidence confidence levels and defining the conditional probability tables, which determined how child variable confidence values were combined by the parent variable.

\subsubsection{Required Development Effort}

Naturally, the level of effort required for the risk identification and analysis activities is directly related to the scope and complexity of the SoS under consideration as well as the number and fidelity of the supporting models. The identification activity would potentially require several workshop sessions in order to elicit sufficient SME information in order to fully populate the risk matrix and define the causal network.

Definition of the BBNs is a labour intensive task requiring SME input requiring the definition of the variable states and the population of the conditional probability tables: again the effort required is driven by the BBN complexity.

Development of the supporting models may be undertaken in parallel with the BBNs and potentially requires the most effort of all activities, although this is also dependent on the number of models and their required fidelity.

When considering the case studies, the first one required risk analysis to be performed within a short time period and potentially in real time. In this instance it is not possible or practical to follow the process, however the process could be undertaken outside an operational situation in order to develop the BBNs and models, which could then be integrated into a tool providing rapid decision supporting CAS risk management (the SME validating application case study 1 identified this as a potential exploitation path for this research). 
The second case was concerned with the assessment of risk over medium and long-term periods hence an extended model development period would be practical. However, as previously discussed, existing model re-use would be essential in this case; it would completely unrealistic to expect a risk management activity to implement models predicting climate change and economic behaviour.

However, the activity of modelling risk in a particular class of complex SoS is educative, so that the modeller may understand better the relationship of causes and outcomes in terms of risk in the system. Hence, the knowledge gained through these activities, which may be undertaken over a relatively long period, can enhance knowledge with which to make decisions in the short-term when similar situations arise in the future.

\subsection{Benefits of Approach}

This section summarises the overall benefits of the proposed SoS risk management approach, which were touched on above in section 10.3.

The process was applied to two application cases studies, which although they could both legitimately be viewed as SoS, were fundamentally different; the CAS case study was an example of an operational, rapidly formed and relatively easily bounded SoS whereas the wheat supply chain study was a relatively stable and large-scale enterprise SoS.

The SoS Sol dimensions (Kinder et al., 2012) were found to be an effective method in establishing the Sol for both case studies, providing further validation of them, beyond that originally provided, as described in the paper attached in Annex A.

The holistic risk categories; hazard, control, opportunity (Hopkin, 2002) were also suitably generic, allowing the proposed risk identification framework to be utilised for both case studies, whilst ensuring risk identification was undertaken through a SoS perspective.

The causal network allowed the relationships between risks and contributing factors to be established, with the network providing a visual representation which afforded an understanding of a complex set of relationships and which enabled this to be effectively communicated to stakeholders.

Modelling the risks and contributing factors using a set of BBNs enabled different mitigation approaches to be assessed, including system enhancements and risk transfer and was extremely effective in demonstrating the effects to stakeholders who could observe changes in probability propagated through the BBN. 
Use of supporting models reduced the level of subjectivity and increased the credibility of the risk analysis process, with the model selection tool identifying techniques that may not have initially been familiar to the practitioner.

The determination of an honest risk confidence was essential in order to establish trust in the analysis results, with the proposed approach allowing all aspects of the analysis to be allocated a confidence level which could then be combined into a single overall assessment, and which also allowed strategies to improve the confidence level to be assessed.

The overall benefit of the approach was the ability to identify and analyse risk in inherently complex situations which, if analysed through entirely subjective, judgement based assessment, would be prone to bias and error and beyond the capability of mental models, thereby breaking the law of requisite parsimony (Miller, 1956).

\subsection{Limitations of Approach}

Whilst section 10.3, provided a detailed critical analysis of the proposed SoS risk management approach, this section highlights the more general limitations.

As previously discussed, one of the proposed approach aims was to reduce the general level of subjectivity within the risk management process. Whilst this was achieved through the introduction of supporting models and the ability to enter known evidence into the BBNs, risk identification, definition of the BBN states and conditional probability tables still relied on subjective judgement. Indeed, the supporting models could also potentially be derived from subjective assumptions. However, this is an accepted limitation of model development in general and is acceptable if assumptions are clearly defined, enabling "honest" validation and confidence assessment.

A limitation of the approach, which became apparent under application case study two, is that risk analysis results are effectively a snapshot of risk likelihood over a short period. Whilst this was acceptable for the CAS study, which considered risks related to a mission undertaken over a short period, the second case study was concerned with risk over medium and long periods, potentially up to several years. In this case, the trend in risk would be more appropriate than a single output.

Case study 2 also illustrated that the complexity of the supporting models may be prohibitive and would require re-use of existing models, or the available output data. 


\subsection{Critique of Research}

Overall, the research was regarded as successful, with several outputs peer reviewed and published, with other outputs identified by SMEs as having real potential future application, for example the CAS SME saw real benefit if the models could be refined and then incorporated into a, potentially handheld, decision support tool, indicating immediate risk such as Fratricide.

A key foundation of the research was the SoS Sol model which introduced the associated dimensions (Kinder et al., 2012) and was published in the conference paper attached in Annex A. The development of the SoS Sol took a qualitative research approach, with a questionnaire to eleven purposefully selected participants. Whilst it is acknowledged that this is a relatively small group the participants possessed current, state of the art knowledge of the SoSE domain and the subsequent workshop allowed further in depth discussion of key issues. Use of the dimension in support of the application studies provided further validation of them. Indeed, their use appears to be gaining traction with reference made from the Systems Engineering Body of Knowledge (SEBoK) online resource (SEBoK, 2017) and various published papers. In addition the SoS Sol dimensions provided a framework for the description of case studies referenced by the T-Area SoS research project (Barot et al., 2013).

However, further work refining the SoS Sol dimensions through the application of additional case studies would be beneficial if undertaken as future research. Indeed, within this thesis it was necessary to provide descriptions of the relationships between the Sol dimensions (section 6.1), as although they were identified in the original paper (Annex A) they were not elaborated upon.

The literature review required three themes to be researched, SoSE, Modelling and Simulation and Risk. The Modelling and Simulation theme included a survey of a wide range of modelling techniques, assessing their applicability to the SoS domain. The results were published in a peer reviewed journal (attached in Annex C), however it was based upon expert judgement and would benefit from a survey of a wide range of participants, specifically when determining relevance to applicability to aspects of SoS. The framework devised to support this survey utilised the Sol SoS dimensions, where each technique was assessed against each dimension thereby providing more structured context in determining their applicability to SoS modelling. Further work could also include an expansion in the number of techniques surveyed. 
The demonstration of the proposed SoS risk management approach was achieved using a mixed method approach through SME consultation and development of models. The first case study was chosen because of the researchers familiarity with the subject matter and the access to a suitable SME with current and detailed knowledge of the military planning and CAS domain. This proved to be a good decision, allowing the research to focus on the proposed process, rather than an unfamiliar domain. The second case study arose through serendipity, as a result of a conference discussion with the UK Met Office who showed interest in the approach and mentioned its applicability to UK Food Security. Whilst this was an unfamiliar area to the researcher, it enabled the process, which was now more mature, to be applied to a radically different case. The second study was successful in highlighting areas where the process could be expanded and provided a worthwhile contrast to the first.

For both case studies input from the SME was initially elicited through unstructured interviews, in order to establish initial requirements. While this was successful it can be said with hindsight that semi-structured interviews may have been more successful in maintaining the discussion focus. However, this lesson was learnt for the application case study validation, resulting in shorter interviews providing results in a comparable format and which captured all required information.

\subsection{Summary of Chapter}

This chapter provided a summary flowchart illustrating the proposed SoS risk management process and showing its relationship to risk management activities. The theoretical basis for the approach is described through a set of principles and identification of underlying theories. The proposed process was critically assessed, where both positive and negative aspects were identified, more general limitations and benefits were also highlighted. A critique of the research approach was also undertaken, which although overall regarded as successful did suggest future improvements and discussed lessons learnt. 


\section{Chapter 11: Conclusions}

This chapter reviews achievement of this research against the original research questions and objectives.

\subsection{Assessment of Objectives}

Each research objective is discussed below with a brief description of the action taken to address it and an assessment of the achievement.

\section{Objective 1: Review of a wide range of modelling techniques applicability to SoS}

Objective 1 was addressed by theme 2 of the literature review, where thirteen modelling and simulation techniques were reviewed and assessed within a framework based the upon the Sol dimensions (Kinder et al., 2012). The results of this survey were published in a peer reviewed journal (Kinder et al., 2014), the paper is attached in Annex C.

The author considers that this objective is met, with the caveat that future research could expand the scope and survey additional techniques using the established framework.

\section{Objective 2: Review current risk management approaches.}

Theme 3 of the literature review surveyed traditional risks management approaches and the limited material available relating to SoS and risk.

The author considers that this objective is met.

\section{Objective 3: Identify differences between system and SoS risk management}

SoS Risk Management was identified as an essential element of SoSE in chapter 2 where several case studies were discussed and it was found that incorrect application may have contributed towards some notable failures. Theme 1 of the literature review compared SE with SoSE, discussing where risk management was appropriate whilst theme 3 surveyed current approaches. Chapter 7 discussed the application of risk management within SoSE, proposed new approaches and defined a model based SoS risk management process.

The author considers that this objective is met.

Objective 4: Identify an initial SoS application to explore whether a risk management model based approach would provide benefit.

Chapter 8 proposed risk management within Close Air Support mission planning as an application case study, consultation with an SME confirmed the validity of this choice. The author considers that this objective is met. 
Objective 5: Apply the SoS Risk Management process to the initial SoS application, developing models using appropriate identified modelling techniques.

The model based risk management process was applied to the Close Air Support mission planning application case study, as described in chapter 8, where BBNs and supporting models were developed. The results of the process were validated using SME input.

The author considers that this objective is met.

Objective 6: Identify a second application (case study) in order to determine the adaptability of using the overall approach in a different domain.

The second application was identified as UK National Food Security and the Wheat Supply Chain, the application of the SoS risk management process is documented in chapter 9 . The scope of the application was reduced in scope, in comparison to the first case study, however it was sufficient to demonstrate the adaptability of the proposed approach. A key finding resulting from the second case study application was that, where the SoS under consideration is subject to high levels of uncertainty the modelling approach is limited in its predictive capability. However, it does provide benefit through greater understanding of the risks and contributing factors, allowing "what if" scenarios to be developed and hence, risk mitigation strategies.

\section{Objective 7: Use model to assess transfer of risk within a SoS.}

The BBNs developed for both application case studies enabled different mitigation options to be evaluated and would enable the impact on other risks to be assessed. However, transfer of risk was not explicitly discussed.

The author considers that this objective is partially met.

Objective 8: Define a SoS risk management methodology in which the above approach can be encapsulated.

A SoS risk management process was proposed in chapter 7 which was refined through application cases studies in chapters 8 and 9 and summarised in chapter 10 .

Accepting this process was developed at a Concept level the author considers that this objective is met.

\subsection{Addressing the Research Questions}

This section considers each research question and assesses the extent to which they were addressed by this research. Although it is implicit that the questions were adequately 
addressed through the research objectives, they are assessed separately in this section in order to provide another viewpoint from which to validate the research.

The top-level question "How can risk be managed using a model based approach for a physical System of Systems in an operational environment?" was addressed through application of the proposed approach and is elaborated through the responses to the lower level questions below.

How is the management of SoS risk different from managing risk at the system level?

Risk Management of SoS requires a holistic view of the interactions between risk components and consequences, it cannot be assumed that if risk is "traditionally" managed for each component system then the aggregated affect will be to mitigate risk at the SoS level. SoS risk management is concerned with the likelihood of the fulfilment of the SoS purpose, which may be prevented through detrimental emergent behaviour, or conversely achievement is enhanced through beneficial emergent behaviour. Whilst a directed, and to some extent acknowledged, SoS (Dahmann \& Baldwin, 2008) will be controlled centrally, collaborative SoS requires a more cooperative approach to risk management.

\section{How is a model based approach beneficial?}

A SoS is inherently complex, the law of requisite parsimony states human beings can only deal simultaneously with between five and nine observations at one time (Miller, 1956), therefore attempting to manage SoS risk from a purely subjective, judgement based approach is likely to exceed the capacity of mental models. A mathematical model is not constrained in the same way, therefore enabling analysis of complex problems whilst providing understanding and prediction.

\section{How can a model based approach be developed and how can it quantify risk?}

A model based approach was developed utilising SoS Sol dimensions (Kinder et al., 2012) and a set of holistic risk types to create a risk identification framework, a causal network was used to represent the relationships between the risks and contributing factors. BBNs were used to model the causal network allowing probabilities to be propagated, thereby quantifying risk levels, with an associated confidence also provided. Supporting models could also be integrated into the BBNs using a Monte Carlo approach, with the choice of modelling technique appropriate to the subject under consideration.

\section{What are the types of risk associated with SoS?}


A Model Based Approach to System of Systems Risk Management

The generic holistic risk types, hazard, control and opportunity (Hopkin, 2002) were used in support of the model based approach, more specific risk types will be related to the SoS purpose and type.

\section{How does the application of risk management relate to the SoS 'lifecycle'?}

How Risk Management integrates into the SoSE approach is driven by the SoS classification; directed, acknowledged, collaborative and virtual (Dahmann \& Baldwin, 2008). Risk Management of SoS does not directly align with the traditional SE lifecycle, a more appropriate lifecycle approach is the DANSE model, comprising the phases, initiation, creation and operations (DANSE Consortium, 2015). During its Initiation phase the SoS comes into existence - risk management at this point will attempt to identify associated risks, which may result in undesired emergent behaviour, thereby preventing the SoS fulfilling its purpose. The creation phase is only applicable when the initial SoS emergent behaviour is severely deficient, this may be a pre-emptive activity as a result of risks identified within the initiation phase. In the Operational Phase the SoS provides the desired emergent behaviour, with risk management continuously undertaken to assess risks, which may impact the new SoS objectives and those that may impact current objectives.

\section{How can the impact of risk transfer be assessed within a SoS?}

The model based approach requires the creation of BBNs and supporting models enabling experimentation to be undertaken whereby different scenarios and mitigation strategies may be assessed. Risks may be owned by different components or dimensions within a SoS hence a mitigation strategy may result in one risk decreasing at the expense of another increasing.

\subsection{Summary of Research Contribution}

Although, this research provided insight into managing risk within a SoS through the development of a model based approach it also defined a novel method for the identification of the SoS Sol and an assessment of the applicability of modelling and techniques to SoS, which formed the basis of a technique selection tool. The key research contributions are summarised below:

- A model based SoS risk management process was defined, with a summary of initial research presented at 10th IEEE International Conference on System of Systems Engineering in 2015, the associated paper is attached in Annex C

- The SoS Sol model defined a set of dimensions and the relationships between them and is described in the paper attached in Annex A, which was presented at the 7th 
A Model Based Approach to System of Systems Risk Management

IEEE International Conference on System of Systems Engineering in 2012. This is a key element of the SoS risk management process, underpinning the risk identification activity and the selection of appropriate M\&S methods.

- A method was defined which supported the integration of heterogeneous model ouputs into a central BBN using a Monte Carlo approach producing an estimate of risk. This is a novel technique which is essential to the modelling of SoS risk.

The following contributions may be regarded as supplementary, each contributing to elements of the model based SoS risk management process:

- The modelling and simulation survey assessed a range of techniques using a framework based upon the SoS Sol dimensions, the results of which were published in the International Journal of Systems of Systems Engineering Vol. 5, No. 2, 2014 with the paper attached in Annex C.

- A spreadsheet-based tool was implemented based on the contribution above, which enabled model requirements to be input and a subset of appropriate techniques identified.

- A framework to support the identification of SoS risks was defined, utilising the SoS Sol and a set of holistic risk types.

- A method to present and interpret quantified SoS risk results was introduced, which allowed risk analysis results to be assessed relatively against best and worst case values.

- A method to determine confidence associated with risk analysis results was defined.

In addition, the following outputs were produced as a result of the case study application:

- A set of causal networks, BBNs and supporting models were produced which supported analysis of SoS risk associated with CAS missions.

- An initial causal network and BBN was produced which supported the analysis of SoS risk associated with food security and the wheat food supply chain.

Key insights gained from the research were:

- Risk Management of SoS requires a different approach to that currently taken for individual systems, if risk is managed for each component system then it cannot be assumed that the aggregated affect will be to mitigate risk at the SoS level. 
- Establishing the SoS Sol is essential for effective SoS risk identification, providing a clear context when eliciting subject matter expertise. The SoS Sol enables risk transfer to be distinguished from mitigation, which is transfer to outside the SoS boundary.

- Due to the complex and heterogeneous nature of SoS, effective modelling requires a range of techniques where suitability is determined by the problem context. A Bayesian modelling approach was found to be suitable for representing and analysing SoS risk.

- The responsibility for SoS Risk Management is related to the overall SoS classification whereby directed and acknowledged SoS allow a single body to perform Risk Management but a collaborative SoS requires an advisory body or a bottom up cooperative approach. However, Risk Management cannot be applied to virtual SoS due to the "lack a central management authority and a centrally agreed upon purpose for the system-of-systems"

This research was also presented at webinars to the INCOSE SOS Working Group and Systems of Systems Engineering Collaborators Info Exchange (SoSCIE).

\subsection{Future Work and Exploitation}

This section identifies future work, taking the outputs from this research as starting points and developing them further. It also identifies potential industrial exploitation paths.

Although the SoS Sol dimensions were found to be an effective approach to establishing a specific Sol, future research could apply further case studies and further refine the underlying definitions, which in some cases were somewhat unclear.

The survey of modelling and simulation techniques covered a reasonable range but further research could extend the scope whilst using the same assessment framework. This could potentially be developed into an online resource, which could also encompass the selection tool.

The first application case study could be further refined. The conditional probability tables would require detailed SME input and the fidelity of the supporting models would require enhancement. The enhanced solution could be exploited further through the development of a handheld tool, which could provide real-time, or near real-time, CAS risk assessments for use in operational situations. 
The scope of the second case study was necessarily reduced because its purpose was purely intended to demonstrate the adaptability of the SoS risk management process. Further research could fully apply the process in the context of the food supply chain and the creation of appropriate supporting models.

It is suggested that further development of this research beyond the concept stage should first revisit and refine the SoS Sol model, and then expand the model technique selection tool to incorporate a wider range of techniques, the case studies could then be developed further in parallel.

Exploitation of the SoS risk management approach could be achieved through further conference presentation and introduction into SOSE guides, for example those produced by INCOSE.

The SoS risk management process is generic and is intended to be applicable to all directed, acknowledged and collaborative (Dahmann \& Baldwin, 2008) SoS. Figure 71 in Section 10.1 provides a flowchart indicating the activities and their sequence comprising the process, which may therefore be followed when applying it to SoS beyond those examined in the two case studies.

\subsection{Concluding Remarks}

The case studies discussed in chapter 2 clearly identified a need for a more effective approach to the management of risk within SoS, with examples of SoS failure resulting in human and financial loss cited where risks were not identified and mitigated.

This research has furthered the understanding of risk management application to SoS and developed a pragmatic model based approach that was demonstrated to be adaptable to different types of SoS. As part of the process, a novel approach enabling the integration of heterogeneous models was developed which has application beyond the bounds of SoS risk management.

Within our highly networked world, SoS are now pervasive, therefore an improved understanding of risk in this context and a practical approach to managing it will hopefully be of great benefit. 


\section{References}

Ackoff, R. L. (1971). Towards a System of Systems Concepts. Management Science. https://doi.org/10.1287/mnsc.17.11.661

Adams, K. M. (2011). Systems principles: foundation for the SoSE methodology. International Journal of System of Systems Engineering, 2(2/3), 120. https://doi.org/10.1504/IJSSE.2011.040550

Aitken, J. M., Alexander, R., \& Kelly, T. (2011). A risk modelling approach for a communicating system of systems. 2011 IEEE International Systems Conference, SysCon 2011 - Proceedings, 442-447. https://doi.org/10.1109/SYSCON.2011.5929099

Alberts, D. S. (2003). Power to the Edge: Command, Control in the Information Age. Information Age. https://doi.org/10.1038/nri2836

Ashby, W. R. (1961). An Introduction to Cybernetics. London, UK: Chapman and Hall Ltd. https://doi.org/10.2307/3006723

Axelband, E., Drezner, J. A., Pernin, C. G., Held, B. J., McMahon, K. S., Dille, B. B., ... Sollinger, J. M. (2012). Lessons from the Army's Future Combat Systems Program. https://doi.org/10.1214/07-EJS057

Balci, O. (1995). Principles and techniques of simulation validation, verification, and testing. Winter Simulation Conference Proceedings, 1995., 147-154. https://doi.org/10.1109/WSC.1995.478717

Barot, V., Henshaw, M., Siemieniuch, C., Sinclair, M., Lim, S. L., Henson, S., ... DeLaurentis, D. (2013). SoA Report Work Package 2, Deliverable D2.1 Revised version. Loughborough.

Bartha, P. (2016). Analogy and Analogical Reasoning. Retrieved December 6, 2016, from https://plato.stanford.edu/cgi-bin/encyclopedia/archinfo.cgi?entry=reasoning-analogy

Berry, B. (1964). Cities as Systems with Systems of Cities. Papers in Regional Science, 13(1), 147-163.

Boardman, J., \& Sauser, B. (2006). System of Systems - the meaning of of. Smc, (April), 1-6. https://doi.org/10.1109/SYSOSE.2006.1652284

Box, G. E. P. (1954). Robustness in the Strategy of Scientific Model Building. Madison, USA.

Bretti, G., Natalini, R., \& Piccoli, B. (2007). A fluid-dynamic traffic model on road networks. Archives of Computational Methods in Engineering, 14(2), 139-172. https://doi.org/10.1007/s11831-007-9004-8

Bristow, M., Fang, L., \& Hipel, K. W. (2012). System of Systems Engineering and Risk Management of Extreme Events: Concepts and Case Study. Risk Analysis, 32(11), 19351955. https://doi.org/10.1111/j.1539-6924.2012.01867.x

British Standards. (2011). BS 15288:2015 BSI Standards Publication Systems and software engineering - Life cycle management, 15288.

Brook, P. (2016). On the Nature of Systems of Systems. In 26th Annual INCOSE International Symposium. Edinburgh. https://doi.org/10.1002/j.2334-5837.2016.00240.x

Brown, S. (2009). Naivety in Systems Engineering Research: are we putting the methodological cart before the philosophical horse. In 7th Annual Conference on Systems Engineering Research 2009. Loughborough.

Carson, J. S. (2005). Introduction To Modeling and Simulation. In Proceedings of the 2005 
A Model Based Approach to System of Systems Risk Management

Winter Simulation Conference (pp. 16-23). https://doi.org/10.1109/WSC.2005.1574235

Comfort, L. K. (2007). Crisis management in hindsight: Cognition, communication, coordination, and control. Public Administration Review, 67, 189-197.

COMPASS Consortium. (2014a). Final Report on Guidelines for SoS Engineering.

COMPASS Consortium. (2014b). Guidelines for Architectural Modelling of SoS.

Conrow, E. (2005). Risk Management for Systems of Systems. Crosstalk: The Journal of Defence Software Engineering, 18(2), 8-12.

Cooper, G. F. (1990). The computational complexity of probabilistic inference using Bayesian belief networks. Artificial Intelligence, 42(2-3), 393-405.

COSO. (2016). Enterprise Risk Management - Aligning Risk with Strategy and Performance.

Creel, R., \& Ellison, B. (2008). System-of-systems influences on acquisition strategy development. Software Engineering Institute.

Creswell, J. (2014). Reserach Design: Qualitative, Quantitative, and Mixed Methods Approaches. Journal of Chemical Information and Modeling (4th ed., Vol. 53). SAGE Publications.

Crotty, M. (2012). The foundations of social research: Meaning and perspective in the research process. Thousand Oaks, CA: SAGE Publications.

Dahmann, J. (2014). System of Systems Pain Points. In INCOSE International Symposium (pp. 108-121). https://doi.org/10.1002/j.2334-5837.2014.tb03138.x

Dahmann, J., \& Henshaw, M. (2016). Introduction to Systems of Systems Engineering. INSIGHT, 19(3), 12-16.

Dahmann, J., \& Roedler, G. (2016). Moving Towards Standardization for System of Systems Engineering. In 2016 11th System of Systems Engineering Conference (pp. 1-6). https://doi.org/10.1109/SYSOSE.2016.7542953

Dahmann, J. S., \& Baldwin, K. J. (2008). Understanding the Current State of US Defense Systems of Systems and the Implications for Systems Engineering. 2008 2nd Annual IEEE Systems Conference, 1-7. https://doi.org/10.1109/SYSTEMS.2008.4518994

DANSE Consortium. (2015). DANSE Methodology V03.

DASDSE. (2015). Risk, Issue, and Opportunity Management Guide for Defense Acquisition Programs, (June), 116. Retrieved from http://www.acq.osd.mil/se

Day, A. L. (2008). Mastering Risk Modelling: A Practical Guide to Modelling Uncertainty with Microsoft Excel. Pearson Prentice Hall.

DeForge, B. R. (2010). Research Design Principles. In Encyclopedia of Research Design (pp. 1253-1259). SAGE Publications. https://doi.org/10.4135/9781412961288

DEFRA. (2006). Food Security and the UK: An Evidence and Analysis Paper. Food Chain Analysis Group, (December), 1-87. Retrieved from http://www.ifr.ac.uk/waste/Reports/Defra-foodsecurity.pdf

Diabat, A., Govindan, K., \& Panicker, V. V. (2012). Supply chain risk management and its mitigation in a food industry. International Journal of Production Research, 50(11), 3039-3050. https://doi.org/10.1080/00207543.2011.588619

DoD. (2014). Chapter 4 - Systems Engineering. Defense Acquisition Guidebook, (May). 
Eisner, H. (2008). Essentials of project and systems engineering management. John Wiley \& Sons, Inc.

Eisner, H., Marciniak, J., \& McMillan, R. (1991). Computer-aided System of Systems (S2) Engineering. IEEE International Conference on Systems, Man, and Cybernetics, 531-7. https://doi.org/10.1109/ICSMC.1991.169739

Environment Food and Rural Affairs Committee. (2015). Food security: demand, consumption, and waste (Vol. II).

Epstein, J. M. (2008). Why Model? Journal of Artificial Societies and Social Simulation, 11(4), 12. Retrieved from http://jasss.soc.surrey.ac.uk/11/4/12.html

FAO. (1996). Rome Declaration on World Food Security. Retrieved May 9, 2017, from http://www.fao.org/docrep/003/w3613e/w3613e00.htm

FERC, \& NERC. (2014). FERC/NERC Staff Report on the September 8, 2011 Blackout.

Ferris, T. L. J. (2009). On the Methods of Research for Systems Engineering. 7th Annual Conferenceon Systems Engineering Research 2009 (CSER 2009), 2009(April), 7.

Finn V. Jensen; Thomas Dyhre Nielsen. (2007). Bayesian Networks and Decision Graphs. New York: Springer.

Flyvbjerg, B., Garbuio, M., \& Lovallo, D. (2009). Deception in Large Infrastructure Projects : California Management Review, 51(2), 170-194. https://doi.org/10.1225/CMR423

Fowler, F. J. (2009). Survey research methods (4th ed.). Thousand Oaks, CA: SAGE Publications.

Friedenthal, S., Moore, A., \& Steiner, R. (2009). A Practical Guide to SysML: The Systems Modeling Language. San Francisco: Morgan Kaufmann Publishers Inc.

Fritzson, P. (2010). System Modeling Methodology and Continuous Model Representation. In Principles of Object-Oriented Modeling and Simulation with Modelica 2.1 (pp. 381403). John Wiley \& Sons, Inc.

Gandhi, S. J., Gorod, A., \& Sauser, B. (2012a). A Systemic Approach to Managing Risks of SoS. Aerospace and Electronic Systems Magazine, (May), 23-27. https://doi.org/10.1109/SYSCON.2011.5929045

Gandhi, S. J., Gorod, A., \& Sauser, B. (2012b). A Systemic Approach to Managing Risks of SoS. Aerospace and Electronic Systems Magazine, 23-27. https://doi.org/10.1109/SYSCON.2011.5929045

Ganguly, A., Mansouri, M., \& Nilchiani, R. (2010). A Risk Assessment Framework for analyzing risks associated with a Systems Engineering Process. 2010 IEEE International Systems Conference, 484-489. https://doi.org/10.1109/SYSTEMS.2010.5482460

Garcia, D., Mavrodiev, P., \& Schweitzer, F. (2013). Social Resilience in Online Communities: The Autopsy of Friendster. In Proceedings of the first ACM conference on Online social networks (pp. 39-50). ACM. https://doi.org/10.1145/2512938.2512946

Garvey, P. R. (2004). Analytical Methods for Risk Management: An Engineering Systems Perspective. In Encyclopedia of Statistical Sciences. John Wiley \& Sons, Inc. https://doi.org/10.1002/0471667196.ess7135

Ghadge, A., Dani, S., Chester, M., \& Kalawsky, R. (2013). A systems approach for modelling supply chain risks. Supply Chain Management-an International Journal, 18(5), 523-538. https://doi.org/10.1108/SCM-11-2012-03661 
Glick, B. (2012). Everybody Lost in NHS IT Disaster. Retrieved December 3, 2016, from http://www.computerweekly.com/blogs/editors-blog/2012/09/everybody-lost-in-nhsit-disas.html

Gorod, A., Gove, R., Sauser, B., \& Boardman, J. (2007). System of Systems Management: A Network Management Approach. 2007 IEEE International Conference on System of Systems Engineering, 1-5. https://doi.org/10.1109/SYSOSE.2007.4304218

Government Accounting Office. (2011). Coast Guard Action Needed As Approved Deepwater Program Remains Unachievable.

Guba, E. G. (1990). The paradigm dialog. Newbury Park, CA: SAGE Publications.

Haimes, Y. Y. (2012). Modeling Complex Systems of Systems with Phantom System Models. Systems Engineering, 15(3), 333-346. https://doi.org/10.1002/sys

Haimes, Y. Y. (2015). Risk modeling, assessment, and management. John Wiley \& Sons, Inc.

Halford, G. S., Baker, R., Mccredden, J. E., Bain, J. D., Halford, G. S., Baker, R., ... Bain, J. D. (2011). How Many Variables Can Humans Process?, 16(1), 70-76. https://doi.org/10.1111/j.0956-7976.2005.00782.x

Hart, C. (1998). The Literature review in research. Doing a Literature Review. SAGE Publications.

Henshaw, M., Siemieniuch, C., Sinclair, M., Henson, S., Barot, V., Jamshidi, M., ... Dogan, H. (2013). Systems of Systems Engineering: A research imperative. Iccse, 389-394. https://doi.org/10.1109/ICSSE.2013.6614697

Hesse, M. (1963). Models and analogies. Models and Analogies in Science. https://doi.org/10.1111/b.9780631230205.2001.00047.x

Hillson, D. (2003). Effective Opportunity Management for Projects: Exploiting Positive Risk. CRC Press.

Hipel, K. W., Fang, L., \& Heng, M. (2010). System of systems approach to policy development for global food security. Journal of Systems Science and Systems Engineering, 19(1), 121. https://doi.org/10.1007/s11518-010-5122-1

Hopkin, P. (2002). Holistic Risk Management in Practice. Witherby \& Co Ltd.

Hubbard, D. W. (2009). The failure of risk management: why it's broken and how to fix it. John Wiley and Sons.

IEEE. (1989). IEEE Standard Glossary of Modeling and Simulation Terminology. IEEE Std 610.3-1989. https://doi.org/10.1109/IEEESTD.1989.94599

INCOSE. (2007). Systems Engineering Vision 2020. Retrieved from http://www.incose.org/ProductsPubs/pdf/SEVision2020_20071003_v2_03.pdf

INCOSE. (2015). Systems Engineering Handbook $4 E$.

International Organization for Standardization. (1989). Information processing systems Open Systems Interconnect - Basic Reference Model: Part 4 - Management Framework,. In ISO/IEC 7498. International Standards Organization.

International Organization for Standardization. (2009a). ISO/IEC 31010:2009 Risk management - Risk assessment techniques. Risk Management, 31010, 92.

International Organization for Standardization. (2009b). PD ISO Guide 73: 2009 BSI Standards Publication Risk management - Vocabulary.

International Organization for Standardization. (2009c). Risk management - Principles and 
Guidelines BS ISO 31000:2009.

ISO/IEC. (2006). International Standard Iso / lec 16085 - Systems and software engineering - Life cycle processes - Risk management (Vol. 25021). https://doi.org/10.1109/IEEESTD.2015.7106438

Jamshidi, M. (2008). System of systems engineering - New challenges for the 21st century. Aerospace and Electronic Systems Magazine, IEEE, 23(5), 4-19. https://doi.org/10.1109/MAES.2008.4523909

John J Schaefer III. (2012). Repsonsive Close Air Support. Joint Force Quarterly, (67), 91-96.

Kahneman, D., Tversky, A., Kahneman, B. Y. D., \& Tversky, A. (2016). Prospect Theory : An Analysis of Decision under Risk Linked references are available on JSTOR for this article : All use subject to http://about.jstor.org/terms E C O N OMETRICA I C I, 47(2), 263-292.

Keating, C. B. (2009). Emergence in system of systems. In M. Jamshidi (Ed.), Systems of Systems Engineering: Innovations for the 21st Century (pp. 169-190). Hoboken, NJ: John Wiley and Sons.

Keating, C. B., \& Katina, P. F. (2011). Systems of systems engineering: prospects and challenges for the emerging field. International Journal of System of Systems Engineering, 2(2), 234-256. https://doi.org/10.1504/IJSSE.2011.040556

Kemp, D., Camm, D., Evans, R., \& Elphick, J. (2013). 2.1.1 Steampunk System of Systems Engineering: A case study of successful System of Systems engineering in $19^{\text {th }}$ century Britain. INCOSE International Symposium, 23(1), 617-633. https://doi.org/10.1002/j.2334-5837.2013.tb03043.x

Kilgour, D. M., \& Hipel, K. W. (2005). The graph model for conflict resolution: Past, present, and future. Group Decision and Negotiation, 14(6), 441-460. https://doi.org/10.1007/s10726-005-9002-x

Kinder, A., Barot, V., Henshaw, M., \& Siemieniuch, C. (2012). System of systems: "Defining the system of interest." In Proceedings - 2012 7th International Conference on System of Systems Engineering, SOSE 2012 (pp. 463-468).

Kinder, A., Henshaw, M., \& Siemieniuch, C. (2014). System of systems modelling and simulation - an outlook and open issues. International Journal of System of Systems Engineering, 5(2), $150 . \quad$ Retrieved from http://www.scopus.com/inward/record.url?eid=2-s2.084907448498\&partnerID=tZOtx3y1

Kinder, A., Henshaw, M., \& Siemieniuch, C. (2015). A Model Based Approach to System of Systems Risk Management. Int. J. of Complex Systems - Computing, Sensing and Control, 3(1), 25-35. https://doi.org/10.1109/SYSOSE.2015.7151940

Korn, J. (2011). Deepwater RIP. Service Lines.

Kovacic, S., Sousa-Poza, A., \& Keating, C. (2008). Complex situations: An alternative approach for viewing a System of Systems. 2008 IEEE International Conference on System of Systems Engineering, SoSE 2008, 1-6. https://doi.org/10.1109/SYSOSE.2008.4724167

Lamothe, D. (2014, September). Investigation: Friendly fire airstrike that killed U.S. Special Forces was avoidable. Washington Post. Washington. Retrieved from https://www.washingtonpost.com/news/checkpoint/wp/2014/09/04/investigationfriendly-fire-airstrike-that-killed-u-s-special-forces-wasavoidable/?utm_term=.cd93f08ce691 
Lampis, M. (2010). Application of Bayesian Belief Networks to system fault diagnostics. Loughborough University.

Lauritzen, S. L., \& Spiegelhalter, D. J. (1988). Local Computations with Probabilities on Graphical Structures and Their Application to Expert Systems. Journal of the Royal Statistical Society. Series B (Methodological), 50(2), 157-224.

Law, A. (2009). How to Build and Valid and Credible Simulation Models. In Proceedings of the 2009 Winter Simulation Conference (pp. 24-33).

Levy, Y., \& Ellis, T. J. (2006). A Systems Approach to Conduct an Effective Literature Review in Support of Information Systems Research. Information Science Journal, 9, 181-212. https://doi.org/10.1049/cp.2009.0961

Lincoln, Y. S., Lynham, S. A., \& Guba, E. G. (2011). Paradigmatic controversies, contradictions, and emerging confluences, revisited. In The Sage handbook of qualitative research 4 (pp. 97-128).

Lipsey, M. W. (1990). Design sensitivity: Statistical power for experimental research. Newbury Park, CA: SAGE Publications.

Lochow, T. (2016). Tool and Techniques - DANSE. INSIGHT, 19(3), 55-57.

Lockheed Martin. (2016). iSMART. Retrieved December 4, 2016, from http://www.lockheedmartin.com/uk/what-we-

do/products/InteroperableSystemsManagementandRequirementsTransformation.html

Lynch, C., Padilla, J., Diallo, S., Sokolowski, J., \& Banks, C. (2014). A multi-paradigm modeling framework for modeling and simulating problem situations. In Proceedings - Winter Simulation Conference (Vol. 2015-Janua, pp. 1688-1699). IEEE. https://doi.org/10.1109/WSC.2014.7020019

Macharis, C. (2000). Hybrid Modeling: System Dynamics combined with Multi-criteria Analysis. Submitted for Publication. Retrieved from http://www.researchgate.net/publication/228800843_Hybrid_Modeling_System_Dyna mics_combined_with_Multi-criteria_Analysis/file/d912f5135ffd5861cf.pdf

Maier, M. W. (1998). Architecting Principles for Systems-of-Systems. Systems Engineering, $1(4), \quad 267-284 . \quad$ https://doi.org/10.1002/(SICI)1520-6858(1998)1:4<267::AIDSYS3>3.0.CO;2-D

Martin, J. N., \& Davidz, H. L. (2007). Systems Engineering Case Study Development. Proc CSER 2007, (May).

Mertens, D. M. (2010). Research and evaluation in education and psychology: Integrating diversity with quantitative, qualitative, and mixed methods (3rd ed.). Thousand Oaks, CA: SAGE Publications.

Metropolis, N., \& Ulam, S. (1949). The Monte Carlo Method. Journal of the American Statistical Association, 44(247), 335-341. https://doi.org/10.1080/01621459.1949.10483310

Miller, G. A. (1956). The magical number seven, plus or minus two: some limits on our capacity for processing information. Psychological Review, 63(2), 81-97. https://doi.org/10.1037/h0043158

MOD. (2012). MOD Architecture Framework. Retrieved December 6, 2016, from https://www.gov.uk/guidance/mod-architecture-framework

Musser, W. N., \& Patrick, G. F. (2002). Musser, Wesley N., and George F. Patrick. "How much 
does risk really matter to farmers? In A comprehensive assessment of the role of risk in US agriculture (pp. 537-556). Springer US.

National Audit Office. (2009). The National Offender Management Information System.

National Audit Office. (2011). The National Programme for IT in the NHS: an update on the delivery of detailed care records systems. Forty-Fifth Report of Session 2010-12, (July), 1-80. Retrieved from papers3://publication/uuid/77BC75EB-2DA4-48EC-8699CEC26891238E

National Audit Office. (2012). AUDITOR GENERAL Restructuring of the National Offender Management Service Key facts. London, UK.

National Police Improvement Agency. (2009). Guidance on Command and Control.

National Weather Service. (2017). FAQ - What is the Meaning of PoP. Retrieved June 25, 2017, from https://www.weather.gov/ffc/pop

NATO. (2005). ATP-3.3.2.1(A) - TACTICS, TECHNIQUES AND PROCEDURES FOR CLOSE AIR SUPPORT OPERATIONS.

NATO. (2008). Standardization Agreement TDL 5516 Ed6.

Neuman, W. L. (2003). Social Research: Qualitative and Quantitative Approaches. Social Research Methods: Qualitative and Quantitative Approaches (7th ed.). Boston: Allyn \& Bacon. https://doi.org/10.1234/12345678

Object Management Group. (2016a). SYSML. Retrieved from http://www.omgsysml.org/index.htm

Object Management Group. (2016b). UML Profile for DoDAF/MODAF Resource Page. Retrieved December 7, 2016, from http://www.omg.org/updm/

Patton, M. (1990). Qualitative evaluation and research methods (2nd ed.). Newbury Park, CA: SAGE Publications.

Pearl, J. (1986). Fusion, propagation, and structuring in belief networks. Artificial Intelligence, 29(3), 241-288. https://doi.org/10.1016/0004-3702(86)90072-X

Pearl, J. (2014). Probabilistic reasoning in intelligent systems: networks of plausible inference. Morgan Kaufmann Publishers Inc.

Pinto, C. A., McShane, M. K., \& Bozkurt, I. (2012). System of systems perspective on risk: towards a unified concept. International Journal of System of Systems Engineering, 3(1), 33. https://doi.org/10.1504/IJSSE.2012.046558

Pinto, R. M. (2010). Mixed Methods Design. In Encyclopedia of Research Design (pp. 813818). SAGE Publications. https://doi.org/10.4135/9781412961288

Popper, K. R. (2002). The logic of scientific discovery. 1959. London: Hutchinson (Vol. 268). https://doi.org/10.1016/S0016-0032(59)90407-7

Public Accounts Committee. (2009). Public Accounts Committee - Fortieth Report The National Offender Management Information System. London, UK. Retrieved from http://www.publications.parliament.uk/pa/cm200809/cmselect/cmpubacc/510/51002 .htm

Qinetiq. (2005). NHS Connecting for Health.

Rabelo, L., Helal, M., Jones, A., \& Min, H.-S. (2005). Enterprise simulation: a hybrid system approach. International Journal of Computer Integrated Manufacturing, 18(6), 498508. https://doi.org/10.1080/09511920400030138 
Robinson, S. (1997). SIMULATION MODEL VERIFICATION AND VALIDATION: INCREASING THE USERS' CONFIDENCE. In The 1997 Winter Simulation Conference (pp. 53-59). Atlanta, Georgia, USA: IEEE Computer Society.

Ross, W., Ulieru, M., \& Gorod, A. (2014). A Multi-Paradigm Modelling \& Simulation Approach for System of Systems Engineering : A Case Study. SoSE, 183-188.

Sargent, R. G. (2015). A tutorial on verification and validation of simulation models. Proceedings of the 2015 Winter Simulation Conference, 1729-1740. Retrieved from http://dl.acm.org/citation.cfm?id=809441

Saunders, M., Lewis, P., \& Thornhill, A. (2009). Research Methods for Business Students. Research methods for business students (5th ed.). Harlow: Pearson Education Limited. https://doi.org/10.1007/s13398-014-0173-7.2

SEBoK. (2017). Systems Engineering Body of Knowledge v1.8. Retrieved December 6, 2016, from http://sebokwiki.org/wiki/Guide_to_the_Systems_Engineering_Body_of_Knowledge_( SEBOK)

Security Service MI5. (2017). THREAT LEVELS. Retrieved June 4, 2017, from https://www.mi5.gov.uk/threat-levels

Shah, P., Davendralingam, N., \& DeLaurentis, D. A. (2015). A conditional value-at-risk approach to risk management in system-of-systems architectures. 2015 10th System of Systems Engineering Conference, SOSE 2015, 457-462. https://doi.org/10.1109/SYSOSE.2015.7151969

Shenhar, A. J. (1995). 2.5.1 a New Systems Engineering Taxonomy. INCOSE International Symposium, 5(1), 723-732. https://doi.org/10.1002/j.2334-5837.1995.tb01933.x

Skinner, R. L. (2007). STATEMENT OF RICHARD L. SKINNER INSPECTOR GENERAL U.S. DEPARTMENT OF HOMELAND SECURITY BEFORE THE COMMITTEE ON TRANSPORTATION AND INFRASTRUCTURE SUBCOMMITTEE ON COAST GUARD AND MARITIME TRANSPORTATION U.S. HOUSE OF REPRESENTATIVES Deepwater: 120-Day Updat. U.S. DEPARTMENT OF HOMELAND SECURITY. Retrieved from https://www.oig.dhs.gov/assets/TM/OIGtm_RLS_061207.pdf

Slovic, P., \& Peters, E. (2006). Risk perception and affect. , 15(6), 322-325. Current Directions in Psychological Science, 15(6), 322-325. https://doi.org/10.1177/03063127067078012

Sousa-Poza, A., Padilla, J. J., \& Bozkurt, I. (2008). Implications of a Rationalist Inductive Approach in System of Systems Engineering Research Concept of Research. System of Systems Engineering, 2008. SoSE'08. IEEE International Conference on, 1-6.

Spyridopoulos, T., Maraslis, K., Tryfonas, T., Oikonomou, G., \& Li, S. (2014). Managing cyber security risks in industrial control systems with game theory and viable system modelling. Proceedings of the 9th International Conference on System of Systems Engineering: The Socio-Technical Perspective, SOSE 2014, 266-271. https://doi.org/10.1109/SYSOSE.2014.6892499

Sterman, J. D. (2002). All models are wrong: Reflections on becoming a systems scientist. System Dynamics Review, 18(4), 501-531. https://doi.org/10.1002/sdr.261

Sulistio, A., Yeo, C. S., \& Buyya, R. (2004). A taxonomy of computer-based simulations and its mapping to parallel and distributed systems simulation tools. Software - Practice and Experience, 34(7), 653-673. https://doi.org/10.1002/spe.585

Taleb, N. N. (2007). The black swan: the impact of the highly improbable. New York: 
Random House.

Teose, M., Lu, Z., Ellner, S. P., Smith, R. L., \& Gomes, C. (2007). Embedding System Dynamics in Agent Based Models for Complex Adaptive Systems. International Joint Conference on Artificial Intelligence Embedding, 2531-2538.

Townsend, F. F. (2006). The Federal Response to Hurricane Katrina: Lessons Learned. Retrieved from https://georgewbush-whitehouse.archives.gov/reports/katrina-lessonslearned/index.html

US Department of Defense. (2006). Risk management guide for DoD Acquisition, (May), 4. Retrieved from http://www.dau.mil/publications/publicationsDocs/RMG 6Ed Aug06.pdf

US Department of Defense. (2008a). Department of Defense Modeling and Simulation Body of Knowledge (BOK). Retrieved from https://www.csiac.org/sites/default/files/DoD M\&S BOK (M\&SCO) 2008_0.pdf

US Department of Defense. (2008b). Systems Engineering Guide for Systems of Systems. Technology (Vol. 36). https://doi.org/10.1109/EMR.2008.4778760

Valerdi, R., \& Davidz, H. L. (2008). Empirical Research in Systems Engineering : Challenges and Opportunities of a New Frontier. Systems Engineering, 12(2), 169-181. https://doi.org/10.1002/sys

Van Der Vorst, J., Da Silva, C. A., \& Trienekens, J. H. (2007). Agro-industrial supply chain management: concepts and applications. Rome.

Von Bertalanffy, L. (1968). General System Theory. Georg. Braziller New York, 1, 289. Retrieved from http://books.google.es/books?id=N6k2mILtPYIC

Weber, P., Medina-Oliva, G., Simon, C., \& lung, B. (2012). Overview on Bayesian networks applications for dependability, risk analysis and maintenance areas. Engineering Applications of Artificial Intelligence, 25(4), 671-682. https://doi.org/10.1016/j.engappai.2010.06.002

Webster, J., \& Watson, R. T. (2002). ANALYZING THE P AST TO PREPARE FOR THE FUTURE : WRITING A LITERATURE R EVIEW. MIS Quarterly, 26(June), xiii-xxxiii.

Zachary Taylor, \& Ranganathan, S. (2013). Designing High Availability Systems: Design for Six Sigma and Classical Reliability Techniques with Practical Real-Life Examples. The Institute of Electrical and Electronics Engineers, Inc.

Zio, E., \& Ferrario, E. (2013). A framework for the system-of-systems analysis of the risk for a safety-critical plant exposed to external events. Reliability Engineering and System Safety, 114(1), 114-125. https://doi.org/10.1016/j.ress.2013.01.005 


\section{Appendix A - SoS SoI Questionnaires}

This appendix provides evidence of the methodology applied in determining the SoS Sol dimensions through the provision of questionnaire results completed by purposefully selected participants. It should be noted that the responses included are as completed and have not been subsequently paraphrased, amended or reformatted.

\section{PARTICIPANT 1}

What are the Dimensions of the System of Interest for a SoS?

\begin{tabular}{|c|c|c|}
\hline Proposed Dimension & $\begin{array}{l}\text { This } \\
\text { dimension is } \\
\text { relevant to } \\
\text { the SOl of a } \\
\text { SoS? }\end{array}$ & Comment \\
\hline Component Systems & 4 & \\
\hline Interactions & 4 & \\
\hline \multicolumn{2}{|l|}{ Time } & \\
\hline - $\quad$ Lifecycle & 3 & \\
\hline - $\quad$ Latency & 3 & \\
\hline$\bullet$ & & \\
\hline Classification $^{2}$ & 3 & \\
\hline Functions / Services & 3 & \\
\hline Enterprise Participants & 3 & \\
\hline Capabilities & 4 & \\
\hline Disciplines & 2 & \\
\hline $\begin{array}{l}\text { Concept of Operation / Use / } \\
\text { Employment }\end{array}$ & 4 & \\
\hline Geographic Distribution & 2 & \\
\hline Emergence & 4 & \\
\hline
\end{tabular}

SoS M\&S Outputs

\begin{tabular}{|l|l|l|}
\hline SoS M\&S Output Dimensions ${ }^{\mathbf{3}}$ & $\begin{array}{l}\text { Which M\&S techniques enable } \\
\text { measurement of this output? }\end{array}$ & $\begin{array}{l}\text { How reliable is this } \\
\text { measurement? }\end{array}$ \\
\hline Emergent Behaviour & & \\
\hline Connectivity & & \\
\hline Interoperability & & \\
\hline Agility & & \\
\hline Cost/ Balance of Investment & & \\
\hline Effectiveness (ability to achieve purpose) & & \\
\hline $\begin{array}{l}\text { Sustainability (deliver level of performance } \\
\text { despite interference) }\end{array}$ & & \\
\hline
\end{tabular}

1 1=Strongly Disagree, 2=Disagree, 3=Neutral, 4=Agree, 5=Strongly Agree

${ }^{2}$ Assume Directed, Acknowledged, Collaborative and Virtual, until something better comes along

${ }^{3}$ Some are re-used from Evaluating SoS Against Mission Requirements, P Johnson 2009, PhD Thesis

${ }^{4}$ 1=Extremely Unreliable, 2=Unreliable, 3=Neutral, 4=Reliable, 5=Extremely Reliable 


\begin{tabular}{|l|l|l|}
\hline $\begin{array}{l}\text { Survivability (to function during and after } \\
\text { environment disturbance) }\end{array}$ & & \\
\hline Risk & & \\
\hline Performance & & \\
\hline Safety (Hazard Analysis) & & \\
\hline Evolution & & \\
\hline Autonomy & & \\
\hline Reliability & & \\
\hline Mission Planning & & \\
\hline Robustness (to environmental factors) & & \\
\hline Capability Planning & & \\
\hline
\end{tabular}

\section{PARTICIPANT 2}

What are the Dimensions of the System of Interest for a SoS?

\begin{tabular}{|c|c|c|}
\hline Proposed Dimension & $\begin{array}{l}\text { This } \\
\text { dimension is } \\
\text { relevant to } \\
\text { the SOl of a } \\
\text { SoS? }\end{array}$ & Comment \\
\hline Component Systems & 5 & May need to think about groups vs. components/subsystems. \\
\hline Interactions & 5 & $\begin{array}{l}\text { Need to consider close/loose coupling, where decisions are } \\
\text { made, control, overriding, etc. }\end{array}$ \\
\hline \multicolumn{3}{|l|}{ Time } \\
\hline \multirow{2}{*}{$\begin{array}{ll}\text { - } & \text { Lifecycle } \\
\text { - } & \text { Latency }\end{array}$} & 3 & \\
\hline & 5 & \\
\hline & & \\
\hline Classification $^{5}$ & 2 & \\
\hline Functions / Services & 4 & \\
\hline Enterprise Participants & 4 & \\
\hline Capabilities & 5 & $\begin{array}{l}\text { What's the difference between function and capability? There } \\
\text { seems at least some overlap. }\end{array}$ \\
\hline Disciplines & 3 & \\
\hline $\begin{array}{l}\text { Concept of Operation / Use / } \\
\text { Employment }\end{array}$ & 3 & \\
\hline Geographic Distribution & 3 & \\
\hline Emergence & 5 & $\begin{array}{l}\text { As automation increases, unwanted emergent properties will } \\
\text { become a big issue due to the loss of smart human 'dampers'. } \\
\text { For example, planned automation developments relating to } \\
\text { flight control around airports could result in multiple fuel- } \\
\text { based disasters as increasing numbers of aircraft are } \\
\text { restacked. }\end{array}$ \\
\hline
\end{tabular}

SoS M\&S Outputs

\begin{tabular}{|c|c|c|}
\hline SoS M\&S Output Dimensions & $\begin{array}{l}\text { Which M\&S techniques enable } \\
\text { measurement of this output? }\end{array}$ & $\begin{array}{l}\text { How reliable is this } \\
\text { measurement? }\end{array}$ \\
\hline Emergent Behaviour & $\begin{array}{l}\text { DEVS, surrogate models, systems } \\
\text { dynamics, agent-based M\&S }\end{array}$ & $4,4,3,2$ \\
\hline Connectivity & Petri Nets & 3 \\
\hline Interoperability & Mdlg language, enterpr arch frwrk & 3,3 \\
\hline Agility & DEVS, agent-based M\&S & 3,3 \\
\hline Cost/ Balance of Investment & DEVS will give you the & 4 \\
\hline
\end{tabular}

${ }^{5}$ Assume Directed, Acknowledged, Collaborative and Virtual, until something better comes along

${ }^{6} 1=$ Extremely Unreliable, 2=Unreliable, 3=Neutral, 4=Reliable, 5=Extremely Reliable 


\begin{tabular}{|c|c|c|}
\hline & $\begin{array}{l}\text { OVERcapacity req'ts to achieve the } \\
\text { required effectiveness, perf, sust \& } \\
\text { surv }\end{array}$ & \\
\hline Effectiveness (ability to achieve purpose) & $\begin{array}{l}\text { DEVS, surrogate models, agent- } \\
\text { based M\&S }\end{array}$ & $4,3,3$ \\
\hline $\begin{array}{l}\text { Sustainability (deliver level of performance } \\
\text { despite interference) \& DAMAGE - LOSS OF } \\
\text { UNITS }\end{array}$ & $\begin{array}{l}\text { DEVS, surrogate models, agent- } \\
\text { based M\&S }\end{array}$ & $4,4,4$ \\
\hline $\begin{array}{l}\text { Survivability (to function during and after } \\
\text { environment disturbance) }\end{array}$ & $\begin{array}{l}\text { DEVS, surrogate models, agent- } \\
\text { based M\&S }\end{array}$ & $5,4,4$ \\
\hline Risk & $\begin{array}{l}\text { DEVS, surrogate models, BBN or } \\
\text { stats mdls, SystemD, Gen Markov } \\
\text { chains }\end{array}$ & $5,4,3,3$ \\
\hline Performance & DEVS, surrogate models & 5,4 \\
\hline Safety (Hazard Analysis) & DEVS, surrogate models & 4,3 \\
\hline Evolution & Mdlg language, enterpr arch frwrk & 3,3 \\
\hline Autonomy & ?? & \\
\hline Reliability & $\begin{array}{l}\text { DEVS, surrogate models, BBN (if } \\
\text { you have the data) }\end{array}$ & $5,4,3$ \\
\hline Mission Planning & DEVS, surrogate models & \\
\hline Robustness (to environmental factors) & $\begin{array}{l}\text { DEVS, surrogate models, agent- } \\
\text { based M\&S }\end{array}$ & $5,4,3$ \\
\hline Capability Planning & $\begin{array}{l}\text { Mdlg language, enterpr arch frwrk, } \\
\text { DEVS, surrogate models, agent- } \\
\text { based M\&S }\end{array}$ & $4,4,3,3,2$ \\
\hline
\end{tabular}

\section{Potential M\&S methods}

These are methods that are either currently, or may be, used to model and/or simulate SoS. Any further suggestions are welcome.

- Modelling Language ( $\underline{\mathrm{UML}}, \underline{\mathrm{SySML}})$

- $\quad$ Enterprise Architecture Framework (e.g. MODAF)

- $\quad$ System Dynamics

- $\quad$ Discrete Event Simulation/DEVS

- $\quad$ Agent Based M\&S

- $\quad$ Bayesian Belief Network

- $\quad$ Artificial Neural Network (ANN)

- $\quad$ Petri Nets

- Cellular Automata

- $\quad$ Generalized Markov Chains

- Surrogate Models

It is possible that some of the techniques above may be combined, e.g. intelligent Agents, where an ANN is embedded in an Agent giving it a learning capability.

Some further comments

The reason that I have put DEVS down so many times is that it can do all these things, by-and-large far better than any other tools ... IF you have the data, and if you have the right DEVS software; this is where the bad news comes in ...

... Highly complex scenarios can only be simulated using the activity-based form of DEVS - I have programmed in both activitybased and the alternative event- and process-based forms - the latter two are extremely difficult to write and debug, as their complexity rises approximately as the square of the number of activities. Worse still, to make these alternative (event \& process-based) forms handle certain conditions, the programmer must use non-simulation botches which make it impossible to maintain and modify the resulting model ... I am speaking from both personal experience of these problems and from conversations with experts.

So, what about the activity-based simulation programming languages? Unfortunately, they were mostly UK-based and, when the US developed 'pretty-picture' simulation modellers and took over most of the simulation markets, the activity-based languages were were lost. There are (as far as I know) no activity-based programming languages currently available. The only one that I managed to locate was developed and used by a US consultancy company. I talked to one of the company's senior managers over the phone several years ago. He said that the simulation program gave them such a large advantage over their competitors that they would not consider releasing it, even as a commercial product.

Most of the discrete simulators currently available are parameter-driven models, not programming languages. Inevitably, these models only have very limited flexibility, and you cannot make them do anything that the model creator did not anticipate. 
PARTICIPANT 3

What are the Dimensions of the System of Interest for a SoS?

\begin{tabular}{|c|c|c|}
\hline Proposed Dimension & $\begin{array}{l}\text { This dimension is } \\
\text { relevant to the SOI } \\
\text { of a SoS? }\end{array}$ & Comment \\
\hline Component Systems & 5 & $\begin{array}{l}\text { Do not see how you can bound your Sol if you cannot } \\
\text { identify the component systems within your Sol boundary } \\
\text { and those with which your Sol will have key interactions or } \\
\text { dependencies in the SoS environment }\end{array}$ \\
\hline Interactions & 5 & See comment above \\
\hline \multirow{3}{*}{$\begin{array}{cc}\text { Time } & \\
\bullet & \text { Lifecycle } \\
\bullet & \text { Latency } \\
\bullet & \end{array}$} & & \multirow{3}{*}{$\begin{array}{l}\text { Time is an important concept for an SoS (eg lifecycle phase, } \\
\text { required period of operation, time available for configuration } \\
\text { etc) but it is not a constant across all types of SoS }\end{array}$} \\
\hline & 3 & \\
\hline & $?$ & \\
\hline Classification & 4 & $\begin{array}{l}\text { I get the feeling that some kind of classification system is } \\
\text { important as I think there are likely similarities and } \\
\text { differences that could be identified as pertaining to one } \\
\text { group or another - but not sure if the current classification is } \\
\text { fit for purpose - maybe we need a multidimensional one }\end{array}$ \\
\hline Functions / Services & 3 & $\begin{array}{l}\text { Yes if functions can be grouped or classified and can fit into } \\
\text { some kind of overall classification as referred to above. } \\
\text { Obviously functionality required and available is critical to } \\
\text { configuring and controlling an SoS but is it a key dimension in } \\
\text { the same sense as the others }\end{array}$ \\
\hline Enterprise Participants & 4 & $\begin{array}{l}\text { If you rephrase this to SoS participants then yes - if you } \\
\text { mean the SoS environment participants then not so much. } \\
\text { But again perhaps we are also looking for some kind of } \\
\text { classification of participants such as controllers, users, } \\
\text { maintainers etc }\end{array}$ \\
\hline Capabilities & 3 & $\begin{array}{l}\text { Same comment as functions until you start looking at } \\
\text { differing overall capability depending on the particular } \\
\text { configuration of an SoS. Maybe this should tie in with } \\
\text { Classification above }\end{array}$ \\
\hline Disciplines & 2 & $\begin{array}{l}\text { Not sure you could capture this in a meaning way that would } \\
\text { add anything to functions/services }\end{array}$ \\
\hline $\begin{array}{l}\text { Concept of Operation / Use / } \\
\text { Employment }\end{array}$ & 4 & $\begin{array}{l}\text { Yes important to understand how different Conops might } \\
\text { impact on the configuration or operation of an SoS }\end{array}$ \\
\hline Geographic Distribution & 5 & $\begin{array}{l}\text { Yes if it is pertinent to the operation or (re)configurability of } \\
\text { an SoS }\end{array}$ \\
\hline Emergence & $?$ & $\begin{array}{l}\text { This is obviously important but what do we mean here. Are } \\
\text { we referring to the potential for emergence (desirable or } \\
\text { undesirable), the impact, the likelihood etc. Think we need } \\
\text { to be more specific }\end{array}$ \\
\hline \multicolumn{3}{|l|}{ Levels of Control } \\
\hline Maintainability & & \\
\hline
\end{tabular}

SoS M\&S Outputs

\begin{tabular}{|c|c|c|}
\hline SoS M\&S Output Dimensions & $\begin{array}{l}\text { Which M\&S techniques enable } \\
\text { measurement of this output? }\end{array}$ & $\begin{array}{l}\text { How reliable is this } \\
\text { measurement? }\end{array}$ \\
\hline Emergent Behaviour & $\begin{array}{ll}\text { - } & \text { Modelling Language } \\
\text { - } & \text { EnL, SySML) } \\
\text { Enterprise Architecture } \\
\text { - } & \frac{\text { Framework (e.g. MODAF) }}{\text { System Dynamics }} \\
\text { - } & \frac{\text { Discrete Event }}{\text { Simulation/DEVS }} \\
\text { - } & \frac{\text { Cellular Automata }}{\text { Generalized Markov }} \\
\end{array}$ & $\begin{array}{l}\text { 4-in terms of input to decision } \\
\text { support. Some of these can be } \\
\text { modelled accurately or } \\
\text { quantatively }\end{array}$ \\
\hline
\end{tabular}




\begin{tabular}{|c|c|c|}
\hline & $\begin{array}{l}\quad \frac{\text { Chains }}{\text { Surrogate Models }} \\
\text { Simulations, Enterprise System } \\
\text { Modelling }\end{array}$ & \\
\hline Connectivity & $\begin{aligned} & \text { - } \text { Modelling Language } \\
& \text { (UML, SySML) } \\
& \text { - } \text { Enterprise Architecture } \\
& \text { - } \text { Framework (e.g. MODAF) } \\
& \text { - } \text { Agent Based M\&S } \\
& \text { - } \text { Bayesian Belief Network } \\
& \text { - } \text { (ANificial Neural Network } \\
& \text { - } \text { Petri Nets } \\
& \text { DFDs, circuit diagrams etc }\end{aligned}$ & 4 for planned, 2 for ad hoc \\
\hline Interoperability & $\begin{aligned} & \text { UML etc } \\
& \text { - } \text { Modelling Language } \\
& \text { (UML, SySML) } \\
& \text { - } \text { Enterprise Architecture } \\
& \text { Framework (e.g. MODAF) } \\
& \text { - } \text { System Dynamics } \\
& \text { Artificial Neural Network } \\
& \text { - } \text { (ANN) } \\
&\end{aligned}$ & 4 for planned, 2 for ad hoc \\
\hline Agility & $\begin{array}{l}\text { Need to define it first - from PJ } \\
\text { thesis remember it was made up of a } \\
\text { series of measurable secondary } \\
\text { factors }\end{array}$ & \\
\hline Cost/ Balance of Investment & Standard Financial Modelling & $\begin{array}{l}4 \text { in terms of straight financial cost, } \\
2 \text { or } 1 \text { for overall added value }\end{array}$ \\
\hline Effectiveness (ability to achieve purpose) & 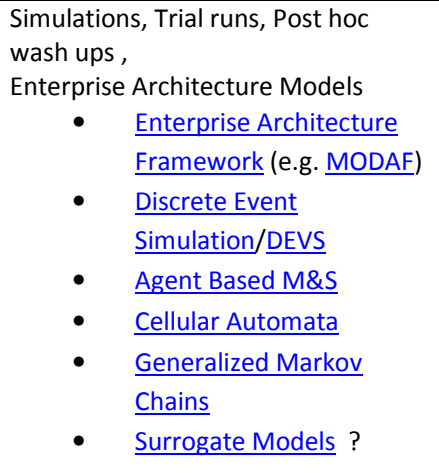 & $\begin{array}{l}4 \\
3-\text { but better than nothing }\end{array}$ \\
\hline $\begin{array}{l}\text { Sustainability (deliver level of performance } \\
\text { despite interference) }\end{array}$ & $\begin{array}{l}\text { Simulations, Trial runs, Post hoc } \\
\text { wash ups, } \\
\text { Enterprise Architecture Models } \\
+ \text { as above }\end{array}$ & 4 \\
\hline $\begin{array}{l}\text { Survivability (to function during and after } \\
\text { environment disturbance) }\end{array}$ & $\begin{array}{l}\text { Simulations, Trial runs, Post hoc } \\
\text { wash ups, } \\
\text { Enterprise Architecture Models } \\
+ \text { as above }\end{array}$ & $\begin{array}{l}4 \\
3-\text { but better than nothing }\end{array}$ \\
\hline Risk & $\begin{array}{l}\text { Do you model risk or identify and } \\
\text { manage it with the previous } 3 \text { used } \\
\text { to establish impact on performance }\end{array}$ & \\
\hline Performance & $\begin{array}{l}\text { Simulations, Trial runs, Post hoc } \\
\text { wash ups, } \\
\text { Enterprise Architecture Models } \\
\text { - } \quad \text { Enterprise Architecture } \\
\text { - } \quad \text { Framework (e.g. MODAF) } \\
\text { Discrete Event } \\
\text { - } \\
\text { - } \\
\text { - } \\
\text { Aimulation/DEVS Based M\&S } \\
\text { Gellular Automata } \\
\text { Generalized Markov }\end{array}$ & \\
\hline
\end{tabular}




\begin{tabular}{|l|l|l|}
\hline & $\bullet \quad$ Chains & \\
\hline Safety (Hazard Analysis) & & \\
\hline Evolution & & \\
\hline Autonomy & & \\
\hline Reliability & & \\
\hline Mission Planning & & \\
\hline Robustness (to environmental factors) & & \\
\hline Capability Planning & & \\
\hline
\end{tabular}

\section{PARTICIPANT 4}

What are the Dimensions of the System of Interest for a SoS?

\begin{tabular}{|c|c|c|}
\hline Proposed Dimension & $\begin{array}{l}\text { This } \\
\text { dimension is } \\
\text { relevant to } \\
\text { the SOI of a } \\
\text { SoS? }\end{array}$ & Comment \\
\hline Component Systems & 3 & Not sure what this means. \\
\hline Interactions & 4 & Ditto. Nature of Interactions? \\
\hline $\begin{aligned} \text { Time } & \\
\bullet & \text { Lifecycle } \\
\bullet & \text { Latency } \\
\bullet & \end{aligned}$ & $\begin{array}{l}5 \\
4 \\
\end{array}$ & \\
\hline Classification & 4 & \\
\hline Functions / Services & 4 & \\
\hline Enterprise Participants & 3 & ? Meaning personnel? \\
\hline Capabilities & 4 & \\
\hline Disciplines & 5 & \\
\hline $\begin{array}{l}\text { Concept of Operation / Use / } \\
\text { Employment }\end{array}$ & 5 & \\
\hline Geographic Distribution & 4 & Will have some influence \\
\hline Emergence & 5 & This will likely determine the modelling approach \\
\hline
\end{tabular}

SoS M\&S Outputs

\begin{tabular}{|l|l|l|}
\hline SoS M\&S Output Dimensions & $\begin{array}{l}\text { Which M\&S techniques enable } \\
\text { measurement of this output? }\end{array}$ & How reliable is this measurement? \\
\hline Emergent Behaviour & Agent, ANN, CA & $\begin{array}{l}4 \text { (unlikely to expose all emergent } \\
\text { behaviours with simplified models) }\end{array}$ \\
\hline Connectivity & EAF, Petri & 5 (true for a snapshot) \\
\hline Interoperability & EAF & 5 (true for a snapshot) \\
\hline Agility & Agent, ANN & 4 \\
\hline Cost/ Balance of Investment & EAF & 5 (but only good for pre-defined \\
& & assemblies of systems) \\
\hline Effectiveness (ability to achieve purpose) & EAF & 5 (if system is already defined) \\
\hline $\begin{array}{l}\text { Sustainability (deliver level of performance } \\
\text { despite interference) }\end{array}$ & Agent & 4 \\
\hline Survivability (to function during and after & Agent & 4 \\
\hline
\end{tabular}




\begin{tabular}{|l|l|l|}
\hline environment disturbance) & & \\
\hline Risk & DEVS & 4 because it is formal in nature \\
\hline Performance & & $\begin{array}{l}\text { In which dimension? Needs a } \\
\text { modelling tool such as matlab }\end{array}$ \\
\hline Safety (Hazard Analysis) & DEVS & \\
\hline Evolution & Agent & \\
\hline Autonomy & Agent, ANN & Possibly - using a FMECA approach \\
\hline Reliability & Bayesian & Not sure what this means \\
\hline Mission Planning & & \\
\hline Robustness (to environmental factors) & & $\begin{array}{l}4-\text { may need to use a combination } \\
\text { of all tthe techniques }\end{array}$ \\
\hline Capability Planning & Agent & Agent/EAF combination \\
\hline
\end{tabular}

\section{PARTICIPANT 5}

What are the Dimensions of the System of Interest for a SoS?

\begin{tabular}{|c|c|c|}
\hline Proposed Dimension & $\begin{array}{l}\text { This } \\
\text { dimension is } \\
\text { relevant to } \\
\text { the SOI of a } \\
\text { SoS? }\end{array}$ & Comment \\
\hline Component Systems & 5 & $\begin{array}{l}\text { Considering the hierarchy of SoS-Systems-Subsystems, do } \\
\text { component systems encapsulate Systems and also Subsystems? }\end{array}$ \\
\hline Interactions & 4 & $\begin{array}{l}\text { Is there an association between interactions and also } \\
\text { interoperability i.e. NCOIC framework. }\end{array}$ \\
\hline \multirow{3}{*}{$\begin{aligned} & \text { Time } \\
& \bullet \text { Lifecycle } \\
& \bullet \text { Latency } \\
&\end{aligned}$} & 5 & \multirow{3}{*}{$\begin{array}{l}\text { Lifecycle of SoS - how much different is this from other lifecycles } \\
\text { e.g. CADMID? }\end{array}$} \\
\hline & 5 & \\
\hline & 4 & \\
\hline Classification & 5 & $\begin{array}{l}\text { The four classifications from the literature - need clear example } \\
\text { to have an understanding of their meaning? Are these } \\
\text { classifications mutually exclusive? }\end{array}$ \\
\hline Functions / Services & 4 & $\begin{array}{l}\text { Does Services already contain functions in addition to } \\
\text { engineering and commercial processes? How is service defined } \\
\text { in this context? It might be a good idea to separate these two. }\end{array}$ \\
\hline Enterprise Participants & 4 & $\begin{array}{l}\text { Enterprise and participants may also need to be separated. } \\
\text { Enterprise in this context could be the organisations and the } \\
\text { supply chain etc. Participants/Stakeholders/Users etc., may need } \\
\text { another term and listed apart from the term Enterprise! }\end{array}$ \\
\hline Capabilities & 4 & $\begin{array}{l}\text { Does capabilities exhibit SoS or vice versa? There are different } \\
\text { viewpoints associated to the term Capability. We may need a } \\
\text { clear definition! Refer to Michael Henshaw paper on Capability } \\
\text { Engineering Perspective Analysis. }\end{array}$ \\
\hline Disciplines & 4 & $\begin{array}{l}\text { Are these role specific disciplines? Disciplines could also be part } \\
\text { o the component systems as each system may comprise a } \\
\text { number of disciplines? }\end{array}$ \\
\hline $\begin{array}{l}\text { Concept of Operation / Use / } \\
\text { Employment }\end{array}$ & 4 & $\begin{array}{l}\text { Are these just processes associated to SoS? CONOPS may also be } \\
\text { part of TEPIDOIL or Component of Capability }(\mathrm{CoC}) \text { i.e. processes. }\end{array}$ \\
\hline Geographic Distribution & 4 & Yes - important. \\
\hline Emergence & 4 & Yes - important. \\
\hline Heterogeneity & 4 & $\begin{array}{l}\text { I introduced this as I think it is relevant to disciplines. } \\
\text { Heterogeneous domain/disciplines may be they right approach! }\end{array}$ \\
\hline $\begin{array}{l}\text { Operational and Managerial } \\
\text { Independence }\end{array}$ & & $\begin{array}{l}\text { Maier's and also Jamshidi's characteristics of SoS can also be } \\
\text { incorporated into these dimensions. It depends on what we } \\
\text { mean by dimension? }\end{array}$ \\
\hline
\end{tabular}

SoS M\&S Outputs

\begin{tabular}{|l|l|l|}
\hline SoS M\&S Output Dimensions & $\begin{array}{l}\text { Which M\&S techniques enable } \\
\text { measurement of this output? }\end{array}$ & How reliable is this measurement? \\
\hline Emergent Behaviour & $\begin{array}{l}\text { Prediction through BNs (Bayesian } \\
\text { Networks) }\end{array}$ & 3 \\
\hline Connectivity & $\begin{array}{l}\text { Social Network Analysis (SNAs) } \\
\text { depends on what you mean by } \\
\text { connectivity? }\end{array}$ & 3 \\
\hline
\end{tabular}




\begin{tabular}{|c|c|c|}
\hline Interoperability & NCOIC framework?? & 3 \\
\hline \multicolumn{3}{|l|}{ Agility } \\
\hline Cost/ Balance of Investment & $\begin{array}{l}\text { COCOMO, is COSYSMO by Boehm } \\
\text { et al. }\end{array}$ & 4 \\
\hline Effectiveness (ability to achieve purpose) & $\begin{array}{l}\text { Key Performance Indicators } \\
\text { (KPIs)??? }\end{array}$ & 3 \\
\hline \multicolumn{3}{|l|}{$\begin{array}{l}\text { Sustainability (deliver level of performance } \\
\text { despite interference) }\end{array}$} \\
\hline \multicolumn{2}{|l|}{$\begin{array}{l}\text { Survivability (to function during and after } \\
\text { environment disturbance) }\end{array}$} & \\
\hline Risk & COSYSMO? & 3 \\
\hline \multicolumn{3}{|l|}{ Performance } \\
\hline \multicolumn{3}{|l|}{ Safety (Hazard Analysis) } \\
\hline \multicolumn{3}{|l|}{ Evolution } \\
\hline \multicolumn{3}{|l|}{ Autonomy } \\
\hline Reliability & $\begin{array}{l}\text { Weibull distribution, Jelinski- } \\
\text { Moranda (JM), Littlewood-Verall, } \\
\text { Musa-Okumoto, Mills Seeding and } \\
\text { Taggin, Markov, Computer-Aided } \\
\text { Software Reliability Estimation } \\
\text { (CASRE) system }\end{array}$ & 4 \\
\hline \multicolumn{2}{|l|}{ Mission Planning } & \\
\hline Robustness (to environmental factors) & PESTLE factors??? & \\
\hline Capability Planning & Audits? & 3 \\
\hline
\end{tabular}

\section{PARTICIPANT 6}

What are the Dimensions of the System of Interest for a SoS?

\begin{tabular}{|c|c|c|}
\hline Proposed Dimension & $\begin{array}{l}\text { This } \\
\text { dimension is } \\
\text { relevant to } \\
\text { the SOI of a } \\
\text { SoS? }\end{array}$ & Comment \\
\hline Component Systems & 5 & \\
\hline Interactions & 5 & $\begin{array}{l}\text { Maybe you can separate them in between and within } \\
\text { component system interactions }\end{array}$ \\
\hline \multirow{3}{*}{$\begin{aligned} \text { Time } & \\
\bullet & \text { Lifecycle } \\
\bullet & \text { Latency } \\
\bullet & \end{aligned}$} & & \multirow{3}{*}{$\begin{array}{l}\text { Maybe more important for a whole system. For example, if } \\
\text { your SOI includes a whole subsystem then the importance of } \\
\text { the dimension may be greater than if the SOI includes a part } \\
\text { of a subsystem. }\end{array}$} \\
\hline & 3 & \\
\hline & & \\
\hline Classification & 5 & $\begin{array}{l}\text { Although the classification that we have at this moment may } \\
\text { not be hugely representative for all types of SoS, the type of } \\
\text { system may inform the way someone establishes or } \\
\text { represents the SOI. }\end{array}$ \\
\hline Functions / Services & 4 & $\begin{array}{l}\text { In my opinion, although they are important, functions can go } \\
\text { sometimes beyond the SOI. }\end{array}$ \\
\hline Enterprise Participants & 5 & \\
\hline Capabilities & & $\begin{array}{l}\text { This can be covered by functions and interactions. } \\
\text { I believe it should belong to another level of dimensions. }\end{array}$ \\
\hline Disciplines & & $\begin{array}{l}\text { This can be covered by component systems, or component } \\
\text { of systems. } \\
\text { The same as capability dimension }\end{array}$ \\
\hline $\begin{array}{l}\text { Concept of Operation / Use / } \\
\text { Employment }\end{array}$ & & Functionality? \\
\hline Geographic Distribution & 4 & Implications for logistics and additional costs. \\
\hline Emergence & 2 & $\begin{array}{l}\text { Don't know if this would be of any help as by looking at the } \\
\text { emergent behaviour of subsystem (looking at } \\
\text { intercomponent linkages) you can't predict the emergent } \\
\text { behaviour of the whole SoS because of the intracomponent }\end{array}$ \\
\hline
\end{tabular}




\begin{tabular}{|l|l|l|}
\hline & & linkages that one my miss. \\
\hline
\end{tabular}

SoS M\&S Outputs

\begin{tabular}{|c|c|c|}
\hline SoS M\&S Output Dimensions & $\begin{array}{l}\text { Which M\&S techniques enable } \\
\text { measurement of this output? }\end{array}$ & How reliable is this measurement? \\
\hline Emergent Behaviour & $\begin{array}{l}\text { Systems Dynamics } \\
\text { Agent-Based Models }\end{array}$ & \\
\hline \multicolumn{3}{|l|}{ Connectivity } \\
\hline \multicolumn{3}{|l|}{ Interoperability } \\
\hline \multicolumn{3}{|l|}{ Agility } \\
\hline \multicolumn{3}{|l|}{ Cost/ Balance of Investment } \\
\hline \multicolumn{3}{|l|}{ Effectiveness (ability to achieve purpose) } \\
\hline \multicolumn{3}{|l|}{$\begin{array}{l}\text { Sustainability (deliver level of performance } \\
\text { despite interference) }\end{array}$} \\
\hline \multicolumn{3}{|l|}{$\begin{array}{l}\text { Survivability (to function during and after } \\
\text { environment disturbance) }\end{array}$} \\
\hline \multicolumn{3}{|l|}{ Risk } \\
\hline Performance & Discrete event simulation & \\
\hline \multicolumn{3}{|l|}{ Safety (Hazard Analysis) } \\
\hline \multicolumn{3}{|l|}{ Evolution } \\
\hline \multicolumn{3}{|l|}{ Autonomy } \\
\hline Reliability & Petri Nets Simulation & \\
\hline \multicolumn{3}{|l|}{ Mission Planning } \\
\hline \multicolumn{3}{|l|}{ Robustness (to environmental factors) } \\
\hline Capability Planning & & \\
\hline
\end{tabular}

\section{PARTICIPANT 7}

What are the Dimensions of the System of Interest for a SoS?

\begin{tabular}{|c|c|c|}
\hline Proposed Dimension & $\begin{array}{l}\text { This } \\
\text { dimension is } \\
\text { relevant to } \\
\text { the SOI of a } \\
\text { SoS? }\end{array}$ & Comment \\
\hline Component Systems & yes & $\begin{array}{l}\text { Defines what is actually in the systems of interest; but the } \\
\text { representation of component systems could be done at the } \\
\text { actual system level or the class/type of system. }\end{array}$ \\
\hline Interactions & Yes & $\begin{array}{l}\text { An SoS exists only because of interactions between } \\
\text { constituent systems, so the interactions must provide some } \\
\text { definition of the SOI. As above, these could be at the } \\
\text { individual interaction level or at the class/type of interaction } \\
\text { level. }\end{array}$ \\
\hline Time & & \multirow{4}{*}{$\begin{array}{l}\text { The SOI at a specific instant in time depends on what Systems } \\
\text { are currently contributing. Frequency of } \\
\text { change/replenishment of systems within the SoS could be a } \\
\text { useful measure (slowly or quickly changing). Life phases, } \\
\text { rather than cycle, could be relevant to indicate current status } \\
\text { of the SoS which is related to SOI }\end{array}$} \\
\hline \multirow{3}{*}{$\begin{array}{ll}\text { - Lifecycle } \\
\text { - } \\
\text { - }\end{array}$} & yes & \\
\hline & Possibly not & \\
\hline & & \\
\hline Classification & Yes & But tighter definition of the types is needed \\
\hline Functions / Services & yes & $\begin{array}{l}\text { This seems relevant but perhaps less important than others. } \\
\text { The services or functions provided by individual systems } \\
\text { seems to indicate something about the content of the SOI. In } \\
\text { terms of building or analysing a SoS, the question what } \\
\text { services should I include in the analysis seems relevant. }\end{array}$ \\
\hline Enterprise Participants & yes & $\begin{array}{l}\text { This may have more relevance for some types than others. } \\
\text { For instance, for virtual, maybe it is a) impossible to know, b) } \\
\text { not much help when you do. However, for collaborative and } \\
\text { acknowledged, there is clearly a need to understand }\end{array}$ \\
\hline
\end{tabular}




\begin{tabular}{|l|l|l|}
\hline & & $\begin{array}{l}\text { participation. Probably also try for Directed, but may be not } \\
\text { such a significant question. }\end{array}$ \\
\hline Capabilities & no & $\begin{array}{l}\text { Probably not, though the SOI might be important information } \\
\text { for deciding on the SOI for a capability. }\end{array}$ \\
\hline Disciplines & moderate & May be helpful, but I don't think it defines the SOI. \\
\hline $\begin{array}{l}\text { Concept of Operation / Use / } \\
\text { Employment }\end{array}$ & Yes & $\begin{array}{l}\text { Given that the individual systems can perform operations } \\
\text { independently, I think the use/mission of the SoS is critical for } \\
\text { defining the SOl for the SoS as opposed to the individual } \\
\text { constituent systems. }\end{array}$ \\
\hline Geographic Distribution & Yes & May be more relevant to some situations than others \\
\hline Emergence & No & $\begin{array}{l}\text { Although emergence is an important property, I don't think it } \\
\text { defines the SOI. }\end{array}$ \\
\hline
\end{tabular}

\section{SoS M\&S Outputs}

\begin{tabular}{|c|c|c|}
\hline SoS M\&S Output Dimensions & $\begin{array}{l}\text { Which M\&S techniques enable } \\
\text { measurement of this output? }\end{array}$ & How reliable is this measurement? \\
\hline Emergent Behaviour & $\begin{array}{l}\text { Simulation } \\
\text { Baysian belief } \\
\text { Agent based }\end{array}$ & $\begin{array}{l}\text { Only as reliable as our } \\
\text { understanding of the problem } \\
\text { space. }\end{array}$ \\
\hline Connectivity & Architecture, simulation, & Depends on complexity \\
\hline Interoperability & architecture & Pretty good if done properly \\
\hline Agility & $\begin{array}{l}\text { System dynamics } \\
\text { Agent based }\end{array}$ & \\
\hline Cost/ Balance of Investment & $\begin{array}{l}\text { Scenario planning } \\
\text { EA } \\
\text { Wisdom of the crowd simulation } \\
\text { tools and calculators }\end{array}$ & \\
\hline Effectiveness (ability to achieve purpose) & $\begin{array}{l}\text { Agent based } \\
\text { Baysian } \\
\text { System dynamic }\end{array}$ & \\
\hline $\begin{array}{l}\text { Sustainability (deliver level of performance } \\
\text { despite interference) }\end{array}$ & $\begin{array}{l}\text { Agent based } \\
\text { DES }\end{array}$ & \\
\hline \multicolumn{3}{|l|}{$\begin{array}{l}\text { Survivability (to function during and after } \\
\text { environment disturbance) }\end{array}$} \\
\hline Risk & $\begin{array}{l}\text { Combination of models within } \\
\text { tradespace } \\
\text { Baysian } \\
\text { Systems dynamics }\end{array}$ & \\
\hline \multicolumn{3}{|l|}{ Performance } \\
\hline Safety (Hazard Analysis) & $\begin{array}{l}\text { Architectures } \\
\text { As risks }\end{array}$ & \\
\hline Evolution & $\begin{array}{l}\text { Scenario planning } \\
\text { architecture }\end{array}$ & \\
\hline Autonomy & Agent based & \\
\hline Reliability & architecture & \\
\hline Mission Planning & $\begin{array}{l}\text { Scenario planning } \\
\text { Architecture }\end{array}$ & \\
\hline Robustness (to environmental factors) & Scenario planning & \\
\hline Capability Planning & $\begin{array}{l}\text { Scenario } \\
\text { Architecture } \\
\text { Cost modelling tools }\end{array}$ & \\
\hline
\end{tabular}




\section{PARTICIPANT 8}

What are the Dimensions of the System of Interest for a SoS?

\begin{tabular}{|c|c|c|}
\hline Proposed Dimension & $\begin{array}{l}\text { This } \\
\text { dimension is } \\
\text { relevant to } \\
\text { the SOI of a } \\
\text { SoS? }\end{array}$ & Comment \\
\hline Component Systems & 5 & $\begin{array}{l}\text { You need to know what systemsa are within the SoS (as far } \\
\text { as you cann determine) }\end{array}$ \\
\hline Interactions & 5 & $\begin{array}{l}\text { Need to know both the interfaces, and the behaviour at the } \\
\text { interfaces, when the systems interact }\end{array}$ \\
\hline \multirow{4}{*}{$\begin{aligned} \text { Time } & \\
\bullet & \text { Lifecycle } \\
\bullet & \text { Latency } \\
\bullet & \text { Variability }\end{aligned}$} & & \multirow{4}{*}{$\begin{array}{l}\text { An SoS may become unstable with large variations in event } \\
\text { times. Camn't remember the paper that discusses this issue. }\end{array}$} \\
\hline & 5 & \\
\hline & 5 & \\
\hline & 5 & \\
\hline Classification & 3 & $\begin{array}{l}\text { DoD } 4 \text { casses somewhat irrelevant - most SoS are a } \\
\text { combination of these classes. }\end{array}$ \\
\hline Functions / Services & 4 & $\begin{array}{l}\text { Not sure what you mean - infrastructural support? Tends to } \\
\text { be overlooked in the literature, but vital in practice }\end{array}$ \\
\hline Enterprise Participants & 5 & $\begin{array}{l}\text { Determines nature of control in SoS. Note, we don't know } \\
\text { how to design the interface between organisations that are } \\
\text { a different technology/lifecycle points. }\end{array}$ \\
\hline Capabilities & 4 & Not as importantas interactions - see above \\
\hline Disciplines & 5 & $\begin{array}{l}\text { The more that re involved in understanding what's going on, } \\
\text { the better. }\end{array}$ \\
\hline $\begin{array}{l}\text { Concept of Operation / Use / } \\
\text { Employment }\end{array}$ & 4 & $\begin{array}{l}\text { Useful for design; less useful for operations, due to effects of } \\
\text { resilience, adaptability over life cycle }\end{array}$ \\
\hline Geographic Distribution & 5 & Vital - cultural and legal issues \\
\hline Emergence & 5 & $\begin{array}{l}\text { Critical issue; need to know and metricate this w.r.t. } \\
\text { intended emergence (that's why you are putting the SoS } \\
\text { together), and highly necessary to estimatethe unwanted } \\
\text { emergence, so that you can build in resilience, adaptabilty. } \\
\text { Latter will probably be insufficient, due to surprises, but may } \\
\text { result in less-costly amelioration efforts. }\end{array}$ \\
\hline
\end{tabular}

SoS M\&S Outputs

\begin{tabular}{|l|l|l|}
\hline SoS M\&S Output Dimensions & $\begin{array}{l}\text { Which M\&S techniques enable } \\
\text { measurement of this output? }\end{array}$ & How reliable is this measurement? \\
\hline Emergent Behaviour & $\begin{array}{l}\text { Most of the oens in the list below } \\
\text { (see comments) will help with } \\
\text { desired emergence; only some will } \\
\text { provide input for } \\
\text { unwanted/unexpected emergence. }\end{array}$ & $\begin{array}{l}\text { I expect } 4 \text { for desired. } 2 \text { for } \\
\text { unexpected. }\end{array}$ \\
\hline Connectivity & Not sure what you mean by this. & \\
\hline Interoperability & & \\
\hline Agility & $\begin{array}{l}\text { None that I can think of. Don't think } \\
\text { any I yourist really get hold of this. }\end{array}$ & \\
\hline Cost/ Balance of Investment & & \\
\hline Effectiveness (ability to achieve purpose) & & \\
\hline $\begin{array}{l}\text { Sustainability (deliver level of performance } \\
\text { despite interference) }\end{array}$ & & \\
\hline $\begin{array}{l}\text { Survivability (to function during and after } \\
\text { environment disturbance) }\end{array}$ & & \\
\hline Risk & & \\
\hline Performance & & \\
\hline Safety (Hazard Analysis) & & \\
\hline Evolution & & \\
\hline Autonomy & & \\
\hline
\end{tabular}




\begin{tabular}{|l|l|l|}
\hline Reliability & & \\
\hline Mission Planning & & \\
\hline Robustness (to environmental factors) & & \\
\hline Capability Planning & & \\
\hline
\end{tabular}

Potential M\&S methods

These are methods that are either currently, or may be, used to model and/or simulate SoS. Any further suggestions are welcome.

- Modelling Language ( $\quad$ MML, SySML) Need these as a lingua franca, but they may need extension to cover SoS-specific aspects. Probably need templates, or something, to cater for complexity of large SoS - too much for humans to dif=gest without somekind of reductive tool

- $\quad$ Enterprise Architecture Framework (e.g. MODAF) OK for ITC systems; badly missing elaborations for organisational aspects. Anns Bruseberg did some work on this aspect, but not enough

- $\quad$ System Dynamics Definitely need something like this;may need some ontologiv=cal extension

- $\quad$ Discrete Event Simulation/DEVS Necessary

- $\quad$ Agent Based M\&S Probably a good way forwards.

- $\quad$ Bayesian Belief Network Yet to be convinced that BBNs are relevant to a resilience domain, as in SoS

- $\quad$ Artificial Neural Network (ANN) Same comment. How do these model resilience and adaptability of the SoS context? Don't think they were designed for tis.

- $\quad$ Petri Nets Likrely to be useful, but need some good tools for this

- $\quad$ Cellular Automata Potentially useful, complementing system dynamics and agent approaches.

- $\quad$ Generalized Markov Chains I think these have a presumption about static environments; doesn't apply to SoS except short-term.

- $\quad$ Surrogate Models Pass.

\section{PARTICIPANT 9}

What are the Dimensions of the System of Interest for a SoS?

\begin{tabular}{|c|c|c|}
\hline Proposed Dimension & $\begin{array}{l}\text { This } \\
\text { dimension is } \\
\text { relevant to } \\
\text { the SOI of a } \\
\text { SoS? }\end{array}$ & Comment \\
\hline Component Systems & Yes & \\
\hline Interactions & yes & \\
\hline \multicolumn{3}{|l|}{ Time } \\
\hline \multirow{3}{*}{$\begin{array}{ll}\text { - } & \text { Lifecycle } \\
\text { - } & \text { Latency } \\
\text { - } & \end{array}$} & May be & \\
\hline & May be & \\
\hline & & \\
\hline Classification & & Not sure what you mean \\
\hline Functions / Services & yes & \multirow{6}{*}{$\begin{array}{l}\text { I think for most of these dimensions, I think you have to } \\
\text { work out first what the purpose of the model and/or } \\
\text { simulation is before you can decide whether or not they are } \\
\text { relevant to your SOI. }\end{array}$} \\
\hline Enterprise Participants & yes & \\
\hline Capabilities & maybe & \\
\hline Disciplines & maybe & \\
\hline $\begin{array}{l}\text { Concept of Operation / Use / } \\
\text { Employment }\end{array}$ & uncertain & \\
\hline Geographic Distribution & Maybe & \\
\hline Emergence & & $\begin{array}{l}\text { This one is tricky as you can include expected emergence } \\
\text { but what about unexpected emergence? If it can be } \\
\text { included, wouldn't that be an output from the } \\
\text { model/simulation rather than something that goes in? }\end{array}$ \\
\hline
\end{tabular}


SoS M\&S Outputs

\begin{tabular}{|c|c|c|}
\hline SoS M\&S Output Dimensions & $\begin{array}{l}\text { Which M\&S techniques enable } \\
\text { measurement of this output? }\end{array}$ & How reliable is this measurement? \\
\hline Emergent Behaviour & $\begin{array}{l}\text { I don't have enough experience of } \\
\text { the techniques to comment. }\end{array}$ & \\
\hline \multicolumn{3}{|l|}{ Connectivity } \\
\hline \multicolumn{3}{|l|}{ Interoperability } \\
\hline \multicolumn{3}{|l|}{ Agility } \\
\hline \multicolumn{3}{|l|}{ Cost/ Balance of Investment } \\
\hline \multicolumn{3}{|l|}{ Effectiveness (ability to achieve purpose) } \\
\hline \multicolumn{3}{|l|}{$\begin{array}{l}\text { Sustainability (deliver level of performance } \\
\text { despite interference) }\end{array}$} \\
\hline \multicolumn{3}{|l|}{$\begin{array}{l}\text { Survivability (to function during and after } \\
\text { environment disturbance) }\end{array}$} \\
\hline \multicolumn{3}{|l|}{ Risk } \\
\hline \multicolumn{3}{|l|}{ Performance } \\
\hline \multicolumn{3}{|l|}{ Safety (Hazard Analysis) } \\
\hline \multicolumn{3}{|l|}{ Evolution } \\
\hline \multicolumn{3}{|l|}{ Autonomy } \\
\hline \multicolumn{3}{|l|}{ Reliability } \\
\hline \multicolumn{3}{|l|}{ Mission Planning } \\
\hline \multicolumn{3}{|l|}{ Robustness (to environmental factors) } \\
\hline Capability Planning & & \\
\hline
\end{tabular}

\section{PARTICIPANT 10}

What are the Dimensions of the System of Interest for a SoS?

\begin{tabular}{|c|c|c|}
\hline Proposed Dimension & $\begin{array}{l}\text { This } \\
\text { dimension is } \\
\text { relevant to } \\
\text { the SOl of a } \\
\text { SoS? }\end{array}$ & Comment \\
\hline Component Systems & 5 & \\
\hline Interactions & 5 & \\
\hline $\begin{aligned} \text { Time } & \\
\bullet & \text { Lifecycle } \\
\bullet & \text { Latency } \\
\bullet & \end{aligned}$ & 5 & \\
\hline Classification & 3 & \\
\hline Functions / Services & 4 & \\
\hline Enterprise Participants & 4 & \\
\hline Capabilities & 5 & The aim of SOS is to achieve some of capability \\
\hline Disciplines & 3 & \\
\hline $\begin{array}{l}\text { Concept of Operation / Use / } \\
\text { Employment }\end{array}$ & 3 & \\
\hline Geographic Distribution & 3 & \\
\hline Emergence & 5 & \\
\hline
\end{tabular}




\begin{tabular}{|c|c|c|}
\hline SoS M\&S Output Dimensions & $\begin{array}{l}\text { Which M\&S techniques enable } \\
\text { measurement of this output? }\end{array}$ & How reliable is this measurement? \\
\hline \multicolumn{3}{|l|}{ Emergent Behaviour } \\
\hline \multicolumn{3}{|l|}{ Connectivity } \\
\hline \multicolumn{3}{|l|}{ Interoperability } \\
\hline \multicolumn{3}{|l|}{ Agility } \\
\hline \multicolumn{3}{|l|}{ Cost/ Balance of Investment } \\
\hline \multicolumn{3}{|l|}{ Effectiveness (ability to achieve purpose) } \\
\hline \multicolumn{3}{|l|}{$\begin{array}{l}\text { Sustainability (deliver level of performance } \\
\text { despite interference) }\end{array}$} \\
\hline \multicolumn{3}{|l|}{$\begin{array}{l}\text { Survivability (to function during and after } \\
\text { environment disturbance) }\end{array}$} \\
\hline \multicolumn{3}{|l|}{ Risk } \\
\hline \multicolumn{3}{|l|}{ Performance } \\
\hline \multicolumn{3}{|l|}{ Safety (Hazard Analysis) } \\
\hline \multicolumn{3}{|l|}{ Evolution } \\
\hline Autonomy & $\begin{array}{l}\text { ALFUS (autonomy levels for } \\
\text { unmanned systems) }\end{array}$ & 4 \\
\hline \multicolumn{3}{|l|}{ Reliability } \\
\hline \multicolumn{3}{|l|}{ Mission Planning } \\
\hline \multicolumn{3}{|l|}{ Robustness (to environmental factors) } \\
\hline Capability Planning & & \\
\hline
\end{tabular}

\section{PARTICIPANT 11}

What are the Dimensions of the System of Interest for a SoS?

\begin{tabular}{|c|c|c|}
\hline Proposed Dimension & $\begin{array}{l}\text { This } \\
\text { dimension is } \\
\text { relevant to } \\
\text { the SOI of a } \\
\text { SoS? }\end{array}$ & Comment \\
\hline Component Systems & 4 & \\
\hline Interactions & 5 & \\
\hline \multirow{4}{*}{$\begin{array}{cl}\text { Time } & \\
\bullet & \text { Lifecycle } \\
\bullet & \text { Latency } \\
\bullet & \end{array}$} & 5 & \\
\hline & 5 & \\
\hline & 4 & \\
\hline & & \\
\hline Classification & 3 & \\
\hline Functions / Services & 4 & \\
\hline Enterprise Participants & 5 & \\
\hline Capabilities & 4 & \\
\hline Disciplines & 3 & \\
\hline $\begin{array}{l}\text { Concept of Operation / Use / } \\
\text { Employment }\end{array}$ & 3 & \\
\hline Geographic Distribution & 4 & \\
\hline Emergence & 5 & \\
\hline
\end{tabular}




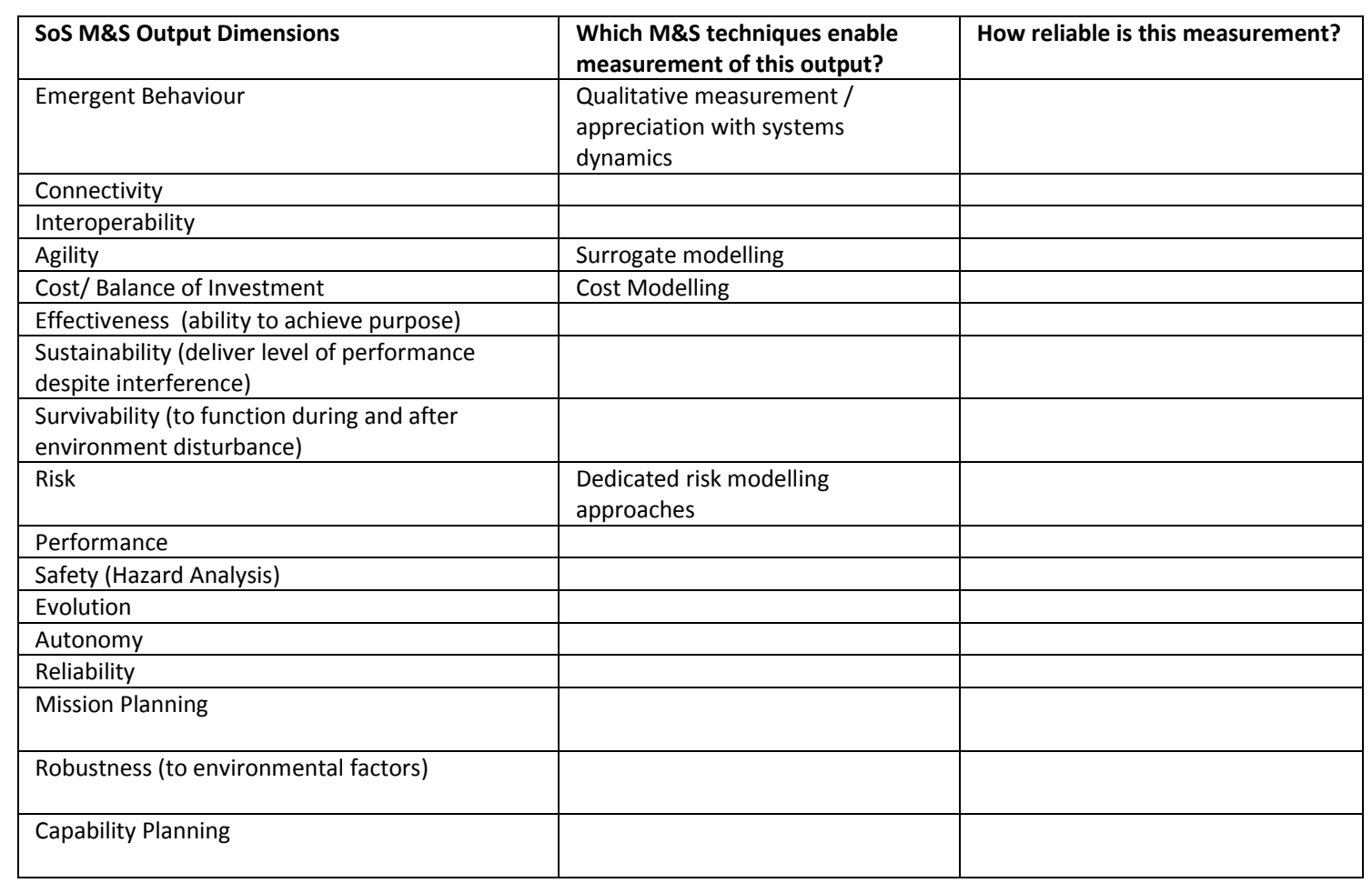




\section{Appendix B - CAS Model Selection Matrix Tool Output}

This appendix provides evidence of the research methodology through the inclusion of screenshots from the SoS model technique selection tool as used in model development for the risk analysis activity in application case study 1, Close Air Support. It also provides artefacts that provide a starting point from which future research may further develop the case study. 


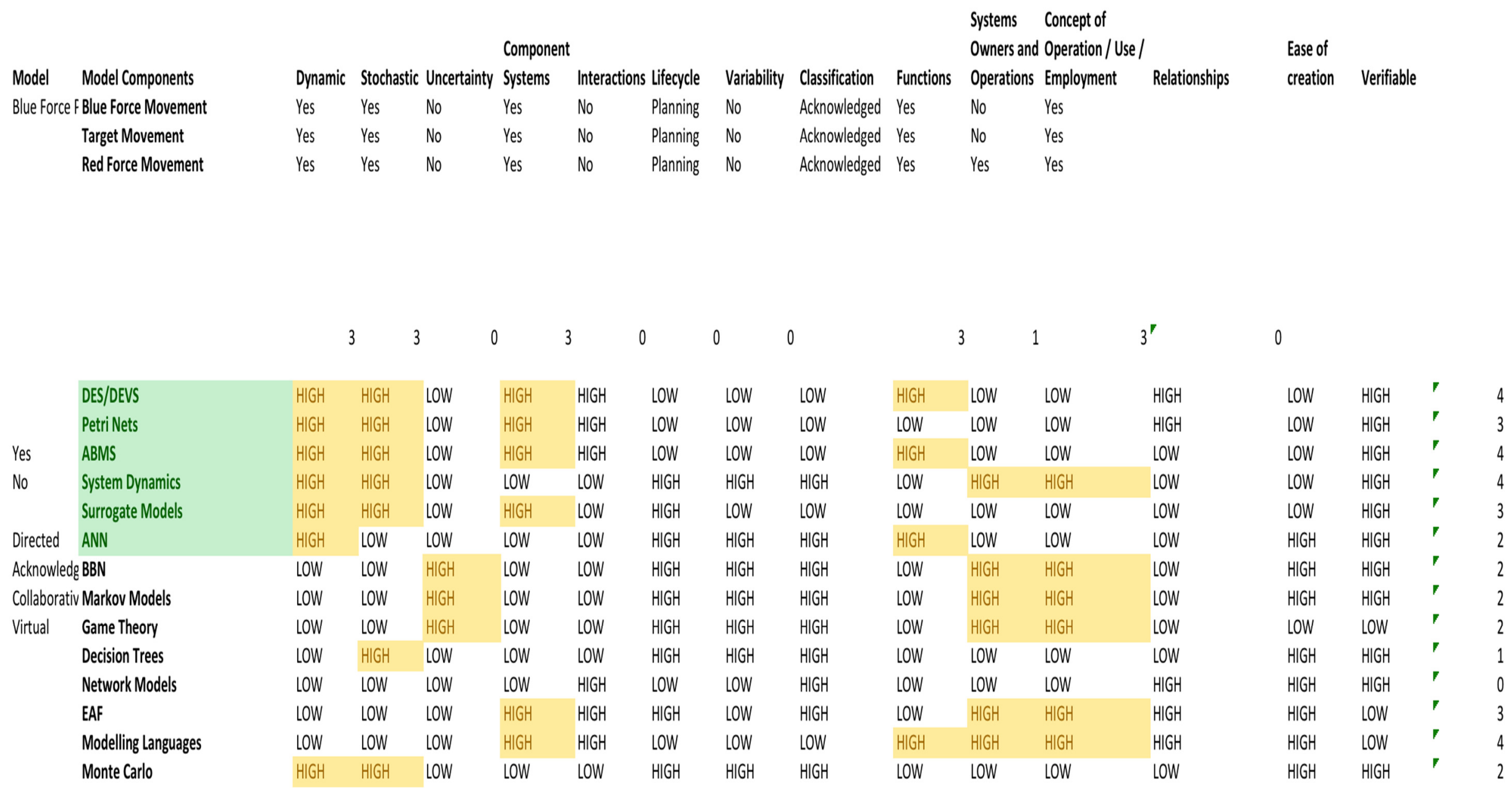

Table B - 1 : M\&S Technique Selection: Blue Force Proximity 


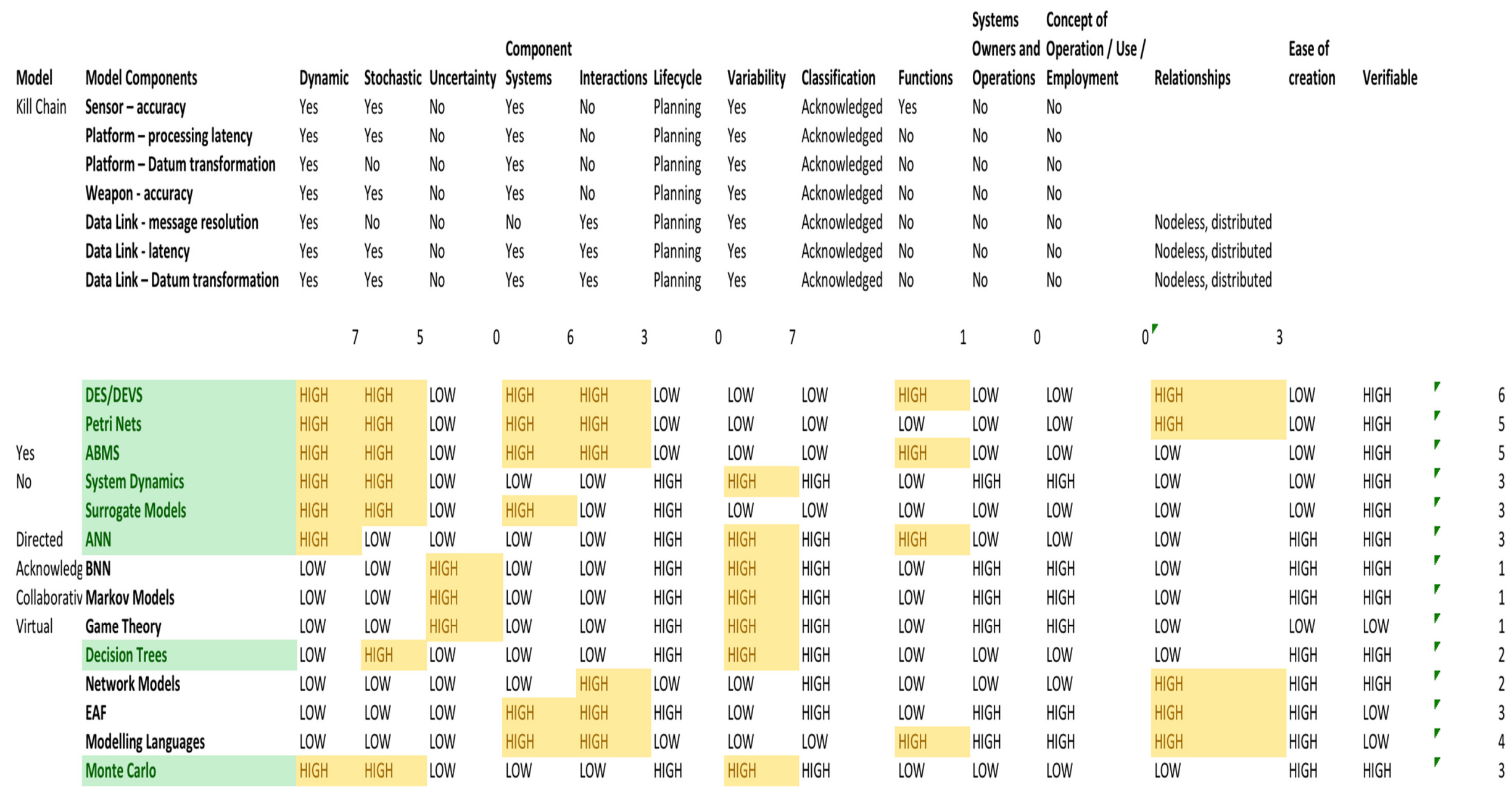

Table B - 2 : M\&S Technique Selection: Kill Chain Accuracy 


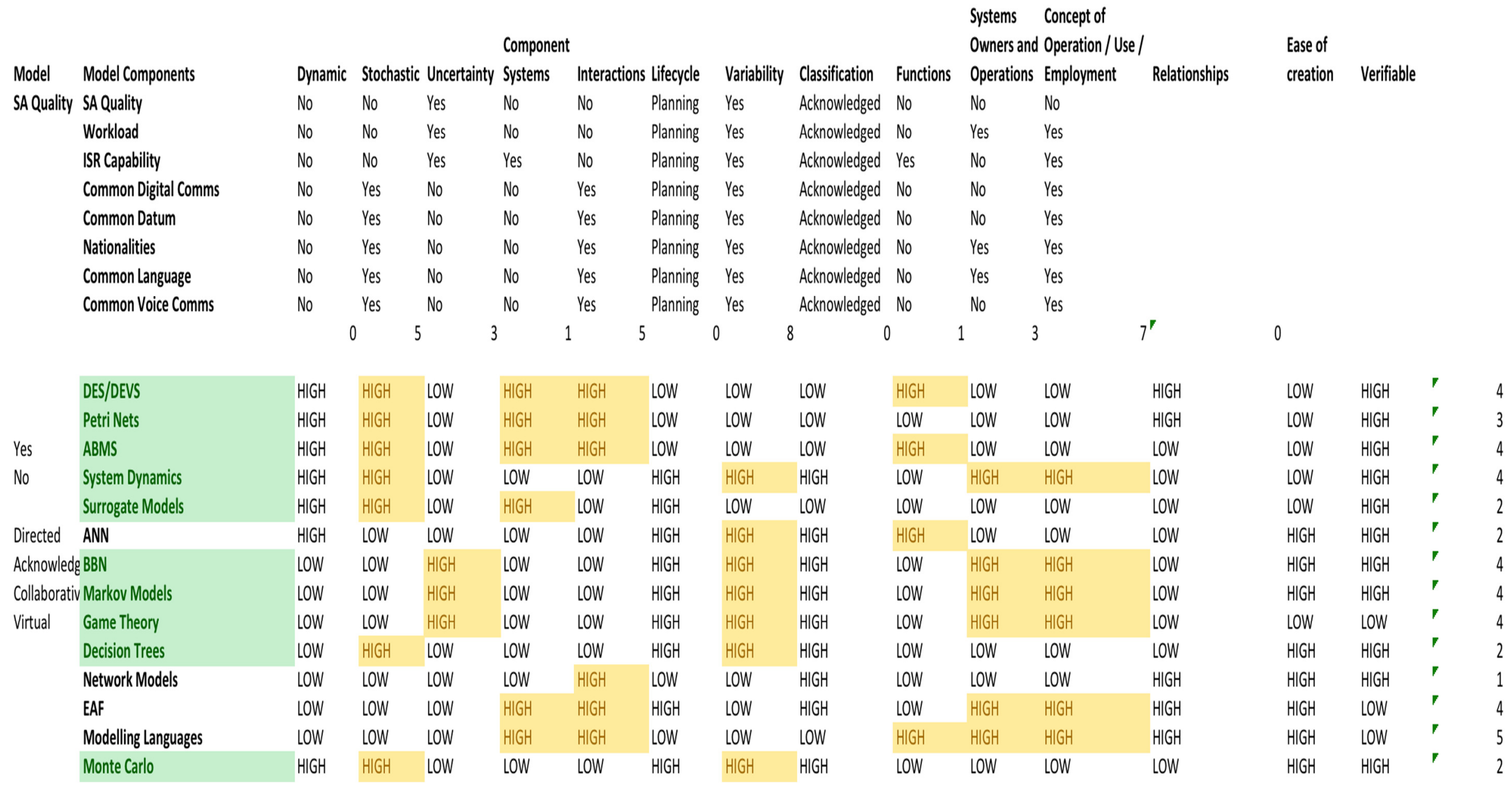

Table B - 3 : M\&S Technique Selection: SA Quality - Initial Analysis 
Systems Concept of

Owners and Operation/Use /

\section{Component}

Dynamic Stochastic Uncertainty Systems Interactions lifecycle Variability Classification Functions Operations Employment

No No Yes

No

No

Planning Yes

Planning Yes

Planning Yes
Acknowledged No

Acknowledged $\mathrm{N} N$

Acknowledged Yes

$\begin{array}{ll}\text { No } & \text { No } \\ \text { Yes } & \text { No }\end{array}$

$\begin{array}{ll}\text { No } & \text { No } \\ \text { Yes } & \text { Yes } \\ \text { No } & \text { Yes }\end{array}$

Ease of creation Verifiable
ISR Capability$$
\text { No }
$$

0

$\begin{array}{lllll}3 & 1 & 0 & 0 & 3\end{array}$

11

$2^{\prime}$

0

$\begin{array}{lllllllllllllll} & \text { DES/DEVs } & \text { HIGH } & \text { HIGH } & \text { LOW } & \text { HIGH } & \text { HIGH } & \text { LOW } & \text { LOW } & \text { LOW } & \text { HIGH } & \text { LOW } & \text { LOW } & \text { HIGH } \\ & \text { Petri Nets } & \text { HIG } & \text { HIGH } & \text { LOW } & \text { HIGH } & \text { HIGH } & \text { LOW } & \text { LOW } & \text { LOW } & \text { LOW } & \text { LOW } & \text { LOW } & \text { HIGH } \\ \text { Yes } & \text { ABMS } & \text { HIGH } & \text { HIGH } & \text { LOW } & \text { HIGH } & \text { HIGH } & \text { LOW } & \text { LOW } & \text { LOW } & \text { HIGH } & \text { LOW } & \text { LOW } & \text { LOW } \\ \text { No } & \text { System Dynamics } & \text { HIGH } & \text { HIGH } & \text { LOW } & \text { LOW } & \text { LOW } & \text { HIGH } & \text { HIGH } & \text { HIGH } & \text { LOW } & \text { HIGH } & \text { HIGH } & \text { LOW } \\ & \text { Surrogate Models } & \text { HIGH } & \text { HIGH } & \text { LOW } & \text { HIGH } & \text { LOW } & \text { HIGH } & \text { LOW } & \text { LOW } & \text { LOW } & \text { LOW } & \text { LOW } & \text { LOW } \\ \text { Directed } & \text { ANN } & \text { HIGH } & \text { LOW } & \text { LOW } & \text { LOW } & \text { LOW } & \text { HIGH } & \text { HIGH } & \text { HIGH } & \text { HIGH } & \text { LOW } & \text { LOW } & \text { LOW } \\ \text { Acknowledg BBN } & \text { LOW } & \text { LOW } & \text { HIGH } & \text { LOW } & \text { LOW } & \text { HIGH } & \text { HIGH } & \text { HIGH } & \text { LOW } & \text { HIGH } & \text { HIGH } & \text { LOW } \\ \text { Collaborativ Markov Models } & \text { LOW } & \text { LOW } & \text { HIGH } & \text { LOW } & \text { LOW } & \text { HIGH } & \text { HIGH } & \text { HIGH } & \text { LOW } & \text { HIGH } & \text { HIGH } & \text { LOW } \\ \text { Virtual } & \text { Game Theory } & \text { LOW } & \text { LOW } & \text { HIGH } & \text { LOW } & \text { LOW } & \text { HIGH } & \text { HIGH } & \text { HIGH } & \text { LOW } & \text { HIGH } & \text { HIGH } & \text { LOW } \\ & \text { Decision Trees } & \text { LOW } & \text { HIGH } & \text { LOW } & \text { LOW } & \text { LOW } & \text { HIGH } & \text { HIGH } & \text { HIGH } & \text { LOW } & \text { LOW } & \text { LOW } & \text { LOW } \\ & \text { Network Models } & \text { LOW } & \text { LOW } & \text { LOW } & \text { LOW } & \text { HIGH } & \text { LOW } & \text { LOW } & \text { HIGH } & \text { LOW } & \text { LOW } & \text { LOW } & \text { HIGH } \\ & \text { EAF } & \text { LOW } & \text { LOW } & \text { LOW } & \text { HIGH } & \text { HIGH } & \text { HIGH } & \text { LOW } & \text { HIGH } & \text { LOW } & \text { HIGH } & \text { HIGH } & \text { HIGH } \\ & \text { Modelling Languages } & \text { LOW } & \text { LOW } & \text { LOW } & \text { HIGH } & \text { HIGH } & \text { LOW } & \text { LOW } & \text { LOW } & \text { HIGH } & \text { HIGH } & \text { HIGH } & \text { HIGH } \\ & \text { Monte Carlo } & \text { HIGH } & \text { HIGH } & \text { LOW } & \text { LOW } & \text { LOW } & \text { HIGH } & \text { HIGH } & \text { HIGH } & \text { LOW } & \text { LOW } & \text { LOW } & \text { LOW }\end{array}$

Table B - 4 : M\&S Technique Selection: SA Quality

\begin{tabular}{|c|c|c|}
\hline LOW & HIGH & $\boldsymbol{r}$ \\
\hline LOW & HIGH & $r$ \\
\hline LOW & $H I G H$ & $r$ \\
\hline LOW & $H \mathrm{HGH}$ & $r$ \\
\hline LOW & $H \mathrm{HGH}$ & I \\
\hline$H G H$ & $H I G H$ & $\boldsymbol{r}$ \\
\hline$H G H$ & $H I G H$ & 7 \\
\hline$H G H$ & $H I G H$ & 7 \\
\hline LOW & LOW & 7 \\
\hline HIGH & $H I G H$ & 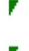 \\
\hline $\mathrm{HIGH}$ & HIGH & $r$ \\
\hline HIGH & LOW & I \\
\hline HIGH & LOW & I \\
\hline$H G H$ & $H I G H$ & $r$ \\
\hline
\end{tabular}




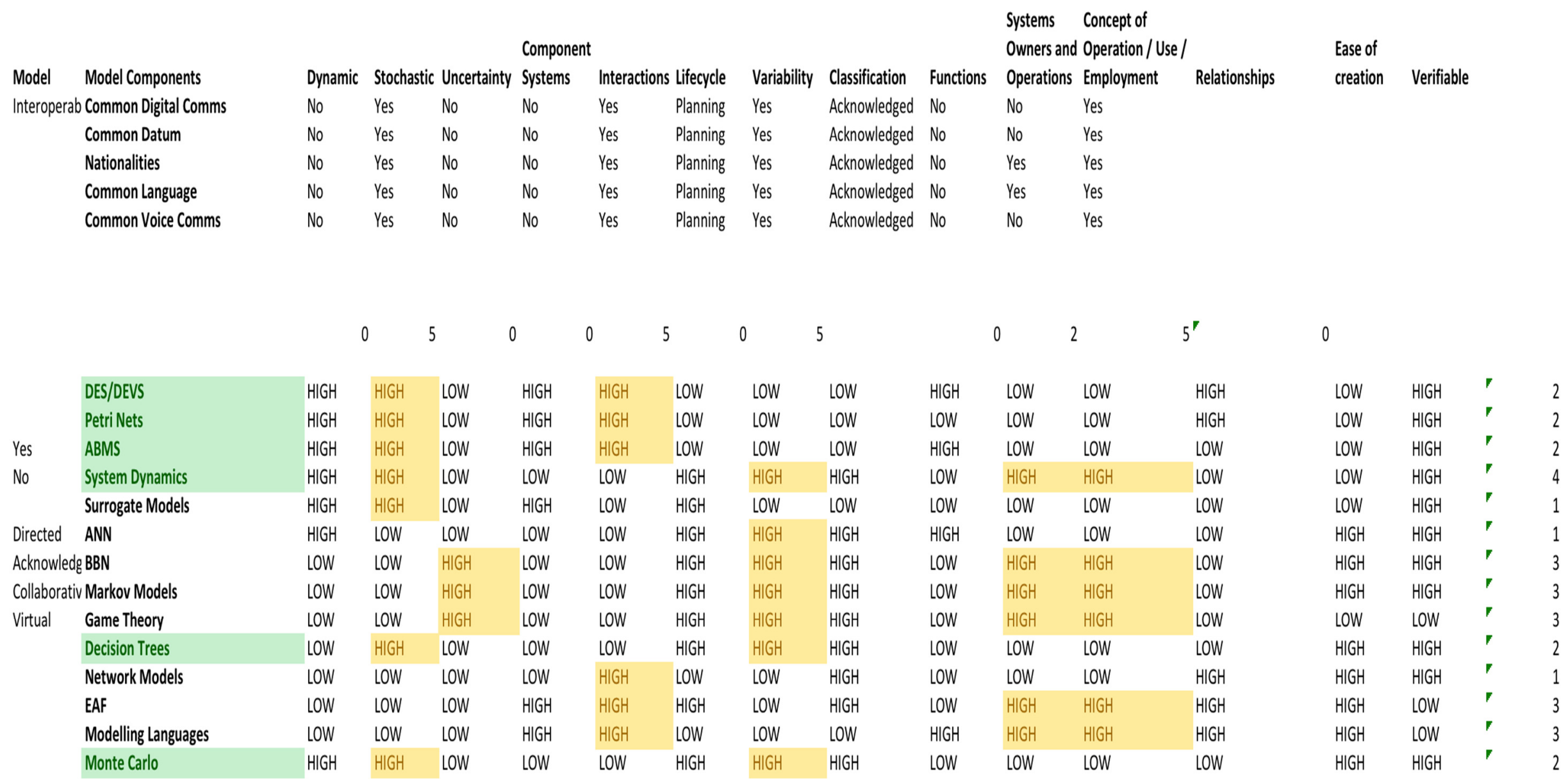

Table B - 5 : M\&S Technique Selection - Interoperability 
Systems Concept of

Component Owners and Operation/Use / Ease of

\begin{tabular}{|c|c|c|c|c|c|c|c|c|c|c|c|c|}
\hline \multirow{2}{*}{$\begin{array}{l}\text { Model } \\
\text { Blue Force } \\
\text { Visibility }\end{array}$} & \multirow{2}{*}{$\begin{array}{l}\text { Model Components } \\
\text { Blue Force Visibility }\end{array}$} & \multirow{2}{*}{$\begin{array}{l}\text { Dynamic } \\
\text { № }\end{array}$} & \multicolumn{3}{|c|}{ Stochastic Uncertainty Systems } & \multicolumn{2}{|c|}{ Interactions lifecycle } & Variability & Classification & Functions & Operations & Employment \\
\hline & & & No & Yes & No & No & Planning & Yes & Acknowledged & № & No & No \\
\hline & Cloud & No & No & Yes & No & No & Planning & No & Acknowledged & № & No & No \\
\hline & Dust & No & No & Yes & No & No & Planning & No & Acknowledged & № & No & No \\
\hline & Light Level & No & No & No & No & No & Planning & No & Acknowledged & № & No & No \\
\hline
\end{tabular}

\begin{tabular}{|c|c|c|c|c|c|c|c|c|c|c|c|c|c|c|c|}
\hline DES/DEVS & $H I G H$ & HIGH & LOW & $\mathrm{HIGH}$ & HIGH & LOW & LOW & LOW & HIGH & LOW & LOW & HIGH & LOW & HIGH & ' \\
\hline Petri Nets & $H I G H$ & HIGH & LOW & HIGH & HIGH & LOW & LOW & LOW & LOW & LOW & LOW & HIGH & LOW & $H G G$ & $r$ \\
\hline ABMS & $H I G H$ & HIGH & LoW & HIGH & HIGH & LOW & LOW & LoW & HIGH & LoW & Low & LOW & Low & $H G H$ & $r$ \\
\hline System Dynamics & $H I G H$ & HIGH & LoW & LOW & Low & HIGH & HIGH & HIGH & LOW & HIGH & HIGH & LOW & LoW & HIGH & 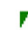 \\
\hline Surrogate Models & $H I G H$ & HIGH & LoW & HIGH & Low & HIGH & LOW & Low & Low & LOW & LoW & LoW & LoW & $H I G H$ & $r$ \\
\hline ANN & HIGH & LoW & LoW & LOW & Low & HIGH & HIGH & HIGH & HIGH & LOW & LoW & Low & HIGH & $H \mathrm{HGH}$ & $r$ \\
\hline gBBN & LOW & LoW & HGH & LOW & Low & HIGH & HIGH & HIGH & Low & HIGH & HIGH & Low & HIGH & $H \mathrm{HGH}$ & $r$ \\
\hline Varkov Models & LOW & LOW & HIGH & LOW & LOW & HGH & HIGH & HIGH & LOW & $\mathrm{HIGH}$ & $H I G H$ & LOW & $H I G H$ & HGH & $r$ \\
\hline Game Theory & LOW & LOW & HIGH & LOW & Low & HIGH & HIGH & HIGH & Low & $\mathrm{HIGH}$ & HIGH & LOW & Low & LOW & $r$ \\
\hline Decision Trees & LOW & $H I G H$ & LOW & LoW & LOW & HIGH & HIGH & HIGH & LOW & LOW & LOW & LOW & $H I G H$ & HGG & $r$ \\
\hline Network Models & LOW & Low & LoW & LoW & HIGH & LOW & LOW & $\mathrm{HIGH}$ & LOW & LOW & LOW & HIGH & HIGH & HGH & $r$ \\
\hline EAF & LOW & LOW & LoW & HIGH & HIGH & HGH & LoW & HIGH & LoW & HIGH & HIGH & HIGH & $H G H$ & LOW & 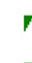 \\
\hline Modelling Languages & LOW & LoW & LOW & HIGH & HIGH & LOW & LoW & LoW & HIGH & HIGH & $H I G H$ & HIGH & HIGH & LOW & $r$ \\
\hline Monte Carlo & $H I G H$ & HIGH & LoW & LOW & Low & HIGH & HIGH & HIGH & LoW & LOW & Low & LoW & $H I G H$ & $H$ HGH & $r$ \\
\hline
\end{tabular}

Table B - 6 : M\&S Technique Selection - Blue Force Visibility 


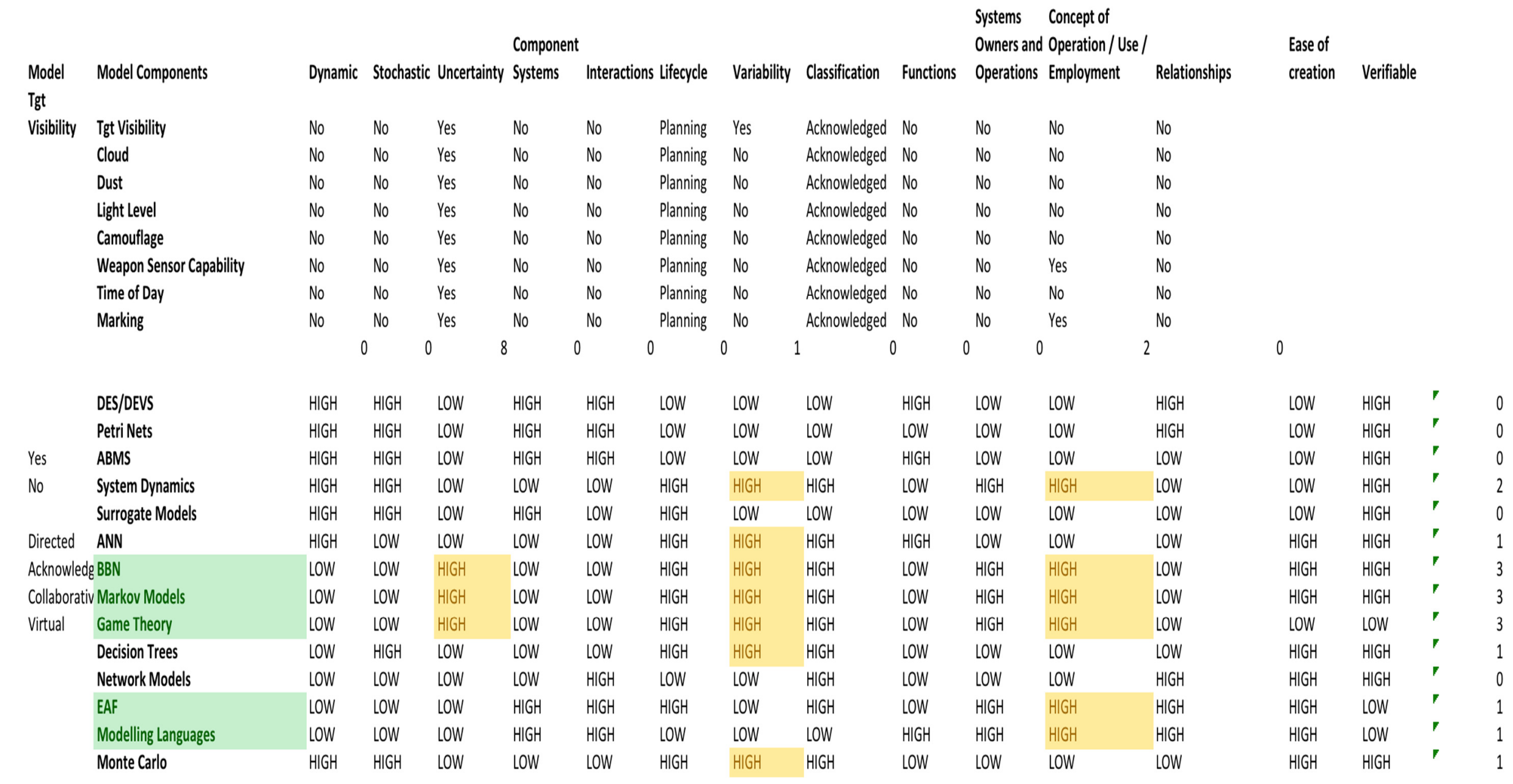


Systems Concept of

Owners and Operation/Use /

Component

Dynamic Stochastic Uncertainty Systems Interactions Lifecycle Variability Classification Functions Operations Employment Relationships

Yes Yes No Yes No Planning No Acknowledged Yes No Yes

Yes Yes No Yes No Planning No Acknowledged Yes No Yes
Ease of

creation Verifiable
Civilian Prox Civilian Movement

Target Movement

\begin{tabular}{|c|c|c|c|c|c|c|c|c|c|c|c|c|c|c|c|c|c|}
\hline & & & 2 & 2 & 0 & 2 & 0 & 0 & 0 & & $?$ & 0 & $2^{\prime}$ & 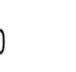 & & & \\
\hline & DES/DEVS & HIGH & $H G H$ & LOW & HIGH & $H G H$ & LOW & LOW & LOW & HIGH & LOW & LOW & HGH & LOW & $H I G H$ & $\boldsymbol{T}$ & 4 \\
\hline & Petri Nets & $H I G H$ & $H I G H$ & LOW & $H G H$ & $H G H$ & LOW & LOW & LOW & LOW & LOW & LOW & HGH & LOW & $H I G H$ & $\boldsymbol{r}$ & 3 \\
\hline Yes & ABMS & HIGH & $H G H$ & LOW & HIGH & $H G H$ & LOW & LOW & LOW & HIGH & LOW & LOW & LOW & LOW & $H I G H$ & $\boldsymbol{T}$ & 4 \\
\hline \multirow[t]{2}{*}{ No } & System Dynamics & HIGH & HGH & LOW & LOW & LOW & HIGH & $H I G H$ & HGH & LOW & HIGH & HGH & LOW & LOW & $H G H$ & $\boldsymbol{V}$ & 3 \\
\hline & Surrogate Models & HIGH & HGH & LOW & $H G H$ & LOW & HIGH & LOW & LOW & LOW & LOW & LOW & LOW & LOW & $H G H$ & $\boldsymbol{r}$ & 3 \\
\hline Directed & ANN & HIGH & LOW & LOW & LOW & LOW & HIGH & HIGH & HIGH & $H I G H$ & LOW & LOW & LOW & $H I G H$ & $H I G H$ & $\boldsymbol{r}$ & 2 \\
\hline \multicolumn{2}{|c|}{ Acknowledg BBN } & LOW & LOW & $H G H$ & LOW & LOW & $H G H$ & $H I G H$ & $H G H$ & LOW & HIGH & HIGH & LOW & $H G H$ & $H \| H$ & $r$ & 1 \\
\hline \multicolumn{2}{|c|}{ Collaborativ Markov Models } & LOW & LOW & $H G H$ & LOW & LOW & HIGH & HIGH & HIGH & LOW & HIGH & HIGH & LOW & $H G H$ & $H \| H$ & $\boldsymbol{r}$ & 1 \\
\hline \multirow[t]{6}{*}{ Virtual } & Game Theory & LOW & LOW & $H G H$ & LOW & LOW & HIGH & HIGH & HIGH & LOW & HIGH & HIGH & LOW & LOW & LOW & $\boldsymbol{r}$ & 1 \\
\hline & Decision Trees & LOW & $H G H$ & LOW & LOW & LOW & $H G H$ & $H I G H$ & HGH & LOW & LOW & LOW & LOW & $H G H$ & $H I G H$ & $\boldsymbol{r}$ & 1 \\
\hline & Network Models & LOW & LOW & LOW & LOW & $H G H$ & LOW & LOW & HGH & LOW & LOW & LOW & HGH & $H G H$ & $H I G H$ & $\boldsymbol{r}$ & 0 \\
\hline & EAF & LOW & LOW & LOW & HGG & $H G H$ & HIGH & LOW & HGH & LOW & HIGH & HIGH & HGH & $H G H$ & LOW & $\boldsymbol{T}$ & 2 \\
\hline & Modelling Languages & LOW & LOW & LOW & HIGH & $H G H$ & LOW & LOW & LOW & $H I G H$ & HIGH & HIGH & HIGH & $H G H$ & LOW & ' & 3 \\
\hline & Monte Carlo & HIGH & $H I G H$ & LOW & LOW & LOW & HIGH & $H I G H$ & HGH & LOW & LOW & LOW & LOW & $H G H$ & $H I G H$ & 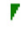 & 2 \\
\hline
\end{tabular}

Table B - 8 : M\&S Technique Selection - Civilian Proximity 
Systems Concept of

Component

Owners and Operation / Use /

Model Model Components

Dynamic Stochastic Uncertainty System

Operations Employment

Ease of

Tgt

Visibility Civilian Visibility

Cloud

Dust

Light Level

$\begin{array}{lll}\text { No } & \text { No } & \text { Yes } \\ \text { No } & \text { No } & \text { Yes } \\ \text { No } & \text { No } & \text { Yes } \\ \text { No } & \text { No } & \text { Yes }\end{array}$

$\begin{array}{ll}\text { No } & \text { No } \\ \text { No } & \text { No }\end{array}$

Interactions Lifecycle

Variability Classification

Function

creation Verifiable

No No

Acknowledged No

Acknowledged No

Acknowledged No

No
No
No
No

No
No
No
No

No

Planning No

Acknowledged №

No

No

\begin{tabular}{|c|c|c|c|c|c|c|c|c|c|c|c|c|c|c|c|c|}
\hline & DES/DEVS & $H I G H$ & $H I G H$ & LOW & HIGH & HIGH & LOW & LOW & LOW & HIGH & LOW & LOW & HIGH & LOW & $H I G H$ & $\boldsymbol{V}$ \\
\hline & Petri Nets & $H \| G$ & $H I G H$ & LOW & HIGH & $H I G H$ & LOW & LOW & LOW & LOW & LOW & LOW & HIGH & LOW & $H I G H$ & $r$ \\
\hline Yes & ABMS & $H I G H$ & HIGH & LOW & HIGH & HIGH & LOW & LOW & LOW & HIGH & LOW & LOW & LOW & LOW & $H I G H$ & $r$ \\
\hline \multirow[t]{2}{*}{ No } & System Dynamics & $H I G H$ & $H I G H$ & LOW & LOW & LOW & HIGH & HIGH & HIGH & LOW & $H I G H$ & HIGH & LOW & LOW & $H I G H$ & $\boldsymbol{T}$ \\
\hline & Surrogate Models & $H I G H$ & $\mathrm{HIGH}$ & LOW & HIGH & LOW & $H G H$ & LOW & LOW & LOW & LOW & LOW & LOW & LOW & $H I G H$ & $r$ \\
\hline Directed & ANN & $H I G H$ & LOW & LOW & LOW & LOW & HIGH & HIGH & HIGH & $H G H$ & LOW & LOW & LOW & $H \mathrm{HGH}$ & $H I G H$ & 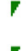 \\
\hline \multirow{2}{*}{\multicolumn{2}{|c|}{$\begin{array}{l}\text { Acknowledg BBN } \\
\text { Collaborativ Markov Models }\end{array}$}} & LOW & LOW & HIGH & LOW & LOW & HIGH & HIGH & HIGH & LOW & $H I G H$ & $H I G H$ & LOW & $H I G H$ & $H I G H$ & $\boldsymbol{r}$ \\
\hline & & LOW & LOW & HIGH & LOW & LOW & HIGH & HIGH & HIGH & LOW & HIGH & HIGH & LOW & $H I G H$ & $H I G H$ & $r$ \\
\hline \multirow[t]{6}{*}{ Virtual } & Game Theory & LOW & LOW & HIGH & LOW & LOW & HIGH & HIGH & HIGH & LOW & HIGH & HIGH & LOW & LOW & LOW & 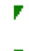 \\
\hline & Decision Trees & LOW & $H I G H$ & LOW & LOW & LOW & $H G H$ & HIGH & HIGH & LOW & LOW & LOW & LOW & $H I G H$ & $H I G H$ & $\boldsymbol{r}$ \\
\hline & Network Models & LOW & LOW & LOW & LOW & HIGH & LOW & LOW & HIGH & LOW & LOW & LOW & HIGH & $H \mathrm{HGH}$ & $H I G H$ & r \\
\hline & EAF & LOW & LOW & LOW & HIGH & HIGH & HIGH & LOW & HIGH & LOW & HIGH & HIGH & HIGH & $\mathrm{HIGH}$ & LOW & $r$ \\
\hline & Modelling Languages & LOW & LOW & LOW & HIGH & $H I G H$ & LOW & LOW & LOW & $H I G H$ & $H I G H$ & HIGH & HIGH & $H I G H$ & LOW & $r$ \\
\hline & Monte Carlo & $H I G H$ & $\mathrm{HIGH}$ & LOW & LOW & LOW & HIGH & HIGH & $H I G H$ & LOW & LOW & LOW & LOW & $H I G H$ & $H I G H$ & $\boldsymbol{T}$ \\
\hline
\end{tabular}

Table B - 9 : M\&S Technique Selection - Civilian Visibility 
Systems Concept of

Owners and Operation/Use /

Component Dynamic Stochastic Uncertainty Systems Interactions lifecycle Variability Classification Functions Operations Employment No No Yes

No

No

Planning No

Acknowledged No

No

No

Targetting Capability

\section{No}

\section{No}

\begin{tabular}{|c|c|c|c|c|c|c|c|c|c|c|c|c|c|c|c|c|c|}
\hline & & & 0 & 0 & 2 & 0 & 0 & 0 & 1 & & & 0 & $1^{\prime}$ & 0 & & & \\
\hline & DES/DEVS & HIGH & HIGH & LOW & HIGH & HIGH & LoW & LOW & LOW & HGH & LOW & LoW & HIGH & LOW & $H G H$ & $r$ & 1 \\
\hline & Petri Nets & $H G H$ & $H G H$ & LOW & HIGH & $H G H$ & LOW & LOW & LOW & LOW & LOW & LOW & HIGH & LOW & $H G H$ & $r$ & 0 \\
\hline Yes & ABMS & $\mathrm{H} G \mathrm{H}$ & $H G H$ & LOW & HIGH & $H I G H$ & LOW & LOW & LOW & HGH & LOW & LOW & LOW & LOW & $H G H$ & $r$ & 1 \\
\hline \multirow[t]{2}{*}{ No } & System Dynamics & $\mathrm{HIGH}$ & $\mathrm{HIGH}$ & LOW & LOW & LOW & $H G H$ & HIGH & HIGH & LOW & HGH & HIGH & LOW & LOW & $H I G H$ & $r$ & 2 \\
\hline & Surrogate Models & $\mathrm{H} G \mathrm{GH}$ & $H G H$ & LOW & HIGH & LOW & $H I G H$ & LOW & LOW & LOW & LOW & LOW & LOW & LOW & $H G H$ & $r$ & 0 \\
\hline Directed & ANN & $H G H$ & LOW & LOW & LOW & LOW & HIGH & HIGH & HIGH & HIGH & LOW & LOW & LOW & $H I G H$ & $H G H$ & $r$ & 2 \\
\hline \multirow{2}{*}{\multicolumn{2}{|c|}{$\begin{array}{l}\text { Acknowledg BBN } \\
\text { Collaborativ Markov Models }\end{array}$}} & LOW & LOW & HIGH & LOW & LOW & HIGH & HIGH & HIGH & LOW & HIGH & HIGH & LOW & $H I G H$ & HIGH & $r$ & 3 \\
\hline & & LOW & LOW & HIGH & LOW & LOW & HIGH & HIGH & HIGH & LOW & HIGH & HIGH & LOW & $H I G H$ & HIGH & $r$ & 3 \\
\hline \multirow[t]{6}{*}{ Virtual } & Game Theory & LOW & LOW & HIGH & LOW & LOW & HIGH & HIGH & HIGH & LOW & HIGH & HIGH & LOW & LOW & LOW & $r$ & 3 \\
\hline & Decision Trees & LOW & $H G H$ & LOW & LOW & LOW & HIGH & HIGH & HIGH & LOW & LOW & LOW & LOW & $H I G H$ & $H I G H$ & $r$ & 1 \\
\hline & Network Models & LOW & LOW & LOW & LOW & HIGH & LOW & LOW & $H I G H$ & LOW & LOW & LOW & HIGH & $H G H$ & $H I G H$ & $r$ & 0 \\
\hline & EAF & LOW & LOW & LOW & HIGH & $H I G H$ & $H G H$ & LOW & $H I G H$ & LOW & $H G H$ & HIGH & HIGH & $H G H$ & LOW & $r$ & 1 \\
\hline & Modelling Languages & LOW & LOW & LOW & HIGH & $H I G H$ & LOW & LOW & LOW & HIGH & $H G H$ & HIGH & HIGH & $H G H$ & LOW & $r$ & 2 \\
\hline & Monte Carlo & $H G H$ & $H G H$ & LOW & LOW & LOW & $H I G H$ & HIGH & $H I G H$ & LOW & LOW & LOW & LOW & $H G H$ & $H \mid G H$ & $r$ & 1 \\
\hline
\end{tabular}


Systems Concept of

Component

Owners and Operation / Use /

Ease of

Model Model Components

Flight Path $\mathrm{F}$ Terrain

Aircraft Height
Dynamic Stochastic Uncertainty Systems

No No Yes $\begin{array}{ll}\text { Systems } & \text { Intera } \\ \text { No } & \text { No }\end{array}$
Interactions lifecycte
Planning No

Planning No
Operations Employment

Relationships

Acknowledged No No No

Acknowledged Yes No Yes creation Verifiable

\begin{tabular}{|c|c|c|c|c|c|c|c|c|c|c|c|c|c|c|c|c|c|}
\hline & & & b & 0 & 2 & 0 & 0 & 0 & 0 & & & 0 & $1^{\prime}$ & 0 & & & \\
\hline & DES/DEVS & $H G H$ & $H G H$ & LOW & $H I G H$ & HIGH & LOW & LOW & LOW & HIGH & LOW & LOW & $H G H$ & LOW & $H \mathrm{HGH}$ & 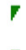 & 1 \\
\hline & Petri Nets & $H I G H$ & $H I G H$ & LOW & $H I G H$ & HIGH & LOW & LOW & LOW & LOW & LOW & LOW & $H G H$ & LOW & HIGH & ' & 0 \\
\hline Yes & ABMS & $H G H$ & $H G H$ & LOW & $H I G H$ & HIGH & LOW & LOW & LOW & HIGH & LOW & LOW & LOW & LOW & HIGH & ' & 1 \\
\hline \multirow[t]{2}{*}{ No } & System Dynamics & $H G H$ & $H G H$ & LOW & LOW & LOW & HIGH & $H I G H$ & HIGH & LOW & HIGH & HIGH & LOW & LOW & HIGH & $r$ & 1 \\
\hline & Surrogate Models & $H G H$ & $H I G H$ & LOW & $H I G H$ & LOW & HIGH & LOW & LOW & LOW & LOW & LOW & LOW & LOW & $H I G H$ & $\boldsymbol{T}$ & 0 \\
\hline Directed & ANN & $H G H$ & LOW & LOW & LOW & LOW & HIGH & $H I G H$ & HIGH & HIGH & LOW & LOW & LOW & HIGH & HIGH & 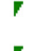 & 1 \\
\hline \multicolumn{2}{|c|}{ Acknowledg BBN } & LOW & LOW & $\mathrm{HIGH}$ & LOW & LOW & HIGH & $H I G H$ & HIGH & LOW & HIGH & HIGH & LOW & HIGH & HIGH & $r$ & 2 \\
\hline \multicolumn{2}{|c|}{ Collaborativ Markov Models } & LOW & LOW & $H G H$ & LOW & LOW & HIGH & $H I G H$ & HIGH & LOW & HIGH & HIGH & LOW & $H G H$ & HIGH & 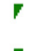 & 2 \\
\hline \multirow[t]{6}{*}{ Virtual } & Game Theory & LOW & LOW & $\mathrm{HIGH}$ & LOW & LOW & HIGH & $H I G H$ & HIGH & LOW & HIGH & $H I G H$ & LOW & LOW & LOW & r & 2 \\
\hline & Decision Trees & LOW & $H G H$ & LOW & LOW & LOW & HIGH & $H I G H$ & HIGH & LOW & LOW & LOW & LOW & $H G H$ & HIGH & $r$ & 0 \\
\hline & Network Models & LOW & LOW & LOW & LOW & HIGH & LOW & LOW & HIGH & LOW & LOW & LOW & HIGH & HGH & HIGH & 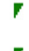 & 0 \\
\hline & EAF & LOW & LOW & LOW & $H I G H$ & HIGH & HIGH & LOW & HIGH & LOW & HIGH & HIGH & HIGH & $H I G H$ & LOW & $r$ & 1 \\
\hline & Modelling Languages & LOW & LOW & LOW & $H I G H$ & HGG & LOW & LOW & LOW & HIGH & HIGH & HIGH & HIGH & HGH & LOW & 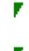 & 2 \\
\hline & Monte Carlo & $H G H$ & $H$ HGH & LOW & LOW & LOW & HIGH & $H I G H$ & HIGH & LOW & LOW & LOW & LOW & $H I G H$ & HIGH & $r$ & 0 \\
\hline
\end{tabular}

Table B - 11 : M\&S Technique Selection: Flight Path Risk 
Systems Concept of

Owners and Operation / Use /

Component

Dynamic Stochastic Uncertainty Systems Interactions lifecycle Variability Classification Functions Operations Employment Relationships

No No Yes Yes No Planning No Acknowledged Yes

No

Yes

Counter Measures

No Yes

Yes

Planning No

Acknowledged Yes

No

Yes
Ease of creation Verifiable

\begin{tabular}{|c|c|c|c|c|c|c|c|c|c|c|c|c|c|c|c|c|c|}
\hline & & & 0 & 0 & 2 & 2 & 0 & 0 & 0 & & ? & 0 & $2^{\prime \prime}$ & 0 & & & \\
\hline & DES/DEVS & $H G H$ & HIGH & LOW & HIGH & HIGH & LOW & LOW & LOW & HIGH & LOW & LOW & HIGH & LOW & $H G H$ & r & 2 \\
\hline & Petri Nets & $H G H$ & $H G H$ & LOW & $H I G H$ & $H G H$ & LOW & LOW & LOW & LOW & LOW & LOW & HGG & LOW & $H \| G$ & $\boldsymbol{T}$ & 1 \\
\hline Yes & ABMS & $H G H$ & $H G H$ & LOW & $H I G H$ & $H G H$ & LOW & LOW & LOW & HIGH & LOW & LOW & LOW & LOW & $H \mid G H$ & $r$ & 2 \\
\hline \multirow[t]{2}{*}{ No } & System Dynamics & $H G H$ & $H G H$ & LOW & LOW & LOW & HIGH & $H G H$ & HIGH & LOW & $H I G H$ & HIGH & LOW & LOW & $H I G H$ & $r$ & 1 \\
\hline & Surrogate Models & $H G H$ & $H G H$ & LOW & HIGH & LOW & $H G H$ & LOW & LOW & LOW & LOW & LOW & LOW & LOW & $H G H$ & $r$ & 1 \\
\hline Directed & ANN & $H G H$ & LOW & LOW & LOW & LOW & HIGH & $H I G H$ & HIGH & HIGH & LOW & LOW & LOW & HIGH & $H I G H$ & $r$ & 1 \\
\hline \multirow{2}{*}{\multicolumn{2}{|c|}{$\begin{array}{l}\text { Acknowledg BBN } \\
\text { Collaborativ Markov Models }\end{array}$}} & LOW & LOW & HIGH & LOW & LOW & HIGH & $H I G H$ & HIGH & LOW & HIGH & HIGH & LOW & $H G H$ & $H G H$ & $\boldsymbol{T}$ & 2 \\
\hline & & LOW & LOW & $H I G H$ & LOW & LOW & HIGH & $H I G H$ & $H I G H$ & LOW & HIGH & HIGH & LOW & HIGH & $H G H$ & $\boldsymbol{T}$ & 2 \\
\hline \multirow[t]{6}{*}{ Virtual } & Game Theory & LOW & LOW & $H I G H$ & LOW & LOW & $H I G H$ & $H I G H$ & HIGH & LOW & $H$ HGH & HIGH & LOW & LOW & LOW & $r$ & 2 \\
\hline & Decision Trees & LOW & $H G H$ & LOW & LOW & LOW & HIGH & $H I G H$ & HIGH & LOW & LOW & LOW & LOW & HIGH & $H G H$ & $\boldsymbol{r}$ & 0 \\
\hline & Network Models & LOW & LOW & LOW & LOW & $H I G H$ & LOW & LOW & HIGH & LOW & LOW & LOW & HIGH & HIGH & $H G H$ & $\boldsymbol{T}$ & 0 \\
\hline & EAF & LOW & LOW & LOW & HIGH & HIGH & HIGH & LOW & HIGH & LOW & HIGH & HIGH & HIGH & HIGH & LOW & $\boldsymbol{T}$ & 2 \\
\hline & Modelling Languages & LOW & LOW & LOW & $H I G H$ & HIGH & LOW & LOW & LOW & HIGH & $H I G H$ & HIGH & HIGH & $H G H$ & LOW & $r$ & 3 \\
\hline & Monte Carlo & $H G H$ & $H G H$ & LOW & LOW & LOW & HIGH & $H G H$ & HIGH & LOW & LOW & LOW & LOW & $H G H$ & $H G H$ & $r$ & 0 \\
\hline
\end{tabular}

Table B - 12 : M\&S Technique Selection: Defensive Capability 


\section{Appendix C - Application Case Study 1 Bayesian Belief Network Probability Tables}

This appendix contains the probability tables, which are associated with application case study 1 (Close Air Support) Bayesian Belief Network (BBN) conditional probability table variables; the following tables were generated by the Netica application, reporting facility, providing evidence of the research methodology through the development of models and a point from which future research may further develop the case study, enabling the BBNs to be reconstructed.

\section{Risk Probability Tables}

Table for "AtmosphericVisibility"

\begin{tabular}{|l|ll|}
\hline Atmospheric Visibility & \multicolumn{1}{|c|}{ CloudLevel } & DustLevel \\
\hline High & LessThanTwoOktas & Low \\
High & LessThanTwoOktas & Medium \\
Low & LessThanTwoOktas & High \\
High & BetweenTwoAndSixOktas & Low \\
Medium & BetweenTwoAndSixOktas & Medium \\
Low & BetweenTwoAndSixOktas & High \\
Medium & GreatThanSixOktas & Low \\
Medium & GreatThanSixOktas & Medium \\
Low & GreatThanSixOktas & High \\
\hline
\end{tabular}

Table for "BlueForceVisibility"

\begin{tabular}{|l|ll|}
\hline BlueForceVisibility & AtmosphericVisibility & \multicolumn{1}{c|}{ Light } \\
\hline Low & Low & Daytime \\
Low & Low & Night \\
Low & Low & Twilight \\
Medium & Medium & Daytime \\
Low & Medium & Night \\
Medium & Medium & Twilight \\
High & High & Daytime \\
Low & High & Night \\
Medium & High & Twilight \\
\hline
\end{tabular}


able for "BlueForceRisk"

\begin{tabular}{|c|c|c|c|c|}
\hline $\begin{array}{l}\text { Lo } \\
\text { w }\end{array}$ & $\underset{\mathrm{m}}{\text { Mediu }}$ & $\begin{array}{c}\mathrm{Hig} \\
\mathrm{h}\end{array}$ & $\begin{array}{c}\text { BlueForceVisibili } \\
\text { ty }\end{array}$ & BlueForceProximity \\
\hline $\begin{array}{l}0.0 \\
5\end{array}$ & 0.25 & 0.7 & Low & LessThan $500 \mathrm{~m}$ \\
\hline $\begin{array}{l}0.2 \\
5\end{array}$ & 0.25 & 0.5 & Low & $\begin{array}{l}\text { Between } 500 \mathrm{mAnd} 100 \\
\text { Om }\end{array}$ \\
\hline 0.5 & 0.3 & 0.2 & Low & GreaterThan $1000 \mathrm{~m}$ \\
\hline 0.2 & 0.3 & 0.5 & Medium & LessThan $500 \mathrm{~m}$ \\
\hline 0.2 & 0.5 & 0.3 & Medium & $\begin{array}{l}\text { Between } 500 \text { mAnd } 100 \\
\text { Om }\end{array}$ \\
\hline 0.5 & 0.4 & 0.1 & Medium & GreaterThan $1000 \mathrm{~m}$ \\
\hline 0.5 & 0.3 & 0.2 & High & LessThan $500 \mathrm{~m}$ \\
\hline 0.7 & 0.2 & 0.1 & High & $\begin{array}{l}\text { Between } 500 \mathrm{mAnd} 100 \\
\text { Om }\end{array}$ \\
\hline 0.8 & 0.15 & 0.05 & High & GreaterThan $1000 \mathrm{~m}$ \\
\hline
\end{tabular}

Table for "OperatorWorkload"

\begin{tabular}{|lll|ll|}
\hline Low & Medium & High & ScenarioDensity & C2SystemCapability \\
\hline 0.6 & 0.3 & 0.1 & Low & Low \\
0.7 & 0.2 & 0.1 & Low & Medium \\
0.95 & 0.03 & 0.02 & Low & High \\
0.1 & 0.55 & 0.35 & Medium & Low \\
0.1 & 0.8 & 0.1 & Medium & Medium \\
0.45 & 0.5 & 0.05 & Medium & High \\
0.01 & 0.09 & 0.9 & High & Low \\
0.01 & 0.39 & 0.6 & High & Medium \\
0.2 & 0.6 & 0.2 & High & High \\
\hline
\end{tabular}

\section{Table for "SurveillanceCapability"}

\begin{tabular}{|lll|ll|}
\hline Low & Medium & High & UAVCapability & ASTORCapability \\
\hline 1 & 0 & 0 & Low & Low \\
0.5 & 0.5 & 0 & Low & Medium \\
0.2 & 0.6 & 0.2 & Low & High \\
0.5 & 0.5 & 0 & Medium & Low \\
0 & 1 & 0 & Medium & Medium \\
0 & 0.5 & 0.5 & Medium & High \\
0.2 & 0.6 & 0.2 & High & Low \\
0 & 0.5 & 0.5 & High & Medium \\
0 & 0 & 1 & High & High \\
\hline
\end{tabular}

Table for "IntelligenceCapability"

\begin{tabular}{|lll|ll|}
\hline Low & Medium & High & SIGINTCapability & VisualCapability \\
\hline 1 & 0 & 0 & Low & Low \\
0.5 & 0.5 & 0 & Low & Medium \\
0.2 & 0.6 & 0.2 & Low & High \\
0.5 & 0.5 & 0 & Medium & Low \\
0 & 1 & 0 & Medium & Medium \\
0 & 0.5 & 0.5 & Medium & High \\
\hline
\end{tabular}

\begin{tabular}{|lll|ll|}
\hline 0.2 & 0.6 & 0.2 & High & Low \\
0 & 0.5 & 0.5 & High & Medium \\
0 & 0 & 1 & High & High \\
\hline
\end{tabular}

ble for "ISRCapability"

\begin{tabular}{|lll|ll|}
\hline $\begin{array}{c}\text { Lo } \\
\mathbf{w}\end{array}$ & $\begin{array}{c}\text { Mediu } \\
\mathbf{m}\end{array}$ & $\begin{array}{c}\text { Hig } \\
\mathbf{h}\end{array}$ & $\begin{array}{l}\text { SurveillanceCapabil } \\
\text { ity }\end{array}$ & $\begin{array}{l}\text { IntelligenceCapabil } \\
\text { ity }\end{array}$ \\
\hline 1 & 0 & 0 & Low & Low \\
0.5 & 0.5 & 0 & Low & Medium \\
0.2 & 0.6 & 0.2 & Low & High \\
0.5 & 0.5 & 0 & Medium & Low \\
0 & 1 & 0 & Medium & Medium \\
0 & 0.5 & 0.5 & Medium & High \\
0.2 & 0.6 & 0.2 & High & Low \\
0 & 0.5 & 0.5 & High & Medium \\
0 & 0 & 1 & High & High \\
\hline
\end{tabular}

Table for "SoSCapability"

\begin{tabular}{|lll|ll|}
\hline Low & Medium & High & OperatorWorkload & ISRCapability \\
\hline 0.6 & 0.4 & 0 & Low & Low \\
0 & 0.8 & 0.2 & Low & Medium \\
0 & 0 & 1 & Low & High \\
0.8 & 0.2 & 0 & Medium & Low \\
0 & 1 & 0 & Medium & Medium \\
0 & 0.3 & 0.7 & Medium & High \\
1 & 0 & 0 & High & Low \\
0.2 & 0.8 & 0 & High & Medium \\
0.1 & 0.3 & 0.6 & High & High \\
\hline
\end{tabular}

Table for "SAQuality"

\begin{tabular}{|lll|ll|}
\hline Low & Medium & High & Interoperability & SoSCapability \\
\hline 1 & 0 & 0 & Low & Low \\
0.5 & 0.5 & 0 & Low & Medium \\
0.2 & 0.6 & 0.2 & Low & High \\
0.5 & 0.5 & 0 & Medium & Low \\
0 & 1 & 0 & Medium & Medium \\
0 & 0.5 & 0.5 & Medium & High \\
0.2 & 0.6 & 0.2 & High & Low \\
0 & 0.5 & 0.5 & High & Medium \\
0 & 0 & 1 & High & High \\
\hline
\end{tabular}


Table for "InformationQuality"

\begin{tabular}{|lll|ll|}
\hline Low & Medium & High & \multicolumn{1}{|c|}{ KillChainAccuracy } & SAQuality \\
\hline 0.2 & 0.6 & 0.2 & LessThan100m & Low \\
0 & 0.5 & 0.5 & LessThan100m & Medium \\
0 & 0.1 & 0.9 & LessThan100m & High \\
0.5 & 0.5 & 0 & Between100mAnd200m & Low \\
0.2 & 0.8 & 0 & Between100mAnd200m & Medium \\
0.1 & 0.45 & 0.45 & Between100mAnd200m & High \\
0.9 & 0.1 & 0 & GreaterThan200m & Low \\
0.5 & 0.5 & 0 & GreaterThan200m & Medium \\
0.2 & 0.6 & 0.2 & GreaterThan200m & High \\
\hline
\end{tabular}

\section{Table for "Fractricide"}

\begin{tabular}{|lll|ll|}
\hline High & Medium & Low & BlueForceRisk & InformationQuality \\
\hline 0 & 0.5 & 0.5 & Low & Low \\
0 & 0.3 & 0.7 & Low & Medium \\
0 & 0.1 & 0.9 & Low & High \\
0.3 & 0.7 & 0 & Medium & Low \\
0 & 1 & 0 & Medium & Medium \\
0 & 0.5 & 0.5 & Medium & High \\
0.9 & 0.1 & 0 & High & Low \\
0.7 & 0.3 & 0 & High & Medium \\
0.3 & 0.6 & 0.1 & High & High \\
\hline
\end{tabular}

Table for "CivilianVisibility"

\begin{tabular}{|l|ll|}
\hline CivilianVisibility & Atmospheric Visibility & LightLevel \\
\hline Low & Low & Daytime \\
Low & Low & Night \\
Low & Low & Twilight \\
Medium & Medium & Daytime \\
Low & Medium & Night \\
Medium & Medium & Twilight \\
High & High & Daytime \\
Low & High & Night \\
Medium & High & Twilight \\
\hline
\end{tabular}

Table for "CivilianRisk"

\begin{tabular}{|lll|ll|}
\hline Low & Medium & High & CivilianVisibility & CivilianProximity \\
\hline 0.1 & 0.2 & 0.7 & Low & LessThan $1 \mathrm{~km}$ \\
0.5 & 0.25 & 0.25 & Low & Between $1 \mathrm{kmAnd} 2 \mathrm{~km}$ \\
0.7 & 0.25 & 0.05 & Low & GreaterThan $2 \mathrm{~km}$ \\
0.1 & 0.3 & 0.6 & Medium & LessThan $1 \mathrm{~km}$ \\
0.45 & 0.35 & 0.2 & Medium & Between $1 \mathrm{kmAnd} 2 \mathrm{~km}$ \\
\hline
\end{tabular}

\begin{tabular}{|lll|ll|}
\hline 0.75 & 0.2 & 0.05 & Medium & GreaterThan2km \\
0.2 & 0.3 & 0.5 & High & LessThan1km \\
0.6 & 0.25 & 0.15 & High & Between1kmAnd2km \\
0.9 & 0.08 & 0.02 & High & GreaterThan2km \\
\hline
\end{tabular}

Table for "CollateralDamage"

\begin{tabular}{|lll|ll|}
\hline High & Medium & Low & CivilianRisk & InformationQuality \\
\hline 0 & 0.5 & 0.5 & Low & Low \\
0 & 0.3 & 0.7 & Low & Medium \\
0 & 0.1 & 0.9 & Low & High \\
0.3 & 0.7 & 0 & Medium & Low \\
0 & 1 & 0 & Medium & Medium \\
0 & 0.5 & 0.5 & Medium & High \\
0.9 & 0.1 & 0 & High & Low \\
0.7 & 0.3 & 0 & High & Medium \\
0.3 & 0.6 & 0.1 & High & High \\
\hline
\end{tabular}

Table for "FlightPathRisk"

\begin{tabular}{|lll|ll|}
\hline Low & Medium & High & \multicolumn{1}{|c|}{ Terrain } & \multicolumn{1}{c|}{ Aircraftheight } \\
\hline 0.7 & 0.2 & 0.1 & Flat & Below500ft \\
0.85 & 0.1 & 0.05 & Flat & Between500and1000ft \\
0.95 & 0.05 & 0 & Flat & Above1000ft \\
0.3 & 0.5 & 0.2 & Undulating & Below500ft \\
0.5 & 0.4 & 0.1 & Undulating & Between500and1000ft \\
0.7 & 0.25 & 0.05 & Undulating & Above1000ft \\
0.1 & 0.2 & 0.7 & Mountainous & Below500ft \\
0.15 & 0.25 & 0.6 & Mountainous & Between500and1000ft \\
0.3 & 0.4 & 0.3 & Mountainous & Above1000ft \\
\hline
\end{tabular}

Table for "EnemyCapabiity"

\begin{tabular}{|lll|ll|}
\hline Low & Medium & High & WeaponType & Targetting \\
\hline 1 & 0 & 0 & Low & Low \\
0.7 & 0.3 & 0 & Low & Medium \\
0.25 & 0.5 & 0.25 & Low & High \\
0.7 & 0.3 & 0 & Medium & Low \\
0.25 & 0.5 & 0.25 & Medium & Medium \\
0.1 & 0.6 & 0.3 & Medium & High \\
0.25 & 0.5 & 0.25 & High & Low \\
0.1 & 0.3 & 0.6 & High & Medium \\
0 & 0 & 1 & High & High \\
\hline
\end{tabular}


Table for "DefensiveCapability"

\begin{tabular}{|lll|ll|}
\hline Low & Medium & High & Jamming & CounterMeasures \\
\hline 1 & 0 & 0 & Low & Low \\
0.7 & 0.3 & 0 & Low & Medium \\
0.25 & 0.5 & 0.25 & Low & High \\
0.7 & 0.3 & 0 & Medium & Low \\
0.25 & 0.5 & 0.25 & Medium & Medium \\
0.1 & 0.6 & 0.3 & Medium & High \\
0.25 & 0.5 & 0.25 & High & Low \\
0.1 & 0.3 & 0.6 & High & Medium \\
0 & 0 & 1 & High & High \\
\hline
\end{tabular}

Table for "PlatformDefence"

\begin{tabular}{|lll|ll|}
\hline Low & Medium & High & EnemyCapabiity & DefensiveCapability \\
\hline 0 & 0.2 & 0.8 & Low & Low \\
0 & 0.1 & 0.9 & Low & Medium \\
0 & 0.01 & 0.99 & Low & High \\
0.55 & 0.4 & 0.05 & Medium & Low \\
0.1 & 0.8 & 0.1 & Medium & Medium \\
0.05 & 0.25 & 0.7 & Medium & High \\
0.9 & 0.1 & 0 & High & Low \\
0.25 & 0.7 & 0.05 & High & Medium \\
0.2 & 0.6 & 0.2 & High & High \\
\hline
\end{tabular}

Table for "PlatformVulnerability"

\begin{tabular}{|lll|ll|}
\hline Low & Medium & High & SAQuality & PlatformDefence \\
\hline 0 & 0.3 & 0.7 & Low & Low \\
0 & 0.8 & 0.2 & Low & Medium \\
0.4 & 0.4 & 0.2 & Low & High \\
0 & 0.5 & 0.5 & Medium & Low \\
0 & 1 & 0 & Medium & Medium \\
0.5 & 0.5 & 0 & Medium & High \\
0.2 & 0.5 & 0.3 & High & Low \\
0.7 & 0.25 & 0.05 & High & Medium \\
0.9 & 0.1 & 0 & High & High \\
\hline
\end{tabular}

Table for "LossOfDeliveryAircraft"

\begin{tabular}{|lll|ll|}
\hline Low & Medium & High & PlatformVulnerability & FlightPathRisk \\
\hline 1 & 0 & 0 & Low & Low \\
0.5 & 0.5 & 0 & Low & Medium \\
0.2 & 0.6 & 0.2 & Low & High \\
0.5 & 0.5 & 0 & Medium & Low \\
\hline
\end{tabular}

\begin{tabular}{|lll|ll|}
\hline 0 & 1 & 0 & Medium & Medium \\
0 & 0.5 & 0.5 & Medium & High \\
0.2 & 0.6 & 0.2 & High & Low \\
0 & 0.5 & 0.5 & High & Medium \\
0 & 0 & 1 & High & High \\
\hline
\end{tabular}

Table for "EnvironmentalVisibility"

\begin{tabular}{|l|ll|}
\hline EnvironmentalVisibility & AtmosphericVisibility & Light \\
\hline Low & Low & Daytime \\
Low & Low & Night \\
Low & Low & Twilight \\
Medium & Medium & Daytime \\
Low & Medium & Night \\
Medium & Medium & Twilight \\
High & High & Daytime \\
Low & High & Night \\
Medium & High & Twilight \\
\hline
\end{tabular}

Table for "Marking"

\begin{tabular}{|l|lll|}
\hline Marking & Smoke & Phosphorous & Laser \\
\hline High & Present & Present & Present \\
Medium & Present & Present & Absent \\
High & Present & Absent & Present \\
Low & Present & Absent & Absent \\
High & Absent & Present & Present \\
Medium & Absent & Present & Absent \\
High & Absent & Absent & Present \\
Low & Absent & Absent & Absent \\
\hline
\end{tabular}

Table for "TargetVisLevel"

\begin{tabular}{|lll|ll|}
\hline Low & Medium & High & Marking & Camouflage \\
\hline 0.3 & 0.4 & 0.3 & Low & Low \\
0.6 & 0.4 & 0 & Low & Medium \\
0.9 & 0.1 & 0 & Low & High \\
0.1 & 0.6 & 0.3 & Medium & Low \\
0.2 & 0.7 & 0.1 & Medium & Medium \\
0.4 & 0.55 & 0.05 & Medium & High \\
0.05 & 0.15 & 0.8 & High & Low \\
0.05 & 0.25 & 0.7 & High & Medium \\
0.1 & 0.4 & 0.5 & High & High \\
\hline
\end{tabular}


Table for "VisualVisibility"

\begin{tabular}{|lll|ll|}
\hline $\begin{array}{c}\text { Lo } \\
\mathbf{w}\end{array}$ & $\begin{array}{c}\text { Mediu } \\
\mathbf{m}\end{array}$ & $\begin{array}{c}\text { Hig } \\
\mathbf{h}\end{array}$ & \multicolumn{1}{|c|}{$\begin{array}{c}\text { EnvironmentalVisibilit } \\
\mathbf{y}\end{array}$} & $\begin{array}{c}\text { TargetVisLeve } \\
\mathbf{~}\end{array}$ \\
\hline 0.9 & 0.1 & 0 & Low & Low \\
0.7 & 0.3 & 0 & Low & Medium \\
0.2 & 0.6 & 0.2 & Low & High \\
0.5 & 0.5 & 0 & Medium & Low \\
0 & 1 & 0 & Medium & Medium \\
0 & 0.5 & 0.5 & Medium & High \\
0.2 & 0.6 & 0.2 & High & Low \\
0 & 0.5 & 0.5 & High & Medium \\
0 & 0 & 1 & High & High \\
\hline
\end{tabular}

Table for "TargetVisibility"

\begin{tabular}{|lll|ll|}
\hline $\begin{array}{l}\text { Lo } \\
\mathbf{w}\end{array}$ & $\begin{array}{c}\text { Mediu } \\
\mathbf{m}\end{array}$ & $\begin{array}{c}\text { Hig } \\
\mathbf{h}\end{array}$ & $\begin{array}{l}\text { WeaponSensorCapabiit } \\
\mathbf{y}\end{array}$ & $\begin{array}{l}\text { VisualVisibilit } \\
\mathbf{y}\end{array}$ \\
\hline 1 & 0 & 0 & Low & Low \\
0 & 0.5 & 0.5 & Low & Medium \\
0.2 & 0.6 & 0.2 & Low & High \\
0.5 & 0.5 & 0 & Medium & Low \\
0 & 1 & 0 & Medium & Medium \\
0 & 0.5 & 0.5 & Medium & High \\
0.2 & 0.6 & 0.2 & High & Low \\
0 & 0.5 & 0.5 & High & Medium \\
0 & 0 & 1 & High & High \\
\hline
\end{tabular}

Table for "TargetDestructionRisk"

\begin{tabular}{|lll|ll|}
\hline High & Medium & Low & TargetVisibility & InformationQuality \\
\hline 0.9 & 0.1 & 0 & Low & Low \\
0.7 & 0.3 & 0 & Low & Medium \\
0.1 & 0.6 & 0.3 & Low & High \\
0.8 & 0.2 & 0 & Medium & Low \\
0.1 & 0.7 & 0.2 & Medium & Medium \\
0.05 & 0.15 & 0.8 & Medium & High \\
0.5 & 0.5 & 0 & High & Low \\
0.05 & 0.2 & 0.75 & High & Medium \\
0.01 & 0.09 & 0.9 & High & High \\
\hline
\end{tabular}

Table for "AdditionalTargetOpportunity"

\begin{tabular}{|lll|ll|}
\hline High & Medium & Low & TargetVisibility & SAQuality \\
\hline 0 & 0 & 1 & Low & Low \\
0 & 0.5 & 0.5 & Low & Medium \\
0.4 & 0.3 & 0.3 & Low & High \\
0 & 0.2 & 0.8 & Medium & Low \\
0 & 1 & 0 & Medium & Medium \\
\hline
\end{tabular}

\begin{tabular}{|lll|ll|}
\hline 0.7 & 0.3 & 0 & Medium & High \\
0 & 0.3 & 0.7 & High & Low \\
0.2 & 0.8 & 0 & High & Medium \\
1 & 0 & 0 & High & High \\
\hline
\end{tabular}




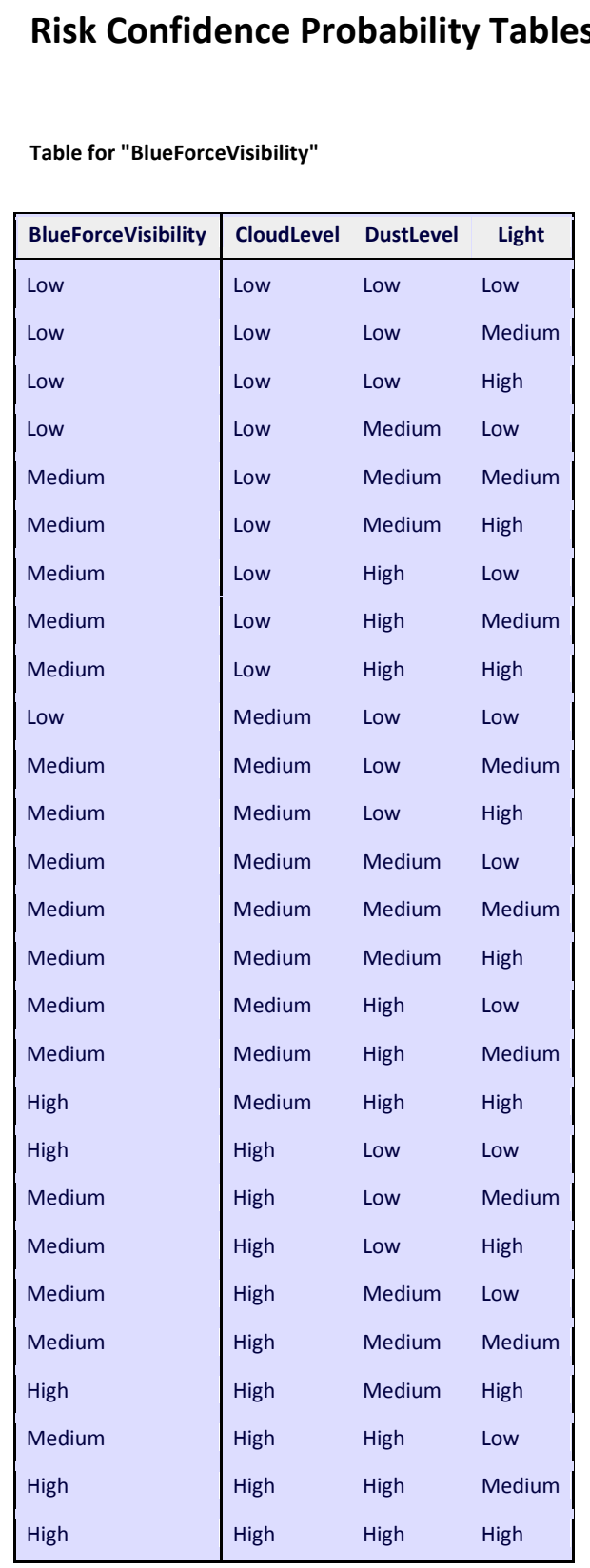

Table for "BlueForceRisk"

\begin{tabular}{|l|ll|}
\hline BlueforceRisk & BlueForceVisibility & BlueforceProximity \\
\hline Low & Low & Low \\
Low & Low & Medium \\
Medium & Low & High \\
Low & Medium & Low \\
Medium & Medium & Medium \\
Medium & Medium & High \\
Medium & High & Low \\
Medium & High & Medium \\
High & High & High \\
\hline
\end{tabular}

Table for "ISRCapability"

\begin{tabular}{|c|c|c|c|c|}
\hline $\begin{array}{c}\text { ISRCapa } \\
\text { bility }\end{array}$ & $\begin{array}{l}\text { UAVCapa } \\
\text { bility }\end{array}$ & $\begin{array}{c}\text { ASTORCapa } \\
\text { bility }\end{array}$ & $\begin{array}{c}\text { SIGINTCapa } \\
\text { bility }\end{array}$ & $\begin{array}{c}\text { VisualCapa } \\
\text { bility }\end{array}$ \\
\hline Low & Low & Low & Low & Low \\
\hline Low & Low & Low & Low & Medium \\
\hline Low & Low & Low & Low & High \\
\hline Low & Low & Low & Medium & Low \\
\hline Low & Low & Low & Medium & Medium \\
\hline Medium & Low & Low & Medium & High \\
\hline Low & Low & Low & High & Low \\
\hline Medium & Low & Low & High & Medium \\
\hline Medium & Low & Low & High & High \\
\hline Low & Low & Medium & Low & Low \\
\hline Low & Low & Medium & Low & Medium \\
\hline Medium & Low & Medium & Low & High \\
\hline Low & Low & Medium & Medium & Low \\
\hline Medium & Low & Medium & Medium & Medium \\
\hline Medium & Low & Medium & Medium & High \\
\hline Medium & Low & Medium & High & Low \\
\hline Medium & Low & Medium & High & Medium \\
\hline Medium & Low & Medium & High & High \\
\hline Low & Low & High & Low & Low \\
\hline Medium & Low & High & Low & Medium \\
\hline Medium & Low & High & Low & High \\
\hline Medium & Low & High & Medium & Low \\
\hline Medium & Low & High & Medium & Medium \\
\hline Medium & Low & High & Medium & High \\
\hline Medium & Low & High & High & Low \\
\hline Medium & Low & High & High & Medium \\
\hline Medium & Low & High & High & High \\
\hline Low & Medium & Low & Low & Low \\
\hline Low & Medium & Low & Low & Medium \\
\hline Low & Medium & Low & Low & High \\
\hline Low & Medium & Low & Medium & Low \\
\hline Medium & Medium & Low & Medium & Medium \\
\hline Medium & Medium & Low & Medium & High \\
\hline Medium & Medium & Low & High & Low \\
\hline Medium & Medium & Low & High & Medium \\
\hline Medium & Medium & Low & High & High \\
\hline Low & Medium & Medium & Low & Low \\
\hline Medium & Medium & Medium & Low & Medium \\
\hline Medium & Medium & Medium & Low & High \\
\hline Medium & Medium & Medium & Medium & Low \\
\hline Medium & Medium & Medium & Medium & Medium \\
\hline Medium & Medium & Medium & Medium & High \\
\hline Medium & Medium & Medium & High & Low \\
\hline Medium & Medium & Medium & High & Medium \\
\hline Medium & Medium & Medium & High & High \\
\hline Medium & Medium & High & Low & Low \\
\hline
\end{tabular}




\begin{tabular}{|c|c|c|c|c|}
\hline $\begin{array}{c}\text { ISRCapa } \\
\text { bility }\end{array}$ & $\begin{array}{c}\text { UAVCapa } \\
\text { bility }\end{array}$ & $\begin{array}{c}\text { ASTORCapa } \\
\text { bility }\end{array}$ & $\begin{array}{l}\text { SIGINTCapa } \\
\text { bility }\end{array}$ & $\begin{array}{c}\text { VisualCapa } \\
\text { bility }\end{array}$ \\
\hline Medium & Medium & High & Low & Medium \\
\hline Medium & Medium & High & Low & High \\
\hline Medium & Medium & High & Medium & Low \\
\hline Medium & Medium & High & Medium & Medium \\
\hline Medium & Medium & High & Medium & High \\
\hline Medium & Medium & High & High & Low \\
\hline Medium & Medium & High & High & Medium \\
\hline High & Medium & High & High & High \\
\hline Low & High & Low & Low & Low \\
\hline Medium & High & Low & Low & Medium \\
\hline Medium & High & Low & Low & High \\
\hline Medium & High & Low & Medium & Low \\
\hline Medium & High & Low & Medium & Medium \\
\hline Medium & High & Low & Medium & High \\
\hline Medium & High & Low & High & Low \\
\hline Medium & High & Low & High & Medium \\
\hline Medium & High & Low & High & High \\
\hline Medium & High & Medium & Low & Low \\
\hline Medium & High & Medium & Low & Medium \\
\hline Medium & High & Medium & Low & High \\
\hline Medium & High & Medium & Medium & Low \\
\hline Medium & High & Medium & Medium & Medium \\
\hline Medium & High & Medium & Medium & High \\
\hline Medium & High & Medium & High & Low \\
\hline Medium & High & Medium & High & Medium \\
\hline High & High & Medium & High & High \\
\hline Medium & High & High & Low & Low \\
\hline Medium & High & High & Low & Medium \\
\hline Medium & High & High & Low & High \\
\hline Medium & High & High & Medium & Low \\
\hline Medium & High & High & Medium & Medium \\
\hline High & High & High & Medium & High \\
\hline Medium & High & High & High & Low \\
\hline High & High & High & High & Medium \\
\hline High & High & High & High & High \\
\hline
\end{tabular}

Table for "OperatorWorkload"

\begin{tabular}{|l|ll|}
\hline OperatorWorkload & ScenarioDensity & C2SystemCapability \\
\hline Low & Low & Low \\
Low & Low & Medium \\
Medium & Low & High \\
Low & Medium & Low \\
Medium & Medium & Medium \\
High & Medium & High \\
Medium & High & Low \\
\hline
\end{tabular}

\begin{tabular}{|l|ll|}
\hline High & High & Medium \\
High & High & High \\
\hline
\end{tabular}

Table for "SAQuality"

\begin{tabular}{|c|c|c|c|}
\hline $\begin{array}{c}\text { SAQualit } \\
y\end{array}$ & $\begin{array}{c}\text { Interoperabilit } \\
y\end{array}$ & $\begin{array}{c}\text { ISRCapabilit } \\
y\end{array}$ & $\begin{array}{c}\text { OperatorWorkloa } \\
\text { d }\end{array}$ \\
\hline Low & Low & Low & Low \\
\hline Low & Low & Low & Medium \\
\hline Low & Low & Low & High \\
\hline Low & Low & Medium & Low \\
\hline Low & Low & Medium & Medium \\
\hline Low & Low & Medium & High \\
\hline Low & Low & High & Low \\
\hline Low & Low & High & Medium \\
\hline Low & Low & High & High \\
\hline Low & Medium & Low & Low \\
\hline Medium & Medium & Low & Medium \\
\hline Medium & Medium & Low & High \\
\hline Medium & Medium & Medium & Low \\
\hline Medium & Medium & Medium & Medium \\
\hline Medium & Medium & Medium & High \\
\hline Medium & Medium & High & Low \\
\hline Medium & Medium & High & Medium \\
\hline Medium & Medium & High & High \\
\hline Low & High & Low & Low \\
\hline Medium & High & Low & Medium \\
\hline Medium & High & Low & High \\
\hline Medium & High & Medium & Low \\
\hline High & High & Medium & Medium \\
\hline High & High & Medium & High \\
\hline High & High & High & Low \\
\hline High & High & High & Medium \\
\hline High & High & High & High \\
\hline
\end{tabular}

Table for "Fractricide"

\begin{tabular}{|c|c|c|c|}
\hline Fractricide & KillChainAccuracy & SAQuality & BlueForceRisk \\
\hline Low & Low & Low & Low \\
\hline Low & Low & Low & Medium \\
\hline Low & Low & Low & High \\
\hline Low & Low & Medium & Low \\
\hline Medium & Low & Medium & Medium \\
\hline Medium & Low & Medium & High \\
\hline Low & Low & High & Low \\
\hline Medium & Low & High & Medium \\
\hline Medium & Low & High & High \\
\hline Low & Medium & Low & Low \\
\hline Medium & Medium & Low & Medium \\
\hline
\end{tabular}




\begin{tabular}{|l|lll|}
\hline Fractricide & KillChainAccuracy & SAQuality & BlueForceRisk \\
\hline Medium & Medium & Low & High \\
Medium & Medium & Medium & Low \\
Medium & Medium & Medium & Medium \\
Medium & Medium & Medium & High \\
Medium & Medium & High & Low \\
Medium & Medium & High & Medium \\
High & Medium & High & High \\
Low & High & Low & Low \\
Medium & High & Low & Medium \\
Medium & High & Low & High \\
Medium & High & Medium & Low \\
Medium & High & Medium & Medium \\
High & High & Medium & High \\
Medium & High & High & Low \\
High & High & High & Medium \\
High & High & High & High \\
\hline
\end{tabular}

\begin{tabular}{|c|c|c|c|}
\hline High & High & High & High \\
\hline
\end{tabular}

Table for "CivilianRisk"

\begin{tabular}{|l|ll|}
\hline CivilianRisk & CivilianVisibility & CivilianProximity \\
\hline Low & Low & Low \\
Low & Low & Medium \\
Medium & Low & High \\
Low & Medium & Low \\
Medium & Medium & Medium \\
Medium & Medium & High \\
Medium & High & Low \\
Medium & High & Medium \\
High & High & High \\
\hline
\end{tabular}

Table for "CivilianVisibility"

\begin{tabular}{|c|c|c|c|}
\hline CivilianVisibility & CloudLevel & DustLevel & Light \\
\hline Low & Low & Low & Low \\
\hline Low & Low & Low & Medium \\
\hline Low & Low & Low & High \\
\hline Low & Low & Medium & Low \\
\hline Medium & Low & Medium & Medium \\
\hline Medium & Low & Medium & High \\
\hline Medium & Low & High & Low \\
\hline Medium & Low & High & Medium \\
\hline Medium & Low & High & High \\
\hline Low & Medium & Low & Low \\
\hline Medium & Medium & Low & Medium \\
\hline Medium & Medium & Low & High \\
\hline Medium & Medium & Medium & Low \\
\hline Medium & Medium & Medium & Medium \\
\hline Medium & Medium & Medium & High \\
\hline Medium & Medium & High & Low \\
\hline Medium & Medium & High & Medium \\
\hline High & Medium & High & High \\
\hline High & High & Low & Low \\
\hline Medium & High & Low & Medium \\
\hline Medium & High & Low & High \\
\hline Medium & High & Medium & Low \\
\hline Medium & High & Medium & Medium \\
\hline High & High & Medium & High \\
\hline Medium & High & High & Low \\
\hline High & High & High & Medium \\
\hline
\end{tabular}

Table for "CollateralDamage"

\begin{tabular}{|c|c|c|c|}
\hline CollateralDamage & KillChainAccuracy & SAQuality & CivilianRisk \\
\hline Low & Low & Low & Low \\
\hline Low & Low & Low & Medium \\
\hline Low & Low & Low & High \\
\hline Low & Low & Medium & Low \\
\hline Medium & Low & Medium & Medium \\
\hline Medium & Low & Medium & High \\
\hline Low & Low & High & Low \\
\hline Medium & Low & High & Medium \\
\hline Medium & Low & High & High \\
\hline Low & Medium & Low & Low \\
\hline Medium & Medium & Low & Medium \\
\hline Medium & Medium & Low & High \\
\hline Medium & Medium & Medium & Low \\
\hline Medium & Medium & Medium & Medium \\
\hline Medium & Medium & Medium & High \\
\hline Medium & Medium & High & Low \\
\hline Medium & Medium & High & Medium \\
\hline High & Medium & High & High \\
\hline Low & High & Low & Low \\
\hline Medium & High & Low & Medium \\
\hline Medium & High & Low & High \\
\hline Medium & High & Medium & Low \\
\hline Medium & High & Medium & Medium \\
\hline High & High & Medium & High \\
\hline Medium & High & High & Low \\
\hline High & High & High & Medium \\
\hline High & High & High & High \\
\hline
\end{tabular}

Table for "PlatformDefence" 


\begin{tabular}{|c|c|c|c|c|}
\hline $\begin{array}{l}\text { PlatformDefe } \\
\text { nce }\end{array}$ & $\begin{array}{c}\text { WeaponTy } \\
\text { pe }\end{array}$ & $\begin{array}{l}\text { Targetti } \\
\text { ng }\end{array}$ & $\begin{array}{l}\text { Jammi } \\
\text { ng }\end{array}$ & $\begin{array}{c}\text { CounterMeas } \\
\text { ures }\end{array}$ \\
\hline Low & Low & Low & Low & Low \\
\hline Low & Low & Low & Low & Medium \\
\hline Low & Low & Low & Low & High \\
\hline Low & Low & Low & $\begin{array}{l}\text { Mediu } \\
\mathrm{m}\end{array}$ & Low \\
\hline Low & Low & Low & $\begin{array}{l}\text { Mediu } \\
\mathrm{m}\end{array}$ & Medium \\
\hline Medium & Low & Low & $\begin{array}{l}\text { Mediu } \\
\mathrm{m}\end{array}$ & High \\
\hline Low & Low & Low & High & Low \\
\hline Medium & Low & Low & High & Medium \\
\hline Medium & Low & Low & High & High \\
\hline Low & Low & Medium & Low & Low \\
\hline Low & Low & Medium & Low & Medium \\
\hline Medium & Low & Medium & Low & High \\
\hline Low & Low & Medium & $\begin{array}{l}\text { Mediu } \\
\mathrm{m}\end{array}$ & Low \\
\hline Medium & Low & Medium & $\begin{array}{l}\text { Mediu } \\
\mathrm{m}\end{array}$ & Medium \\
\hline Medium & Low & Medium & $\begin{array}{l}\text { Mediu } \\
\mathrm{m}\end{array}$ & High \\
\hline Medium & Low & Medium & High & Low \\
\hline Medium & Low & Medium & High & Medium \\
\hline Medium & Low & Medium & High & High \\
\hline Low & Low & High & Low & Low \\
\hline Medium & Low & High & Low & Medium \\
\hline Medium & Low & High & Low & High \\
\hline Medium & Low & High & $\begin{array}{l}\text { Mediu } \\
\mathrm{m}\end{array}$ & Low \\
\hline Medium & Low & High & $\begin{array}{l}\text { Mediu } \\
\mathrm{m}\end{array}$ & Medium \\
\hline Medium & Low & High & $\begin{array}{l}\text { Mediu } \\
\mathrm{m}\end{array}$ & High \\
\hline Medium & Low & High & High & Low \\
\hline Medium & Low & High & High & Medium \\
\hline Medium & Low & High & High & High \\
\hline Low & Medium & Low & Low & Low \\
\hline Low & Medium & Low & Low & Medium \\
\hline Low & Medium & Low & Low & High \\
\hline Low & Medium & Low & $\begin{array}{l}\text { Mediu } \\
\mathrm{m}\end{array}$ & Low \\
\hline Medium & Medium & Low & $\begin{array}{l}\text { Mediu } \\
\mathrm{m}\end{array}$ & Medium \\
\hline Medium & Medium & Low & $\begin{array}{l}\text { Mediu } \\
\mathrm{m}\end{array}$ & High \\
\hline Medium & Medium & Low & High & Low \\
\hline Medium & Medium & Low & High & Medium \\
\hline Medium & Medium & Low & High & High \\
\hline Low & Medium & Medium & Low & Low \\
\hline Medium & Medium & Medium & Low & Medium \\
\hline Medium & Medium & Medium & Low & High \\
\hline
\end{tabular}

\begin{tabular}{|c|c|c|c|c|}
\hline $\begin{array}{l}\text { PlatformDefe } \\
\text { nce }\end{array}$ & $\begin{array}{l}\text { WeaponTy } \\
\text { pe }\end{array}$ & $\begin{array}{l}\text { Targetti } \\
\text { ng }\end{array}$ & $\begin{array}{c}\text { Jammi } \\
\mathrm{ng}\end{array}$ & $\begin{array}{c}\text { CounterMeas } \\
\text { ures }\end{array}$ \\
\hline Medium & Medium & Medium & $\begin{array}{l}\text { Mediu } \\
\mathrm{m}\end{array}$ & Low \\
\hline Medium & Medium & Medium & $\begin{array}{l}\text { Mediu } \\
\mathrm{m}\end{array}$ & Medium \\
\hline Medium & Medium & Medium & $\begin{array}{l}\text { Mediu } \\
\mathrm{m}\end{array}$ & High \\
\hline Medium & Medium & Medium & High & Low \\
\hline Medium & Medium & Medium & High & Medium \\
\hline Medium & Medium & Medium & High & High \\
\hline Medium & Medium & High & Low & Low \\
\hline Medium & Medium & High & Low & Medium \\
\hline Medium & Medium & High & Low & High \\
\hline Medium & Medium & High & $\begin{array}{l}\text { Mediu } \\
\mathrm{m}\end{array}$ & Low \\
\hline Medium & Medium & High & $\begin{array}{l}\text { Mediu } \\
\mathrm{m}\end{array}$ & Medium \\
\hline Medium & Medium & High & $\begin{array}{l}\text { Mediu } \\
\mathrm{m}\end{array}$ & High \\
\hline Medium & Medium & High & High & Low \\
\hline Medium & Medium & High & High & Medium \\
\hline High & Medium & High & High & High \\
\hline Low & High & Low & Low & Low \\
\hline Medium & High & Low & Low & Medium \\
\hline Medium & High & Low & Low & High \\
\hline Medium & High & Low & $\begin{array}{l}\text { Mediu } \\
\mathrm{m}\end{array}$ & Low \\
\hline Medium & High & Low & $\begin{array}{l}\text { Mediu } \\
\mathrm{m}\end{array}$ & Medium \\
\hline Medium & High & Low & $\begin{array}{l}\text { Mediu } \\
\mathrm{m}\end{array}$ & High \\
\hline Medium & High & Low & High & Low \\
\hline Medium & High & Low & High & Medium \\
\hline Medium & High & Low & High & High \\
\hline Medium & High & Medium & Low & Low \\
\hline Medium & High & Medium & Low & Medium \\
\hline Medium & High & Medium & Low & High \\
\hline Medium & High & Medium & $\begin{array}{l}\text { Mediu } \\
\mathrm{m}\end{array}$ & Low \\
\hline Medium & High & Medium & $\begin{array}{l}\text { Mediu } \\
\mathrm{m}\end{array}$ & Medium \\
\hline Medium & High & Medium & $\begin{array}{l}\text { Mediu } \\
\mathrm{m}\end{array}$ & High \\
\hline Medium & High & Medium & High & Low \\
\hline Medium & High & Medium & High & Medium \\
\hline High & High & Medium & High & High \\
\hline Medium & High & High & Low & Low \\
\hline Medium & High & High & Low & Medium \\
\hline Medium & High & High & Low & High \\
\hline Medium & High & High & $\begin{array}{l}\text { Mediu } \\
\mathrm{m}\end{array}$ & Low \\
\hline Medium & High & High & $\begin{array}{l}\text { Mediu } \\
\mathrm{m}\end{array}$ & Medium \\
\hline
\end{tabular}




\begin{tabular}{|l|llll|}
\hline $\begin{array}{c}\text { PlatformDefe } \\
\text { nce }\end{array}$ & $\begin{array}{l}\text { WeaponTy } \\
\text { pe }\end{array}$ & $\begin{array}{c}\text { Targetti } \\
\text { ng }\end{array}$ & $\begin{array}{c}\text { Jammi } \\
\text { ng }\end{array}$ & $\begin{array}{c}\text { CounterMeas } \\
\text { ures }\end{array}$ \\
\hline High & High & High & $\begin{array}{l}\text { Mediu } \\
\text { m }\end{array}$ & High \\
Medium & High & High & High & Low \\
High & High & High & High & Medium \\
High & High & High & High & High \\
\hline
\end{tabular}

Table for "FlightPathRisk"

\begin{tabular}{|l|ll|}
\hline FlightPathRisk & Terrain & Aircraftheight \\
\hline Low & Low & Low \\
Low & Low & Medium \\
Medium & Low & High \\
Low & Medium & Low \\
Medium & Medium & Medium \\
High & Medium & High \\
Medium & High & Low \\
High & High & Medium \\
High & High & High \\
\hline
\end{tabular}

Table for "LossOfDeliveryAircraft"

\begin{tabular}{|c|c|c|c|}
\hline $\begin{array}{c}\text { LossOfDeliveryAircr } \\
\text { aft }\end{array}$ & $\begin{array}{c}\text { SAQualit } \\
y\end{array}$ & $\begin{array}{c}\text { PlatformDefen } \\
\text { ce }\end{array}$ & $\begin{array}{l}\text { FlightPathRi } \\
\text { sk }\end{array}$ \\
\hline Low & Low & Low & Low \\
\hline Low & Low & Low & Medium \\
\hline Low & Low & Low & High \\
\hline Low & Low & Medium & Low \\
\hline Low & Low & Medium & Medium \\
\hline Low & Low & Medium & High \\
\hline Low & Low & High & Low \\
\hline Low & Low & High & Medium \\
\hline Low & Low & High & High \\
\hline Low & Medium & Low & Low \\
\hline Medium & Medium & Low & Medium \\
\hline Medium & Medium & Low & High \\
\hline Medium & Medium & Medium & Low \\
\hline Medium & Medium & Medium & Medium \\
\hline Medium & Medium & Medium & High \\
\hline Medium & Medium & High & Low \\
\hline Medium & Medium & High & Medium \\
\hline Medium & Medium & High & High \\
\hline Low & High & Low & Low \\
\hline Medium & High & Low & Medium \\
\hline Medium & High & Low & High \\
\hline Medium & High & Medium & Low \\
\hline High & High & Medium & Medium \\
\hline High & High & Medium & High \\
\hline
\end{tabular}

\begin{tabular}{|l|lll|}
\hline High & High & High & Low \\
High & High & High & Medium \\
High & High & High & High \\
\hline
\end{tabular}

Table for "EnvironmentalVisibility"

\begin{tabular}{|c|c|c|c|}
\hline EnvironmentalVisibility & Light & CloudLevel & DustLevel \\
\hline Low & Low & Low & Low \\
\hline Low & Low & Low & Medium \\
\hline Low & Low & Low & High \\
\hline Low & Low & Medium & Low \\
\hline Medium & Low & Medium & Medium \\
\hline Medium & Low & Medium & High \\
\hline Low & Low & High & Low \\
\hline Medium & Low & High & Medium \\
\hline Medium & Low & High & High \\
\hline Low & Medium & Low & Low \\
\hline Medium & Medium & Low & Medium \\
\hline Medium & Medium & Low & High \\
\hline Medium & Medium & Medium & Low \\
\hline Medium & Medium & Medium & Medium \\
\hline Medium & Medium & Medium & High \\
\hline Medium & Medium & High & Low \\
\hline Medium & Medium & High & Medium \\
\hline High & Medium & High & High \\
\hline Low & High & Low & Low \\
\hline Medium & High & Low & Medium \\
\hline Medium & High & Low & High \\
\hline Medium & High & Medium & Low \\
\hline Medium & High & Medium & Medium \\
\hline High & High & Medium & High \\
\hline Medium & High & High & Low \\
\hline High & High & High & Medium \\
\hline High & High & High & High \\
\hline
\end{tabular}

Table for "TargetVisLevel"

\begin{tabular}{|l|ll|}
\hline TargetVisLevel & Marking & Camouflage \\
\hline Low & Low & Low \\
Low & Low & Medium \\
Low & Low & High \\
Medium & Medium & Low \\
Medium & Medium & Medium \\
Medium & Medium & High \\
\hline
\end{tabular}




\begin{tabular}{|l|ll|}
\hline TargetVisLevel & Marking & Camouflage \\
\hline Medium & High & Low \\
High & High & Medium \\
High & High & High \\
\hline
\end{tabular}

Table for "VisualVisibility"

\begin{tabular}{|l|ll|}
\hline VisualVisibility & EnvironmentalVisibility & TargetVisLevel \\
\hline Low & Low & Low \\
Low & Low & Medium \\
Medium & Low & High \\
Low & Medium & Low \\
Medium & Medium & Medium \\
Medium & Medium & High \\
Medium & High & Low \\
Medium & High & Medium \\
High & High & High \\
\hline
\end{tabular}

Table for "TargetVisibility"

\begin{tabular}{|l|ll|}
\hline TargetVisibility & WeaponSensorCapabiity & VisualVisibility \\
\hline Low & Low & Low \\
Medium & Low & Medium \\
Medium & Low & High \\
Low & Medium & Low \\
Medium & Medium & Medium \\
Medium & Medium & High \\
Medium & High & Low \\
Medium & High & Medium \\
High & High & High \\
\hline
\end{tabular}

Table for "TargetDestruction"

\begin{tabular}{|l|lll|}
\hline $\begin{array}{l}\text { TargetDestructio } \\
\mathbf{n}\end{array}$ & \multicolumn{1}{|c|}{$\begin{array}{l}\text { KillChainAccurac } \\
\mathbf{y}\end{array}$} & $\begin{array}{c}\text { SAQualit } \\
\mathbf{y}\end{array}$ & \multicolumn{1}{c|}{$\begin{array}{c}\text { TargetVisibilit } \\
\text { Low }\end{array}$} \\
Low & Low & Low & Low \\
Low & Low & Low & Medium \\
Low & Low & Low & High \\
Medium & Low & Medium & Low \\
Medium & Low & Medium & Medium \\
Low & Low & Medium & High \\
Medium & Low & High & Low \\
Medium & Low & High & Medium \\
Low & Medium & High & High \\
Medium & Medium & Low & Low \\
Medium & Medium & Low & Medium \\
Medium & Medium & Medium & Low \\
Medium & Medium & Medium & Medium \\
\hline
\end{tabular}

\begin{tabular}{|l|lll|}
\hline \multicolumn{1}{|c|}{$\begin{array}{l}\text { TargetDestructio } \\
\mathbf{n}\end{array}$} & \multicolumn{1}{|c|}{$\begin{array}{l}\text { KillChainAccurac } \\
\text { Medium }\end{array}$} & \multicolumn{1}{c|}{$\begin{array}{c}\text { SAQualit } \\
\text { Medium }\end{array}$} & \multicolumn{1}{c|}{ TargetVisibilit } \\
Medium & Medium & Medium & High \\
High & Medium & High & Low \\
Low & Medium & High & High \\
Medium & High & Low & Low \\
Medium & High & Low & Medium \\
Medium & High & Low & High \\
Medium & High & Medium & Low \\
High & High & Medium & Medium \\
Medium & High & Medium & High \\
High & High & High & Low \\
High & High & High & Medium \\
\hline
\end{tabular}




\section{Appendix D - CAS Hypothetical Scenario Description}

This appendix provides evidence of the mixed method research methodology with the provision a hypothetical CAS scenario description from which the BBN and models were derived, as used in the risk analysis activity for application case study 1.

It also provides artefacts that provide a point from which future research may further develop the case study.

The mission is a pre-planned mission, 24 hours in advance of execution, which will commence at $1000 \mathrm{hrs}$ the following day. Visibility is expected to be good with full daylight and no cloud or wind forecast; hence dust will not impact visibility levels.

The scenario density is likely to be low, less than 20 tracks expected to be present.

E3-D C2 and Sentinel aircraft will be available, providing good situational awareness and interoperability (via Link 16). The Sentinel provides a powerful ASTOR capability.

It is probable that UAV support will be available although they may be diverted to other tasks.

Signal Intelligence and visual capability are likely to be high, with assets placed in the target vicinity for visual reporting and signal analysis systems available.

On the ground the forward air controller will utilise laser target marking which is compatible with the Tornado GR4 targeting system. However, it is expected the target will be well camouflaged. Target position will be derived from a laser rangefinder sensor and reported on the VMF data link. The Tornado GR4 is equipped with jamming and countermeasure systems. The attack weapon is planned to be a "smart" missile. The mission flight path will be at low level over flat terrain.

The mission will be undertaken by a coalition of UK, US and French forces, with approximately half connected via tactical data links and secure voice.

Blue forces are predicted to be $5 \mathrm{~km}$ west of the red forces, although there is an uncertainty of $2 \mathrm{~km}$ for this estimate. Civilians are initially expected to be $12 \mathrm{~km}$ west and $2 \mathrm{~km}$ north of red forces but are likely to be moving towards them, again there is a $2 \mathrm{~km}$ uncertainty associated with these values. 


\section{Appendix E - Further CAS Risk Analysis and Handling}

This appendix provides evidence of the mixed method research methodology with the remaining risk analysis and handling activity descriptions undertaken in application case study 1.

It also provides artefacts that provide a point from which future research may further develop the case study.

\section{E.1. Analysis and Handling - Target Destruction Risk}

The causal network relating to target destruction risk, i.e. the risk of failing to destroy the target, is shown below in Figure E - 1 with the primary contributing factors identified as Kill Chain Accuracy, SA Quality and Target Visibility.

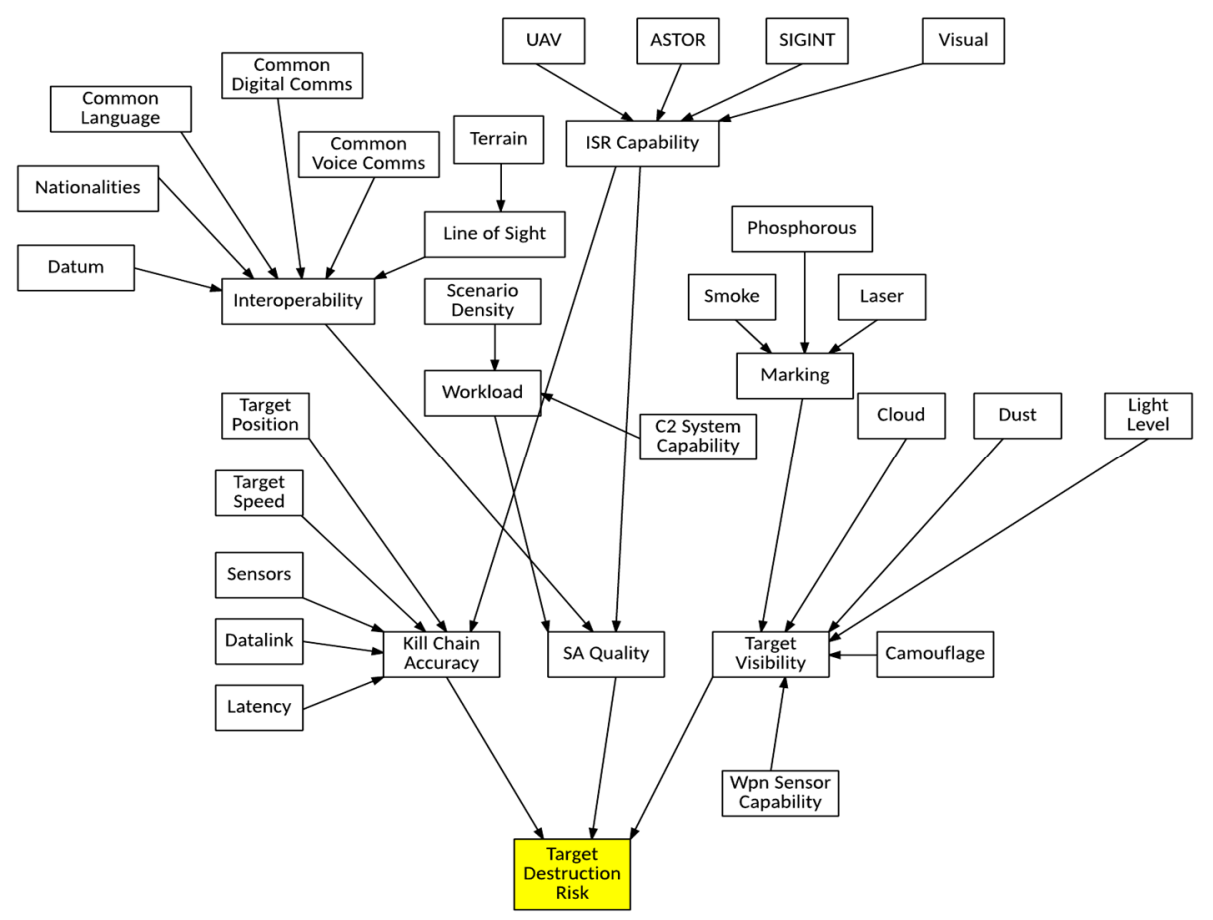

Figure E - 1 : Target Destruction Risk Causal Network

\section{E.1.1 Model Technique Selection}

Kill Chain Accuracy and SA Quality were discussed previously as contributing factors to Fratricide Risk, hence the same logic was applied for the derivation of supporting model types, i.e. Interoperability represented using System Dynamics and Kill Chain as a DES model, with SA Quality represented as a BBN. The remaining primary contributing factor, Target 
Visibility was analysed using the selection tool spreadsheet (Appendix B, Table B - 7) with BBN, Markov Chain or Game Theory identified as appropriate modelling methods; of these BBN was selected, allowing direct incorporation into the central BBN.

\section{E.1.2 Simplified Causal Network}

With appropriate supporting models identified, the simplified causal network was created and is shown below in Figure E - 2. It was not deemed necessary to define intermediate model architecture in this case because both supporting models and their relationship with the central BBN had been determined under the Fratricide Risk analysis.

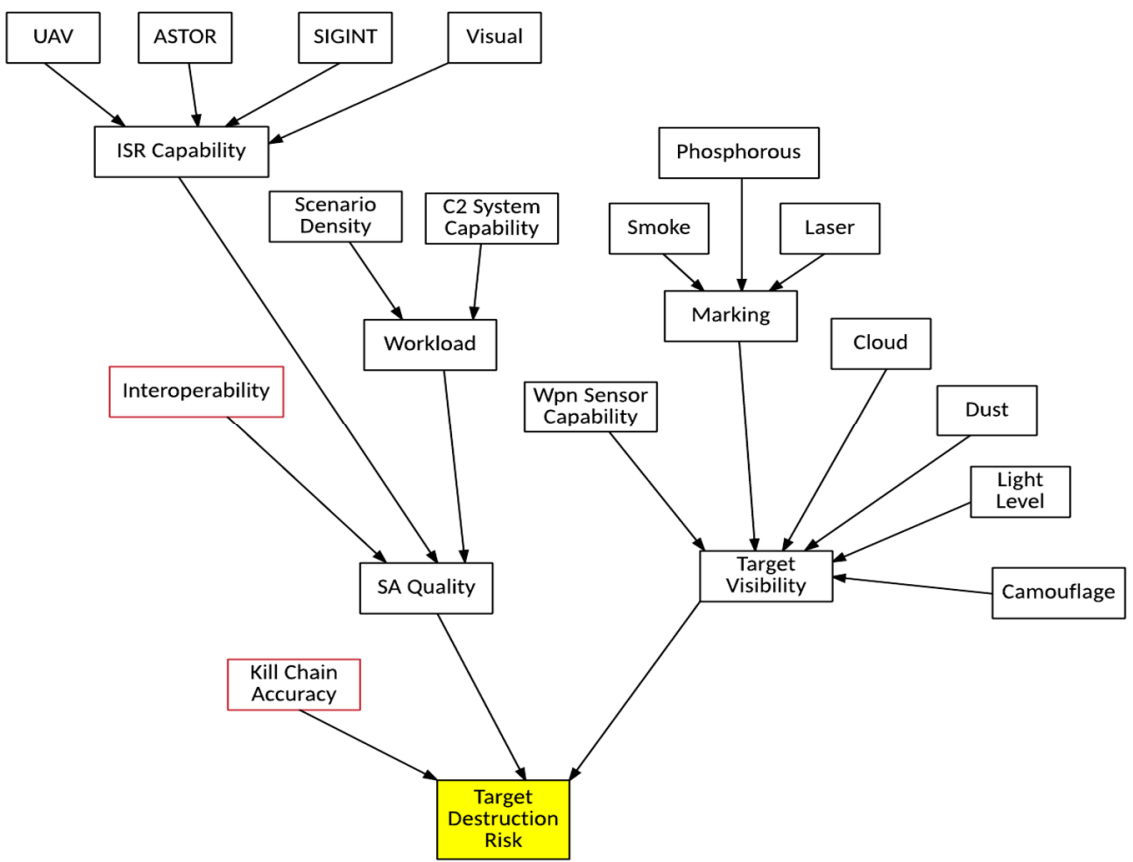

Figure E - 2 : Target Destruction Risk - Simplified Causal Network

\section{E.1.3 Target Destruction Risk BBN}

Having established the model architecture, the Target Destruction Risk BBN was implemented in accordance with the Causal Network and is shown below in Figure E - 3, the variables that are not already defined previously in relating to the Fratricide risk BBN are described in Table E - 1. 


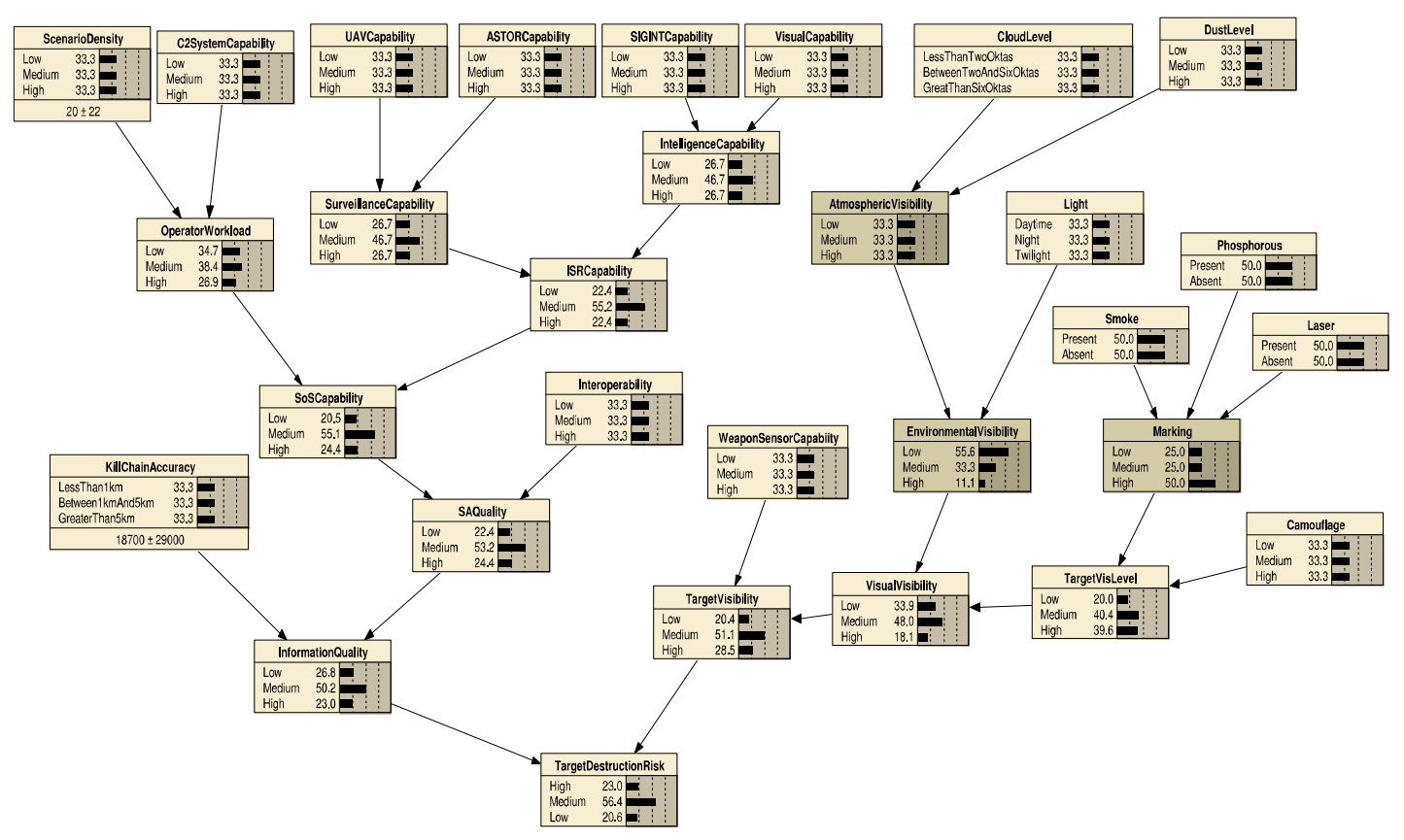

Figure E - 3 : Target Destruction Risk BBN

\begin{tabular}{|c|c|c|}
\hline Variable & Description & States \\
\hline Smoke & $\begin{array}{l}\text { Smoke used as a target } \\
\text { marker }\end{array}$ & $\begin{array}{l}\text { Present } \\
\text { Absent }\end{array}$ \\
\hline Phosphorous & $\begin{array}{l}\text { Phosphorous used as a } \\
\text { target marker }\end{array}$ & $\begin{array}{l}\text { Present } \\
\text { Absent }\end{array}$ \\
\hline Laser & $\begin{array}{l}\text { Laser used as a target } \\
\text { marker }\end{array}$ & $\begin{array}{l}\text { Present } \\
\text { Absent }\end{array}$ \\
\hline Marking & $\begin{array}{l}\text { Combined target marking } \\
\text { capability }\end{array}$ & $\begin{array}{l}\text { Low: No target marking } \\
\text { available or smoke only } \\
\text { Medium: Phosphorous } \\
\text { marking available possibly } \\
\text { augmented by smoke } \\
\text { High: Laser Marking available } \\
\text { possible augmented by } \\
\text { Phosphorous or smoke }\end{array}$ \\
\hline Camouflage & $\begin{array}{l}\text { Target } \\
\text { capability }\end{array}$ & $\begin{array}{l}\text { Low: Target is not } \\
\text { camouflaged } \\
\text { Medium: Target has } \\
\text { rudimentary camouflage } \\
\text { High: Target is highly } \\
\text { camouflaged }\end{array}$ \\
\hline WeaponSensorCapability & $\begin{array}{l}\text { Friendly weapon onboard } \\
\text { sensor capability }\end{array}$ & $\begin{array}{l}\text { Low: No sensor capability, a } \\
\text { "dumb" weapon }\end{array}$ \\
\hline
\end{tabular}




\begin{tabular}{|c|c|c|}
\hline Variable & Description & States \\
\hline & & $\begin{array}{l}\text { Medium: Radar or heat } \\
\text { seeking sensor, requires initial } \\
\text { target co-ordinates } \\
\text { High: Sophisticated sensor } \\
\text { capability }\end{array}$ \\
\hline TargetVisibility & $\begin{array}{l}\text { Overall target position } \\
\text { visibility }\end{array}$ & $\begin{array}{l}\text { Low: Poor visual and sensor } \\
\text { visibility } \\
\text { Medium: Limited visibility } \\
\text { High: Target easily } \\
\text { distinguishable }\end{array}$ \\
\hline TargetDestructionRisk & $\begin{array}{l}\text { The risk of failing to } \\
\text { destroy the primary target } \\
\text { of a CAS mission }\end{array}$ & $\begin{array}{l}\text { Low: Destruction of the target } \\
\text { is highly likely } \\
\text { Medium: It is possible the } \\
\text { target may not be destroyed, } \\
\text { options for reducing the risk } \\
\text { should be considered } \\
\text { High: Destruction is the target } \\
\text { is unlikely, risk mitigation is } \\
\text { required }\end{array}$ \\
\hline
\end{tabular}

Table E - 1 : Target Destruction Risk BBN Variables

The following additional mediating variables were required in order to simplify the internal probability tables:

- TargetVisLevel

- VisualVisibility

- $\quad$ Surveillance Capability

- Intelligence Capability

- ISR Capability

- SoS Capability

- Information Quality

- $\quad$ Atmospheric Visibility

\section{E.1.4 Interpretation of Results}

The BBN was updated with evidence derived from the hypothetical scenario, described in Appendix D, and learnt from the output of the Kill Chain and Interoperability supporting models. The updated BBN is shown in Figure E - 4, indicating High Risk at 15.8\%, Medium Risk at $27.0 \%$ and Low Risk at $57.2 \%$. 
The BBN was then updated the worst and best case scenario evidence, as shown in Figure E 5 and Figure $\mathrm{E}-6$ respectively.

The worst-case scenario relating to the failure to destroy the mission target required evidence to be entered reflecting a high scenario density with a low C2 system capability thereby resulting in a high operator workload, poor ISR capability and Interoperability providing a low quality SA picture, which was combined with high error growth within the kill chain. In addition, target marking and visibility was poor, with the weapon having no independent sensor capability. The resulting risk levels were; High $=84.4 \%$, Medium $=15.0 \%$ and Low $=0.6 \%$.

The best-case scenario required low workload, high ISR capability, good Interoperability, providing a high quality SA picture with minimal error within the kill chain, with good target visibility and weapon sensor capability; the resultant risk levels were; High = 1.69\%, Medium $=10.9 \%$ and Low $=87.5 \%$.

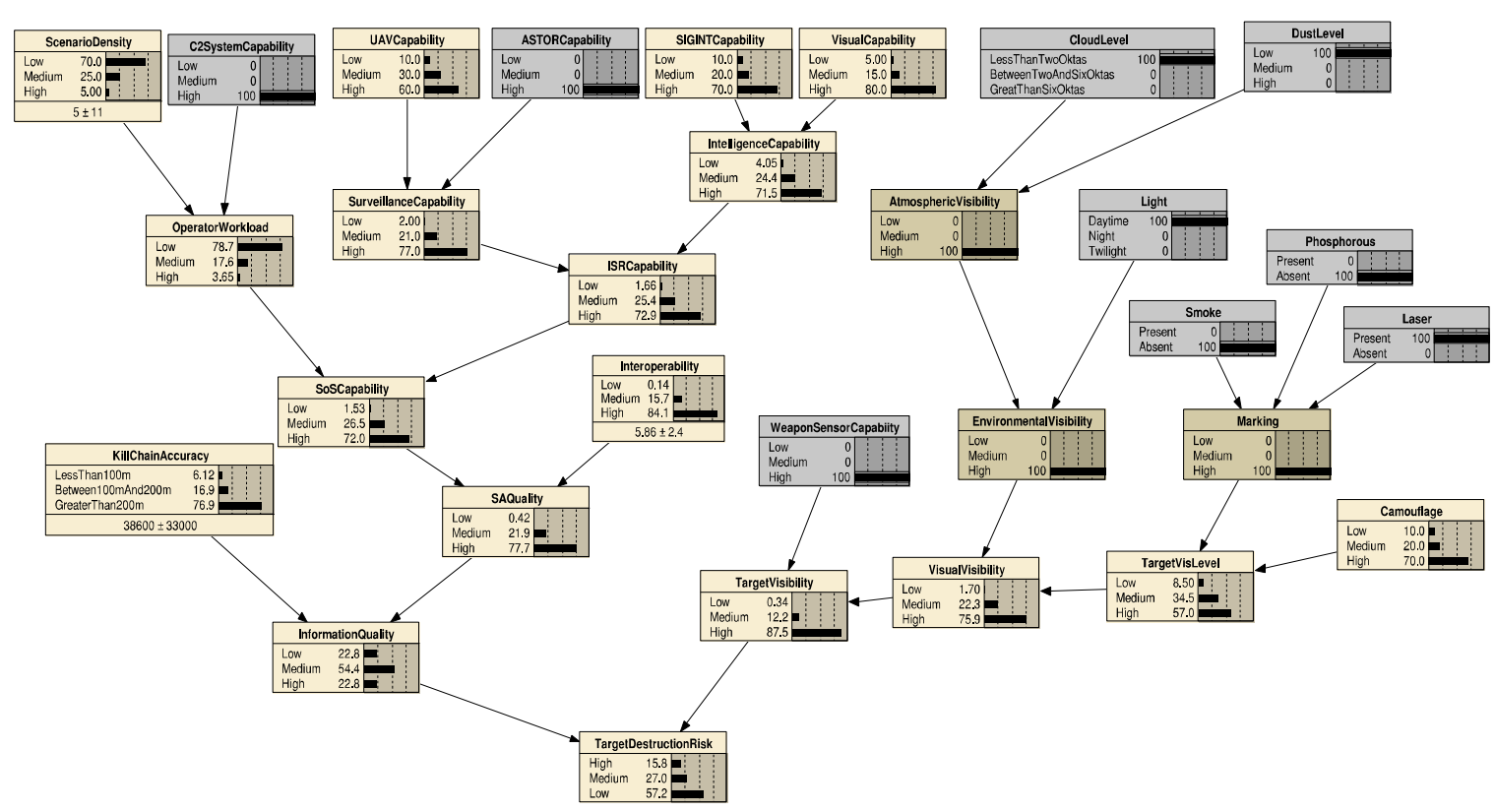

Figure E - 4 : Target Destruction Risk BBN Post Learning and Evidence 


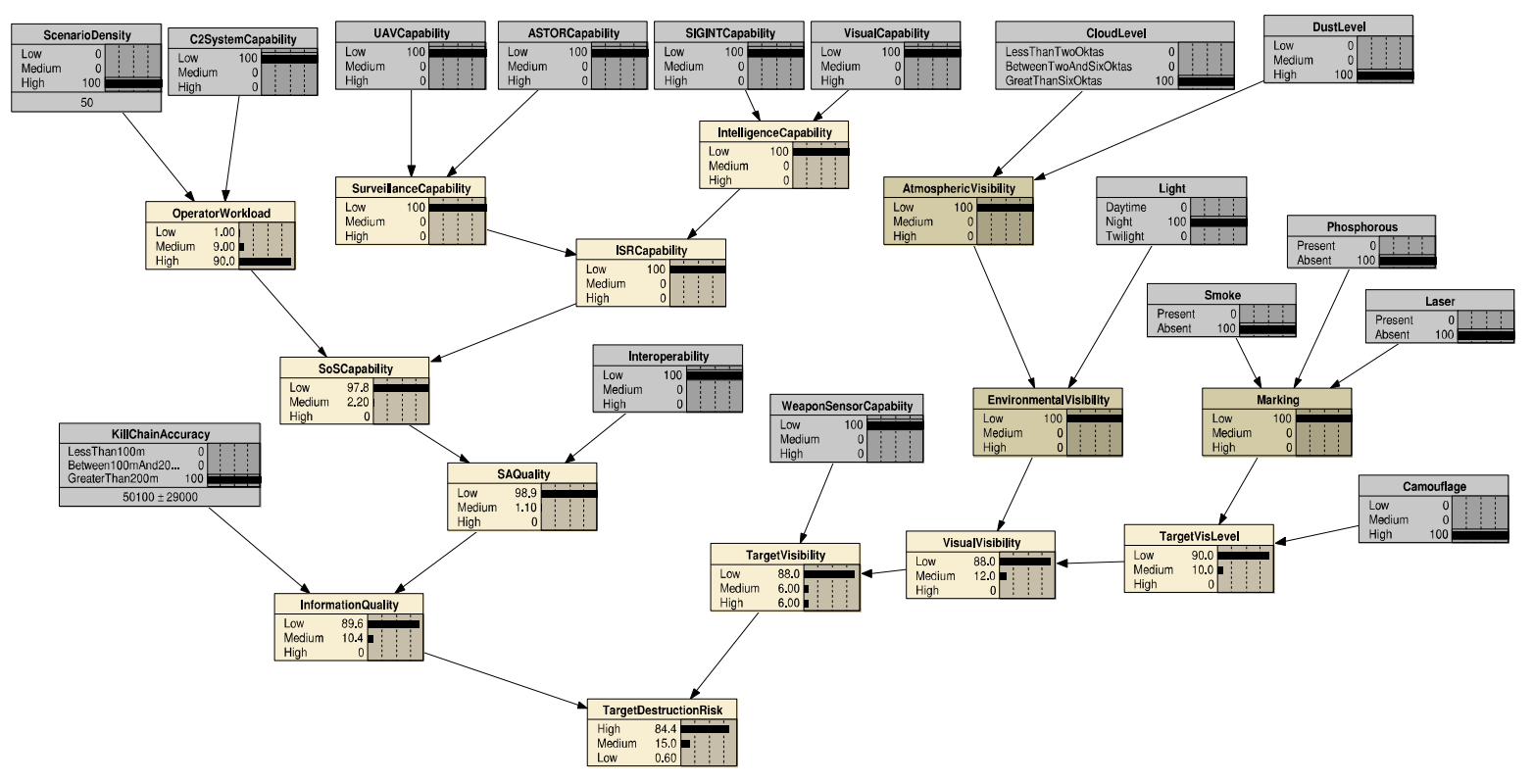

Figure E - 5 : Target Destruction Risk BBN Worst Case

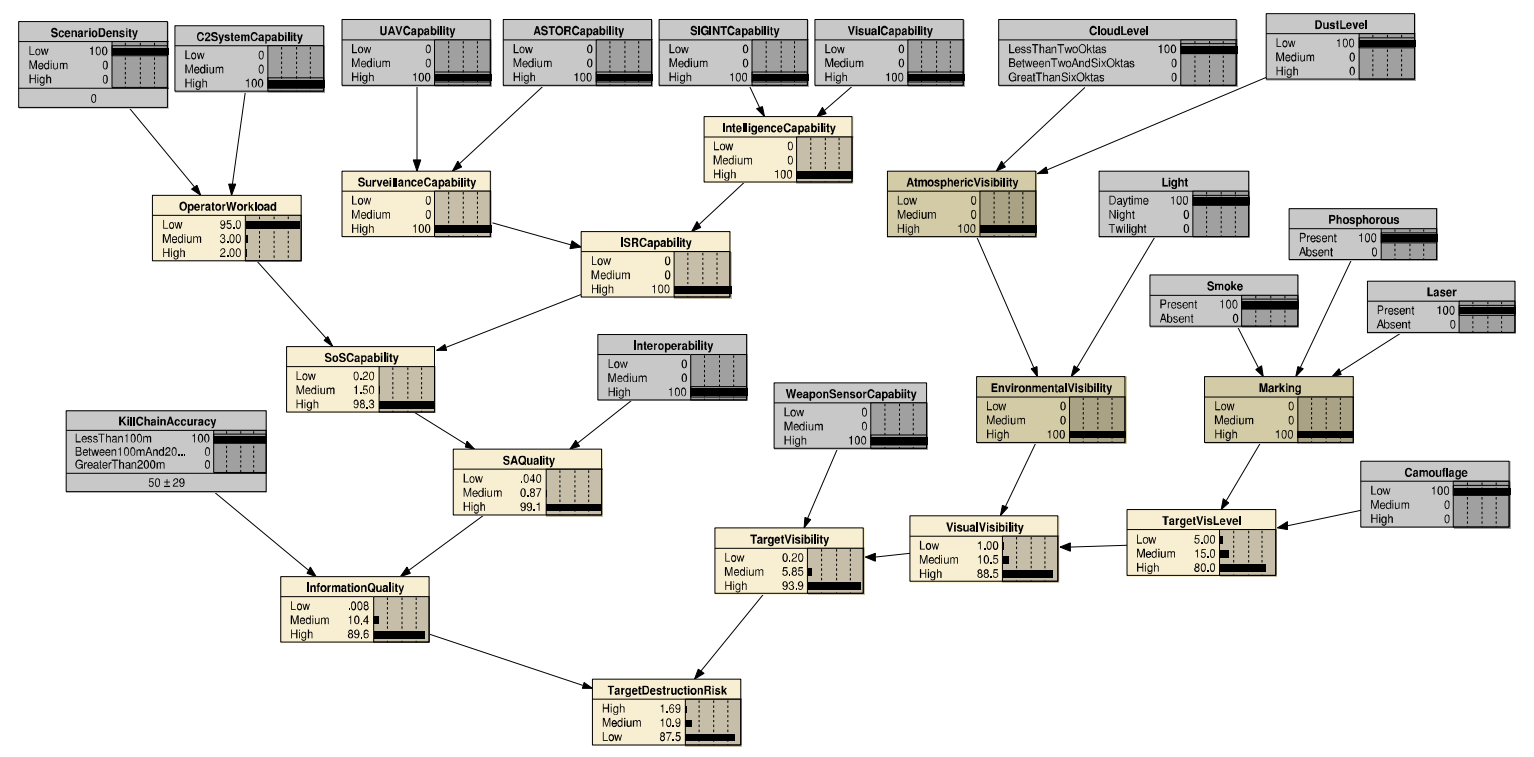

Figure E - 6 - Target Destruction Risk BBN Best Case

These risk levels were compared with the best and worse case levels, as shown below in Figure $E-7$, providing context for the meaningful interpretation of the results and indicated that although the results were more aligned to the best-case results the probability of a low chance of target destruction failure was $30 \%$ lower than the optimum value. It was therefore concluded that the level of risk was acceptable but any potential mitigation should be explored. 


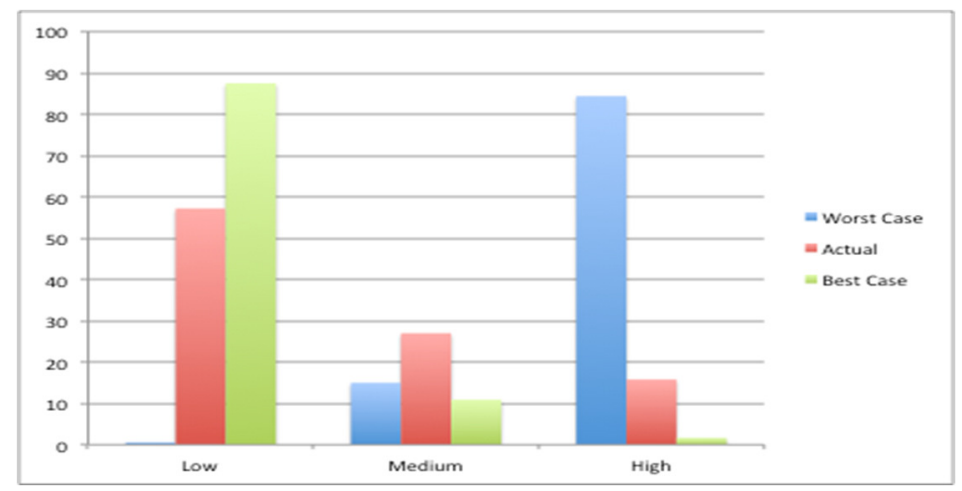

Figure E - 7 : Target Destruction Risk Comparison

Examining the BBN it was noted that the inaccuracy associated with the kill chain was contributing to the reduced probability of low risk of fratricide, as it also similarly had for fratricide risk. Using the same approach as taken previously, the BBN was updated by setting the probability for minimum kill chain error to $100 \%$, improving the overall risk level, which was now closely comparable with the best case values, as shown in Figure E - 8, therefore implying that further analysis of the kill chain component systems with an aim to reducing error growth would be beneficial.

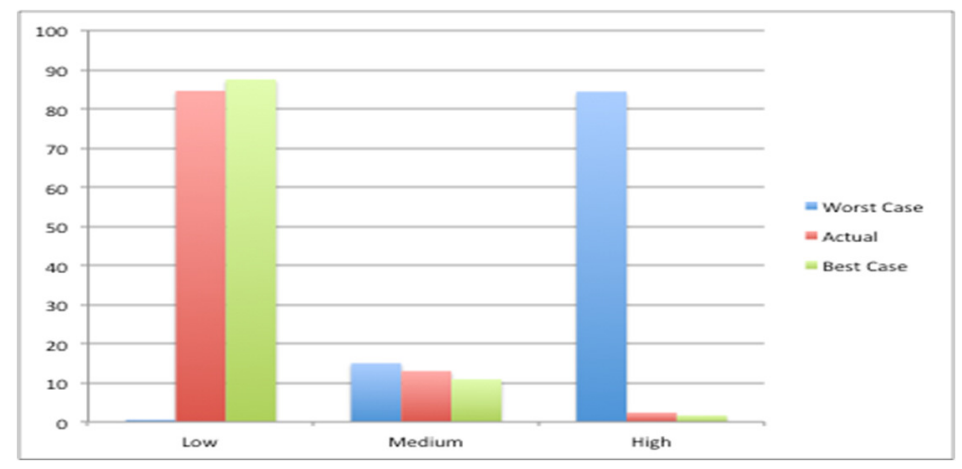

Figure E - 8 : Target Destruction BBN with High Accuracy Kill Chain

\section{E.1.5 Risk Confidence}

Again, the final stage in the analysis process was the derivation of the associated confidence, requiring the construction of an equivalent confidence BBN as shown in Figure $E-9$, with the rationale, not previously provided, for the BBN variable confidence estimates provided in Table E - 2 .

\begin{tabular}{|l|l|l|}
\hline Variable & Input Type & Confidence Estimate \\
\hline Marking & Evidence & $\begin{array}{l}\text { High: Marking capability of ground systems } \\
\text { is known }\end{array}$ \\
\hline Camouflage & Evidence & $\begin{array}{l}\text { High: Current intelligence images available } \\
\text { of target }\end{array}$ \\
\hline
\end{tabular}




\begin{tabular}{|l|l|l|}
\hline WeaponSensorCapability & Evidence & $\begin{array}{l}\text { High: All available weapon types have same } \\
\text { sensor capability }\end{array}$ \\
\hline
\end{tabular}

Table E - 2 - Target Destruction Risk Confidence

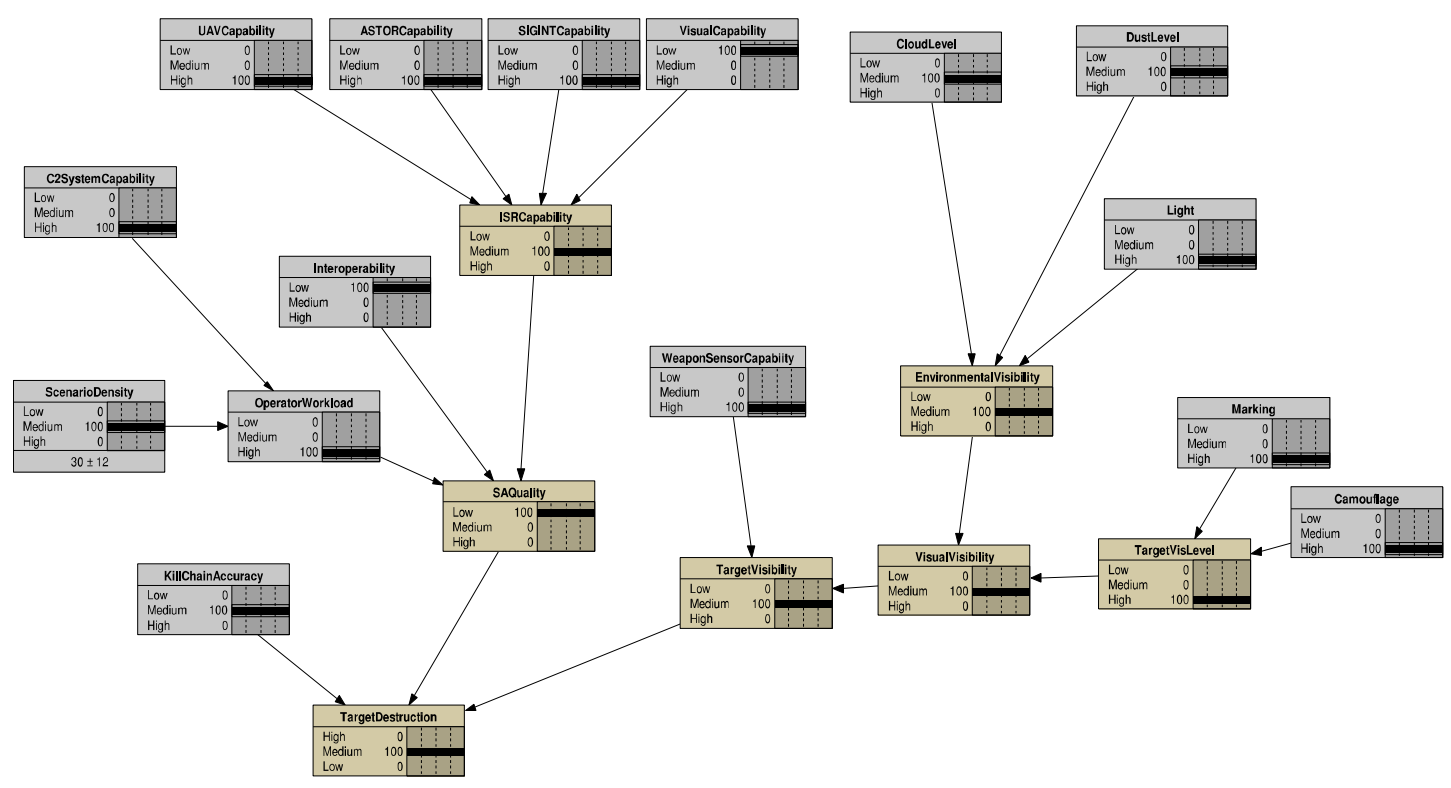

Figure E - 9 : Target Destruction Risk Confidence BBN

\section{E.2. Analysis and Handling - Collateral Damage Risk}

The Collateral Damage Risk was extracted from the overall CAS causal network and is shown below in Figure E - 10. In the case the primary contributing factors were; Kill Chain Accuracy, SA Quality, Civilian Proximity and Civilian Visibility.

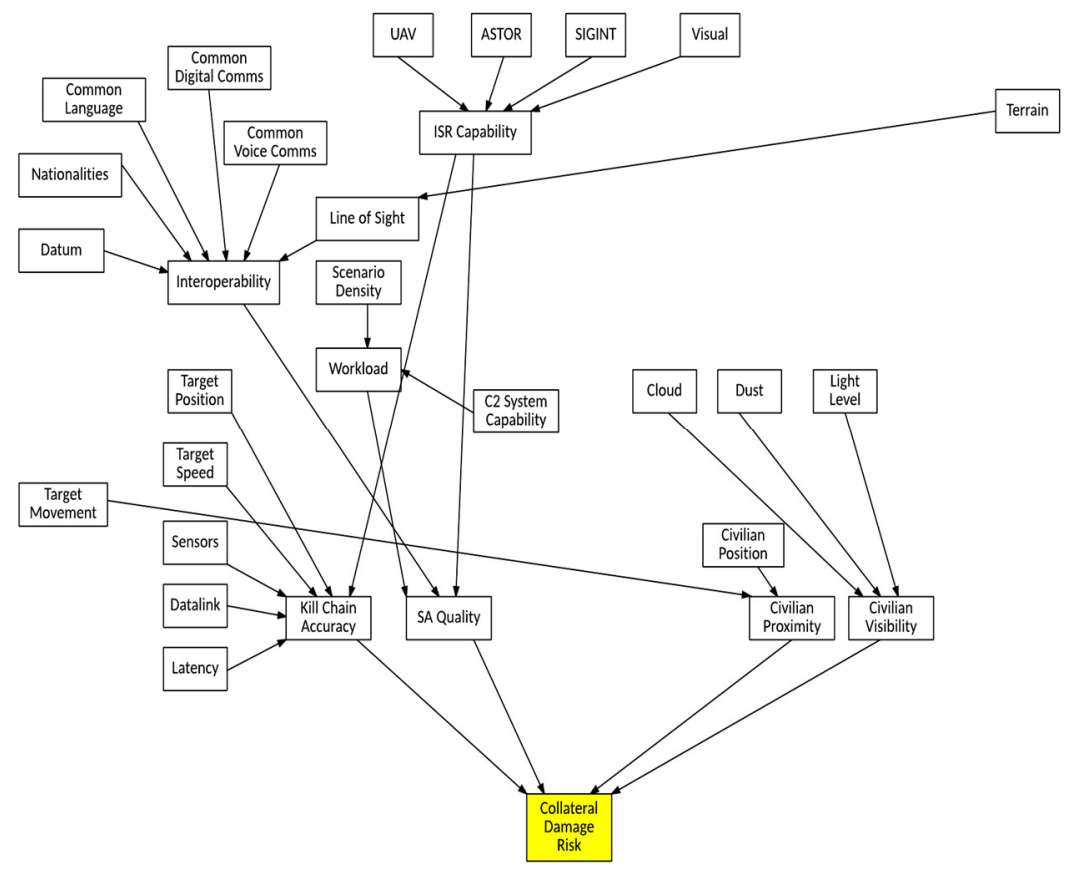

Figure E - 10 : Collateral Damage Risk Causal Network 


\section{E.2.1 Model Technique Selection}

Kill Chain Accuracy and Interoperability were again determined as DES and System Dynamics supporting models respectively, with SA Quality directly modelled within the central BBN based on the analysis performed for the Fratricide and Target Destruction Risks. Civilian Proximity (to the target blast zone) was influenced by target movement and civilian position; the selection tool analysis identified DES and ABMS as suitable approaches (Appendix B, Table B - 8). It was decided to use ABMS because a very similar model, produced for Blue Force Proximity could be easily adapted to model Civilian Proximity, thereby reducing the development effort required. The final Collateral Damage contributing factor to be considered was Civilian Visibility, whilst it could be assumed that a probabilistic modelling approach would be suitable, selection tool analysis was performed for consistency, which confirmed the initial assumption (Appendix B, Table B - 9). As determined for the risks analysed previously, a Bayesian approach was utilised, providing straightforward integration into the central BBN. In this case, a new supporting model was identified, Civilian Proximity, with its context within the overall model architecture shown below in Figure E - 11 .

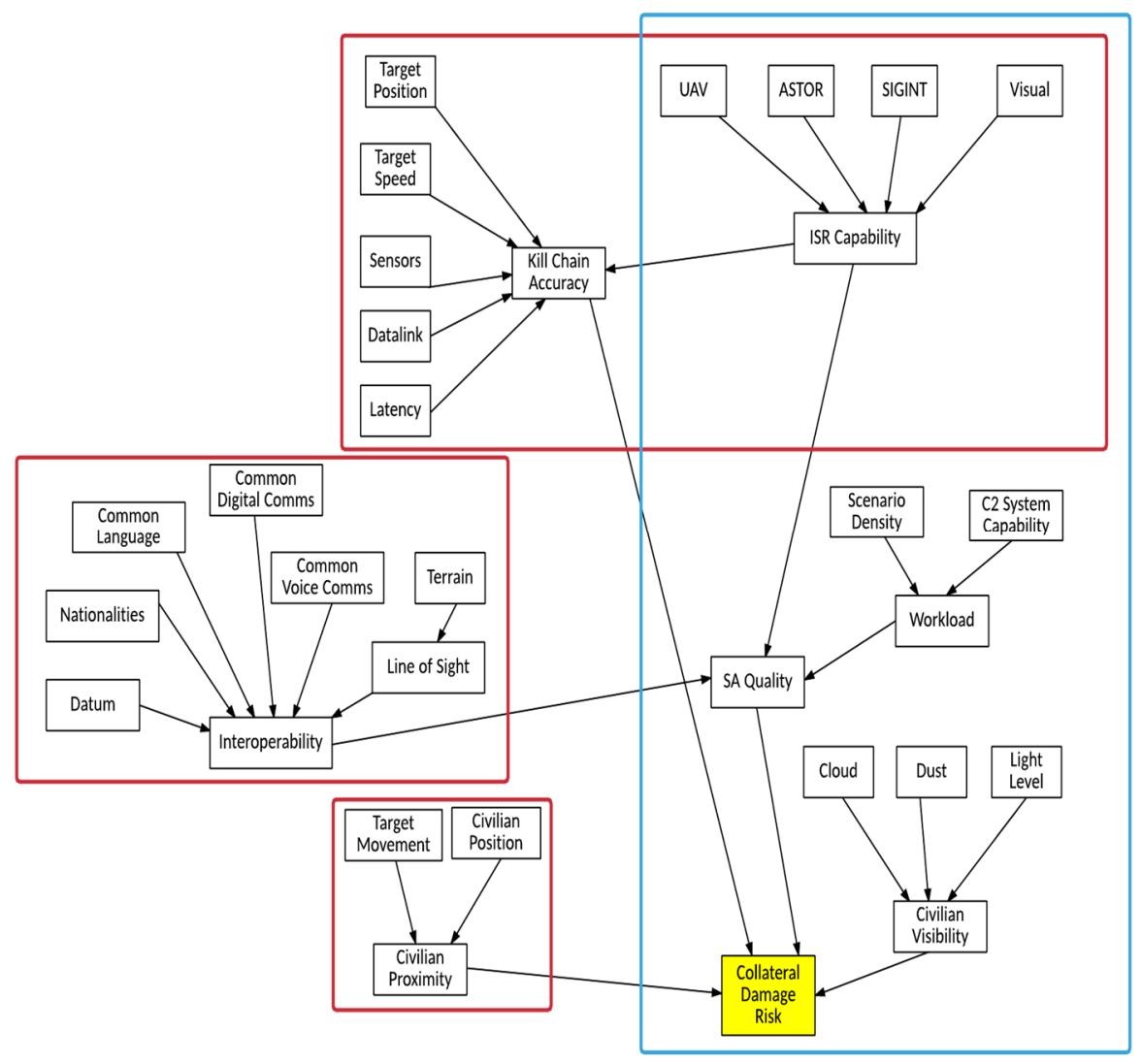

Figure E - 11 : Collateral Damage Model Architecture 


\section{E.2.2 Simplified Causal Network}

Removing nodes that were subsumed into the supporting models, allowed the causal network to be simplified, and is shown below in Figure E - 12, providing the basis of the central BBN.

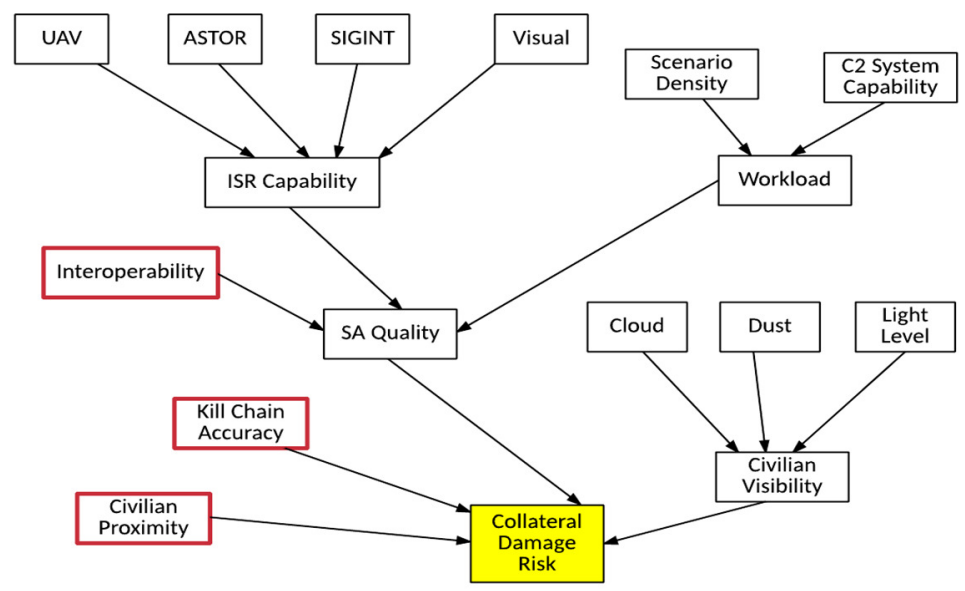

Figure E - 12 : Collateral Damage Simplified Causal Network

\section{E.2.3 Collateral Damage Risk BBN}

Having established the model architecture the Collateral Damage Risk BBN was implemented in accordance with the Causal Network and is shown in Figure E - 13. Definitions of BBN variables not previously described are provided below in Table E - 3 .

\begin{tabular}{|l|l|l|}
\hline Variable & Description & States \\
\hline CivilianProximity & $\begin{array}{l}\text { Distance of Civilians from } \\
\text { target }\end{array}$ & $\begin{array}{l}\text { Less than 1km } \\
\text { Between 1km and 2Km } \\
\text { Greater than 2Km }\end{array}$ \\
\hline CollateralDamage & $\begin{array}{l}\text { The risk of civilian injury } \\
\text { or loss of life as a result of } \\
\text { the CAS mission weapon } \\
\text { impact }\end{array}$ & $\begin{array}{l}\text { Low: Collateral Damage is } \\
\text { extremely unlikely to occur, } \\
\text { no further risk mitigation is } \\
\text { required } \\
\text { Medium: There is a potential } \\
\text { for Collateral Damage, care } \\
\text { must be exercised or the risk } \\
\text { mitigated } \\
\text { High: There is a significant } \\
\text { potential for Collateral } \\
\text { Damage, risk mitigation is } \\
\text { necessary before mission } \\
\text { commencement }\end{array}$ \\
\hline
\end{tabular}

Table E - 3 : Collateral Damage Risk BBN Variables 
In addition to the variables identified above, the following mediating variables (beyond those previously identified) were required in order to simplify the internal probability tables:

- CivilianRisk

- CivilianVisibility

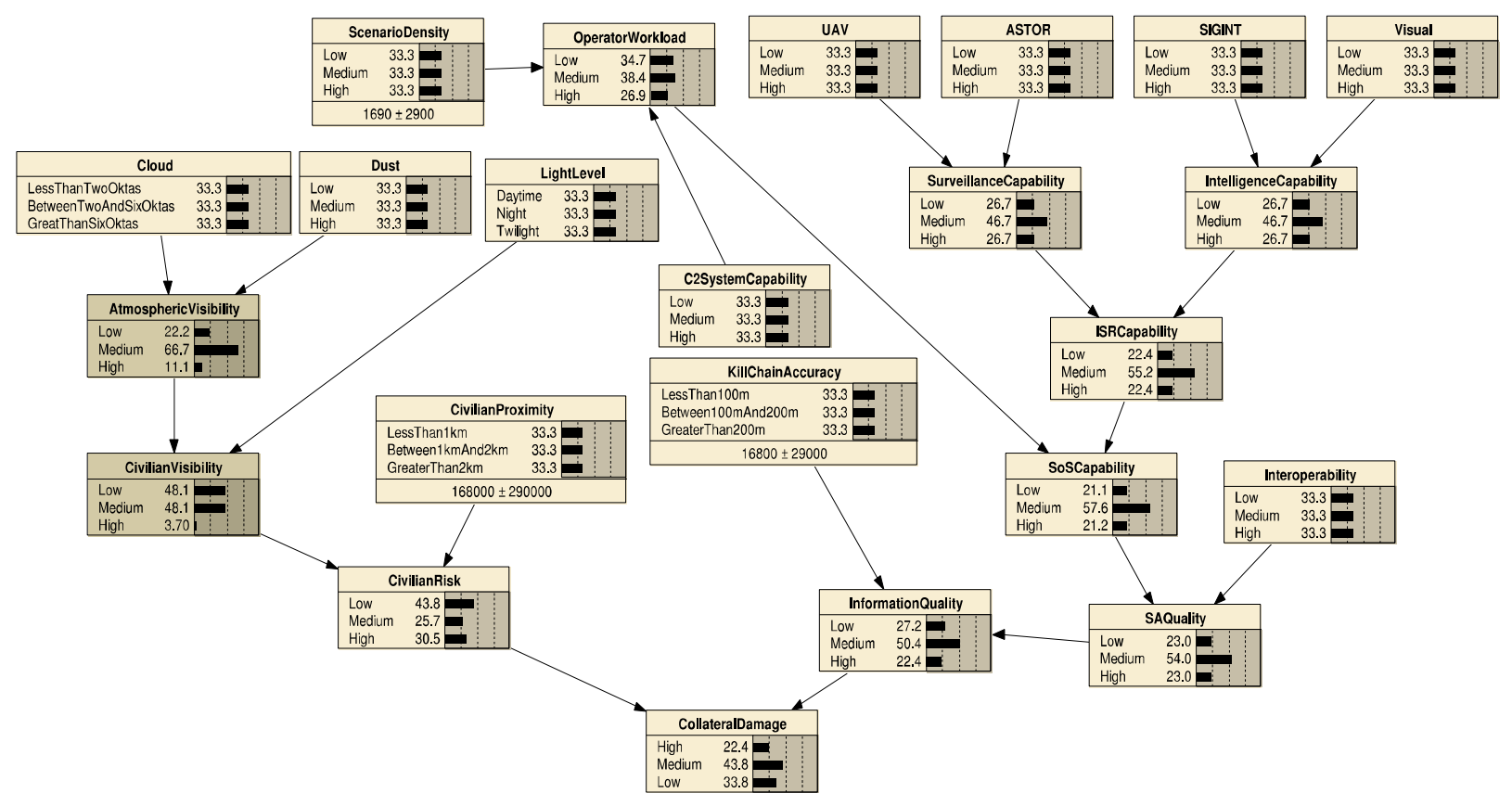

Figure E - 13 : Collateral Damage BBN

\section{E.2.4 Interpretation of Results}

The BBN was then updated with evidence derived from the hypothetical scenario, described in Appendix D, and learnt from the output of the Kill Chain, Civilian Proximity and Interoperability supporting models. The updated BBN is shown in Figure E - 16, indicating High Risk at $4.2 \%$, Medium Risk at 36.4\% and Low Risk at 59.4\%.

In common with the previous risk analysis, the BBN was instantiated with evidence derived from worst-case and best-case scenarios, the results of which are provided in Figure E - 17 and Figure E - 18 respectively.

The side-by-side comparison the actual, best-case and worst-case results are shown below in Figure E - 14, revealing that although the actual results were more closely aligned to the best-case values, the probability of low risk was over $20 \%$ lower. 


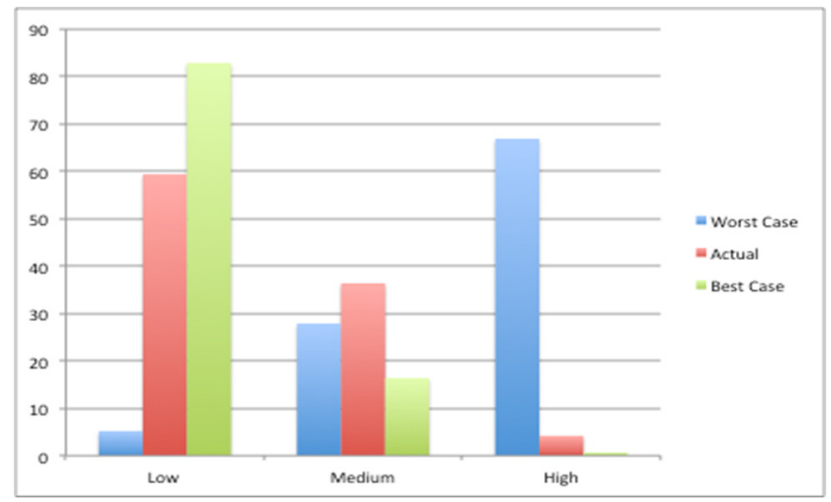

Figure E - 14 : Collateral Damage Risk Comparison

As observed previously, the kill chain was contributing to the reduced probability of low risk of collateral damage, as it similarly had for fratricide risk. Again, the BBN was updated by setting the probability for minimum kill chain error to $100 \%$, improving the overall risk levels to close to those of the best case values, as shown in Figure E-15, hence implying that a reduction in kill chain error growth would be beneficial.

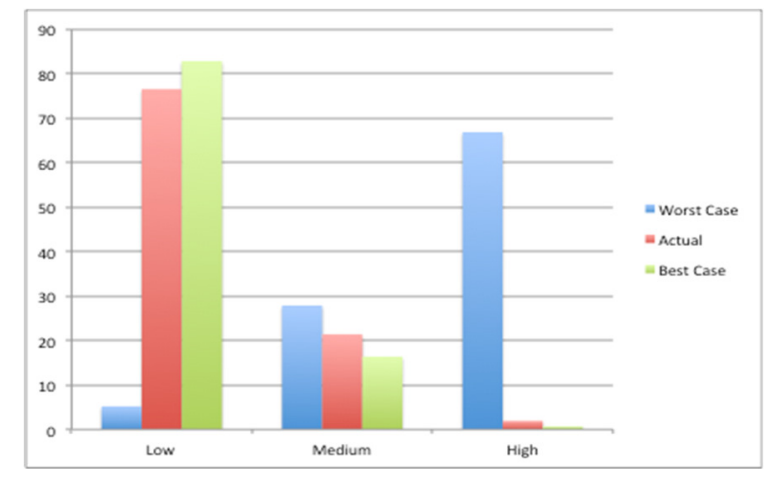

Figure E - 15 : Collateral Damage Risk with High Accuracy Kill Chain

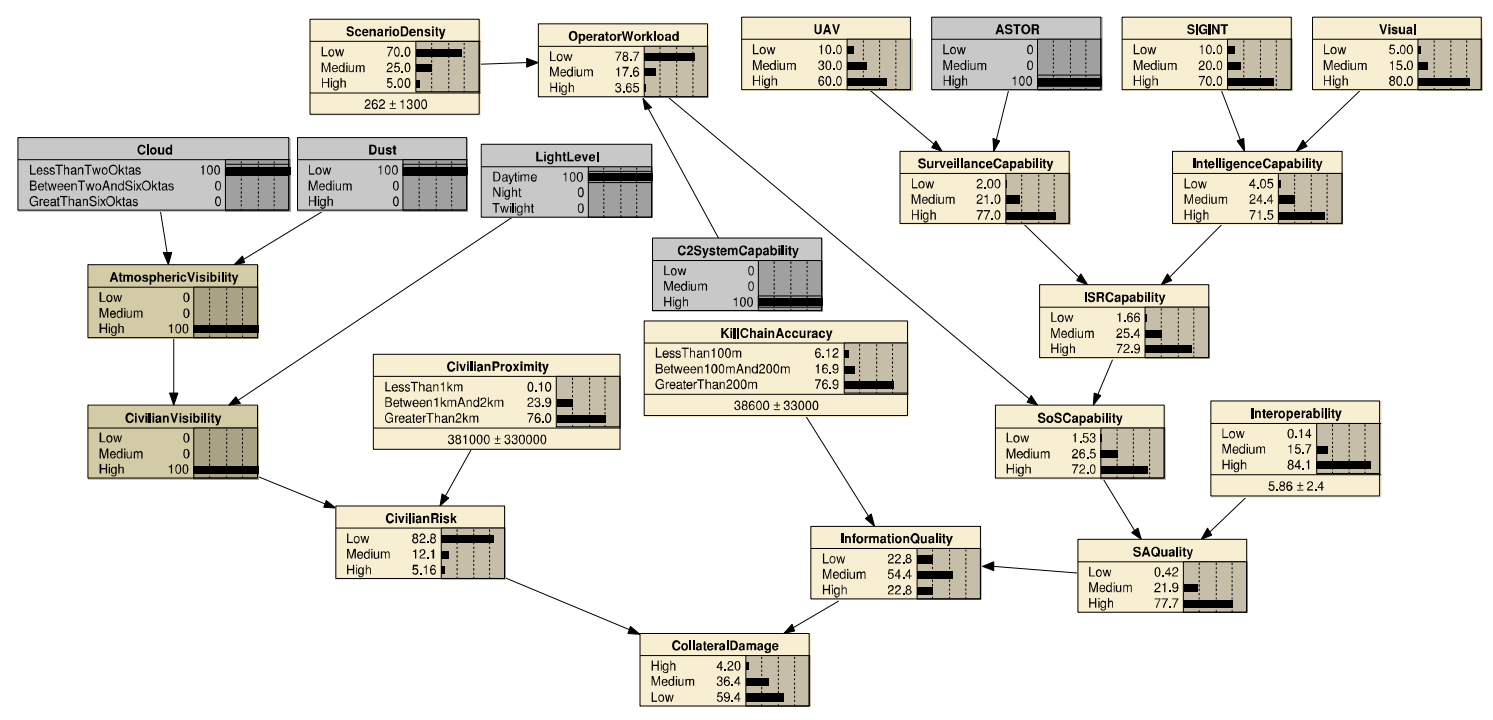

Figure E - 16 : Collateral Damage Risk Post Learning and Evidence 


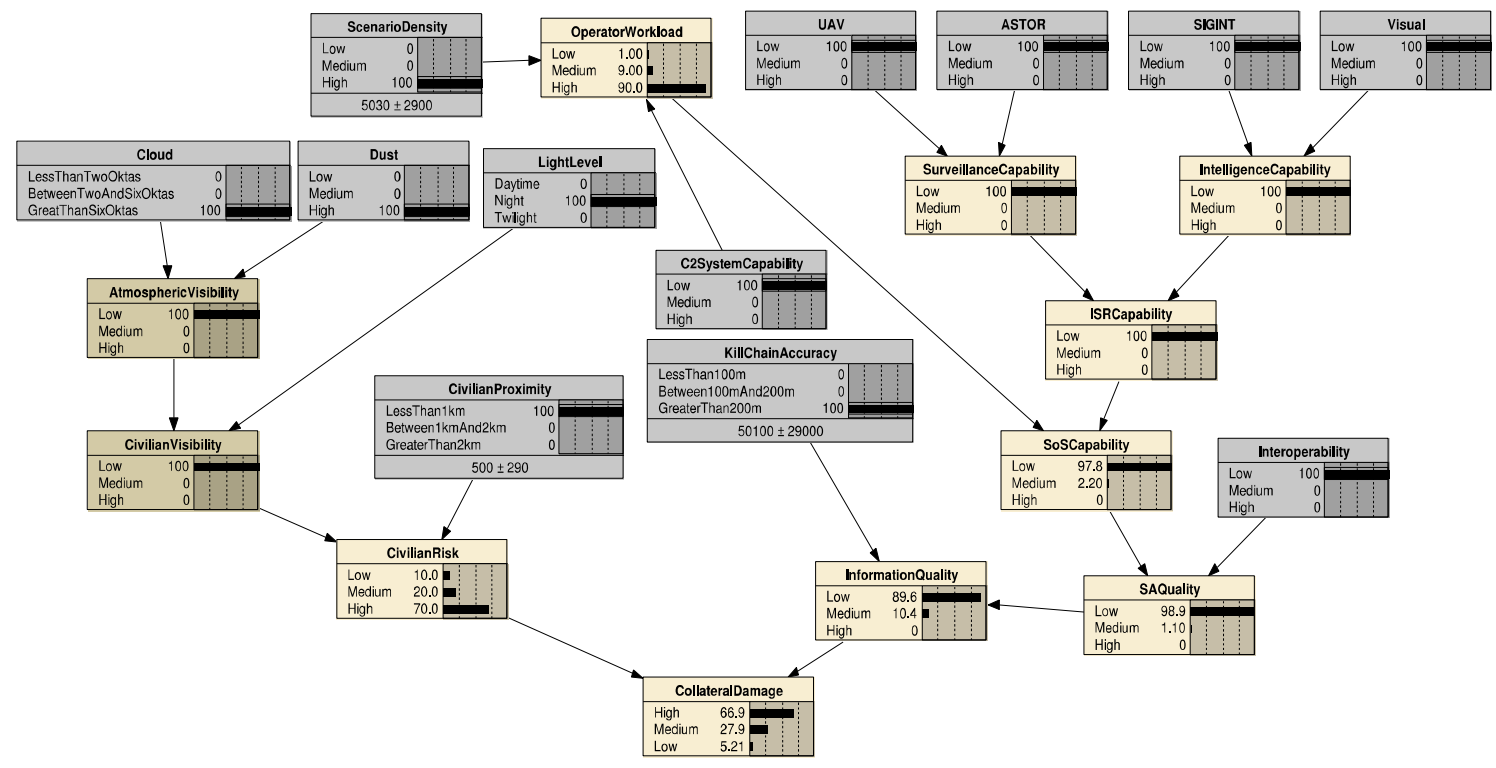

Figure E - 17 : Collateral Damage Risk Worst Case

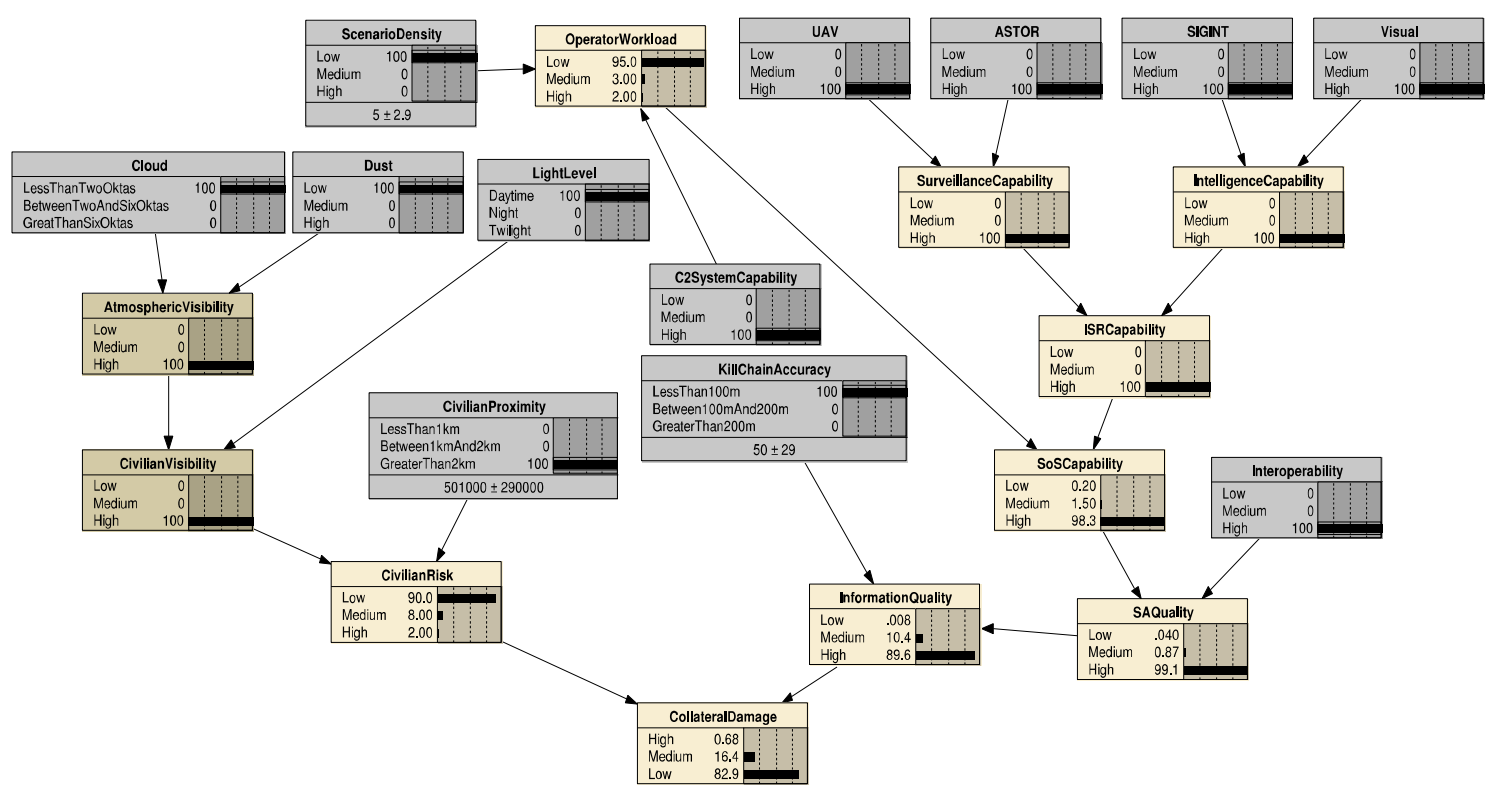

Figure E - 18 : Collateral Damage Risk Best Case

\section{E.2.5 Risk Confidence}

In accordance with the analysis process an equivalent confidence BBN was implemented as shown in Figure E - 19, with the rationale, not previously provided, for the BBN variable confidence estimates provided in Table E - 4 .

\begin{tabular}{|l|l|l|}
\hline Variable & Input Type & Confidence Estimate \\
\hline CivilianProximity & Supporting Model & Low: Model is simplistic \\
\hline
\end{tabular}

Table E - 4 : Collateral Damage Risk Confidence 


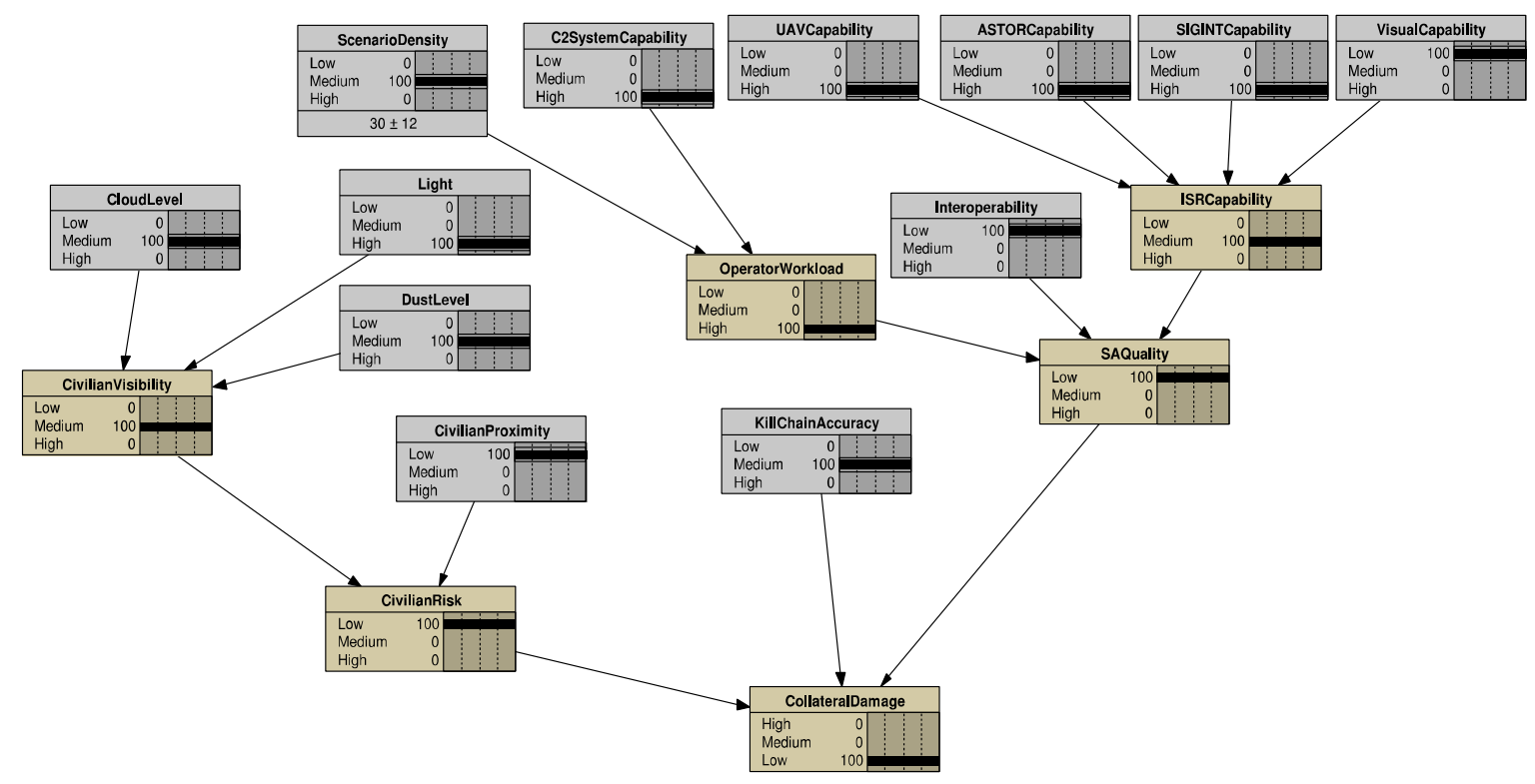

Figure E - 19 - Collateral Damage Risk Confidence

\section{E.3. Analysis and Handling - Additional Target Opportunity}

The Additional Target Opportunity Risk network was extracted from the overall CAS causal network and is shown below in Figure E - 20. In this case there were two primary contributing factors, SA Quality and Target Visibility, which were both analysed previously under Fratricide Risk and Target Destruction Risk respectively and resulted in the identification of an Interoperability System Dynamics supporting model with Bayesian representation of SA Quality and Target Visibility.

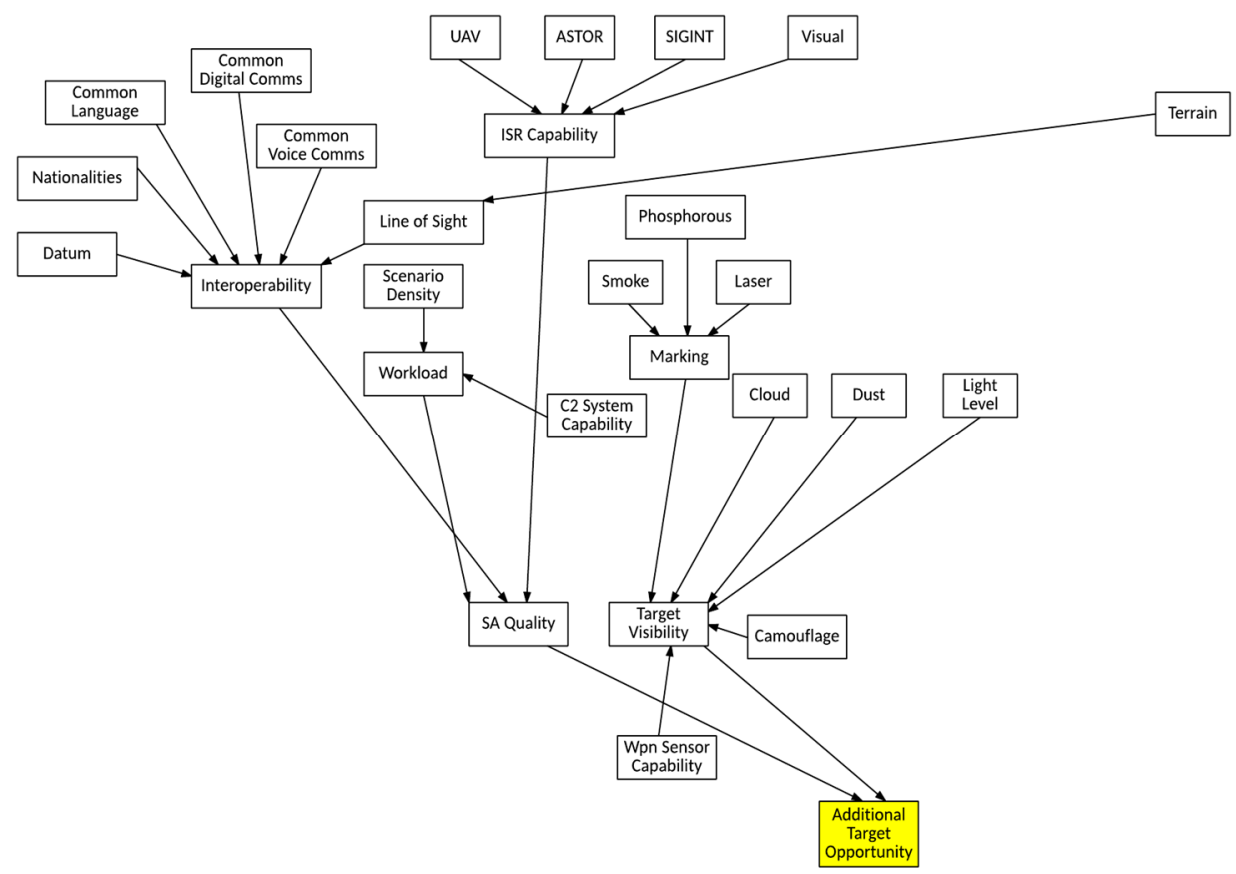

Figure E - 20 : Additional Target Opportunity Causal Network 


\section{E.3.1 Simplified Causal Network}

Having determined that only an Interoperability supporting model was required, it was not deemed necessary to produce a model architecture diagram before production of the simplified causal network, shown in Figure E - 21.

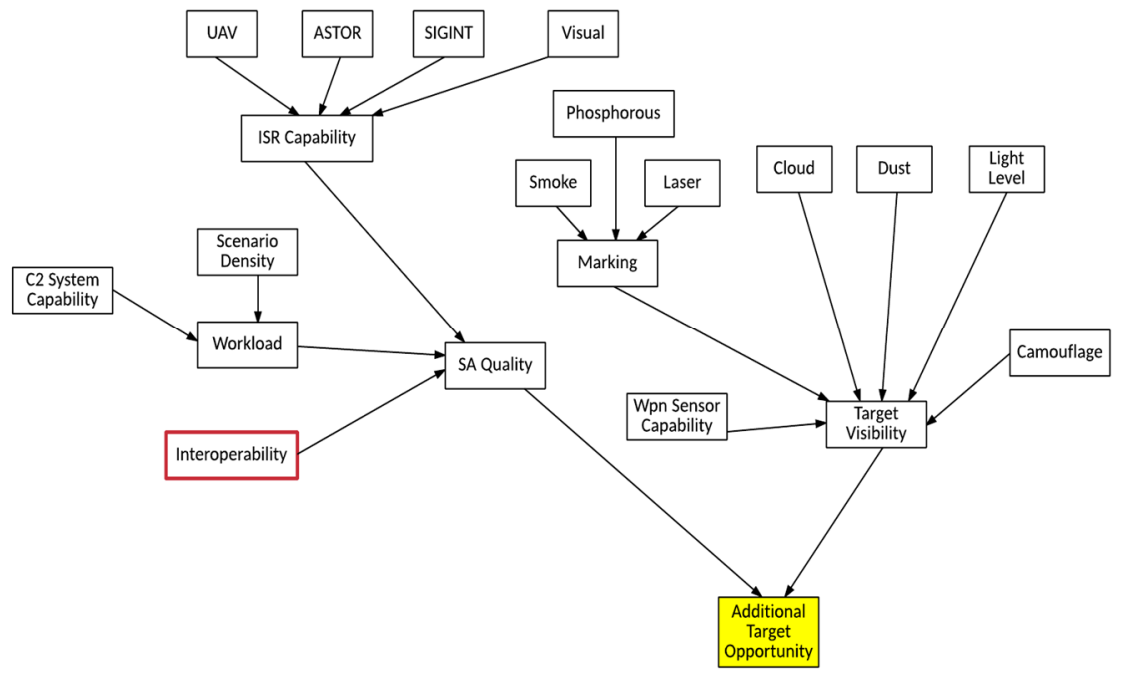

Figure E - 21 : Additional Target Opportunity Risk - Simplified Causal Network

\section{E.3.2 Additional Target Opportunity Risk BBN}

The Additional Target Opportunity Risk BBN was implemented in accordance with the Causal Network and is shown below in Figure E - 22. In this case only one additional BBN variable was introduced, AdditionalTargetOpportunity, the remaining definitions and mediating variables were described within the previous risk analysis.

Definition of BBN the single variable not previously described is provided below in Table E 5.

\begin{tabular}{|l|l|l|}
\hline Variable & Description & States \\
\hline AdditionalTargetOpportunity & $\begin{array}{l}\text { Opportunity to prosecute } \\
\text { a target in addition to the } \\
\text { primary mission target }\end{array}$ & $\begin{array}{l}\text { Low: Likelihood of locating } \\
\text { and prosecuting an additional } \\
\text { target is low } \\
\text { Medium: There is a potential } \\
\text { for the location and } \\
\text { prosecution of an additional } \\
\text { target } \\
\text { High: There is a significant } \\
\text { potential the location and } \\
\text { prosecution of an additional } \\
\text { target }\end{array}$ \\
\hline
\end{tabular}

Table E - 5 - Additional Target Opportunity BBN Variables 


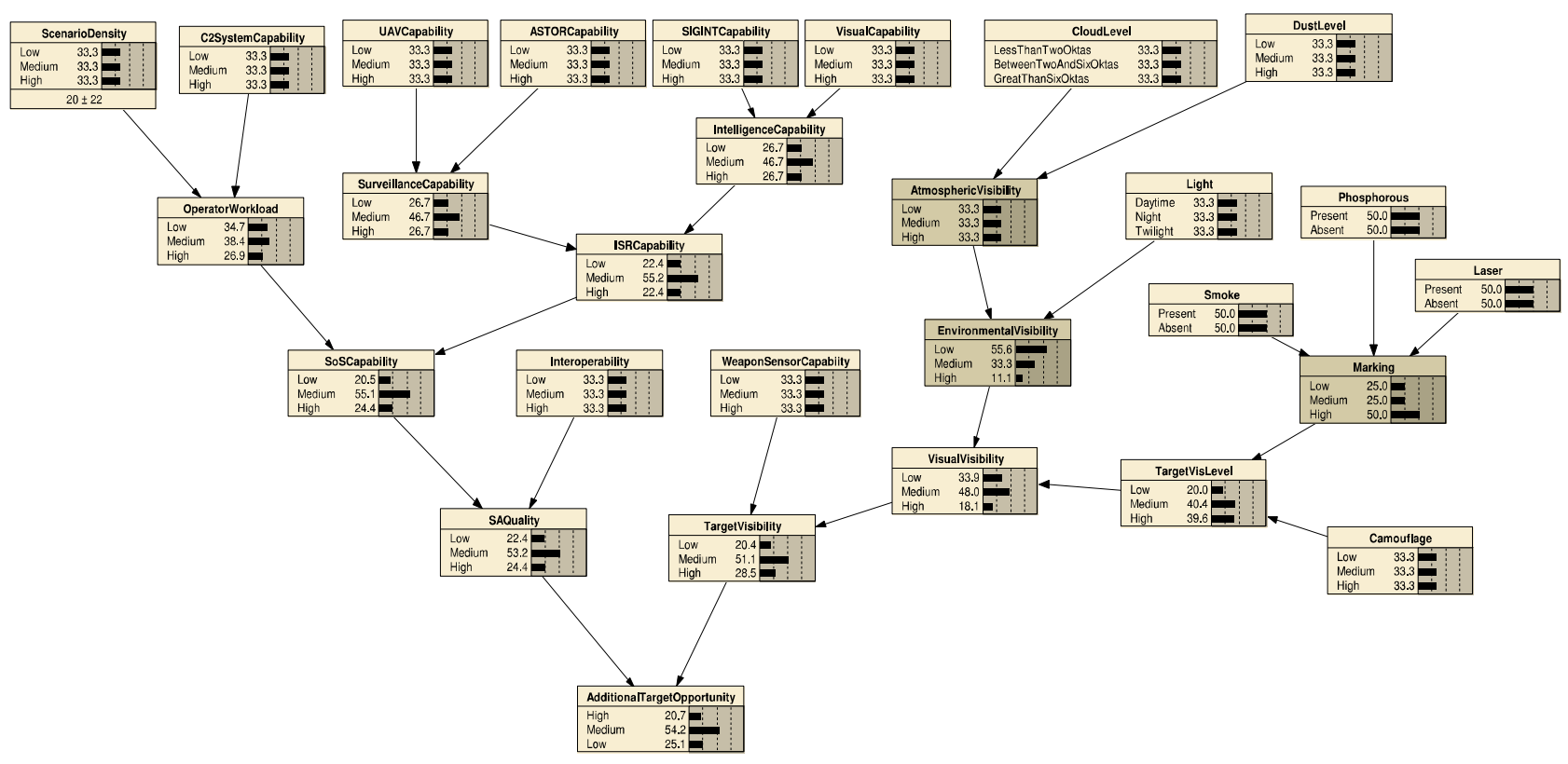

Figure E - 22 : Additional Target Opportunity BBN

\section{E.3.3 Interpretation of Results}

In accordance with the analysis process, the BBN first was instantiated with evidence derived from the hypothetical scenario, described in Appendix $D$, and output from the Interoperability supporting model; the resultant BBN is shown in Figure E - $\mathbf{2 5}$ and with worst and best case evidence, shown in Figure E - 26 and Figure E - 27 respectively. The sideby-side comparison is shown below in Figure E - 23.

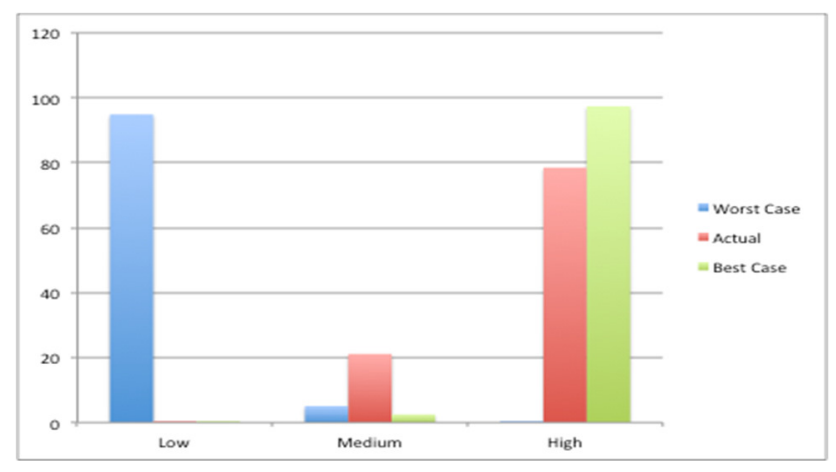

Figure E - 23 : Additional Target Opportunity Comparison

These results revealed a relatively close correlation between the actual and best-case results, although further experimentation was undertaken in order to identify potential approaches to improvement in the actual risk profile. Initially the UAV "high" capability was set to $100 \%$ resulting in a marginal improvement in the risk profile, as shown in Figure E - 24. 


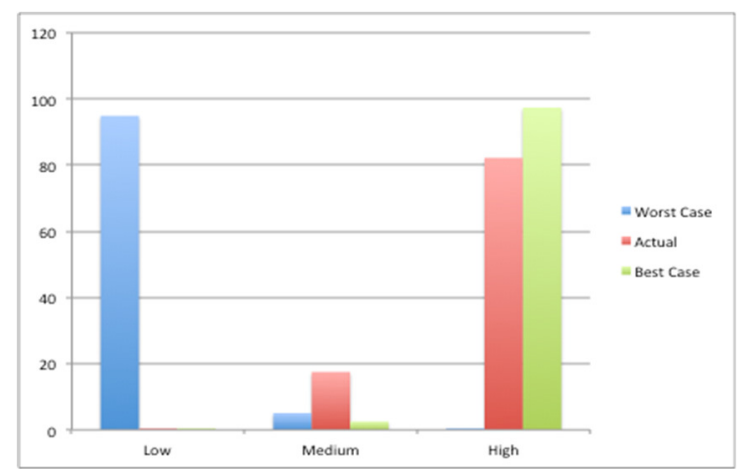

Figure E - 24 : Additional Target Opportunity with High UAV Capability

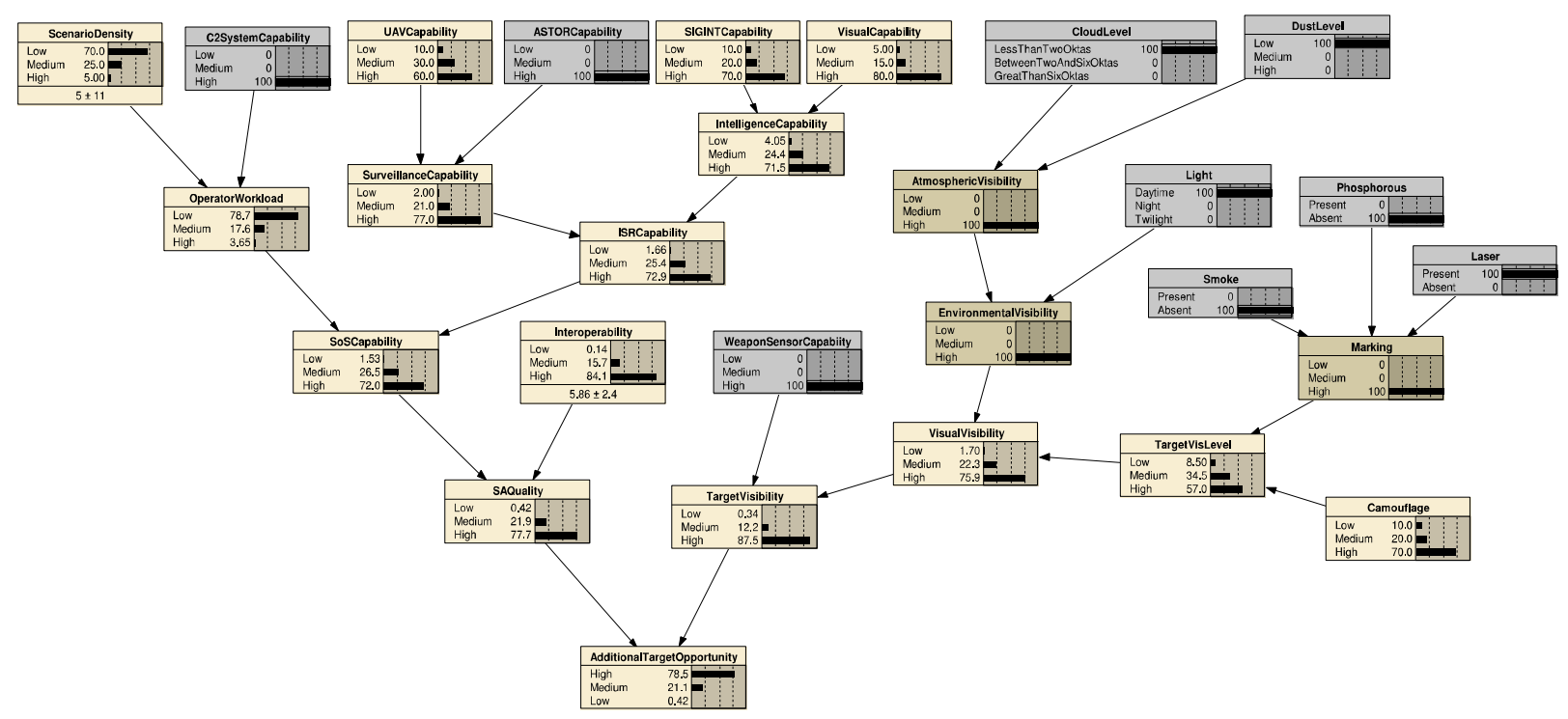

Figure E - 25 : Additional Target Opportunity Post Learning and Evidence

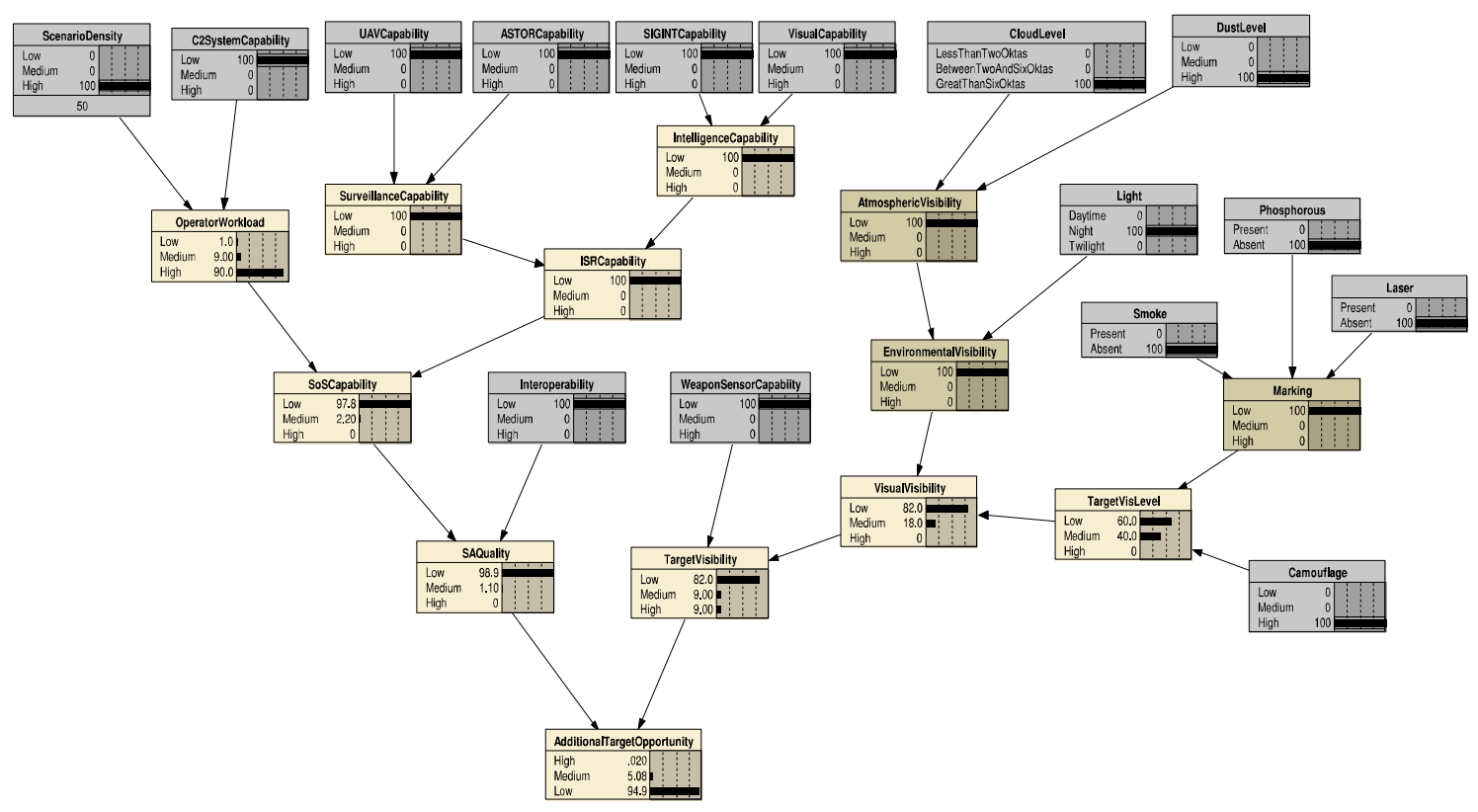

Figure E - 26 : Additional Target Opportunity Worst Case 


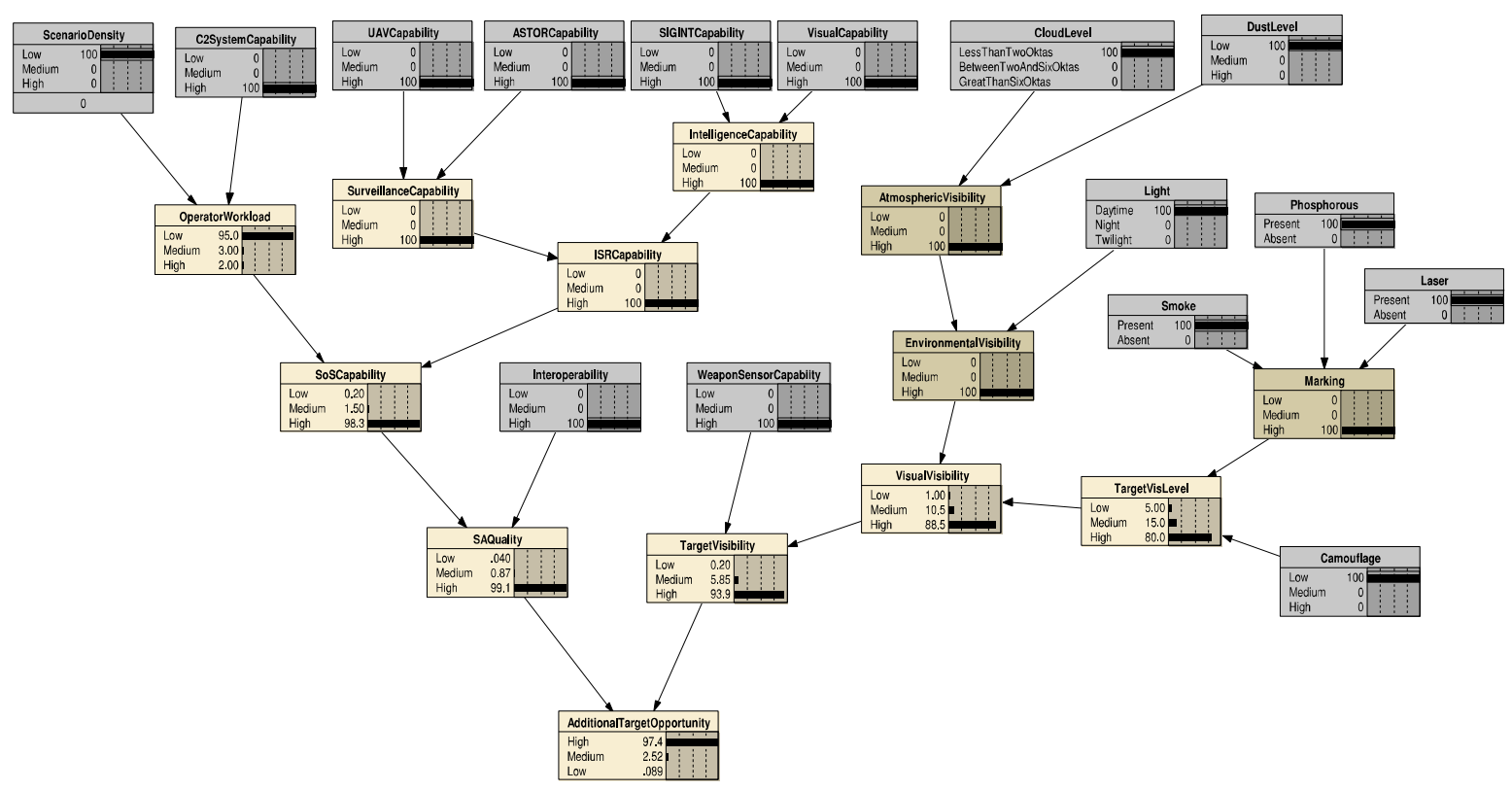

Figure E - 27 : Additional Target Opportunity Best Case

Setting the IO capability to "high", in addition to the updated UAV variable, resulted in a more marked improvement, as shown in Figure E - 28. However, in practice more detailed analysis of the 10 model would be necessary in order to determine how overall IO could be improved, whilst improvement in UAV capability was potentially more straightforward, if assets were available, it resulted in a minimal improvement in risk profile.

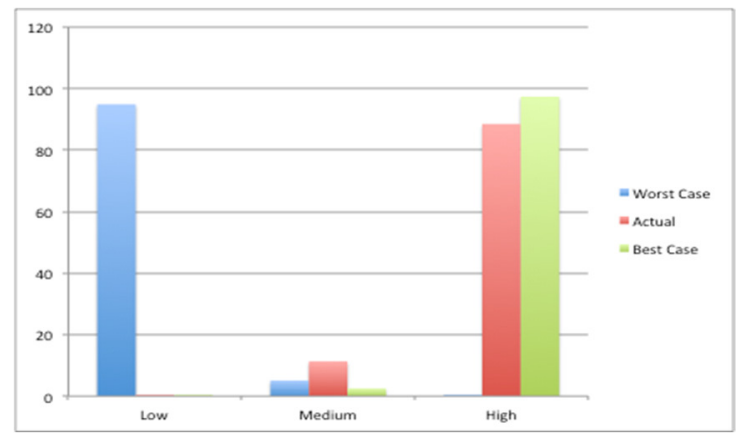

Figure E - 28 : Additional Target Opportunity with High UAV Capability and Good IO

\section{E.3.4 Risk Confidence}

In accordance with the analysis process an equivalent confidence BBN was implemented as shown in Figure E - 29; in this case the rationale for the confidence levels entered into the network are covered within the previous risk analysis. 


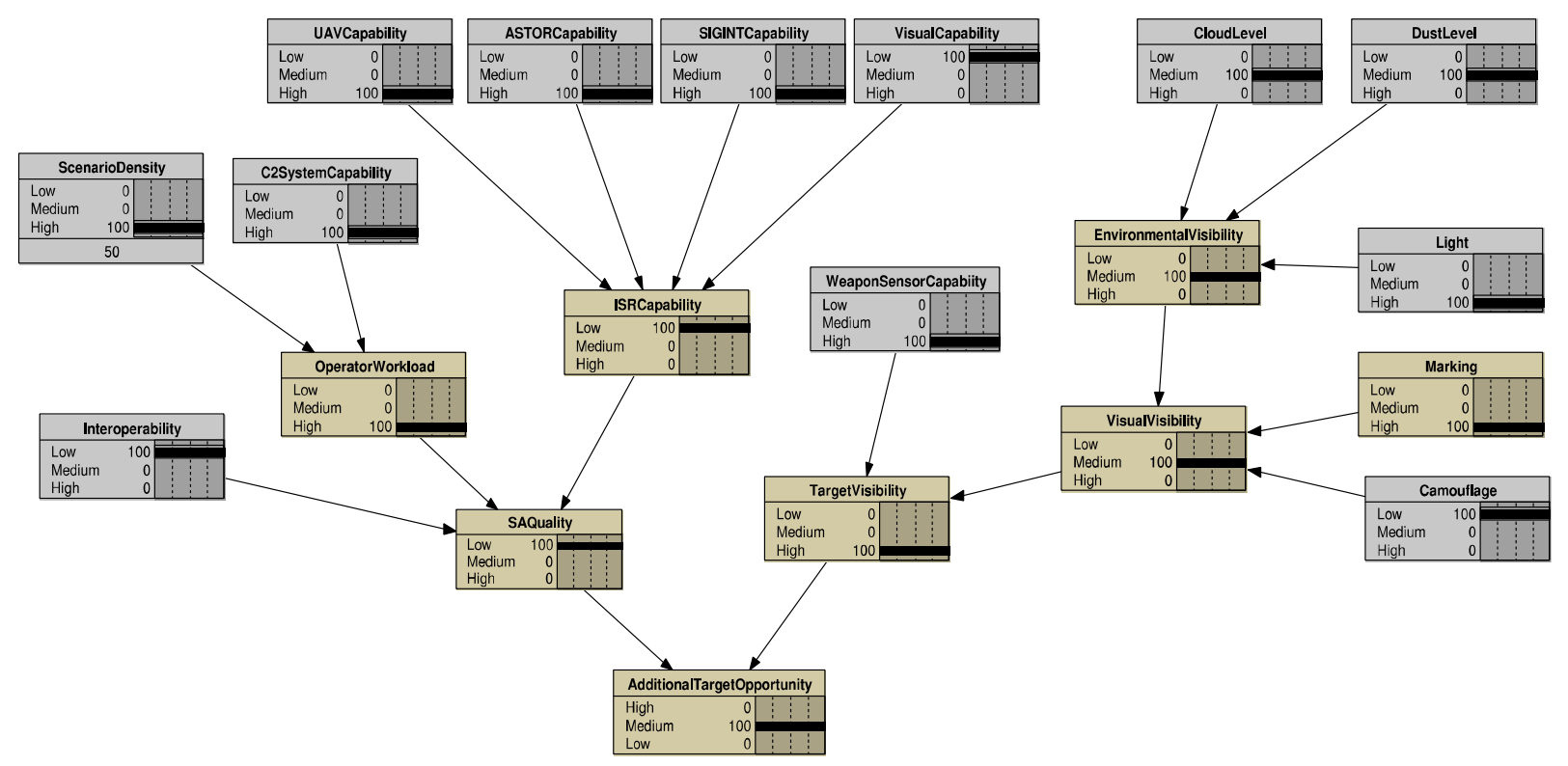

Figure E - 29 : Additional Target Opportunity Confidence

\section{E.4. Analysis and Handling - Loss of Aircraft Risk}

Loss of Aircraft Risk was influenced by four primary contributing factors, SA Quality, Enemy Capability, Flight Path Risk and Defensive Capability. As discussed previously, SA Quality could be represented as a BBN with an associated Interoperability supporting model, the remaining contributing factors were unique to Loss of Aircraft Risk and therefore analysed further, as follows.

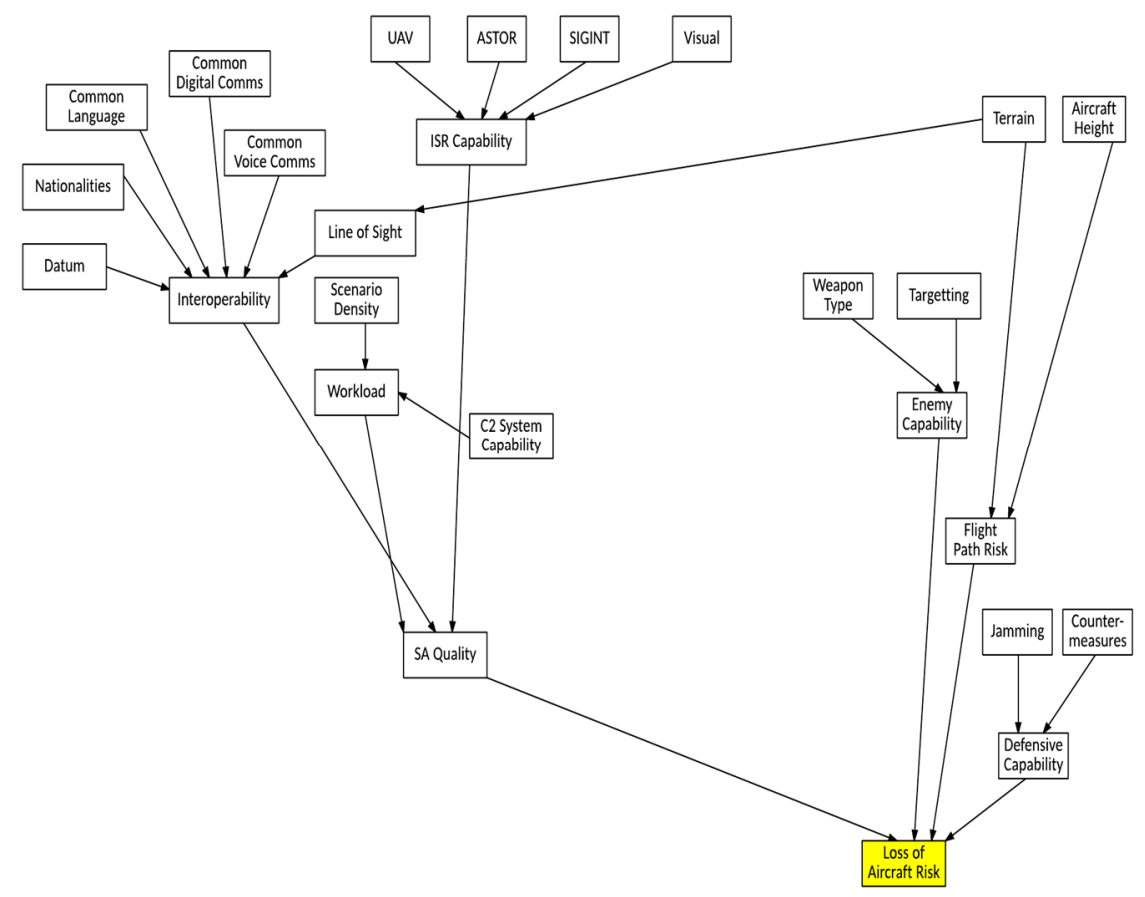

Figure E - 30 : Loss Of Aircraft Risk Causal Network 


\section{E.4.1 Model Technique Selection}

Enemy Capability and the associated contributing factors were analysed using the selection tool (Appendix B, Table B - 10), indicating that a probabilistic modelling approach would be appropriate. It was decided that, as with the previously discussed risk analysis, a BBN would allow direct incorporation into the central BBN.

The same process was applied to Flight Path risk (Appendix B, Table B - 11), again indicating that a probabilistic modelling approach would be appropriate with a BBN selected for the same rationale as described above.

Finally, the modelling requirements for defensive capability (of CAS aircraft) were considered (Table B - 12), in this case "Modelling Languages" (e.g. UML, SysML) was the most highly rated selected option followed by probabilistic techniques (BBN, Markov Models, Game Theory) and EAF (e.g. MODAF). However, although a technique such as SysML, would provide a detailed representation of jamming and counter measures capability it does not enable uncertainty to be modelled, which is essential where not all information is available and may actually provide more detail than necessary. A Bayesian approach allows uncertainty to be modelled and where defensive capabilities are well understood, this information may be entered as evidence into the BBN. It was therefore decided to model defensive capability with a simple BBN approach, which could be directly incorporated into the overall risk $\mathrm{BBN}$.

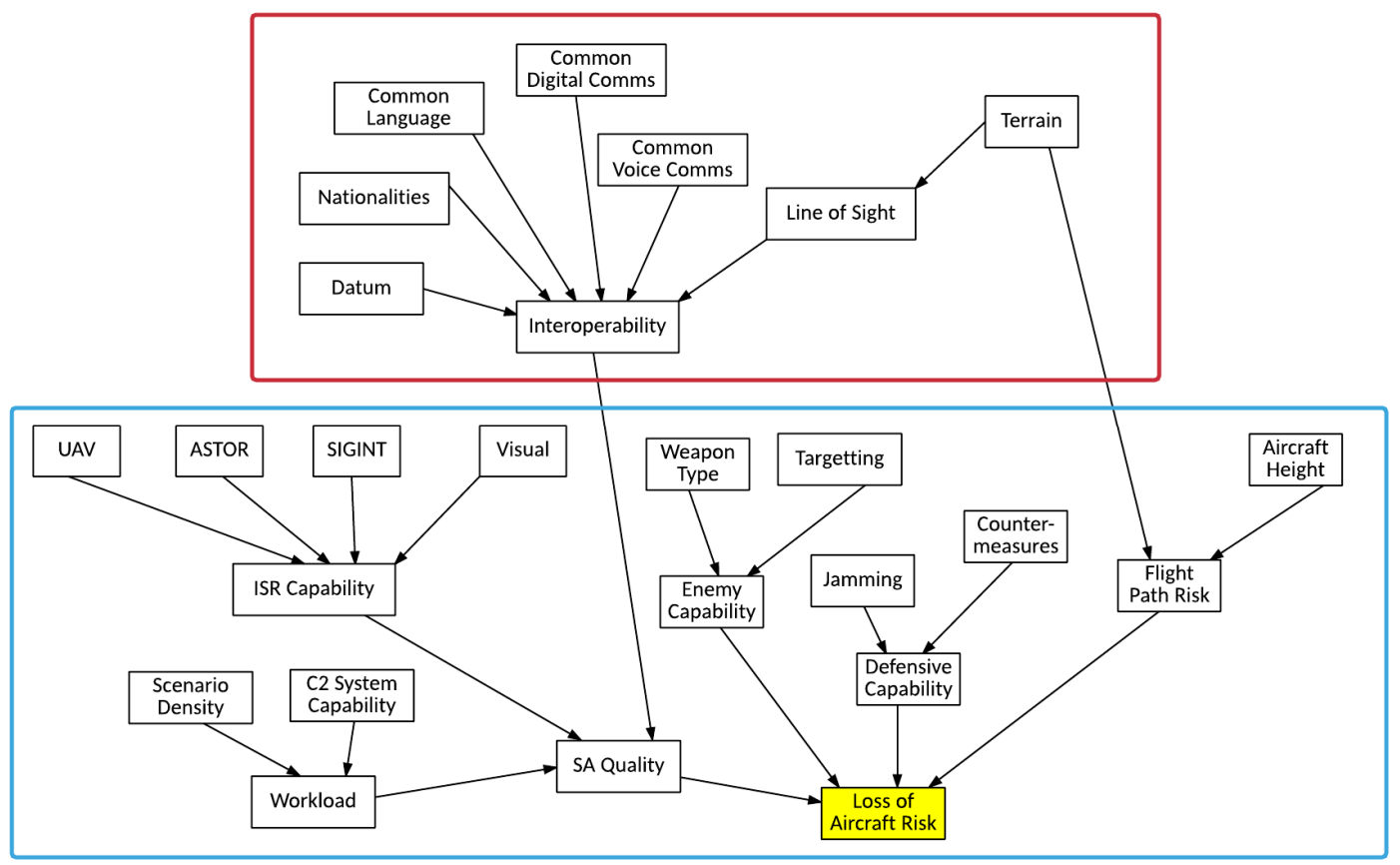

Figure E - 31 : Loss of Aircraft Risk Model Architecture 


\section{E.4.2 Simplified Causal Network}

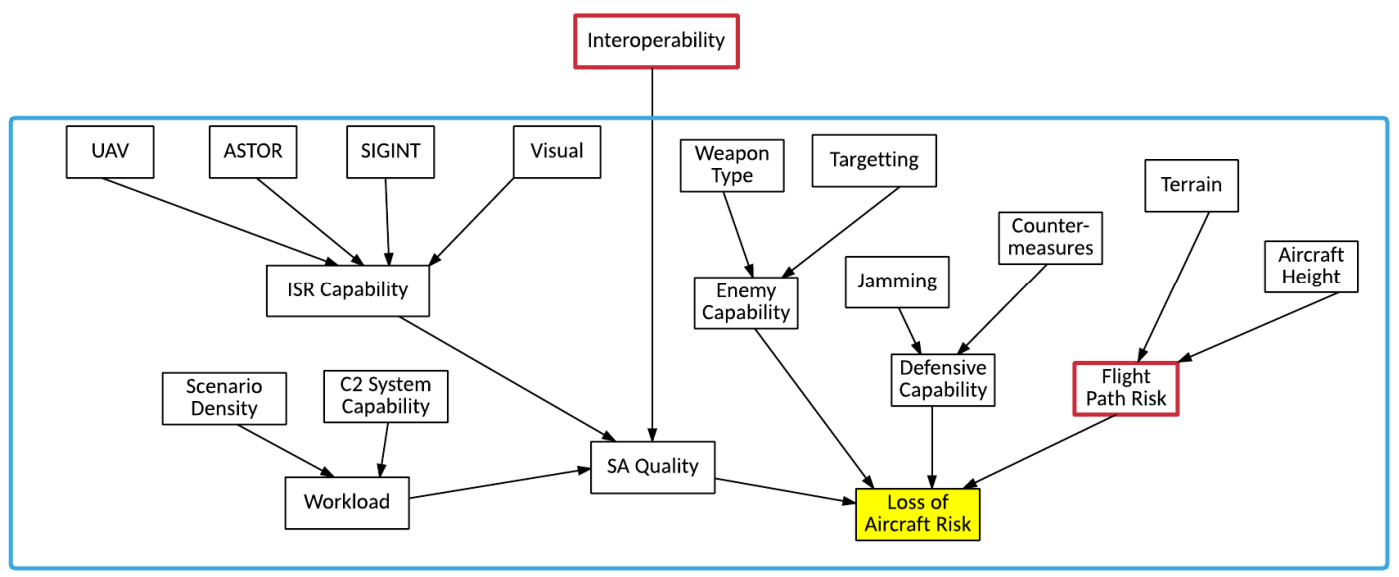

Figure E - 32 : Loss of Aircraft Risk Simplified Causal Network

\section{E.4.3 Loss of Aircraft Risk BBN}

Having established the model architecture the Loss of Aircraft Risk BBN was implemented in accordance with the Causal Network and is shown below in Figure E - 33. Definitions of BBN variables not previously described are provided below in Table E - 6 .

\begin{tabular}{|l|l|l|}
\hline Variable & Description & States \\
\hline WeaponType & $\begin{array}{l}\text { Hostile ground to air } \\
\text { weapon capability }\end{array}$ & $\begin{array}{l}\text { Low: No ground to air } \\
\text { capability except gunfire } \\
\text { Medium: Basic ground to air } \\
\text { capability, short range missile } \\
\text { High: Sophisticated medium } \\
\text { range ground to air capability }\end{array}$ \\
\hline Targeting & $\begin{array}{l}\text { Hostile target tracking } \\
\text { capability }\end{array}$ & $\begin{array}{l}\text { Low: Only visual target } \\
\text { tracking } \\
\text { Medium: Short range radar } \\
\text { tracking } \\
\text { High: Medium, long range high } \\
\text { fidelity tracking }\end{array}$ \\
\hline EnemyCapability & $\begin{array}{l}\text { Overall hostile defensive } \\
\text { capability }\end{array}$ & $\begin{array}{l}\text { Low: Defensive capability } \\
\text { poses very low risk } \\
\text { Medium: Defensive capability } \\
\text { exhibits some threat } \\
\text { High: Forces defensive } \\
\text { capability is significant posing } \\
\text { major threat to weapon } \\
\text { delivery aircraft }\end{array}$ \\
\hline
\end{tabular}




\begin{tabular}{|c|c|c|}
\hline Variable & Description & States \\
\hline Jamming & $\begin{array}{l}\text { Weapon delivery aircraft } \\
\text { jamming capability }\end{array}$ & $\begin{array}{l}\text { Low: No jamming capability } \\
\text { Medium: Basic, short range } \\
\text { jamming } \\
\begin{array}{l}\text { High: High r power, } \\
\text { sophisticated } \\
\text { capability }\end{array}\end{array}$ \\
\hline CounterMeasures & $\begin{array}{l}\text { Weapon delivery aircraft } \\
\text { countermeasures (e.g. } \\
\text { Chaff, Flare) capability }\end{array}$ & $\begin{array}{l}\text { Low: No countermeasures } \\
\text { Medium: Chaff or flare } \\
\text { High: Chaff and flare }\end{array}$ \\
\hline DefensiveCapability & $\begin{array}{l}\text { Overall weapon delivery } \\
\text { aircraft } \\
\text { capability }\end{array}$ & $\begin{array}{l}\text { Low: No countermeasures or } \\
\text { jamming capability } \\
\text { Medium: } \\
\text { countermeasures or jamming } \\
\text { High: Both countermeasures } \\
\text { and jamming }\end{array}$ \\
\hline Terrain & $\begin{array}{l}\text { Description of the terrain } \\
\text { encountered in the } \\
\text { mission }\end{array}$ & $\begin{array}{l}\text { Flat } \\
\text { Undulating } \\
\text { Mountainous }\end{array}$ \\
\hline AircraftHeight & $\begin{array}{l}\text { Weapon delivery aircraft } \\
\text { altitude throughout attack } \\
\text { run in }\end{array}$ & $\begin{array}{l}\text { Below } 500 \mathrm{ft} \\
\text { Between500and100ft } \\
\text { Above1000ft }\end{array}$ \\
\hline FlightPathRisk & $\begin{array}{l}\text { Overall risk of } \\
\text { combination of terrain } \\
\text { type and aircraft altitude }\end{array}$ & $\begin{array}{l}\text { Low: Likelihood of aircraft loss } \\
\text { due to terrain and altitude is } \\
\text { very low, no risk mitigation } \\
\text { required } \\
\text { Medium: Potential for aircraft } \\
\text { loss, change of flight path } \\
\text { should be considered if } \\
\text { possible } \\
\text { High: Increased likelihood of } \\
\text { aircraft loss, risk mitigation or } \\
\text { re-assessment of the model is } \\
\text { required }\end{array}$ \\
\hline
\end{tabular}




\begin{tabular}{|l|l|l|}
\hline Variable & Description & States \\
\hline LossofDeliveryAircraft & $\begin{array}{l}\text { Risk of loss of weapon } \\
\text { delivery aircraft as a result } \\
\text { of the CAS mission }\end{array}$ & $\begin{array}{l}\text { Low: Risk of loss of aircraft is } \\
\text { low, no risk mitigation } \\
\text { required } \\
\text { Medium: Potential of aircraft } \\
\text { loss, risk mitigation is } \\
\text { recommended } \\
\text { High: Significant risk of aircraft } \\
\text { loss, risk mitigation is essential }\end{array}$ \\
\hline
\end{tabular}

Table E - 6 : Loss of Aircraft Risk BBN Variables

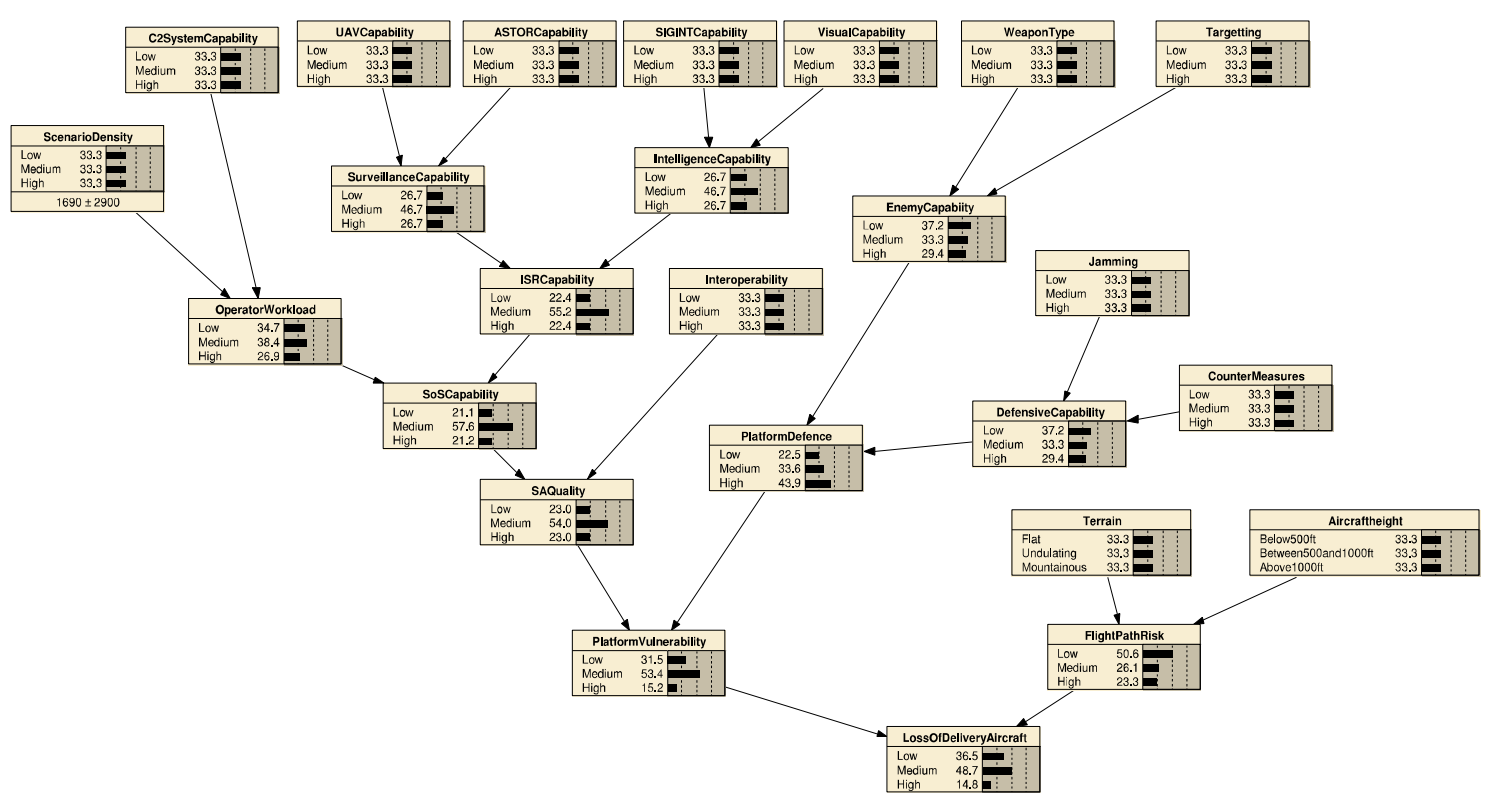

Figure E - 33 - Loss of Aircraft Risk BBN

\section{E.4.4 Interpretation of Results}

In accordance with the analysis process, the BBN first was instantiated with evidence derived from the hypothetical scenario, described in Appendix $D$, and output from the Interoperability supporting model; the resultant BBN is shown in Figure E-36. Worst and best case evidence BBN instantiations are shown in Figure E - 37 and Figure E - 38 respectively. The side-by-side comparison shown below in Figure E - 34 and revealing that, although the analysis results aligned more closely to the best case risk profile, the probability of low risk was approximately $30 \%$ lower than the optimum level, indicating that further risk mitigation was desirable. 


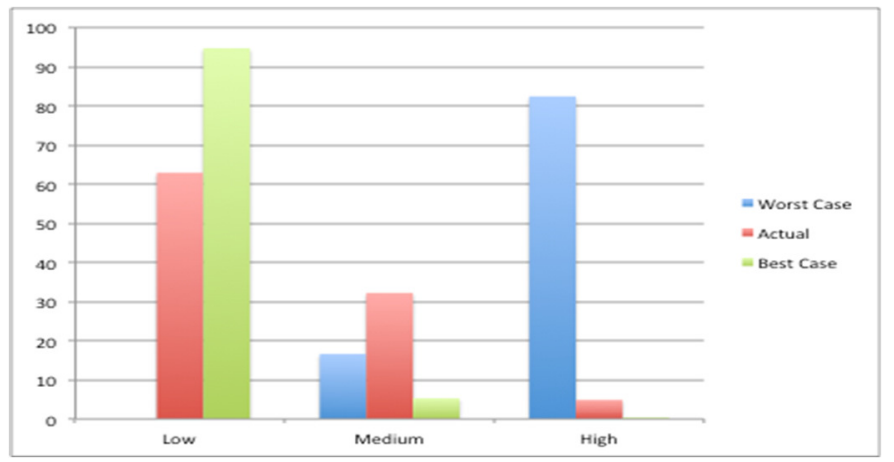

Figure E - 34 : Loss of Aircraft Risk Comparison

A potentially straightforward risk mitigation approach in this case was to raise the mission flight altitude of over 1000ft, resulting in an improved risk profile, reducing the gap between best-case and actual low risk level to $15 \%$. However, the applicability of this mitigation would be dependent on the limitations of weapons launched from the aircraft.

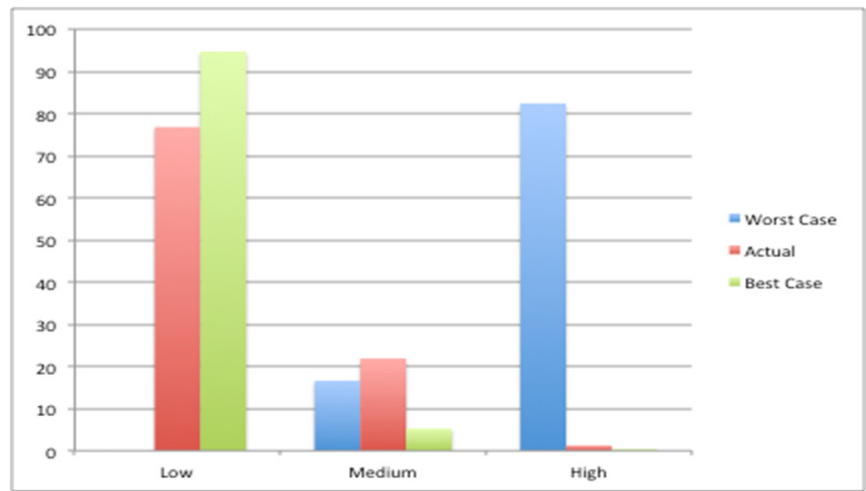

Figure E - 35 : Loss of Aircraft Risk - Altitude of $1000 \mathrm{ft}$

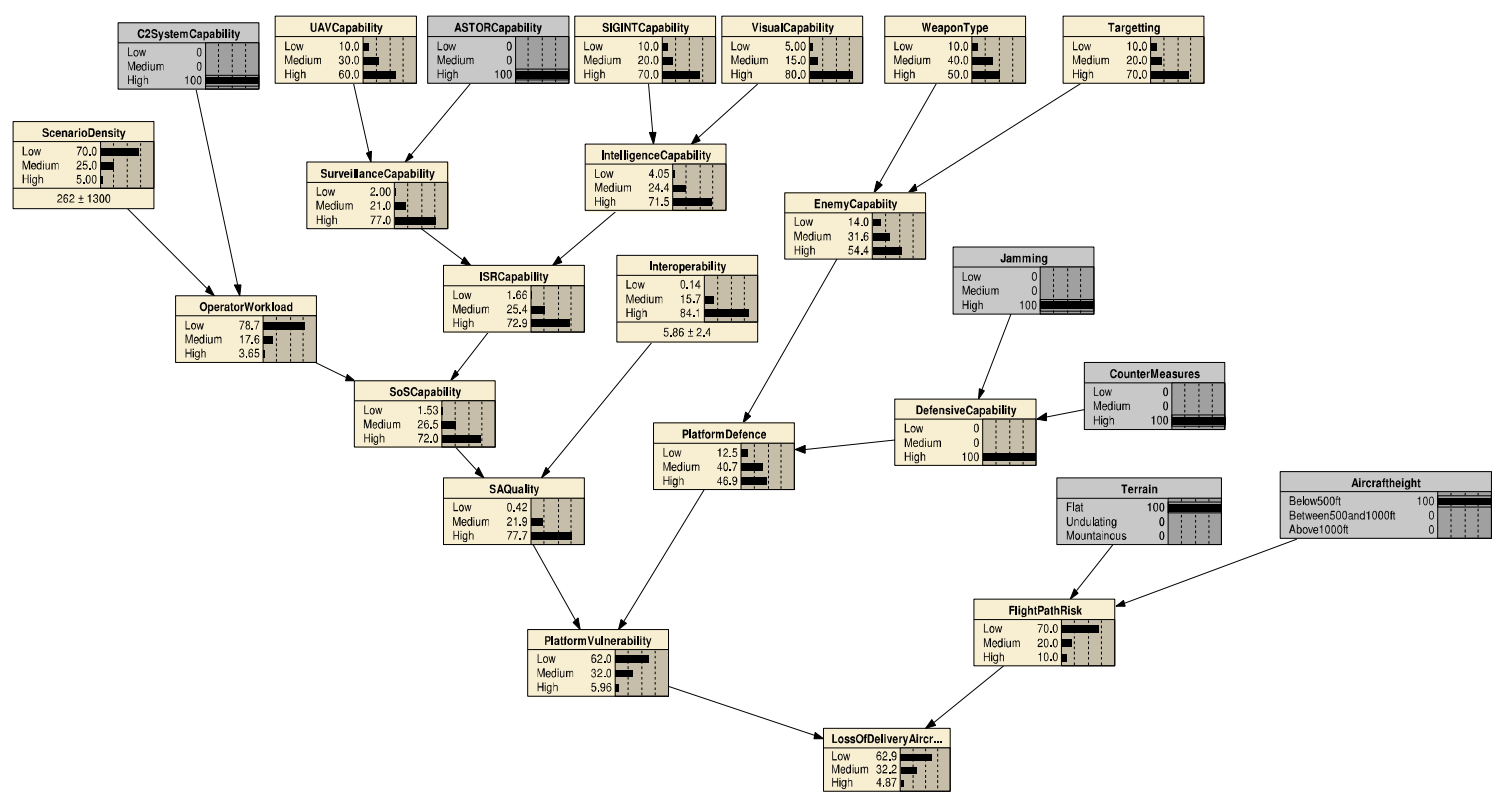

Figure E - 36 : Loss of Aircraft Risk Post Learning and Evidence 


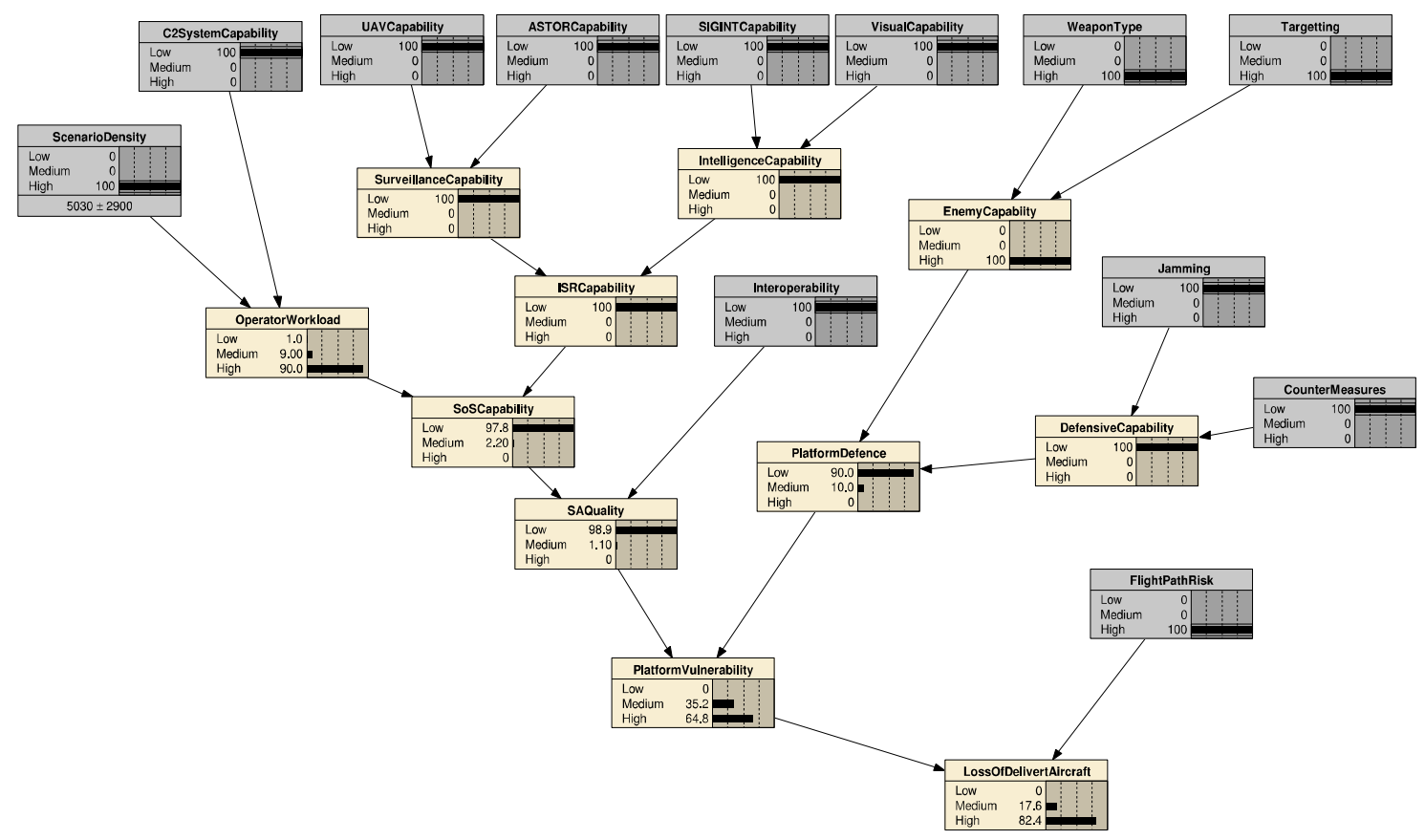

Figure E - 37 : Loss of Aircraft Risk Worst Case BBN

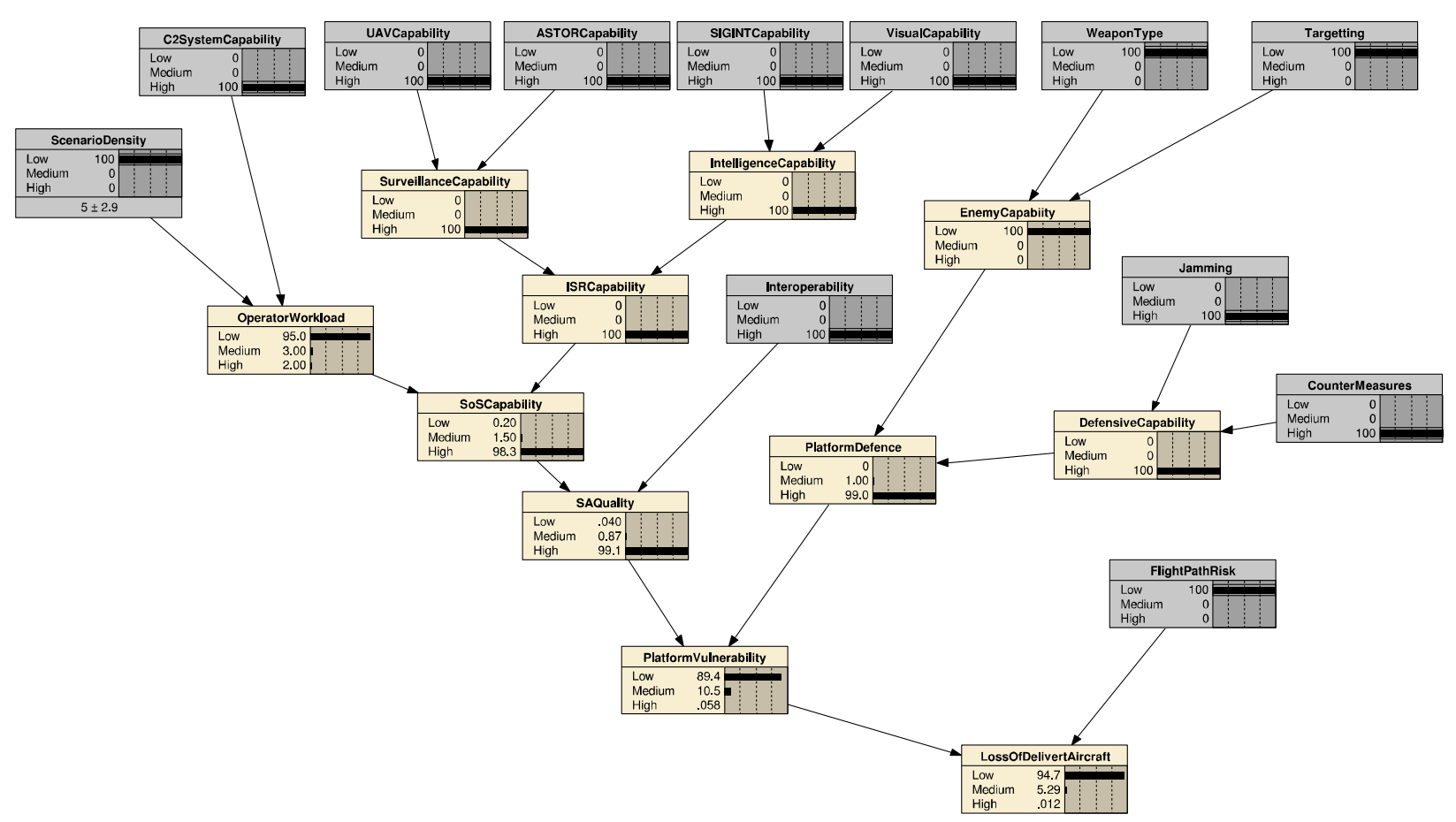

Figure E - 38 : Loss of Aircraft Risk Best Case BBN

\section{E.4.5 Risk Confidence}

Finally, the associated confidence was derived, through the construction of an equivalent confidence BBN as shown in Figure E - 39, with the rationale, not previously provided, for the BBN variable confidence estimates provided in Table E - 7 . 


\begin{tabular}{|l|l|l|}
\hline Variable & Input Type & Confidence Estimate \\
\hline WeaponType & Estimate & $\begin{array}{l}\text { Low: based on intelligence reports that are } \\
\text { unconfirmed }\end{array}$ \\
\hline Targeting & Estimate & $\begin{array}{l}\text { Low: based on intelligence reports that are } \\
\text { unconfirmed }\end{array}$ \\
\hline Jamming & Evidence & $\begin{array}{l}\text { High: all available weapon delivery aircraft } \\
\text { capabilities are similar and known }\end{array}$ \\
\hline Terrain & Evidence & $\begin{array}{l}\text { High: all available weapon delivery aircraft } \\
\text { capabilities are similar and known }\end{array}$ \\
\hline AircraftHeight & Evidence & $\begin{array}{l}\text { High: the terrain in the vicinity of the target } \\
\text { area is known }\end{array}$ \\
\hline
\end{tabular}

Table E - 7 - Loss of Aircraft Risk Confidence

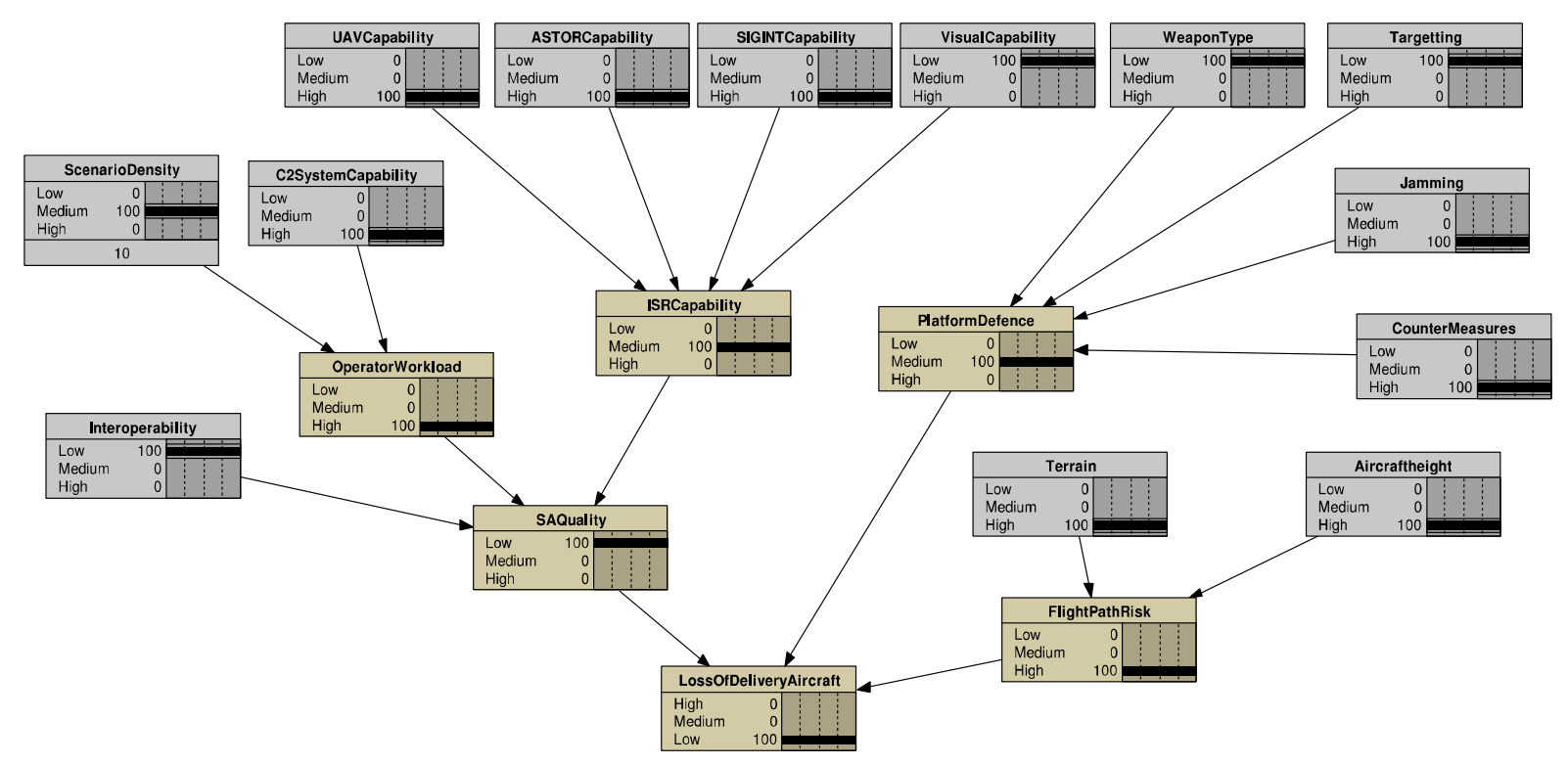

Figure E - 39 : Aircraft Loss Risk Confidence 


\section{Appendix F - Supporting Models}

This appendix provides evidence in support of the research methodology through the development of the supporting models and information to facilitate future development of the research outputs.

It should be noted that, in order to maintain the scope of this research at a manageable level, the specification and development of the supporting models was deliberately basic, but was sufficient to exercise the model-based risk management concept.

\section{F.1 Kill Chain Accuracy Model}

\section{F.1.1 Requirements}

The key requirement for the Kill Chain Accuracy model was to simulate the accumulation of spatial error from "sensor" to "shooter".

A target position may be determined from a land-based sensor, such a laser range finder, which is then transmitted through a series of systems via interconnecting data links to the weapon delivery aircraft and potentially to the weapon itself, depending on its capability.

Specifically, the model was required to:

a) Allow definition of a specific target position

b) Represent a sensor whereby a target position is input and realistic positional error and latency distributions are introduced.

c) Represent Tactical Data Links whereby a target position is input and realistic positional error (e.g. registration errors) and latency distributions are introduced.

d) Represent a Command and Control whereby a target position is input and realistic positional error and latency distributions are introduced.

e) Represent a Weapon Delivery Platform whereby a target position is input and realistic positional error and latency distributions are introduced.

f) Represent a Weapon whereby a target position is input and realistic positional error and latency distributions are introduced.

g) Allow the kill chain component sub-models to be connected serially.

h) Allow the kill chain model to run for a set number of iterations.

\section{F.1.2 Model Assumptions}

The assumptions made when developing the Kill Chain Model were as follows: 
1. Sensor was assumed to have an average $0.02 \%$ range error and a $1^{\circ}$ azimuth error, providing generally realistic performance.

2. Data link $A$ was assumed to introduce an average $100 \mathrm{~m}$ registration error (introduced by differences in GPS and sensor co-ordinate systems) and an average latency of 6 seconds.

3. The command and control system was assumed to introduce an average $20 \mathrm{~m}$ position error (caused by internal co-ordinate transformations) and a latency of 6 seconds.

4. Data link B was assumed to introduce an average $50 \mathrm{~m}$ registration error and an average latency of 3 seconds.

5. The weapon delivery platform was assumed to introduce an average $5 \mathrm{~m}$ position error (caused by internal co-ordinate transformations) and a latency of 1 second.

6. The weapon was assumed not to be a "smart" weapon introducing an average $100 \mathrm{~m}$ positional error and a latency of 5 seconds.

\section{F.1.3 Design and Implementation}

The model was implemented using Simulink, a graphical programming language, the toplevel view of the Simulink model is shown below in Figure F - 1.

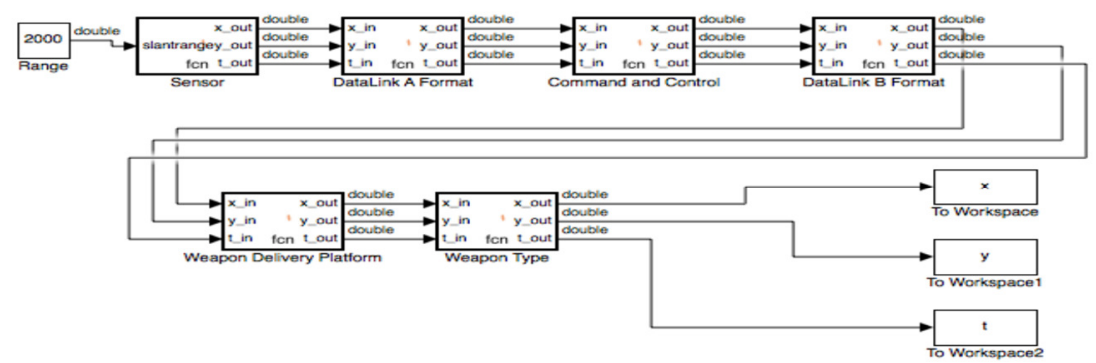

Figure F - 1 : Kill Chain Error Simulink Model

The code contained within each model block is provided below.

\section{Sensor Block}

function [x_out,y_out, t_out] = fcn(slantrange)

\%\#codegen

\%slant range metres

range_error $=0.02 ; \% 2 \%$ 
azimuth_error $=1$; \%degree

range_error = range_error*randn;

azimuth_error = azimuth_error*randn;

slantrange $=$ slantrange + slantrange ${ }^{*}$ range_error;

x_out = slantrange ${ }^{*} \operatorname{cosd}($ azimuth_error);

y_out $=$ slantrange ${ }^{*}$ sind(azimuth_error);

t_out = 0;

\section{DataLink A Format}

function [x_out,y_out,t_out] $=f \mathrm{cn}\left(x_{-}\right.$in, y_in, $t$ _in)

\%\#codegen

registration_error $=100$; $\%$ metres

registration_error_x = registration_error*randn;

registration_error_y = registration_error*randn;

latency $=6 *$ rand;

$\% y=u+a b s($ randn*200);

$\mathrm{x}$ _out $=\mathrm{x}$ _in + registration_error_x;

y_out $=y \_$in + registration_error_y;

t_out $=\mathrm{t}$ _in+latency; $\%$ seconds

\section{Command and Control}

function [x_out,y_out,t_out] $=\mathrm{fcn}\left(\mathrm{x}_{-}\right.$in, $y \_i n, t$ _in $)$ \%\#codegen

trans_error $=20$; $\%$ metres

trans_error_x = trans_error*randn;

trans_error_y $=$ trans_error*randn;

latency $=6^{*}$ rand;

$\% y=u+a b s($ randn*200);

$x \_$out $=x \_$in + trans_error_ $x$;

y_out $=y \_$in + trans_error_y;

$\mathrm{t}$ _out $=\mathrm{t}$ _in+latency; $\%$ seconds

\section{DataLink B Format}

function [x_out,y_out,t_out] = $f c n\left(x \_i n, y \_i n, t\right.$ in $)$

\%\#codegen

registration_error $=50$; $\%$ metres

registration_error_x = registration_error*randn;

registration_error_y = registration_error*randn;

latency $=3$ *and;

$\% y=u+a b s($ randn*200);

$\mathrm{x}$ _out $=\mathrm{x}$ _in + registration_error_x;

y_out $=y \_$in + registration_error_y;

$\mathrm{t} \_$out $=\mathrm{t} \_$in + latency; $\%$ seconds 


\section{Weapon Delivery Platform}

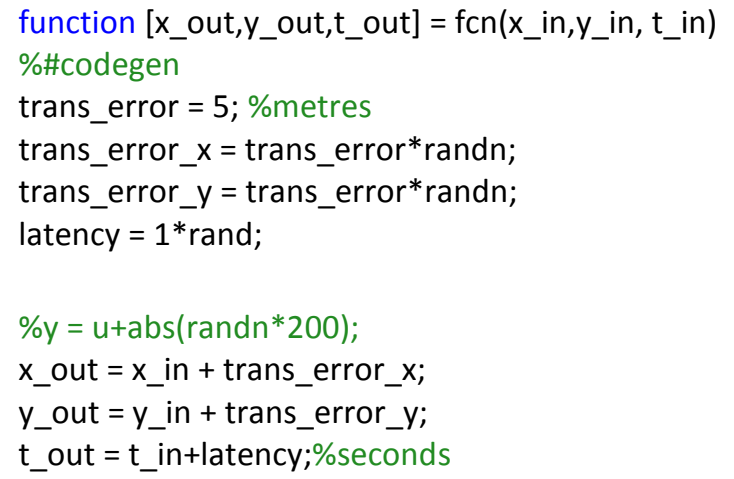

\section{Weapon Type}

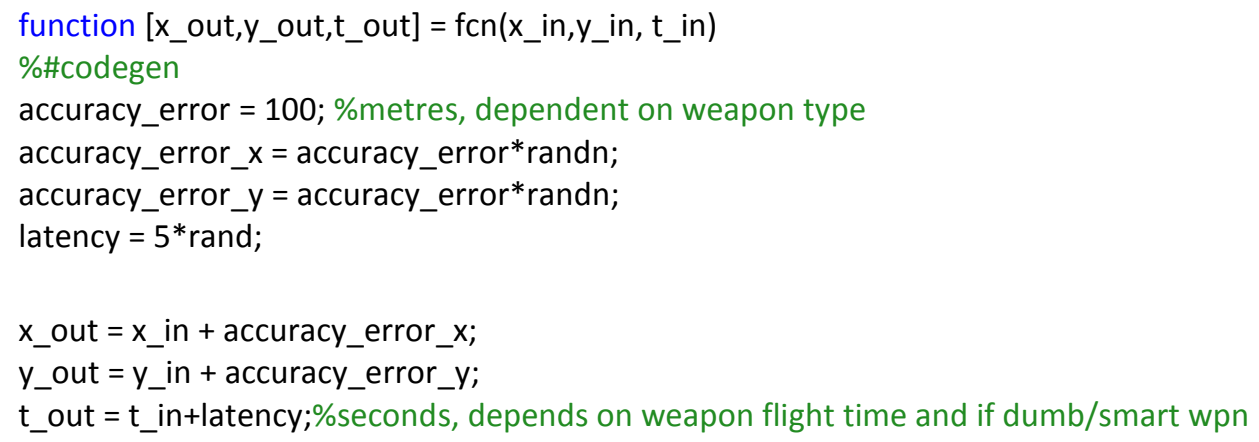

\section{F.1.4 Validation and Verification}

Initial verification was performed through code inspection followed by running the model over a single iteration. Initial basic errors were identified and the model corrected, with confidence in the model it was subsequently run over a limited (100) iterations, the results of which were exported to a spreadsheet enabling a visual check of the output to be performed.

\section{F.1.5 Model Output}

The model was executed over 5000 iterations (i.e. a Monte Carlo simulation), where the output consisting of $x, y$ co-ordinates and latency was written to the Simulink/MATLAB ${ }^{\circledR}$ workspace allowing it to be manually copied into an Excel spreadsheet (Figure F - 2), where the distance of the position from target "truth" (indicated in red) was calculated for each. Finally, the distance results were copied to a text file, formatted such that the Netica BBN application could "learn" from it. 


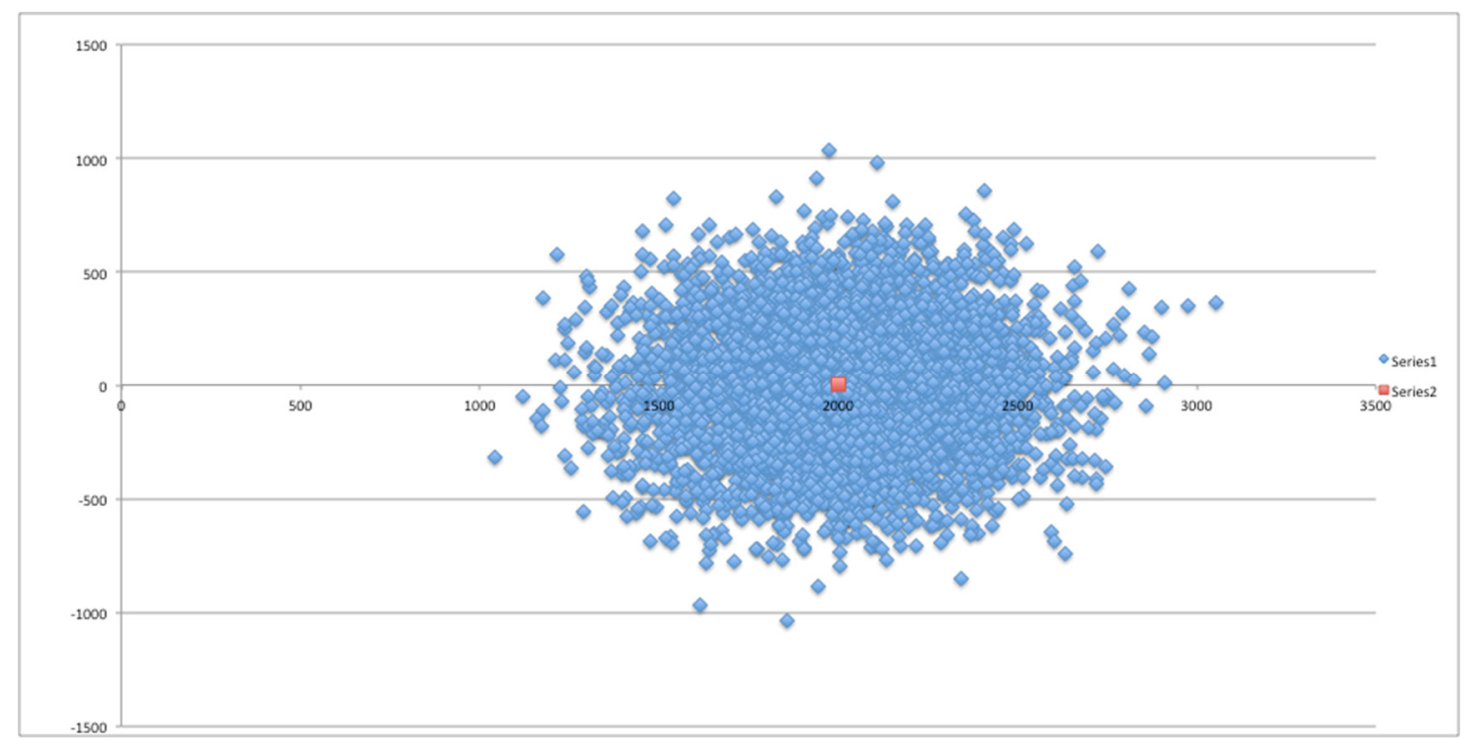

Figure F - 2 : Kill Chain Model Output

\section{F.2 Interoperability Model}

\section{F.2.1 Requirements}

The Interoperability System Dynamics model key requirement was to represent overall levels of 10 throughout the SoS from a generalised perspective, considering aggregated behaviour rather than modelling individual information exchanges.

The model was required to represent the following factors, their relationships and the impact upon IO:
a) Voice Communications
b) Tactical Data Link Communications
c) Language
d) Nationality
e) Geodetic Datum
f) Line of Sight

A further requirement was to allow the model to run over multiple iterations and to enable the modelling of uncertainty associated with the identified 10 factors.

\section{F.2.2 Model Assumptions}

The assumptions made when developing the Interoperability Model were as follows:

1. Between $70 \%$ and $100 \%$ of participants have a common spoken language.

2. Between $10 \%$ and $60 \%$ of participants operate using common procedures.

3. Tactical data link (TDL) average range is $500 \mathrm{~km}$.

4. The average number of tactical data link networks is 3 . 
5. Between 50 and $100 \%$ of participants utilise TDLs.

6. Between 50 and $100 \%$ of participants utilise secure voice communications.

7. In determining an overall interoperability score all aspects are equally weighted.

\section{F.2.3 Sensor Design and Implementation}

The causal model is shown below in Figure F - 3 and was implemented using the Vensim ${ }^{\circledR}$ application.

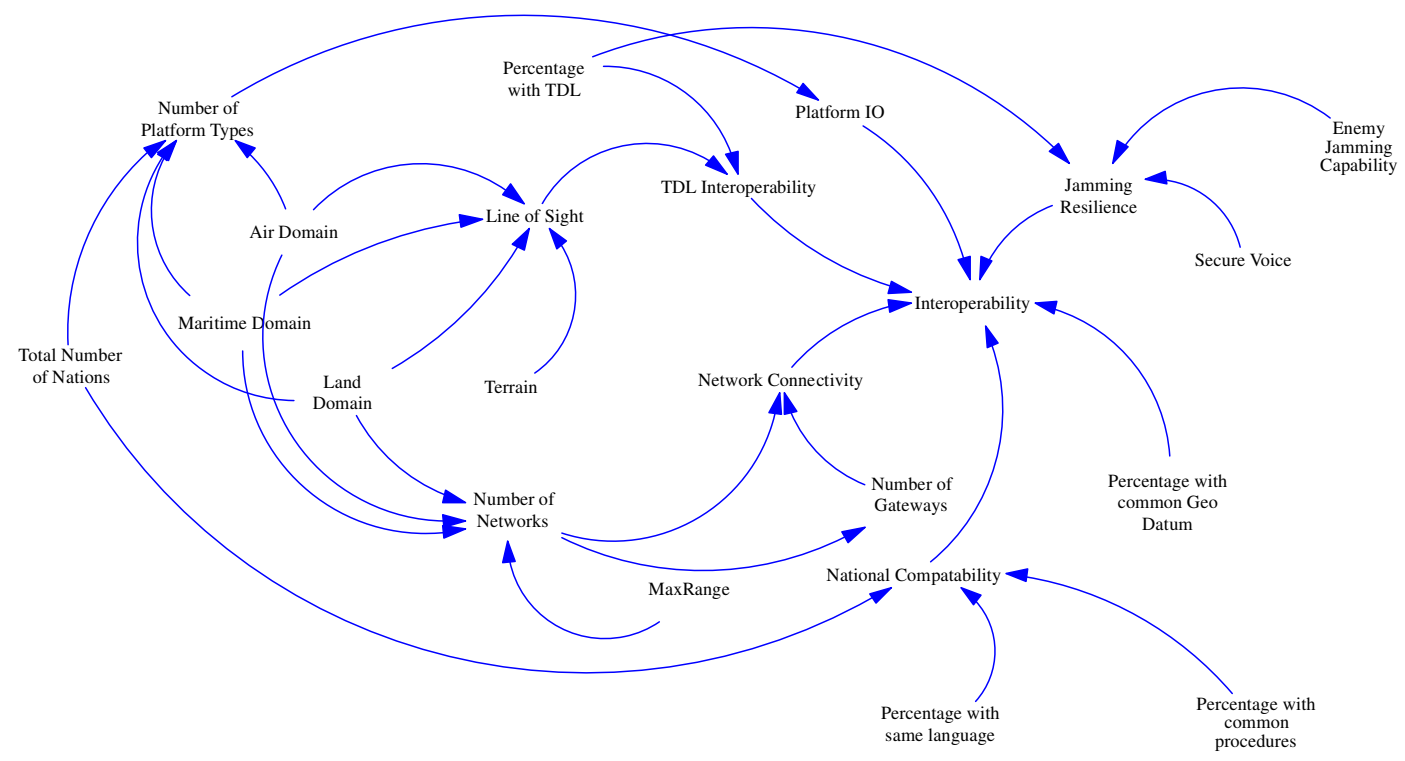

Figure F - 3 : Interoperability Model

The Vensim tool also allowed the export of all variables, including the equations defined for each and is included below.

(01) Air Domain $=\operatorname{ABS}(\operatorname{RANDOM} \operatorname{UNIFORM}(0,1,6))$

Units: **undefined $* *[0,1]$

(02) Enemy Jamming Capability= RANDOM UNIFORM $(0,0.5,31)$

Units: **undefined**

0 - low 1 - high

(03) FINAL TIME $=5000$

Units: Month

The final time for the simulation.

(04) INITIAL TIME $=0$

Units: Month

The initial time for the simulation. 
(05) Interoperability $=$ Jamming Resilience + National Compatability + Network Connectivity+Percentage with common Geo Datum+TDL Interoperability+Platform IO

Units: **undefined $* *$

(06) Jamming Resilience $=\operatorname{MIN}(($ Percentage with TDL+Secure Voice $) *(1$-Enemy Jamming Capability),1)

Units: **undefined $* *$

0 - low 1 - high Limit output to a max of 1

(07) Land Domain=

ABS(RANDOM UNIFORM $(0,1,4))$

Units: **undefined $* *[0,1,1]$

(08) Line of Sight= $($ Air Domain+Maritime Domain+(Land Domain*Terrain $)) / 3$

Units: **undefined $* *$

0 - NO LINE OF SIGHT 1 - FULL LOS

(09) Maritime Domain $=\operatorname{ABS}(\operatorname{RANDOM} \operatorname{UNIFORM}(0,1,5))$

Units: **undefined $* *[0,1]$

(10) $\quad$ MaxRange $=$ RANDOM NORMAL $(300,1000,500,100,1)$

Units: **undefined**

(11) National Compatability= IF THEN ELSE( Total Number of Nations $<5$, (Percentage with common procedures +Percentage with same language) $/ 2$, (Percentage with common procedures+Percentage with same language)/4 )

Units: **undefined**

Assume that IO is not degraded if less than 5 nations, if more then assume IO problems will occur

(12) Network Connectivity=IF THEN ELSE(Number of Gateways $>=($ Number of Networks-1), 1 , Number of Gateways

/Number of Networks )

Units: **undefined**

$1=$ GOOD CONNECTIVITY $0=$ BAD CONNECTIVITY

(13) Number of Gateways $=($ RANDOM UNIFORM $(0$, Number of Networks $+1,28))$

Units: **undefined $* *$ 
(14) Number of Networks=ABS((MaxRange/300))*(Air Domain+Land Domain+Maritime Domain)

Units: **undefined**

(15) Number of Platform Types=Total Number of Nations*(Air Domain+Land Domain+Maritime Domain)*RANDOM UNIFORM

$(1,5,4)$

Units: **undefined**

(16) Percentage with common Geo Datum=RANDOM UNIFORM $(0,1,14$ )

Units: **undefined $* *$

(17) Percentage with common procedures=RANDOM UNIFORM $(0.1,0.6,17)$

Units: **undefined $* *$

(18) Percentage with same language $=$ RANDOM UNIFORM $(0.7,1,15)$

Units: **undefined $* *$

(19) Percentage with TDL=RANDOM UNIFORM $(0.5,1,1)$

Units: **undefined $* *$

(20) Platform IO=1-(MIN((Number of Platform Types-1)/20,1))

Units: **undefined $* *$

Assumes IO degrades with more platform types, over $20 \mathrm{IO}=0$

(21) $\quad$ SAVEPER $=$ TIME STEP

Units: Month [0,?]

The frequency with which output is stored.

(22) Secure Voice=RANDOM UNIFORM $(0.5,1,27)$

Units: **undefined $* *$

0 - low 1 - high

(23) TDL Interoperability=Line of Sight*Percentage with TDL

Units: **undefined $* *$

(24) Terrain $=1$ 
Units: **undefined $* *$

$0=$ Mountainous $1=$ Relatively Flat

(25) TIME STEP $=1$

Units: Month [0,?]

The time step for the simulation.

(26) Total Number of Nations=ABS(RANDOM UNIFORM $(1,5,4)$ )

Units: **undefined $* *[1,5,1]$

\section{F.2.4 Validation and Verification}

Initial verification was performed through code inspection followed by running the model and examining the state of each variable. The value of each variable was assessed in the context of its inputs with several errors identified and corrected as a result. The model was updated to allow the generation of outputs for best and worst case scenarios, this activity also proved to be a useful approach in the validation of the model, again identifying several errors which were corrected.

\section{F.2.5 Model Output}

A System Dynamics normally models causal effect over a period of time, however in this case a more unconventional approach was taken, where each time step was used to generate a different 10 "level" as a result of the randomness defined for the model variables. The model was run over 5000 time steps, thereby generating a suitable sized result set which was reformatted into textual format and then input, i.e. learned, by the BBNs.

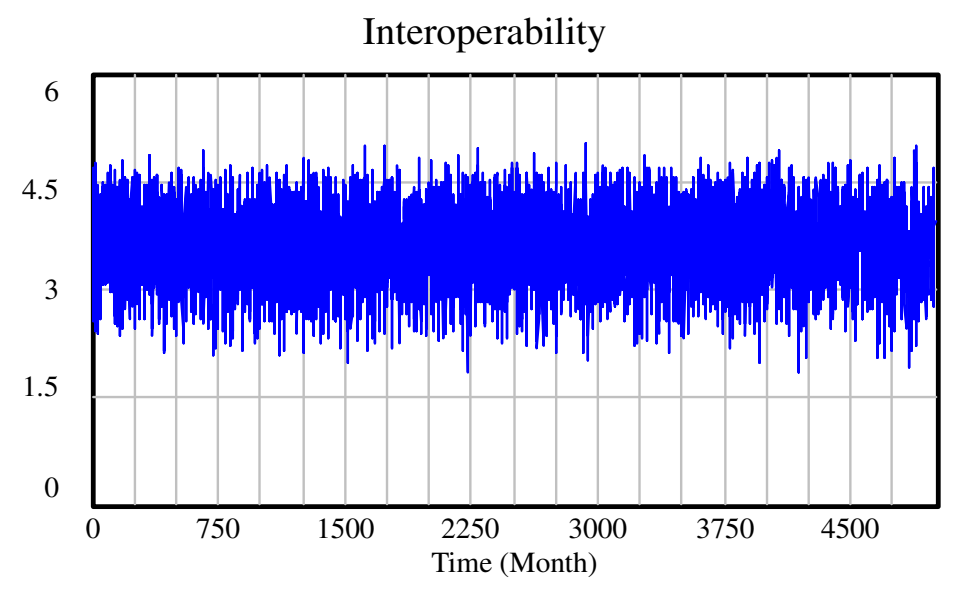

Interoperability : Current

Figure F - 4 : Interoperability Model Output 


\section{F.3 Blue Force Proximity and Civilian Proximity Model}

\section{F.3.1 Requirements}

The key requirement of the Blue Force Proximity and Civilian Proximity model was to simulate the movement of a target, blue forces and civilians, based on intelligence data.

Specifically, the model was required to:

a) Allow the initial position of a target to be defined, randomised within an area of probability.

b) Move the target in accordance with predicted behaviour if available with an element of randomness as applicable.

c) Allow the initial position of blue forces to be defined, randomised within an area of probability.

d) Move the blue forces in accordance with predicted behaviour if available with an element of randomness as applicable.

e) Allow the initial position of civilians to be defined, randomised within an area of probability.

f) Move the civilians in accordance with predicted behaviour if available with an element of randomness as applicable.

\section{F.3.2 Model Assumptions}

The assumptions made when developing the Proximity Model were as follows:

1. Initial position of the blue forces was $(-10,0)$ with an uncertainty of $2 \mathrm{~km}$ and a heading of $45^{\circ}$.

2. Initial position of the red forces was $(-10,5)$ with an uncertainty of $2 \mathrm{~km}$.

3. Initial civilian position was $(-12,12)$.

4. Blue forces move towards red forces.

5. Civilians move towards blue forces.

\section{F.3.3 Design and Implementation}

The model was written in ReLogo, an ABMS language, using the Repast Simphony toolset; a screen shot of the initialised state is shown below in Figure $F-5$. 


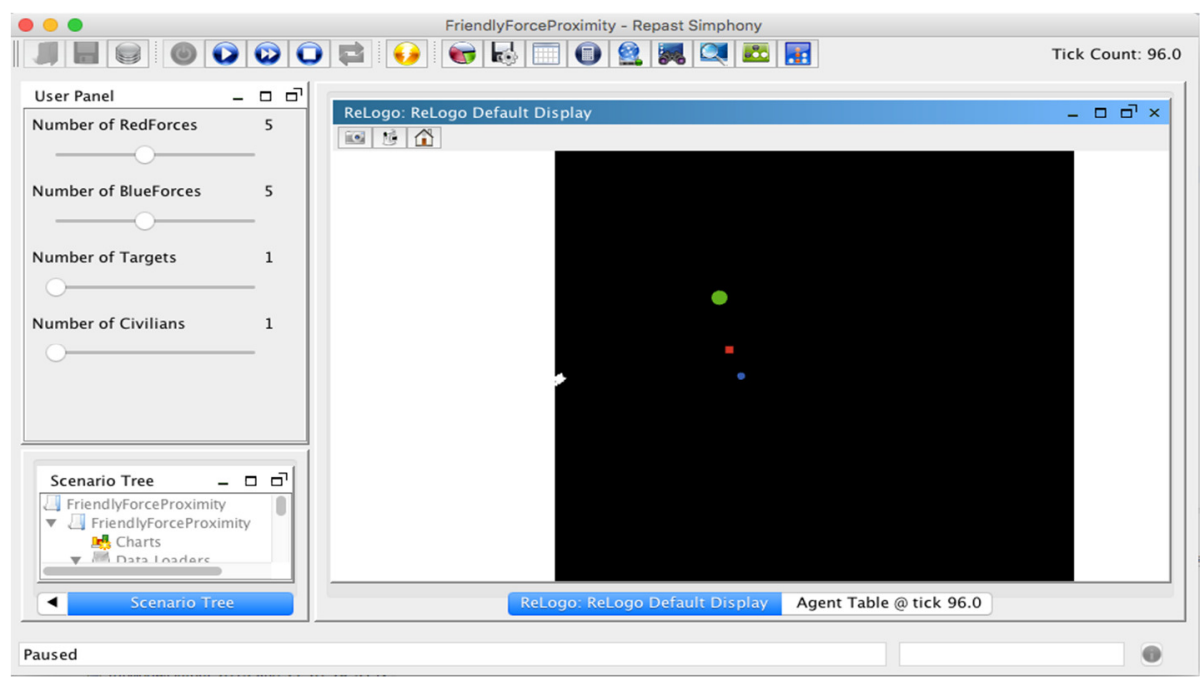

Figure F - 5 : Blue Force and Civilian Proximity Model

The ReLogo code for the control and movement of the model agents is included below. class UserObserver extends ReLogoObserver\{

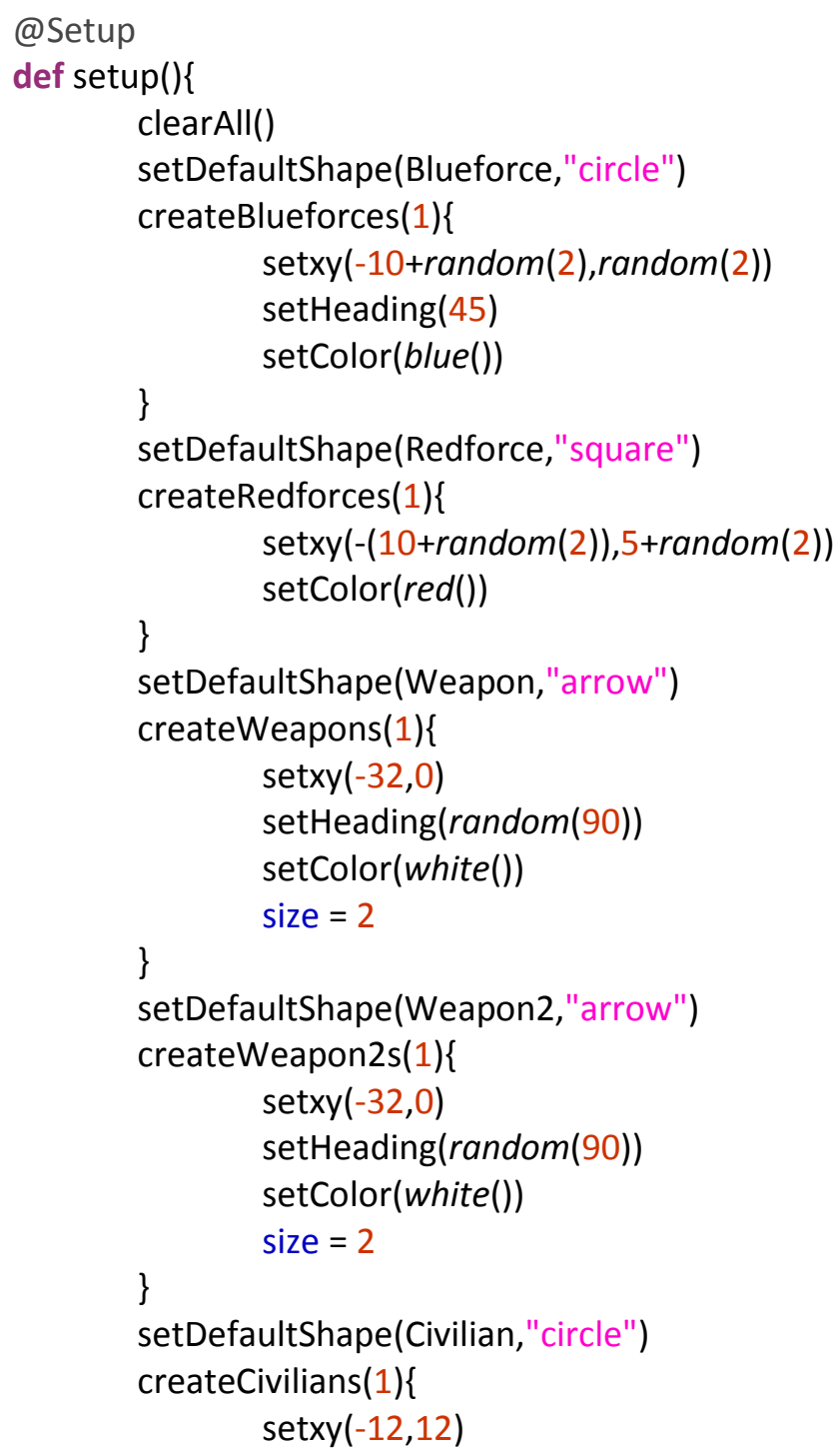




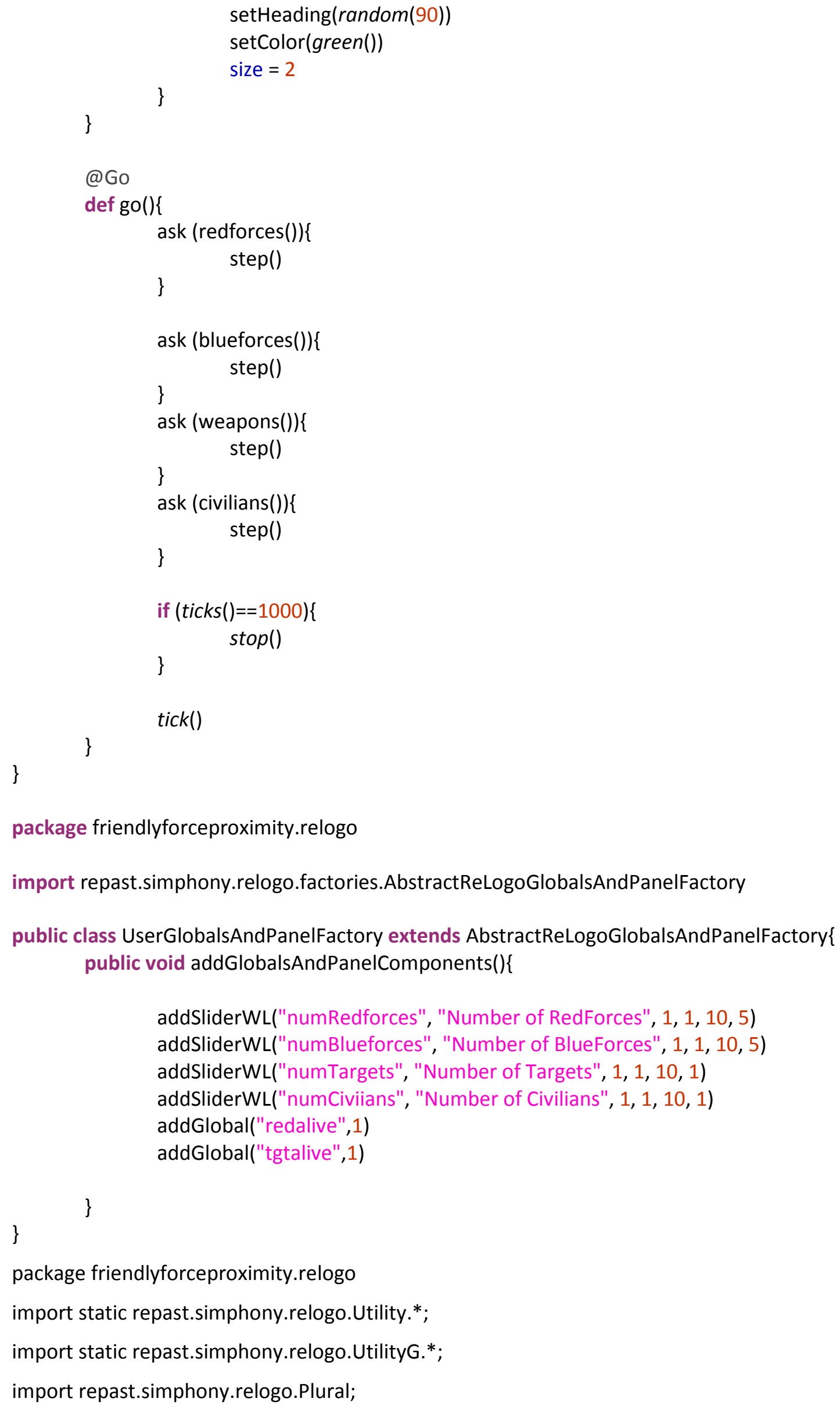




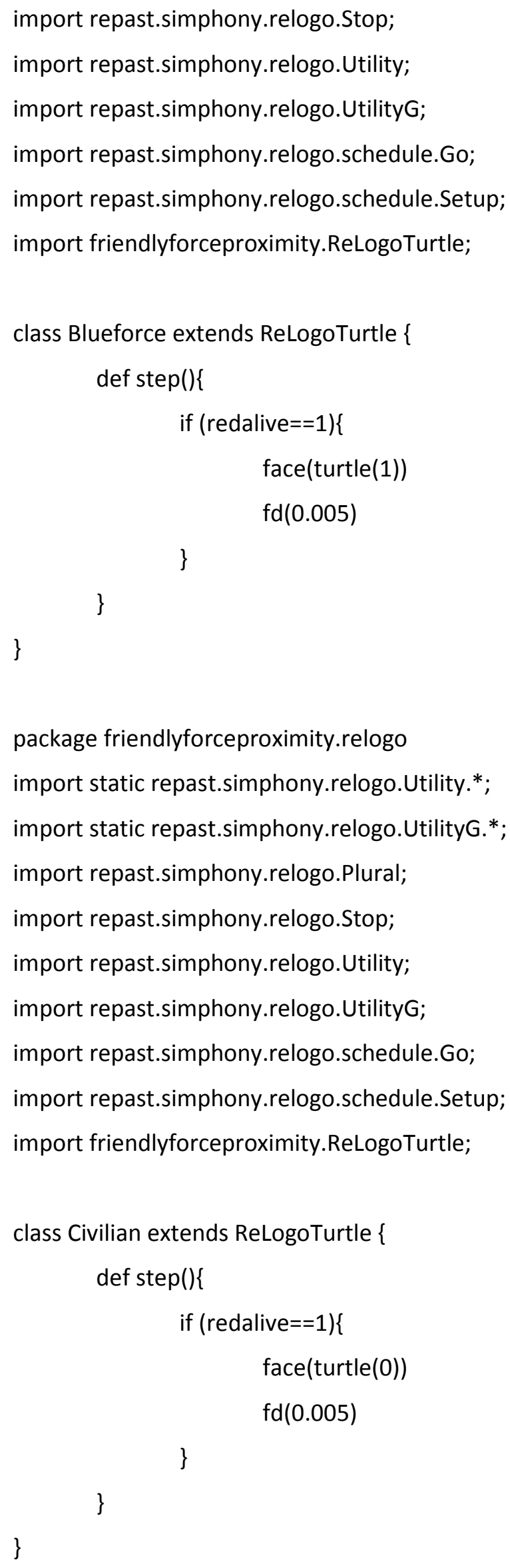




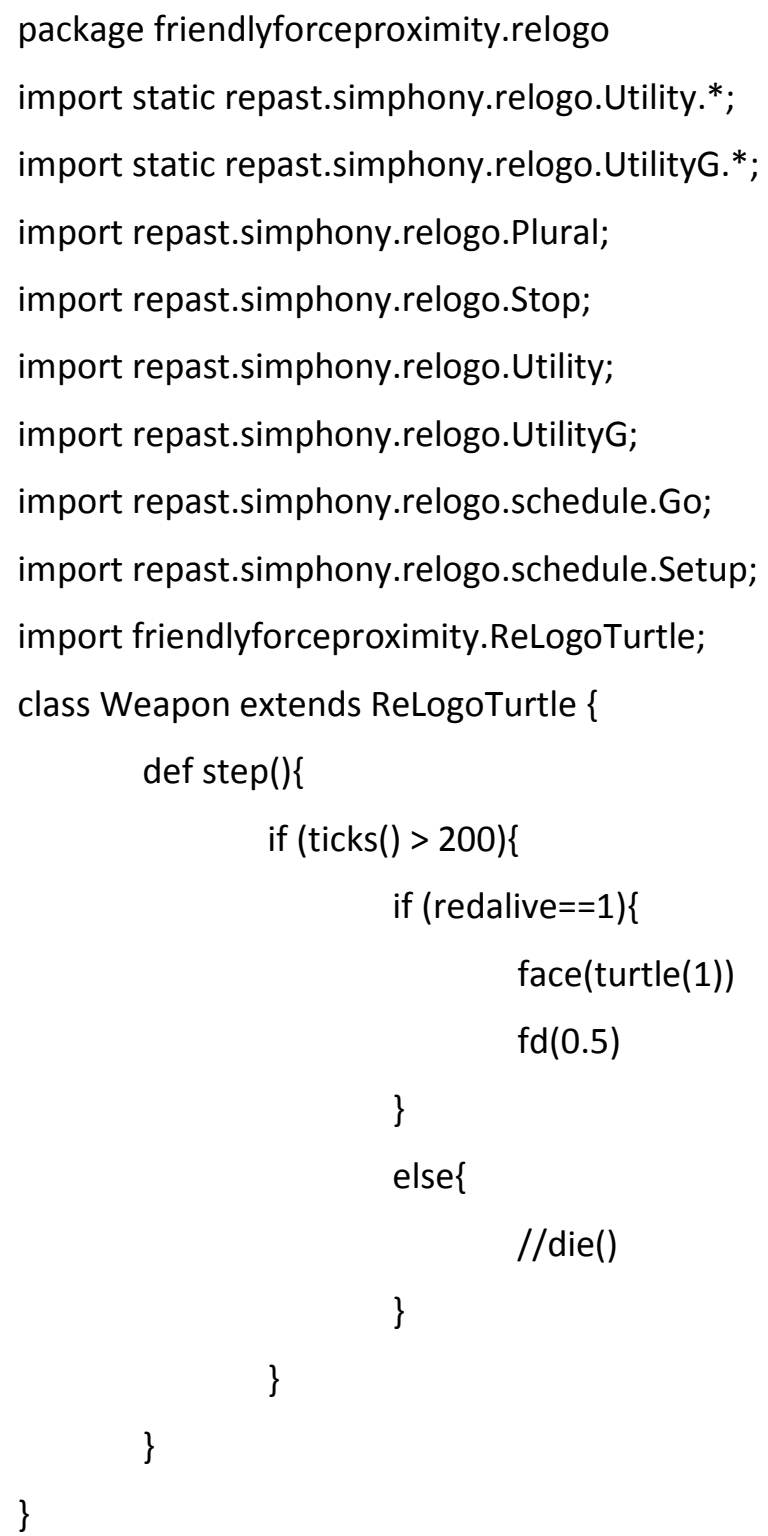

\section{F.3.4 Validation and Verification}

Initial verification was performed through code inspection followed by individual simulation runs where movement was observed visually. The true accuracy of the model is more dependent on the availability of accurate intelligence data. Further validation of the model was performed using "batch" processing, enabling the model to be executed over multiple iterations and checking the output data in an excel data format.

\section{F.3.5 Model Output}

The model was run 1000 times with the output data exported into text files for BBN learning. It should be noted that the number of model iteration was limited to 1000 because higher numbers appeared to inhibit the creation of output data files. 

Annex A: Paper 1 - System of Systems: "Defining the System of Interest” 


\section{System of Systems: "Defining the System of Interest"}

\author{
Mr. Andrew Kinder \\ Lockheed Martin UK IS\&S Ltd, \\ Chester House, Farnborough Aerospace \\ Centre, GU14 6TD, UK. \\ Andrew.M.Kinder@1mco.com
}

Dr. Vishal Barot, Prof. Michael Henshaw, Dr. Carys Siemieniuch

School of Electronic, Electrical and Systems Engineering,

Engineering Systems of Systems (ESoS) Group

Loughborough University, LE11 3TU, Loughborough, UK

V.Barot@lboro.ac.uk, M.J.d.Henshaw@lboro.ac.uk,

C.E. Siemieniuch@lboro.ac.uk

Abstract - This paper proposes a multi-dimensional framework approach to defining the System of Interes (SOI) for System of Systems (SoS). A number of dimension are described in detail, enabling development of a more comprehensive model that illustrates the relationships between these dimensions in SoS. The resultant framework model is evaluated using 2 practical case studies in the domains of Defence and ICT to indicate the applicability and suitability of the proposed approach. The potential benefits of the framework are described within the context of the case studies, in conjunction with the limitations of this initial framework. A brief description of intended further research is provided giving some insight into future development of the framework.

Keywords: System of Systems, System of Interest, dimension, emergent behaviour, systems boundary, SoS lifecycle

\section{Introduction}

A distinguishing feature between Systems Engineering and System of Systems Engineering is the difficulty of defining the System of Interest (SOI) [1]. But failure to properly identify the SOI is one of the causes of unpredicted emergent behaviour which usually arises from the combined actions and interactions of the constituents of a SoS. A framework approach for comprehensive and sufficient definition of the systems boundary for SoS of all four types [2] is proposed in this paper. For a SoS the boundary is rarely static; this framework is intended to capture the dynamic nature of this by clearly defining the relationship between the SoS lifecycle and other dimensions. The definition of the SoS boundary will contribute to the ability to predict some aspects of emergent behaviour.

This article defines SOI through a number of dimensions; component systems (including specific and general system types), interactions (including types), lifecycle, variability, classification, functions, system owners and operations, concept of operations / use / employment, and nature of relationships. Using these dimensions a conceptual model has been constructed illustrating the relationships between them. The generic approach to SOI definition, and the associated model, enables a rich, dynamic definition of a SoS providing a common framework in which related SoS Engineering activities may be undertaken.

\section{System of Interest (SOI)}

The International Council on Systems Engineering (INCOSE) defines the SOI for a system [4] as; "The system whose life cycle is under consideration".

Wasson elaborates upon the definition [5]; "The system consisting of a MISSION SYSTEM and its SUPPORT SYSTEM(s) assigned to perform a specific organizational mission and accomplish performance-based objective(s) within a specified time frame."

ISO/IEC 15288 [7] takes a hierarchical view; "the top system in the system structure is called a system-of-interest and consists of lower level systems. Except the lowest level is identified as being made up of system elements."

Hitchens [8] provides an alternative definition; "It [the SOI] contains intra-connected sub-systems, which are systems in their own right, existing within their own

Whilst these system level definitions can be interpreted with reference to the SoS SOI (particularly with reference to multiple component systems and system lifecycle), they do not fully address the relationship between emergent behaviour and the SOI. An extension to the INCOSE SOI definition is therefore proposed;

SoS SOI: The system of systems whose life cycle is under consideration described by all dimensions that contribute to the resultant emergent behaviour.

\section{The SoS SOI Dimensions}

The challenge to be addressed is to identify the relevant parameters through which a $\mathrm{SoS}$ is adequately defined. Whilst trivial to state, the complexity of many SoS make this challenge a remarkably difficult undertaking and it is not clear that there is a generalised approach that may be applied to all SoS. To attempt the derivation of a environment." 
generalised approach, this research has simultaneously used a top down approach, in which the many definitions of SoS [3] have been analysed, and a bottom up approach based on analysis of specific SoS case studies. The proposed SoS SOI definition makes reference to dimensions; these are now described in more detail, allowing the initial high level statement to be developed into a more comprehensive model.

\subsection{Component Systems (including specific and general system types)}

By definition, specific component systems (also known as constituent systems) will be identified for the directed SoS ("those in which the integrated system-of-systems is built and managed to fulfill specific purposes") [6] and to some extent the acknowledged SoS ("the constituent systems retain their independent ownership, objectives, funding, and development and sustainment approaches") [6] earlier in the SoS lifecycle than those classified as collaborative (the component systems interact more or less voluntarily to fulfill agreed upon central purposes) [6] and virtual (lack a central management authority and a centrally agreed upon purpose for the system-of-systems) [6], however, it is possible that generic system types may be identified for all classifications. Indeed, for a collection of systems collaborating on an unplanned basis, it may not be possible to identify all specific systems at any point in time but in order for the systems to interact there must be a certain commonality between them allowing an abstracted general system type to be inferred. The level of abstraction is dependent on the scope of the commonality and the system purpose. The "Component Systems" dimension captures this distinction by allowing the definition of both specific systems and generic system types.

The generic system types are dependent upon SoS under consideration; e.g. for an SoS concerned with the processing and management of information [9] the generic component system types may include

- Management information system

- Executive information system.

- Executive support system

- Decision support system.

- Group decision support system

- Electronic meeting system.

- Organizational decision support system

- Expert system

- Office information system.

- Intelligent organizational information system

\subsection{Classification}

The SoS classifications [6]; directed, acknowledged, collaborative and virtual, are used to populate this particular dimension. However, a particular SoS may exhibit the behaviours of more than one classification depending on the make-up of component systems and the current point in the lifecycle. As shown in the NHS case study (section 5.2), the classification may evolve over time.

\subsection{Interactions (including types)}

A SoS exists only because of interactions between constituent systems. With no interaction the SoS merely becomes a set of independent systems exhibiting no overall emergent behaviour, so the interactions must provide some definition of the SoS SOI

The interactions may be initially defined at a generic level and subsequently at a more specific level which will identify both the interaction medium and the nature of the interaction. This allows a typical top down approach to be taken, progressing from the abstract to the specific where interaction contents, protocol and media are identified. Considering a financial SoS, a requirement to exchange customer bank account details may exist between component systems, this provides the general interaction type, i.e. "Customer Account Information". As the SoS is developed design decisions are taken which elaborate upon this general type until a point is reached whereby the precise data contents and format, communication protocol and medium are identified.

The interaction type will therefore be influenced by the requirements derived from the SoS purpose. Considering another example; a SoS whose purpose is to perform a Search and Rescue mission will require a combination of surveillance and co-ordination information exchanged between component systems. In a military environment this may be accomplished through the utilisation of Tactical Data Links (TDL) and voice communications. The SOI description may then be further refined to identify specific TDLs (e.g. Link 11 [10] or Link16 [11]) and voice communication frequencies enabling interoperability between component systems. The ability of the SoS to fulfil its purpose is dependent upon the effectiveness and availability of these interactions. Using the search and rescue case it is clear that the performance will be compromised if communications are degraded because component systems are not interoperable. Simplistically, this may occur if correct voice frequencies are not used or platforms have incompatible TDL fits with no "gateway" to link them, compromising the exchange of situational awareness data.

The availability of interactions is important and related to performance and agility, i.e. a SoS may be formed or reconfigured relatively quickly if component systems share a common means to interact. For two component systems to interact there must be at least one homogeneous interaction medium, if there is none then an intermediate system must act as a "translator" or gateway. Interactions can be also 
constrained by geographical dispersion, therefore this should be considered.

\subsection{Nature of Relationships}

The Nature of Relationships dimension is distinct from interactions (section 3.3) which are more concerned with the inter-connections between systems. This dimension is used to define the category of relationship between component systems, such as 'peer-to-peer', hierarchical control or distributed control.

\subsection{Lifecycle}

This dimension considers the lifecycle of the SoS as a whole as well as the respective timelines of the component systems, providing an insight into dynamism within the SOI. For some SoS, it may not be possible to define a cycle, as such, but rather an evolution in which the SoS passes through identifiable phases.

ISO 15288 [7] considers the lifecycle of single systems, it does allude to the lifecycle of "enabling systems" (e.g. a concept system, development system, production system, utilization system, support system or retirement system [7]) but these are distinct from SoS component systems. It lists typical lifecycle stages applicable to a single system as;

- Concept stage - Development stage - Production stage - Utilization stage - Support stage Retirement stage

A directed SoS which is "built and managed"[6], and therefore developed in a more structured manner, aligns more readily to these suggested stages with the traditional System Engineering lifecycle being applicable to each component system. As the SoS classification moves away from the more managed type and to the ad-hoc type these stages become far less applicable. Alternative stages may include

- Planning - Convergence - Collaboration Execution - Evolution - Dispersion

However, where the SoS has no agreed central purpose and behaviour emerges through a process of self-discovery among the component systems (a virtual SoS [6]) the lifecycle becomes more an observation of the evolution of the SoS rather than an imposed structure permitting a degree of management.

It is also established that there is a link between the point in the lifecycle and the ability to populate a dimension. Table 1 shows the population of the dimensions for two case studies. For example, until the execution phase of a SoS the actual component systems may not be known but the types should be. Consider a military SoS whose purpose is to recover a downed pilot; it will require a surveillance component system. There are several possible systems that could fulfil this role, depending on suitability and availability this will be instantiated with a specific type when the SoS is executing its task, a potential type being an E3-D Sentry aircraft. It is argued that the lifecycle is an overarching dimension, affecting all other dimensions. As the SoS passes through the lifecycle phases the dimensions evolve both in relation to each other and to external influences.

\subsection{Variability}

The frequency of change of a SoS is considered to be a critical dimension. This is linked to stability and also agility. Variability is related to time in the sense that it may reveal some sort of characteristic frequency representing change in the SoS. Stability is linked to the behaviours of the SOI such as "Stable over time", "Unstable, rapidly grows" and "Boundary changes". Factors such as an evolving purpose or a response to environmental factors will influence the variability.

\subsection{Functions}

A SoS performs a combination of functions in order to accomplish its purpose. Functions may be performed by individual component systems, by subsets of component systems or by the entire SoS. The Function dimension defines both specific functions and more generic function types. The aggregate effect of the functions gives the SoS emergent behaviour, it is this behaviour that should fulfil the SoS purpose. Analysis of these functions may enable unexpected behaviour to be anticipated.

\subsection{Systems Owners and Operations}

This dimension is closely related to organisations, management and enterprise (multi-organisational) relationships. It is dependent on the classification type, for example within a directed SoS the owners and operations will be well defined but for a more ad-hoc SoS this dimension will not be so well defined. However, the definition of System Owners and Operations includes generic types enabling the dimension to be defined to a certain extent for less rigidly defined systems.

\subsection{Concept of Operation / Use / Employment}

Given that the individual systems can perform operations independently the use/mission of the SoS is critical for defining the SOI for the SoS as opposed to the individual constituent systems. This dimension is potentially the starting point for populating the SOI dimensions. 


\section{Relationships between Dimensions}

The SOI dimensions should not be considered in isolation, Figure 1 illustrates the relationships between the dimensions. It is the combination of the identification of the individual dimensions and the relationships that gives this perspective its inherent power. There are numerous, well documented, accounts of SoS failures ${ }^{1}$, with a common theme of systems and/or dimensions being developed independently using traditional SE techniques. The SOI provides an holistic view of the SoS rather than a set of unconnected viewpoints.

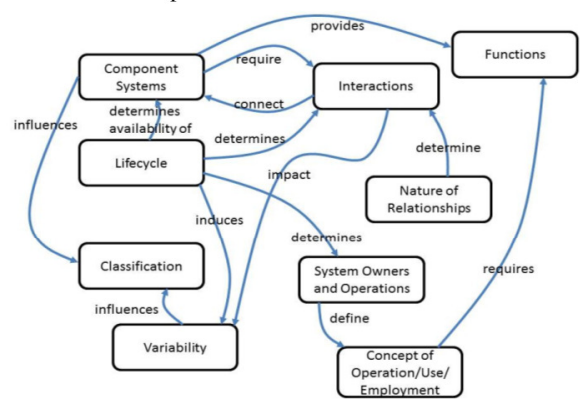

Figure 1: Dimension Relationships

\section{Case Study Evaluation}

Two case studies in the domains of defence and ICT have been initially selected to illustrate the applicability and significance of the proposed framework. These case studies are "Counter Air Mission" and "National Programme for IT in the NHS (NPfIT)". Each case study has been analyzed in terms of the SOI dimensions, Table 1 presents the results.

\subsection{Counter Air Mission}

This case study uses a typical example of a military Counter Air Mission [12], as shown in Figure 2, the purpose of which is to provide protection from airborne threats, either offensively or defensively. In this case the SoS will be required to perform offensive pre-emptive strikes against known hostile airborne assets as well reacting defensively to unpredictable airborne attack. The component systems exchange tactical information through a combination of voice and tactical data links (TDL), both Link 11 [10] and Link 16 [11], utilizing radio and satellite communications with gateways to extend range and provide interoperability.

E.g. the National Programme for IT in the NHS which is examined as a case study in this paper.

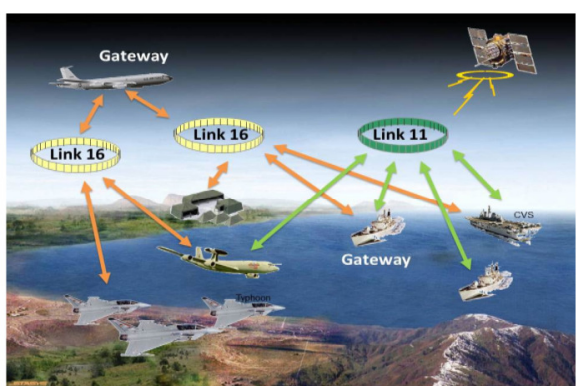

Figure 2 - Typical Counter Air Mission

5.2 National Programme for IT in the NHS (NPfIT)

The overall purpose of the NPfIT system [13], as shown in Figure 3, was to use Information Technology (IT) to help deliver better patient care by moving towards a single, centrally-mandated electronic care record for patients and to connect 30,000 GPs to 300 hospitals, providing secure and audited access to these records by authorized health professionals. The Core of the programme was going to be NHS Care Records Service (i.e. "the Spine"), which would have made relevant parts of a patient's clinical record available to whoever needs it to care for the patient. However, progress has been poor with completion date for some component systems now forecast to be 2014-15, at best. Other component systems are in use but only operating independently. Overall, this is an excellent example of SoS failure.

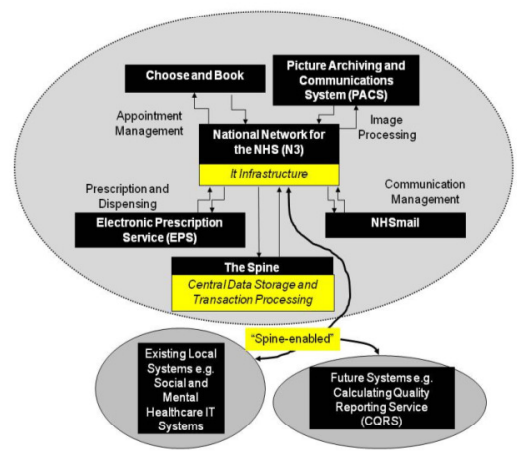

Figure 3 - National Programme for IT in the NHS 


\begin{tabular}{|c|c|c|}
\hline \multirow{2}{*}{ Dimensions } & \multicolumn{2}{|l|}{ Case Studies } \\
\hline & Counter Air Mission & National Programme for IT in the NHS (NPfIT) \\
\hline $\begin{array}{l}\text { Component } \\
\text { Systems }\end{array}$ & $\begin{array}{l}\text { Counter Air Aircraft (Typhoons) } \\
\text { Command and Control Unit (E3-D, TACC) } \\
\text { JRE Gateway (KC-135) } \\
\text { TDL gateway (Type 45) } \\
\text { Air Defence Artillery (Type 45) } \\
\text { ISR (E3-D) } \\
\text { CVS Aircraft Carrier } \\
\text { Satellite (Skynet 5) }\end{array}$ & $\begin{array}{l}\text { Choose and Book } \\
\text { Electronic Prescription Service (EPS) } \\
\text { National Network for the NHS (N3) } \\
\text { NHS Mail } \\
\text { Picture Archiving and Communication System } \\
\text { (PACS) } \\
\text { The Spine }\end{array}$ \\
\hline Interactions & $\begin{array}{l}\text { Digital Communications (Link 11/16, Satellite) } \\
\text { Voice Communications } \\
\text { Track Data } \\
\text { Command and Control } \\
\text { Imagery }\end{array}$ & $\begin{array}{l}\text { N3 Network } \\
\text { eMAIL } \\
\text { Appointment Data } \\
\text { Image Processing } \\
\text { Patient records } \\
\text { Prescriptions (electronic) } \\
\end{array}$ \\
\hline Lifecycle & $\begin{array}{l}\text { ment, Planning, Assembly, Execution } \\
\text { ion }\end{array}$ & $\begin{array}{l}\text { Concept, Design and Development, Operation, } \\
\text { Reconfigure/Upgrade, Disposal }\end{array}$ \\
\hline Variability & $\begin{array}{l}\text { Assets may change during mission. } \\
\text { The purpose may evolve as the mission progresses. } \\
\text { This SoS may exhibit a high level of variability. }\end{array}$ & $\begin{array}{l}\text { Intuitively one assumes variability is low because } \\
\text { of the directed nature of this SoS. However, due to } \\
\text { the poor definition of requirements component } \\
\text { systems were frequently changed which resulted in } \\
\text { a higher level of variability than would perhaps be } \\
\text { expected. }\end{array}$ \\
\hline Classification & $\begin{array}{l}\text { Ledged. } \\
\text { Link } 11 / 16 \text { networks }\end{array}$ & $\begin{array}{l}\text { Originally Directed but later moved towards } \\
\text { Acknowledged. }\end{array}$ \\
\hline Functions & $\begin{array}{l}\text { Surveillance, Reconnaissance, Targeting, Detect, } \\
\text { Identify, Intercept, Destroy, Aircraft Control, } \\
\text { Battle Management. }\end{array}$ & $\begin{array}{lccc}\text { Choose and } & \text { Book } & \text { appointment, } & \text { Change } \\
\text { appointment, Produce } & \text { prescription, } & \text { Picture } \\
\text { Archiving } & & & \\
\end{array}$ \\
\hline $\begin{array}{l}\text { Systems } \\
\text { Owners and } \\
\text { Operations }\end{array}$ & $\begin{array}{l}\text { Nations (Governments), Services (Air force, Navy, } \\
\text { Army), Overarching control, e.g. NATO. }\end{array}$ & $\begin{array}{l}\text { Project Team, NHS Connecting for Health } \\
\text { Local Authority / Strategic Health Authority } \\
\text { Project Board, Board of Directors / PCT } \\
\text { Executive Group }\end{array}$ \\
\hline $\begin{array}{l}\text { Concept of } \\
\text { Operation }\end{array}$ & $\begin{array}{l}\text { The SoS provides protection from air and missile } \\
\text { threats. }\end{array}$ & $\begin{array}{l}\text { Provide a single, centrally-mandated electronic } \\
\text { care record for patients and to connect } 30,000 \text { GPs } \\
\text { to } 300 \text { hospitals. }\end{array}$ \\
\hline $\begin{array}{l}\text { Nature of } \\
\text { Relationships }\end{array}$ & Hierarchical (military C2) & $\begin{array}{l}\text { Plural system of procurement. } \\
\text { Client-Server. }\end{array}$ \\
\hline
\end{tabular}

Table 1: Mapping of Case Study Data to SOI Dimensions

\section{Benefits of the Proposed SOI Framework}

Having shown that the dimensional framework "fits" selected SoS case studies, we examine some of the benefits that application of this framework could bring in each case.

Regarding the first case study, a common problem that often besets military operations is a lack of interoperability between component systems. The proposed framework shows the relationship between component systems and interactions, emphasising the need for interoperability. Whilst this in itself can already be determined with current frameworks (e.g. MODAF (Ministry of Defence Architecture Framework)), the proposed framework allows further elaboration of each system, in accordance with the life cycle and the SoS purpose and shows the relationships to other aspects of the SoS.

The second case study regarding the National IT Programme for the NHS has not, as yet, entirely fulfilled its purpose for a number of reasons, some of which are;

a. Those closest to the healthcare delivery were not able to provide adequate input into the SoS requirements definition, resulting in specific local requirements not being met. The SoS SOI links Lifecycle, System Owners and Operations, Concept of Operation / Use / Employment and Functions. 
This relationship shows that system owners indirectly influence the functions provided by the SoS. By definition all health trusts (System Owners) are part of the SOI and, therefore, in accordance with the proposed model, would be provided with input into the definition of Lifecycle, System Owners and Operations, Concept of Operation / Use / Employment.

b. Incompatibility between systems. For example, a Patient Administration System in a particular NHS trust was delayed when found to be incompatible with the Choose and Book System. The SoS SOI links Component Systems, Interactions and Lifecycle. The SOI allows component system types and interaction types to be defined early in the lifecycle and, if correctly modelled, should enable any incompatibilities to be detected and prevented. The relationship of Lifecycle in this case is intended to enable component systems at different phases of development to form part of the SOI

c. A common issue was that the NPfIT system was implemented with insufficient flexibility in terms of range of locations from where data could be collected. As described in response to the issue ' $a$ ' above, the SOI should ensure that all System Owners have sufficient input to the system definition, allowing a greater range of locations to be specified. In addition, the interactions may identify a geographical element.

\section{Limitations}

The proposed framework has been exercised on a small number of case studies, two of which are given by example herein. Generalisability of the framework approach has clearly not been demonstrated in the work conducted so far. However, the purpose of this activity is to provide a practical and pragmatic approach for SoS Engineers that will enable such activities as modelling and simulation to be conducted with greater confidence. To this extent, the next stages of the research will be to formalise the approach and to exercise it on a wider set of case studies.

\section{Conclusions}

This paper shows the benefit of a multi-dimensional SoS SOI description for enabling the impact of interdependencies between the dimensions of a SoS to be considered through all phases of the SoS lifecycle. The use of factual case studies is used to assess the applicability of the dimensions beyond a theoretical environment into practice. Further research is planned to formalize the approach as a tool for SoS modelers as a means of enabling greater confidence in the application of modeling and simulation techniques for SoS

\section{Acknowledgements}

The authors would like to acknowledge EU T-AREA-SoS FP7 support action (Grant agreement no: 287593), the Loughborough University Engineering SoS group and other collaborative experts for their kind support.

\section{References}

[1] Bjelkemyr, M., Semere, D.T., Lindberg, B. (2009) Definitions, classification, and methodological issues of systems of systems, ch. 7 in Systems of Systems Engineering Principles and Applications, Jamshidi, M.

[2] Dahmann, J., Baldwin, K.J. and Rebovich, G. (2009), Systems of Systems and Net-Centric Enterprise Systems, 7 th ann. Conf. on Sys. Eng. Res., Loughborough.

[3] Jamshidi, M. (2005), Theme of the IEEE SMC 2005 , Waikoloa Hawaii, UUSA, http://iecesmc2005.unm.edu/

[4] INCOSE Systems Engineering Handbook v. 3.2.1, January 2011.

[5] System Analysis, Design, and Development Concepts, Principles, and Practices, Charles S. Wasson, John Wiley \& Sons, Jan 2006

[6] Dahmann, J., and K. Baldwin. 2008. "Understanding the Current State of US Defense Systems of Systems and the Implications for Systems Engineering." Paper presented at IEEE Systems Conference, 7-10 April, Montreal, Canada

[7] ISO/IEC 15288:2008 System life cycle processes.

[8] Basic Models for System Thinking, Hitchins, D, http://www.hitchins.net/SysMods.html

[9] G Mentzas, "A Functional Taxonomy of Computerbased Information Systems", International Journal of Information Management (1994), 14 (397-410).

[10] NATO Standardization Agreement TDL 5511 Ed 6.

[11] NATO Standardization Agreement TDL 5516 Ed 6.

[12] Counterair Operations, Air Force Doctrine Document 21.1, October 2008, http://www.dtic.mil/doctrine/jel/ service_pubs/ afdd2_1_1.pdf

[13] NHS The National Programme for IT in the NHS: an update on the delivery of detailed care records systems, Report by the Comptroller and Auditor General HC 888, Session 2010-2012, 18 may 2011 
\begin{tabular}{l} 
Annex B: Paper 2 - System of \\
Systems Modelling and Simulation - \\
an Outlook and Open Issues \\
\hline
\end{tabular} 


\section{System of systems modelling and simulation - an outlook and open issues}

\section{Andrew Kinder*, Michael Henshaw and Carys Siemieniuch}

Engineering Systems of Systems (ESoS) Group,

School of Electronic, Electrical and Systems Engineering,

Loughborough University,

LE11 3TU, Loughborough, UK

E-mail: A.M.K.Kinder2@lboro.ac.uk

E-mail: M.J.d.Henshaw@lboro.ac.uk

E-mail: C.E.Siemieniuch@lboro.ac.uk

*Corresponding author

Abstract: System of systems (SoS) engineering is an emerging sub-discipline, within which modelling and simulation is a key area. The failure of many SoS endeavours can be attributed to the inappropriate application of systems engineering processes, including modelling approaches. Selection of suitable modelling approaches is critical to ensuring representative models are created, ensuring reliable SoS analysis and prediction of emergent behaviour is possible. Essential to SoS modelling is the dynamic element, enabling the generation of emergent behaviour, appropriate approaches including; discete perion, event system specification, Petri apts, agent-based modelling and system dynamics. Bayesian belief networks, Markov models and game theory provide a static probabilistic approach, suitable for strategic, decision and risk models.
Enterprise architecture frameworks and modelling languages are typically used Enterprise architecture frameworks and modelling languages are typically used
for static modelling of SoS. A combination of static and dynamic approaches for static modelling of SoS. A combination of static and dynamic approaches
should be utilised, providing a range of perspectives, giving an insight into the should be utilised, providing a
complexity inherent in all SoS

Keywords: system; system of systems; SoS; system of systems engineering; modelling; simulation; discrete event; agent-based model; neural network; Bayesian; Markov; enterprise architecture; game theory; surrogate model; decision tree.

Reference to this paper should be made as follows: Kinder, A., Henshaw, M. and Siemieniuch, C. (2014) 'System of systems modelling and simulation an outlook and open issues', Int. J. System of Systems Engineering, Vol. 5 , No. 2, pp.150-192.

Biographical notes: Andrew Kinder is a PhD candidate within the Engineering Systems of Systems (ESoS) group at Loughborough University, where he is
researching a model-based approach to system of systems risk management. researching a model-based approach to system of systems risk management.
The research is being performed concurrently with his role as a Tactical Data The research is being performed concurrently with his role as a Tactical Data
Link Consultant for JCSys Ltd., who provide specialist technical support to the Link Consultant for JCSys Ltd., who provide specialist technical support to the
UK MoD and other nations. He has over 25 years of experience working in the defence industry in systems, software, research and consultancy engineering roles. 
Michael Henshaw is a Professor of Systems Engineering; he is the Head of the Systems Division at Loughborough University, UK, and leads the Engineering Systems of Systems (EsoS) Research Group. His research focuses on integration and management of complex socio-technical systems, with a particular emphasis on the challenges of through-life mair in System systems and capabilities. He was appointed to a Chair in Systems Engineering at Loughborough in 2006 to direct the large multi-university, multi-disciplinary programme, NECTISE, that ran from 2005-2009. This was a $£ 4 \mathrm{M}$ investment by EPSRC and BAE Systems to research network enabled capability (NEC).

Carys Siemieniuch is a Professor of Enterprise Systems Engineering in the School of Electronic, Electrical and Systems Engineering. She has worked as a Systems Ergonomist for 27 years in the manufacturing, automotive and aerospace domains. As a member of the Institute of Ergonomics and Human Factors, she has expertise across the full range of systems and systems of systems related human factors topics. As a PI/CoI on a range of EPSRC (8), $\mathrm{EU}$ (6) and $\mathrm{MoD} /$ Industry (7) funded projects, she has developed new understandings about enterprise systems modelling. enterprise systems architectures; human and organisationat performance measuenent; and emergent system behaviours.

\section{Introduction}

System of systems (SoS) engineering (SoSE) is an emerging sub-discipline, within which modelling and simulation (M\&S) is a key area. The failure of many SoS endeavours can be attributed to the inappropriate application of systems engineering processes, including modelling approaches, within the SoS domain because of the mistaken belief that a SoS can always be regarded as a single large, or complex, system.

Examples of high profile and high cost SoS, which are generally regarded as unsuccessful for these reasons, include the U.S. Future Combat Systems (FCS) programme, the U.K. National Programme for IT (NPfIT) in the National Health Service (NHS), the U.S. Coastguard Integrated Deepwater System Program and U.K. National Offender Management System (NOMIS).

A RAND lessons learnt review (Pernin et al., 2012) examining the FCS programme found that M\&S demonstrations focussed on the system rather than the SoS. Engineers and analysts used different M\&S tools along with processes with different timescales that inhibited the use of one to support the other, $M \& S$ support for decision makers was often untimely and tended to lag decisions.

Regarding the NPfIT project the UK House of Commons Committee of Public Accounts (2011) noted "a multiplicity of failure factors" including a failure to use and populate the correct project models; improperly populated project models, along with suitably skilled staff. The NHS lacked "a patient-oriented, informatics-driven health and social care information architecture". with the proposed information architecture design described as poor. Further issues were raised in an earlier review (QinetiQ, 2005), which stated that "we found no documented process setting out a systematic approach for selecting the most beneficial course of action where alternatives existed" and "there was no evidence that an architectural design process had been defined, documented or deployed". 
In a report to Congress the following problems relating to $\mathrm{M} \& \mathrm{~S}$ with the Deepwater programme were identified (O'Rourke, 2007); the models used to measure operational performance lacked the fidelity to determine whether improvements were attributable to Coast Guard or contractor actions, the Coast Guard incorrectly assumed "full operational capability of assets and communications and did not account for actual asset operating data" when initialising operational effectiveness models.

Within the NOMIS programme there was an underestimation of the project complexity and a lack of stakeholder input to requirements (Comptroller and Auditor General, 2009). Also, the modelling appears to have been limited to static enterprise architecture approaches, which is generally typical of large scale IT projects.

The theme running through these examples is inappropriate choice of models. It is now widely accepted that $\mathrm{SoS}$ require differing engineering approaches to those used for single systems (Jamshidi, 2008; Dahmann and Baldwin, 2008), and model choices may be one such difference. Guidance is required on how to make such choices among the many and varied modelling approaches.

M\&S within SoSE will provide the potential to predict and analyse emergent behaviour. The predicted behaviour may be as expected, however, equally it may not. Revealing this unexpected behaviour, will allow designers or operators to determine how to exploit or mitigate this ahead of SoS construction or operation.

Depending upon the context and the question the modelling approach is required to answer, different M\&S techniques and approaches are required. Selection of appropriate techniques is not a trivial exercise, because it requires comparative knowledge of a wide range of modelling approaches and their relationship to the different aspects of SoS. Consequently there is a need for a reference guide and more formalised selection approach of appropriate $\mathrm{M} \& \mathrm{~S}$ techniques.

There has been much research into individual SoS modelling techniques, e.g., the comprehensive modelling and analysis for advanced systems of systems (COMPASS) project (Coleman et al., 2012), discrete event systems specification (DEVS) (Zeigler, 2003).

\subsection{Aim of the paper}

The aim of this paper is to analyse the applicability of a wide range of M\&S methods for SoS in order to provide guidance to SoSE practitioners on choice of method according to context and decision needs.

Whilst there is valuable research that has directly compared techniques, such as discrete event simulation (DES) with system dynamics (Tako and Robinson, 2009) or DES with agent-based simulation (Siebers et al., 2010), the survey described in this paper is intended to provide a broader comparison of a wide range of $M \& S$ methods, assessing their suitability and applicability in the SoS domain, through the use of a case study; the result of which is intended to aid the selection of suitable approaches.

The survey of modelling techniques is performed within a framework, enabling particular modelling techniques to be assessed in relation to particular SoS aspects and SoS types. The framework is based upon a SoS system of interest (SoI) model (Kinder et al., 2012). The SoS SoI identifies a series of dimensions against which techniques can be assessed, providing a method for identifying appropriate techniques in relation to a particular problem or question. The approach taken was to initially consider how each technique could have been applied to the case study: the techniques were then 
reconsidered from a more general viewpoint by assessing their applicability to each SoS SoI dimension.

The M\&S methods surveyed in this paper were selected through a review of current approaches and extant research in the systems and SoS domains. Methods were selected to ensure that a variety of different types were compared, including static, dynamic, bottom up, top down, deterministic, stochastic and probabilistic. The survey revealed a wide of range of methods from multiple disciplines applicable to SoS modelling. For example; artificial neural networks (ANNs), fall within the artificial intelligence domain, but when ANNs are combined with agent-based modelling, they provide an extremely powerful SoS modelling capability. The methods listed below are assessed.

1 graph-based models to create structure:

enterprise architecture framework (EAF)

modelling languages

Petri nets

2 inference testing for consistency, patterns, and completeness of models above

ANNs

network models (graph theory)

3 probabilistic simulation based on directed acyclic graphs (DAGs)

Bayesian belief networks (BBNs)

Markov models

decision trees

4 system simulation

DES

DEVS

agent-based modelling and simulation (ABMS)

system dynamics

surrogate models

game theory.

The paper itself is structured as follows: the introductory section is followed by a description of firstly (Section 2) the methodology used for M\&S assessment and secondly

(Section 3 ) the case study used as a context for comparison of the various M\&S methods; Section 4 provides a description of a range of M\&S techniques, assessing their applicability to the case study and more general SoS modelling; Section 5 discusses the evaluation results followed by a summary of conclusions from this work in Section 6.

\section{Methodology}

The assessment of each M\&S method is judgement-based; utilising experience gained through close involvement with $M \& S$ activities related to a case study and the associated problem domain meant it was possible to realistically consider the suitability and 


\section{A. Kinder et al.}

potential application of each $\mathrm{M} \& \mathrm{~S}$ technique. For some techniques basic models were constructed, however it was not possible to create models using every technique. A further assessment was performed regarding each technique's suitability for more general SoS modelling.

\subsection{The common model}

The following SoS SoI dimensions (Kinder et al., 2012) comprise a common SoS mode and were used as a basis for the assessments, allowing each technique's suitability to specific $\mathrm{SoS}$ aspects to be considered:

Component systems: the individual, independent systems comprising the $\mathrm{SoS}$

Interactions: a SoS exists only because of interactions between constituent systems. With no interaction the SoS merely becomes a set of independent systems exhibiting no overall emergent behaviour.

Functions: a SoS performs a combination of functions in order to accomplish its purpose. Functions may be performed by individual component systems, by subsets of component systems or by the entire SoS.

Nature of relationships: this dimension is used to define the category of relationship between component systems, such as 'peer-to-peer', hierarchical control or distributed control.

Lifecycle: this dimension considers the lifecycle of the $\mathrm{SoS}$ as a whole as well as the respective timelines of the component systems, providing an insight into dynamism within the SOI. For some SoS, it may not be possible to define a cycle, as such, but rather an evolution in which the SoS passes through identifiable phases.

Classification: The SoS classifications (Dahmann and Baldwin, 2008): directed, acknowledged, collaborative and virtual, are used to populate this particular dimension. However, a particular SoS may exhibit the behaviours of more than one classification depending on the make-up of component systems and the current point in the lifecycle.

Variability: the frequency of change of a SoS is considered to be a critical dimension. This is linked to stability and also agility. Variability is related to time in the sense that it may reveal some sort of characteristic frequency representing change in the SoS.

System owners and operations: this dimension is closely related to organisations, management and enterprise (multi-organisational) relationships. It is dependent on the classification type, for example within a directed SoS the owners and operations will be well defined but for a more ad-hoc SoS this dimension will not be so well defined.

Concept of operation/use/employment: given that the individual systems can perform operations independently the use/mission of the SoS is critical for defining the SOI for the SoS as opposed to the individual constituent systems. This dimension is potentially the starting point for populating the SOI dimensions. 
Although the above are described as dimensions, they are not independent. One could consider these to be a set of interrelated characteristics of SoS that may be used to distinguish a particular SoS from another. Figure 1 illustrates the SoS SOI model, which describes the relationships between dimensions.

Figure 1 SoS SOI relationships (see online version for colours)

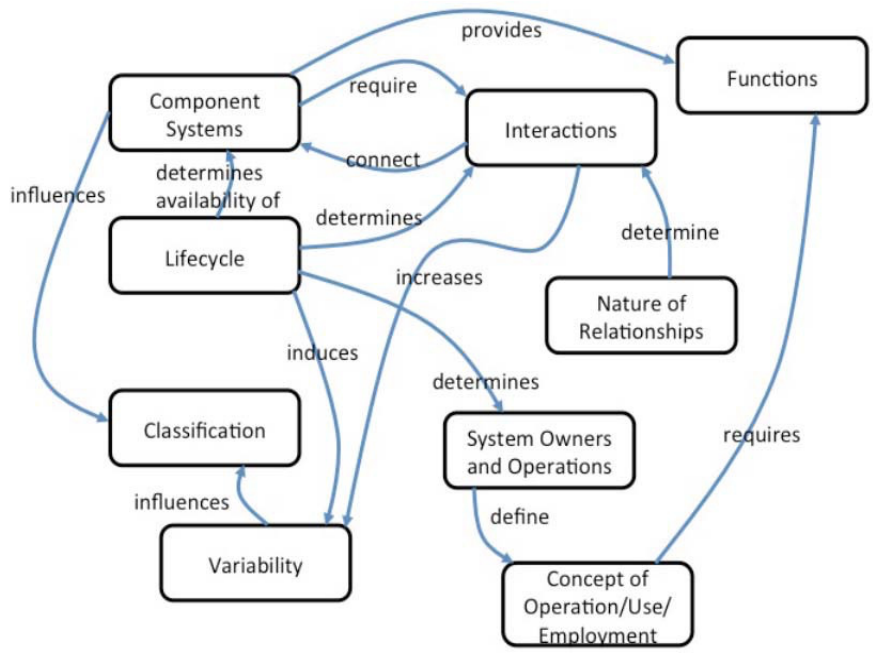

Source: Kinder et al. (2012)

2.2 Technique assessment

Using these SoS SoI dimensions as an assessment framework, each selected modelling or simulation technique was initially analysed in the context of a data looping case study, which was limited in scope, providing partial coverage of the SoS SOI dimensions and is described in Section 3 below. Each M\&S technique was then assessed by considering their suitability for all aspects of the dimensions, with the intention of providing a more general SoS modelling analysis. From these two considerations a traffic light assessment was produced that maps the techniques against the dimensions and then, separately, against three properties: dynamic (whether the technique produces time-dependent results), ease of creation (a measure of complexity and hence effort required to create the model), and verifiability (whether the results can be formally verified). 


\section{Case study: data looping}

This review of SoS M\&S is based around a case study approach, namely data looping in network-enabled capability (NEC) environments, an acknowledged SoS problem in the military communications domain.

Figure 2 illustrates a typical military scenario where tactical information is exchanged, in near real time, across several tactical data link (TDL) networks. TDL networks (in this case Link 11 and Link 16) utilise a series of fixed format messages to convey tactical, command and intelligence data, e.g., the position of an enemy aircraft or a command to intercept a specific target. Both Link 11 (NATO, 2008a) and Link 16 (NATO, 2008b) exchange fixed format digital messages; Link 11 networks operate through a polling mechanism whereas Link 16 utilises a time division multiple access (TDMA) architecture. The purpose of a TDL is to enable the sharing of a common situational awareness (SA) picture amongst all participants of a network. For example, Fighter Aircraft A sensors will locate tracks and pass them over the TDL to Fighter Aircraft B and vice versa. This will result in both fighters displaying the same SA picture in their respective cockpits; essentially the TDL maintains synchronisation between the system track databases.

Figure 2 Counter air mission data loop example

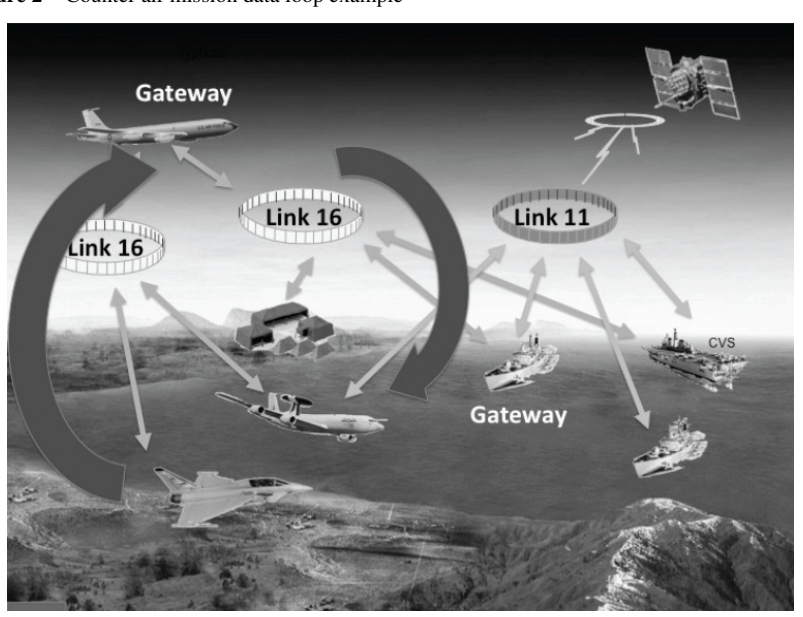

The provision of a NEC typically requires multiple networks to be 'connected', these may be multiple Link 16 networks or the combination of different types such as Link 11, as shown in Figure 2. 'Forwarding units' are utilised to connect these networks, which translate from one message standard to another following relatively simple rules, in accordance with the current NATO forwarding standard (NATO, 2010). The differing 
TDL types have arisen due to the stove piped development of these systems in the air, naval and land domains. A data loop occurs when, through network topology, a message is looped back onto the originating network through a series of connected networks. The dark arrows in Figure 2 indicate the occurrence of a data loop. This inevitably increases network traffic, due to the continuous recirculation of 'stale' data, resulting in excessive latency and corruption of the tactical situational awareness picture; for instance, a target representation on a tactical display may move erratically if a 'current' target report is received followed by 'stale' data which contains a previous position, causing the displayed track to jump backwards. The scenario depicted in Figure 2 was used as a test case for the $M \& S$ techniques described in this paper.

Typically, a complete model of the data looping scenario will require a representation of each system, the communication links between them, representation of the targets and data exchanged between the systems. The model should enable the potential for data loops to be assessed and the quantification of the effect if they do occur. The single integrated air picture (SIAP) metrics (Votruba et al., 2001) are suitable for measuring the effects; in particular;

completeness (the percentage of live objects existing as track objects; $100 \%$ indicates all objects are reported)

clarity (measures the number spurious reported tracks)

continuity (a measure of the consistency and persistence of the track reports).

It should be noted that a combination of simulation techniques may be required in order to create a complete model. Table 1 contrasts data looping $M \& S$ requirements (relating to the dimensions provided in Figure 1) to more general SoS requirements, providing a framework for the assessment of different M\&S techniques in the context of both the data looping case study and more generic SoS modelling. This demonstrates the boundary of this M\&S assessment, imposed by use of the data looping case study, highlighting the fact that it represents only part of the full SoS domain.

\section{Evaluation of M\&S methods}

For each M\&S technique this section:

provides a brief description of the technique and current application to SoS modelling

assesses the technique in the context of the data looping case study

assesses the technique in the context of general SoS modelling

provides an overall conclusion the techniques suitability.

The assessment for each M\&S technique is presented in the consistent format shown in Figure 3. 
Figure 3 Technique assessment format

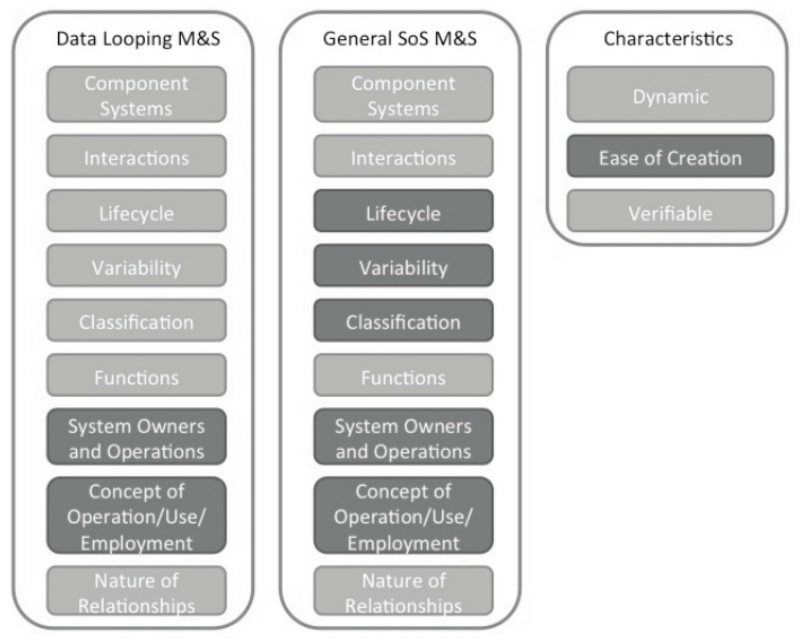

The M\&S techniques are assessed in the context of the data looping case study and general SoS M\&S, as described in Table 1 . Where a technique is determined as highly suited to modelling in the context of a particular dimension, it is shaded light grey, conversely where it is considered as of minimal suitability it is dark grey.

Table 1 Modelling and simulation requirements

\begin{tabular}{lll}
\hline SoS dimensions & \multicolumn{1}{c}{ Data looping $M \& S$ requirements } & \multicolumn{1}{c}{ General SoS $M \& S$ requirements } \\
\hline $\begin{array}{l}\text { Component } \\
\text { systems }\end{array}$ & $\begin{array}{l}\text { Define a platform type to an entity, } \\
\text { where an entity is a component } \\
\text { system. }\end{array}$ & $\begin{array}{l}\text { Any system; either generic or } \\
\text { specific. }\end{array}$ \\
$\begin{array}{l}\text { Define independent spatial } \\
\text { behaviour for each entity. }\end{array}$ & \\
Interactions & $\begin{array}{l}\text { Exchange information between } \\
\text { simulated entity TDL formats, } \\
\text { typically; }\end{array}$ & $\begin{array}{l}\text { Any interaction; either generic or } \\
\text { specific. }\end{array}$ \\
& Link 11 & \\
& Link 16 & \\
& Link 22 \\
& variable message format (VMF) & \\
\hline
\end{tabular}


Table 1 Modelling and simulation requirements (continued)

\begin{tabular}{|c|c|c|}
\hline SoS dimensions & Data looping $M \& S$ requirements & General SoS $M \& S$ requirements \\
\hline Lifecycle & $\begin{array}{l}\text { Operational (or execution) phase } \\
\text { only }\end{array}$ & $\begin{array}{l}\text { All applicable lifecycle stages, e.g., } \\
\text { planning } \\
\text { convergence } \\
\text { collaboration } \\
\text { execution } \\
\text { evolution } \\
\text { dispersion }\end{array}$ \\
\hline Variability & $\begin{array}{l}\text { Within each test scenario the } \\
\text { component systems do not change. }\end{array}$ & $\begin{array}{l}\text { Model change in component } \\
\text { systems throughout lifecycle. }\end{array}$ \\
\hline Classification & $\begin{array}{l}\text { Acknowledged with directed } \\
\text { elements, e.g., Link } 11 / 16 \text { networks }\end{array}$ & All SoS classifications. \\
\hline Functions & $\begin{array}{l}\text { Correlation (of tracks) } \\
\text { Reporting (on allocated TDL) of } \\
\text { tracks detected by sensor } \\
\text { Track forwarding between } \\
\text { allocated TDLs }\end{array}$ & $\begin{array}{l}\text { All functions applicable to } \\
\text { modelling requirement. }\end{array}$ \\
\hline $\begin{array}{l}\text { Systems owners } \\
\text { and operations }\end{array}$ & $\begin{array}{l}\text { Scenarios should be realistic but } \\
\text { this dimension is not explicitly } \\
\text { modelled. }\end{array}$ & $\begin{array}{l}\text { Model all relevant system owners } \\
\text { and operations. }\end{array}$ \\
\hline $\begin{array}{l}\text { Concept of } \\
\text { operation/use/ } \\
\text { employment }\end{array}$ & $\begin{array}{l}\text { Each scenario reflects a different } \\
\text { concept of operation, e.g., } \\
\text { close air support } \\
\text { refuelling } \\
\text { suppression of enemy air defence } \\
\text { search and rescue }\end{array}$ & $\begin{array}{l}\text { Model relevant concept of } \\
\text { operation/use/employment }\end{array}$ \\
\hline Relationships & Node-less network & $\begin{array}{l}\text { All applicable relationship types, } \\
\text { including: } \\
\text { peer-to-peer } \\
\text { hierarchical control } \\
\text { distributed control }\end{array}$ \\
\hline
\end{tabular}

4.1 DES and DEVS

DES models a system through the representation of system states and the change of these states at precise times. A DES does not run in real time (although activity ordered DES breaks time into a series of events and can give the impression of running in real time), it transitions between states, enabling system output and behaviour to be determined 
rapidly. For example, a queuing system may be modelled, with the simulation stepping through events, which add or remove entities from the queue but not replicating the time spent in the queue thereby drastically reducing the simulation execution time. Nance (1993) defines DES as follows:

"Discrete event simulation utilizes a mathematical/logical model of a physical system that portrays state changes at precise points in simulated time. Both the nature of the state change and the time at which the change occurs mandate precise description. Customers waiting for service, the management of parts inventory or military combat are typical domains of discrete event simulation."

DEVS is a mathematical formalism, which enables the hierarchical and modular specification of a discrete event system or simulation, since its inception a wide range of applications has employed DEVS concepts (Sarjoughian and Cellier, 2001), e.g., TDL testing and industrial control process analysis.

The key characteristics of DEVS are:

it is based on mathematical formalism using system theoretic principles

it provides a mathematical formalism for systems and DES

the underlying conceptual framework separates experimental frame, source system, model and simulator

a system is modelled by a combination of atomic models, which capture system

behaviour, and coupled models, which capture system structure.

The concept of creating a large complex model from simpler atomic models could be regarded as the creation of a 'system of models' and it is this characteristic of DEVS that lends itself to SoS modelling. Current research in this area includes the DEVS unified process (DUNIP) which uses XML-based DEVS modelling language (DEVSXML) framework which enables models be defined and implemented in a variety of languages (Mittal et al., 2008). A service-oriented architecture (SOA) is employed to enable distributed and remote execution. Another area of interest is the mapping of the DoD architectural framework (DoDAF) to DEVS models with the aim of providing an 'executable architecture' capability (Zeigler and Mittal, 2005).

4.1.1 Application to data looping case study

The behaviour of a forwarding unit, being event driven, is particularly suited to modelling using DEVS, i.e., an input message will unconditionally result in the generation of an output message, for a standard forwarder (NATO, 2010). When modelling 'smart' forwarding rules, the generation of an output will be conditional, dependent on the existence of a track on the output network. However, full implementation of the forwarding rules within DEVS would be extremely complex because of the nature of the multi-stage transactional correlation requirements, which retain knowledge of previous states, such as the number of correlation attempts, in order to determine the subsequent state and output.

\subsubsection{DEVS SoS M\&S assessment}

Figure 4 provides an assessment of DEVS suitability for SoS M\&S. 
Figure 4 DEVS M\&S suitability

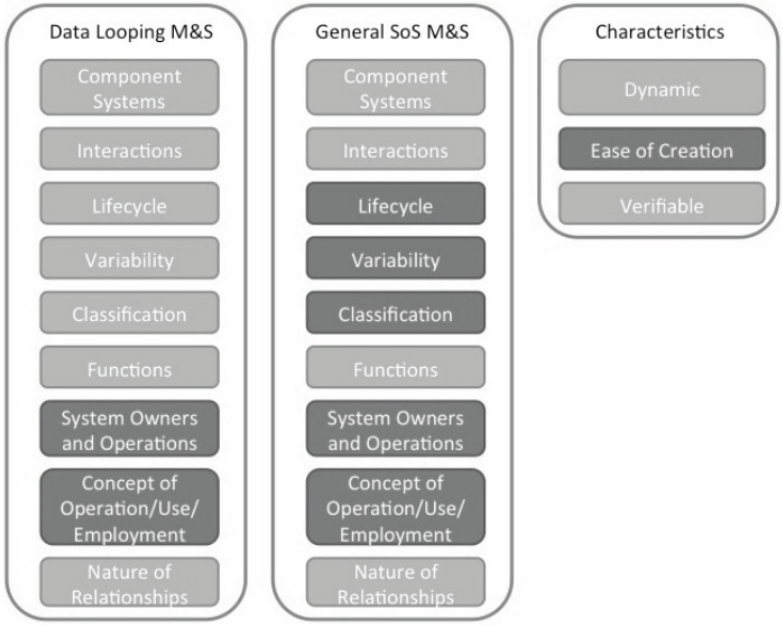

4.1.3 Application strengths and limitations of DES and DEVS

DES and DEVS should be considered 'core' M\&S techniques with regard to SoS Discrete event modelling is applicable to the complex, detailed modelling of SoS components and interactions; it is less suited to the modelling of aggregate and strategic behaviour. Detailed information regarding the SoS under consideration is required for model implementation. Because DEVS is a mathematical formalism, the correctness of the model can be proved.

Using the DEVS formalism to directly to define the model mathematically requires specialist knowledge, which could be outside the knowledge domain of many modellers/programmers. However, there are applications available that enable models to be created using a graphical interface removing the requirement to directly use the mathematical representation.

DES and DEVS are limited to the execution/operational phase of a mission. This restricts the technique to situations where all component systems are known a priori. DEVS will enable implementation of a scenario but is too low level (i.e., working at too detailed a level) to enable definition of a concept of operations at a more abstract level.

\subsection{Petri nets}

Petri nets enable the analysis, design and modelling of discrete event systems, with the specific advantage that the same model may be used for the analysis of behavioural properties and performance evaluation. Petri nets may be described both graphically, 
allowing designs to be communicated from engineer to customer, and mathematically, enabling formal analysis of a model (Zurawski and Zhou, 1994). They comprise basic constructs enabling the simulation of parallel and distributed systems (Murata, 1989), namely:

places (symbolising states or conditions)

transitions (symbolising actions)

arcs (connecting places to transition and vice versa).

Places may contain tokens, which are moved to connected places, via transactions when 'fired'. Each arc has an associated weighting (where the weighting is not shown it should be assumed the value is 1). A transition is enabled if each input place contains at least as many as the weighting of the connection arc. On firing the number of tokens equal to the weighting is transferred from the input place to each output place. This is demonstrated in the simple example shown in Figure 5, which depicts the Petri net 'marking' transition following firing. There are various extensions to Petri nets, including: Timed Petri nets, stochastic Petri nets, coloured Petri nets, hybrid Petri nets, hierarchical Petri nets, agent Petri nets (University of Hamburg, 2014).

Figure 5 Petri marking transition

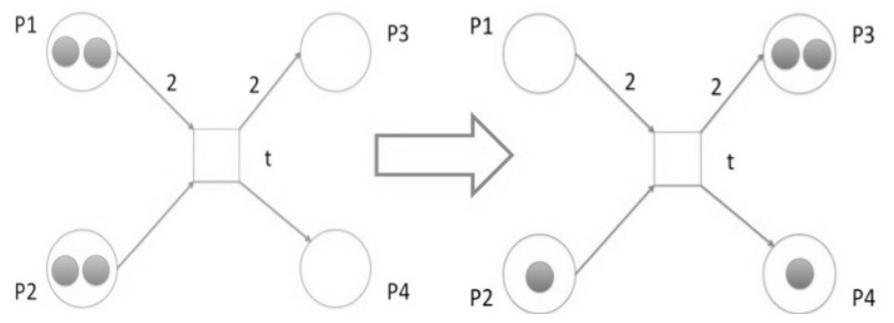

4.2.1 Application to data looping case study

Of particular interest to the data looping case study and SoS modelling in general is the coloured Petri net. This extension allows each token to be assigned data values, known as token 'colour'. Data types and operation may also be defined. It can be seen that tokens could be defined as discrete data link messages, looping within the model could be reproduced. The use of timed Petri nets is also applicable, allowing delays to be introduced in the model, which would reflect the cyclic transmission cycle of data link messages.

Coloured Petri nets have been utilised in the development of executable architecture through an extension to DoDAF ( $\mathrm{Li}$ et al., 2012), providing a dynamic element to the static models currently produced by DoDAF (and MoDAF). 
4.2.2 Petri net SoS M\&S assessment

Figure 6 provides an assessment of Petri net suitability for SoS M\&S.

Figure 6 Petri net M\&S suitability

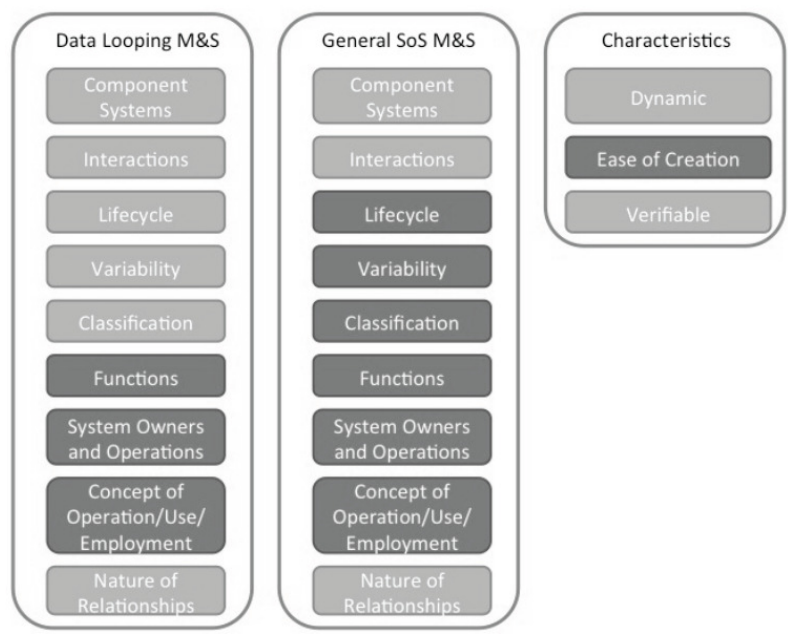

4.2.3 Application strengths and limitations of Petri nets

Petri nets are essentially a type of discrete event model and are therefore similarly suited to the low level modelling of interactions between SoS component systems. Detailed knowledge of the component systems is required for model implementation. Petri nets are less well suited to the modelling of aggregate and strategic behaviour.

In assessing ease of creation of the model, it is assumed that this is linked to the complexity of the model and that detailed modelling of the SoS will include greater complexity.

\subsection{Agent-based modelling and simulation}

ABMS is a relatively new approach to modelling systems comprising autonomous interacting components. Where the components are themselves independent systems then it follows that ABMS may also be applied to the modelling of a SoS. Examples (Macal and North, 2008) of the use of ABMS include the deregulation of the electric supply industry and the financial markets. In both of these examples individual agents make independent decisions, which have an effect on the over-arching system, such as an individual selecting an energy supplier based on the lowest price. 


\section{A. Kinder et al}

Through implementing relatively simple rules in individual agents it is possible to replicate and study emergent system behaviour within an ABM. Indeed it can be argued that as emergent behaviour is not always predictable more traditional scripted simulation methods are unsuitable for investigating this behaviour, because in order to simulate behaviour there must be knowledge of it. In effect, this is a 'chicken and egg' situation. ABMS surmounts this by taking a bottom up approach, only requiring knowledge of the constituent or component systems but allowing them to interact and hence generate emergent behaviour holistically. However, the ability to predict emergent behaviour is dependent on the programmed behaviour of the agents.

It should be noted that the modeller would generally use such techniques to understand better the way in which behaviours emerge, than to attempt prediction of all emergent behaviour available to the SoS in question.

\subsubsection{The properties of an agent}

Although there does not seem to be a generally agreed definition of an agent there are a common set of properties and attributes, which are described below:

Autonomous: an agent can function independently of its environment and other agents.

Modular: an agent is an identifiable, discrete entity with a well-defined boundary.

Interacting: agents interact with other agents and their environment. Protocols are defined which enable this interaction.

Goal-directed: an agent has goals to achieve related to its behaviour, i.e. an agent will have a set of goals with which it can compare its current effectiveness against causing it to modify its behaviour if necessary.

Flexible: an agent may have the ability to learn and adapt its behaviour over time.

Resource attributes: agents may store the level of current 'stock' of a resource, e.g., wealth, information, etc.

\subsubsection{Application to data looping case study}

ABMS potentially allows relatively simple representations of data looping scenarios to be created entirely within ABMS applications, such as NetLogo@. Another benefit is scalability; generally an ABM will contain many agents although this is dependent on the complexity of the agent behaviour and the processing power available.

\subsubsection{ABMS SoS M\&S assessment}

Figure 7 provides an assessment of ABMS suitability for SoS M\&S. 
Figure 7 ABMS M\&S suitability

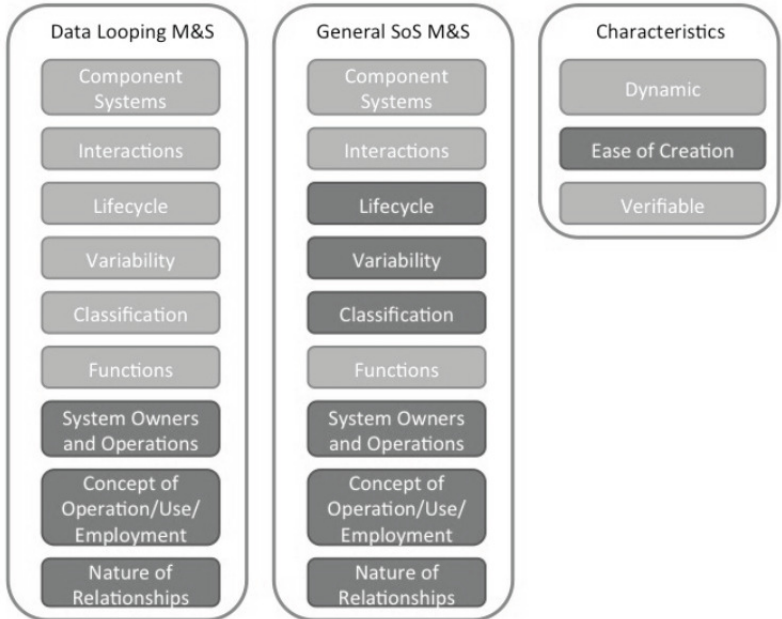

4.3.4 Application strengths and limitations of ABMS

Of all the techniques surveyed ABMS is most likely to reveal unanticipated emergent behaviour due to its bottom up approach. However, the behaviour observed is clearly dependent upon the implementation of the agents (component systems), therefore determining the correct level of fidelity and identifying which systems characteristics to model within each agent is key and is, in itself, a complex activity.

ABMs are highly scalable and models can be constructed with simplified agent behaviour. Modularity of agents enables extension of behavioural complexity if required. Full implementation of all processing rules would increase complexity of the agents thereby severely increasing processing load. It is noted that within the SoS domain, Baldwin et al. (2012) have demonstrated the use of the Schelling model as a basis for validation of a SoS model.

4.4 System dynamics

System dynamics is founded on control theory and the theory of nonlinear dynamics and is currently used across a wide range of domains, including: business administration, engineering (numerous disciplines, including systems), biology, ecology, economics, education, medicine, policy design, law, psychology, sociology and defence (Sterman, 2000). 
It provides a 'systems thinking' viewpoint and a toolset enabling the analysis of complex systems, and by inference, analysis of SoS. System dynamics enables a holistic, top down, approach to analysis, with aggregation of data and information represented as stocks and flows between component systems and the effects of positive and negative feedback as a result of causal linkages modelled. The aggregation of data, rather than individually identifiable 'tokens' or 'packets' makes this approach suitable to more abstract applications, such as the effect of governance or policy on a complex system, or a SoS.

Models are continuous rather than discrete event driven; the system behaviour is demonstrated through the dynamic nature of the model.

\subsubsection{Application to data looping case study}

Whilst not applicable to the direct modelling of TDL networks, which requires the unique identification of messages being passed between component systems, system dynamics could be suitable for modelling the effects of data looping mitigation techniques on the impact on the situational awareness picture, and possibly be utilised in determining the cost effectiveness of implementing these solutions.

Additional applications include network planning, where the effect of network plans (allocations of time slots and use of forwarding units) could be assessed in the context of overall system effect.

4.4.2 System dynamics M\&S assessment

Figure 8 provides an assessment of system dynamics suitability for SoS M\&S.

Figure 8 System dynamics M\&S suitability

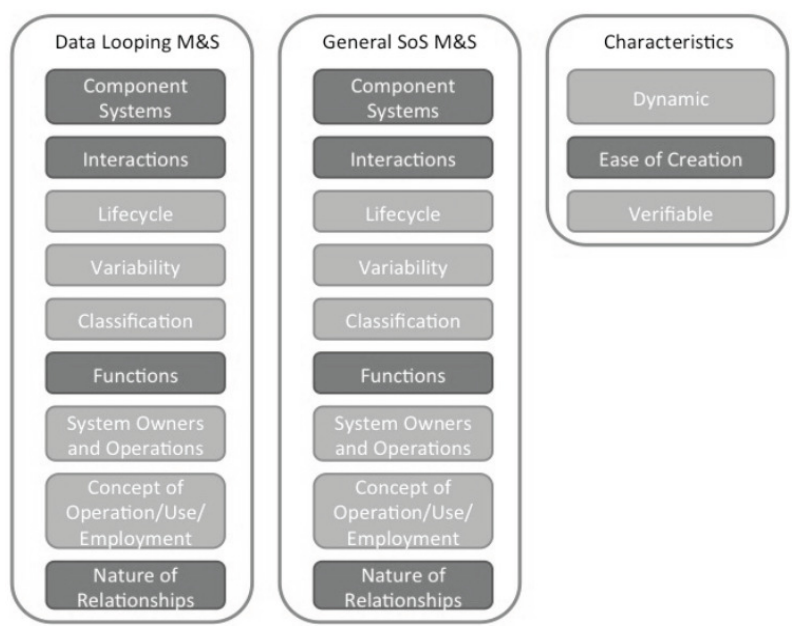


4.4.3 Application strengths and limitations of system dynamics

System dynamics provides top down modelling of SoS allowing the aggregate behaviour to be analysed and is therefore more suited to the modelling of more strategic problems. This can be used to complement the bottom up, detailed modelling approaches such as DES or Petri nets, which are more suited to modelling operational problems. Because systems dynamics models aggregate behaviour; therefore changes in individual component systems do not necessarily affect the model.

\subsection{Surrogate models}

A surrogate model is a data driven, bottom up model that models an approximated output of a system rather than modelling the actual system itself (Forrester et al., 2008). Indeed, surrogate models are also referred to as 'data driven models'.

This approach enables highly efficient system models to be created, which reduce the processing and time burdens when running the model, which, potentially, can be prohibitive. This is particularly true in the SoS domain where, by definition, the SoS under investigation will be complex. Several common approaches, described below, are taken to surrogate modelling, however response surfaces and ANNs are the most popular for SoS analysis (Biltgen, 2008):

Response surface methodology (RSM): a statistical approach to exploration of the relationship of several explanatory variables to response variables. Experiments are designed to optimise a response (output) variable, which is influenced by a number of independent (input) variables. The dependence of the response variable on the independent variables is approximated using a least-squares regression approach to determine unknown model coefficients (Box and Draper, 1987). Response surface equations are easily created but may perform poorly when modelling nonlinear or discontinuous responses, which are typical of SoS (Biltgen, 2008)

\section{$A N N$ : see Section 4.6}

Kriging: the prediction of a function at a particular point by using the weighted average of known values at nearby points. Kriging originated in the field of geostatics and has become a generic term for a set of least-squares methods that provide best linear unbiased predictions and some nonlinear types of prediction (Oliver and Webster, 2013).

Support vector machines: a pattern recognition approach using learning algorithms, which output an optimal hyperplane enabling new examples to be classified (Cortes and Vapnik, 1995).

Of particular interest to SoS modelling is the use of ANN's within a surrogate model, several research papers have been published utilising this approach, in particular to perform ballistic missile defence (BMD) architecture analysis (Ender et al., 2010) and capability-based quantitative technology evaluation (Biltgen and Mavris, 2007).

\subsubsection{Application to data looping case study}

The effects of data looping may be quantified thorough a set of metrics (e.g. completeness, redundancy, quality of information, bandwidth and kinematic accuracy); 
using surrogate models it would be possible to generate the metrics on a dynamic basis, thereby modelling the aggregate behaviour of the SoS. This output could potentially be used to assess factors such as workload and the impact of data looping on mission success.

\subsubsection{Surrogate model SoS M\&S assessment}

Figure 9 provides an assessment of surrogate models suitability for SoS M\&S.

Figure 9 Surrogate model M\&S suitability

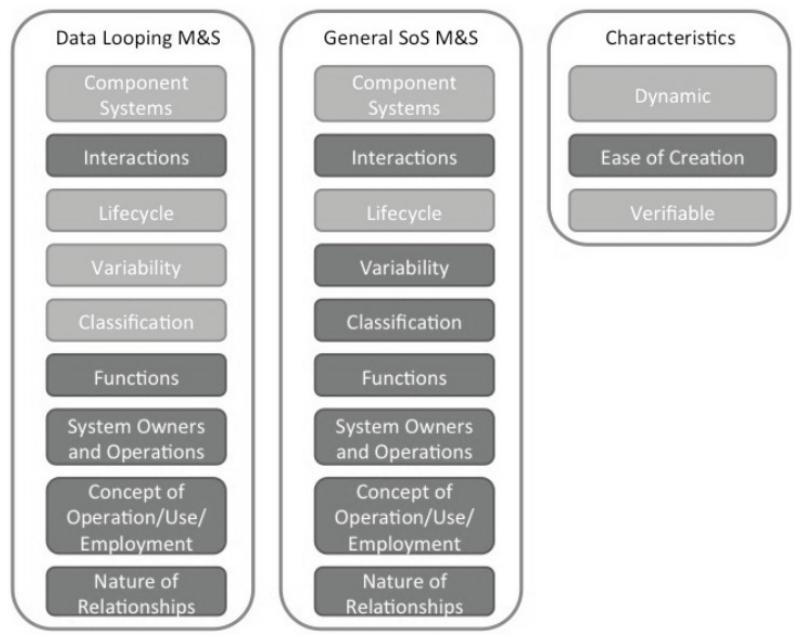

4.5.3 Application strengths and limitations of surrogate models

Surrogate models are potentially an extremely valuable technique in SoS modelling, in conjunction with other techniques such as ANN (Biltgen and Mavris, 2007; Ender et al., 2010). Surrogate models may be embedded within agent-based simulations, reducing the complexity that would be required for high fidelity system representations.

Surrogate models are, by definition, simplifications of systems being modelled, therefore ease of creation will be greater than an equivalent detailed model using techniques such as DES/DEVS or ABMS. This depends on the complexity and behaviour of the function. 
4.6 Artificial neural networks

An ANN is a computer program supposedly designed to model the human brain and its ability to learn tasks (Haykin, 1999). Of course, it is recognised that the human brain functions in a completely different way to a digital computer, based on the Von Neuman architecture, typically partitioned as; processing unit, memory, control unit an input/output. An example of a typical multilayer feedforward network is shown below in Figure 10.

Figure 10 Multilayer feed forward network

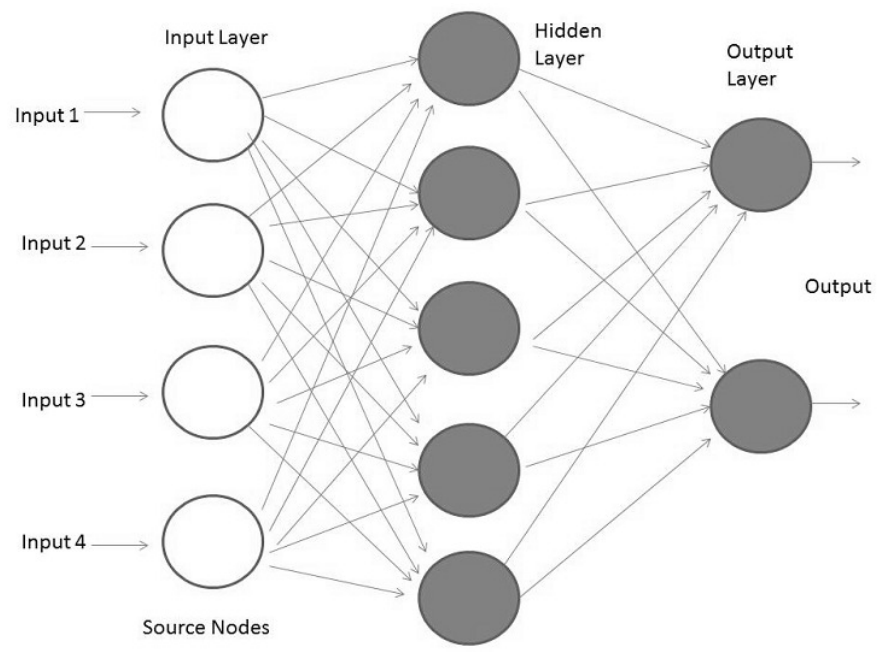

4.6.1 Application to data looping case study

A key part of the data looping research involved the adjustment of correlation gates in order to optimise the reduction in looped tracks without incurring any loss of valid data. This was achieved through a trial and error approach and tended to be scenario dependent. It is possible that the use of an ANN could have automated this process with the ANN dynamically altering the correlations gates until the optimum settings were found.

4.6.2 ANN SoS M\&S assessment

Figure 11 provides an assessment of ANN suitability for SoS M\&S 
Figure 11 ANN M\&S suitability

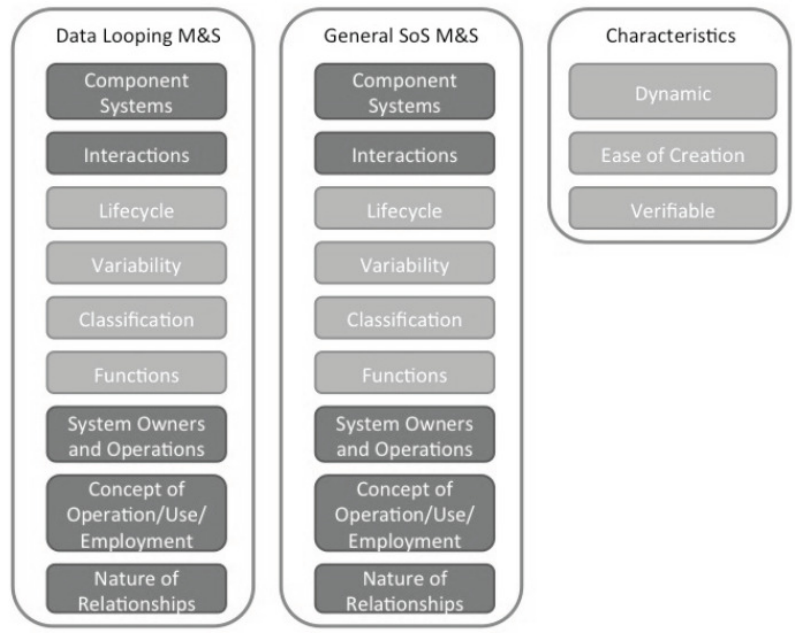

4.6.3 Application strengths and limitations of ANNS

ANN modelling may be regarded as a meta-modelling approach, and is particularly suited to supporting surrogate models (Ender et al., 2010) enabling behaviour to be learnt from available data. The most significant issue with this approach is lack of availability of appropriate data. An ANN can model system behaviour, embedded in an agent or surrogate model and there is the potential for application in decision support. An ANN may be embedded in a dynamic model.

\subsection{Bayesian belief networks}

A BBN is a graphical representation of the probabilistic relationships between a set of random variables using a DAG (Charniak, 1991). A network consists of a set of interconnected nodes with each interconnection representing the causal relationship between nodes with each node having multiple states. The probabilities relate to the likelihood of a particular state occurring in relation to the states of the connected nodes.

4.7.1 Application to data looping case study

The application of BBNs to modelling is more abstract than the approach taken with more traditional modelling techniques (e.g., DEVS) but could provide a useful decision support capability. For example, a BBN could be constructed to determine the probability of data looping occurring in specific scenarios. BBNs have a useful practical application 
as a network planning tool, although it was considered too abstract for application in the data looping case study. Within the SoS domain there has been research into the application of BBNs into assessing vulnerabilities in tactical data wireless networks (Chan et al., 2010). Another area of interest is the modelling of phased missions (Andrews et al, 2008) where the use dynamic Bayesian networks (DBNs) appears to be applicable.

\subsubsection{BBN SoS M\&S assessment}

Figure 12 provides an assessment of BBN suitability for SoS M\&S.

Figure 12 BBN M\&S suitability

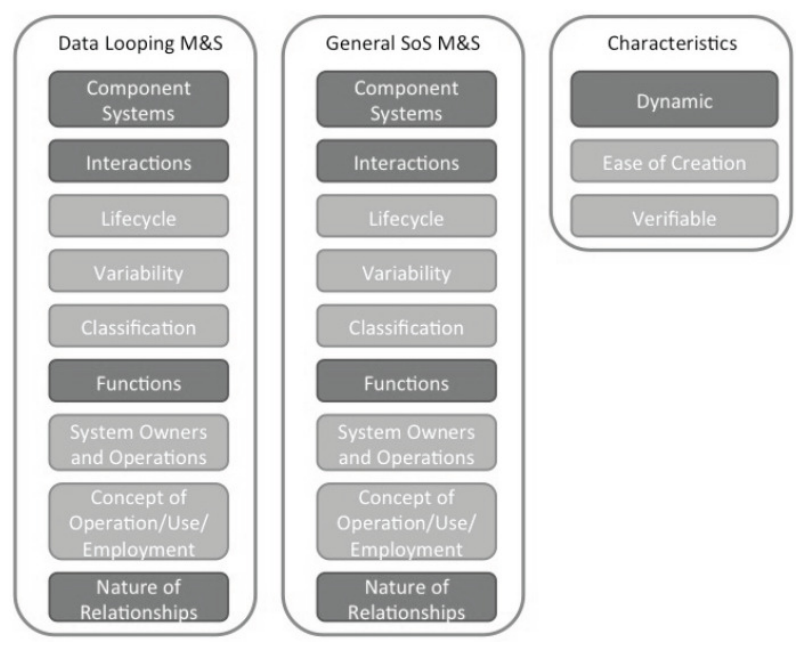

4.7.3 Application strengths and limitations of BBNs

BBNs enable the creation of top down, probabilistic SoS models. This potentially enables risk to be quantitatively determined early in the SoS lifecycle, before data is available regarding all component systems. The identification of appropriate nodes and population of state probability tables within BBNs can be extremely subjective; hence reliance on the results of models in this situation must be tempered with caution. The general applicability of BBNs is enhanced because not all elements in SoS need to be known a priori, however, there is a subjective aspect because completion of the probability tables has to be the result of a qualitative assessment or derived from historical data, which presumes that this is a good indication of future behaviour. BBNs do not have an executable element and the specific functions cannot be directly verified using this modelling technique. 
4.8 Markov model

A Markov model can be used to model any situation where a system moves probabilistically between states (European Mathematical Society, 2012). A Markov model is 'memory-free', the probability of transitioning to an alternate state is only dependent on the current state. A Markov system will always reach a fixed equilibrium regardless of initial conditions. There are similarities to DBNs, however Markov processes model the overall system state whereas DBN's model variables.

One application of Markov models is to determine when previous states have no effect on a system, or SoS, when compared to a model that does take previous states into account. Markov models may be applicable to analysing 'variability' of a SoS.

\subsubsection{Application to data looping case study}

Research has been undertaken to model system component system development and the effect on the overall SoS, enabling the impact of using less, or more, mature systems to be assessed (Mane et al., 2011). This may have applications in producing a 'dynamic' TDL roadmap, for instance, for assessing the impact of the out of service date of a particular TDL.

4.8.2 Markov model SoS M\&S assessment

Figure 13 provides an assessment of Markov models suitability for SoS M\&S

Figure 13 Markov model M\&S suitability

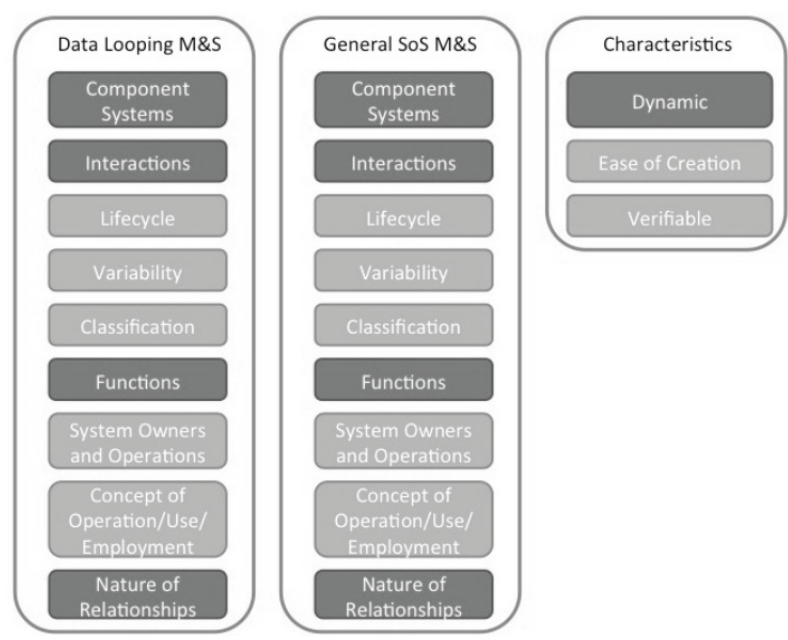


4.8.3 Application strengths and limitations of Markov models

Markov models enable the creation of top down, probabilistic SoS models at the strategic level. As with BBN modelling, there can be a subjective element. However, previous research (Mane et al., 2011) has illustrated that Markov models do certainly have potential applications in the SoS modelling domain.

\subsection{Game theory}

Game theory enables the modelling and study of strategic situations amongst multiple parties, allowing analysis of payoffs and determination of the equilibrium giving optimum pay-off to each party (Osborne, 2003). Typical applications include analysis of business competition, political voting behaviour, bidders competing in an auction and in biology, the behaviour of predators and prey. A basic distinction is made between cooperative and non-cooperative games. Cooperative games require a contract or agreement between parties whereas in non cooperative games the parties are independent.

Applications within the SoS, domain could potentially include assessment of design decisions to determine which give maximum benefit and cost-effectiveness, i.e., the payoffs providing most benefits to all parties. There is also potential to use this technique to formally assess the payoffs between industry and the customer base regarding the adoption of open systems. In particular, the use of game theory has been researched in the context of a smart energy grid SoS (Cooksey and Mavris, 2011). Also, within the SoS domain, game theory has been used in the identification of a SoS unification framework (Wojcik and Hoffman, 2006).

\subsubsection{Application to data looping case study}

This approach may have applications at the strategic level, through the determination of payoffs amongst parties regarding the implementation of data looping mitigation techniques.

4.9.2 Game theory SoS M\&S assessment

Figure 14 provides an assessment of game theory suitability for SoS M\&S.

\subsubsection{Application strengths of game theory}

Game theory will enable pay-offs and equilibrium between both component systems and users to be modelled, potentially enabling SoS behaviour to be analysed. However, a word of caution is sounded. Green (2005) found that game theorists were only equally as successful as a group of students in predicting the outcome of a pre-defined set of situations. However, the unreliability of game theory appears to be associated with economic forecasting. Using game theory to calculate pay-offs in the SoS domain, potentially enabling different SoS solutions to be compared, may be less susceptible to unpredictability and therefore provide more reliable results. 
Figure 14 Game theory M\&S suitability

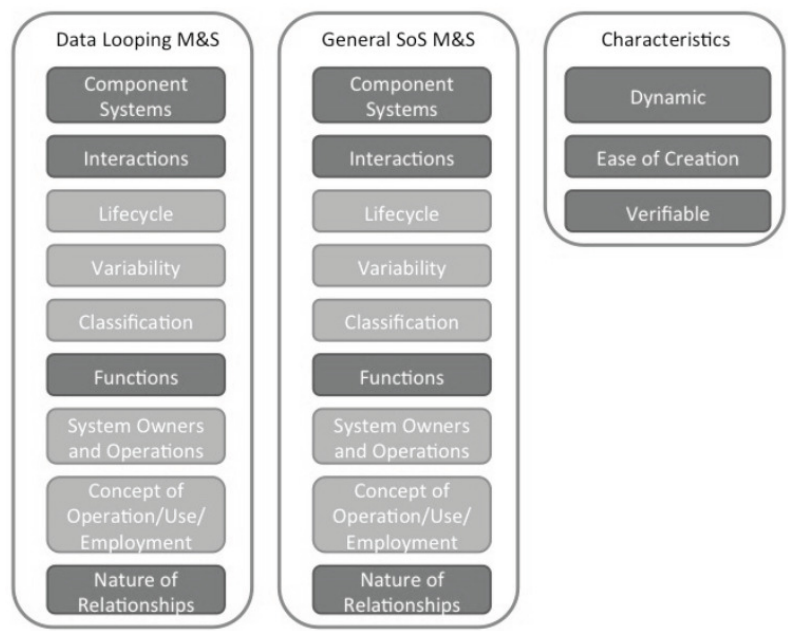

\subsection{Decision trees}

Decision trees enable the modelling of complex decision-making processes potentially allowing the effects of specific decision alternatives to be predicted (Quinlan, 1986). They are typically employed in decision analysis when determining the optimum strategy in relation to a particular goal.

4.10.1 Application to data loop case study?

A potential application would be the assessment of strategy for the implementation of a potential data looping mitigation solution and the selection of candidate platforms for the introduction of this capability.

4.10.2 Decision trees $\operatorname{SoS} M \& S$ assessment

Figure 15 provides an assessment of decision trees suitability for SoS M\&S.

4.10.3 Application strengths of decision trees

Of relevance to SoS modelling is the research carried out into decision making within a phased mission, relating to adaptability and agility of systems (Andrews et al., 2008). 
Figure 15 Decision tree M\&S suitability

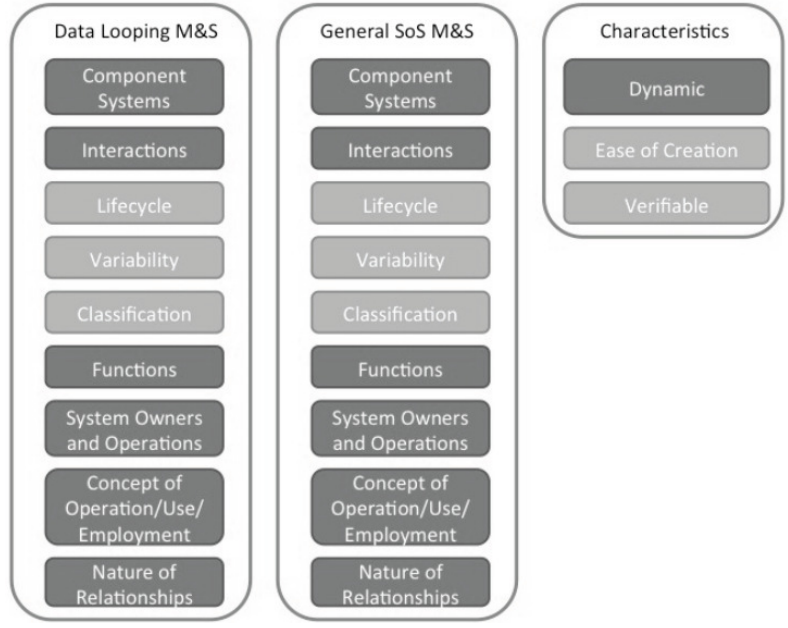

4.11 Network models (graph theory)

In this context, 'network models' means the use of graph theory to model networks, types of graph include (Chartrand, 1984);

flow graphs

bipartite graphs

multi-layered graphs.

Different types of networks may be modelled, e.g., small-world networks where most nodes are not directly connected but can be connected via a series of 'hops' and scale-free networks, which include many highly connected nodes. Percolation models may be used to assess networks robustness to loss of nodes.

Within the SoS domain research has been carried into the application of the clustering coefficient, generally used as a measure of the level of connectivity within a group of people, to determine connectivity between component systems (Dahlgren, 2007).

4.11.1 Application to data looping case study

The obvious application for network modelling and graph theory is connectivity between component systems within a SoS. Percolation models may be used to assess the robustness of multi-TDL networks. 
4.11.2 Network models SoS M\&S assessment

Figure 16 provides an assessment of network models suitability for SoS M\&S.

Figure 16 Network models M\&S suitability

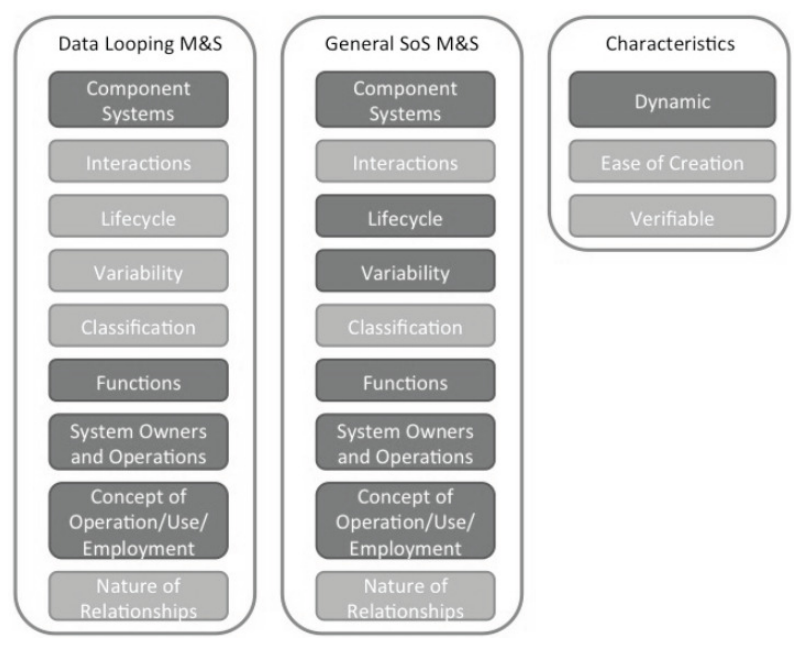

4.11.3 Application strengths of network models

Network models are, by definition, highly applicable to the modelling of interaction within a SoS. They are suitable for modelling all types of SoS, from the highly specified directed to the more adhoc collaborative or virtual (Dahmann and Baldwin, 2008), such as social networks.

\subsection{Enterprise architecture framework}

EAFs are typically used to represent SoS, particularly in the defence domain, with MoDAF (Ministry of Defence, UK, n.d.) being the UK variant. Whilst they do provide a suitable structure, enabling the modelling of abstract to concrete concepts, the models are purely static with no dynamic capability, severely limiting the capability to predict emergent behaviour and model causal relationships.

Generally the development of a MODAF model is based on numerous scenarios, however, this implies that component systems are known, as least at a generic level, meaning applicability is limited to directed and acknowledged types of SoS [as defined by Dahmann and Baldwin (2008)]. In order to add an executable element to DoDAF, the US equivalent to MoDAF, research is being undertaken to add a dynamic view through the use of coloured Petri nets (Li et al., 2012). 
4.12.1 Application to data looping case study

Scenarios may be developed using MoDAF or DoDAF, with scenarios defined in the operational views $(\mathrm{OV})$. The OV-1a view utilises a pictorial format enabling communication between the model developers and the operational community. Once the scenarios are agreed and deemed to be operationally realistic, further operational views enable the nodes within the scenario to be elaborated and the information exchanges to be defined. The model may then be instantiated with specific systems through the system views (SV) allowing detailed analysis of data flows between the systems, including the presence of data loops.

\subsubsection{EAFs SoS M\&S assessment}

Figure 17 provides an assessment of EAFs suitability for SoS M\&S. EAFs are shown to be applicable to most of the dimensions, except 'functions' and 'variability'. EAFs range of different viewpoints provides this close match with the dimensions, although they do not generally enable detailed modelling of functions, and due to their static nature are not suited to modelling variability or emergent behaviour within a SoS. Verification is generally limited to subject matter expert review.

Figure 17 EAF M\&S suitability

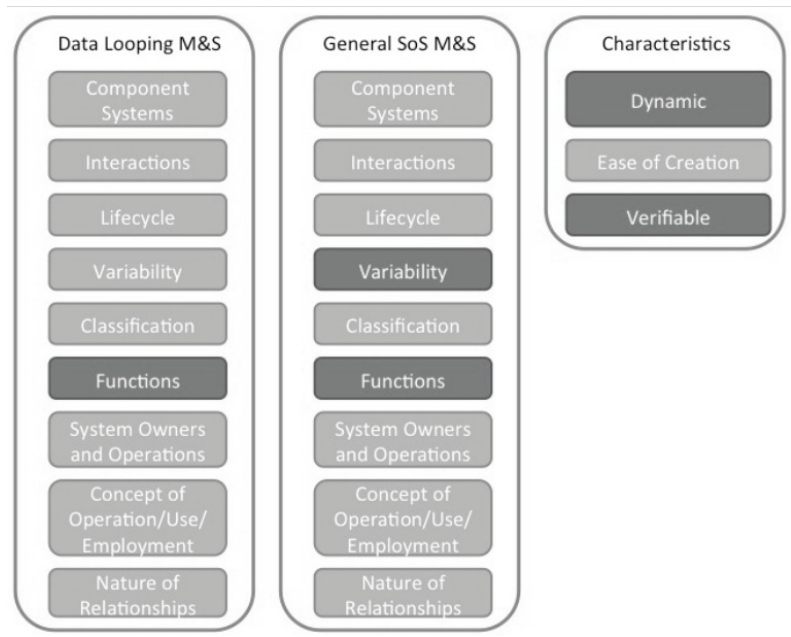


4.12.3 Application strengths of EA frameworks

EA frameworks are commonly used to create static SoS models and are an essential technique in this domain. However, they are unsuited to identifying emergent behaviour because of their static nature, although a dynamic element is the subject of current research ( $\mathrm{Li}$ et al., 2012). At present functions can be identified by EAs, but their behaviour cannot be modelled.

\subsection{Modelling languages}

Within the discipline of software engineering the Unified Modelling Language (UML) (OMG, n.d.b) is the currently the industry standard for software design, within the systems domain the Systems Modelling Language (SySML) (OMG, n.d.a) is used, and is an extension of a subset of UML. These modelling languages are not directly executable. However, executable UML is available, which derives code from the model to be used in implementation, requiring a very high level of detail to be captured in the model. The model effectively becomes the system.

The model-based systems engineering (MBSE) approach may be applicable to a directed SoS, where the component systems are known and the SoS is designed. For a more ad-hoc SoS it is less applicable being more appropriate to the design of the component systems. The COMPASS project (Coleman et al., 2012) is carrying out research into defining a SoS modelling language, COMPASS Modelling Language (CML), which has an executable element.

\subsubsection{Application to data looping case study}

Sequence diagrams were used to demonstrate the information flow between systems and networks. Additionally, the same diagrams were used to analyse the processing flow within the requirement transactions. Use case helped to define both the scenarios and the forwarding requirements. A potential application would be the assessment of strategy for the implementation of data looping mitigation forwarding rules and the selection of candidate platforms for the introduction this capability.

4.13.2 Modelling language SoS M\&S assessment

Figure 18 provides an assessment of modelling language suitability for SoS M\&S.

4.13.3 Application strengths of modelling languages

Whilst UML is generally utilised in the software domain, it is possible the apply elements when specifying and designing SoS, SySML is possibly more applicable as it is intended to enable modelling of physical systems. CML is likely to become an important technique, as it is specifically intended for SoS modelling. 
Figure 18 Modelling language M\&S suitability

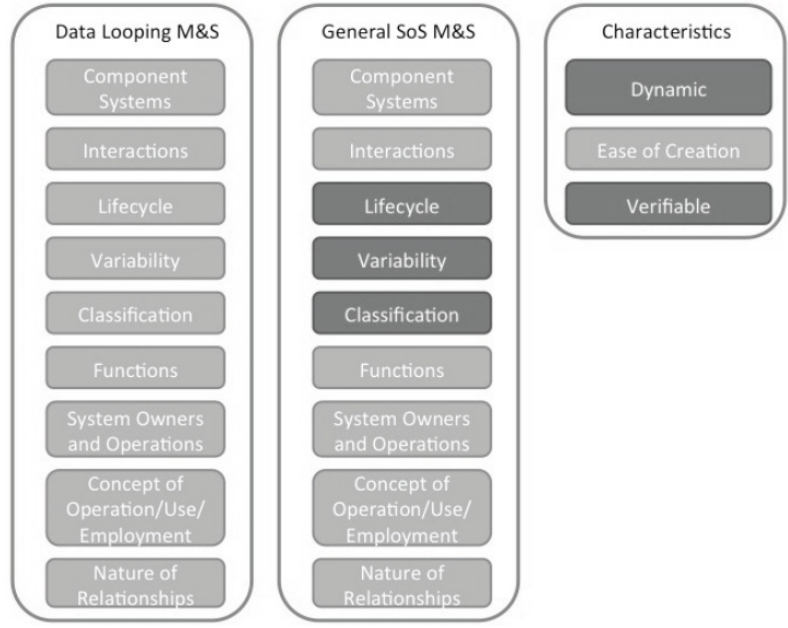

\section{Summary of modelling technique comparison}

This section collects the above results together in order to enable a side-by-side comparison of the modelling techniques assessed in this report.

\subsection{Modelling technique case study comparison}

Table 2 summarises the suitability of the assessed M\&S techniques to the data looping case study. As the case study considers an operational scenario with fixed component systems, the lifecycle, variability and classification are constrained to a limited set of states; this results in all techniques being indicated as applicable for all of these dimensions. Techniques utilising a bottom up approach enable component systems to be modelled, although spatial behaviour is limited to those with a dynamic capability. It is noted that although EAF and modelling languages enable component systems to be modelled, their spatial behaviour cannot be replicated, due to their static nature. Bottom up techniques such as DEVS, Petri nets and ABMS are less suitable for more strategic, high level dimensions; system owners and concept of operation. 


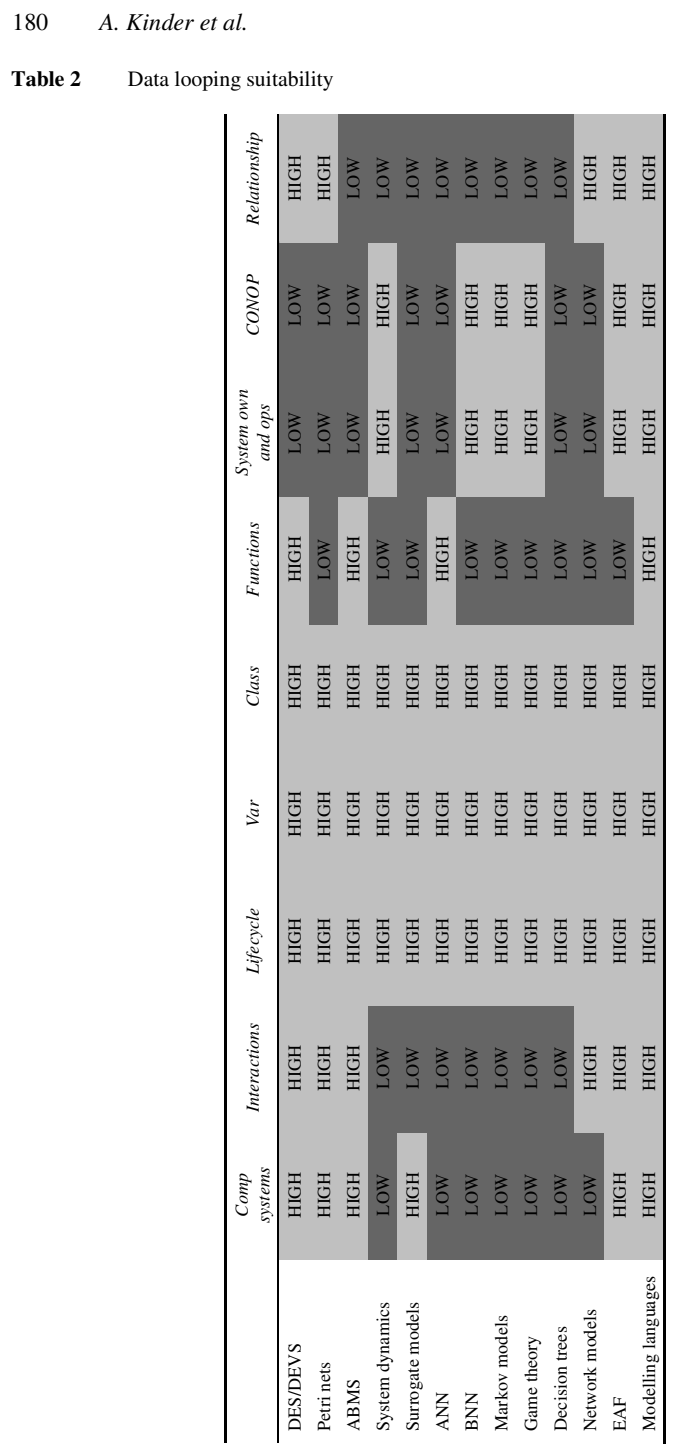


Table $3 \quad$ SoS modelling

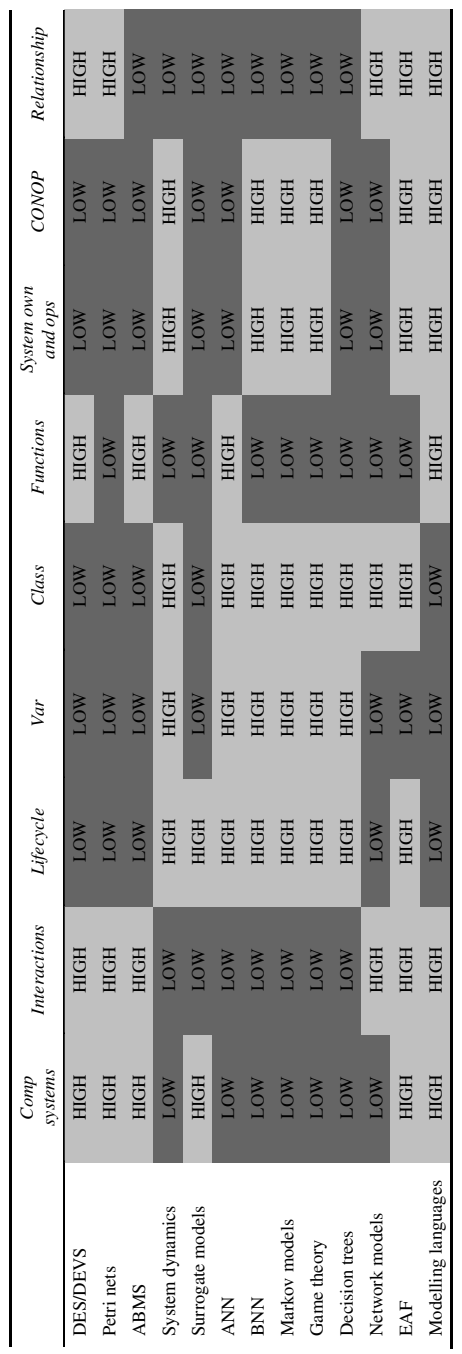


5.2 Modelling technique SoS assessment

Table 3 summarises the suitability of the assessed M\&S techniques to more general SoS modelling, considering all SoS classifications and all phases of the lifecycle. This demonstrates that some bottom up techniques, such as DEVS, Petri nets and ABMS, whilst being highly applicable to the case study, which was concerned with the operation (or execution) phase, are less appropriate throughout the lifecycle. These techniques are also less suited to SoS with higher variability. This is because individual component systems are modelled; as a result if these systems change in the SoS lifetime then the model becomes redundant, exhibiting low resilience to variability. However, top down techniques, such as system dynamics are generally used to model more strategic dimensions rather than individual component systems and hence are more resilient to variability.

\subsection{General $M \& S$ comparison}

Table 4 provides a comparison of the surveyed M\&S using general characteristics. For emergent behaviour to be generated by a model it generally must be dynamic. It can be seen that dynamic models tend to be more difficult to implement, based on the assumption that the complexity is greater than those of static models. However, the testability of these models is also greater as, being dynamic, they may be run against test cases.

Table 4 General M\&S attributes

\begin{tabular}{lcc|c}
\hline & Dynamic & Ease of creation & Verifiable \\
\hline DES/DEVS & HIGH & LOW & HIGH \\
Petri nets & HIGH & LOW & HIGH \\
ABMS & HIGH & LOW & HIGH \\
System dynamics & HIGH & LOW & HIGH \\
Surrogate models & HIGH & LOW & HIGH \\
ANN & HIGH & HIGH & HIGH \\
BNN & LOW & HIGH & HIGH \\
Markov models & LOW & HIGH & HIGH \\
Game theory & LOW & LOW & LOW \\
Decision trees & LOW & HIGH & HIGH \\
Network models & LOW & HIGH & HIGH \\
EAF & LOW & HIGH & LOW \\
Modelling languages & LOW & HIGH & LOW \\
\hline
\end{tabular}

\subsection{Modelling technique classification}

Table 5 shows how each modelling technique, described in this report, related to a basic M\&S taxonomy. Some entries in the table may appear contradictory, e.g., DEVS being both continuous and discrete. However, this does reflect the extensibility of DEVS, as the 
formalism is intended to capture both continuous and discrete paradigms. In other cases, model techniques may be extended to include a stochastic element for example.

Table 5 Modelling technique classification

\begin{tabular}{lcccccc}
\hline & Continuous & Discrete & Deterministic & Stochastic & Static & Dynamic \\
\hline DES/DEVS & $\checkmark(4)$ & $\checkmark$ & $\checkmark$ & $\checkmark$ & & $\checkmark$ \\
Petri nets & $\checkmark$ & $\checkmark$ & $\checkmark$ & $\checkmark$ & & $\checkmark$ \\
ABMS & & $\checkmark$ & $\checkmark$ & $\checkmark$ & & $\checkmark$ \\
System dynamics & $\checkmark$ & & $\checkmark$ & $\checkmark(1)$ & & $\checkmark$ \\
Surrogate models & $\checkmark$ & $\checkmark$ & $\checkmark$ & $\checkmark$ & & $\checkmark$ \\
ANN & $\checkmark$ & & $\checkmark$ & & $\checkmark$ & $\checkmark(2)$ \\
BNN & & & $\checkmark$ & & $\checkmark$ & \\
Markov models & & & $\checkmark$ & & $\checkmark$ & \\
Game theory & & & $\checkmark$ & & $\checkmark$ & \\
Decision trees & & & & $\checkmark$ & $\checkmark$ & $\checkmark(3)$ \\
Network models & & & $\checkmark$ & & $\checkmark$ & \\
EAF & & & & $\checkmark$ & \\
Modelling languages & & & & & $\checkmark$ & \\
\hline
\end{tabular}

Notes: (1) SD models are generally deterministic but a stochastic element may be incorporated.

(2) May be incorporated into a dynamic model, e.g., the behaviour of an agent may be implemented through an ANN

(3) May have a temporal aspect.

(4) Time may be divided into discrete steps thereby approximating a continuous simulation.

\subsection{Technique and SoS lifecycle relationship}

Within the SoS lifecycle each modelling technique is applicable at a particular time or period, However, the SoS lifecycle is not generally a linear sequence of events, this is reflected in the lifecycle defined in the Designing for adaptability and evolution in system of systems engineering (DANSE) methodology (DANSE, 2013). For this reason, Figure 19 uses the DANSE SoS lifecycle, showing the increase in SoS data availability through the 'initiation' 'creation' and 'operation' phases. As the SoS begins to evolve within the Operation Phase the data availability will fluctuate as component systems enter or leave the SoS with resultant changes in the architecture.

Figure 20 shows modelling technique data 'requirements' in relation to one another. The graph-based techniques (e.g., UML or SySML) are used early and often to reduce complexity due to the potential combinations of plausible architectures, whereas a 'bottom up' technique, such as DES, requires a higher level of certainty of the SoS architecture to enable a more complete representation. 
184 A. Kinder et al

Figure 19 SoS lifecycle and data availability

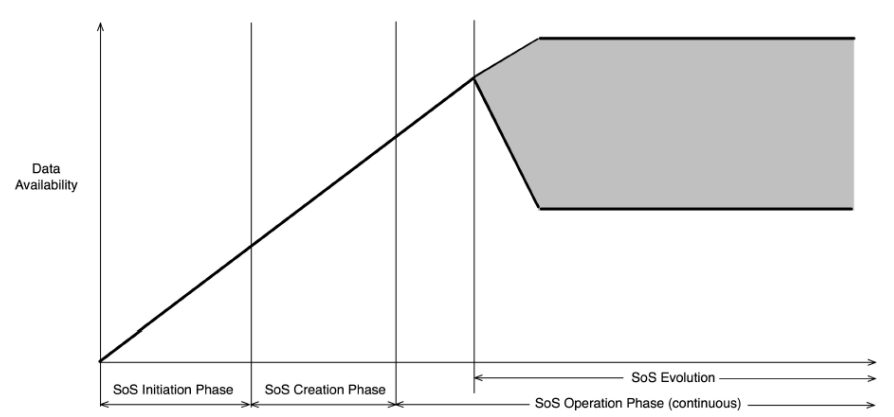

Figure 20 M\&S method data requirements

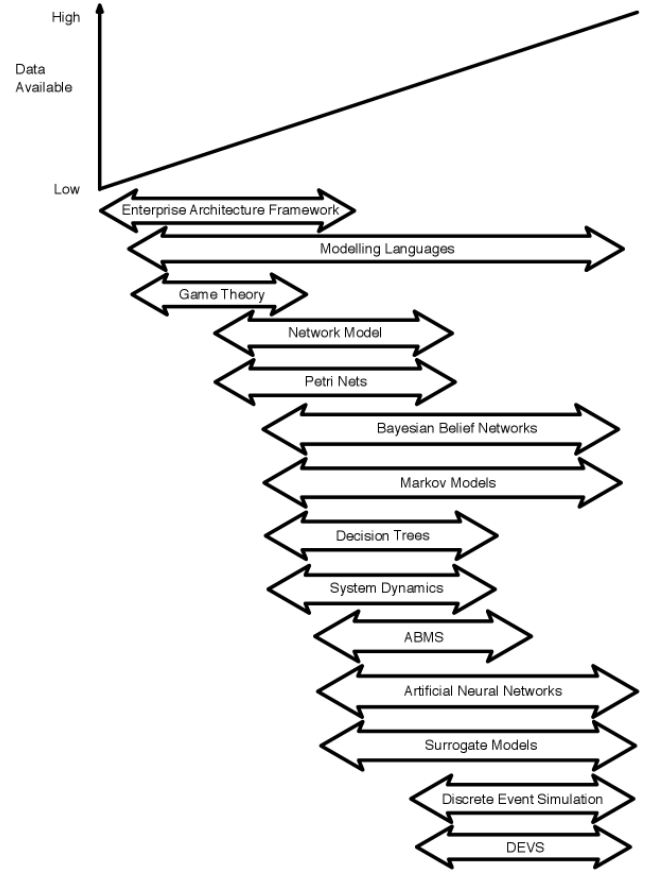




\subsection{Limitations}

As stated previously in Section 2, the review of each technique is a qualitative assessment based on previous experience of modelling the problem presented in the data looping case study and wider experience of SoS modelling and research. The purpose of this activity is to provide a pragmatic and practical approach for SoS engineers, enabling appropriate techniques to be selected when modelling a SoS, at any point the lifecycle.

\section{Concluding remarks}

\subsection{General comments on the $M \& S$ tools}

The generation of emergent behaviour requires a model to have a dynamic element DES/DEVS and Petri nets provide a dynamic, highly structured bottom up approach to modelling and are both extensible, providing extensions for stochastic and continuous capability. DEVS is more developed in the SoS domain with processes available which support model development, e.g., DUNIP (Mittal et al., 2008).

Petri nets are a more specialised modelling technique and their selection will likely depend on the practitioner's experience. However, the use of Petri nets for the creation of executable DoDAF views (Li et al., 2012) represents an important development, which may become an essential SoS modelling approach. The structured nature of DES/DEVS and Petri nets does require a model to implement all component systems and interactions between them.

ABMS provides a more flexible approach, through the definition of agents which abide by a set of behavioural rules, the interactions will then naturally occur as a result of these rules, potentially revealing unanticipated emergent behaviour. However, these techniques may be combined, i.e., an agent may be implemented using DEVS or Petr nets. ABMS is potentially more suited to the modelling of ad hoc SoS (collaborative, virtual) than DEVS and Petri nets, since models with different combinations of agent may be created and run relatively rapidly. A surrogate model generates an approximated nonlinear output of a system rather than modelling the actual system itself, ANNs may be utilised to generate these nonlinear models, as has been demonstrated in the BMD domain (Ender et al., 2010). Surrogate models themselves may be incorporated within agents, providing an efficient and flexible modelling solution. This approach should be considered where a high fidelity modelling is impractical due to complexity or the processing overhead would be excessive.

System dynamics provides a dynamic, top down modelling capability, which can compliment the bottom up approaches and is more suited to modelling high level, strategic dimensions and behaviour, as opposed to low level modelling of individual component systems.

BBNs and Markov models both provide another alternative probabilistic approach, suitable for strategic, decision and risk models. There is potential for lower model outputs to generate probability distributions as input to these models. Numerous software applications are available to enable the creation of these models, making them a practical option, however selection of nodes and determination of probability values does tend to be subjective and can result in less reliable results. 
Game theory does require more specialist knowledge to implement models and is relatively immature in the SoS domain but it is unique in its capability to analyse pay-offs between component systems at the strategic level. Decision trees may have some use in assessing combinations of component systems and providing decision support, but BBNs, Markov and game theory models appear to be more applicable for SoS.

Network models are, by definition, limited to the analysis of interactions within SoS However, the interactions between the component systems are what enable the existence of a SoS; therefore network models are highly relevant and are also unique in supporting detailed analysis of these connections.

EAFs are currently used most widely in modelling SoS, particularly DoDAF and MODAF in the military domains. These enable multiple views of SoS architecture and enable communication between operational and engineering communities. However, although this is a valuable approach, the models produced are static and therefore not able to generate emergent behaviour (noting the proposed executable view as discussed above). It is suggested that, generally, SoS modelling should utilise both static and dynamic models. EAFs and modelling languages are closely related, for example certain DoDAF/MODAF views may be specified in UML or SySML format. Again, modelling languages are typically static, although some tool vendors do provide an executable capability. Of major interest is the COMPASS modelling language, CML; although relatively immature it is specifically designed for SoS modelling.

6.2 Selection of M\&S methods

The survey of the M\&S methods using the SOI dimensions provides a framework for the selection of methods appropriate for a given context. The process for method selection, utilising this framework, is described below.

\subsubsection{Data looping M\&S method selection}

For the data looping case study, a model is required to reproduce the recirculation of data through multiple networks and demonstrate the effect.

The analysis of Table 1 shows that the key dimensions to be considered in this case are; component systems, interactions and functions.

From Table 2, it is observed that DES/DEVS, ABMS and modelling languages are the only methods that are applicable to all three key dimensions.

To demonstrate the effect of data looping, the model is required to be dynamic, eliminating the use of modelling languages, as indicated in Table 4.

Consulting the general descriptions of the M\&S techniques in Section 4 , it is suggested that DES/DEVS is the most appropriate modelling option, as it is more suited to the queuing and transmission of data between component systems. However, although a modelling language (e.g., UML) is ruled out as a final M\&S option it could be applied in the design of DES/DEVS model. 
6.2.2 General SoS M\&S method selection

The following general process for M\&S method selection is proposed:

determine model requirements, i.e., what is its purpose?

cross-reference the model requirements to the SOI dimensions and determine the key dimensions

from Table 4, select $M \& S$ techniques, which are applicable across dimensions

from the model requirements determine if dynamic behaviour is required; re-assess the list of selected models using Table 4 information.

The following typical SoS problems are considered, demonstrating the application of the proposed process.

\subsubsection{Achieving interoperability}

Achieving interoperability within a SoS, e.g., in a close air support military scenario, requiring communication between ground and air forces.

Model purpose: assess communication capabilities of component systems and identify where gateways are required. Assume component systems are identified.

Key dimensions: component systems, interactions.

Applicable M\&S methods (Table 3): DES/DEVS, Petri nets, ABMS, EAF, modelling language.

Dynamic behaviour required: no. A static analysis of the communication media will suffice.

Final M\&S method selection: EAF, modelling language.

\subsubsection{Delivery of a directed SoS}

Delivery of a directed SoS; requiring coordinated component system development, e.g., a national healthcare system, enabling the exchange and storage of patient information, online booking and prescription management.

Model purpose: provide analysis of the maturity of component systems, enabling the determination of the current overall SoS maturity, highlighting risk of delivery failure, early in the lifecycle.

Key dimensions: lifecycle, system owners and operations, concept of operation, variability, classification.

Applicable M\&S methods (Table 3): system dynamics, BNN, Markov models, game theory.

Dynamic behaviour required: Not essential.

Final M\&S method selection: BNN, Markov models, game theory. 


\subsubsection{Situational awareness}

Achieving adequate situational awareness (SA) within a SoS, e.g. in a disaster relief scenario amongst cooperating agencies.

Model purpose: determine level of SA within an operational SoS.

Key dimensions: component systems, interactions, functions, nature of relationships, system, concept of operation/use/employment, owners an operations.

Applicable M\&S methods (Table 3): modelling language.

Dynamic behaviour required: no.

Final M\&S method selection: modelling language.

\subsubsection{Emergent behaviour}

Predicting emergent behaviour within a SoS, e.g. behaviour of traffic flows in an integrated platform system.

Model purpose: analysis of traffic flow and bottlenecks in SoS under varying load conditions.

Key dimensions: component systems, interactions, functions.

Applicable M\&S methods (Table 3): DES/DEVS, ABMS, modelling languages.

Dynamic behaviour required: yes.

Final M\&S method selection: DES/DEVS, ABMS.

\subsection{Future work}

This paper is intended to be a starting point for a wider survey of modelling techniques applicable to SoS, providing the basis for a structured technique selection process, depending on SoS type and context.

Currently, the survey is limited to a single case study, however the results will be further enhanced through the examination of different case studies, including examples of different types of SoS across multiple domains. This will lead to more specific selection criteria for SoS modelling technique.

As well as increasing the scope of case studies, additional modelling techniques should also be considered, such as the following:

1 Monte Carlo simulation

2 multi-criteria decision analysis

3 failure modes and effects analysis

4 highly optimised tolerance (HOT) (Wojcik, 2004)

5 cost modelling

constructive systems engineering cost model (COSYSMO) (Valerdi, 2005)

standard financial modelling (Benninga, 1997) 
6 risk modelling

dedicated risk modelling approaches (Rasmussen, 1997)

7 reliability models

Jelinski-Moranda (JM) (Jelinski and Moranda, 1972)-reliability model

Littlewood-Verall (Littlewood and Verrall, 1974)

computer-aided software reliability estimation (CASRE)

(Lyu and Nikora, 1992)

system Weibull distribution (Kapur and Lamberson, 1977)

Whilst undertaking this survey it became apparent that a general resource does not currently exist outlining SoS modelling techniques and their applicability. It is hoped that the work described in this paper will form the basis of a just such a resource, which can be expanded and refined as the SoSE discipline matures.

\section{Acknowledgements}

The authors would like to thank Mr. Robert Garrett, Chief Engineer for Modelling and Simulation at the Missile Defence Agency, for his encouragement and invaluable input to this paper.

\section{References}

Andrews, J., Prescott, D. and Remenyte-Prescott, R. (2008) 'A systems reliability approach to decision making in autonomous multi-platform systems operating a phased mission', Reliability and Maintainability Symposium, IEEE, Las Vegas, pp.8-14.

Baldwin, W., Boardman, J. and Sauser, B. (2012) 'Model, expanding a system of systems model with the schelling segregation', Systems Research and Behavioral Science Syst. Res., Vol. 30, No. 1, pp.65-75.

Benninga, S. (1997) Financial Modeling, MIT Press, Cambridge, MA.

Biltgen, P. and Mavris, D. (2007) 'Capability-based quantitative technology evaluation for systems-of-systems', International Conference on System of Systems Engineering, IEEE, Atlanta, pp.1-6.

Biltgen, P.T. (2008) 'Technology evaluation for system of systems', in Jamshidi, M. (Eds.) Systems of Systems Engineering: Principles and Applications, pp.133-158, CRC, Boca Raton.

Box, G.E. and Draper, N.R. (1987) Empirical Model-building and Response Surfaces, Wiley Series in Probability and Mathematical Statistics, John Wiley \& Sons, Oxford.

Chan, P., Mansuri, M. and Man, H. (2010) 'Applying systems engineering in tactical wireless network analysis with Bayesian networks', 7th International Conference on System of Systems network analysis with Bayesian
Engineering, pp.208-215, IEEE.

Charniak, E. (1991) 'Bayesian networks without tears', AI Magazine.

Chartrand, G. (1984) Introductory Graph Theory, Dover Publications, New York.

Coleman, J., Malmos, A., Larsen, P., Peleska, J., Hains, R., Andrews, Z. et al. (2012) 'COMPASS tool vision for a system of systems collaborative development environment', 7 th International Conference on System of Systems Engineering, pp.451-456, IEEE, Genoa.

Comptroller and Auditor General (2009) The National Offender Management Information System, National Audit Office. 
Cooksey, K. and Mavris, D. (2011) 'Game theory as a means of modeling system of systems viability and feasibility', Aerospace Conference, IEEE, Big Sky, pp.1-11.

Cortes, C. and Vapnik, V. (1995) 'Support-vector networks', Machine Learning, Vol. 20, No. 3, pp.273-297.

Dahlgren, J. (2007) 'Developing a framework for exploring clustering coefficient to evaluate system coupling', 12th ICCRTS.

Dahmann, J. and Baldwin, K. (2008) 'Understanding the current state of US defense systems of systems and the implications for systems engineering', SysCon 2008 - IEEE International Systems Conference, IEEE, Montreal, pp.1-7.

DANSE (2013) DANSE Methodology V2, from DANSE - Designing for Adaptability and EvolutioN in System of Systems Engineering, 7 November [online] http://www.danseip.eu/home/images/deliverables/danse_d4.3_methodology_v2.pdf (accessed 1 June 2014).

Ender, T., Leurck, R., Weaver, B., Miceli, P., Blair, W., West, P. et al. (2010) 'Systems-of-systems analysis of ballistic missile defense architecture effectiveness through surrogate modeling and simulation', IEEE Systems Journal, Vol. 4, No. 2, pp.156-166.

European Mathematical Society (2012) Markov Chain, Encyclopedia of Mathematics [online] http://www.encyclopediaofmath.org/index.php?title=Markov_chain\&oldid=14607 (accessed September 2012).

Forrester, A., Sobester, A. and Keane, A. (2008) Engineering Design via Surrogate Modelling: A Practical Guide, Wiley, West Sussex, UK. Green, K. (2005) 'Game theory, simulated interaction, and unaided judgement for forecasting
decisions in conflicts: further evidence', International Journal of Forecasting, Vol. 21, No. 3, pp.463-472.

Haykin, S. (1999) Neural Networks A Comprehensive Foundation, Pearson Education Inc., Saddle River.

House of Commons Committee of Public Accounts (2011) The National Programme for IT in the NHS: An Update on the Delivery of Detailed Care Records Systems, The Stationery Office Limited, London.

Jamshidi, M. (2008) 'System of systems engineering - new challenges for the 21st century', Aerospace \& Electronic Systems Magazine, pp.4-19.

Jelinski, Z. and Moranda, P. (1972) 'Software reliability research', in Freiberger, F. (Ed.) Statistical Computer Performance Evaluation, pp.465-484, Academic Press, New York.

Kapur, K. and Lamberson, L. (1977) Reliability in Engineering Design, John Wiley and Sons, Inc., New York.

Kinder, A., Barot, V., Henshaw, M. and Siemieniuch, C. (2012) 'System of systems: 'defining the system of interest', 7th International Conference on System of Systems Engineering, IEEE, Genoa, pp.463-468.

Li, L., Dou, Y., Ge, B., Yang, K. and Chen, Y. (2012) 'Executable system-of-systems architecting based on DoDAF meta-model', 7th International Conference on System of Systems Engineering, IEEE, Genoa, pp.362-367.

Littlewood, B. and Verrall, J. (1974) 'A Bayesian reliability model with stochastically monotonous failure rate', IEEE Trans. Reliability, Vol. R-23, No. 2, pp.108-115.

Lyu, M. and Nikora, A. (1992) 'CASRE: a computer-aided software reliability estimation tool', Fifth International Workshop on Computer-Aided Software Engineering, IEEE, pp.264-275.

Macal, C. and North, M. (2008) 'Agent-based modeling and simulation: ABMS examples', Simulation Conference, pp.101-112, IEEE, Austin.

Mane, M., DeLaurentis, D. and Frazho, A. (2011) 'A Markov perspective on system-of-systems complexity', IEEE International Conference on Systems, Man, and Cybernetics, IEEE, pp.1238-1243. 
Ministry of Defence, UK (n.d.) MOD Architecture Framework (MODAF) [online] http://Mnw.mod.uk/Defencelnternet/AboutDefence/WhatWeDo/InformationManagement/MO DAF/ (accessed September 2012).

Mitta, S., Zeiger, B., Risco Matin, J.L., Sahin, F. and Jamshidi, M. (2008) 'Modeling and simulation for systems of systems engineering', in Jamshidi, M. (Ed.): System of Systems Engineering - Innovations for the 21 st Century, Wiley, Hoboken.

Murata, T. (1989) 'Petri nets: properties, andysis and apd ications', Proceedings of the IEEE.

Nanœ, R.E. (1993) A History of Discrete Event Programming Languages, Virginia Pdytednnic Institute and State University, Computer Science, Bladksourg.

NATO (2008a) Tactical Data Exchange - Link 11/Link 11B, Ed8.

NATO (2008b) Tactical Data Exchange - Link 16, Ed 6.

NATO (2010) Standardization Agreement TDL Forwarding 5616, Ed 6.

O'Rourke, R. (2007) Coast Guard Deepwater Program: Background, Oversight Issues, and Options for Congress, Congressional Research Service, Washington.

Oliver, M. and Webster, R. (2013) A Tutorial Guide to Geostatistics: Computing and Modelling Variograms and Kriging, CATENA.

OMG (n.d.a) OMG Systems Modeling Language, The Offidid OMG SysML [online] http://wnw.omgsysml.org (accessed September 2012).

OMG (n.d.b) Uml® Resource Page, Unified Modding Language ${ }^{T M}$ [online] http://unw.uml.org (accessed September 2012.

Osborne, M. (2003) An Introduction to Game Theory, Oxford University Press, New Y ork.

Pernin, C.G., Axelbend, E., Drezner, J.A., Dille, B.B., Gordon IV, J., Held, B.J. et d. (2012) Lessons from the Army's Future Combat Systems Program, RAND Corparation, Santa Monica.

QinetiQ (2005) NHS Connecting for Health Process Capability Appraisal, National Audit Office, QinetiQ.

Quinlan, J. (1986) 'Induction of decision trees', Machine Learning, Vd. 1, No. 1, pp.81-106.

Rasmussen, J. (1997) 'Risk management in a dynamic sodiety: a moddling prodlem', Safety Science, Vd.27, No. 2, po.183-213

Sajoughian, H.S. and Cellier, F.E. (2001) Discrete Event Modeling and Simulation Technologies: A Tapestry of Systems and AI-Based Theories and Methodologies, Saringer $\forall$ erlag, NY.

Siebers, P., Macd, C., Garnett, J., Buxton, D. and Pidd, M. (2010) 'Discrete-event simulation is dead, Iong live agenttbased simulation', Journal of Operational Research Society, Vd. 4 , No. 3, po.204-210.

Sterman, J. (2000) Business Dynamics: Systems Thinking and Modeling for a Complex World, MoGraw Hill/1rwin, Boston.

Tako, A, and Robinson, S. (2009) 'Comparing discrete-event simulation and system dynamics' users' perceptions', Journal of the Operational Research Society, Vd. 60, No. 3, pp.296-312

University of Hamburg (2014) Petri Nets World [online] http://hww.infarmatik.unihamburg.de/TGI/PetriNets/ (accessed 16 Mach 2014).

Valerdi, R. (2005) COSYSMO: A Systems Engineering Cost Model, PhD, University of Southern California.

Votruba, P., Nisley, R. and Rothrock, R. (2001) Single Integrated Air Picture (SIAP) Metrics Implementation, The Joint Staff, Washington.

Wojcik, L. (2004) A Highly-Optimized Tolerance (HOT)-Inspired Model of the Large Scale Systems Engineering Process, Student Papers, Complex Systerms Summer Schod, The MITRE Corporation, SantaFe.

Wojcik, L. and Hoffman, K. (2006) 'Systems of systems engineering in the enterprise context: a unifying framework for dynamics', International Conference on System of Systems Engineering, IEEE, Los Angdes. 


\section{$192 \quad$ A. Kinder et al.}

Zeigler, B. and Mittal, S. (2005) 'Enhancing DoDAF with a DEVS-based system lifecycle', Systems, Man and Cybernetics, 2005 IEEE International Conference on, pp.2991-2998, IEEE, Waikoloa.

Zeigler, B.P. (2003) 'DEVS today: recent advances in discrete event-based information technology', Proceedings of the 11th IEEE/ACM International Symposium on Modelling, Analysis and Simulation, IEEE Computer Society.

Zurawski, R. and Zhou, M. (1994) 'Petri nets and industrial applications: a tutorial', IEEE. Transactions on Industrial Electronics, Vol. 41, No. 6, pp.567-583. 


\section{Annex C: Paper 3 - A Model Based Approach to System of Systems Risk Management}

\section{A Model Based Approach to System of Systems Risk Management}

\author{
Mr. Andrew Kinder \\ JCSys Ltd \\ 20 Gong Hill Drive, \\ Lower Bourne, Farnham, Surrey, GU10 3HQ \\ Andy.kinder@j.jcsys.co.uk
}

Prof. Michael Henshaw, Prof. Carys Siemieniuch School of Electronic, Electrical and Systems Engineering, Engineering Systems of Systems (ESoS) Group Loughborough University, LE11 3TU, Loughborough, UK. M.J.d.Henshaw@1boro.ac.uk, C.E.Siemieniuch@lboro.ac.uk

\begin{abstract}
This paper discusses the approaches required for risk management of 'traditional' (single) Systems and System of Systems (SoS) and identifies key differences between them. When engineering systems, the Risk Management methods applied tend to use qualitative techniques, which provide subjective probabilities and it is argued that, due to the inherent complexity of SoS, more quantitative methods must be adopted. The management of SoS risk must be holistic and should not assume that if risks are managed at the system level then SoS risk will be managed implicitly. A model-based approach is outlined, utilizing a central Bayesian Belief Network (BBN) to represent risks and contributing factors. Supporting models are run using a Monte Carlo approach, thereby generating results, which may be 'learnt' by the BBN, reducing the reliance on subjective data.
\end{abstract}

Keywords: System, System of Systems, System of Systems Engineering, risk, uncertainty, risk management modeling, simulation.

\section{Introduction}

System of Systems (SoS) Engineering (SoSE) is an emerging sub-discipline of which Risk Management is a critical, but immature, element. When engineering 'traditional' systems, the likelihood of risk is typically determined through qualitative approaches resulting in the allocation of subjective (as opposed to objective) probability [1].

Pinto [2] argues that, when engineering traditional systems, the tools and methodologies are available to address defined problems and, as the system boundaries are fixed and expected behavior is known, scoping these problems and the associated risks, is relatively straightforward. However, the SoS boundary is not necessarily static, the component systems may not all be identified and behavior is emergent: therefore new tools and methodologies are required.

In addition, Conrow [3] identifies the following SoS Risk Management issues, which are specific to this domain: multiple stakeholders and risk management processes, long life cycles, disparate technical risk assessment, integration risks, and functional performance and interface complexity.

This paper briefly discusses the nature of risk before examining the different approaches required for Risk Management, when engineering 'traditional' systems and SoS. A framework for allocating SoS Risk Type is proposed combining a holistic classification [4] with a SoS System of Interest (SoI) definition [5]. It is argued that a quantitative approach is essential for effective SoS Risk Management, due to the inherent complexity of this process and that this can only be achieved through the implementation of appropriate models.

A model-based approach to SoS Risk Management is introduced; allowing heterogeneous models to be integrated into a central risk network and ensuring appropriate modeling techniques are selected [6].

\section{Risk}

\subsection{What is Risk?}

The ISO Guide relating to risk management vocabulary [7] defines risk simply as;

"the effect of uncertainty on objectives".

Where "effect" is defined as, "a deviation from the expected - positive and/or negative" and "uncertainty" is defined as,

"the state, even partial, of deficiency of information related to understanding or knowledge of an event, its consequence, or likelihood".

Whilst this definition may capture current practice, whereby risks are often identified on a subjective basis, often without quantification, it also highlights a common pitfall: the confusion of risk and uncertainty. A key point that this definition does raise is that risk encompasses both detrimental and beneficial effects [21] 
However, the DoD definition [8] does link risk to probability (or likelihood), thereby clearly distinguishing risk from uncertainty, identifying three components:

- A future root cause (yet to happen), which, if eliminated or corrected, would prevent a potential consequence from occurring

- A probability (or likelihood) assessed at the present time of that future root cause occurring, and

- The consequence (or effect) of that future occurrence."

\subsection{Perception of Risk}

Risk is frequently determined as a subjective estimate of likelihood utilising experience of an individual or team, which is subject to intuitive biases and emotion. Slovic and Peters [9] introduced the concept of the "affect heuristic", which suggests that the assessment of risk is related to the perceived "goodness" or "badness" of an activity. It is further hypothesised that the positive or negative correlation between risk and benefit is dependant upon the state of the affect heuristic. Hence, if feelings towards an activity are negative, the benefits are perceived to be low and risk high, whereas a positive feeling will elicit a perception of high benefit and low risk. The implication of this is that risk is often judged on an emotional basis as well as a purely rational one and that the same risk will not always be consistently assessed.

The "conspiracy of optimism" [10] is another example of the perception of risk influenced by extraneous factors. In this case the likelihood or impact of a risk may be In this case the likelihood or impact of a risk may be pressures. Although the term "conspiracy" implies a deliberate deception, the underestimation of risk arises more through the subconscious awareness of these pressures; for example the assumptions made when determining a project schedule may be biased in favour of the desired outcome.

\subsection{Types of Risk}

The INCOSE SE Guide [11] identifies the following set of risk categories: Technical, Programmatic, Schedule and Cost. Whilst these are valid within the Systems Engineering domain, when considering SoS risk they are less applicable. Consider a Search and Rescue scenario: a SoS will be formed rapidly to fulfill a specific aim, possibly including some ad-hoc component systems, e.g. shipping in the vicinity of a casualty. In this case only the Technical risk category and to some extent Schedule risk apply. It is therefore concluded that SoSE required a new approach regarding the classification of risk.
Hopkin [4] takes a more holistic view of risk and suggests the following broad categories, which appear to be more appropriate to SoS:

- Hazard Risks (e.g. Threat and perils)

- Control Risks (e.g. Doubt about mission achievement, project management control)

- Opportunity Risks (Opportunity to enhance or inhibit mission achievement)

In order to relate these broad categories to more specific SoS attributes, it is suggested the SoS System of Interest model ( $\mathrm{SoI}$ ) [5], as shown in Figure 1 below, is used to identify risk types through the consideration of SoS 'dimensions', which can then be considered against the holistic classification. [4]. The SoS SoI model is intended to identify all aspects (dimensions) of a SoS that must be considered in order to determine the SoI, it also classifies the relationships between these dimensions.

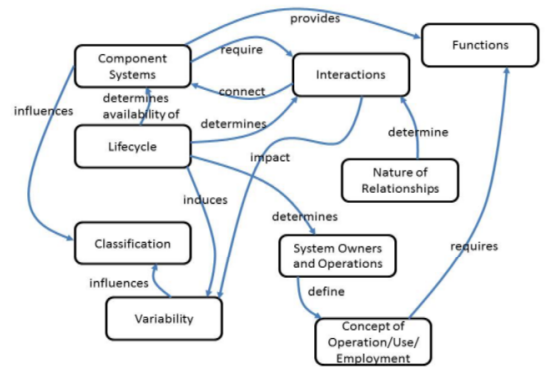

Figure 1 SoS System of Interest Dimensions [5]

\section{Risk Management}

\subsection{System Risk Management}

The Risk management ISO 31000 standard [7] outlines the following activities in the Risk Management process:

- Communication and Consultation

- Establish the context

- Risk identification

- Risk analysis

- Risk evaluation

- Risk treatment

- Monitoring and Review

These activities should be performed on an iterative basis, with risks continuously identified and managed. This process can be generically applied, however the implementation of each activity differs dramatically between disciplines, e.g. engineering, finance, insurance, or indeed within disciplines themselves. This paper is 
primarily concerned with the Risk analysis and evaluation stages, where both qualitative and quantitative approaches are taken. When developing systems, risks are often analysed and assessed through subjective scoring techniques, rather than rigorous and scientific techniques, which as Hubbard [12] comments, are still not widespread. This results in the use of subjective (as opposed to objective) probabilities when engineering systems [1]. However, the requirement for more rigorous techniques is dependent upon the complexity of the system and risk context under consideration. Statistical modelling approaches typically used for the quantification of risk include Monte Carlo simulation, Bayesian Belief Networks, Markov analysis and Decision trees [13]

\subsection{System of Systems Risk Management}

Although the DoD SoSE guide [14] is somewhat acquisition focussed, it does succinctly describe the subtle distinction between system and SoS risk management;

"Risk management for a SoS begins with the identification of SoS objectives and the identification of the risks that threaten the achievement of those objectives. While it is true that minor individual program risks could be major risks to the SoS, it is also true that significant system risks may have little or no impact on the SoS functionality. Furthermore there may be risk to a set of SoS objectives which are not risks to the constituent systems (e.g., unwanted emergent behaviour, infrastructure, integration risks, cost risk)."

A common perception is that if all SoS component system risks are managed, then any SoS risks will be implicitly managed without further intervention. The quote above demonstrates that SoS risk may be decoupled from individual system risk and should be managed separately.

SoS risk management requires a holistic approach, rather than bottom up and at the component system level. By definition, a SoS will exhibit a high level of complexity, it is therefore argued that the subjective risk management approaches, discussed earlier, are not generally suitable and that a more quantitative approach is required.

Another critical factor differentiating SoS Risk Management is the timeliness associated with the process. Whereas a 'traditional' system will developed over an extended period of time, typically months or years, a SoS may be formed rapidly in response to an urgent need, such as disaster relief. In this case risk management may be required to become a near real-time, decision support activity. Indeed, Haimes [15] states, "Assessing and managing risk should be a part of the decision-making process within the lifecycle of SoS" and Aitken et al [16] make the case for dynamic risk assessment in Network Enabled SoS

\section{A Proposed Model Based Approach to System of Systems Risk Management}

\subsection{Why a Model Based Approach?}

As discussed above, a SoS is inherently complex. Currently, risks identified in the engineering of systems are typically quantified through, subjective expert opinion, which will be derived from a mental model of the problem. Research has demonstrated that human processing of problems involving four variables "showed a significant decline in accuracy and speed of solution" from those involving three variables, whilst, problem solving involving five variables was at "chance level." [17]. When considering risks relating to a SoS, many variables will need to taken into account and certainly more than five! Emotional response and bias, regarding the perception of risk, are also factors, which may impair the objective risk, are also factors, which may impair

It is therefore proposed that, in order to provide, rigorous and trustworthy quantified SoS risk assessment, a modelling approach must be taken, removing the reliance on subjective judgement and mental models of complex causal relationships between risk factors.

However, it must be acknowledged that, as Box famously stated; "all models are wrong, but some are useful" [18]. Models are abstractions and simplifications intended to answer specific questions and their output should be questioned and compared against available historical data where possible, allowing them to be refined over time. Over reliance on poorly tested models, based on false assumptions, providing the illusion of a sophisticated risk management method is described as the "worst" case by Hubbard [12], even more so than qualitative, "soft" methods. However, he deems the "best" case to be the use of proven, quantitative models.

\subsection{The System of Systems Risk Model}

A modelling approach has been developed to reflect the holistic nature of SoS Risk, which allows the interaction of risks to be modelled and enables the integration of heterogeneous modelling techniques, ensuring the use of methods appropriate to individual risk characteristics, as opposed to a 'one size fits all' approach.

However, if risk models are to provide quantified risk, i.e. objective probability values, then the decision makers reliant upon it must trust this information. Confidence values associated with the objective risk values are deemed an appropriate mechanism to achieve this, a low confidence output will suggest that the result should be questioned and an alternative assessment sought, if possible. 


\subsubsection{Risk Identification}

Risk assessment should be initially undertaken using a more traditional approach involving subject matter expert input. Appropriate modelling techniques may then be exploited in order to reveal emergent behaviour and identify associated 'emergent' risks. To enable the selection of appropriate supporting model methods, Kinder et al [6] propose a framework for selecting methods in the context of SoS modeling. The purpose of the model is linked to the relevant SoS SoI dimension(s) [5], the requirement for dynamic modeling assessed and an appropriate set modeling techniques output. Potential methods identified [6], include, but are not limited to: Discrete Event Simulation, Agent-Based Modelling and Simulation, System Dynamics, Bayesian Belief Networks, Bayesian Belief Networks and Decision Trees.

\subsubsection{Central Bayesian Model}

To enable the dependency between risks and contributing factors throughout a SoS to be modelled it is proposed that these are represented using a Bayesian Belief Network (BBN).

A BBN is a graphical representation of the probabilistic relationships between a set of random variables using a directed acyclic graph [19]. A simple example is shown below in Figure 2, which shows how the probability of the weather state affects the probability of a sprinkler being on or off and, in turn, the probability of a lawn being either wet or dry.

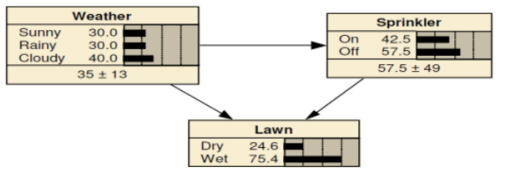

Figure 2 - Bayesian Belief Network example

A network consists of a set of interconnected nodes, each interconnection represents the causal relationship between nodes, and with each node having multiple states. Each state is allocated a probability, which indicates the likelihood of that state occurring in relation to the states of the connected nodes. Each node may represent an attribute of a risk. The probabilities within the BBN may be populated using historical data and updated with new information, if available. If historical data does not exist the probabilities may be determined subjectively, enabling risks to be assessed where gaps in data are present, a situation, which is often faced by risk analysts. Although this is an advantage of BBN's, this subjectivity can also be viewed as a disadvantage resulting in an apparently quantitative assessment of risk being based on a qualitative source; precisely what Hubbard [12] identified as the worst-case risk management approach.
$\mathrm{BBNs}$ are identified in the Risk Management Assessment Techniques ISO standard [13] as a suitable approach to analyse causal relationships in the context of risk and to determine the effect of intervention, although the description of their use is limited to a generic description of a BBN. It is also stated that BBNs are currently used in "medical diagnosis, image modelling, genetics, speech recognition, economics, space exploration and in web search engines".

\subsubsection{Monte Carlo Simulation}

In order to address the issue highlighted above, regarding the subjectivity associated with $\mathrm{BBNs}$, it is proposed a Monte Carlo approach will be utilised. Monte Carlo Simulations will generate probability distributions relating to the finite set of states for specific nodes within the BBN, which can then be "learnt" (i.e. imported) by these nodes thereby updating their internal probability tables.

Monte Carlo simulation is perhaps the most widely used technique in risk management; the model contains random elements and is run iteratively (e.g. 10,000 cycles) thereby generating a spread of output values, e.g. the accuracy of a sensor within a model may be defined as a Gaussian distribution over the specified error range. This is Gaussian distribution over the specified error range. This is
an extremely powerful, practical technique allowing uncertainty within complex systems to be modelled realistically.

\subsubsection{Supporting Models}

The Monte Carlo approach, described above, permits heterogeneous models to be executed, providing data to be learnt by appropriate nodes in the BBN. This enables modeling techniques to be selected, which provides the most suitable method for modeling a particular factor contributing to a SoS risk, rather than being artificially constrained to a single method. Haimes [15] reinforces this view when considering complex systems and SoS, stating that "Clearly, no single model can ever attempt to capture the essence of such systems - their multiple dimensions and perspectives". Figure 3 visualizes the generic framework for the proposed modeling approach, showing how supporting models, of any suitable technique, may be run within a Monte Carlo simulation, the output of which will allow a central BBN to 'learn' from the results. $\Lambda$ suitable modeling approach may be selected as discussed in 4.2.1.

\section{Case Study Evaluation}

A single case study has been initially selected in order to demonstrate the applicability of the proposed model based SoS Risk Management approach. This initial case study is related to Close Air Support and is using the proposed approach to develop a Risk Management tool, which is intended to support planning activities. 


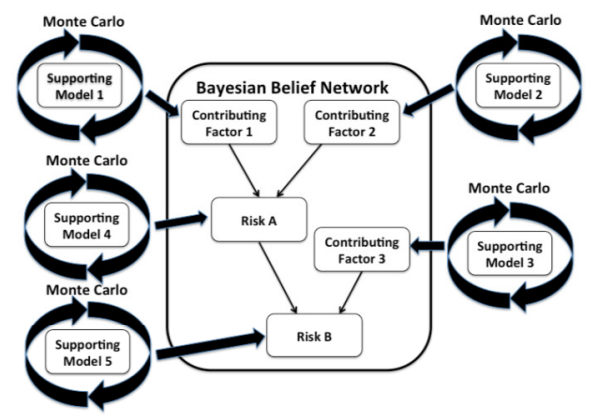

Figure 3 - SoS Risk Management Modelling Framework

\subsection{Close Air Support Risk Management} Support Tool

The NATO publication; Tactics, Techniques and Procedures for Close Air Support Operations [20] defines Close Air Support (CAS) as;

“..air action against hostile targets which are in close proximity to friendly forces and requires detailed integration of each air mission with the fire and movement of those forces."

CAS missions are differentiated between 'immediate' and 'pre-planned'. An immediate CAS mission will require an urgent response in a situation where it is probable troops are under fire. The case study is focussed upon pre-planned missions, that fit into the typical planning cycle (usually in the order of 24 hours), where more time is available for planning and the suitability of platforms and munitions may be tailored to match the mission requirements. A CAS mission will comprise multiple elements, including troops on the ground requiring support, a forward air controller who guides the aircraft into position, tactical headquarters co-ordinating operations, a command and control aircraft controlling smaller fighter aircraft which will all communicate via radio (voice), tactical data link or satellite. These component systems comprise an overall SoS.

Using subject matter input and enterprise architecture modelling, the operational requirement for the CAS Risk Management tool was defined as:

To model and quantify the risks associated with the CAS mission, including; fratricide, failure to eliminate the threat, collateral damage and loss of weapon delivery aircraft.

The tool is currently under development; at this stage an initial, comprehensive BBN has been defined, using the Netica application, which is intended to capture the primary risks and the numerous contributing factors. Figure 4 illustrates a simplified segment of the BBN, illustrating some factors, which impact the risk of Fratricide.

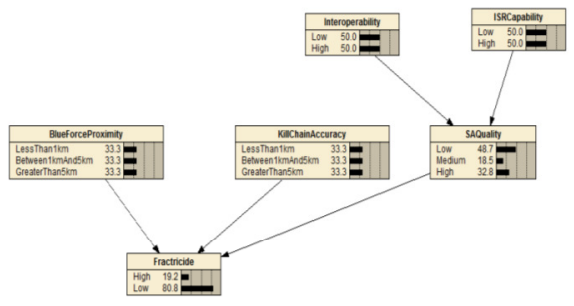

Figure 4 - Close Air Support BBN Segment

Design and implementation of appropriate supporting models is at an early stage of development. Figure 5 shows an initial, basic Simulink model, which determines kill chain accuracy, derived from sensor accuracy and communication link error propagation. Each module in the Simulink model introduces an error into the positional target data, either through sensor error, co-ordinate conversion, data link message resolution or latency.

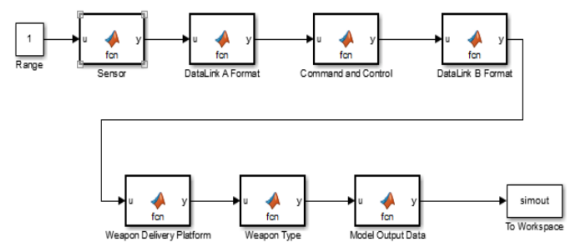

Figure 5 - Basic Kill Chain Model

The Simulink model is run from a MATLAB script, allowing multiple iterations to be executed, the output consists of a single multistate variable, which is written into a text file, the format of which is compatible with the Netica application. This data is then 'learnt' by the BBN, the change in probability values pre and post learning is illustrated in Figure 6, demonstrating the change in probability values following import of the simulation results. This describes just one supporting model; others will he implemented, using appropriate techniques, as the tool is developed.

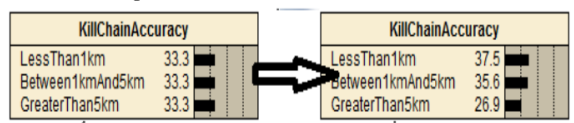

Figure 6 - Node probabilities, pre and post learning

This output demonstrates that the proposed modeling concept does allow BBN nodes, representing risks and their contributing factors, to be updated using data generated from appropriate models with a Monte Carlo simulation. 


\section{Conclusions}

This paper has discussed the approaches required for 'traditional' System and SoS Risk Management and identified key differences. Whilst Risk Management in the engineering of systems tends to use qualitative techniques providing subjective probabilities, it is argued that, due to the SoS inherent complexity, quantitative methods must be adopted. The management of SoS risk must be holistic and not assume that if risks are managed at the system level then SoS risk will be managed implicitly.

A model-based approach is outlined, utilizing a central BBN to represent risks and contributing factors. To enable the probabilities within the BBN to be populated objectively, rather than subjectively, the use of supporting models is proposed, which are run using a Monte Carlo approach, thereby generating results, which may be 'learnt' by the BBN.

A Close Air Support Risk Management tool is described, which utilizes the proposed approach. The tool is currently in the early stages of development, therefore this paper focuses on the conceptual modeling approach rather than describing and analysing the tool output. Additional heterogeneous supporting models will be implemented which will further demonstrate the general modeling concept.

Further research will identify additional case studies, which will be selected in order to demonstrate the generic applicability of the proposed approach.

\section{References}

[1] Garvey, Paul R. "Analytical methods for risk management: A systems engineering perspective," CRC Press, 2008.

[2] Pinto, C. Ariel, Michael K. McShane, and Ipek Bozkurt. "System of systems perspective on risk: towards a unified concept," International Journal of System of Systems Engineering 3.1, 33-46, 2012

[3] Conrow, Edmund H. "Risk management for systems of systems." CrossTalk 18.2, 2005

[4] Hopkin, P Holistic Risk Management in Practice, Witherby \& Co Ltd, 2002

[5] Kinder, A.; Barot, V.; Henshaw, M.; Siemieniuch, C. "System of Systems: "Defining the system of interest", System of Systems Engineering (SoSE), 2012 7th International Conference on, vol., no., pp.463, 468, 16-19 July 2012

[6] Kinder, A.; Henshaw, M.; Siemieniuch, C., System of systems modelling and simulation - an outlook and open issues, International Journal of System of Systems Engineering, Vol 5, Issue 2, pp 150-192, September, 2014

[7] ISO 31000:2009 Risk management -- Principles and guidelines

[8] Department of Defense, Risk Management Guide for DoD Acquisition, Sixth Edition, Version 1.0, 2006

[9] Slovic, P., Finucane, M. L., Peters, E., \& MacGregor, D. G. The affect heuristic. European Journal of Operational Research, 177(3), 1333-1352, 2012

[10] Lyvbjerg, Bent, Massimo Garbuio, and Dan Lovallo "Delusion and deception in large infrastructure projects: two models for explaining and preventing executive disaster." California Management Review, vol. 51, no. 2 Winter, pp. 170-193, 2009

[11] INCOSE Systems Engineering Handbook v. 3.2.1, January 2011

[12] Hubbard, Douglas W. The failure of risk management: why it's broken and how to fix it. John Wiley and Sons, 2009.

[13] ISO 31010:2009 Risk management -- Risk assessment techniques

[14] DoD. Systems Engineering Guide for Systems of Systems, version 1.0. Washington, DC, USA: US Department of Defense (DoD) 2008.

[15] Haimes, Yacov Y. "Modeling complex systems of systems with phantom system models." Systems Engineering 15.3: 333-346, 2012

[16] Aitken, Jonathan M., Robert Alexander, and Tim Kelly. "A case for dynamic risk assessment in NEC systems of systems." System of Systems Engineering (SoSE), 2010 5th International Conference on. IEEE, 2010

[17] Halford, Graeme S., et al. "How many variables can humans process?" Psychological science 16.1: 70-76, 2005

[18] Box, G. E. P., and Draper, N. R., Empirical Mode Building and Response Surfaces, John Wiley \& Sons, New York, NY, 1987

[19] Charniak, E. 'Bayesian networks without tears', AI Magazine, 1991

[20] NATO publication; Tactics, Techniques and Procedures for Close Air Support Operations, NATO, 2005

[21] White, Brian E. "Enterprise opportunity and risk." Chapter 5 in Enterprise systems engineering: Advances in the theory and practice. CRC Press, 2010 

Annex D: System of Interest for System of Systems Dimensions: Workshop Report 
System of Interest for System of Systems Dimensions: Workshop Report

Held 22 February 2012 


\section{Contents}

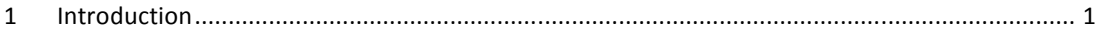

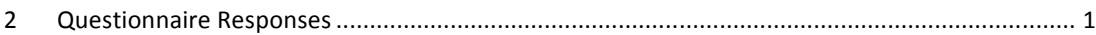

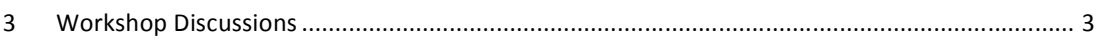

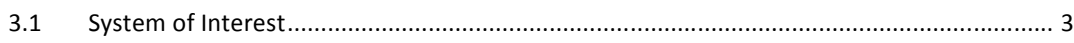

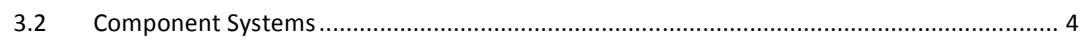

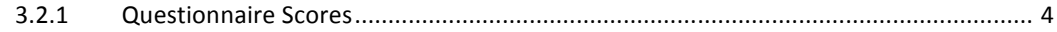

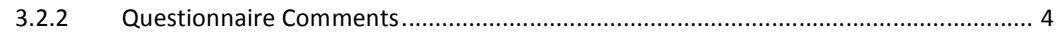

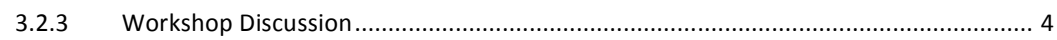

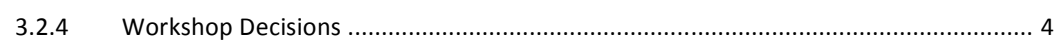

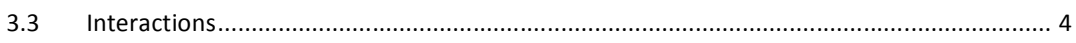

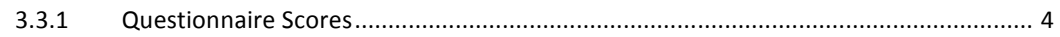

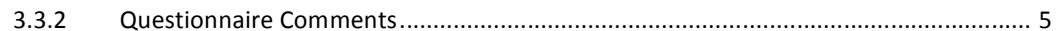

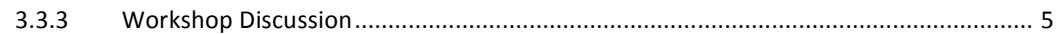

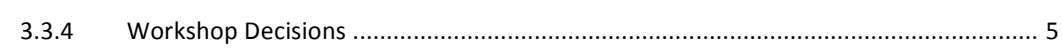

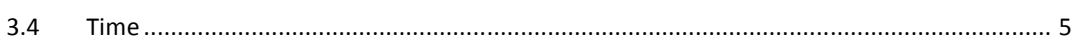

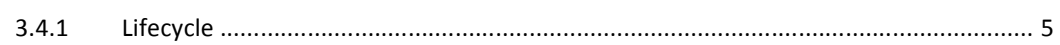

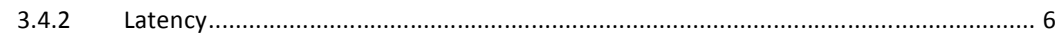

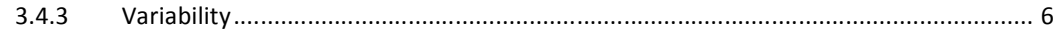

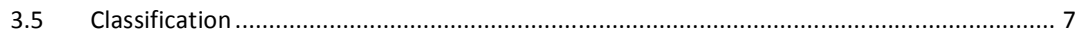

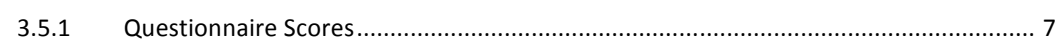

3.5.2 Questionnaire Comments ...................................................................................... 7

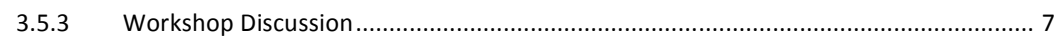

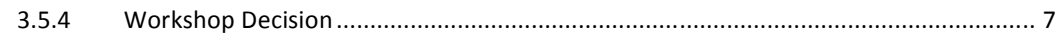

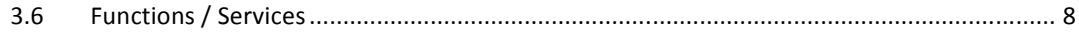

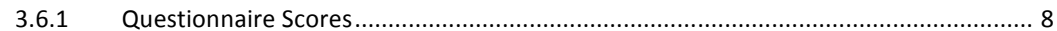

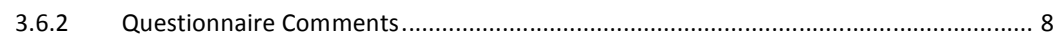

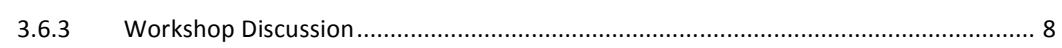

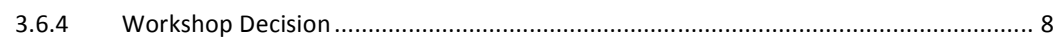

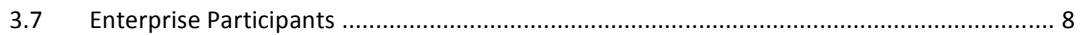

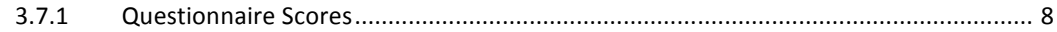

3.7.2 Questionnaire Comments ..................................................................................... 9

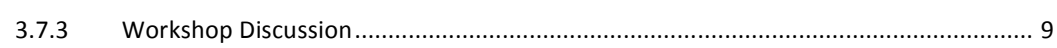




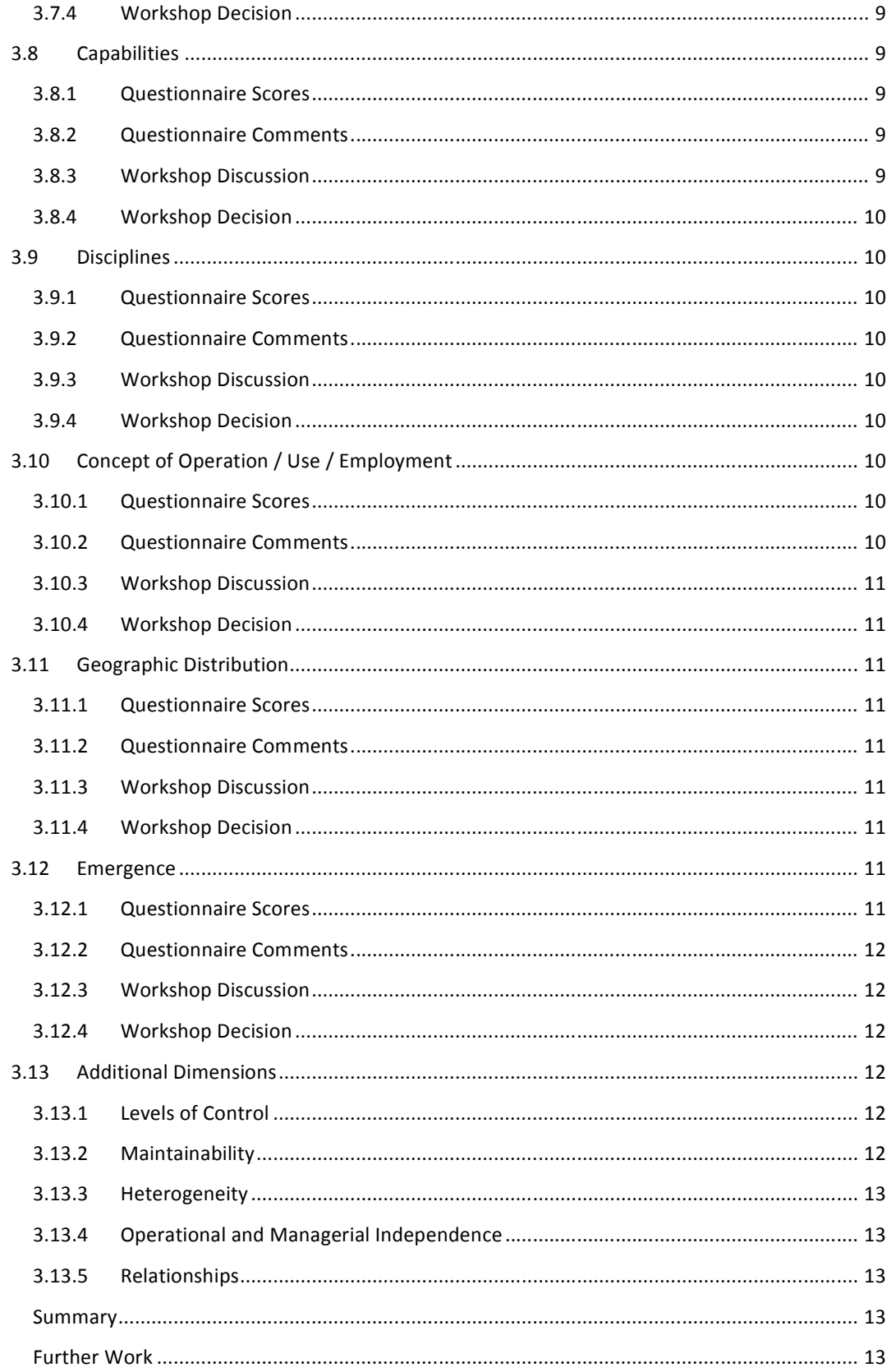




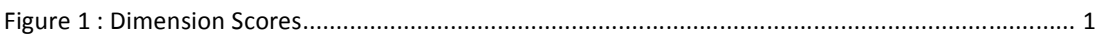

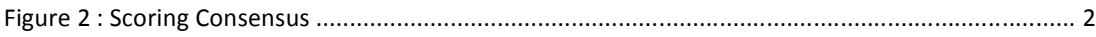

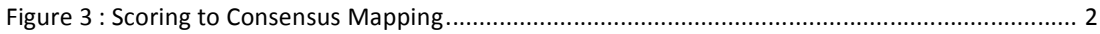

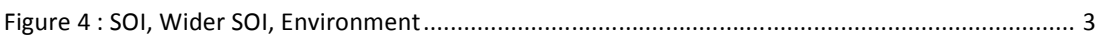

Figure 5 : Component Systems Scores .................................................................................... 4

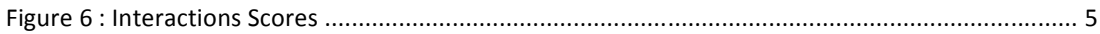

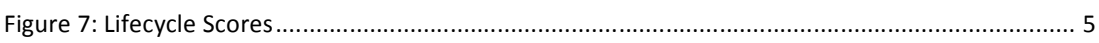

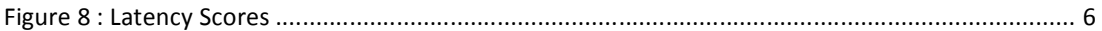

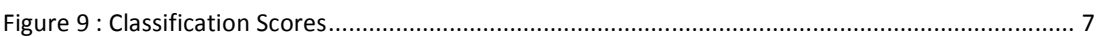

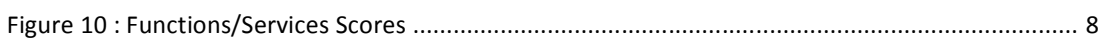

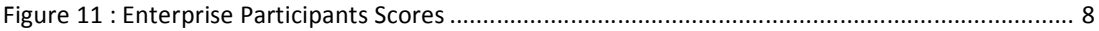

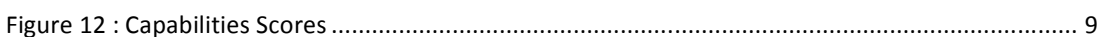

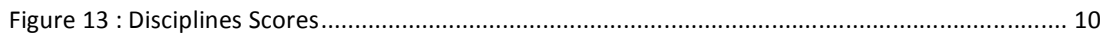

Figure 14 : Concept of Operation / Use / Employment ..................................................................... 10

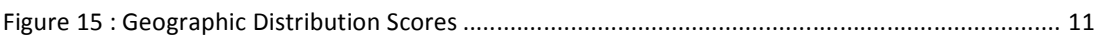

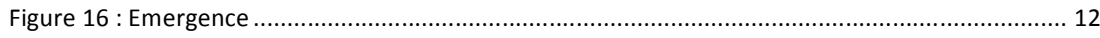

1 


\section{Introduction}

The System of Interest (SOI) of a SoS and its dimensions were first discussed at the ESoS Academic Retreat in 2011. This workshop was held in order to discuss and progress these initial SOI dimensions and definitions. In this report I have analysed the voting from the questionnaires, compiled all comments received and recorded the results of the discussions from the workshop.

As explained in the questionnaire, I intend to use the SOI dimensions as an aid to scope my own research into SoS Modelling and Simulation (M\&S). However it is hoped their definition will assist the ESoS group as a whole.

Thank you, for the responses relating to SoS M\&S. As expected, within the time constraints of the workshop, we barely had time to discuss each dimension. So, although we did not discuss SoS M\&S directly the information you provided is still of great use.

\section{Questionnaire Responses}

A high proportion ${ }^{1}$ of the questionnaires were completed, which was very much appreciated. I have analysed the results by, firstly, just summing the response ratings and, secondly, looking at the variance of the responses for each dimension. The plot of variance is intended to show consensus amongst the responses, i.e. the lower the variance the greater the consensus.

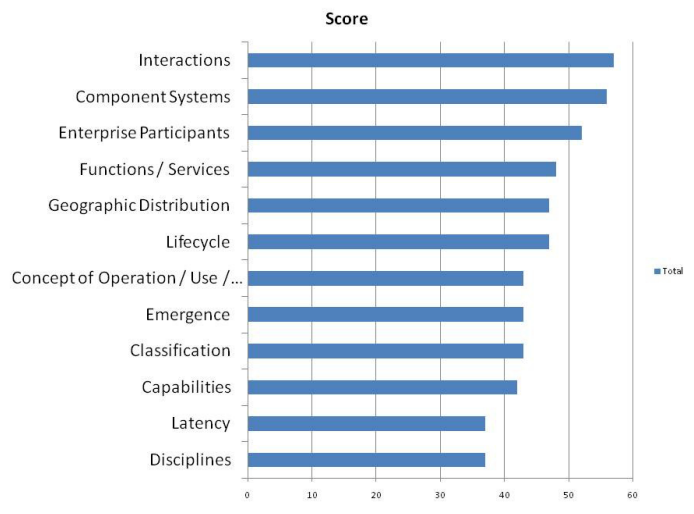

Figure 1 : Dimension Scores

${ }^{1} 75 \%$ of the questionnaires were completed. 


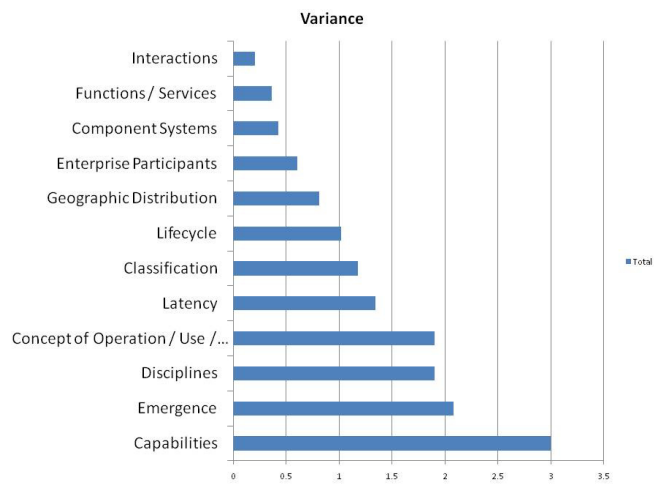

Figure 2 : Scoring Consensus

Below, I have shown the relationship between the overall score of a dimension and the consensus. Perhaps predictably, the most 'popular' dimensions exhibit a higher level of consensus.

\section{Score}

Consensus

- Interactions

- Component Systems

- Enterprise Participants

- Functions / Services

- Geographic Distribution

- Lifecycle

- Concept of Operation

- Classification

- Emergence

- Capabilities

- Latency

- Disciplines $\rightarrow$ Interactions

- Functions / Services

Component Systems

- Enterprise Participants

$\longrightarrow$ Geographic Distribution

$\rightarrow$ Lifecycle

- $\rightarrow$ Classification

- Latency

- Concept of Operation

Disciplines

Emergence

- Capabilities

Figure 3 : Scoring to Consensus Mapping 


\section{Workshop Discussions}

The workshop attendees were;

- Professor Michael Henshaw

- Luminita Ciocoiu

- Huazhong Fu

- Sharon Henson

- Allan Hodgson

- Andy Kinder

\subsection{System of Interest}

The workshop began by considering the meaning of the SOI in the context of a SoS. It was agreed that the SOI of a SoS encloses everything that contributes to the SoS. This was then refined to, everything that contributes to the SoS emergent behaviour. The key is to determine the relevant part of the SoS as opposed to the environmental factors.

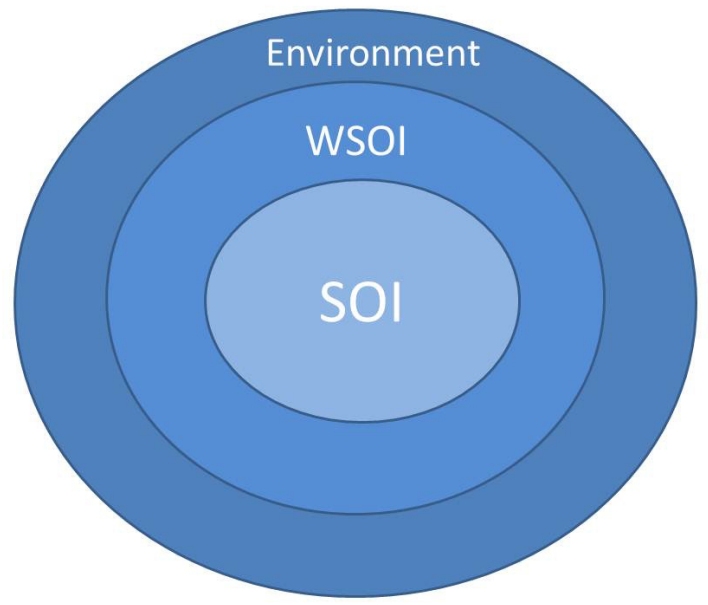

Figure 4 : SOI, Wider SOI, Environment

A SoS example was suggested, allowing the elements and dimension to be visualized.

Purpose is to transport entities between London and Bristol.

- Locomotion (trains)

- Containers (trucks, carriages)

- Mechanism (rail)

- $\quad$ Control (signals)

- Also ticketing and timetabling. 


\subsection{Component Systems}

3.2.1 Questionnaire Scores ${ }^{2}$

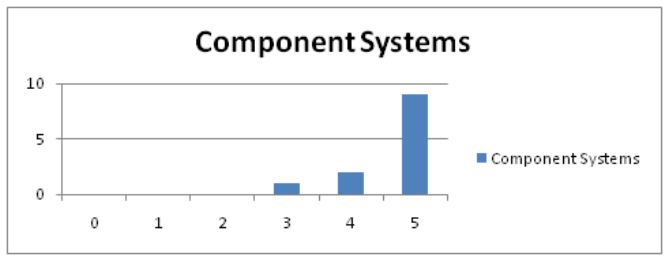

Figure 5 : Component Systems Scores

3.2.2 Questionnaire Comments

- You need to know what systems are within the SoS (as far as you can determine)

- Do not see how you can bound your Sol if you cannot identify the component systems within your Sol boundary and those with which your Sol will have key interactions or dependencies in the SoS environment

- Considering the hierarchy of SoS-Systems-Subsystems, do component systems encapsulate Systems and also Subsystems?

- May need to think about groups vs. components/subsystems.

- Defines what is actually in the systems of interest; but the representation of component systems could be done at the actual system level or the class/type of system.

\subsubsection{Workshop Discussion}

By definition, all the component systems will only be known for a SoS classified as directed ${ }^{3}$ and to some extent acknowledged. In addition to specific systems it will be necessary to identify types of component systems, enabling this dimension to be determined for a collaborative or virtual SoS. However, further work is required to define these types of systems. We should attempt use established definitions.

It was agreed that this is a key dimension, which is in line with the questionnaire responses.

A link was identified between the level of abstraction of the dimensions and the phase in the SoS lifecycle.
3.2.4 Workshop Decisions
- Keep this dimension.
- Establish Component System type definitions.

\subsection{Interactions}

\subsubsection{Questionnaire Scores}

\footnotetext{
${ }^{2} \mathrm{Y}$ axis $=$ total score, $\mathrm{x}$ axis $=$ likert scale numerical value

${ }^{3}$ Although the SoS classification, Virtual, Collaborative, Acknowledged and Directed is referred the workshop agreed that this definition is inadequate and required further refinement.
} 


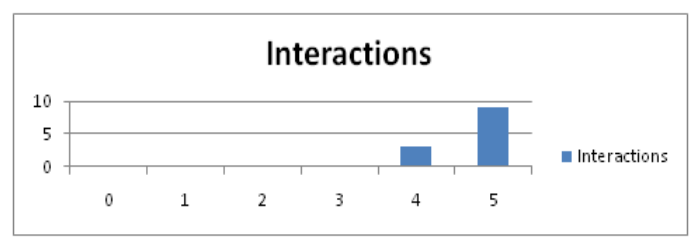

Figure 6 : Interactions Scores

\subsubsection{Questionnaire Comments}

- Need to know both the interfaces, and the behaviour at the interfaces, when the systems interact

- Is there an association between interactions and also interoperability i.e. NCOIC framework.

- Need to consider close/loose coupling, where decisions are made, control, overriding, etc.

- An SoS exists only because of interactions between constituent systems, so the interactions must provide some definition of the SOI. As above, these could be at the individual interaction level or at the class/type of interaction level.

- Maybe you can separate them in between and within component system interactions

\subsubsection{Workshop Discussion}

The availability of interactions is important and related to performance and agility. The type of interactions also needs to be defined. There was some argument regarding the "order" of populating the dimensions of an SoS, i.e. do the component systems (types) need to be established before Interactions are defined, or vice-versa. An aid to establishing this "order" could be helped by the creation of a system dynamics model showing the relationships between the dimensions.

\subsubsection{Workshop Decisions \\ - Keep this dimension. \\ - Define Interaction Types.}

\subsection{Time}

\subsubsection{Lifecycle}

\subsubsection{Questionnaire Scores}

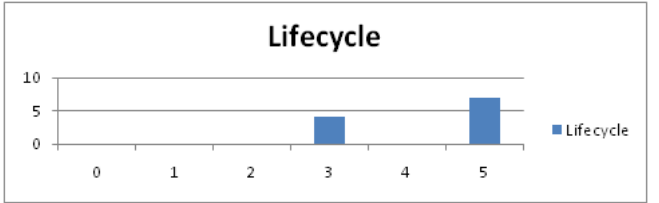

Figure 7: Lifecycle Scores

\subsubsection{Questionnaire Comments}

- Time is an important concept for an SoS (eg lifecycle phase, required period of operation, time available for configuration etc) but it is not a constant across all types of SoS

- Lifecycle of SoS - how much different is this from other lifecycles e.g. CADMID? 
- The SOI at a specific instant in time depends on what Systems are currently contributing. Frequency of change/replenishment of systems within the SoS could be a useful measure (slowly or quickly changing). Life phases, rather than cycle, could be relevant to indicate current status of the SoS which is related to SOI

- Maybe more important for a whole system. For example, if your SOI includes a whole subsystem then the importance of the dimension may be greater than if the SOI includes a part of a subsystem.

\subsubsection{Workshop Discussion}

The respective timelines of the component systems needs to be considered, i.e. dynamism. It was also established that there is link between the point in the lifecycle and the ability to populate a dimension. E.g, as stated earlier, until the construction phase of an SoS the actual Component Systems may not be known but the types should be. It was argued that the lifecycle is an overarching dimension, affecting all other dimensions; the SOI effectively becomes 3-dimensional. Again, the lifecycle phases need to be defined.

\subsubsection{Workshop Decisions}

- Keep this dimension.

\subsubsection{Latency}

3.4.2.1 Questionnaire Scores

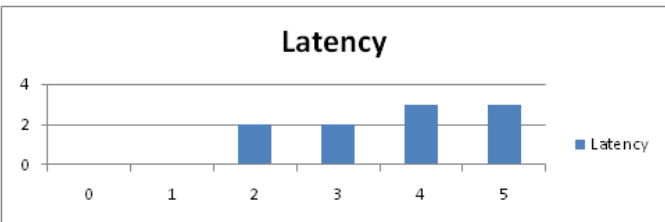

Figure 8 : Latency Scores

3.4.2.2 Questionnaire Comments

None.

3.4.2.3 Workshop Discussion

It was agreed latency is covered by interactions so can be discarded.

\subsubsection{Workshop Decision}

- Discard this dimension.

3.4.3 Variability

3.4.3.1 Questionnaire Scores

$\mathrm{N} / \mathrm{A}$ - this dimension was an additional suggestion.

\subsubsection{Questionnaire Comments}

- A SoS may become unstable with large variations in event times. Can't remember the paper that discusses this issue.

\subsubsection{Workshop Discussion}


Rate of change of systems can be high, engineering techniques required in design to handle this.

The frequency of change of an SoS and also the rate of change need to be considered. This is linked to stability and also agility. i.e. variability, can be related to time in the sense that we might be interested in some sort of characteristic frequency representing change in the SoS

Stability was linked to the following behaviour of the SOI;

- $\quad$ Stable over time

- Unstable, rapidly grows

- Boundary changes

3.4.3.4 Workshop Decision

- Add this dimension.

\subsection{Classification}

\subsubsection{Questionnaire Scores}

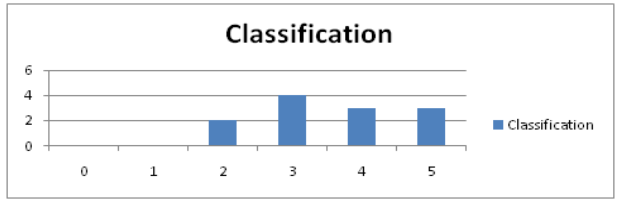

Figure 9 : Classification Scores

3.5.2 Questionnaire Comments

- DoD 4 cases somewhat irrelevant - most SoS are a combination of these classes.

- I get the feeling that some kind of classification system is important as I think there are likely similarities and differences that could be identified as pertaining to one group or anotherbut not sure if the current classification is fit for purpose - maybe we need a multidimensional one

- The four classifications from the literature - need clear example to have an understanding of their meaning? Are these classifications mutually exclusive?

- But tighter definition of the types is needed

- Although the classification that we have at this moment may not be hugely representative for all types of SoS, the type of system may inform the way someone establishes or represents the SOI.

3.5.3 Workshop Discussion

It was agreed that a single classification cannot define a SoS, it is better represented by an combination and that the published definitions are inadequate and need to be developed.

The classification may be derived from the other dimensions.

If dimensions are given a range then these will indicate classification. A SoS could then be classified by having proportions of directed, acknowledged, etc.

\subsubsection{Workshop Decision}

- Keep this dimension. 
- A single classification alone cannot always categorize a SoS.

\subsection{Functions / Services}

3.6.1 Questionnaire Scores

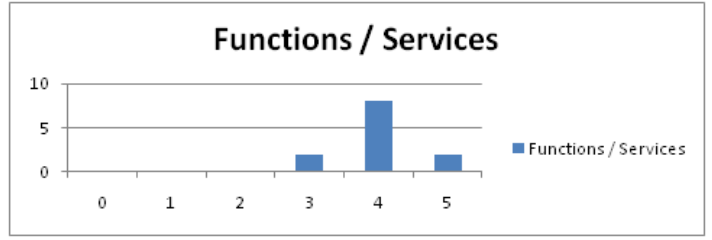

Figure 10 : Functions/Services Scores

3.6.2 Questionnaire Comments

- Not sure what you mean - infrastructural support? Tends to be overlooked in the literature, but vital in practice

- Yes if functions can be grouped or classified and can fit into some kind of overall classification as referred to above. Obviously functionality required and available is critical to configuring and controlling an SoS but is it a key dimension in the same sense as the others

- Does Services already contain functions in addition to engineering and commercial processes? How is service defined in this context? It might be a good idea to separate these two.

- This seems relevant but perhaps less important than others. The services or functions provided by individual systems seems to indicate something about the content of the SOI. In terms of building or analysing a SoS, the question what services should I include in the analysis seems relevant.

- In my opinion, although they are important, functions can go sometimes beyond the SOI.

\subsubsection{Workshop Discussion}

It was decided that it is better to just refer to functions, as services potentially imply Service Oriented Architecture. 'Functions' is a more general term.

\subsubsection{Workshop Decision}

- Keep this dimension but rename it to just 'Functions'.

\subsection{Enterprise Participants}

3.7.1 Questionnaire Scores

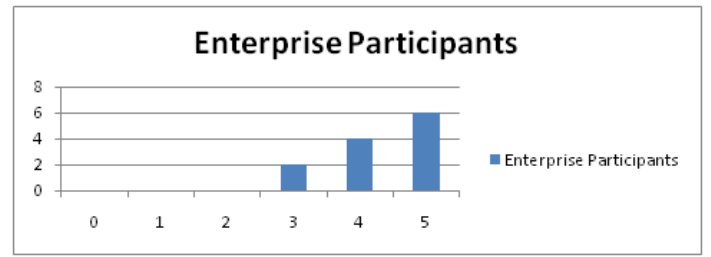

Figure 11 : Enterprise Participants Scores 
3.7.2 Questionnaire Comments

- Determines nature of control in SoS. Note, we don't know how to design the interface between organisations that are a different technology/lifecycle points.

- If you rephrase this to SoS participants then yes - if you mean the SoS environment participants then not so much. But again perhaps we are also looking for some kind of classification of participants such as controllers, users, maintainers etc

- Enterprise and participants may also need to be separated. Enterprise in this context could be the organisations and the supply chain etc. Participants/Stakeholders/Users etc., may need another term and listed apart from the term Enterprise!

- This may have more relevance for some types than others. For instance, for virtual, maybe it is a) impossible to know, b) not much help when you do. However, for collaborative and acknowledged, there is clearly a need to understand participation. Probably also try for Directed, but may be not such a significant question.

- ? Meaning personnel?

\subsubsection{Workshop Discussion}

This is really about organisations and management, it was felt is would be better renamed to Systems Owners and Operations.

\subsubsection{Workshop Decision}

Keep this dimension but rename it to Systems Owners and Operations.

\subsection{Capabilities}

\subsubsection{Questionnaire Scores}

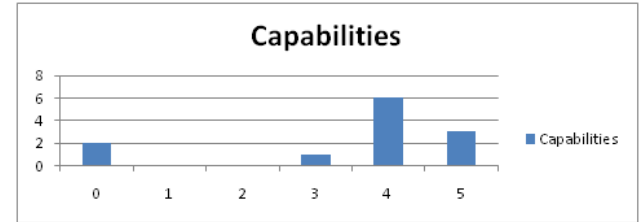

Figure 12 : Capabilities Scores

3.8.2 Questionnaire Comments

- Not as important as interactions - see above

- The aim of SOS is to achieve some of capability

- Same comment as functions until you start looking at differing overall capability depending on the particular configuration of an SoS. Maybe this should tie in with Classification above

- Does capabilities exhibit SoS or vice versa? There are different viewpoints associated to the term Capability. We may need a clear definition! Refer to Michael Henshaw paper on Capability Engineering Perspective Analysis.

- Probably not, though the SOI might be important information for deciding on the SOI for a capability.

- This can be covered by functions and interactions.

- I believe it should belong to another level of dimensions.

\subsubsection{Workshop Discussion}

It was agreed that this is adequately covered by the "Functions" dimension. 
3.8.4 Workshop Decision

Discard this dimension.

\subsection{Disciplines}

3.9.1 Questionnaire Scores

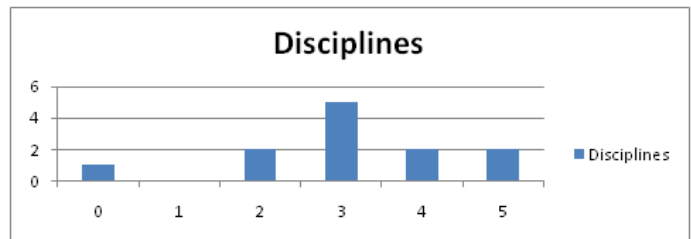

Figure 13 : Disciplines Scores

3.9.2 Questionnaire Comments

- The more that re involved in understanding what's going on, the better.

- Not sure you could capture this in a meaning way that would add anything to functions/services

- Are these role specific disciplines? Disciplines could also be part o the component systems as each system may comprise a number of disciplines?

- May be helpful, but I don't think it defines the SOI.

- This can be covered by component systems, or component of systems.

- I believe it is subordinated to another dimension.

3.9.3 Workshop Discussion

It was agreed, in line with several questionnaire comments, that this is not an applicable dimension, although it is related to the component systems and the overall SoS is does not help define the SOI.

3.9.4 Workshop Decision

Discard this dimension.

3.10 Concept of Operation / Use / Employment

3.10.1 Questionnaire Scores

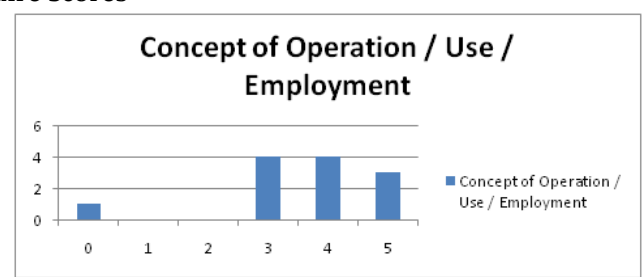

Figure 14 : Concept of Operation / Use / Employment

3.10.2 Questionnaire Comments

- Useful for design; less useful for operations, due to effects of resilience, adaptability over life cycle 
- Yes important to understand how different Conops might impact on the configuration or operation of an SoS

- Are these just processes associated to SoS? CONOPS may also be part of TEPIDOIL or Component of Capability ( $\mathrm{CoC})$ i.e. processes.

- Given that the individual systems can perform operations independently, I think the use/mission of the SoS is critical for defining the SOI for the SoS as opposed to the individual constituent systems.

- This does not belongs to functionality.

3.10.3 Workshop Discussion

It was agreed that this is a valid dimension.

3.10.4 Workshop Decision

Keep this dimension.

\subsection{Geographic Distribution}

3.11.1 Questionnaire Scores

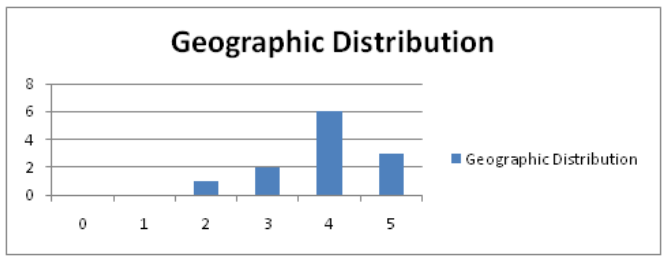

Figure 15 : Geographic Distribution Scores

3.11.2 Questionnaire Comments

- Vital-cultural and legal issues

- Yes if it is pertinent to the operation or (re)configurability of an SoS

- May be more relevant to some situations than others

- Implications for logistics and additional costs.

3.11.3 Workshop Discussion

After a fairly lengthy debate it was agreed that this dimension can be covered by 'Interactions'. It was agreed that cultural and legal issues were important but are more $2^{\text {nd }}$ order issues than direct SOl dimensions.

3.11.4 Workshop Decision

Discard this dimension.

\subsection{Emergence}

\subsubsection{Questionnaire Scores}




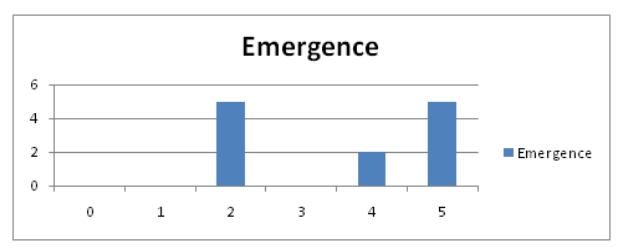

Figure 16 : Emergence

\subsubsection{Questionnaire Comments}

- Critical issue; need to know and metricate this w.r.t. intended emergence (that's why you are putting the SoS together), and highly necessary to estimate the unwanted emergence, so that you can build in resilience, adaptability. Latter will probably be insufficient, due to surprises, but may result in less-costly amelioration efforts.

- This is obviously important but what do we mean here. Are we referring to the potential for emergence (desirable or undesirable), the impact, the likelihood etc. Think we need to be more specific

- As automation increases, unwanted emergent properties will become a big issue due to the loss of smart human 'dampers'. For example, planned automation developments relating to flight control around airports could result in multiple fuel-based disasters as increasing numbers of aircraft are restacked.

- This one is tricky as you can include expected emergence but what about unexpected emergence? If it can be included, wouldn't that be an output from the model/simulation rather than something that goes in?

- Although emergence is an important property, I don't think it defines the SOI.

- Don't know if this would be of any help as by looking at the emergent behaviour of subsystem (looking at intercomponent linkages) you can't predict the emergent behaviour of the whole SoS because of the intracomponent linkages that one my miss.

- This will likely determine the modelling

\subsubsection{Workshop Discussion}

Whilst it was agreed Emergence is a critical issue with regards to a SoS it was decided that is a characteristic rather than a SOI dimension. In terms of modelling, if the dimension were inputs to a model then the emergent behaviour would be the output. It was re-iterated that if the SOI dimensions are fully defined then they will result in the SoS emergent behaviour and the SOI can be regarded as "complete".

\subsubsection{Workshop Decision}

Discard this dimension.

\subsection{Additional Dimensions}

This section includes dimension that were suggested additions to the list originally circulated in the questionnaire.

\subsubsection{Levels of Control}

It was agreed that this is covered by the new 'Relationships' dimension.

3.13.2 Maintainability

This was deemed to be more of a characteristic. 
3.13.3 Heterogeneity

This was deemed to be more of a characteristic and is implied by the component types.

3.13.4 Operational and Managerial Independence

This is covered by other dimensions.

\subsubsection{Relationships}

The workshop decided to introduce a new dimension; 'Relationships', which is distinct from interactions which is more concerned with the inter-connections between systems. This could include types such as 'peer-to-peer'.

\section{Summary}

The list of dimensions agreed by the workshop was;

- Component Systems (including specific and general system types ${ }^{4}$ )

- Interactions (including types)

- Lifecycle

- Variability

- Classification

- Functions

- Systems Owners and Operations

- Concept of Operation / Use / Employment

- Relationships

\section{Further Work}

- Produce system dynamic model of dimension showing dependencies and causal effects.

- Test dimensions against the SoS exemplars (potentially T-area SoS).

- Further elaboration of each dimension definition.

${ }^{4}$ System Types requires definition 